\title{
Simulation for Improvement of Dynamic Path Planning in Autonomous Search and Rescue Robots
}

by

Michael Douglas Hasler, B.E. Hons

\author{
A thesis \\ submitted to the University of Canterbury \\ in partial fulfilment of the \\ requirements for the degree of \\ Master of Engineering \\ in Electrical \& Computer Engineering. \\ University of Canterbury \\ 2009
}





\begin{abstract}
To hasten the process of saving lives after disasters in urban areas, autonomous robots are being looked to for providing mapping, hazard identification and casualty location. These robots need to maximise time in the field without having to recharge and without reducing productivity. This project aims to improve autonomous robot navigation through allowing comparison of algorithms with various weightings, in conjunction with the ability to vary physical parameters of the robot and other factors such as error thresholds/limits.

The lack of a priori terrain data in disaster sites, means that robots have to dynamically create a representation of the terrain from received sensor range-data in order to path plan. To reduce the resources used, the affect of input data on the terrain model is analysed such that some points may be culled. The issues of identifying hazards within these models are considered with respect to the effect on safe navigation.

A modular open-source platform has been created which allows the automated running of experimental trials in conjunction with the implementation and use of other input types, node networks, or algorithms. Varying the terrains, obstacles, initial positions and goals, which a virtual robot is tasked with navigating means that the design, and hence performance, are not tailored to individual situations. Additionally, this demonstrates the variability of scenarios possible. This combination of features allows one to identify the effects of different design decisions, while the use of a game-like graphical interface allows users to readily view and comprehend the scenarios the robot encounters and the paths produced to traverse these environments. The initially planned focus of experimentation lay in testing different algorithms and various weightings, however this was expanded to include different implementations and factors of the input collection, terrain
\end{abstract}


modelling and robot movement. Across a variety of terrain scenarios, the resultant paths and status upon trial completion were analysed and displayed to allow observations to be made.

It was found that the path planning algorithms are of less import than initially believed, with other facets of the robotic system having equally significant roles in producing quality paths through a hazardous environment. For fixed view robots, like the choice used in this simulator, it was found that there were issues of incompatibility with $\mathrm{A}^{*}$ based algorithms, as the algorithm's expected knowledge of the areas in all directions regardless of present orientation, and hence they did not perform as they are intended. It is suggested that the behaviour of such algorithms be modified if they are to be used with fixed view systems, in order to gather sufficient data from the surroundings to operate correctly and find paths in difficult terrains.

A simulation tool such as this, enables the process of design and testing to be completed with greater ease, and if one can restrain the number of parameters varied, then also with more haste. These benefits will make this simulation tool a valuable addition to the field of USAR research. 


\section{Acknowledgements}

Many thanks to Paul Gaynor for providing academic oversight, with guidance in the writing process and encouraging submissions to conferences, as well as help and suggesting ideas when the stress and low-level details overwhelmed the ability to consider the big picture and simpler solutions.

Thanks also go to Professor Dale Carnegie of Victoria University for his help and guidance. His work with robotics and branching into the field of Urban Search and Rescue, being the starting point for the Honours project which preceded this and led subsequently on to this project, to create a tool to aid his research and the research of others.

Additionally the Author would like to extend thanks to postgraduate colleagues and friends, who were there to listen to or discuss frustrating bugs in the code or the search for mathematical techniques and solutions. 


\section{Contents}

Glossary $\quad$ xxi

1 Introduction 1

2 Background $\quad 5$

2.1 Urban Search and Rescue . . . . . . . . . . . . . . 5

2.2 Robot types . . . . . . . . . . . . . . . 7

2.2 .1 Shape shifters . . . . . . . . . . . . . 11

2.2.2 Marsupial teams . . . . . . . . . . . . . 13

2.2.3 Robot swarms . . . . . . . . . . . . . . . . . 14

2.2.4 Confined space exploration . . . . . . . . . . 15

2.3 Analysis of Robot failures . . . . . . . . . . . . 16

2.4 Competitions . . . . . . . . . . . . . . . 16

2.5 Simulators . . . . . . . . . . . . . . . . 21

2.6 Autonomous Navigation . . . . . . . . . . . . 22

2.7 Terrain modelling . . . . . . . . . . . . . . 23

$2.7 .1 \quad \operatorname{LIDAR} \ldots \ldots \ldots \ldots \ldots \ldots$

2.7 .2 Tessellation . . . . . . . . . . . . . 24

2.7 .3 Data culling . . . . . . . . . . . . 26

2.7.4 Simultaneous localisation and mapping . . . . . . . 28

2.8 Path Planning Algorithms . . . . . . . . . . . . . . . . . . 29

$2.8 .1 \quad$ A* Algorithm . . . . . . . . . . . . . . . . 31

$2.8 .2 \quad \mathrm{D}^{*}$ Algorithm . . . . . . . . . . . . 31 
2.8.3 LPA* Algorithm . . . . . . . . . . . . 32

2.8 .4 D* Lite Algorithm . . . . . . . . . . . . . . . 33

2.8.5 Multi-resolution Field D* Algorithm . . . . . . . . . 33

3 Simulator Design $\quad 35$

3.1 Simulator . . . . . . . . . . . . . . . 37

3.2 Modelling LIDAR input . . . . . . . . . . . . 38

3.3 Terrain Modelling and Data Storage . . . . . . . . . . . 40

3.4 Tessellation . . . . . . . . . . . . . . . . . 42

3.4.1 Iterative case-based tessellation . . . . . . . . . . 42

3.4.2 Recursive tessellation . . . . . . . . . . . . . . . . 49

3.4.3 CGAL based tessellation . . . . . . . . . . 51

3.5 Point Culling . . . . . . . . . . . . . . . . 52

3.6 Hazard Identification . . . . . . . . . . . . . . . . 56

3.7 Creation and linking of Node Points/Networks . . . . . . . . . . 58

3.7.1 Centroid based networks . . . . . . . . . . . 61

3.7.2 Border based network . . . . . . . . . . 61

3.7.3 Fixed resolution grids . . . . . . . . . . 63

3.7 .4 Occupancy grids . . . . . . . . . . . . . 63

3.8 Data Objects . . . . . . . . . . . . . . . . . . . . . . . 64

3.8.1 Coords, Triangles \& Edges . . . . . . . . . . . . . . . 64

3.8.2 Points \& Face handles . . . . . . . . . . . . . . 66

3.8 .3 Nodes . . . . . . . . . . . . . . . . . 66

3.9 Path Planning Algorithms . . . . . . . . . . . . . . . 68

3.9.1 Edge Hugger . . . . . . . . . . . . . . . . . . . . 69

3.9 .2 State model . . . . . . . . . . . . . . . . . 69

3.9.3 Breadth First Search and Depth First Search . . . . . . . . 70

3.9 .4 A* Algorithm . . . . . . . . . . . . . 70

3.9.5 LPA* Algorithm . . . . . . . . . . . . . 72

3.9.6 D* and D* Lite Algorithms . . . . . . . . . . 74

3.10 Automation and Configuration . . . . . . . . . . 76 
3.11 Path Analysis . . . . . . . . . . . . . . . . . . 78

4 Experimental Design and Pilot Tests of Path Planning 81

4.1 Hardware . . . . . . . . . . . . . . . . . . . 81

4.2 Variables ...................... 82

4.2 .1 Simulator .................... 82

4.2.2 Algorithms .................. 83

4.2 .3 Robot . . . . . . . . . . . . . . 83

4.2 .4 World . . . . . . . . . . . . . . 83

4.3 Procedure . . . . . . . . . . . . . . . . . 84

4.4 Analysis . . . . . . . . . . . . . . . . . . 84

4.5 Pilot Tests . . . . . . . . . . . . . . . . . 85

4.5.1 $\mathrm{A}^{*}$ algorithm ................ 86

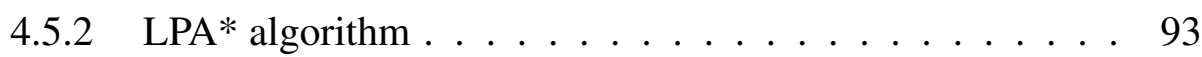

4.5.3 Miscellaneous Configurations . . . . . . . . . . 93

5 Path Planning Results $\quad 95$

5.1 Config_combos 0,1 and $2 \ldots \ldots \ldots 96$

5.2 Algorithms . . . . . . . . . . . . . . . . 96

$5.2 .1 \quad \mathrm{~A}^{*}$ Algorithm . . . . . . . . . . . . . 96

$5.2 .2 \quad$ LPA $^{*}$ Algorithm . . . . . . . . . . . . . . 97

5.2.3 State-based Algorithm . . . . . . . . . . . . 98

5.2.4 Comparison of Algorithms . . . . . . . . . . . . . 99

$5.3 \mathrm{H}$-value weightings . . . . . . . . . . . . . . . 101

5.3.1 A* Algorithm . . . . . . . . . . . . . . . . . 101

5.3.2 LPA* Algorithm . . . . . . . . . . . . . . 105

5.4 A-Series Trial Sets . . . . . . . . . . . . . . . . 107

5.5 Trial Sets D, E, $\mathrm{H} \ldots \ldots \ldots \ldots \ldots$

5.6 Parameter comparisons . . . . . . . . . . . . . . . . . 120

5.6.1 Trial sets A1 vs. C . . . . . . . . . . . . 120

5.7 Noise . . . . . . . . . . . . . . . . . . 122 
6 Discussion $\quad 125$

6.1 Design and Capabilities . . . . . . . . . . . . . . . . 125

6.2 Comparison of Parameters . . . . . . . . . . . . . 127

6.3 Flaws and Bugs . . . . . . . . . . . . . . . . . 127

6.4 Insight . . . . . . . . . . . . . . . . . . . . 128

6.4.1 Config_combos . . . . . . . . . . . . . . 128

6.4.2 Movement/Step size . . . . . . . . . . . . . . . . 129

6.4 .3 View/input . . . . . . . . . . . . . 130

6.4 .4 Hazard identification . . . . . . . . . . . . 132

6.4 .5 Network . . . . . . . . . . . . . . . . 134

6.5 Algorithms . . . . . . . . . . . . . . . 136

7 Conclusion $\quad 137$

7.1 Future work . . . . . . . . . . . . . . . . 138

A Source Code for Simulation System 147

A.1 Algorithms . . . . . . . . . . . . . . . . . . 147

A.2 Algorithms.h . . . . . . . . . . . . . . . 147

A.3 Display . . . . . . . . . . . . . . . . 171

A.3.1 Display.h . . . . . . . . . . . . 171

A.3.2 Display.cpp . . . . . . . . . . . . . . 172

A.4 Hier_Triangulation . . . . . . . . . . . . . . . 177

A.4.1 Hier_Triangulation.h . . . . . . . . . . . . 177

A.4.2 Hier_Triangulation.cpp . . . . . . . . . . . . 181

A.5 Nodes . . . . . . . . . . . . . . . . . . . . . 190

A.5.1 Nodes.h . . . . . . . . . . . . . . . . 190

A.5.2 Nodes.cpp . . . . . . . . . . . . . . . . . 194

A.6 Robot . . . . . . . . . . . . . . . . . . . 215

A.6.1 Robot.h . . . . . . . . . . . . . 215

A.6.2 Robot.cpp................ . . 221

A.7 SimulationEnvironment . . . . . . . . . . . . . . . 233

A.7.1 SimulationEnvironment.h . . . . . . . . 233 
CONTENTS ix

A.7.2 SimEnv.cpp . . . . . . . . . . . . . . . . 240

A.8 World . . . . . . . . . . . . . . . . . 287

A.8.1 World.h . . . . . . . . . . . . . 287

A.8.2 World.cpp . . . . . . . . . . . . . . 342

A.9 adv_math . . . . . . . . . . . . . . . . . . 343

A.9.1 adv_math.h . . . . . . . . . . . . . . . 343

A.9.2 adv_math.cpp . . . . . . . . . . . . . . . 360

B Simulation Results $\quad 387$

B.1 Statistical Data . . . . . . . . . . . . . . . . 387 


\section{List of Figures}

2.1 The OmniTread, an example of an articulated serpentine robot design [4] . . . . . . . . . . . . . . . . . . . . . . . . . . . . . . . 8

2.2 A variety of tracked robot designs. A - Basic tracked setup. B - Fixed form climber. C - Flipper arms climber in low profile shape. D - Flipper arms climber in climb configuration. E - Tank like tracks capable of climbing in either direction of movement.

2.3 A bipedal research robot used to study human motion, viewed from two angles $[5] \ldots \ldots \ldots$

2.4 A multilegged robot, as commercially available, shown in two positions [6]. . . . . . . . . . . . . . . . . 10

2.5 The M-TRAN III, a serpentine shape shifting robot, in a variety of permutations [9]. . . . . . . . . . . . . . . . . 12

2.6 An example of an articulated-pair shape shifting robot design. The Amoeba-II shown in two states [7]. . . . . . . . . . . . . . 13

2.7 Tree diagram categorising areas of failure of mobile robots [12]. . 16

2.8 The three arenas of the NIST competition [14] . . . . . . . . 19

2.9 The three arenas of the MeSci competition [14] . . . . . . . . 20

2.10 Comparison of a terrain model against results from applying data culling techniques [22]. Left: without pre-processing; Centre: with proximity suppression; Right: with coplanar point elimination. 27

2.11 Diagram highlighting the accuracy of measurements within a model but variance from real world locations. . . . . . . . . . . . 30 
2.12 Multi-resolution Field D* produces direct, low-cost paths (in blue/dark gray) through both high-resolution and low-resolution areas [36] . . . . . . . . . . . . . . . . . . . . . . 34

3.1 A UML depiction of the interconnection between objects in the simulation. . . . . . . . . . . . . . 36

3.2 Simulator screenshot depicting Robot approaching terrain containing obstacles. . . . . . . . . . . . . . . 38

3.3 An example tessellation of a cube placed a flat plane, with good triangles shown in yellow while an erroneous triangle is coloured pink. . . . . . . . . . . . . . . . . . 47

3.4 Triangle meshes generated by the CGAL (left) and Iterative (right) based tessellations, given the same series of inputs. . . . . . . . 52

3.5 An example of comparing two new points, A and B, to the existing triangle mesh in order to determine whether to insert the new points or cull them. . . . . . . . . . . . . . 5 54

3.6 Example of Hazard and Map Node creation, showing only a portion of the potential links. The blue triangles being traversable and all others colours being hazards. . . . . . . . . . . . 62

3.7 An example terrain model of a fixed resolution grid, where green nodes are traversable and red ones are not. Given the boundaries of hazard obstacles, shown as grey lines, the arbitrary alignment of the grid with respect to the terrain may produce different models, with the left one having fewer traversable points for the same area of terrain. . . . . . . . . . . . . . . . . . . . 64

3.8 Example of Occupancy Grids in use by a wheelchair collision avoidance system approaching an elderly man with a walking frame [44]. 65

3.9 A hierarchy tree representation of the relationship between the Node objects . . . . . . . . . . . . . . . . . 67 
3.10 The contents of a simulation/environment configuration file. The first three columns of numbers being Cartesian coordinates for positions and the additional three columns associated with the cameras being the target positions at which to be aimed. . . . . . 76

3.11 An obstacle configuration file for adding four obstacles to a terrain. 77

3.12 Four combinations of Obstacle and Simulation configurations inside a control file, allowing two different obstacles files to be each used with two simulation configurations. . . . . . . . . 77

3.13 Two test scenarios, consisting of gentle slopes and two obstacles (top) and steeper slopes, numerous obstacles and an unreachable goal (bottom), displayed beside the overlaid paths generated from trials conducted on them using the $\mathrm{A}^{*}$ algorithm. The point objects are the robot, the white are the goals and the grey objects are additional obstacles. . . . . . . . . . . . . . . . . . . . 79

4.1 UML diagram showing the branching of different trial sets. . . . . 86

4.2 Visual depictions of nearby hazard triangles within the set identified by the robot. $\mathrm{A} \rightarrow \mathrm{C}$ were produced as the robot moved to the right, while $\mathrm{D}$ is from later on, when the robot has explored to the left of the initial position of A. . . . . . . . . . . . . 92

5.1 Comparison of the summed/generalised results produced using each configuration file. . . . . . . . . . . . . . . . 96

5.2 Left: Completion status for A* algorithm, on Config_combos_0, Config_combos_1 and Config_combos_2. Right: The average number of movements occurring for each completion status. . . . . . . 98

5.3 Left: Completion status for LPA* algorithm, on Config_combos_0 and Config_combos_2. Right: The average number of movements occurring for each completion status. . . . . . . . . . . . . . . . 99

5.4 Overlaid paths generated by a simple state-based obstacle avoider for Config_combos_2. . . . . . . . . . . . . . . . . 100 
5.5 Config_combos_0 terrain overview plus overlaid paths generated by the $\mathrm{A}^{*}, \mathrm{LPA}^{*}$ and State-based algorithms, given as B, C and D, respectively. . . . . . . . . . . . . . . . 101

5.6 Config_combos_1 terrain overview plus overlaid paths generated by $\mathrm{A}^{*}$ algorithm, given as B and C, respectively. . . . . . . . . . . 102

5.7 Config_combos_2 terrain overview plus overlaid paths generated by $\mathrm{A}^{*}$ and LPA* algorithms, given as B and C, respectively. . . . 102

5.8 Left: Completion status of various h-value weightings for $\mathrm{A}^{*}$ algorithm, on Config_combos_0. Right: The average number of movements occurring for each completion status. . . . . . . . . 104

5.9 Left: Completion status of various h-value weightings for the $\mathrm{A}^{*}$ and LPA* algorithms, on Config_combos_1. Right: The average number of movements occurring for each completion status. . . . 104

5.10 Left: Completion status of various h-value weightings for $\mathrm{A}^{*}$ algorithm, on Config_combos_2. Right: The average number of movements occurring for each completion status. . . . . . . . . 105

5.11 Left: Completion status of various h-value weightings for the LPA* algorithm, on Config_combos_0. Right: The average number of movements occurring for each completion status. . . . . . . 106

5.12 Left: Completion status of various h-value weightings for the LPA* algorithm, on Config_combos_1. Right: The average number of movements occurring for each completion status. . . . . . . 106

5.13 Left: Completion status of various h-value weightings for the LPA* algorithm, on Config_combos_2. Right: The average number of movements occurring for each completion status. . . . . . . 107

5.14 Left: Completion status for the A-series of alterations with the A* algorithm, on Config_combos_0. Right: The average number of movements occurring for each completion status. . . . . . . . . 108

5.15 Left: Completion status for the A-series of alterations with the A* algorithm, on Config_combos_1. Right: The average number of movements occurring for each completion status. . . . . . . . 109 
5.16 Left: Completion status for the A-series of alterations with the A* algorithm, on Config_combos_2. Right: The average number of movements occurring for each completion status. . . . . . . . . 109

5.17 Overlaid paths generated by the A-series of variation, with the $\mathrm{A}^{*}$ Algorithm on Config_combos_0. (A) Terrain overview (B) Set A0 paths, (C) Set A1 paths and (D) Set A2 paths. . . . . . . . . . . . 110

5.18 Overlaid paths generated by A-series variation with A* Algorithm on Config_combos_1. (A) Terrain overview (B) Set A0 paths, (C) Set A1 paths and (D) Set A2 paths. . . . . . . . . . . . . . . 111

5.19 Overlaid paths generated by A-series variation with A* Algorithm on Config_combos_2. (A) Terrain overview (B) Set A0 paths, (C) Set A1 paths and (D) Set A2 paths. . . . . . . . . . . . . . . . 112

5.20 Left: Completion status for sets $\mathrm{D}, \mathrm{E}$ and $\mathrm{H}$ with the $\mathrm{A}^{*}$ algorithm, on Config_combos_0. Right: The average number of movements occurring for each completion status. . . . . . . . . . . . . . . 114

5.21 Left: Completion status for sets $\mathrm{D}, \mathrm{E}$ and $\mathrm{H}$ with the $\mathrm{A}$ * algorithm, on Config_combos_1. Right: The average number of movements occurring for each completion status. . . . . . . . . . . . . 115

5.22 Left: Completion status for sets D, E and $\mathrm{H}$ with the $\mathrm{A}^{*}$ algorithm, on Config_combos_2. Right: The average number of movements occurring for each completion status. . . . . . . . . . . . . . 116

5.23 Overlaid paths generated by $\mathrm{A}^{*}$ Algorithm in trial sets $\mathrm{D}, \mathrm{E}$ and H, for Config_combos_0. (A) Terrain overview, (B) Set D paths, (C) Set E paths and (D) Set H paths. . . . . . . . . . . . . . 117

5.24 Overlaid paths generated by $\mathrm{A}^{*}$ Algorithm in trial sets D, E and H, for Config_combos_1. (A) Terrain overview, (B) Set D paths, (C) Set E paths and (D) Set H paths. . . . . . . . . . . . . . 118

5.25 Overlaid paths generated by $\mathrm{A}^{*}$ Algorithm in trial sets $\mathrm{D}, \mathrm{E}$ and H, for Config_combos_2. (A) Terrain overview, (B) Set D paths, (C) Set E paths and (D) Set H paths. . . . . . . . . . . . . . . 119 
5.26 Left: Completion status for sets with small step sizes (set A1) and larger step sizes (set $\mathrm{C}$ ), using the $\mathrm{A}^{*}$ algorithm on Config_combos_0. Right: The average number of movements occurring for each completion status of the sets $\mathrm{A} 1$ and $\mathrm{C}$ plus a set adjusted set to account for the movements of set $\mathrm{C}$ being 5 times larger than A1. . . . . . . . . . . . . . . . . . . . 121

5.27 Left: Completion status for sets with small step sizes (A1) and larger step sizes (C), using the A* algorithm on Config_combos_1. Right: The average number of movements occurring for each completion status of the sets $\mathrm{A} 1$ and $\mathrm{C}$ plus a set adjusted set to account for the movements of set $\mathrm{C}$ being 5 times larger than $\mathrm{A} 1$. . 121

5.28 Left: Completion status for sets with small step sizes (A1) and larger step sizes $(\mathrm{C})$, using the A* algorithm on Config_combos_2. Right: The average number of movements occurring for each completion status of the sets $\mathrm{A} 1$ and $\mathrm{C}$ plus a set adjusted set to account for the movements of set $\mathrm{C}$ being 5 times larger than A1. . 122

6.1 Altering Terrain Tessellation as the Robot moves towards bottom right corner (sequence $\mathrm{A} \rightarrow \mathrm{B} \rightarrow \mathrm{C} \rightarrow \mathrm{D}$ ). . . . . . . 133 


\section{List of Algorithms}

3.1 TessellatePoints ()$\ldots \ldots \ldots \ldots$. . . . . . . . . . . . . . . . . . . . . . . . 44

3.2 ProcessEmptyTriangles( $\ldots \ldots \ldots$. . . . . . . . . . 45

3.3 CompareTriangles ()$\ldots \ldots . \ldots . \ldots . \ldots 48$

3.4 World::AddPoint () . . . . . . . . . . . . . . 55

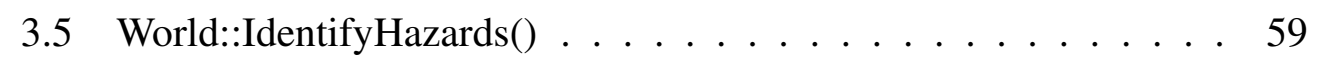

3.6 World::CheckWall() . . . . . . . . . . . . . . 60

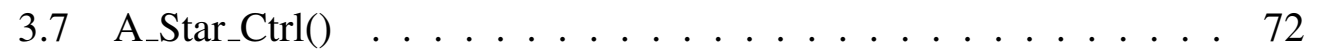

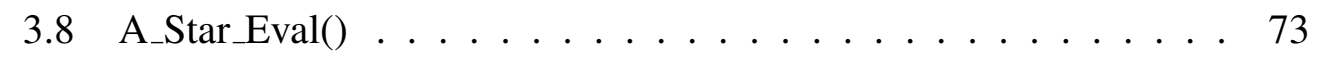

3.9 LPA_Star() . . . . . . . . . . . . . . . . 74

3.10 UpdateLPA ()$\ldots \ldots \ldots \ldots$. . . . . . . . . . . . . . . . . 


\section{List of Tables}

4.1 Combined results of first two configurations within Config_combos_0. 87

4.2 Results from third configuration within Config_combos_0. . . . . 87

4.3 Results from the fourth configuration within Config_combos_0. . . 88

4.4 Config_combos_2. . . . . . . . . . . . . 88

4.5 Config_combos 2 A. . . . . . . . . . . . . . 89

4.6 Config_combos 2 B. . . . . . . . . . . . . . 89

4.7 Config_combos_2B re-simulated. . . . . . . . . . . . 90

4.8 Config_combos_2C . . . . . . . . . . . . . 90 


\section{Glossary}

A

A $^{*} \quad$ A heuristic algorithm, calculating path values based on the distance necessary to traverse to reach a point and proximity of the point to the destination., p. 31.

a priori In advance of a start time. Before examination., p. i.

$\mathbf{C}$

CGAL The Computational Geometry Algorithms Library. A library of mathematical functions and structures, helpful in producing tessellations from sets of points., p. 51.

circumcircle The circle created through joining three points such that they all lie on its edge., p. 25.

circumsphere The sphere which links four points in 3D, such they all lie on its surface., p. 25.

CRASAR Center for Robot-Assisted Search and Rescue., p. 6.

D

D* A heuristic algorithm derived from $A^{*}$, focused on working backwards from the destination to the start position., p. 31 . 
$\mathbf{D}^{*}$ Lite An enhancement of the $\mathrm{D}^{*}$ algorithm, aimed to use a re-use approach much like the LPA* modification of the A* algorithm., p. 33.

Delaunay tessellation A method of generating a set of non-overlapping triangles from a given set of points. The set of triangles being the dual of the voronoi graph of the points., p. 25.

$\mathbf{L}$

LIDAR Light Detection and Ranging. A sensor/technique for gathering precise relative measurements., p. 23.

LPA* A re-use modification of the $A^{*}$ algorithm, limiting re-calculation of path values over successive iterations through a heuristic basis., p. 32 .

M

Marsupial Robot teams which involve a larger unit carrying or housing one or more smaller robots, in order to enable transportation across difficult terrain., p. 13.

MeSci The National Museum of Emerging Science and Innovation., p. 17.

$\mathbf{N}$

NIST National Institute of Standards and Technology., p. 17.

$\mathbf{P}$

Polymorphic Robots which are capable of re-configuring modules so that the form factor of the robot is altered., p. 11. 
$\mathbf{S}$

SLAM Simultaneous localisation and mapping., p. 28.

Swarms Groups of interacting robots which approach tasks from a co-operative stance., p. 14.

$\mathbf{U}$

USAR Urban Search and Rescue., p. 2.

$\mathbf{V}$

Voronoi graph Voronoi graphs are comprised of cells formed around the points within a set, so that all edges in the graph are of equal distance to the nearest two points and hence any position inside a cell is closer to the encapsulated point than any other point in the set., p. 25. 


\section{Chapter 1}

\section{Introduction}

The first few hours following a disaster are the most critical, with the chance of saving lives being at its greatest. However, in the case of collapsed infrastructure and buildings, the first few hours are also the most dangerous to send rescuers into the site of the disaster. Hence, the concept of using unmanned exploratory vehicles suited to this kind of terrain is being embraced, to map the area and locate individuals trapped in the rubble. Then once it is deemed safe for rescuers to enter the area, they can get straight to saving people instead of wasting crucial time searching.

Many research groups across the globe are now developing task forces of robots which can be used to help in such Search and Rescue operations. This project aims to provide a useful tool for such groups in establishing which algorithms and parameters provide the most appropriate autonomous path planning for their specific purposes or scenarios before a physical robot has been built and without need of a disaster site or mock up, on which to test. This will be achieved through the design and implementation of a software based system to allow a virtual robot to autonomously navigate across a disaster site simulation with the choice from a variety of path planning algorithms and a multitude of variables such as robot dimensions, traversable terrain ascent angles, and data structures. The data structures referred to here include, different types of nodes for use by path planning algorithms, various types of terrain models, restricted and flexible 
potential path networks, and also a variety of data storage methods for current and past terrain knowledge.

In order to identify the optimal path through the disaster environment, the robot needs to be able to identify features of the landscape which could be hazardous or insurmountable and determine the shortest path to the destination given the gathered data. As the robot travels and receives new data it will have to dynamically re-evaluate the previously determined hazards and path choice.

Producing a modular open-source design, allows development to be furthered by any interested parties, to reflect design choices they wish to follow or constraints which they have imposed. The addition of new modules greatly expands the potential combinations and scenarios which can be investigated without requiring the replication of the work necessary to produce a platform on which these combinations can be possible.

The motivation which brought about this project's inception, was the creation of a task force of robotic units at Victoria University for the purpose of Urban Search and Rescue (USAR), under the supervision of Dale Carnegie and with the cooperation of other Universities within New Zealand. As such, the selection of features currently present and the basis for decisions such as default values and input method have been guided by that larger project.

To ascertain that the Simulator is both easy to use and can produce usable results, the Simulator tests an individual robot interacting with a varying terrain containing multiple additional obstacles. Values used for the dimensions of this robot and its ability to handle different terrain gradients, are based on a moderately sized four wheel low-profile robot. The focus during testing was on the navigation (and hence also the mapping) of the robot when placed in different locations and tasked with reaching specific goals, with additional exploration not being encouraged more than was necessary to reach the goal.

The original non-graphical prototype from which this simulator progressed, was presented at the 2006 Electronics New Zealand Conference (ENZCon '06), exploring the potential of a USAR simulator, and demonstrating basic behaviour and usage. From the work completed during this thesis, a paper was generated 
outlining the design considerations, hindrances and process of creating and implementing a novel simulation platform such as this one. This paper was accepted by and subsequently presented at the 7th IEEE International Conference on Control \& Automation (ICCA '09), alongside other papers on the design, simulation and interfacing of other autonomous vehicles and robots. 


\section{Chapter 2}

\section{Background}

\subsection{Urban Search and Rescue}

Research into the use of robotic units in Urban Search and Rescue began in Japan after a very strong earthquake hit the regions of Kobe and Osaka on the 17th of January 1995 [1]. In the U.S. it was after the Oklahoma Bombings on the 19th of April the same year, that research first began [1]. The 9/11 terrorist attacks were the first major disaster where teleoperated robots were used to try to aid Search and Rescue teams [1]. This globally watched disaster also gave more impetus and research funding, while greatly expanding the number of institutes involved in developing robotics for post-disaster humanitarian efforts. International competitions have also been established to encourage innovation and provide comparison of design and effectiveness.

This field of research has seen numerous different design paths taken, each design choice with its own benefits and flaws. There are various means of robot locomotion, methods of overcoming or avoiding obstacles and techniques or sensors for detecting human casualties. Due to the hazardous nature of the environment, being robust yet cheap has been a primary objective, with self-righting mechanisms and stair/ledge climbing designs also being tried in attempts to minimise the limitations of movement caused by different hazards or insurmountable obstacles. 
Tethered teleoperated robots have been the primary choice for USAR robots as using a human agent is considered more reliable to make decisions and because the tether provides the reliable high-bandwidth necessary for live video which a human requires[2]. Autonomous robots can dispense with transmitting a videofeed and as such have more freedom with which to move as they have no tether to get caught and are not limited by the tether length. A tether also provides additional strain/resistance as it must be pulled along, an added means of disorientation (if tension is applied or released to the tether when the robot turns) and a tether could pose a danger as it may trip a human rescuer or if being pulled against/around rumble it may cause a shift or rock fall. One benefit of tethers, is that if robots delve into crevasses and fall or get stuck they can potentially be pulled out via the tether acting as a safety line.

USAR robots can provide replacements for humans and canines in dangerous situations, such as when after-shocks or secondary affects may cause further collapse, or when fires or toxic gases are present, thus avoiding further loss or injury. However, their usage and capabilities in this capacity are far from perfected and this is not the only task where they could provide benefit. Due to being a developing and unproven field, the humans involved with search and rescue are often wary of the presence of robots and trusting the information they may produce [3]. Robots can be viewed by rescue teams as a nuisance/distraction and another obstacle to deal with and which may provide a hazard, in an already dangerous environment. In spite of a distrust of the robots in their conceptual role, Search and Rescue members have found alternative purposes for them such as lowering tethered robots down holes to act as a remote camera/sensor to access structural integrity of lower levels and likelihood of whether they may collapse further.

Alternative ways in which USAR robots can provide assistance to rescuers have been taken into account, with the Center for Robot-Assisted Search and Rescue (CRASAR) developing the use of robots as a delivery system for fluids, medical supplies and sensors, in addition to two-way communication.

One finding of the application of USAR robots in real disaster scenarios has been that designs driven by competition results have been lacking in many ways, 
such that they are not suited to real USAR due to the robots not being robust enough and the path planning etc. not being compatible with robots which were[3]. The sensors and detection systems of USAR robots need to be small and robust, however the useful ones are often too large and complex, with the potential to be damaged in a realistic scenario.

\subsection{Robot types}

There are four common types of locomotion used in ground based exploration. These are wheeled, tracked, walking and snake-like units, which move via undulation of multiple jointed segments. The hybridisation of the jointed segment design with one of the other means of locomotion is referred to as serpentine and an example of such a robot is shown in Figure 2.1 [4]. Wheeled and tracked robots are well suited to autonomous operation with the likelihood of tipping over being much less than walking units and the control being much easier than with walking and snake-like or serpentine robots. However, the much greater freedom of movement of a snake-like and serpentine does allow for very adverse terrain to be traversed, with Figure 2.1 showing one such robot overcoming a piece of terrain no other design would have. Aerial robots can also be used in terrain mapping and have a variety of mobility methods which mimic planes, gliders, helicopters and dirigibles. In disaster scenarios, land based units tend to face a greater range of problems than aerial ones, including shifting terrain, hazardous drops, nontraversable slopes and limited fields of view. However, they can provide greater levels of information due to their closer proximity, especially with regards to location of human casualties.

The manner in which a robot moves and the amount of clearance necessary to do so, and what situations are hazardous or insurmountable, is dependant on the choice of locomotion and hence different robot types may be simulated differently or require different values for some parameters. The intended behaviour of a robot, such as working individually versus co-operatively, or exploring a region versus reaching a specific destination, also affects the design of a simulator and 


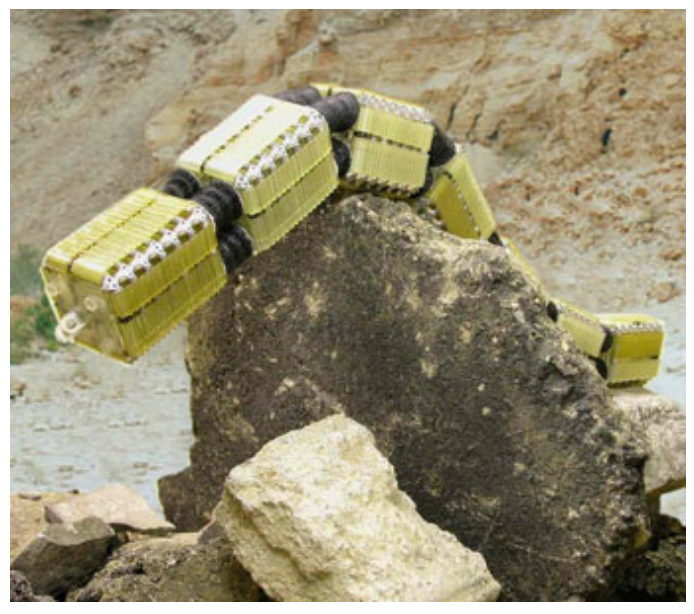

Figure 2.1: The OmniTread, an example of an articulated serpentine robot design [4].

the choice of features present within it.

Of these traversal methods available to ground based units, there are a few specifics which differentiate them from robots used in other scenarios such as office buildings, factories or entertainment/toys. Unlike the standard arrangement in most wheeled vehicles, wheeled robots for USAR purposes usually have fixed position wheels which are differentially driven, rather than having a pair of wheels which change orientation/angle to steer the vehicle.

All fully tracked vehicles are differentially driven as the tracks do not allow for the wheels to alter direction however the USAR robots have a few track designs which are not common to vehicles. Track designs can be simple, such as the flat layout of a Bulldozer labelled A in Figure 2.2, or may account for desirable behaviour as the trapezium layout of tracks on a Tank does, through allowing for bi-directional traversable over top of obstacles. For some purposes the fourth set of wheels for bi-directional obstacle climbing may be removed to produce an obtuse triangular layout, given as layout B of Figure 2.2, as unidirectional climbing capabilities may be sufficient. In addition to these relatively basic track layouts, another design which is possible for robots is having four sets of tracks, as depicted by designs $\mathrm{C}$ and $\mathrm{D}$ of Figure 2.2, with two being used for locomotion 


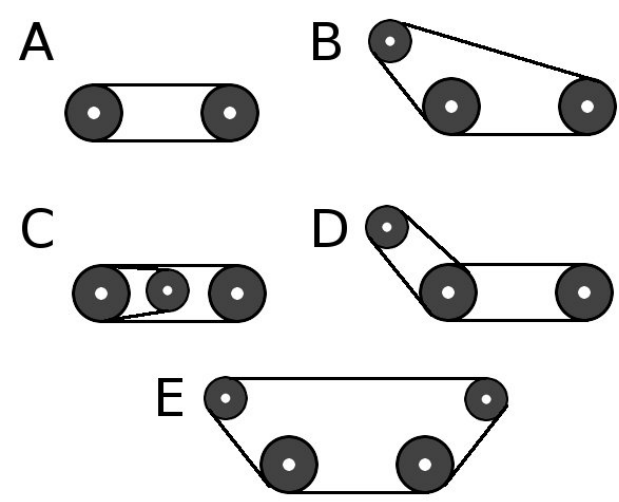

Figure 2.2: A variety of tracked robot designs. A - Basic tracked setup. B - Fixed form climber. C - Flipper arms climber in low profile shape. D - Flipper arms climber in climb configuration. E - Tank like tracks capable of climbing in either direction of movement.

and the others being pivoting arms which can be used to raise the front or rear higher as well as for stair climbing like the obtuse triangle approach. Additionally these flipper arms can be used to right the robot if it rolls or falls, and find itself upside down. Another potential though seeming unutilised approach would be to provide higher point of view for sensors/cameras when the front is raised, to allow them to view past obstacles.

Robots which have legs can either attempt to mimic humans with bipedal motion or adopt a more stable multilegged design akin to an insect or spider, though unlike animals they do not necessarily follow a symmetrical design and can have an odd number of legs. Figures 2.3 and 2.4 show examples of robots designed for bipedal and multilegged motion, respectively. While having more legs provides more points of contact and hence greater stability, each additional leg is another item which must be controlled though often only in terms of extension and placement as the unit will follow an overall guiding pattern of when to move each one in order to move in the desired direction. Designs which have the legs attached radially rather than in two parallel sets, can be omnidirectional as they have no clear front however often the placement of cameras and sensors may dictate a preferential direction. 

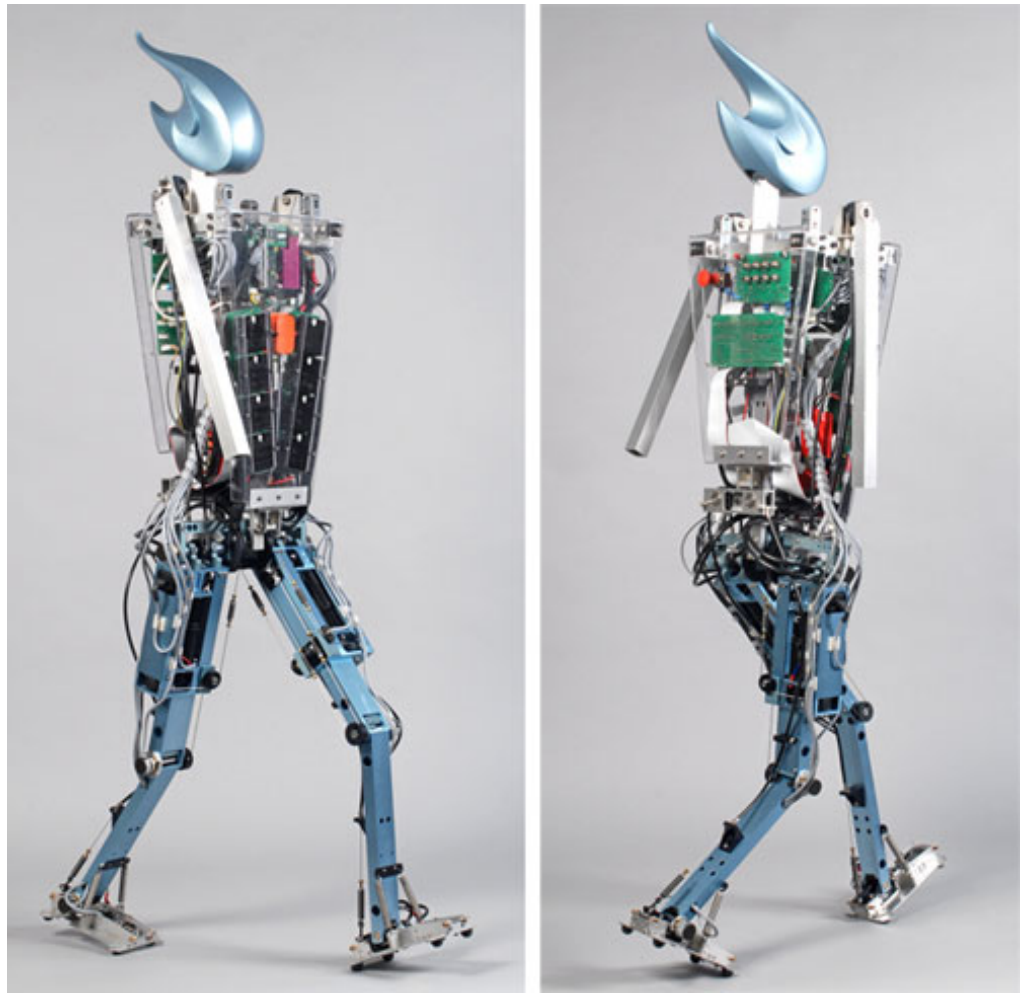

Figure 2.3: A bipedal research robot used to study human motion, viewed from two angles [5].
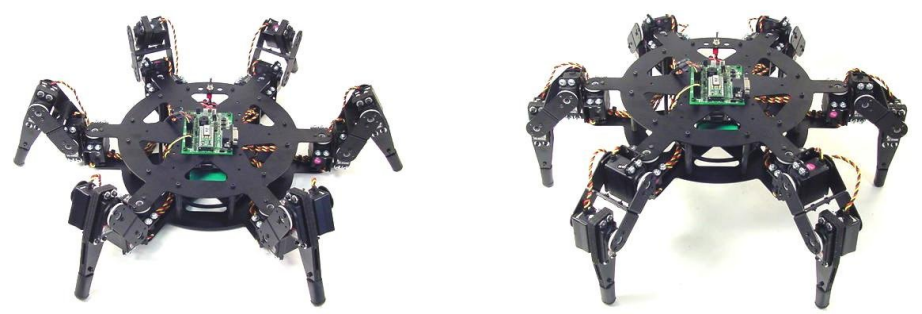

Figure 2.4: A multilegged robot, as commercially available, shown in two positions [6]. 
The choice of locomotion design effects the navigation in terms of potential obstacles and paths. A common point amongst multilegged walkers, tracked robots, differential-drive wheeled robots and potentially some bipedal robots, is that they are capable of changing direction on the spot. This factor is beneficial for both path planning and simulation as there is no turning circle to account for or need for manoeuvres such as 3-point turns. For serpentine robots, wheeled robots without differential drive, and most bipedal designs, small adjustments in direction are perfectly within their capabilities however drastic changes in orientation can require more room than may be available or manoeuvres like 3 point turns.

Approaches which attempt to climb over obstacles affect the process of hazard identification as they introduce the potential for walls of limited height to not be obstacles. However these stair climber mechanisms hold no substantial benefit with traversing steep slopes other than providing recourse in the event the robot rolls on attempting such a slope.

To deal with the complexities of disaster sites, some research groups have opted to redesigned the whole robotic unit rather than including novel functionality, such as stair climbing, through the addition of modules to standard robot designs. The results of these design branches include robots which can transform into different configurations or contort and shape-shift, so that they can overcome obstacles or fit through tight spaces. Other approaches have seen teams of split purpose robots which aim to specialise in different tasks associated with USAR.

\subsubsection{Shape shifters}

Taking solutions to a new level are shape shifting robots, which rather than having mechanisms or modules added to a design to overcome certain obstacle types, have the whole unit designed around the idea of crossing the most difficult terrain. Polymorphic robots initially tended to be of a serpentine or centipede-like design as shown in Figure 2.5, which gave them a high degree of freedom, but also meant control was difficult and movement often consumed a lot of power [7]. Balancing efficiency and ease of control against space required to change shape, the next evolution in design were units such as the Amoeba-II, which is shown in Figure 


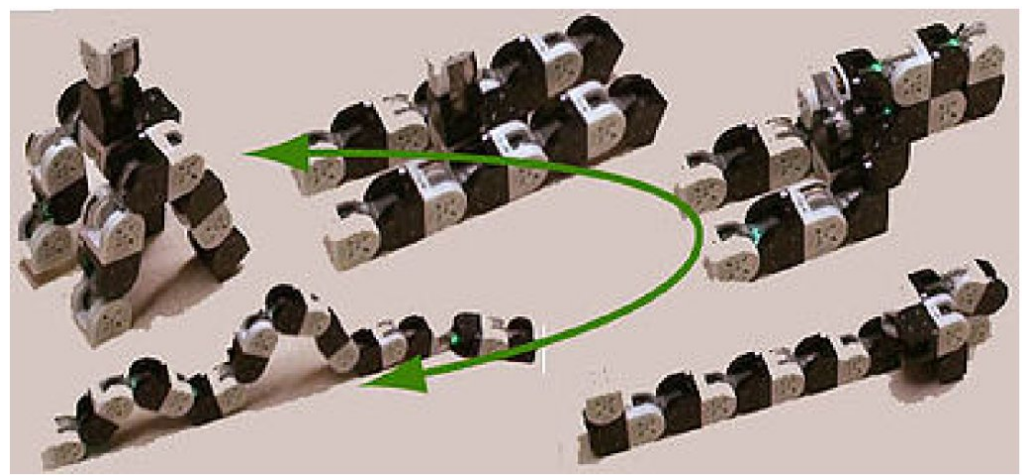

Figure 2.5: The M-TRAN III, a serpentine shape shifting robot, in a variety of permutations [9].

2.6, that can be likened to articulated truck-trailer pairs [7]. These articulated pairs provide a level of compromise, giving the ability for some shape changing but not so much as to be too complex to control, while maintaining much of the efficiency of wheeled or tracked robots. One issue which arises with these simple shape changers is that the space required for them to transform from one mode to another can be much greater. Complex serpentine robots have enough degrees of freedom that the right combination of moves can readjust it in almost any situation. However, for basic designs with few joints, the turning circle/angles required for transformations could make it difficult to find appropriate areas in which to do so. This is especially difficult if the robot is to be autonomous.

While having various configurations in which the robot can traverse across terrain increases the variety of situations which can be handled, these advances in design provide an additional layer of complexity to the already problematic task of autonomous navigation. What the design and focus of current shape shifter research comes down to is the balance of versatility versus power consumption and ease of control for teleoperated robots, with the combination of autonomy and transforming designs being much further off [8]. 


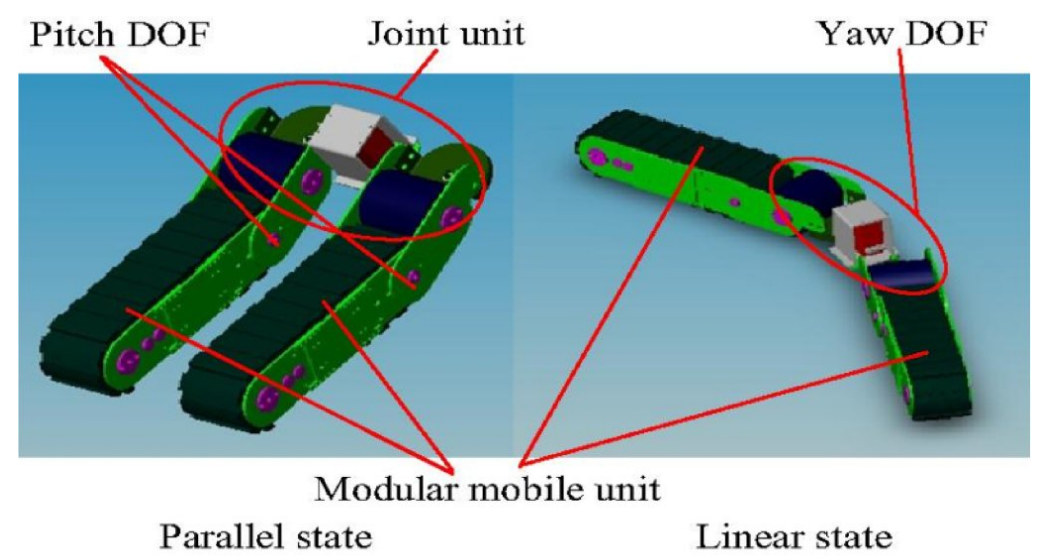

Figure 2.6: An example of an articulated-pair shape shifting robot design. The Amoeba-II shown in two states [7].

\subsubsection{Marsupial teams}

'Marsupial' robots involve features and abilities being broken down across a set of robots of varying size and shape, with larger ones generally transporting smaller ones [8]. With the separation of tasks, functionality and cost can both be improved, as smaller robots with sensors for finding human life signs can get into tighter spots while safely covering great distances to get to these spots thanks to a larger more robust unit acting as a carrier. To cover a vast area, a swarm of these smaller units can be taken deep into the site and then allowed to radiate out from the carrier, or alternatively be dropped while crossing terrain, to spread out orthogonally from the carrier's path. Thanks to the shelter of the carrier, these smaller units need not be particularly robust and as such can be much cheaper, to the point of even being financially disposable, with losses due to accidents and hazards being tolerable[10].

Unlike polymorphic designs which increase the possibilities and hence complexity of autonomous path planning, marsupial teams are complimentary with autonomous navigation. Each class of robot may have different parameters reflecting their size, hardware and purpose but can use the same approach to navigation. Smaller robots delivered by a large carrier may move in smaller increments and 
hence cover a very restricted region, as well as having lower quality sensors which can not detect points as far away. Operating in a smaller region the robots may use a higher resolution/lower scale model, however as they are likely to possess limited memory and processing power, then only relatively simple algorithms can be employed.

\subsubsection{Robot swarms}

One concept which is reliant on autonomous navigation is using 'Swarms' of robot clones, where information is passed between each unit to improve the modelling of the terrain and also ensure greater coverage of the area without a lot of overlap[2]. Due to the nature of having so many robots on a site, having humans controlling each robot is impractical and would require many expensive workstations and trained operators. Autonomous USAR robots provide a practical solution to this problem. With robot swarms the advantages of autonomous navigation are further embraced, using the ability to transfer information and insert it into other robot's models to build more insightful maps and coordinate activities. Swarms also allow for the positions of individual robots to be triangulated with respect to neighbouring robots and hence over an entire swarm and multiple iterations they can calculate fairly accurate relative positions for all, with any error that does occur being uniform/common to all[2]. This method of localisation via neighbours takes advantage of the more accurate ranging sensors to overcome issues of low quality odometry sensors which may be found in small disposable robots and issues of positional slip which odometry sensors are unable to detect.

As already mentioned, there are issues with the use of wireless communication in disaster sites but these are not overly problematic for swarms due to the different nature of what is being transmitted compared to teleoperated robots. Swarms require lower bandwidth as they are sending specific/quality/valuable data rather than streaming video, would be closer to recipient units, have redundant/multiple communication paths and the data does not have to be received in real-time, with robots still able to operate without interaction, hence wireless issues do not become a concern. 
This swarm of clones differs from marsupial teams where the smaller units may communicate only with the central 'mother' unit, being analogous to an ad hoc network as opposed to a network with a central access point. The swarm can be efficient in that the nearer units are more likely to benefit from interaction with the robot, however a central point can provide greater resources for processing and analysing data.

\subsubsection{Confined space exploration}

One of most dangerous and also difficult places for humans to explore are the spaces created within/underneath fallen buildings and other civil structures, thus specific robots for delving into these tight and dangerous spaces are important [11]. In these instances the danger to a rescuer lies in becoming trapped themselves, coming across gas leaks/pockets and the rubble shifting/collapsing on them thus turning them from rescuer into victim/casualty. Sending robots into these situations not only avoids endangering rescuers but also provides an ability to detect dangerous gases that may be found within the rubble as well as offering a wider range of means to detect humans through the gas/air analysis. The same detection systems, such as nondispersive infrared sensors, which can be used to detect deadly gases could also identify concentration of $\mathrm{CO}_{2}$, a by-product of human respiration. As well as the outlined danger, it is also highly difficult for a human to delve very far into the rubble without laborious effort or the aid of heavy machinery (something one is disinclined to send onto a disaster site until late in the rescue due to the weight of the machinery shifting rubble and potentially causing more trouble than benefit). The tight, rough and winding tunnels one may find in the interconnecting nooks and caverns within rubble pose little obstacle for robots of a serpentine design, which are both slender and have many points of freedom such that they can contort into a variety of shapes[4]. In some instances, such as the rubble remaining in large pieces, low profile tracked or wheeled robots may also be able to explore much of the region between rubble [11]. In these confined spaces, the line of sight is often drastically reduced due to winding tunnels as well as low cavern/tunnel ceilings (the height of which may vary in addition to the vari- 


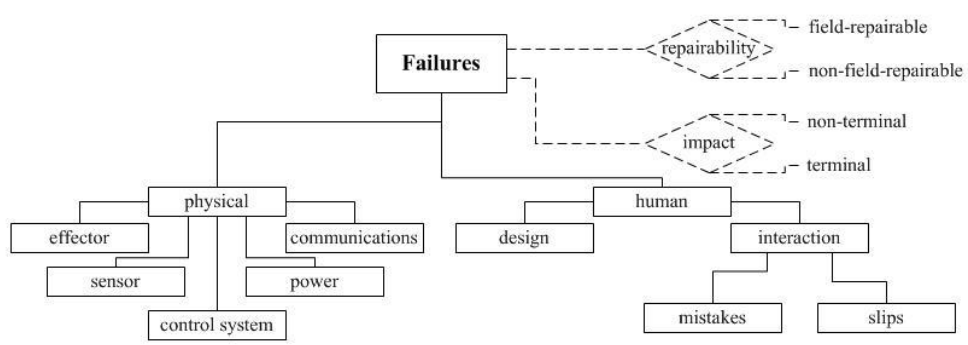

Figure 2.7: Tree diagram categorising areas of failure of mobile robots [12].

ance in the ground upon which the robot is situated). As such, in these regions of occlusion, accurate localisation becomes much more important as the limited line of sight makes it hard to detect errors or disorientation.

\subsection{Analysis of Robot failures}

Much of the analysis of robot failures focuses on the ruggedness of the physical aspects of the robot and/or its ability to avoid potentially damaging situations [12]. The physical component of the failure tree diagram shown in Figure 2.7, is not overly relevant to this project due to being based in the virtual world. With the human branch of the diagram, which is split between mistakes, slips in control and design flaws, it is mainly the design flaws that are of interest and more so in relation to damage avoidance rather than ruggedness.

\subsection{Competitions}

In the past much of the focus in locomotive robot design has been on agility and robustness, aimed at exploration vehicles for places such as the moon or Mars where there is a great deal of preparation for a task and numerous people available to work as a team to control a robot. The usefulness of exploratory robots in other applications has been seized upon, as are using them as the basis for developing artificial intelligence. A number of organisations now hold international competi- 
tions involving robots [1]. These competitions are aimed at creating more public interest in robotics and allowing comparison between the designs and techniques into which different institutes and research groups have been delving. Some competitions or branches thereof, focus on the human-robot interface and the ability to control the robot in complex tasks, while others focus on the robot's autonomy and cognition.

One of the major competitions being run involving rescue robots, is held by the US based National Institute of Standards and Technology (NIST), which has three different arenas representing disaster site scenarios of differing complexity level [13]. The National Museum of Emerging Science and Innovation (MeSci) of Japan hosts similar competitions to NIST but interestingly, due to the country of origin the materials and scenarios encountered vary quite a lot [14]. The NIST arenas involve typical materials of sky scrapers such as rubble from concrete blocks, tile flooring and carpet. The different architectural styles and cityscapes of Japan mean that sheet rock and wooden building materials can be found in the disaster mock-up along with tatami mats which would be extremely uncommon in North America. Figure 2.8 shows the three of the arenas used in the NIST competitions, while the arenas present in the MeSci competition can be seen in Figure 2.9, with it possible to notice some of the similarities and differences in the materials used for each competition.

For both competitions the three arenas are colour coded as yellow, orange and red in order of increasing complexity. The yellow arenas are large and designed to test the sensors and algorithms used in mapping and planning. Some areas of the arena can be altered in real-time, for example closing doors or adding blockages to passages, to test how the robots react to changes in known regions. Overall the yellow arenas, as shown in Figures 2.8 and 2.9, look like evacuated buildings rather than the sites which have sustained damage from some of a disaster.

With the orange arenas the focus shifts to testing the agility of the robots. Various flooring materials which may affect locomotion or odometry, are used across the arena as Figure 2.8 shows and there are some raised sections such as the one in Figure 2.9 which are added to create a multi-tiered environment. In 
addition to ramps, more difficult means of reaching the upper level, such as via stairs or a ladder, are present to encourage and test various climbing techniques. In addition to simple obstacles hindering robots, the orange arena contains hazards which pose a danger to the robots, such as items which if disturbed could cause a minor collapse or holes in the upper level into which a robot could fall.

The most difficult scenarios are present in the red arenas, which as can be see in Figures 2.8 and 2.9, begin to resemble actual disaster environments. Building materials are strewn around creating a terrain which is not only difficult to traverse but also can be problematic for sensors and mapping due to translucent items such as plastic sheeting or discontinuous surfaces like wire meshes, gratings/vents or pallets. In addition to items which if knocked could collapse around or on a robot, the red arena includes flooring which is unstable and may potentially collapse underneath a robot. This type of collapsing hazard provides a significant threat to designs which may attempt to approach USAR through heavy duty robustness and an over abundance of sensors, resulting in heavy robots.

These competitions provide a base platform which allows USAR designs to be benchmarked and compared, however the scenarios are far from realistic. Robots which can survive and negotiate the arenas, will not necessarily be capable of surviving or traversing a real disaster site and the algorithms/automation is in general not portable to other robots which are suited to being used on real disasters.

The most widely known string of competitions have been the ones sponsored by the Defense Advanced Research Projects Agency(DARPA), a US Military research group, with the competitions being the Grand Challenge and the Urban Challenge [15]. The robotic side of the competitions is not as clear to some due to standard vehicles being used, which outwardly do not match public perception of what a robot is. However, these vehicles have been outfitted with many sensors and other robotic controls in order to attempt to autonomously traverse a rugged terrain.

The RoboCup is a group of competitions which are more aligned with public perception of robotics, with units being of a much smaller scale than a vehicle and usually having a much more science fiction like appearance [16]. The first 


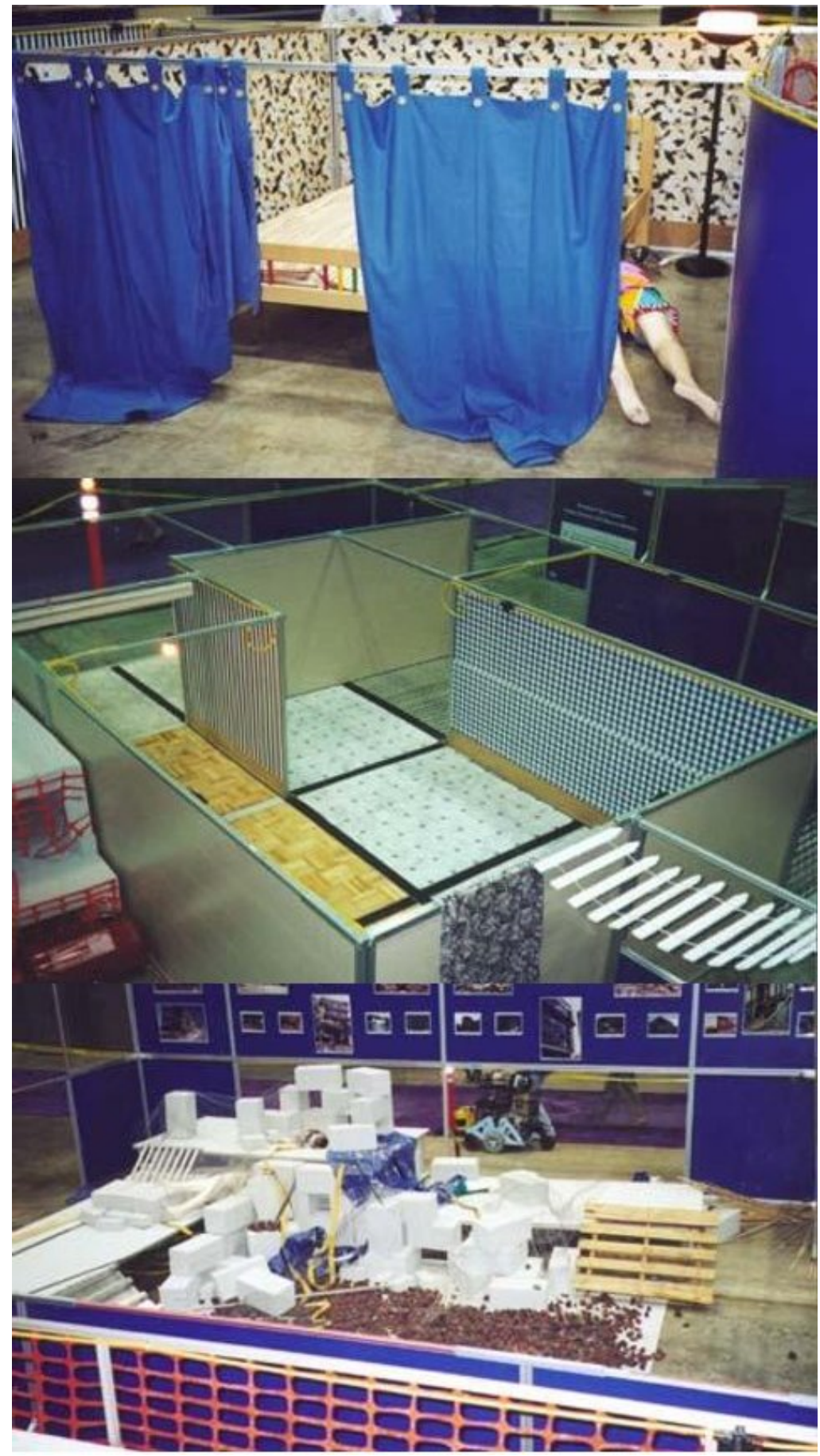

Figure 2.8: The three arenas of the NIST competition [14]. 


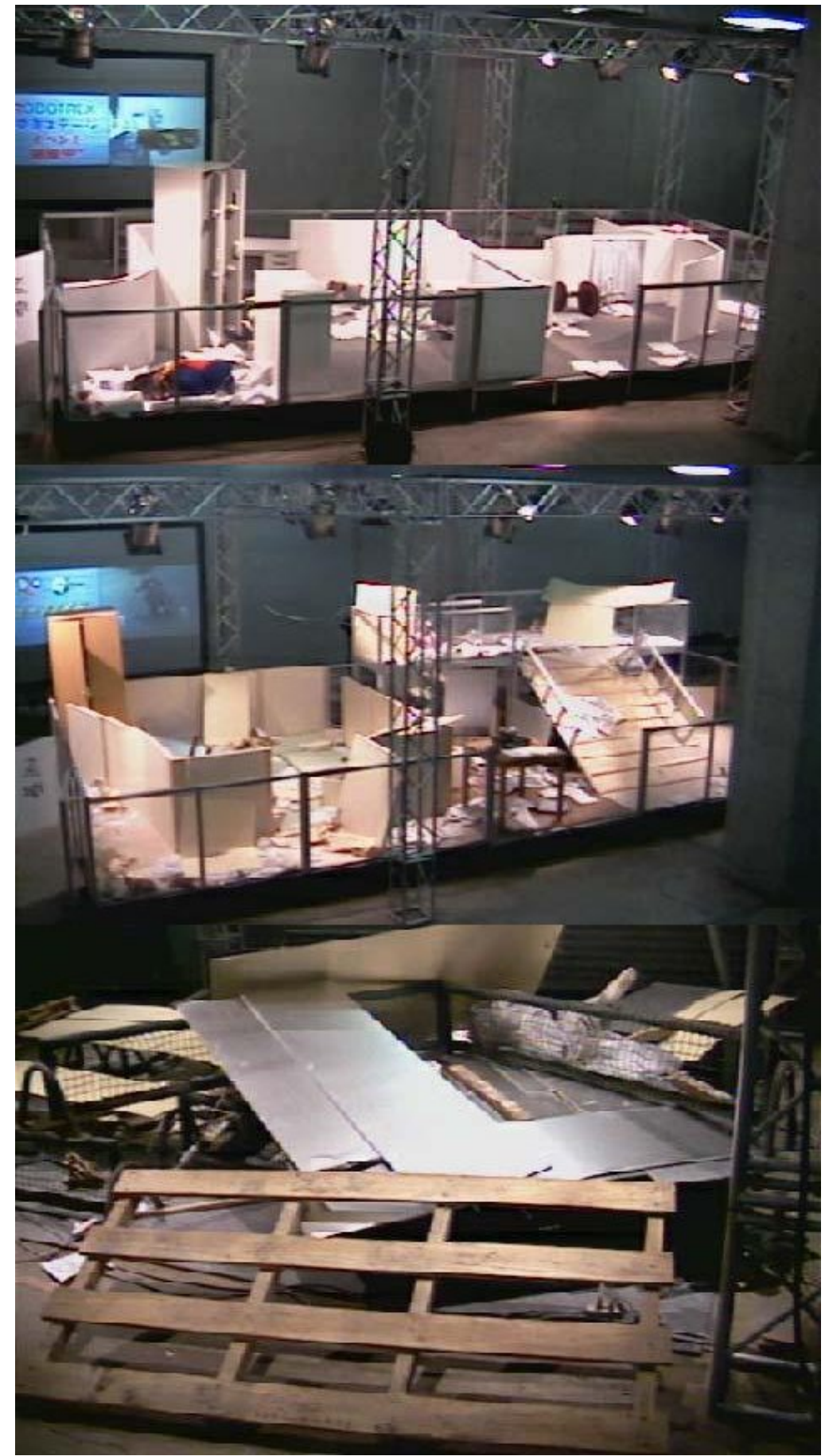

Figure 2.9: The three arenas of the MeSci competition [14]. 
competitions involved playing soccer and were split into different size categories, with a humanoid league later being added as well as competitions focussed on USAR.

\subsection{Simulators}

In terms of 3D Robot Simulators a number of commercial alternatives exist, with a number of very limited open source ones also available [17]. Simulations have in past been limited to a numerical basis (such as those based upon Matlab) with limited or no graphical visualisation, but 3D simulators have over time been adopted by many. In terms of graphics, many have used custom rendering engines which require their own upkeep, rather than widely used pre-existing ones which are likely to stay current with improvements in technology via large development teams or communities. However, there is a trend of moving towards commercial graphics engines or pre-existing open source engines, which has made the uptake of 3D graphical simulations easier as it no longer requires a team/number of developers to implement the graphical side of a simulator.

Most of the simulators are purpose built for certain robots or specific test arenas and in general are not of much use for the wider research community. Instances of simulators used to recreate specific arenas, like the three NIST arenas for example, are analogous to implementing an analogue solution upon digital equipment in that they replicate solutions which had been restricted by limited capabilities and discard all the benefits which the modern technology can provide. Running in the virtual world, a computer simulator allows for drastic alterations to be made to test environments without the time, labour or material costs associated with altering or adapting a physical test arena for each new layout to be tested.

Except for one instance, all the search and rescue simulators encountered have been designed for the purpose of testing teleoperation capabilities and interfaces, focussing on improving the interaction aspect of the failure tree shown in Figure 2.7 , with research into and testing of, autonomous navigation being an ignored area of potential application for simulators. At present the only autonomous nav- 
igation simulator found by the Author, is the Unified System for Automation and Robot Simulation (USARSim)[18], which was not encountered until the completion of this project. USARSim uses the Unreal Tournament (UT) game engine, which it requires to be purchased separately, and has been the basis for the RoboCupRescue virtual robot competition. While providing a very picturesque high-fidelity simulation for free and purporting to being compatible with Unixbased systems, USARSim appears to only be readily available as a pre-compiled executable file for Windows or as source code developed for Windows, and requires the purchase of the proprietary UT graphics engine. So as at present no other modular or truly open source simulation platforms could be found.

\subsection{Autonomous Navigation}

The primary constraint of almost every mobile object is energy, be they baseballs, mobile phones, humans, cars, or robots. The object's capacity to store energy, the frequency of events or locations at which more can be acquired and the efficacy of use, all determine how an object behaves. Some robots have automated recharge points which they periodically must find and return to, other robots will have humans replace or recharge battery banks and then there are a few robots such as the Mars Rovers which once unable to obtain more energy are simply left where they lie. In all cases, the paths travelled by the robots and how they are chosen play an important role, from ensuring the robot finds the recharge point to allowing the robot to operate for as long as possible before it is useless.

The environment robots are placed in are often unknown, with data being received from sensors which give relative range data. The lack of a priori data means the robot has to dynamically create a representation of the terrain, so finding perfect paths is unlikely. Dead-ends will occur in the terrain, so the robot will at times need to back-track and thus the robot must keep data about the terrain for some time, rather than discarding upon passing.

In order to identify suitable paths through the rubble, a robot needs to be able to identify features of the landscape which could be hazardous or insurmountable 
and determine the shortest path to the destination given the known data. As a robot travels and receives new data it will have to dynamically re-evaluate the previously determined hazards and path choices.

\subsection{Terrain modelling}

One section crucial to both identifying hazards and planning paths is the interpretation of data from the input sensors, which is dependant on the nature of the sensors. Sensors may be omnidirectional or have a limited area of detection. In instances where the received data comes from a forward facing directional sensor, such as some form of range finder, regions can often be occluded by obstacles or rises in closer areas and so when ascending a section of terrain the following descent is likely to be unknown. How the height value of an unknown XY coordinate is interpolated depends on the method of modelling the terrain. Models are generally derived from the point cloud of data received about the real terrain, and may involve turning the points into a non-overlapping mesh representing the surface. For omnidirectional sensors occlusion may also occur, however as a robot moves and new data are received, an omnidirectional sensor may view an occluded region more times and from a greater range of angles, giving it more opportunities to detect the previously occluded region.

\subsubsection{LIDAR}

One method of determining distances is through using Light Detection and Ranging (LIDAR). While live camera feeds are highly beneficial to humans teleoperating a robot, they are not intrinsically of benefit to an autonomous robot which would need to process the images for edge detection and try to extrapolate relative distances based on the visual expansion or diminishment of points and knowing its own motion. So LIDAR may be used instead to gather sets of relative measurements from which to build a model. LIDAR measures the distance to the first object a laser encounters in a straight line path and knowing the orientation and 
inclination of the laser, this measurement can be used to calculate the relative position of the object the laser intersected. The principles behind LIDAR are much the same as with radar systems but with LIDAR operating within the ultraviolet, visible light and infrared parts of the electromagnetic spectrum as opposed to using microwaves or radio waves. The benefit of using shorter wavelengths such as IR is that tiny objects can be detected/imaged, thus differentiating the potential uses for LIDAR from radar, which is better suited to tracking large objects such as cars, planes or ships for which fidelity/resolution is of little importance.

While LIDAR is good for monitoring the proximity of obstacles, due to measurements being relative to the robot, the building up of a terrain map can suffer problems if the robot's motion is imprecisely known, it slips or is disorientated. The potential for errors can be dealt with through coupling LIDAR with absolute positioning systems such as GPS or determining a localised position through model matching, the proximity with other robots or active beacons.

The basic process by which distances are determined with LIDAR is through pulsing a laser and then judging the return flight time based on the phase difference between transmission and receipt, and knowing the speed of the signal. There is a limited range within which measurements can be made due to the signal strength weakening and limitations of detection.

\subsubsection{Tessellation}

To create a terrain mesh and hence a model which can be interacted with to determine surfaces, their gradients and collisions with them, the point cloud of input data has to be transformed into a set of non-overlapping shapes. This process is referred to as tessellation and can produce sets of polygons of various sizes and shapes, or sets of specific shapes such as triangles, which is also known as triangulation. As all polygons can be broken down into submeshes of triangles, both paths of modelling are closely related. It is preferred to use triangles for rendering large interactive terrains due to the simplicity provided through the uniform number of vertices and neighbours. There are numerous ways to join any given set of points and hence there are many methods of tessellation, however one of the most 
widely used and thoroughly tested is the technique put forward by Boris Delaunay in 1934[19]. Delaunay's approach has had much mathematical scrutiny and has been found to produce quality meshes which are excellent for the purposes of graphical modelling.

\section{Delaunay}

Delaunay tessellation is a well established method of turning any arbitrary model in two or three dimensions into a set of triangles or tetrahedra, respectively. The act of transforming a set of points/vertices into a set of non-overlapping triangles or non-intersecting tetrahedra which fully embody the model is certainly not a simple one. A vast number of possible combinations/permutations exist and as such Delaunay tessellation is based on a number of mathematical properties. A benefit of Delaunay triangulation is that due to the mathematic principles involved, it attempts to maintain optimal internal angles for triangles and tetrahedra which are hence produced in more uniform shape. As such, two dimensional meshes are usually devoid of slivery triangles, however in three dimensions slivery tetrahedra may still occur dependant on the terrain. At the core of the Delaunay tessellation is the feature that the defined set of edges is the dual of the Voronoi graph for the input points. A Voronoi graph being a set of non-overlapping cells, each position around a point such that any position inside the cell is closer to the enclosed point than any other point within the set. Following on from this property, no point may lie within the circumcircle or circumsphere of a triangle or tetrahedra. This characteristic makes the incremental addition of new points much simpler, as it provides a test for determining which triangles/tetrahedra are affected by the new point's insertion and thus identifies which set of points needs to be re-processed to maintain a valid Delaunay tessellation. A number of algorithms exist to build Delaunay tessellations in two or three dimensions, with the principle applying to higher orders as well but with the complexity of ascertaining the validity of tessellation and hence the complexity of creation greatly rising with the inclusion of each additional dimension. A popular method of constructing Delaunay tessellations is the Bowyer-Watson algorithm, which starts with a large all encompass- 
ing triangle/tetrahedra that tessellates as points are incrementally inserted within it[20]. There are two criteria which must be maintained for validity, firstly that no degenerative cases exist whereby a loss of precision leads to ambiguity with respect to whether additional points exist on a circumcircle/sphere. The other criteria requires that the polygon/polyhedron created by the set of affected points must be 'point convex', whereby the points/edge form a convex hull. A convex hull being when the hull or boundary enclosing a set of points is convex and has no regions within it not being covered by triangles.

A modification to Delaunay tessellation is when a set of edges are given which must not be broken or removed. Because of the restrictions imposed by this, this is referred to as a constrained Delaunay tessellation. Constrained tessellation further increases the complexity of tessellation and as such is currently only possible with two-dimensions, as an equivalent method for three has not been discovered. The benefit of these constrained tessellations would be having them coupled with edge detection algorithms, which determine the presence of edges from visual input. The lines found by the algorithms would help to ensure a greater accuracy in the depiction of the environment [21].

\subsubsection{Data culling}

To ensure the quality and accuracy of models, a large quantity of points are required. However, the amount of data required to gain confidence in the accuracy of a model and subsequent alteration to it, can be excessive in terms of data storage. In order to reduce the resources used, selective filtering of points is necessary in judging which to use and keep/insert into the model.

Dependant on the terrain being viewed, the data or groups of points within the data, may be densely grouped when faced with an upwards slope or an obstacle is nearby while a downwards sloping surface or being at the top of a ridge/cliff may result in the points being spread over a greater area. To ensure having enough data about a region in the latter case, the sensor/scan increments need to be smal$1 /$ fine and hence for the former case there is an excess of data, which needs to be culled. Eliminating points based on proximity, in order to produce a more 


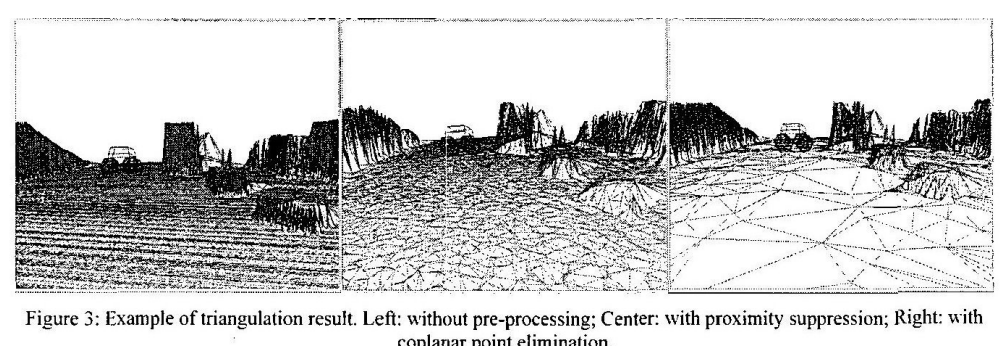
coplanar point elimination.

Figure 2.10: Comparison of a terrain model against results from applying data culling techniques [22]. Left: without pre-processing; Centre: with proximity suppression; Right: with coplanar point elimination.

desirable level of fidelity/resolution, is one method of culling the data which is very effective. Large flat surfaces may potentially exist within a terrain, with little value being provided through these being tessellated by numerous triangles, so combining neighbouring triangles which exist within the same plane, can help reduce the number of points used within a model. However, establishing with certainty whether a surface is flat and whether modelling it with a few large triangles is accurate, is difficult as it may only appear so due to the presence or combination of, sensor error/accuracy, over zealous data culling or occlusion of points due to angle/position. Sensor errors/noise can also conversely result in a flat surface being modelled by triangles which are not coplanar, so tolerance/precision factors must be involved with the removal of data, which thus provides another aspect of the modelling and culling which may lead to erroneously judging a surface to be flat, or potentially judging a coplanar region to be larger than it is. Figure 2.10 demonstrates the benefits of applying pre-processing data culling methods to a terrain model, with the frames (going from left to right) containing progressively fewer triangles while producing a model of the same terrain. The effect of eliminating points which are coplanar is particularly noticeable for the large flat region in the foreground of the models.

In addition to culling received data so as to minimise the resources allocated/necessary for producing an accurate terrain model, further filtering and analysing already held data to limit the size/expanse of the terrain model, provides further 
benefits. Having survived the filtering upon initial reception, data culled at a later stage will have had some value, so attempts to extract/acummulate/concentrate that value into less data may occur, rather than simply discarding/deleting data.

Relaxing the conditions/thresholds of retention of coplanar points, when the points are outside a distance of likely re-encountering/interest such that groups of coplanar faces/triangles are recombined is one such method of decreasing the quantity of data without significant impact on the quality, however dependant on the model or method of tessellation, removal of points may be processor intensive.

\subsubsection{Simultaneous localisation and mapping}

“The 'solution' of the SLAM problem has been seen as a 'holy grail' for the mobile robotics community as it would provide the means to make a robot truly autonomous [23]."

Simultaneous localisation and mapping (SLAM) is an area of research which attempts to use relative measurements of the terrain to both map the region and determine the robot's position within the terrain. As building an accurate terrain model requires knowing the location/relative position and localisation requires accurate maps, SLAM is somewhat of a 'chicken and the egg' conundrum. The eventual aim is to be capable of handling the 'correspondence problem', which involves being able to identify/recognise a set of points common to two or more views/images of the same object/area. In the case of mobile robots this means identifying previously encountered terrain when approached from a different angle and position, by comparing the held map/model with the current view/data. A simple example of the goal for this field of research is the idea of a robot entering a previously visited room via an alternate door, such that the room and contents are now being viewed from the side rather than front, recognising that it had been there and hence both determining its location and further improving/building upon the terrain map. When applied in two dimensions online scan matching which compares held data with scanned data to identify patterns in order to estimate the robot's position is not too difficult. However, when applied to 3D terrains it becomes much more problematic as the positioning (height) and orientation(incline) 
of a robot has to also be accounted for [11].

While this project does not aim to solve the SLAM problem, the style of map building is similar to other autonomous systems such as the type created for this project and highlights the kind of issues which may be faced when mapping a terrain using relative measurements. Maps are built which revolve around the geometrical relevance/consistency (shapes, size, spacing etc.) of the terrain and being as accurate as possible in this respect, as opposed to identifying topological features such as altitudes/contour lines which are more relevant to large scale approximate mapping. Over time, as features are observed from various positions, the accuracy of estimates/modelling increases. Because all measurements within the model are relative, it is not just the re-observed features which improve but also other previously viewed regions, as the affect of the improved estimates propagates throughout the model. This propagation/convergence, means that localised maps provide for high levels of accuracy in terms of relative measurements within a model, however they can lack accuracy with regards to where they believe they, and the terrain, fit on a larger scale, with Figure 2.11 demonstrating this characteristic. Potentially, scanning matching with maps from other robots maybe necessary to reconcile global positioning (as mentioned in swarms/ad-hoc networks). An issue which can be present when using relative positioning to build maps, is that the environment may alter over time and the disparity between the held model and real terrain can flow through to affect the robot's localisation. Using a probabilistic approach allows for variance and subsequently adjustment over time, allowing the model and localisation to eventually converge with the (newly altered) real terrain. For non-SLAM based systems it may be necessary to instead discard all the data and begin again or to have some measure of tracking trust/confidence about regions.

\subsection{Path Planning Algorithms}

There are multiple types of path planning, which are commonly split into two main categories. The first category is topological planning, which is when the terrain 


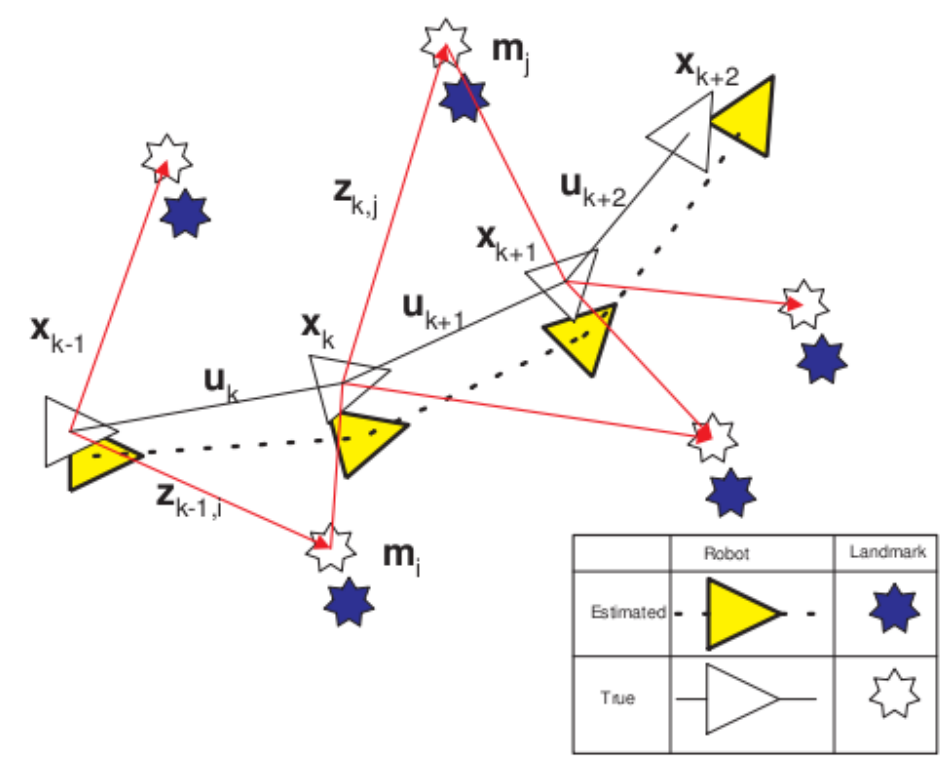

Figure 2.11: Diagram highlighting the accuracy of measurements within a model but variance from real world locations.

to be crossed is known in advance. In most situations this is the preferred style as it produces shorter paths. However, in exploratory applications, topological planning is not possible, so a second category known as Dynamic planning or Sensor Based planning is used. Dynamic planning is where little to no data are known a priori and thus this style can result in substantial back-tracking due to encountering dead ends [24, 25, 26, 27].

There are many different algorithms which can be used in path planning to meet many different objectives [28, 29]. Some algorithms are very simplistic and designed to find any route through an environment, while some aim to find the perfect path with no consideration of memory size or processor limitations. There are also those in between which make different concessions in order to fit different limitations or desires. An algorithm which tries every combination of points is called a Blind Search and will find a path but can take a long time to run as, "For hard combinatorial problems the search fringe often grows exponentially with the search depth [28]." Heuristic algorithms use one or more predefined criteria to find 
acceptable solutions to a problem, an example being comparing sizes and placing larger items in a box first when trying to pack [30]. A related set of algorithms are some metaheuristic ones which are referred to as Greedy algorithms [31]. At each step of reaching a solution, a Greedy algorithm will choose the locally optimal option in the hope that this will lead to attaining an optimal result. For path planning, metrics are chosen so as to compare or estimate potential paths in order to reduce search time by prioritising the evaluation of paths which are likely to be shorter or more optimal. In general, "Heuristic searches will (usually) find ANY path, but will do so faster (usually) than blind search [29]." This has led to a range of heuristic algorithms being adopted by or developed for autonomous navigation, such as A*, Dijkstra's, D* and D* Lite [28, 29].

\subsubsection{A* Algorithm}

The A* algorithm is a heuristic approach to path planning which involves calculating the distance travelled within the network in reaching a node ( $g$-value) and the direct line distance to the goal from that node (h-value). These two values are then combined as the node's f-value and used as a basis for prioritising which potential paths are evaluated first. The $\mathrm{A}^{*}$ algorithm is best suited to quickly generating reasonably optimal paths in known environments, requiring less processing time than many greedy algorithms. The $\mathrm{A}^{*}$ algorithm has the benefit of being able to recover and choose less optimal intermediate steps if a locally optimal choice does not lead to any solution. Due to the nature of the algorithm, any changes in the network require full reprocessing of the planned/potential paths [32, 33].

\subsubsection{D* Algorithm}

One adaptation of $A^{*}$ is the Focussed Dynamic $A^{*}\left(D^{*}\right)$ algorithm which is designed to accommodate changes in the potential path network and as such reduce the amount of recalculation which occurs on subsequent searches [34]. D* can be considered a reuse approach, whereas $A^{*}$ purely involves replanning, thus making $\mathrm{D}^{*}$ more suitable for environments in which dynamic variations are likely. 
A key difference of the $\mathrm{D}^{*}$ approach is that it works in reverse to $\mathrm{A}^{*}$, finding paths from the goal to the current location. This reversal is especially beneficial when alterations occur nearer to the current position than to the goal, as the need to recalculate values is propagated from the point of change back to the current position [34].

\subsubsection{LPA* Algorithm}

Another approach which looks to modify $\mathrm{A}^{*}$, in order to minimise reprocessing when changes are encountered, is the Lifelong Planning A* (LPA*) algorithm [33]. This speed enhancement is achieved through combining the original A* algorithm with an incremental search algorithm. The particular incremental search used within LPA is the Fixed Point Problem version of the Dynamic Strict Weakly Superior Function (DynamicSWSF-FP) [33]. Much like A* reduces processing through prioritising which paths are more probable to lead to an optimal solution, the incremental search identifies which paths are relevant to the modification such that only they are reprocessed.

The means of identifying which paths should be prioritised for recalculation, is the introduction of a new variable called the rhs-value. The rhs-value is the minimum value produced when the neighbours of a selected node, have their gvalue summed with the distance between the node and the neighbour. This value acts as a one-step look-ahead and can thus can be used to determine whether the node's g-value is no longer consistent with its surrounding neighbours. If the rhs is lower than the g-value then the corresponding neighbour becomes the node's predecessor or if the rhs is higher then the g-value of the node is made infinite and the local area needs to be evaluated. Nodes being evaluated get placed on a stack, in order of $[\operatorname{Min}(g, r h s)+h) ; \min (g, r h s)]$. Eventually a path to the goal is found and made consistent, any nodes left in the queue are left with infinite g-values and not recalculated as they are of non-consequence as their f-value is guaranteed to be higher than paths around them $[33,35]$.

If changes occur close to the position of the robot, then the LPA* algorithm can be worse in terms of processing than the $\mathrm{A}^{*}$ algorithm. This scenario requires 
extensive updating and re-analysis in conjunction with the propagation of updated values, which is more complex and can be more time consuming than analysing the whole network with reset values.

\subsubsection{D* Lite Algorithm}

Building upon the LPA algorithm's vision to combine a heuristic algorithm with an incremental search algorithm, the $\mathrm{D}^{*}$ Lite algorithm enhances $\mathrm{D}^{*}$ so as to further reduce processing time [35]. $\mathrm{D}^{*}$ lite is a derived version of the $\mathrm{D}^{*}$ algorithm, with the two having a relationship similar to the one between the LPA* and A* algorithms. With $\mathrm{D}^{*}$ Lite the program flow is much simpler and the processing involved in path analysis is substantially less than the original $\mathrm{D}^{*}$. One enhancement over LPA* is that the priority queue requires less reprocessing to reorder as values held within are relative, so values added when the robot is in a different position can be equated on equal terms to those older values. An improvement upon $\mathrm{D}^{*}$ is that only one tie-breaker is necessary to judge nodes of similar value during comparison [35].

\subsubsection{Multi-resolution Field D* Algorithm}

As many implementations of the aforementioned heuristic algorithms are based on a uniform grid of nodes, there is the difficult task of balancing the resolution, and hence accuracy, of the model versus the required data storage and processing for a set number of nodes. Without moving to a fully flexible unconstrained network of nodes, some improvement can be made through allowing varying resolution levels to be used in different regions [36]. This modification to $\mathrm{D}^{*}$ allows areas of greater danger or interest to be held/modelled with more nodes than regions which have a more homogenous and more easily modelled shape. In addition to producing networks of nodes which provide equivalent quality at reduced resource cost, in some case the paths generated are more optimal. The improvement in the paths lies with there being a greater range of paths/angles which may occur between two points as is shown in Figure 2.12 [36]. 


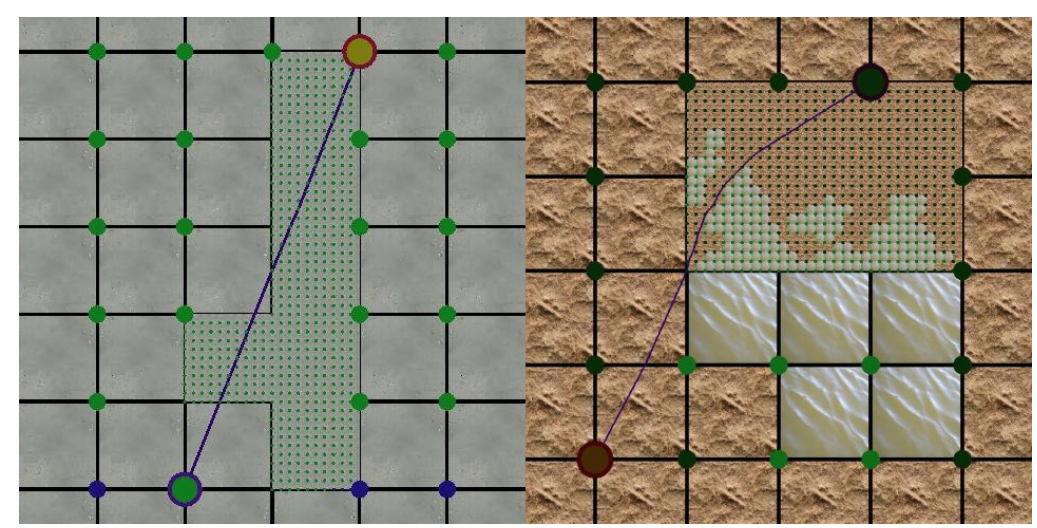

Figure 2.12: Multi-resolution Field $\mathrm{D}^{*}$ produces direct, low-cost paths (in blue/dark gray) through both high-resolution and low-resolution areas [36]. 


\section{Chapter 3}

\section{Simulator Design}

With research into USAR robots and the testing of designs often being very time consuming and hard to do in a realistic manner, virtual simulations are necessary. The purpose of this simulator is to provide an open modular platform on which researchers can simulate the response of different robots and path planning system under various scenarios. To be of maximum benefit in understanding the behaviour of a robot in these situations, the simulator provides a number of means through which the movement of the robot can be visually displayed and as such transfer situational awareness to the researchers. The irrlicht graphics engine, which is described further in Section 3.1, not only provides the visual front-end component of this system but also provides realistic input to the robot. The design of the system is tailored towards the use of a single robot tasked with reaching a given position. For instances where the goal is to cover as much area as possible and thus expand the mapped region, this simply requires the path planning algorithm to dynamically choose and adapt/alter the robot's destination. The system would also be capable of simulating multiple robots on the same terrain with some very minor modifications to the code, such as creating more robot objects and converting the current robot pointer held by the simulator to an array of pointers to the multiple instances.

The inter-relation of the objects used within the simulation program is shown in Figure 3.1. The overall control of the simulation lies within the Simulator 


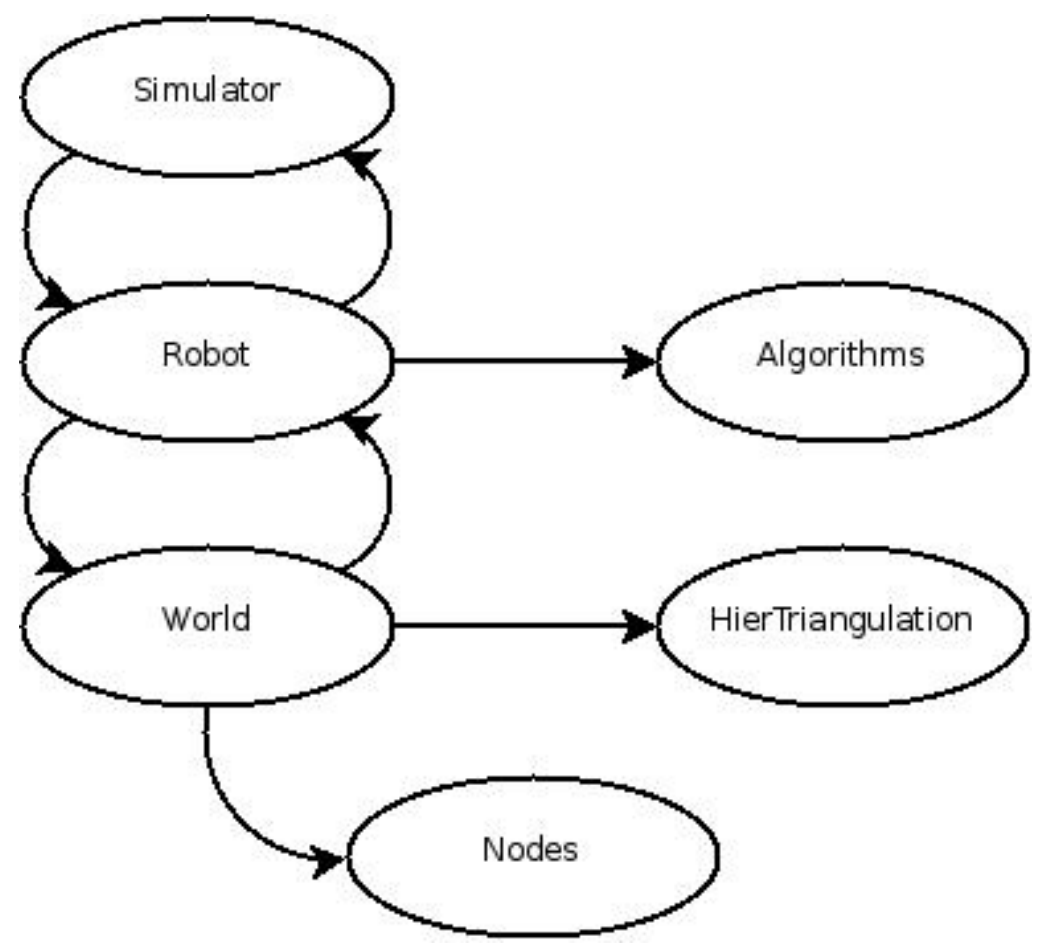

Figure 3.1: A UML depiction of the interconnection between objects in the simulation.

object, which setups up the graphical elements of the simulator and controls the calls to functions within the Robot object. The Robot object is tasked with passing the data generated by the Simulator, to the World object which creates an internal model of the terrain. The process of moving the robot within the simulation is also carried out by the Robot object based on the path planned by Algorithm functions. The terrain model created by the World object uses the CGAL library, and then after analysis of the model, sets of Node objects are created. The purpose of the Node objects are to provide a simplified and reduced network with which the path planning can work to produce potential paths to follow. The full source code for this project can be found in the Appendix, with pieces of pseudo-code being given within this chapter in order to illustrate the nature and operation of functions. 


\subsection{Simulator}

Other simulation undertakings have focussed on the user interface and the replication of specific environments, for testing of tele-operation against standard benchmarks such as NIST USAR Arenas [37]. Human-Robot interfaces have also been of interest with real world tele-operated or semi-autonomous robots, where increasing levels of freedom, sensors and data can lead to cognitive overload for an operator [38]. However, for this project the focus was more on the methods of interaction of the robot with the simulator. As such the simulation environment was mainly an abstract and modular base on to which specifics could be added or altered, with the human interfacing with the robot being superficial, simply providing visual feedback on general navigation such as data relating to hazard identification, path planning and the path implementation/chosen.

In addition to the shortened development time of using a pre-made graphics engine for simulation, the game-like nature of the graphics engine gives familiarity to allow easier understanding of behaviour and also lends itself to later interface interaction/design. The particular graphics engine chosen for this project was Irrlicht [39] as it is open-source, cross-platform and was found to be easy to learn and use. The simulation consists of three camera views, of which one is a static bird's eye view and the other two are dynamic. The dynamic views allow for a better sense of what the robot encounters, with one following the robot's view and the other being available to the user to navigate in order to watch the robot from different locations and perspectives. A greyscale bitmap is loaded by the simulation as a heightmap, from which the graphics engine creates a terrain mesh onto which additional obstacles or items may be added. This terrain mesh takes the place of the real world and functions both as the basis for the graphical representation so a user can see the environment and also as the model of the real environment, with the simulated input being derived from this model. Figure 3.2 is a screenshot of the simulation from basic obstacle avoidance testing and shows the robot, modelled as a car, approaching a section of terrain which is littered with a number of large insurmountable grey objects. A translucent white marker 


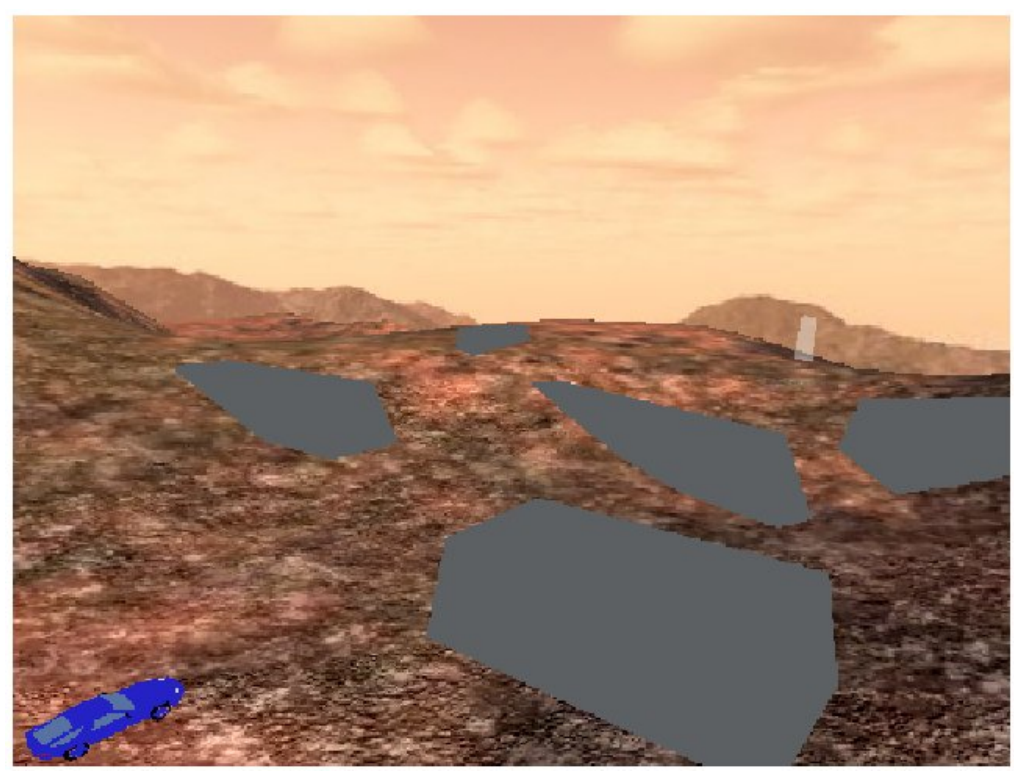

Figure 3.2: Simulator screenshot depicting Robot approaching terrain containing obstacles.

is visible, the purpose of which is to indicate to a user where the robot's goal is located. Due to the resolution of the images and the texture/material applied to the obstacles, it is hard to clearly identify the vertices and edges of the obstacles from Figure 3.2. However, through the robot's perception the position of vertices and edges, and hence the exact shape, can be easily detected if the areas were to be scanned.

\subsection{Modelling LIDAR input}

The common method to build up a set of LIDAR measurements for a position, is to alter the direction and inclination of the laser in fixed discrete increments using pivoting points and mirrors. The result is that these points are dispersed/spread radially and as such the method through which the simulator scans the terrain and provides data to the robot needs to reflect this. Additionally, if the laser does not intersect a surface within a set range then no measurement can be produced, as 
the signal strength upon return would be too weak to detect.

An alternative method for collecting LIDAR measurements involves using a lens to disperse/spread the laser beam and then the data points are taken through sampling discrete points in the sensor behind the lens. Due to the lack of moving parts this provides a more robust input method, suitable for USAR purposes. However, both cases can be modeled in the same way.

The number of input points and their angular dispersal, will depend on the increments by which the laser is adjusted and through which range/arc, or by the lens shape and sensors.

The scanning of the terrain was designed to produce points which emulate the kind of dispersal expected from light passing through a lens, whereby points will be closer spaced in the centre and become less so at the periphery. The range within which data points can be detected has been designed to be variable so it can be set according to any specific sensor, as can the dispersal of points.

Having the horizontal increments being fixed across all vertical increments meant greater spread at edges. The vertical increments increased in sized as they spread out from the sensor in order to cover a greater range but avoiding having to deal with too many data points. This choice of increasing increments as opposed to fixed ones, could be considered the first stage of culling data. This accounts for the affect of vehicle incline due to the variation in gradient of surface at the times when it gathers more data, plus when terrain in front of the robot is sloping up or down significantly. The downward view for close points is particularly important as it is necessary to detect steep drops in front of the robot. It is less important to obtain many points in the upward view as it will usually only produce data when the robot is angled downwards and facing a nearby rise.

An offset to account for the camera's height with respect to the simulated terrain was necessary (since the position coordinates held by robot relate to the point of contact on the terrain surface) to get downward/nearby points for flat or declining planes, otherwise only obstacles will be detected.

The retrieval of scanned input points occurs at regular/periodic intervals, specifically after each movement. The simulation has the robot pause when it gathers the 
new data, because this was seen as a safer options because it means the robot does not risk hitting a hazard while it analyses the data and choose the next movement. This stop-start technique may introduce some jitter, however scanning while moving would also be likely to produce jitter as well as the robot rocks and sway while crossing variations in the terrain.

\subsection{Terrain Modelling and Data Storage}

A key part of navigation is the model used to represent the environment; how is data for it gathered, how it is interpreted, what data points are discarded or kept and when is the decision to remove them made. Many different methods of representing the terrain and storing the data were considered because "If you get the data structures right, the effort will make development of the rest of the program much easier [40]." The most simplistic 'model' is to only consider that which is currently being viewed and involves constantly determining if what is visible consists of traversable terrain or hazards/obstacles. This situation is comparable to a human with absolutely no short term memory. At the other end of the spectrum is building up a near perfect model, taking advantage of a robot's precision and recall. Though simplistic, the first model is highly adaptive and can easily handle random errors in the input data as well as issues such as slipping or sudden dis/reorientation. A detailed robotic model may be able to successfully distinguish errors in input but this will require a significant amount of processing and comparison with input points around the error. The issues of orientation and position are extremely difficult for a precision model to overcome, requiring immense time to process or leading to substantial errors in judgement.

These different methods require different forms of "memory" or data storage. A current view model requires virtually no memory whereas a precision model may require large amounts of memory in the form of lists or multidimensional arrays of data.

A simple model is more robust against errors and sudden dis/reorientation and might overall use less resources (processing \& memory), however a precision 
model is more likely to give optimal results. The level of detail and complexity one chooses is important as it impacts on the design of the path planning algorithm in how much data it will have to base decisions upon, how adaptive it needs to be, and how robust against error it must be made.

In computer games, terrain models tend to be meshes of non-overlapping polygons as this method cuts down on the amount of memory needed to store similar amounts of detail and also lowers processing and search times. As any polygon with three or more sides can be represented as the summation of multiple triangles[41], meshes often consist solely of triangles, as the uniformity of shape aids simplicity to the design of functions or algorithms, especially in computational geometry. Using simple compact structures is also beneficial for hardware rendering of graphics.

Work with search algorithms has more commonly involved a priori data and often glossed over the practicalities of collating and analysing data, such that environments are frequently modelled as perfect grids with details abstracted to being traversable or not and having uniform distances between them irrespective of the affect of heights [33]. This type of modelling is beneficial as no separate network of paths needs to be generated as the model in of itself provides that functionality and in a very simple manner as only a fixed number of neighbours can exist and in preknown locations, the only variance being in whether they are considered traversable or not. However, the practicalities of attaining such a uniform/regular grid model are not realistic for a dynamic environment due to a number of factors. One such factor is the non-linear spread of angles through a lens meaning a single lens can only ensure the $\mathrm{x}$ or $\mathrm{y}$ component of data taken directly in front of it, with all other data points occurring radially from the point of view and their distance being dependent on when they encounter a rise/object in the line of sight. Regular and precise movement would also be required in order to build up a uniform grid of data points. As such, a regular grid model is best obtained in advance and from an aerial unit or extensive coverage by ground units, which removes the dynamic nature of the situation.

In addition to being incompatible with dynamic generation from a single ground 
robot, there are two key drawbacks to the grid network. Firstly, the resource requirements associated with this method of modelling increase rapidly with limited/little gain in information in response. Secondly, the potential paths drawn from a grid model are also limited, with only eight directions of movement and are not optimised or specific to the presence of obstacles in different terrains.

It should be noted that the terrain model which a robot requires, representing its understanding of the world, is distinctly separate from the terrain model produced by the simulator. For the purpose of computer simulation, the simulator's terrain model replaces the real physical world.

\subsection{Tessellation}

Delaunay triangulation is often used as the means of turning a set of points into a mesh of triangles. Unfortunately the complexity of the mathematic processes involved, when applied to more than two dimensions, meant that an implementation could not be programmed from scratch. Suitable open source libraries could not initially be found within a reasonable time frame which meant that a less elegant method had to be coded.

The initial concept was to use points which were nearest neighbours to form triangles, however it was quickly realised that this was not a suitable approach. Creating an optimal tessellation would require discerning, for any given set of points, how many neighbouring points should be linked to a specific point and how their angular spacing should affect their choice. Thus this method would likely be almost as mathematically complex as the Delaunay approach but without the vigorous mathematical analysis and testing Delaunay's technique has received due to prominence and usage.

\subsubsection{Iterative case-based tessellation}

The initial attempts at the nearest neighbour based tessellation involved considering the possible combinations of triangles which could form around a single point 
and how triangles could potentially overlap. This led to looking at the finite number of ways in which two triangles may interact in three dimensions, which subsequently developed into a case-based approach of iteratively going through the potential combinations such that a triangulation was formed devoid of overlap. The idea was to consider every combination of points and discard ones which encompassed another point, formed a line or slivery triangle, or overlapped the triangles bordering the cavity being tessellated, as outlined by the pseudo-code of TessellatePoints (Algorithm 3.1). Empty triangles were then compared to each other in the ProcessEmptyTriangles() function, given as pseudo-code in Algorithm 3.2, which subsequently calls CompareTriangles() (Algorithm 3.3), to choose triangles which were of a better shape and thus formed a better mesh, as well as determining whether an overlap occurred between triangles. During these comparisons triangles may have a status flag set to identifiy them as being blacklisted due to a better triangle overlapping them. TessellatePoints() calls ProcessEmptyTriangles() twice, so it can reconsider triangles which were blacklisted the first time through, to ensure no holes exist in the mesh as triangles which (through comparison) may have lead to another being blacklisted, may have later been blacklisted themselves. The quality of shape was determined based on the internal area of the triangles compared to the distance of the vertices from the centroid (equilateral triangles being the best and the worst being long thin ones). Testing for overlap between triangles was split based on the number of vertices two triangles had in common. For two points in common, the primary test was seeing if the additional point of each triangle was on the same side of the shared line, thus indicating overlap. When there were no common points between two triangles, the edges of each triangle were tested for intersections with the other. In the case of a single vertex being shared, edge intersections were also tested, comparing the edges off the vertex to the edge of the other triangle adjacent to the vertex. Each of the different overlap testing functions, would then decide which triangle to keep based on internal results or if the way the triangles intersected did not indicate a superior triangle, then size and shape were used.

The mathematics used in the different functions employed a top down, XY- 


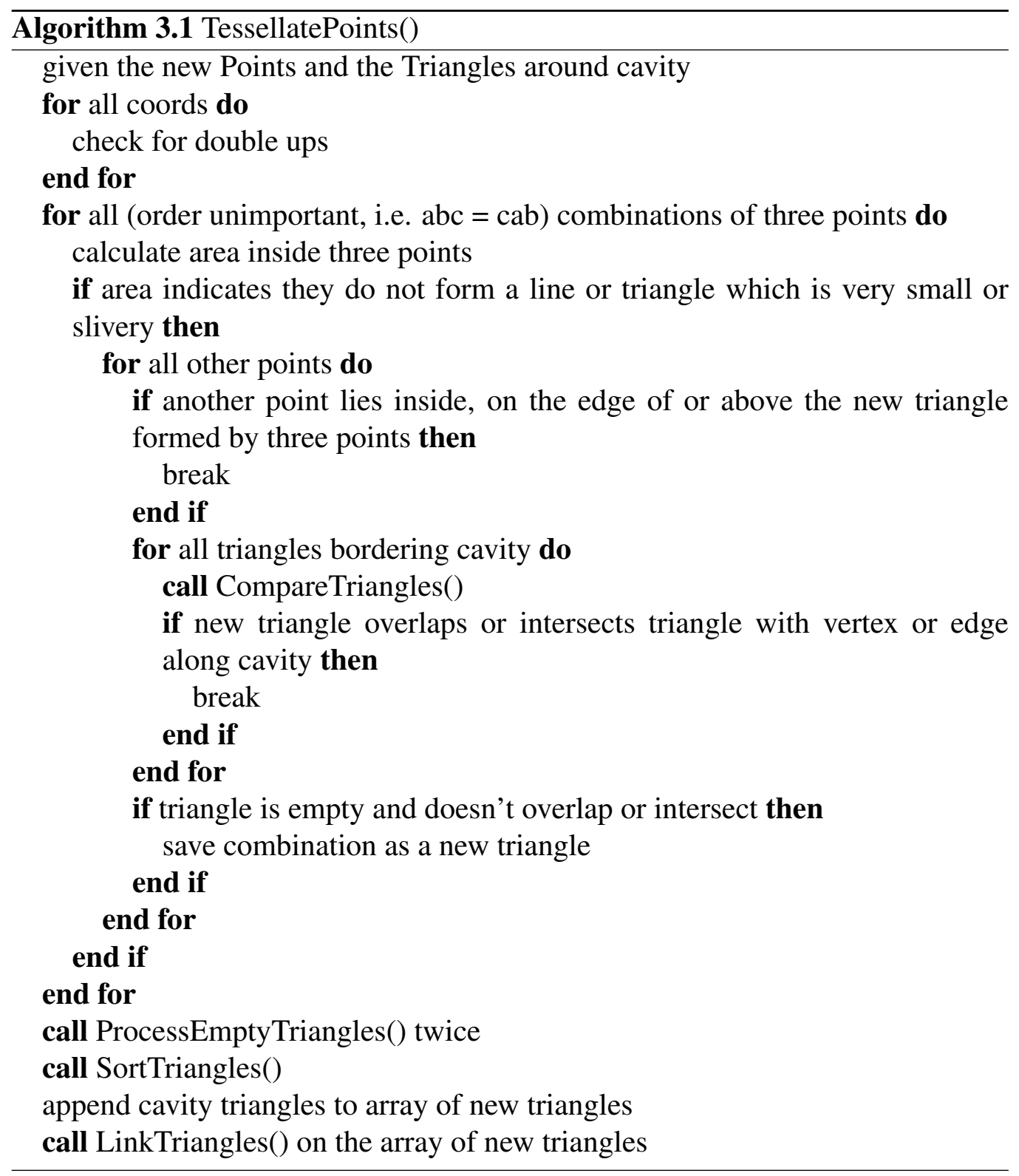




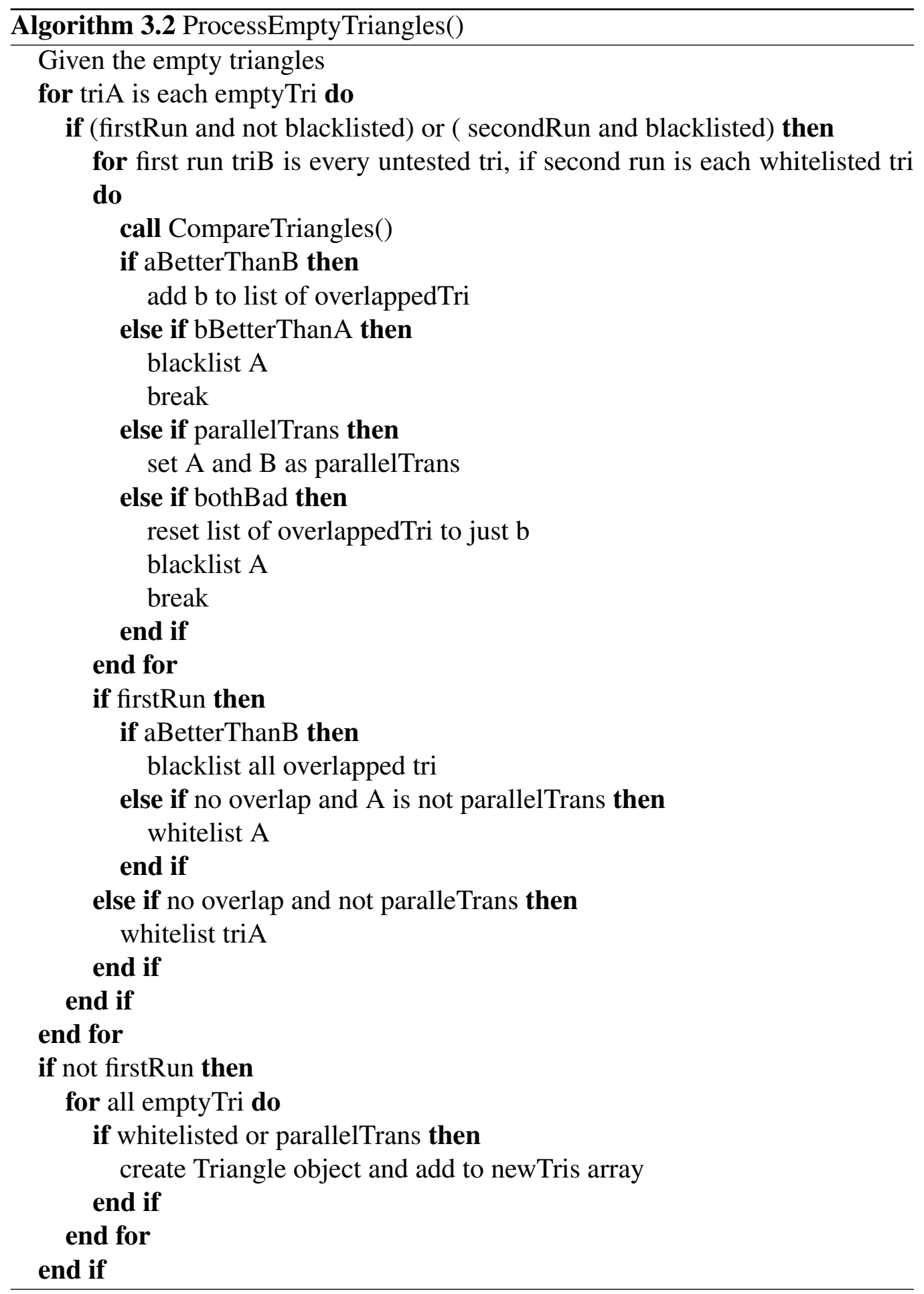


axis approach, to identify overlaps and to judge validity. Triangles purely in the vertical plane caused numerous issues and required many small modifications to be made as new scenarios in which they altered results were detected during testing. Due to the more complex situations which could be encountered with triangles which were perfectly vertical, when encountered during comparison they were skipped unless they were being compared to another vertical triangle, in which case they were temporarily transformed to the XY-axis so tests for overlapping could occur. In all other instances vertical triangles were omitted from comparisons until the linking occurred. Vertical triangles could be erroneous, generated off the sides of obstacles and hence expanding their impact on paths, when a point at the base of the obstacle and another point higher up on the obstacle happened to form a line in the XY-plane with a point on the ground. As shown in Figure 3.3, these triangles can be identified through having no neighbour along the top (skywards facing) edge but having two neighbours on each of the other edges, with labels $\mathrm{A}$ and $\mathrm{B}$ showing the two neighbours of the pink triangle along its bottom edge. When the triangles were all being linked, vertical ones were not considered until the very end (having been placed at the end of the array by SortTriangles()) which allowed erroneous ones to be detected easily due to their existence bordering a ground triangle which already had a neighbour along the same edge. To aid recognising valid triangles the PARALLEL_VERT_TRANS status was used, which indicated that a matching triangle exists in parallel, an occurence common with cubic or prismic obstacles and uncommonly occurring with erroneous triangles.

The inclusion of software to display the triangle meshes rather than reading the lists of coordinates forming triangles and having to picture them mentally or draw them by hand, sped testing up considerably and also allowed white box testing as opposed to just the black box testing of comparing input and final output. Three dimensional displays with a rotateable view would be the best option, however for ease of implementation the Simple DirectMedia Layer (SDL) library was used to display the triangles in the view of the $\mathrm{XY}, \mathrm{XZ}$ and $\mathrm{YZ}$ planes.

The tessellation proved a large stumbling block and mental drain, and as time 


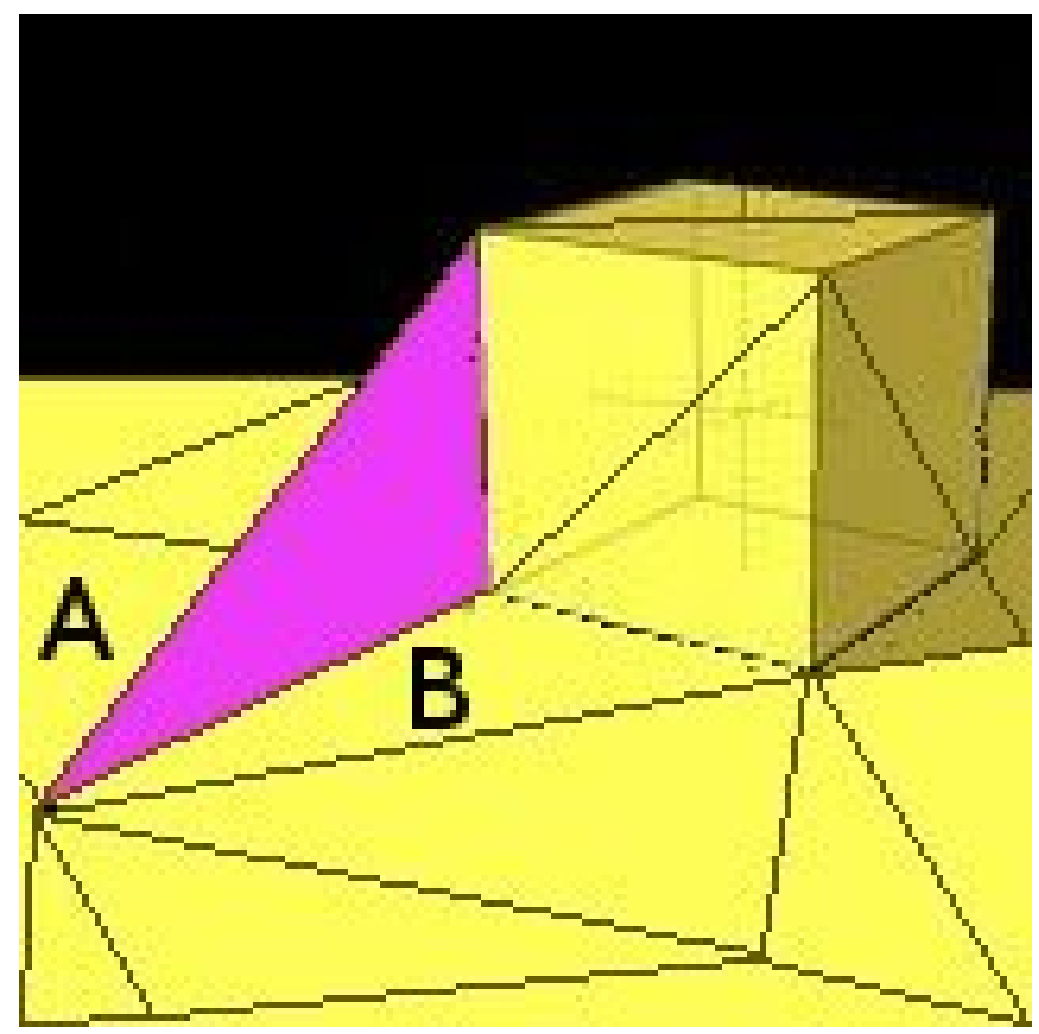

Figure 3.3: An example tessellation of a cube placed a flat plane, with good triangles shown in yellow while an erroneous triangle is coloured pink. 


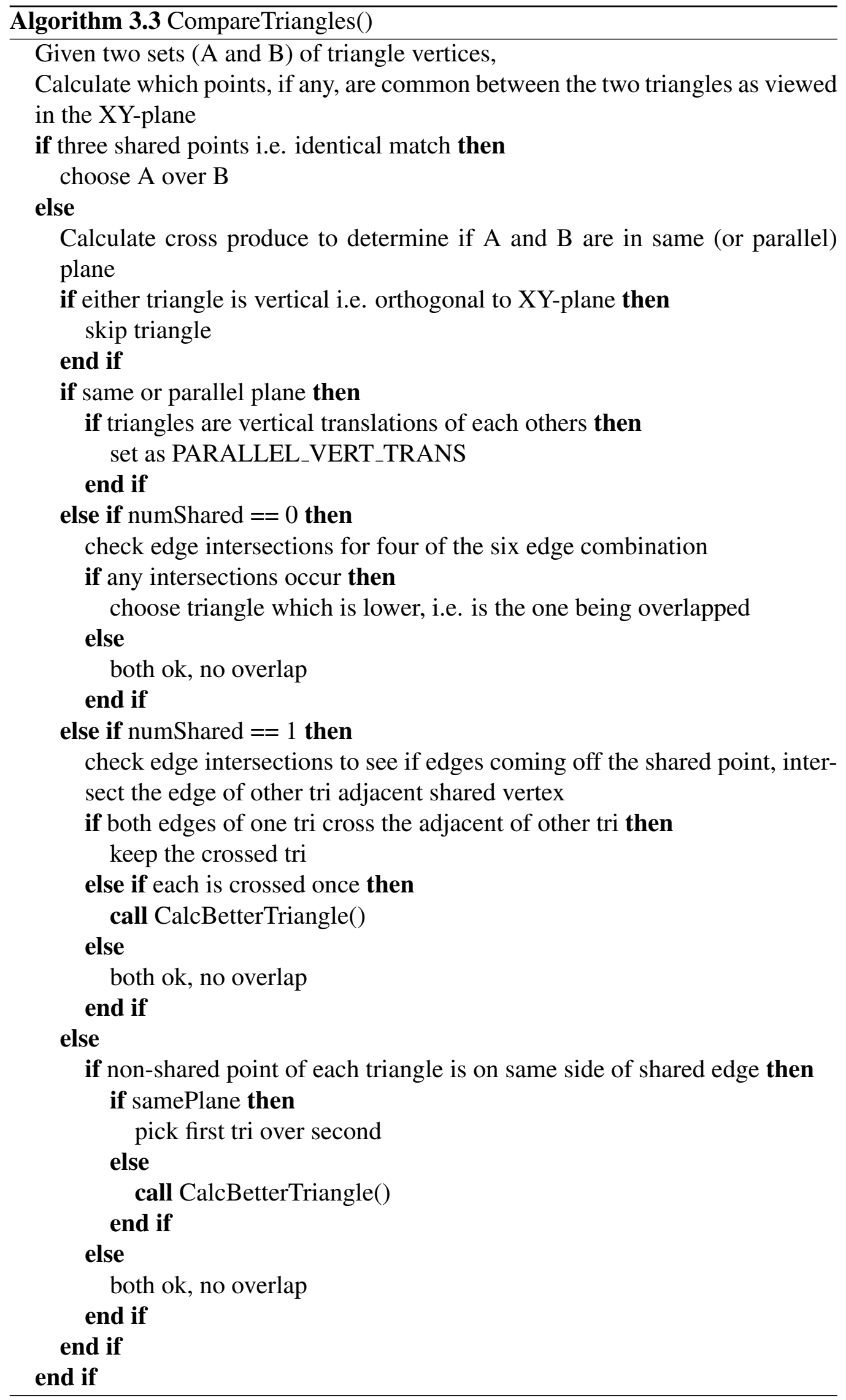


progressed alternative approaches were explored, such as the 'robot' being given pre-tessellated triangle meshes from the graphics engine's model of the terrain. This would remove the effects on the path planning of variance in tessellation as the simulation had very uniform meshes which perfectly modelled the terrain, thus removing some realism but allowing for completion of the project. A compromise of using the pre-tessellated triangles as the input points for the tessellation process proved fruitful as the better dispersal of points meant triangles were generally of a better shape and size, so that errors or invalid choices by the program were more obvious. Being better able to identify issues with the tessellation, the algorithm was eventually completed and was able to handle the original terrain scanning input which was more realistic.

As the tessellation had to be incremental, to minimise processing each time a new set of scanned data was received, for new points lying within known areas it was necessary to identify triangles which would be affected by the new point's addition to the terrain. A cavity within the tessellation was formed by removing these triangles which were affected. This area to be retessellated could be troublesome if it was concave, as new triangles formed through looking at all combinations of points in the cavity, may produce a triangle which partially overlaps triangles outside of the cavity. Keeping track of the triangles which bordered the cavity, in order to test newly formed triangles against them, ensured that there was no overlapping and helped to handle concave edges. Infrequent issues were still observed but with time and testing these were eventually dealt with. Though designed to approach the problem in a simple and quick manner, the iterative casebased tessellation and quest for optimal triangle meshes became a bottleneck in progress.

\subsubsection{Recursive tessellation}

Due to the lengthy processing time required by the Iterative Tesselation Method, ways to increase the speed were considered. At the beginning of this project, tessellation had been viewed as going directly from a set of vertices to a set of complete triangles. After having used 'Edge' objects in an attempt to deal with 
the issue of convex cavities, the value of the intermediate step of creating edges from vertices, from which triangles could be composed, became clear. With this in mind the nearest neighbour approach was revisited, with speed of processing as the primary goal rather than maximising the quality of shape and size.

Starting with an arbitrarily chosen edge rather than a single point, cut down the decisions and processing as there can only be one triangle on either side of an edge as opposed to a point which could have any number of triangles formed off of it. It was felt that recursion was the best method of implementing this approach since the initial edge was branched out from - much like forming a binary search tree [42]. This proved to be much faster to code as there were few possible scenarios that could be encountered during the tessellation, with overlapping triangles being the main concern but being simple and quick to test. With edges keeping track of which sides already had triangles formed on them it was easy to check for overlaps. Also, as the tessellation expands outwards in an approximately geographical sense, it was less likely to encounter points already used, as opposed to going through points based on the input order as was the case with the previous iterative approach. This approach was much faster but often produced a number of slivery triangles in the meshes, which were hard to deal with during later stages of the path planning, such as hazard identification.

One reason why slivery triangles are difficult to work with lies in their poor shape making it hard to determine their angle with respect to the XY-plane. They are also problematic when forming compound hazards from multiple triangles. When expanding the search by moving between neighbours, reaching nearby triangles of a good size and shape may require crossing numerous slivery triangles which could be coming off a single point. This can mean a lot of processing to cover a small region, which over the entire area can added up to a very significant increase in processing requirements. Judging when to halt during this expansion across neighbours can also be problematic as the centroids of the slivery triangles may be outside the region of interest but may be bordering othr triangles of a better shape.

Adding a new point to the terrain tessellation can also take longer. When 
finding the encompassing triangle of a new point, the closest existing point is found and the search begins expanding from there. Though the new and existing points might be within a short distance of each other, there could be numerous slivery triangles between them.

\subsubsection{CGAL based tessellation}

Upon completion of the iterative method and with the recursive tessellation going through final testing, a software library called the Computational Geometry Algorithms Library(CGAL) was found via a paper on mapping terrains, which mentioned using Delaunay triangulation [43].

This library meet all of the criteria which had initially been searched for; being open source for research/non-commercial purposes, written in $\mathrm{C}++$, and doing Delaunay Triangulations in three dimensions.

Utilising this package proved quite difficult due to the vast number of functions provided by the library. Identifying which objects and functions suited the project's application was difficult. The library lacks comments and does not follow good naming conventions, which, given that the code largely consists of templates, meant it was hard to determine the purpose and usage of many objects, functions, and variables. Comprehending a single item often required searching for information scattered throughout the reference manual, which consists of over 3500 pages.

The different Triangulation objects of the CGAL library provide both two and three dimensional triangulations and come in different forms, such as regular, Delaunay or constrained. The regular triangulation simply splits the encompassing triangle when a new point is added whereas the Delaunay tries to satisfy the condition of no triangle's circumcircle encompassing another point, which may require more than just the encompassing triangle to be deconstructed. The constrained triangulations are ones which are required to retain certain edges/vectors and could be of use if future filtering was conducted on the input data to detect edges. The triangulations can be used as a mesh or placed inside a Triangulation_hierarchy object which have an internal structuring designed to allow for faster and more 


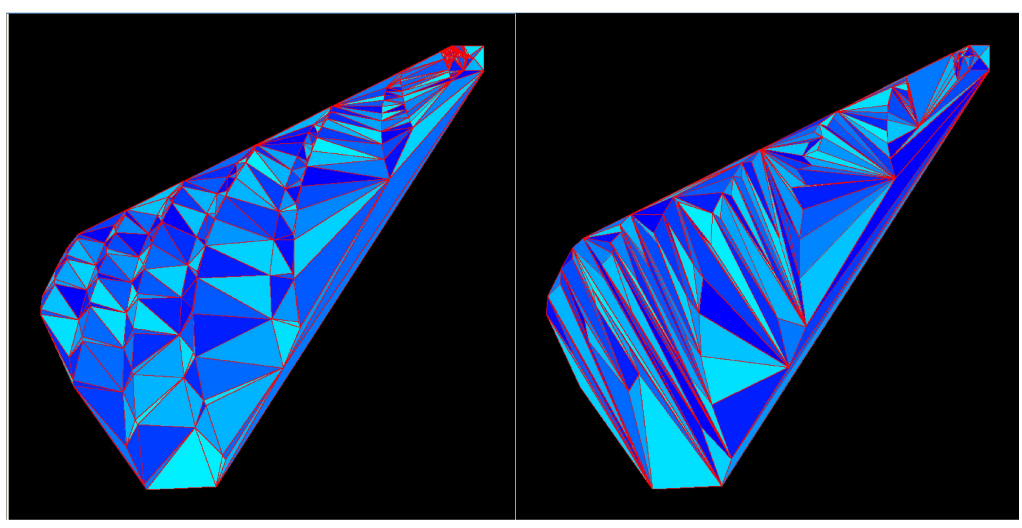

Figure 3.4: Triangle meshes generated by the CGAL (left) and Iterative (right) based tessellations, given the same series of inputs.

efficient point location.

When the resultant tessellations using CGAL were compared to the iterative and recursive approaches, they were found to be of a better overall quality and were produced significantly faster than the Iterative case-based approach. Example triangle meshes generated by the Iterative and CGAL approaches are shown in Figure 3.4, with some similarity being visible between the two but with the Iterative mesh containing numerous undesirable long thin triangles and the CGAL triangles being far more uniform in shape and arrangement.

\subsection{Point Culling}

The volume and quality of input data to a program is a major factor in determining the quality of the resultant output, as even the best processing can do little when only given a small number of good input values or many unreliable values. Though the precision of measurements taken by an autonomous robot whilst gathering data about its environment can affect the quality of input, this is of a fixed nature and can only be improved by upgrading to better sensors. Increasing the accuracy of sensors and attaining more data does improve the quality of output possible, however this can be costly in terms of both the hardware and the 
processing time. To achieve the same high level of quality in a less expensive manner, input data needs to be dynamically condensed down to a smaller set of more valuable data through the appropriate culling of data.

With the terrain data, the preliminary processing consists of checking whether or not a new datum point lies within the known area. Points inside the known region are evaluated with regards to the triangle which encompasses them, looking first at whether the point is coplanar to the triangle or if its height is within an acceptable level of deviation from the interpolated value of the triangle. If it is outside the accuracy tolerance a point is added, and if not then the proximity of the point to other known points is assessed. The red lines in Figure 3.5 show the distances which would be calculated to determine whether point $\mathrm{A}$ is too close to the existing points. For a coplanar point, if any known point is within a minimum distance threshold then the point is disregarded. This means that even if the encompassing triangle is very large, the new point is only added if it is sufficiently far away from all the vertices, for example point B may not be added to the model shown in Figure 3.5. The same action occurs for non-coplanar points which are within the tolerance of accuracy however the minimum distance threshold is half as much. The reasoning for the different distance thresholds is that non-coplanar points immediately affect the terrain, possibly providing new information or possibly being inaccuracies. A coplanar point is added as a precaution, to avoid losing information and becoming more inaccurate in the case where the triangle does not accurately match the terrain. For example, a sudden rise or drop occurring within the triangle's area may appear as a gentle slope if nearer points are not included. This process takes place within the World object, specifically in the AddPoint function, which is presented as pseudo-code in Algorithm 3.4.

As a robot progresses, the value of keeping old data declines. The precise heights of a section of terrain far away from the robot has little to no impact unless back-tracking occurs. Even in cases of reapproaching previously encountered terrain, events may have occurred that have caused the data to be less accurate or perhaps even erroneous. These occurrences could include positional slips or disorientation which may have befallen the robot, or alternately the terrain having 


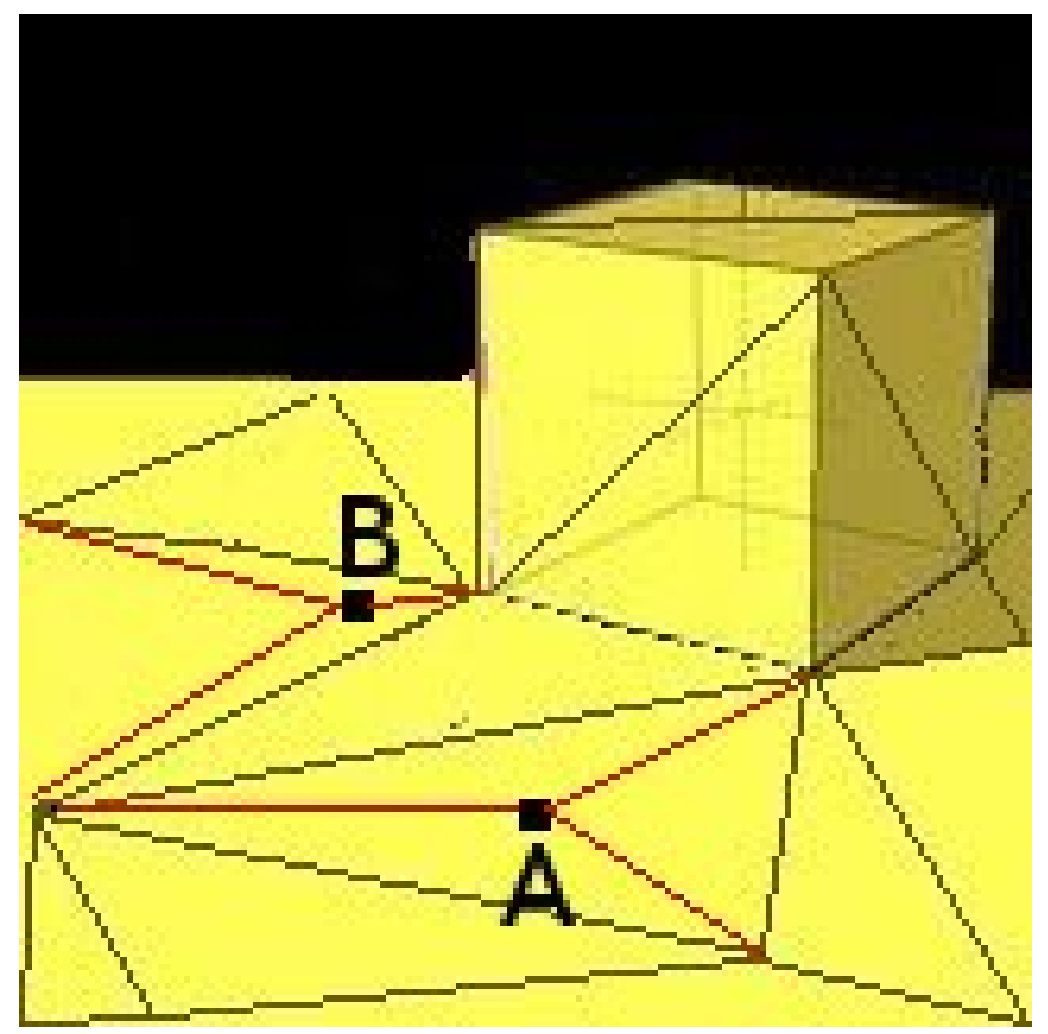

Figure 3.5: An example of comparing two new points, A and B, to the existing triangle mesh in order to determine whether to insert the new points or cull them. 


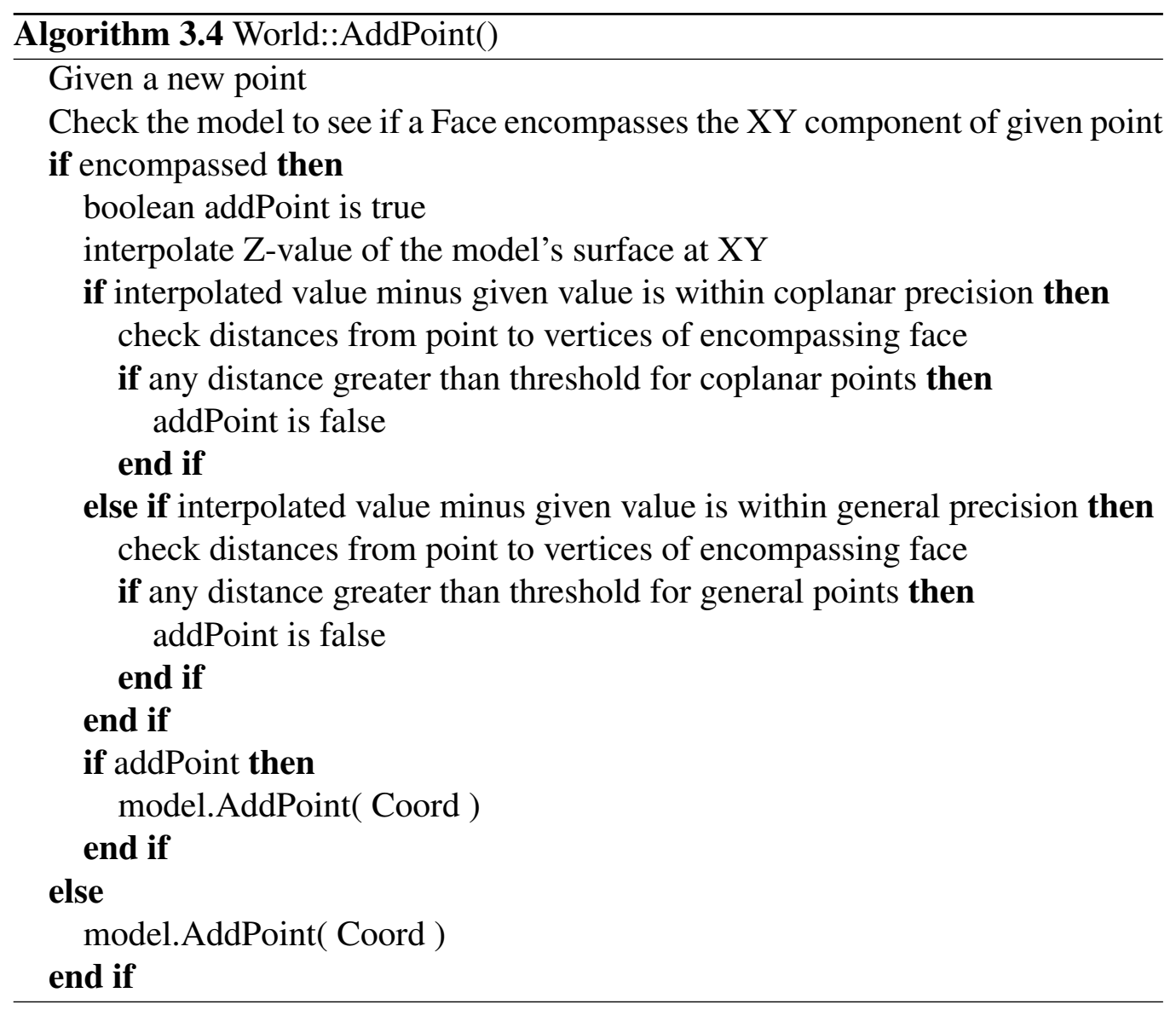


altered due to the movement of unstable rubble or perhaps even the robot's own traversal over it. A means of limiting the resources required by old data such that it is more inline with the potential value it may serve, is to abstract the terrain to a simple dichotomy of being hazardous or traversable. Once specific height data is no longer being looked at, one can view all information about traversable regions as being superfluous as they can be defined as the regions which are not hazardous. Creating simple objects to be retained, which outline the locations of hazards and their general size and shape, while discarding the vast quantity of height data greatly reduces the data storage and processing resources used.

An alternative method of dealing with information loss can be the use of confidence levels, which alter a triangle's associated confidence value when points which lie within it are added or culled. A confidence level indicates the likelihood that the triangle is an accurate representation of the terrain covered, rather than needing to keep a dense coverage of points to feel assured. On top of this, a decay factor can be added which over time will lower the confidence level unless points reinforcing its accuracy are received. This approach is useful in dealing with errors in location or orientation as the terrain dynamically changes over time, meaning the terrain model is more robust as any mismatch between the new data and old data is slowly filtered out. This has not yet been implemented and is recommended for future work, with data points currently being removed based on distance from the robot instead, which is not as beneficial if backtracking needs to occur. For points which lie outside the known area, their proximity to known points it used as a guide to whether they should be added.

\subsection{Hazard Identification}

The hazards a robot faces while navigating a disaster site can be divided into the two categories of static and dynamic hazards, with dynamic ones (such as falling rubble) being harder to detect and avoid. The static hazards such as holes, cliffs, crevasses, and insurmountable inclines which a robot comes across are largely related, with the common factor being the overall slopes and relative angles, which 
greatly reduces the complexity of identifying hazards. Clearances of traversable spaces between hazardous regions can also be a problem causing a robot to get jammed or be blocked, however they are not considered terrain hazards but rather navigational problems.

Dividing static hazards into, slopes which can not be ascended but could be travelled down, and walls which are completely insurmountable, allows some freedom and risk taking in the path planning, as a robot may decide to risk going down a slope it could not climb back up, in order to reach its goal. What is considered a slope or wall is determined by input parameters for each robot, consisting of what angles it is powerful enough to climb, the size of its wheels, and at which angle it would topple or roll. As long as a slope is no longer than the wheel base of the robot or if the height of a wall is less than the the wheel radius, then they can be considered as safe.

One difficult instance to classify is when a section of terrain is at an insurmountable incline, but is not big enough to be considered a wall by and in of itself, and is surrounded by what would be labelled a slope. The overall affect of the section on its surrounds has to be considered to determine whether, in combination with it, the surrounding region should be classified as a wall. There are also problems associated with triangles which are quite large, as an area much greater than a present hazard may appear to be a non-traversable incline due to the model being a poor fit. Additional data which may clarify the status of such areas is unlikely to be received as the robot will likely avoid the region, which in the event of the region being traversable is unsatisfactory as it discards potential paths. Through setting an upper size threshold (hazard_tri_threshold) above which triangles' hazard status are ignored, resolution errors are overcome. If better paths exist they are followed and it is irrelevant whether the sparse region is traversable or not. Otherwise, the region may be orientated or moved towards, and gathering further data will either repulse the robot in search of other paths or show the region to be safe to traverse.

The generalised processes involved in the hazard identification section of the World object, as split across IdentifyHazards and CheckWall, are given as pseudo- 
code in Algorithms 3.5 and 3.6.

Crevasses are an extremely problematic hazard as their danger is based on relative orientation. Depending on the size of the robot's tyres, crevasses can be traversable in a direction orthogonal to that in which they run while they are likely to catch and trap the wheel if they are encountered from an incident angle closer to that direction is which they run. The difficult of dealing with this direction based danger is exacerbated by the fact that identifying crevasses from directions at which they are traversable is practically impossible at a distance, only being identifiable once very close and with sensors aimed sharply downwards such that their line of sight can judge the depth. Approaching roughly parallel to a crevasse, a sensor may be able to ascertain a significant portion of the crevasses depth but is likely to be dangerously close to having a wheel slip into the crevasse before it manages to accumulate enough data points to identify it as a hazard. Since it is not until a robot is much closer that it will be able to identify how deep a slope goes, whether it is a small dip or in fact a crevasse, this affects the quality of path planning and the safety of the robot.

\subsection{Creation and linking of Node Points/Networks}

The decision of which points in a terrain model are the most valuable to the path planning process and how many points to have is highly important. The number of nodes that will be used to generate a possible path network affects computation time at two points. Firstly, the point when the nodes must be tested to decide if they should be linked, and secondly, the point when the navigation algorithm analyses the possible path network to find its estimate of the best potential path. The basis on which the nodes are created is also important in terms of what the risk of encountering hazards will be, and how optimal the paths will be.

Upon developing the simulator towards implementing a greater range of algorithms, it was found that most examples given for more advanced algorithms were based on a simplified grid-based fixed-distance/resolution network of nodes as opposed to flexible unrestricted networks [33]. To allow the algorithms to run 


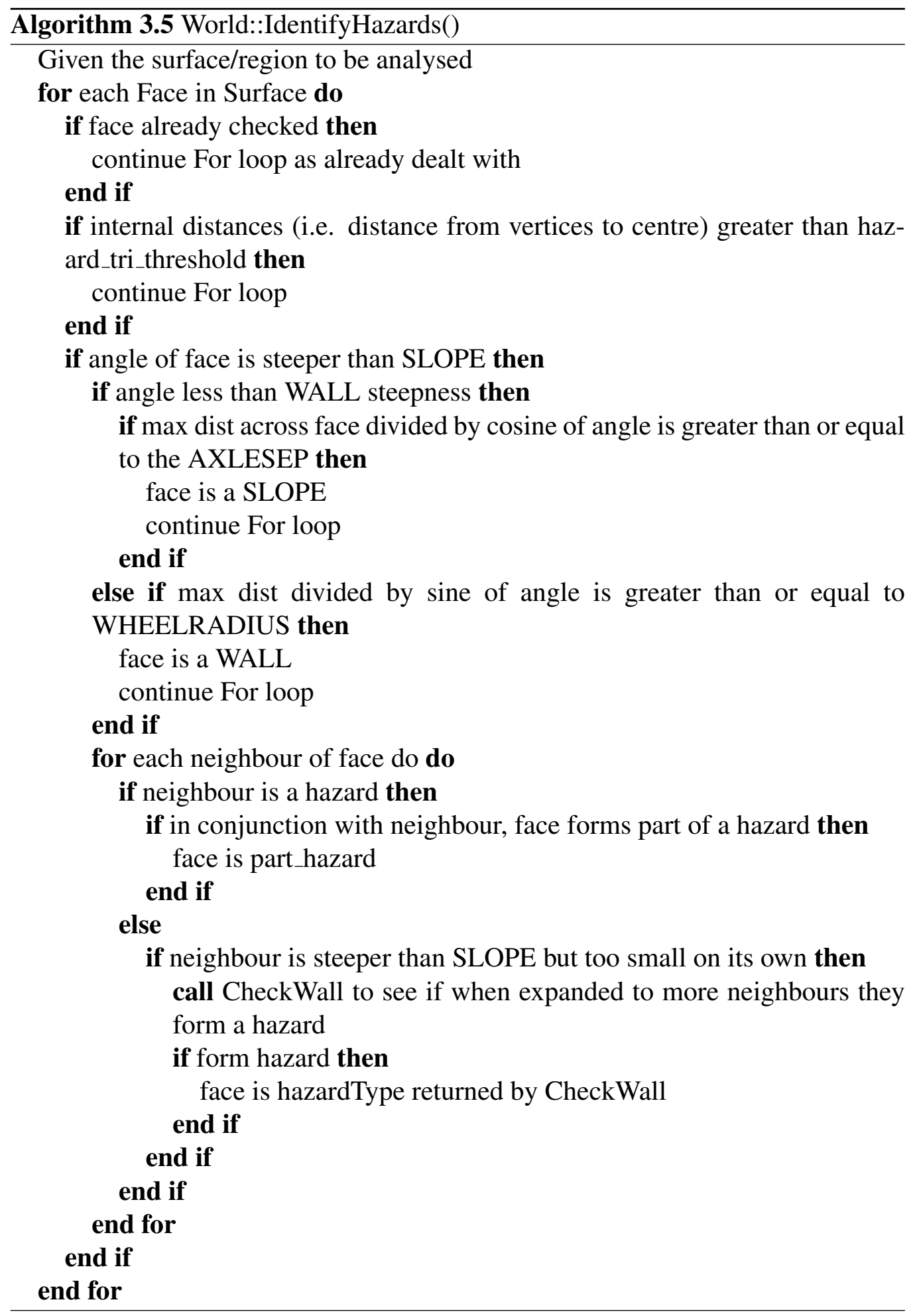




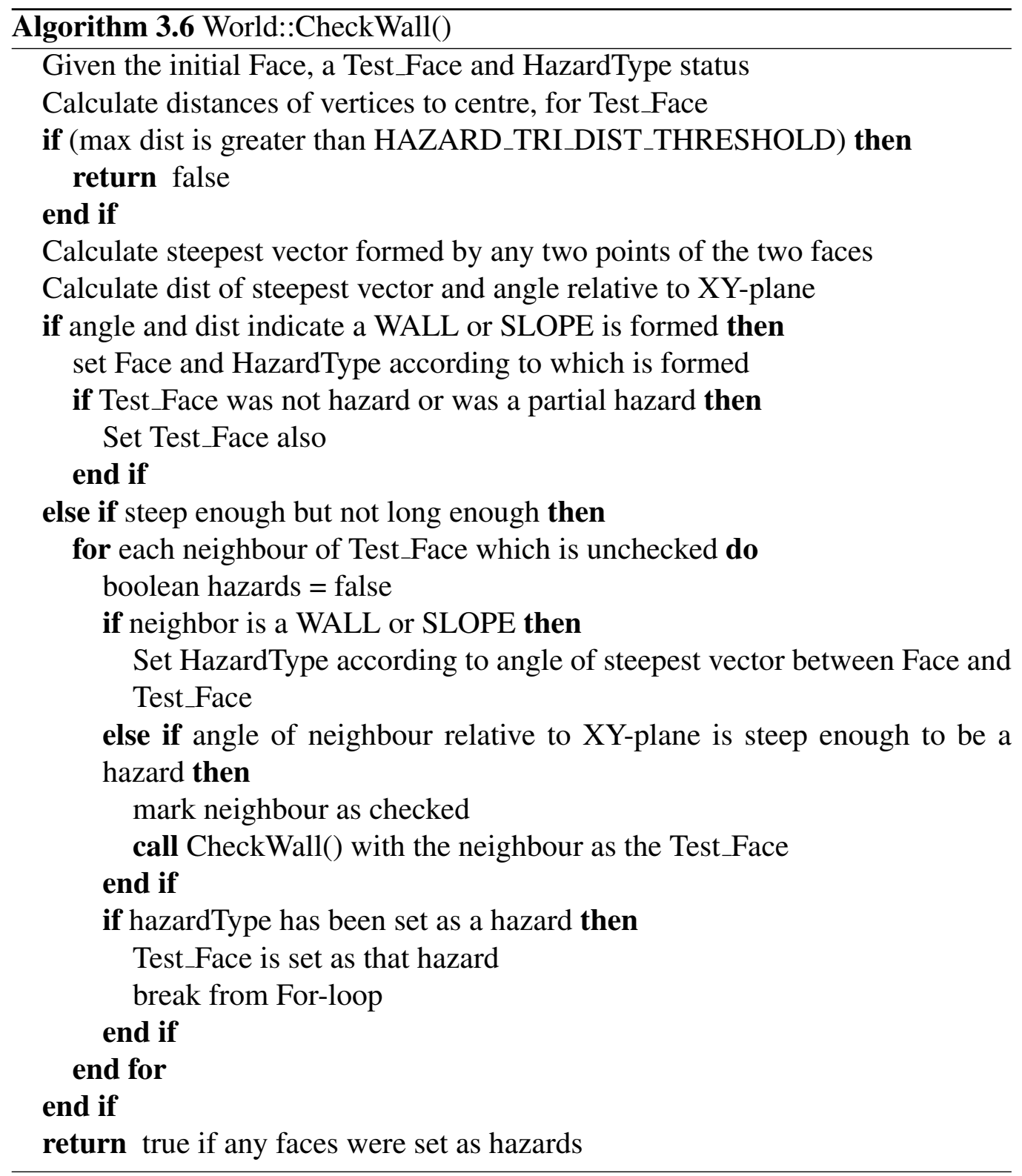


on their originally presented grid-networks, the project was branched to allow an alternate method of node creation and linking, such that the performance of both types of path networks could be compared to identify if there were any short comings when using the algorithms on less uniform or restricted networks of potential paths. Functions and objects were created and included, but full functionality could not be implemented due to time constraints and more pressing issues/features.

\subsubsection{Centroid based networks}

Using the centroids of traversable triangles and linking them only to their traversable neighbours is one approach which can produce a good network of potential paths and removes the cost of testing all combinations of points for linking. The large number of nodes in the network does mean a path planning algorithm may run slowly depending on its design and also it is hard to determine if a node is actually safe to move to once one takes into account the physical dimensions of the robot. Another issue likely to arise from a network based on the centroid's of triangles is that paths will zig-zag since a straight line of centroids through multiple adjacent triangles is unlikely. If the mesh was created from other polygons such as squares or rectangular, the path between two unobstructed points would be less likely to oscillate. However, as outlined already, triangles provide the greatest overall benefit for modelling and as such alternate methods of network creation which are compatible with triangle meshes are advisable.

\subsubsection{Border based network}

Alternative methods were considered which focussed on the border between hazardous and traversable triangles. Linking the midpoints of the edges which create this boundary explicitly allows the robot to know where a hazard is and thus to avoid such nodes by a buffer distance. However, calculations of distances between nodes which some algorithms may do as part of their analysis will have varying levels of inaccuracy when compared to actual path distances the robot may travel. 


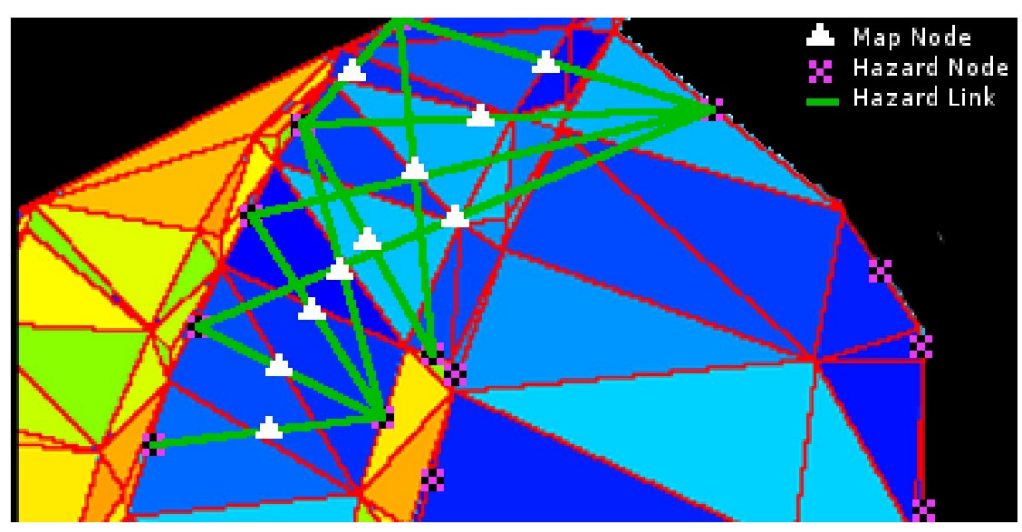

Figure 3.6: Example of Hazard and Map Node creation, showing only a portion of the potential links. The blue triangles being traversable and all others colours being hazards.

Creating nodes a set buffer distance from the border midpoints overcomes this inaccuracy but decreases certainty that a hazard will not be encountered as the node offset from one edge may cross the path/offset of another node and become close to a nearby hazard edge. The method which was chosen involves trying to place the map nodes in the middle of clear regions of terrain, and thus also looks at edges bordering regions which are as yet unknown. Clear central points are found through taking all the edge midpoints, trying to link them and looking at the distance the link covers. If the distance is over a certain threshold then the mid point of the link is used to create a map node. The mid points of these hazard/unknown links can be quite close to each other when links cross, so the set of map nodes needs to be checked for their relative proximity in order to discard superfluous nodes. Figure 3.6 helps to illustrate this point, showing a terrain which has hazards along the left and in the bottom centre while the right side is the limit of the known terrain. Even with only half the links from which map nodes can be created being displayed, overlapping occurs frequently, often placing midpoints beside each other. Once filtered down, the map nodes are then all linked together, which means the process of linking nodes is performed twice. However, this is on a relatively low number of points and provides a more robust network of potential paths. 


\subsubsection{Fixed resolution grids}

While fixed grid node networks are often simply derived from having a grid based terrain model double as the node network, they can also be easily generated for other types of models. To begin, with the initial position and an orientation vector are chosen, often the robot's location and either north, the direction the robot is facing or the direction of the destination is specified. A grid network is formed by sampling the heights for set XY-coordinates, which are separated by fixed increments in four directions (up, right, down and left) (0, 90, 180 and 270 degrees relative to the direction vector). With nodes being uniformly distributed, they can be linked to either 4 or 8 neighbouring nodes, dependant on whether diagonal moves/links are to be included/allowed.

Much like using fixed resolution grids for terrain modelling, this method of network creation is fairly simple but does not produce results specific to the position of obstacles. Consequently, fixed grids may miss potential paths between obstacles due to alignment of the grid with the obstacles and the clear section, with how this may occur being demonstrated in Figure 3.7, as a slight shift of the obstacles with respect to the grid points, resulting in more points being classified as traversable (green) as opposed to hazardous (red).

\subsubsection{Occupancy grids}

An enhancement on using fixed resolution grids is to move from solely using the vertices of where the grid lines intersect to considering the area within square sections of the grid and then using the centre of these squares as the nodes. The terrain bounded by these square sections can be analysed to produce a probability of being hazardous/not traversable based on the proportion of area which is classified as such. This probability can be considered a risk-value, which can be used by algorithms to shape their behaviour in terms of weighting different links/paths based on perceived risk. A threshold below which they may consider a section traversable could also be used and as such negate issues with the alignment of the grid with respect to the position of paths through obstacles. This basis of using 


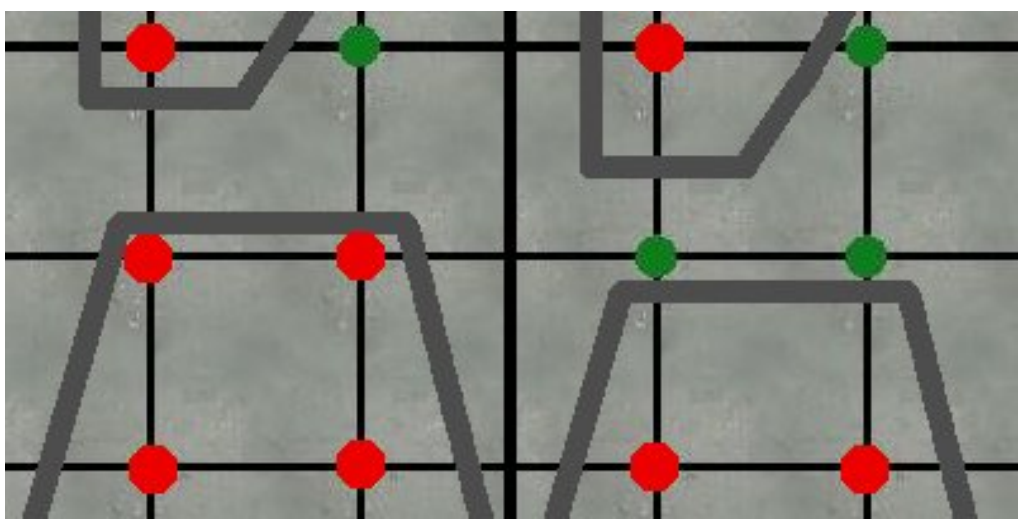

Figure 3.7: An example terrain model of a fixed resolution grid, where green nodes are traversable and red ones are not. Given the boundaries of hazard obstacles, shown as grey lines, the arbitrary alignment of the grid with respect to the terrain may produce different models, with the left one having fewer traversable points for the same area of terrain.

probabilities and thresholds is also beneficial in dealing with noise introduced by input sensors and errors caused through inaccurate positioning, similar to the use of confidence \& decay levels already mentioned.

For display purposes probabilities can be depicted in greyscale, with black indicating the definite presence of an obstacle, white indicating no obstacle, and the lighter or darker shades of grey indicating a lower or higher likelihood of an obstacle existing at that location. Figure 3.8 presents an example of occupancy grids being used to create maps of a unit's view from specific locations.

\subsection{Data Objects}

\subsubsection{Coords, Triangles \& Edges}

The initial Abstract Data Type (ADT) used to represent directions and positions was called a Coord. Coords held $\mathrm{x}, \mathrm{y}$ and $\mathrm{z}$ coordinates and had a number of associated mathematical functions. In addition to the basic mathematical functions such as addition and subtraction, Coords also had Cross Product, Dot Product 


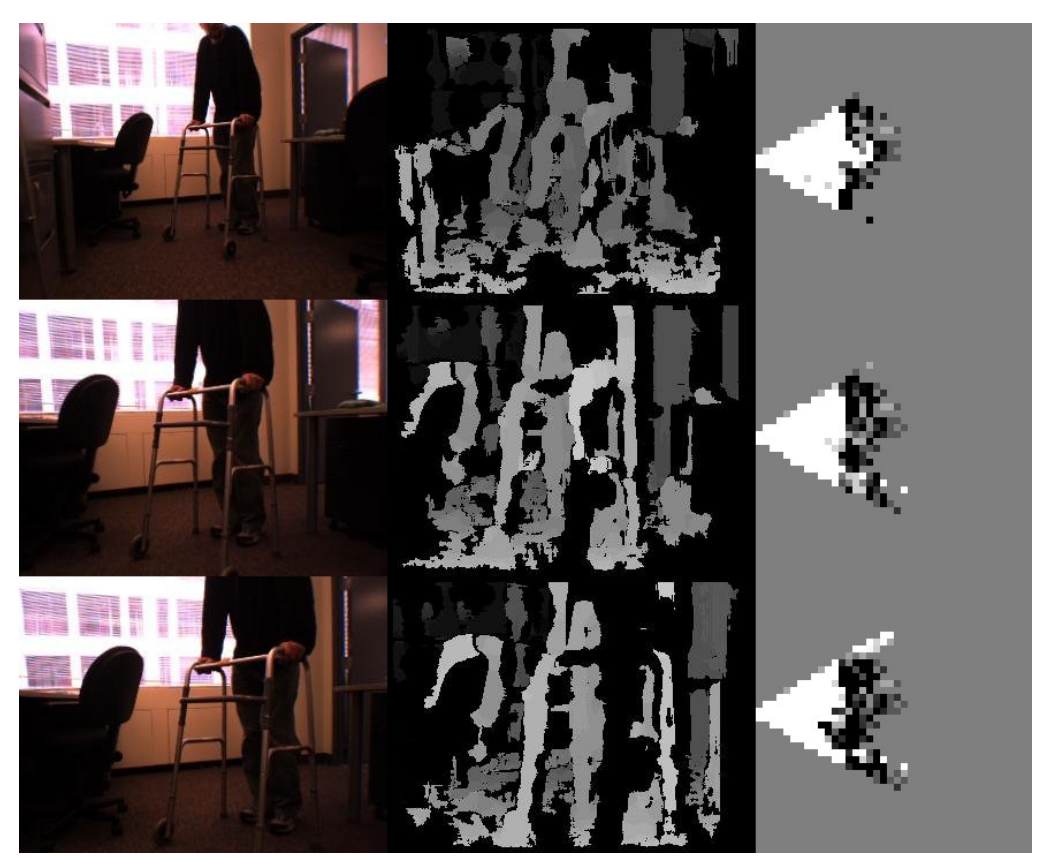

Figure 3.8: Example of Occupancy Grids in use by a wheelchair collision avoidance system approaching an elderly man with a walking frame [44].

and a number of comparative functions. The next layer of objects were Triangles which formed the mesh representing the terrain. Triangles contained links to three Coords which represented the Triangle's vertices as well as links to the three other Triangles which neighboured it. Most of the object functions for Triangles involved comparison to other triangles, to check for points or neighbours in common or if they were different instances of the same Triangle.

Coords and Triangles acted as one of the base underlying objects throughout the design stages of both the iterative and recursive tessellation, with Edges only being utilised in the recursive approach. As one would expect, Edges had two Coords however they only had one neighbouring Triangle as only the first one was of import during the tessellation process. To identify whether triangles had been created on either side of the edge there were two Booleans, these also acted to identify which side the neighbouring triangle lay on when only one had yet been created. 


\subsubsection{Points \& Face handles}

Using CGAL to do the terrain modelling required that a number of other objects types were used in order to successfully interface/interact with the triangulation it created. The primary objects for CGAL triangulations are Points and Face_handles, which are analogous to the previously outlined Coords and Triangles. To accommodate these objects, conversion functions were created, as well as many other functions being templated to allow either Coords and Triangles or Points and Face_handles to be used. Modification of the Coord and Triangle objects so that variables and functions had names matching their CGAL counterparts was also necessary, in order to use either type in template functions. Face handles were also altered to include the hazard status of the face/triangle.

\subsubsection{Nodes}

The potential path network on which path planning occurs, consists of numerous interlinked Node objects. The network can consist of either MapNodes or ASTAR_MapNodes, which are each associated with different types of path planning algorithms. However, before the network is created HazardNodes are used as an intermediary stage in determining the positions of MapNodes or ASTAR_MapNodes.

Though the simulator is not fully capable of supporting grid based networks at this point in time, GridNodes and ASTAR_GridNodes ADTs were created in parallel to MapNodes and ASTAR_MapNodes, looking ahead to the eventual use in grid based networks. A UML diagram depicting the hierarchy of the Nodes is shown in Figure 3.9.

\section{HazardNode}

The faces comprising the CGAL terrain mesh are processed to identify which ones are within visible distance of the robot's current position, as these are the ones which may have been affected by the latest set of terrain/scan input data. This set of faces is then further filtered to identify hazardous faces with traversable neighbours, thus establishing the boundaries of the traversable regions. HazardNodes 


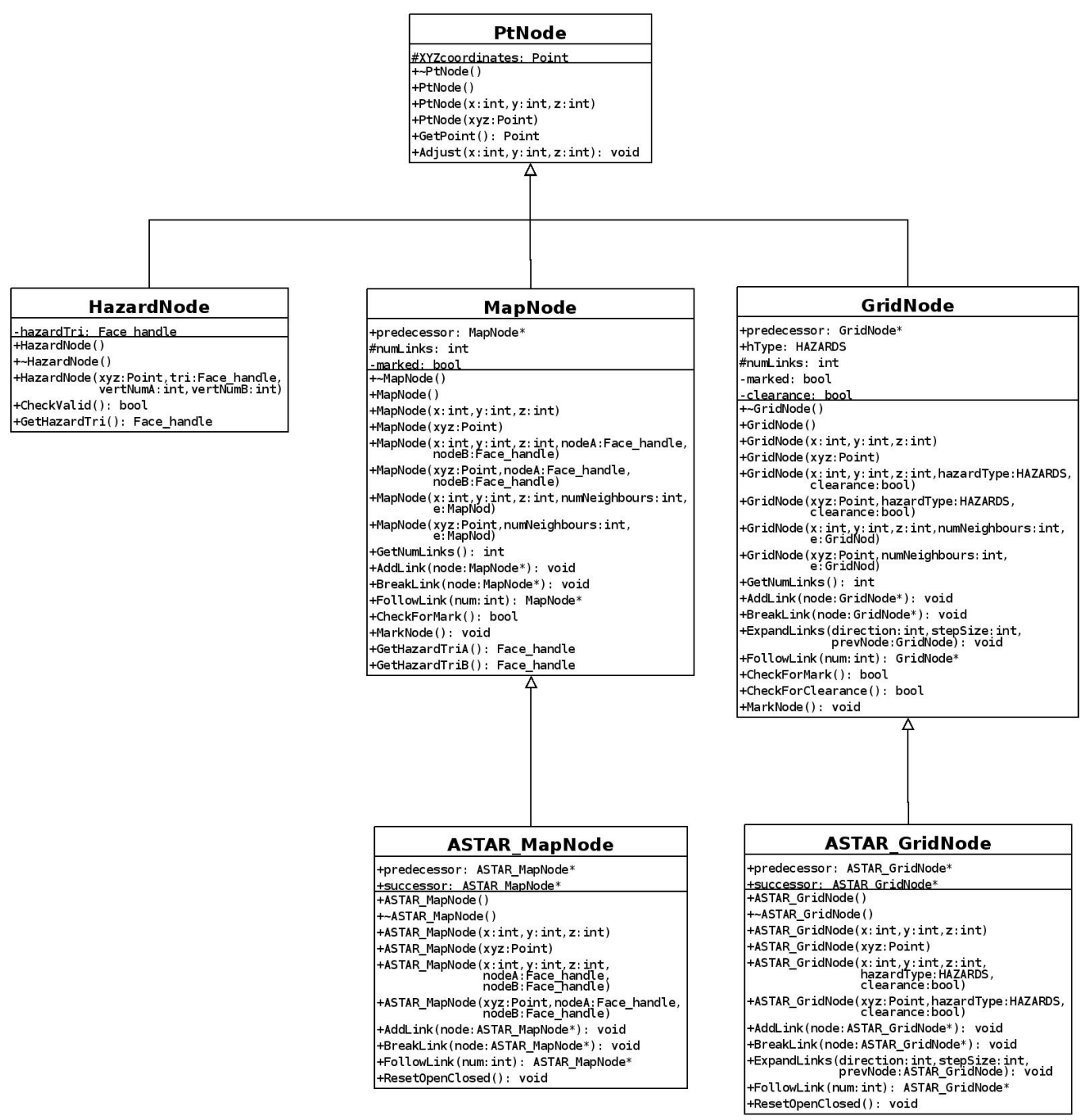

Figure 3.9: A hierarchy tree representation of the relationship between the Node objects 
are created at the mid-point of the shared edges and store pointers to the points at either end of the edge/border.

\section{MapNode}

All combination of HazardNodes are checked to see if they have a clear path between them which is at least wide enough to accommodate the robot passing between them. If it is determined to met the criteria, a new MapNode is created at the mid point between the two HazardNodes.

\section{ASTAR_MapNode}

An ASTAR_MapNode is created through the same process as a MapNode and are in most ways identical to MapNodes, however they differ in what variables are included for use by path planning algorithms. The ASTAR_MapNodes are used by the $A^{*}$ algorithm and its derivatives, including $\mathrm{f}, \mathrm{g}$ and $\mathrm{h}$-value variables used by the algorithms (see 3.9.4).

\subsection{Path Planning Algorithms}

Building upon the tessellation and data structures, the implementation of a number of path planning algorithms was undertaken. The complexity of these algorithms varied greatly, as did the success in implementation. A great deal of difficultly was encountered with the LPA* and D* Lite algorithms since all of the information regarding the specifics of their implementations was based on using an eight direction grid-based network of nodes, an approach which had been discounted in this project due to being sub-optimal in terms of resource requirements. The core of the problems lay in the updating of the network on successive iterations. This action does not occur with the $\mathrm{A}^{*}$ algorithm as it recomputes the entire network upon each iteration. 


\subsubsection{Edge Hugger}

The first algorithm implemented was a simple edge hugger. This approach does not require the same level of processing as the rest of the algorithms as it does not require a network of potential paths to be created and instead relies solely on collision detection. Collision detection being the analysis of whether moving forward in a certain direction would cause the robot to collide with a hazard, thus allowing the robot to choose an alternate direction, parallel to the edge of the hazard to be skirted. This algorithm provided great benefit in not only testing the collision detection function but also the hazard identification process which the collision detection relied on to have analysed and identified the hazards which it would be attempting to avoid.

As the path planning system progressed and the design changed, obsolete and incompatible functions were removed or altered. The shift from points and triangles to coords and faces, along with implementation of the node network creation and linking, led to the Edge Hugger no longer being compatible with the system and as such was not used further for any experimental trials.

\subsubsection{State model}

A simplistic state-based path planning function was produced to provide edgehugger/wall follower like behaviour while using the node network. The states chosen were Forward, Right, Back and Left, with each being the direction relative to facing/centring on the goal. An additional state controlled how the set of states were progressed through, either going in a clockwise (Forward, Right, Back, Left) or anti-clockwise (Forward, Left, Back, Right) manner. This ability to alternate between clockwise and anti-clockwise exploration was added to allow the robot to break free from being trapped in enclosed corners/sections/regions in which paths may be ignored due to favouring left over right or vice-versa. While not overly intelligent this algorithm intrinsically deals with dead ends and the need for back tracking/move away from the goal, in order to reach it. 


\subsubsection{Breadth First Search and Depth First Search}

The very basic search algorithms of Breadth First (BFS) and Depth First (DFS) were considered for usage and comparison within the system. The BFS algorithm places all newly encountered nodes into an array, considering and expanding the search from the oldest entries first, which means that all neighbours of a node are considered before moving on to their neighbours. The DFS algorithm adds nodes in the same manner as the BFS, however it investigates the newest entries first, which leads to it exhausting a branch of searching before considering the neighbours. However, upon implementing and testing Depth First Search it was realised that for open unbounded scenarios the algorithm would continue on regardless of the goal location. Being a blind search algorithm there was no heuristic to draw it towards the goal and hence, without a maze-like bounding box and finite/discrete positions, there was little chance of ever reaching the goal. While a Breadth First Search may eventually find the goal, through its nature of fanning out in all directions, it would take a great deal of time and require vast amounts of resources when there was no bounding/restricting border/box.

\subsubsection{A* Algorithm}

Starting at the node representing the current location of the robot, the $\mathrm{A}^{*}$ algorithm investigates each of the linked nodes/neighbours and calculates their $\mathrm{f}, \mathrm{g}$ and h-values and sets the current node as their predecessor, before placing them into the open set and the current node into the closed set. The g-values are the known distances it is necessary to travel in order to reach any given node from the starting position while only travelling along nodal links. The direct calculated Cartesian distance between any given node and the goal is a node's h-value and is where weighting may be applied to affect the expanse/scope of investigation. Summing both the $\mathrm{g}$ and $\mathrm{h}$-value for a node gives its f-value, which is used by the algorithm to judge the potential likelihood of being an optimal path.

The open and closed sets represent the nodes which have been already investigated, with those in the open set still having potential while the closed set contains 
ones which have had all their links fully exhausted. The nodes within the open set are sorted relative to their f-values so that they are checked relative to their merit as opposed to a first-in first-out or last-in first-out strategy as applied by BFS or DFS, respectively. With the current node being fully expanded and placed in the closed set, a new node is taken from the open set and the process is repeated until the goal is reached or all potential paths have been exhausted. The current pick of the nodes may have links to nodes which are already in the open or closed sets. Nodes in the closed set can be ignored however those in the open set are re-evaluated to see if having the current choice as their predecessor would lower the nodes g-value, in which case they are relinked and have their values adjusted accordingly.

In order to deal with cases where no path is found, on each loop through the path analysis, the current pick is compared to previous ones in order to determine which node has the lowest h-value and hence is closest to the goal. A pointer to the closest node is kept such that when no path to the goal is found, it can be used as a temporary destination in the hopes further traversal will increase knowledge of the terrain and open a path to the robot.

Starting at the goal (or closest node), the predecessor is taken and placed in an array, as is the predecessor's predecessor and so on until the path between location and destination is reconstructed and held within the array. The direction of the next move is calculated and the robot passes this on to the simulator which updates the robot's position.

The assumption within $\mathrm{A}^{*}$ that nodes have a clear link to the goal node unless proven otherwise was found to be problematic in this implementation, especially due to the potential for criss-crossing links. For instances where the goal node was significantly far away, the process of confirming whether hazards existed between the goal and a specific node was very intensive and also problematic when regions of unknown terrain were encountered as the discontinuity was incompatible with the confirmation technique which involved moving through neighbours. The solution used, was to only consider linking the goal node to other nodes which were within a set/visible distance of it. This approach meant the process was quicker 
and it was less likely that while traversing a link it would be found that the terrain had altered significantly and required reanalysis/linking.

Due to points not automatically existing in certain positions and not being able to assume their presence outside the visible region, as occurs with the fixed grid approach, two temporary points were necessary to ensure the algorithms were presented with paths around obstacles even when very little data had been gathered for the region. These points were taken as the limits of the visible region in terms of distance and angle from the centre to both the left and right. Upon traversing either way around an obstacle the point would become unnecessary as new data would be gained through movement in that direction.

An attempt was made to implement a version of the $A^{*}$ algorithm which was restricted to eight directions of movement, however the grid network was not at a reliable state to support the implementation.

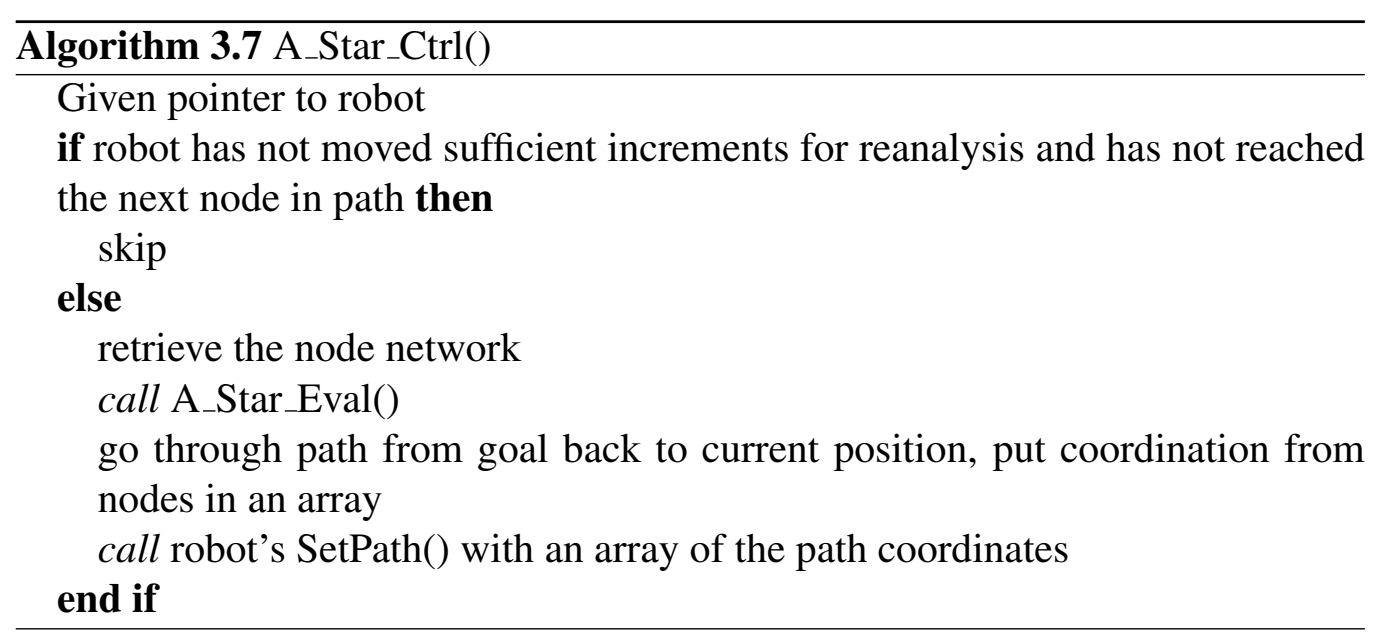

\subsubsection{LPA* Algorithm}

The previously implemented A* algorithm was taken as a base for the LPA* algorithm, being modified to retain the Open and Closed sets across multiple iterations and with functions added to identify changes between iterations and update the successors or predecessors as required. Unlike grid-based networks where nodes 


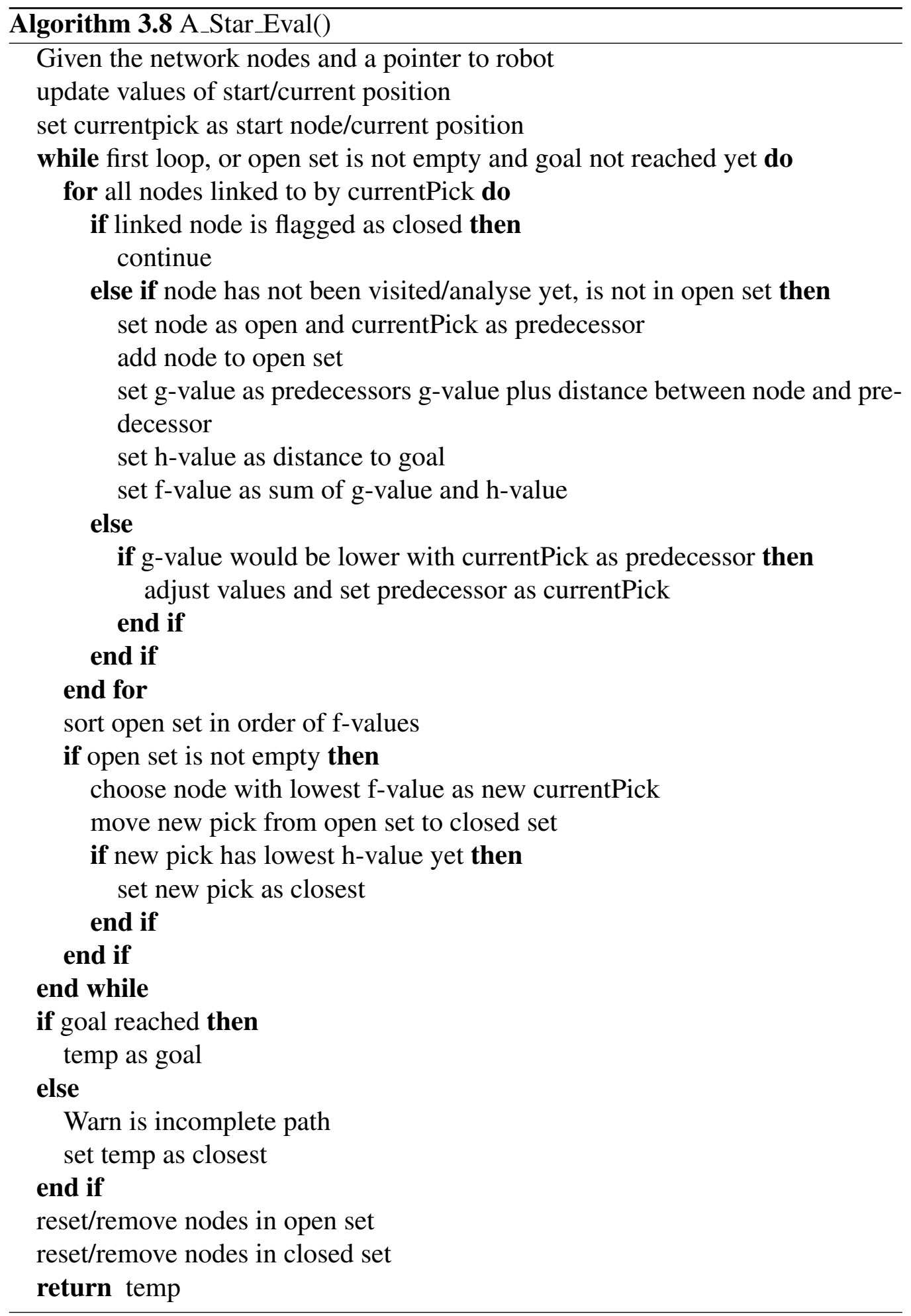


always exist and may only change in terms of their status as being traversable or not, the flexible system used as the primary form of node networks resulted in some nodes no longer existing upon a successive iteration. This facet of flexible networks caused some additional difficultly to the updating of the links as propagating a change could encounter a disconnect in a previous path due to a node's removal. Successfully determining a new predecessor or successor requires that all nodes upstream from the node being relinked have been updated and changes fully propagated, otherwise some nodes may appear locally inconsistent and be relinked when unnecessary. Incorrectly pointing to a node as the successor of another, could lead to cyclic paths when other updates were propagated due to incorrect f-values being compared.

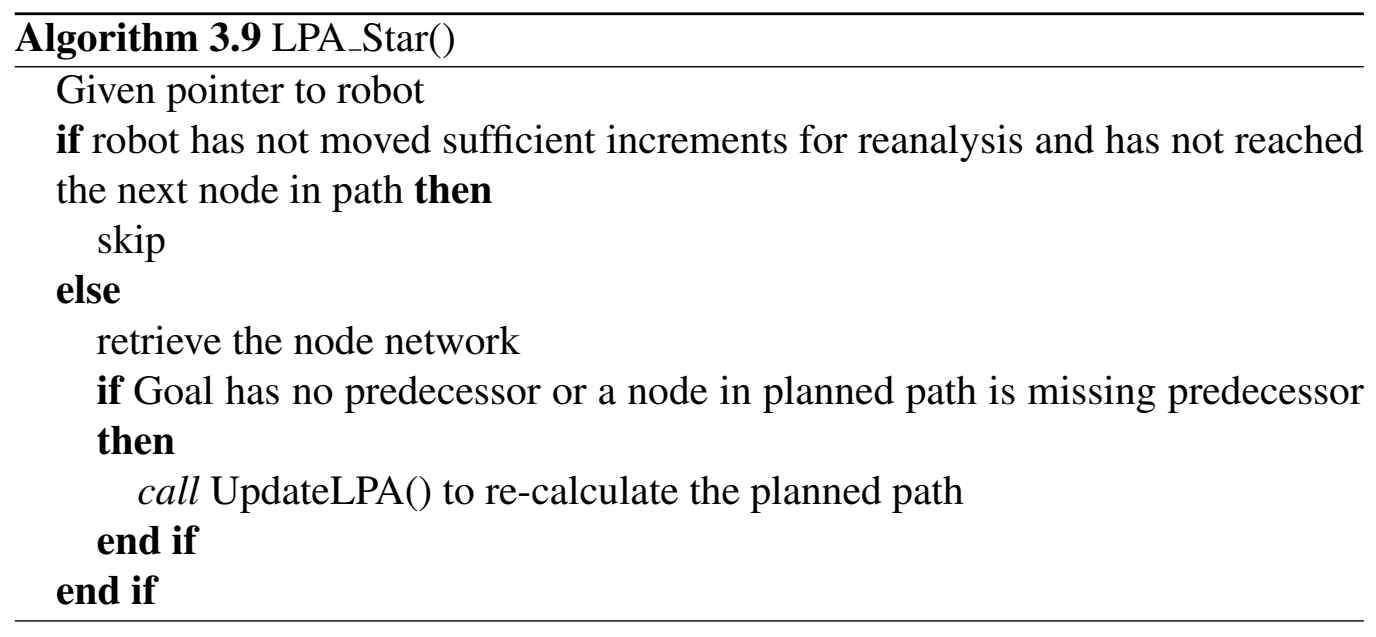

\subsection{6 $\mathrm{D}^{*}$ and $\mathrm{D} *$ Lite Algorithms}

Due to the superior performance of $\mathrm{D}^{*}$ Lite, as outlined by Koenig[35], in terms of having fewer tie-break conditions and being a re-use rather than re-plan algorithm, the implementation of $\mathrm{D}^{*}$ was forgone in favour of $\mathrm{D}^{*}$ Lite. Upon delving into how the $\mathrm{D}^{*}$ Lite algorithm works, it was found to be a little too complex to implement for the flexible node network without greater familiarity with the mathematical basis and principles of the algorithm. The idea of modifying an existing implementation of the algorithm was investigated, however upon downloading a 


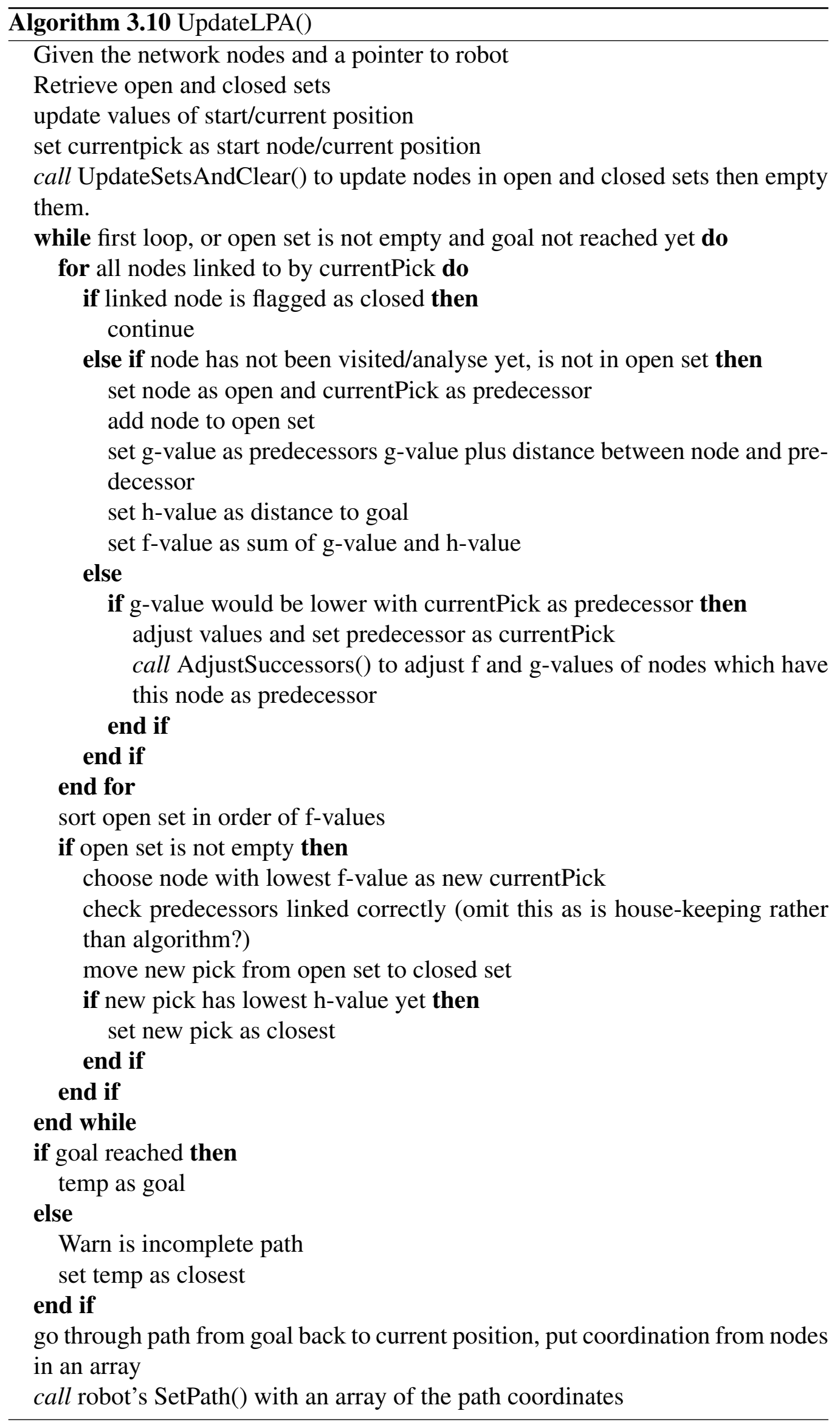




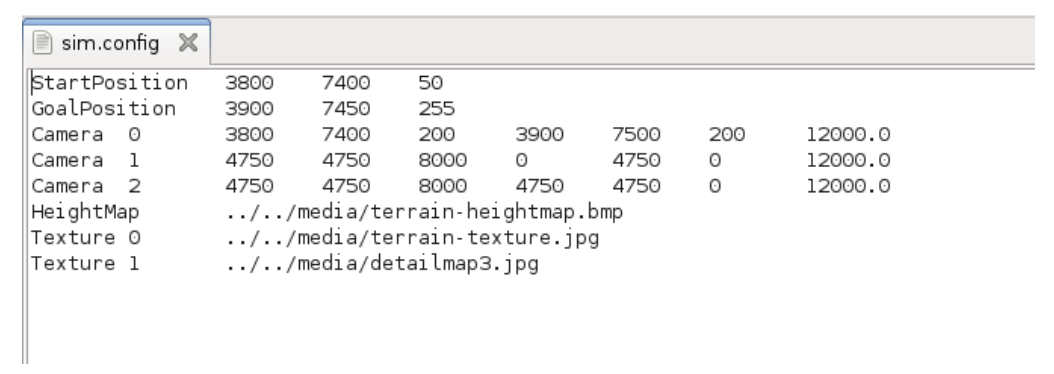

Figure 3.10: The contents of a simulation/environment configuration file. The first three columns of numbers being Cartesian coordinates for positions and the additional three columns associated with the cameras being the target positions at which to be aimed.

couple of implementations of the algorithm, it was realised this still required a better understanding of the algorithm as the underlying data structures between the current system and downloaded algorithm would need to be interfaced and altered.

\subsection{Automation and Configuration}

As with all scientific investigation, repetition is an important factor which adds confidence to findings. Automation of experiments is beneficial to researchers as it provides the ability to repeat trials with little effort, usually no more than entering the number of times to be repeated, and allows them to focus their attention elsewhere.

To further enhance data generating capabilities, the use of configuration files allows a program to switch between running trials that have setups which may differ substantially, with minimal effort on the operator's behalf. After the initial creation of configuration files, which are often just copies of other configuration files with some parameters altered, changing the filepath/name inputted to a simulator is all that is required to run various trials. The automated processes and configuration files allow the program to be left for extended periods, during which it will to go through various trials. 


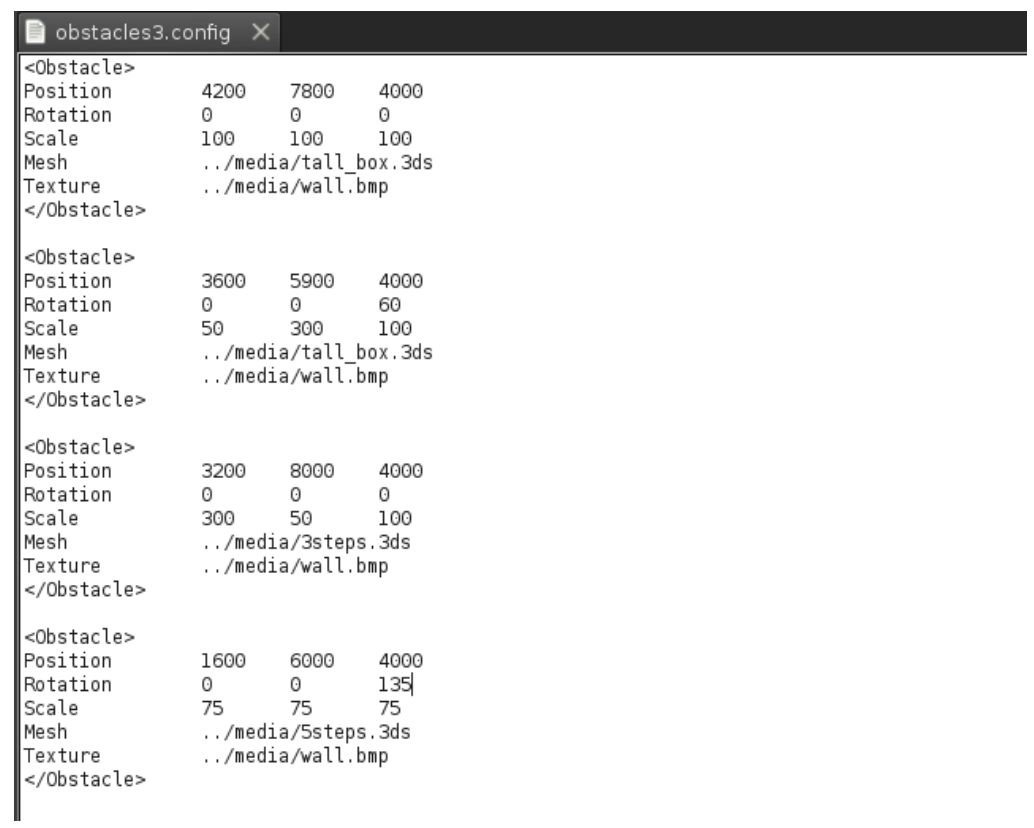

Figure 3.11: An obstacle configuration file for adding four obstacles to a terrain.

\section{config_combos.txt}

\begin{tabular}{|c|c|}
\hline $\begin{array}{l}\ldots / \text { sim. config } \\
\ldots / \text { sim. config } \\
\ldots / \text { siml. config } \\
\ldots / \text { siml, config }\end{array}$ & $\begin{array}{l}\text {. /obstacles, config } \\
\text {. /obstaclesl, config } \\
\text {. /obstacles, config } \\
\text {./obstaclesl, config }\end{array}$ \\
\hline
\end{tabular}

Figure 3.12: Four combinations of Obstacle and Simulation configurations inside a control file, allowing two different obstacles files to be each used with two simulation configurations. 
The simulator created for this project uses three configuration files, with examples given in Figures 3.10, 3.11 and 3.12. The first two configuration files provide Environment/Simulator settings (Figure 3.10) and Obstacle settings (Figure 3.11) while the third file (Figure 3.12) controls which specific files are used for those configurations. The Environment settings provide information required by the graphics engine to produce the simulation, such as which heightmaps are to be used, the position and orientation of cameras, where the robot model should initially be placed and where to put the goal marker. The position, orientation and scale of obstacles to be added to the terrain are outlined in the obstacle's configuration file, along with the filepaths of which models are to be used as the obstacles.

\subsection{Path Analysis}

To aid both later analysis of results as well as confirming correct behaviour in initial testing it is important to have methods to display and demonstrate the results, as they can be difficult to comprehend in numerical form only. As such, a path viewing module which displays overlaid paths was created. Figure 3.13 shows the results from two sets of trials, one set from a simple terrain and one containing many more obstacles and slopes near the limit of the robots traversal capabilities. Another module has also been implemented which allows a set of movements to be replayed. This replay function runs significantly faster and hence is better suited to viewing than when the robot is actively path planning. Replays simply re-enact the movements while not having to scan the environment, analyse the terrain or calculate paths, which are the time consuming steps of the simulation.

To ensure the data to be displayed always fit in the display/viewing window, while showing as much detail as possible, the display is scaled both in the $\mathrm{X}$ and $\mathrm{Y}$ axis based on the bounding box of the points given. The relationship between the $\mathrm{X}$ and $\mathrm{Y}$ axis varies when different sets of data are displayed and as such displayed paths may appear stretched in one direction; this must be kept in mind when comparing paths to the terrain. 


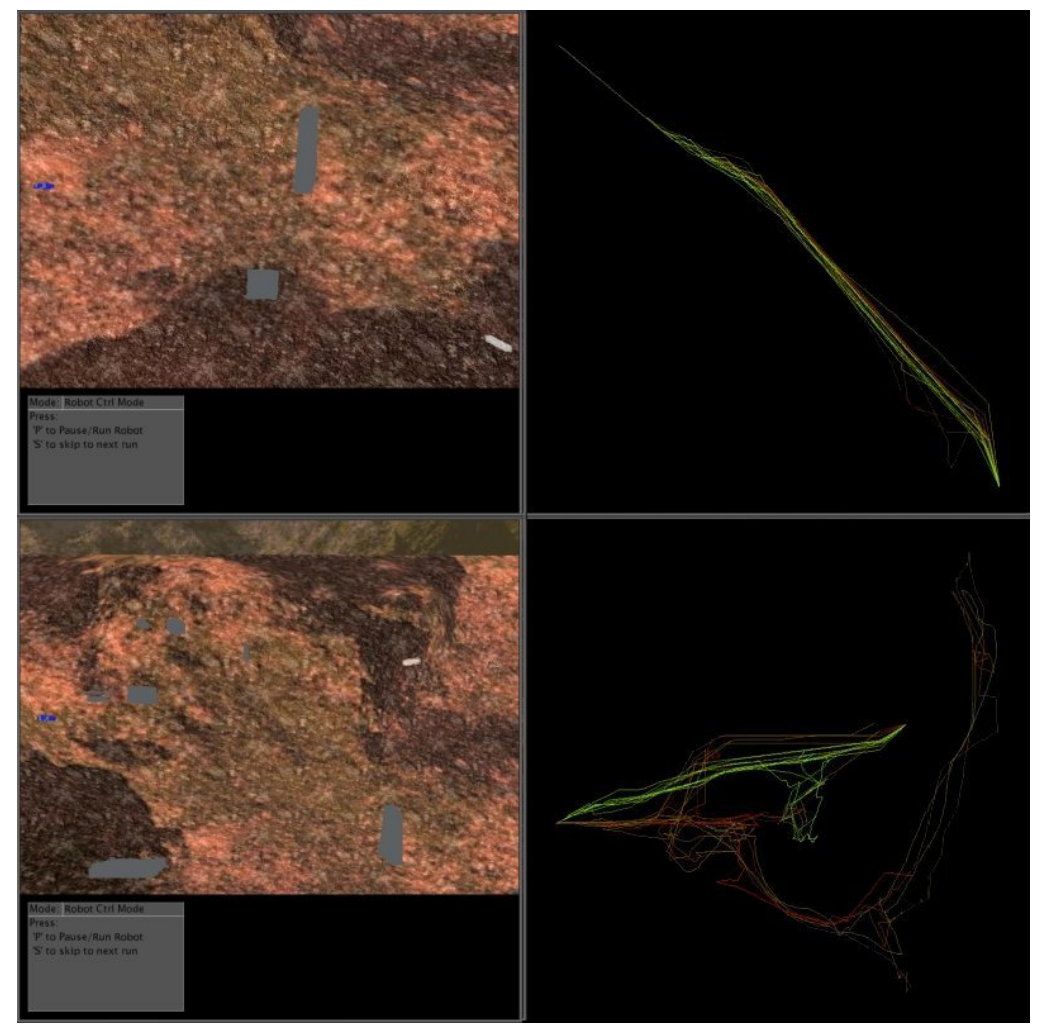

Figure 3.13: Two test scenarios, consisting of gentle slopes and two obstacles (top) and steeper slopes, numerous obstacles and an unreachable goal (bottom), displayed beside the overlaid paths generated from trials conducted on them using the $\mathrm{A}^{*}$ algorithm. The point objects are the robot, the white are the goals and the grey objects are additional obstacles. 


\section{Chapter 4}

\section{Experimental Design and Pilot Tests of Path Planning}

Simulations with varied robot parameters and terrain configurations were run in order to evaluate the Simulator and show that is was capable of being used for experimental investigation. In particular that the Simulator is especially useful to employ on prolonged simulations, where a variety of options are available to alter and that it produces results in a usable form and with sufficient information about the simulated trials so as to identify variance and be able to draw conclusions. Pilot testing was carried out to ascertain whether the design and setup chosen to run experimental trials was viable, to identify and correct any issues before extensive trial runs, and check that the output of the simulator was sufficient for scientific analysis.

\subsection{Hardware}

The computer on which pilot trials were run, had an Intel Core2 Duo 6400 processor at $2.13 \mathrm{GHz}$ and 2.0 GB of RAM. The operating system used was release 5.0.2 (Lenny) of Debian, the display manager used was GNOME 2.22.3, and they were running on the Linux 2.6.26-2-686 kernel. Creating the executables the GNU $\mathrm{C}++$ compiler $(\mathrm{g}++)$ version 4:4.3.2-2 was used, along with the $\mathrm{C}$ standard library 
version 2.7-18, MESA 3D (an open-source computer graphics library providing the OpenGL implementation) version 7.0.3-7, the Irrlicht graphics engine version 1.5, CGAL version 3.3.1-4 and SDL gfx-package version 2.0.13-4 (equiv of SDL 1.2.13-4).

For the extended trials, eight computers in the undergraduate computer lab were initially used, but later a further four also ran simulations. These computers were chosen as they were unused over the holiday period and because the graphical requirements of the simulator excluded it from being able to run on the postgraduate computing cluster. Each computer, had an Intel Core2 Quad 6400 processor at $2.40 \mathrm{GHz}$ and $3.9 \mathrm{~GB}$ of RAM. The operating system used was release 10 (Cambridge) of Fedora, the display manager used was GNOME 2.24.3 and they were running on the Linux 2.6.27-12 kernel. Creating the executables the GNU C++ compiler ( $\mathrm{g}++$ ) version 4:4.3.2-2 was used, along with the $\mathrm{C}$ standard library version 2.7-18, MESA 3D version 7.0.3-7, the Irrlicht graphics engine version 1.5 and CGAL version 3.3.1-4. SDL was not present on the computers and thus some features were omitted, however the omitted features are not needed to generate results, only being for graphically displaying information upon operator request. As the home directory for these computers is on a network drive, results were recorded to the local /tmp/ drive in order to avoid excessive overworking of the input/output of the home drive with the high load of multiple computers writing to it for an extended time period.

\subsection{Variables}

\subsubsection{Simulator}

The quantity of points within the emulation of a LIDAR scan is one of the major variables available to alter, along with the dispersal and angle of these points. 


\subsubsection{Algorithms}

At present the only variable parameter relating to the algorithms is the float H_WEIGHTING, which affects $\mathrm{A}^{*}$ based algorithms and alters the weighting of the h-values in producing f-values.

\subsubsection{Robot}

The variables which reflect the physical properties of the robot are the integers ROBOTLENGTH_MM, ROBOTWIDTH_MM, AXLESEP_MM (distance between axles), WHEELWIDTH_MM, WHEELRADIUS_MM and CAMERA_HEIGHT (height of the camera off the ground) plus VISIBLE_DIST_MM (maximum distance LIDAR can view) which is a float.

Characteristics of the robot's interaction with the world are defined by hazard avoidance parameters, movement parameters and algorithm choice parameters. The SLOPEANGLE and WALLANGLE are used to categorise the gradient of surfaces while CLEARANCEBUFFER_MM stipulates the clearance from hazards to be observed for safety purposes. The robot's movement is controlled by the movement step size and maximum number of movements between the algorithm reanalysing the potential path network, which are MOVE_INCREMENTS_MM and STEPS_BETWEEN_EVAL, respectively. The choice of algorithm is set within the robot object and ALG_NODE_TYPE defines which node type should be used.

\subsubsection{World}

Within the world object point culling is controlled by MEASUREMENT_PRECISION and DIST_THRESHOLD for general additions, while COPLANAR_PRECISION and COPLANAR_DIST_THRESHOLD cover instances where the point is coplanar with its encompassing triangle. Other variables are HAZARD_TRI_DIST_THRESHOLD (Maximum internal distance to centre for which a hazard triangle can be reliably trusted), MAPNODE_PROXIMITY_THRESHOLD, KEY_RATIO (Distance past visible region which nodes should still be held on to) and MIN_INCIDENT_ANGLE 
(Minimum angle of incidence for links between hazardNodes and the border on which a hazardNode lies).

\subsection{Procedure}

The parameters to be altered during testing were recorded and the trials were given designations/reference codes to indicate which variable was being altered and to what value. For a set of trials, the variable of interest would be set in the source code and then an executable file compiled. From the the commandline the simulation executable would be called and passed a configuration file containing the settings of various scenarios, on which the specific set of trials should be tested. When it was desired to run multiple executables, shell scripts were written to iterate through calling the executables and to feed them the same configuration file.

As the computer on which the trials were being run was also needed for other purposes, the ' $\mathrm{i}$ ' key was pressed to set the simulator to continue running even when it was not the active window. Upon completion of a set of trials the resultant files would be moved into a new appropriately directory, either manually or by the shell script if used, so that the results from new sets did not get appended to the past results.

\subsection{Analysis}

The results as listed in SimResults.txt were grouped by scenario and the termination status were tabulated to give an indication of how the different sets performed. In some cases the steps taken were compared by scanning over the outputted steps the robot had taken, but this was generally only of use when the trials ended early, otherwise graphically overlaying the paths was easier and was a greater aid in understanding the overall behaviour of a set of trials. To give a faster indication of how a set fared, for the experimental results histograms were favoured over tables, with the precise values of statistical information such as the median or mean being 
generated and available if needed but otherwise left up to the charts to convey.

One factor which was considered of interest but did not end up being specifically compared was the time taken to complete the trials. The reason this characteristic was not analysed was due to an inability to regulate or monitor the varying loads on the computer from other tasks, the state of network connections and other factors not related to the simulation, which may affect the time taken.

\subsection{Pilot Tests}

For the pilot testing, each configuration file being considered for use in experimental trials and each parameter to be altered and its associated set of values, was trialled in order to confirm correct operation and detect any changes which may be necessary. Ten repetitions were run for each pair of terrain and obstacle settings, resulting in twenty trials for each weighting tested. The majority of trials were run using the $\mathrm{A}^{*}$ algorithm due to greater confidence in the version of it ported to be used on the flexible node network, over that of the modified LPA* algorithm. It was felt the LPA* algorithm required confirmation of correct behaviour and validity, through comparison of performance on both a grid-based node network and flexible node network but the ability to create and use a grid-based network was not completed. Hence it was felt conclusions drawn from the A* algorithm would be more reliable. An overview of how the different trial sets are inter-related is shown in Figure 4.1, with the three algorithms along the top and solid arrows denoting a subset which involved a variation of parameters, while the dotted arrows indicate some similarity between sets.

Depending on which parameters were varied and to which values, on which scenario they were run, as well as the level of success and number of steps before failures, between 15 and 20 trial runs were usually completed in a 24 hour period. The theme across parameters chosen to investigate was vision and movement, as it was expected these may show some of the strongest interactions. With regards to the scenarios created for the robot to deal with, the choices of start positions, goal positions and obstacle locations were largely arbitrary and chosen to provide 


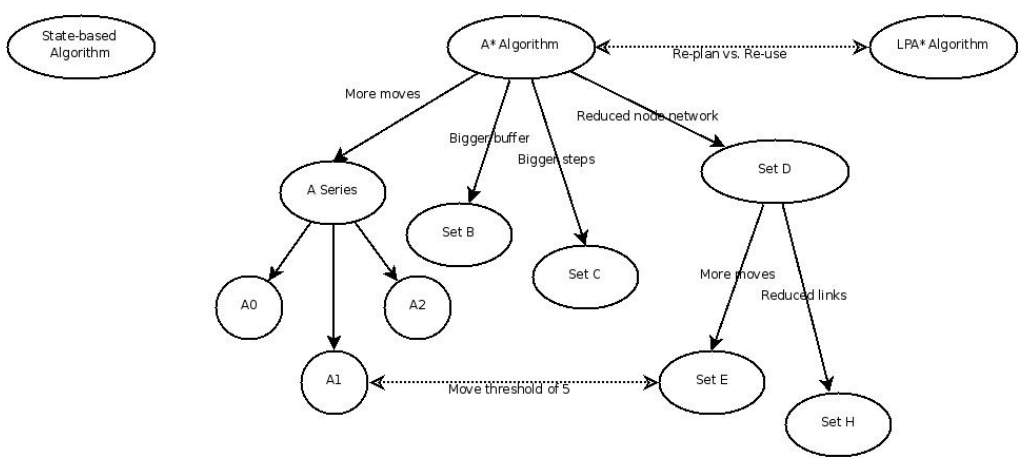

Figure 4.1: UML diagram showing the branching of different trial sets.

some variety.

\subsubsection{A* algorithm}

\section{Config_combos_0}

The first set of trials conducted used the $\mathrm{A}^{*}$ algorithm and looked at the impact of varying the weighting of the h-value used by the algorithm. Trials were conducted on four different combinations of locations and obstacles, using the config_combos_0 configuration file, with each run occurring ten times. The terrain heightmap used by this configuration file (and config_combos_2) was one provided with the irrlicht graphics engine, being chosen due to its size and the reasonable level of variety within the terrain produced. The values of the altered h-value weighting were 1.0, 1.05, 1.1, 1.15, 1.2, 1.3, 1.4, 1.5 and 2.0, with Table 4.1 listing the completion status of each set of trials. For the first two scenarios within the configuration the results across all weightings, except for 2.0, were not significantly different/varied little. This lack of variance is not surprising considering the simple nature of these scenarios which were expected to have minimal hazard avoidance required. The other two scenarios within Config_combos_0 were highly unsuccessful in comparison, as indicated in Tables 4.2 and 4.3. It occurred frequently that the robot, for the second pair of scenarios, encountered a hazard or reached a point from which it was not able to determine a potential path to the 


\begin{tabular}{|c|l|l|l|}
\hline & Success & \multicolumn{2}{|c|}{ Failure } \\
\hline H-value weighting & Goal reached & No path to goal & Hazard traversed \\
\hline \hline 1.0 & 20 & 0 & 0 \\
\hline 1.05 & 20 & 0 & 0 \\
\hline 1.1 & 20 & 0 & 0 \\
\hline 1.15 & 20 & 0 & 0 \\
\hline 1.2 & 20 & 0 & 0 \\
\hline 1.3 & 20 & 0 & 0 \\
\hline 1.4 & 20 & 0 & 0 \\
\hline 1.5 & 20 & 0 & 0 \\
\hline 2.0 & 0 & 20 & 0 \\
\hline
\end{tabular}

Table 4.1: Combined results of first two configurations within Config_combos_0.

\begin{tabular}{|c|l|l|l|}
\hline & Success & \multicolumn{2}{|c|}{ Failure } \\
\hline H-value weighting & Goal reached & No path to goal & Hazard traversed \\
\hline \hline 1.0 & 8 & 2 & 0 \\
\hline 1.05 & 0 & 1 & 9 \\
\hline 1.1 & 0 & 2 & 8 \\
\hline 1.15 & 0 & 3 & 7 \\
\hline 1.2 & 0 & 3 & 7 \\
\hline 1.3 & 1 & 1 & 8 \\
\hline 1.4 & 0 & 6 & 4 \\
\hline 1.5 & 0 & 10 & 0 \\
\hline 2.0 & 0 & 10 & 0 \\
\hline
\end{tabular}

Table 4.2: Results from third configuration within Config_combos_0.

goal. In the case of the final set some trials were not completed due to errors.

The first pair of scenarios within Config_combos_0 consisted of a flat terrain and a small number of obstacles, with a ridge running off to the right side when positioned at the starting location and orientated towards the goal. For the other scenarios within Config_combos_0 the starting location was atop a plateau, on which additional obstacles were placed. The goal was located on a lower region past the obstacles, with a steep slope present between the goal and the obstacles. Config_combos_2 was the same as the second pair of scenarios in Config_combos_0 but with the start and finish points reversed.

\section{Config_combos_2}

Upon following trials, the various weightings of h-values were limited to $1,1.1$, $1.2,1.4$ and 2 as the variance between results from different weighting appeared 


\begin{tabular}{|c|l|l|l|l|}
\hline & Success & \multicolumn{2}{|c|}{ Failure } & Incomplete \\
\hline H-value weighting & Goal reached & No path to goal & Hazard traversed & Error \\
\hline \hline 1.0 & 3 & 5 & 2 & 0 \\
\hline 1.05 & 1 & 3 & 4 & 2 \\
\hline 1.1 & 0 & 3 & 4 & 3 \\
\hline 1.15 & 3 & 6 & 1 & 0 \\
\hline 1.2 & 0 & 5 & 4 & 1 \\
\hline 1.3 & 0 & 7 & 3 & 0 \\
\hline 1.4 & 0 & 0 & 3 & 7 \\
\hline 1.5 & 0 & 10 & 0 & 0 \\
\hline 2 & 0 & 10 & 0 & 0 \\
\hline
\end{tabular}

Table 4.3: Results from the fourth configuration within Config_combos_0.

\begin{tabular}{|c|l|l|l|l|}
\hline & Success & \multicolumn{3}{|c|}{ Failure } \\
\hline H-value weighting & Goal reached & Became stationary, no path & $\begin{array}{l}\text { Stuck alternating between } \\
\text { points }\end{array}$ & Hazard traversed \\
\hline \hline 1.0 & 15 & 1 & 1 & 3 \\
\hline 1.1 & 15 & 4 & 1 & 0 \\
\hline 1.2 & 17 & 3 & 0 & 0 \\
\hline 1.4 & 2 & 18 & 0 & 0 \\
\hline 2.0 & 0 & 20 & 0 & 0 \\
\hline
\end{tabular}

Table 4.4: Config_combos_2.

less significant than anticipated. Config_combos_2 was the next configuration to be used, containing one location-destination setting which was used with two different obstacle configurations. The results from Config_combos_2 were found to be more varied across weightings, but less so between the two different obstacle setups, which appear to have had limited affect, so their results were combined and are given in Table 4.4 .

\section{Trial sets A, B and C}

Using Config_combos_2 again (as from the previous trials it was considered a more interesting combination), other facets of the simulation were then varied. Initially the number of steps occurring between compulsory path re-evaluations were varied. These trials were given the suffix of A0, A1 and A2 which were 2, 5 and 10 steps, respectively. Next the number of steps was reset to one and the clearance buffer the robot required around itself was increased from $100 \mathrm{~mm}$ to $300 \mathrm{~mm}$. The trials were repeated and given the designation/label of B. Across 


\begin{tabular}{|c|c|c|c|c|c|}
\hline & Success & \multicolumn{3}{|c|}{ Failure } & \multirow{2}{*}{$\begin{array}{l}\text { Incomplete } \\
\text { Error }\end{array}$} \\
\hline H-value weighting & Goal reached & $\begin{array}{l}\text { Became stationary, no } \\
\text { path }\end{array}$ & $\begin{array}{l}\text { Stuck alternating be- } \\
\text { tween points }\end{array}$ & Hazard traversed & \\
\hline \multicolumn{6}{|c|}{ A0 } \\
\hline 1.0 & 7 & 6 & 0 & 7 & 0 \\
\hline 1.1 & 9 & 8 & 1 & 2 & 0 \\
\hline 1.2 & 13 & 3 & 0 & 4 & 0 \\
\hline 1.4 & 0 & 20 & 0 & 0 & 0 \\
\hline 2.0 & 0 & 20 & 0 & 0 & 0 \\
\hline \multicolumn{6}{|c|}{$\overline{\mathrm{A} 1}$} \\
\hline 1.0 & 14 & 2 & 2 & 2 & 0 \\
\hline 1.1 & 11 & 0 & 0 & 2 & 7 \\
\hline 1.2 & 13 & 2 & 4 & 1 & 0 \\
\hline 1.4 & 0 & 20 & 0 & 0 & 0 \\
\hline 2.0 & 0 & 20 & 0 & 0 & 0 \\
\hline \multicolumn{6}{|c|}{$\mathrm{A} 2$} \\
\hline 1.0 & 9 & 1 & 5 & 0 & 5 \\
\hline 1.1 & 19 & 0 & 1 & 0 & 0 \\
\hline 1.2 & 3 & 1 & 0 & 0 & 16 \\
\hline 1.4 & 0 & 20 & 0 & 0 & 0 \\
\hline 2.0 & 3 & 17 & 0 & 0 & 0 \\
\hline
\end{tabular}

Table 4.5: Config_combos_2A.

\begin{tabular}{|c|l|l|l|l|}
\hline & Success & \multicolumn{3}{|c|}{ Failure } \\
\hline H-value weighting & Goal reached & Became stationary, no path & $\begin{array}{l}\text { Stuck alternating between } \\
\text { points }\end{array}$ & Hazard traversed \\
\hline \hline 1.0 & 12 & 2 & 2 & 4 \\
\hline 1.1 & 17 & 3 & 0 & 0 \\
\hline 1.2 & 14 & 4 & 1 & 1 \\
\hline 1.4 & 1 & 19 & 0 & 0 \\
\hline 2.0 & 0 & 20 & 0 & 0 \\
\hline
\end{tabular}

Table 4.6: Config_combos_2B.

this variety of parameters it was noted, from the results shown in Tables 4.5 and 4.6, that the weightings of 1.5 and 2.0 were unsuccessful in general and as such the specific paths they had attempted were inspected.

A minor alteration was made to the behaviour of the $A^{*}$ algorithm when no paths were found, after the specific steps involved in the paths of set 8 showed no movement occurred/paths were attempted in almost every trial. As such, the same sets of trials for Config_combo_2B were re-simulated and produced the following results, as shown in Table 4.7, which show improvement in that for the weighting of 1.4 and 2.0 a number of successful runs occured. 


\begin{tabular}{|c|l|l|l|l|}
\hline & Success & \multicolumn{3}{|c|}{ Failure } \\
\hline H-value weighting & Goal reached & Became stationary, no path & $\begin{array}{l}\text { Stuck alternating between } \\
\text { points }\end{array}$ & Hazard traversed \\
\hline \hline 1.0 & 14 & 0 & 2 & 4 \\
\hline 1.1 & 17 & 0 & 2 & 0 \\
\hline 1.2 & 16 & 1 & 0 & 0 \\
\hline 1.4 & 14 & 2 & 4 & 0 \\
\hline 2.0 & 14 & 0 & 0 & 6 \\
\hline
\end{tabular}

Table 4.7: Config_combos_2B re-simulated.

\begin{tabular}{|c|l|l|l|l|l|}
\hline & Success & \multicolumn{2}{|c|}{ Failure } & Incomplete \\
\hline H-value weighting & Goal reached & $\begin{array}{l}\text { Became stationary, no } \\
\text { path }\end{array}$ & $\begin{array}{l}\text { Stuck alternating be- } \\
\text { tween points }\end{array}$ & Hazard traversed & Error \\
\hline \hline 1.0 & 1 & 9 & 2 & 0 & 8 \\
\hline 1.1 & 0 & 0 & 20 & 0 & 0 \\
\hline 1.2 & 5 & 7 & 2 & 0 & 6 \\
\hline 1.4 & 13 & 2 & 4 & 0 & 1 \\
\hline 2.0 & 3 & 6 & 4 & 0 & 7 \\
\hline
\end{tabular}

Table 4.8: Config_combos_2C.

Another variation was tested, which was increasing the step sizes to $500 \mathrm{~mm}$. This trial was labelled $\mathrm{C}$ and was chosen for comparison with A (specifically A1, which was 5 moves of $100 \mathrm{~mm}$ ) to determine whether performance was better with many small steps or with fewer large movements. The results generated by these sets of trials are given in Table 4.8. Note: The results for this trial were produced on a different computer, which had become available for running some simulations, to the other pilot trials.

Following the modification of the incomplete path behaviour, the trials were also altered in the range of weightings used for testing. The original values were chosen to rise in growing increments, so as to give more coverage to closer values but also include values of much greater difference. Upon the second set of trials, where only every second weighting value was used, it was noted that the first few values followed a power of two pattern in their variance from the original weighting. It was decided to follow this pattern through and as such the final two weightings were changed from 1.5 and 2.0 to 1.6 and 1.8 , respectively.

It was also found that the end condition of trials were not always being printed to file, as when exiting due to being caught alternating between two points the 
relevant exit status was not being passed.

\section{Trial sets D, E, F and $\mathrm{H}$}

Additional trial sets were tested which matched up with previous sets but with the basis for the network node creation modified to result in fewer nodes being produced or retained and hopefully less re-processing occurring between successive iterations. Set D is the same as the default settings but with network changes of reducing the visible distance and increasing the minimum allowable distance between nodes, being applied. Sets E and F correspond to sets A1 and C, respectively, having the same settings except for the aforementioned changes to the basis from which the network node creation occurs. Set $\mathrm{H}$ was related to the default setting and Set $\mathrm{D}$, with the maximum distance across which nodes could be linked being reduced below the level of the visible distance. Like previous sets, these trials were tested on Config_combos_2. No issues were detected with the results, with the previous sets appearing to have covered all areas in which the trials may wish to be altered.

\section{Config_known}

Another configuration was Config_known, which provided a flat obstacle free landscape that enabled the use of pre-known/made nodes. The pre-made nodes were used to isolate the path planning section from the earlier hazard identification and node network creation stages. The separation of modules allowed comparison of performance when using the sections compared to omitting them and using idealised/pre-known node positions. These pre-made nodes could potentially be used to test the algorithms with a grid network, however in this instance they were not put to that use.

This configuration was used to visually monitor the performance of the hazard identification and the network node creation, and it was found that both tasks appeared to function as expected. Figure 4.2 contains a few screen shots taken during this testing, which demonstrate the ability to correctly set the hazard status of ter- 


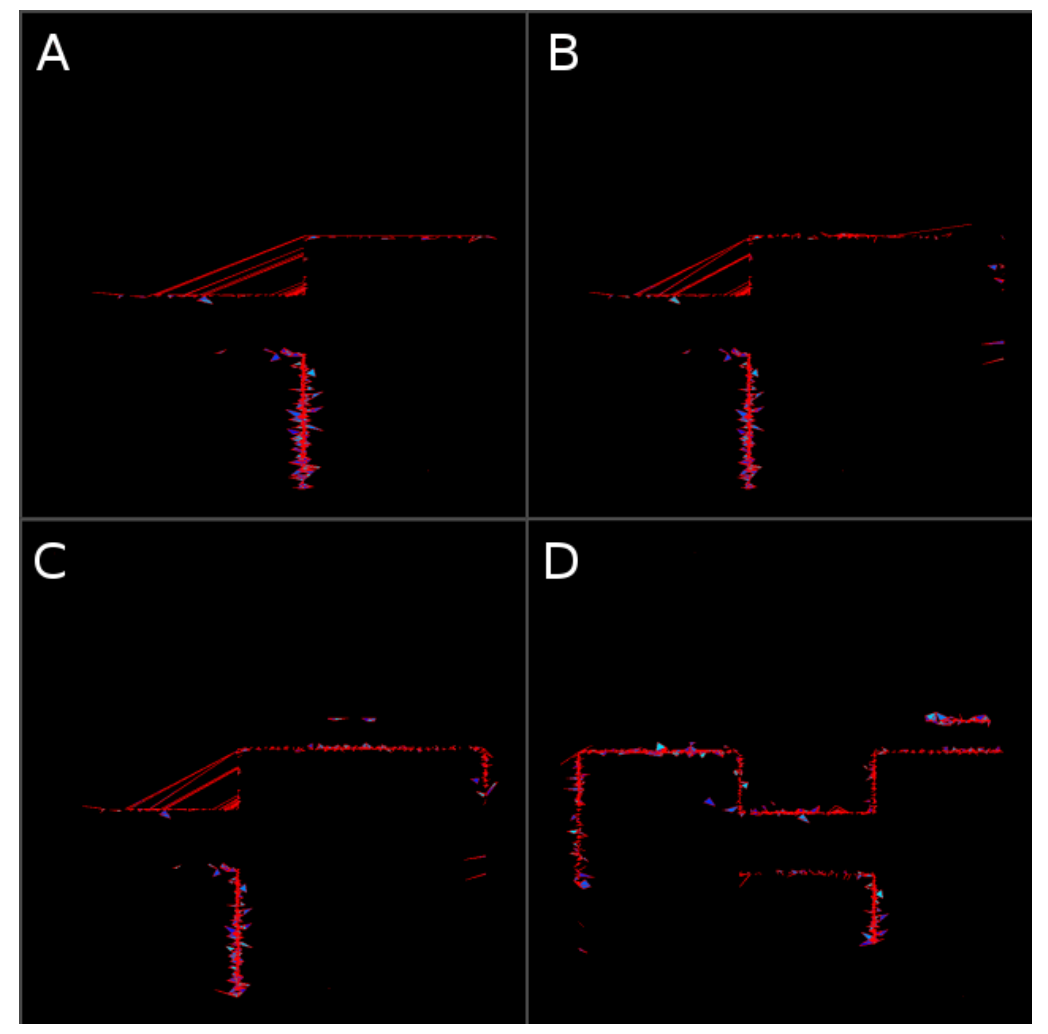

Figure 4.2: Visual depictions of nearby hazard triangles within the set identified by the robot. $\mathrm{A} \rightarrow \mathrm{C}$ were produced as the robot moved to the right, while $\mathrm{D}$ is from later on, when the robot has explored to the left of the initial position of A.

rain faces, retrieve this information and through motion inferred by sequence, the avoidance of hazardous regions. Frames $\mathrm{A} \rightarrow \mathrm{C}$ of Figure 4.2, follow the Robot as it explores to the right, while Frame D is from later in the simulation when it has exhausted its search of the right and has entered a region to the left to further explore potential paths. The robot's node network of potential paths was also monitored and found to have a gradual expansion as expected, as it moved into previously unknown regions. Correct linking was found to occur within current network nodes and between current nodes and the nodes from previous iterations/positions. 


\section{Config_combos_5}

One configuration which was briefly tested involved a gentle rolling terrain, with regular positioning of multiple obstacles. This involved more obstacles than had been included in other scenarios and as such lead to the discovery of a bug in the code, due to a missing break statement, which could cause objects to be written to a full array before it had been reallocated more memory. With the addition of the omitted break statement, the bug was fixed as one of the final acts of the pilot testing session, before moving on to gathered experimental results.

\subsubsection{LPA* algorithm}

Due to an intermittent bug existing somewhere within the memory management of the LPA* algorithm, trials using it had each scenario run as two lots of five rather than one set of ten, so as to avoid the risk of the bug being encountered and hence the simulation exiting before completing the whole set.

\subsubsection{Miscellaneous Configurations}

Some other configurations which were tested but not chosen for extended testing were Config_combos_3 and Config_combos_4, which were flat terrains on which a single large maze obstacle and a larger alternate maze obstacle, were each placed. The mazes had been created using Blender 3D (a modelling suite), and exported as $.3 \mathrm{~d}$ s objects, however there were some issues with adding them and their meshes to the simulation terrain. Subsequently the configurations were omitted as they could not be relied on to run correctly. 
94CHAPTER 4. EXPERIMENTAL DESIGN AND PILOT TESTS OF PATH PLANNING 


\section{Chapter 5}

\section{Path Planning Results}

After the pilot tests were completed, full experimental testing was undertaken with the number of repetitions for each configuration pair within a config_combo being extended to 50, producing 100 trials for each weighting.

The initial configuration file of Config_combos_0, consisted of four scenarios, produced through combining two terrain settings and two obstacle settings. Due to the similarities between the results for the first pair of scenarios (having the same terrain file but differing obstacle files) and between the results of the second pair of scenarios, but differences between the pairs, it was decided to separate the second pair out of Config_combos_0 and into another configuration file, so as to better group the outputted results. This decision was made part way through the generation of experimental results while beginning to calculate and plot the statistics for some of the trial sets, as such for some trials the data was outputted into a combined file. For these combined results only the number of occurrences of different end conditions can be provided for Config_combos_0 and Config_combos_1 (extracted from SimResults.txt) as filtering the data within the Actual_Steps.txt file is too time consuming and difficult to ensure it is done correctly.

Of primary interest within the results from these simulations, are the mixture of completion status, the average number of movements occurring for successful completions and also the general nature or pattern of paths when overlaid. 


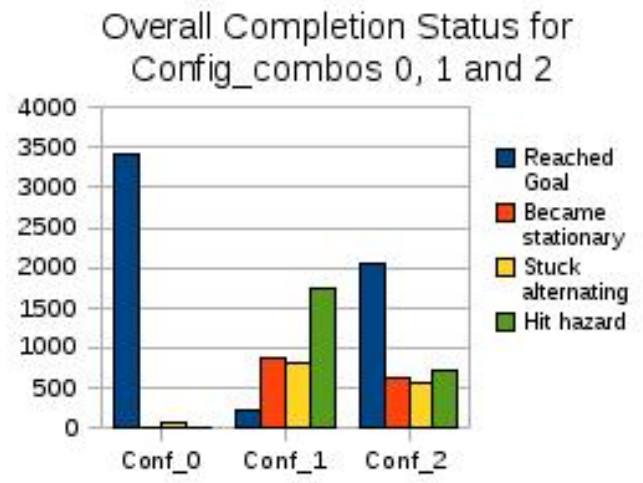

Figure 5.1: Comparison of the summed/generalised results produced using each configuration file.

\subsection{Config_combos 0,1 and 2}

A general idea of the complexity of each of the scenarios is demonstrated in Figure 5.1, which gives the completion status for the results generated using each configuration file, combined together according to the respective file used. Config_combos_0 can be seen to be the least difficult configuration, through the clear predominance of successful completions as the most frequent end condition. Directly contrasting the success rate of Config_combos_0, Config_combos_1 is shown to have a very low occurrence of success, with the three alternative reasons for completing a simulation all occurring many times.

\subsection{Algorithms}

\subsubsection{A* Algorithm}

An observation about the simulation runs for the default parameter setting and $\mathrm{A}^{*}$ algorithm, was that based on external factors it would appear they potentially ran slower than some of the modified sets. This observation was made due to the local temporary directory in which data were recorded, clearing some of the earlier files which it is expected was due to age and inactivity, possibly in combination 
with scheduled cleans. The simulations were run from the local /tmp/ directory of each computer, with data temporarily recorded there in order to avoid excessive $\mathrm{read} / \mathrm{write}$ operations on the home directory as it was a network drive. Upon completion, the shell file which had called the Simulations proceeded to transfer the results on to the network drive, however this occurred too long after the earliest data were recorded and trial sets 0 for all three configurations were lost and required to be re-simulated. These losses were an oversight, with research into the exact basis the computers were using for clearing the tmp directory needing to have been investigated.

Comparing the results of the $\mathrm{A}^{*}$ algorithm under default settings and across all three configuration files, as shown in Figure 5.2, configuration 0 and 1 produce results which are polar opposites, respectively being highly successful and very poor. The results generated for Config_combos_2 provide a mixture of completion status but are distinctly closer to Config_combos_0 than to Config_combos_1. In terms of steps necessary to conclude a trial, Figure 5.2 indicates that trial runs on Config_combos_2 consisted of a much higher number of movements. While the greatest straight line distance between the start position of the robot and the goal location is for Config_combos_0, the other two configurations require greater deviations to avoid regions which are not traversable and hence will generally consist of more movements.

\subsubsection{LPA* Algorithm}

Because of time constraints and in light of earlier results, it was decided to limit the experimental trials of the LPA algorithm to configuration files zero and two, and reduce the number of h-value weightings tested to three (1.0, 1.2 and 1.8). The simulations of the LPA* trial sets occurred after completing all the initial simulations for the $\mathrm{A}^{*}$ trial sets. At this point the computers began to perform poorly, with the linux graphical desktop and the file manager becoming slower to respond, in some cases to the point of requiring logging out and in again or a reboot. As the current installation of Fedora on these computers had not been used before (being the under graduate computer lab), it is not clear whether this 

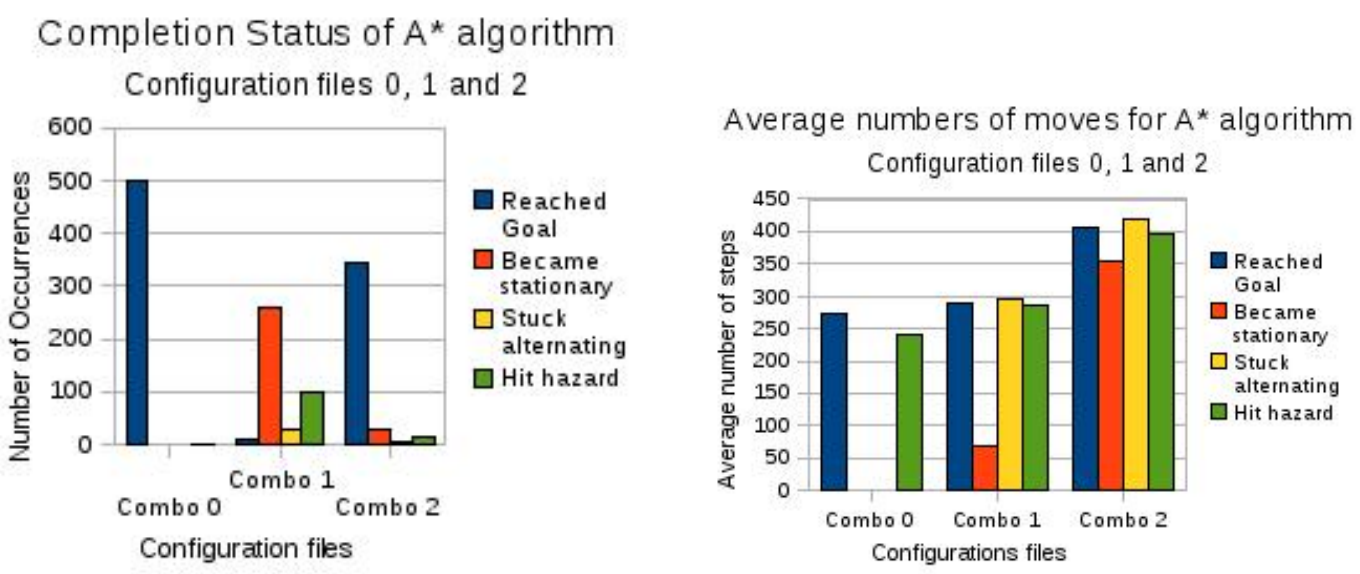

Figure 5.2: Left: Completion status for A* algorithm, on Config_combos_0, Config_combos_1 and Config_combos_2. Right: The average number of movements occurring for each completion status.

was a side-effect of prolonged usage, multiple log in sessions of the same user or the simulation tool. However, as the network administrator had mentioned that there were issues with the installation in terms of the graphical desktop and the file manager, plus regular system messages informing that a package dependency could not be resolved, it is felt the problems are fully or at least significantly due to issues external to the simulator.

The LPA* algorithm performed well on Config_combos_0 with a high number of successful completions, while in contrast the results produced with Config_combos_2, given in Figure 5.3, having few successes and instead have the robot becoming stationary most of the time.

\subsubsection{State-based Algorithm}

The simple state-based algorithm was briefly tested upon Config_combos_0 and Config_combos_2, with very poor results. It was found that, as shown in Figure 5.4, three paths were followed for Config_combos_2, of which two were almost identical save for a slight deviation, while the third path ran quite closely to the other two. With about 70 moves on average, all three of these paths were fairly 
Completion Status of LPA* algorithm

Configuration files 0,1 and 2

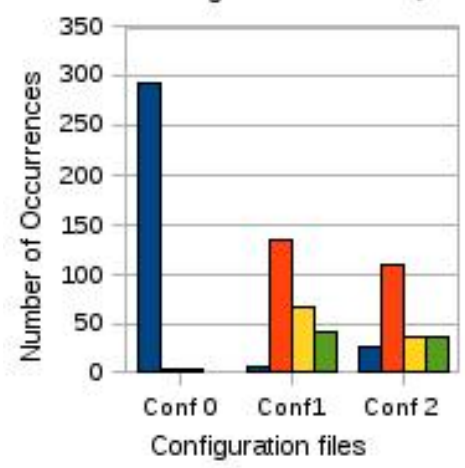

Average number of moves for LPA* algorithm

Reached Goal $\square$ Became stationary $\square$ Stuck alternating $\square$ Hit hazard

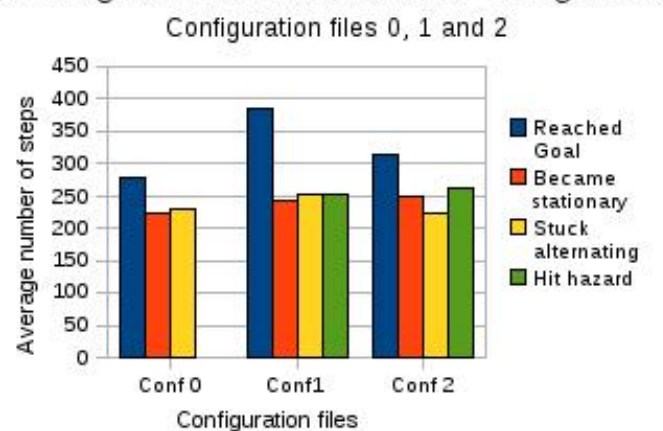

Figure 5.3: Left: Completion status for LPA* algorithm, on Config_combos_0 and Config_combos_2. Right: The average number of movements occurring for each completion status.

short in comparison to the paths generated by the other algorithms which even in cases of not finding the goal, would travel upwards of 150 steps on average. For Config_combos_0 the results were somewhat better, with the robot reaching the goal on nine out of forty seven occasions, which can be seen in the overlay of paths, as shown in the bottom right panel of Figure 5.5.

These poor results are likely due to the rushed conception and design of the algorithm with no performance based testing, and are unlikely to reflect the general performance of other case-based algorithms. However, the fact that in the simpler scenarios provided by Config_combos_0, this quick and simple design was capable of inter-operating with the other modules of the simulator to avoid an obstacle and reach a path, is testimony to how the preparation of data and creation of node network is a significant part of path navigation.

\subsubsection{Comparison of Algorithms}

Over the regions the three configuration files covered, the A* and LPA* algorithms were respectively run with 5 and 3 values of h-value weighting. The State-based algorithm had no equivalent variable for which the value could be weighted. As 


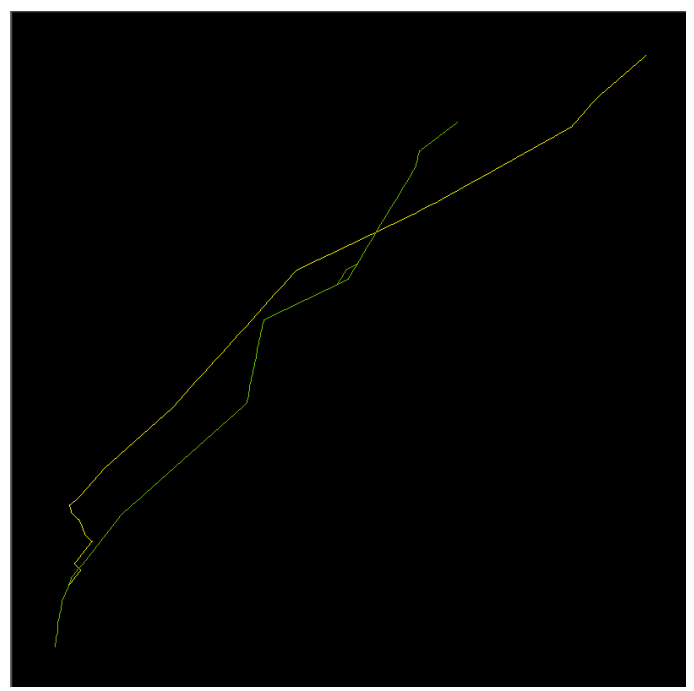

Figure 5.4: Overlaid paths generated by a simple state-based obstacle avoider for Config_combos_2.

seen in Figure 5.5, the $\mathrm{A}^{*}$ and LPA* algorithms produced very similar paths when run on Config_combos_0, which is not surprising. For the LPA* results, the paths appear to be grouped slightly tighter but this is most likely due to the quantity of trials being lower, with the $\mathrm{A}^{*}$ algorithm having been run with 5 weightings compared to LPA* being trialled with only 3 . The results from the State-based algorithm were quite different to those of the other two algorithms. However, there is a region in the centre of all the State-based results which appears to be similar in part, to the results produced by the A* and LPA* algorithms. This region of similarity does not continue on for the State-based algorithm, as it did with the other algorithms, as it would appear the state-based algorithm failed to negotiate getting past the obstacle.

For Config_combos_1 the sets of paths produced by the A* algorithms, presented in Figure 5.6, were not as tightly bunched as had been in seen with Config_combos_0. The presence of two regions containing hazardous obstacles is quite clearly defined in the overlaid paths, with the absence of paths due to skirting the areas. Looking at Figure 5.7, the results for the $A^{*}$ algorithm are a combination of some tightly grouped paths reaching the goal and some other paths 


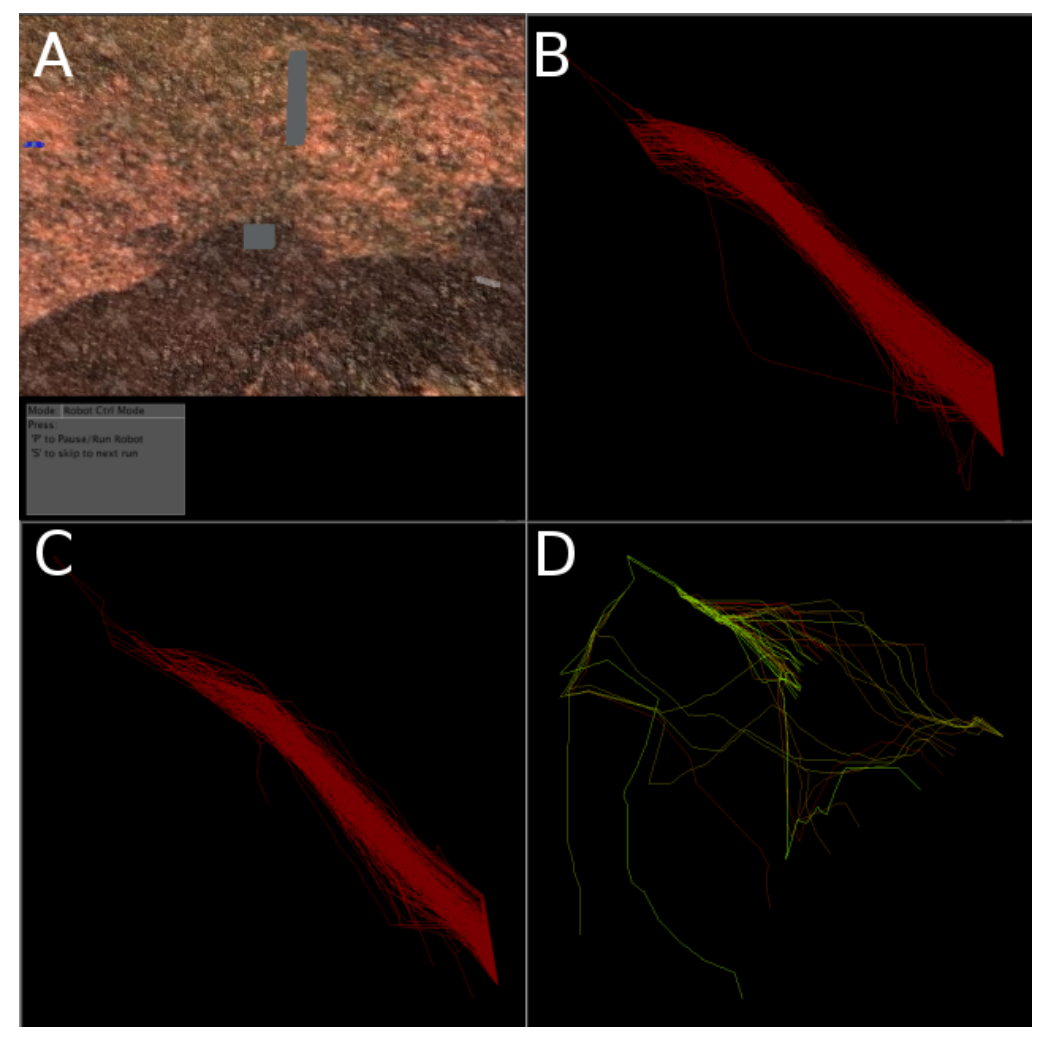

Figure 5.5: Config_combos_0 terrain overview plus overlaid paths generated by the A*, LPA* and State-based algorithms, given as B, C and D, respectively.

which spread out in a diffuse manner as they search for a suitable path to the goal, while with the LPA* algorithm all the path lead directly to the goal with very little divergence from the core path.

\subsection{H-value weightings}

\subsubsection{A* Algorithm}

All trials involving the $\mathrm{A}^{*}$ algorithm were run under the 5 different $\mathrm{h}$-value weightings of 1.0, 1.1, 1.2, 1.4, and 1.8. Looking at the results from individual weightings there is almost no variation between the number of occurrences of the different completion status, as is clearly visible in Figures 5.8, 5.9 and 5.10. The av- 


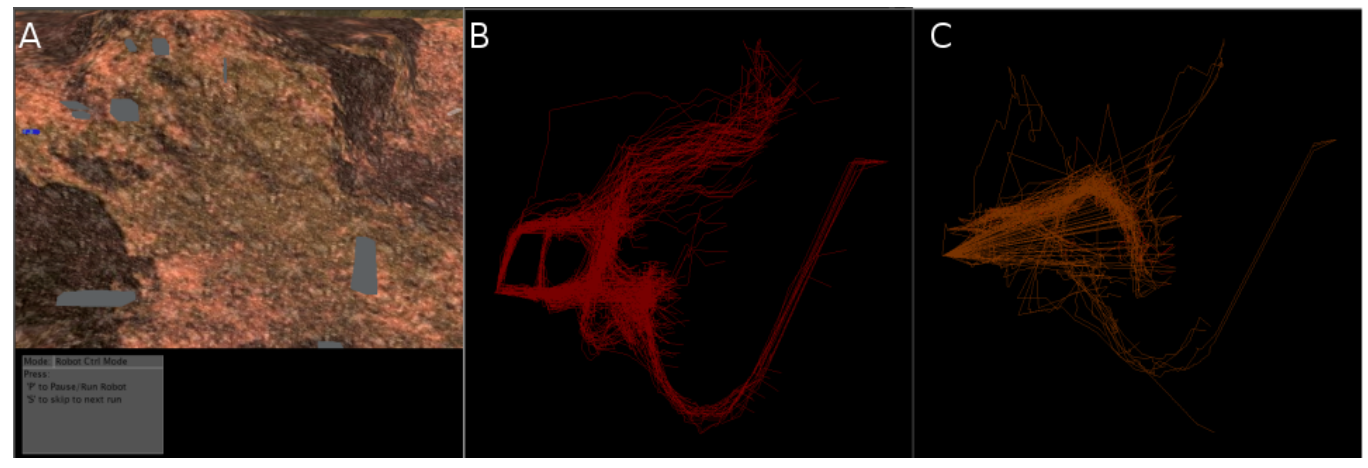

Figure 5.6: Config_combos_1 terrain overview plus overlaid paths generated by $\mathrm{A}^{*}$ algorithm, given as $\mathrm{B}$ and $\mathrm{C}$, respectively.

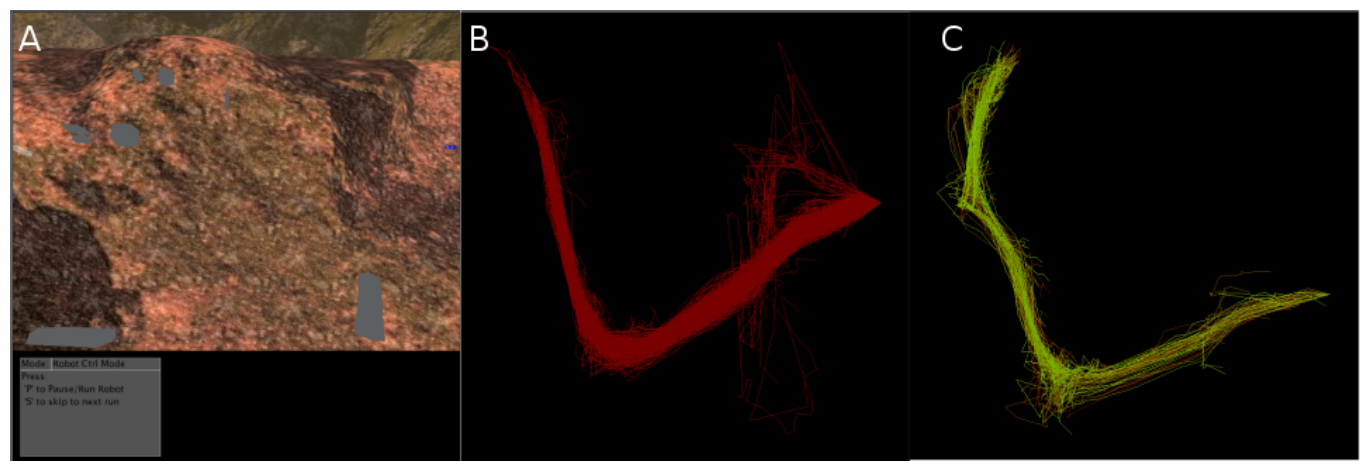

Figure 5.7: Config_combos_2 terrain overview plus overlaid paths generated by A* and LPA* algorithms, given as B and C, respectively. 
erage number of steps taken before the trials exited were also very similar across the different weightings, with the only notable difference being for weightings of 1.2 and 1.4 in Figure 5.9. The results indicate that the goal was reach after a much greater number of steps for the h-value weightings of 1.2 and 1.4, in comparison to the successful cases of a weighting of 1.8 and all the other cases of weightings 1.2 and 1.4 , but as there were very few occurrences no conclusion had be drawn with confidence about the successful paths under those weightings. With Config_combos_2 the average number of steps taken for unsuccessful completions appears to decrease as the h-value weighting increases, which potentially suggests that weighting the h-value is a hindrance but once again the number of occurrences is too low to add any confidence to perceived trends.

These other trials also produced results which showed no notable difference between the specific results from the different values of the h-value weighting. Therefore, upon further testing the weightings were grouped together and presented as a single set due to the similarity across all other trials.

For the two scenarios within Config_combos_1 the results were quite different in that for the first one the same path was chosen each time and failure occurred at the same point, becoming stationary due to being unable to determine/detect any paths. As such, the number of occurrences for the status of becoming stationary is very high, which when comparing Figure 5.9 to the results produced for Config_combos_1 by others sets, gives a distinctly different result to the trend of completion status whereby hitting a hazard is the most common occurrences for this configuration. As the second scenario, which involved the same region of terrain but with different obstacles placed within it, produced results akin to the other trials it must be surmised that this early halting was specific to the scenario rather than being an overriding behaviour.

Note: The results for the h-value weighting of 1.0 were lost for the $\mathrm{A}^{*}$ algorithm as previously mentioned but unfortunately re-simulations were not completed in time for inclusion. 
Config_combos_0Completion Status

Various $h$-value weighting for the $A^{*}$ Algorithm
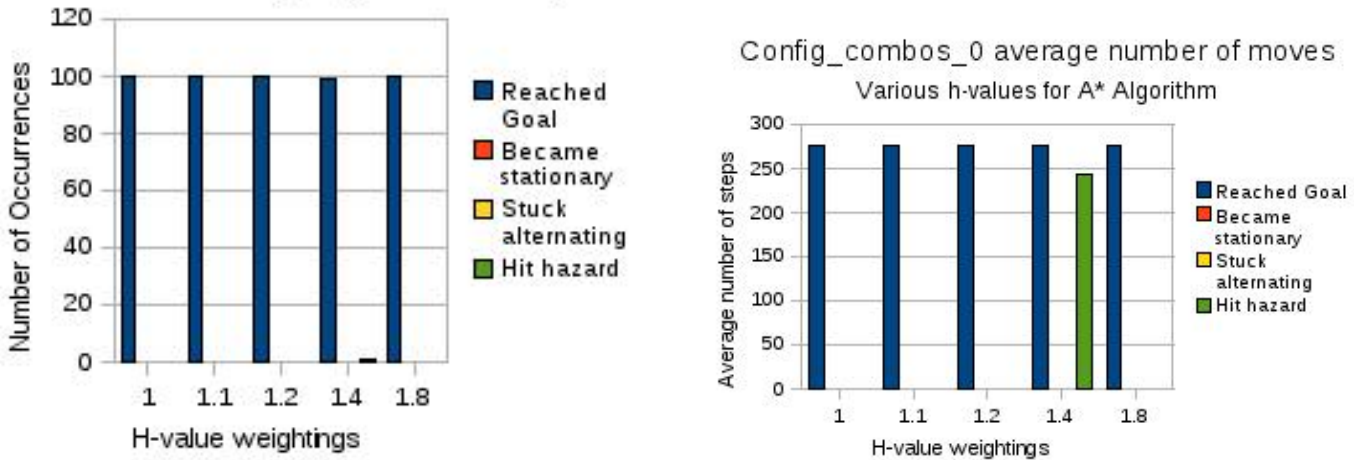

Figure 5.8: Left: Completion status of various $\mathrm{h}$-value weightings for $\mathrm{A} *$ algorithm, on Config_combos_0. Right: The average number of movements occurring for each completion status.

Config_combos_1 Completion Status

Various $h$-value weighting for the $A^{*}$ Algorithm
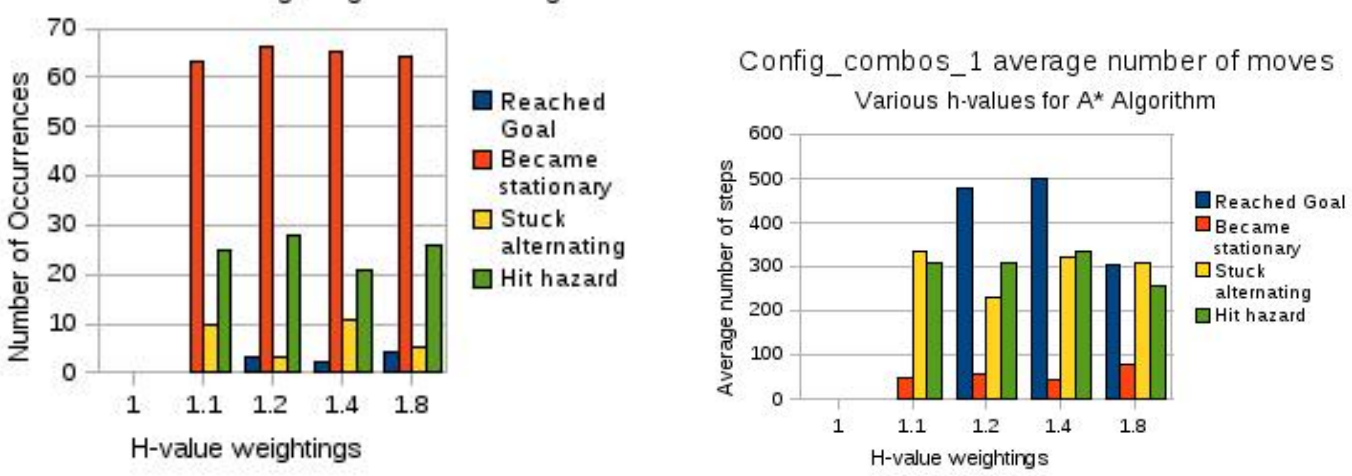

Figure 5.9: Left: Completion status of various h-value weightings for the $\mathrm{A}^{*}$ and LPA* algorithms, on Config_combos_1. Right: The average number of movements occurring for each completion status. 


\section{Config_combos_2 Completion Status}

Various $h$-value weighting for the $\mathrm{A}^{*}$ Algorithm
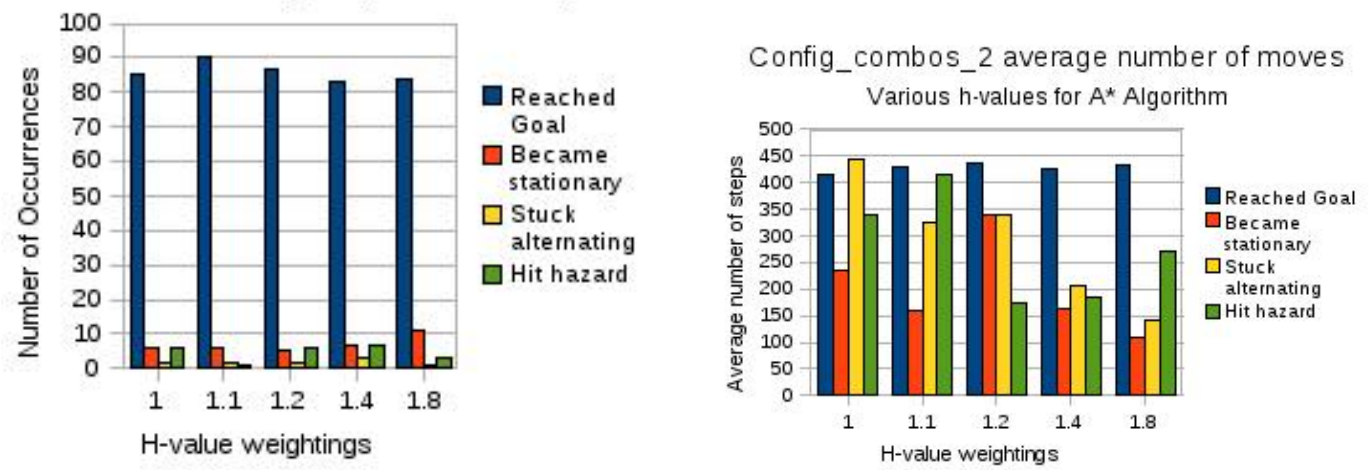

Figure 5.10: Left: Completion status of various h-value weightings for $\mathrm{A}^{*}$ algorithm, on Config_combos_2. Right: The average number of movements occurring for each completion status.

\subsubsection{LPA* Algorithm}

As mentioned previously, the trial sets for the LPA* algorithm involved three different h-value weightings of 1.0, 1.2, and 1.8, and were trialled on Config_combos 0 and 2. The results from the trials when the h-value weighting was 1.0 and Config_combos 2 was used, were lost when they did not get copied in to results directory and were left on the /tmp drive, where they were later deleted. This loss is somewhat significant as fewer weightings had been trialled, however from Figures 5.11, 5.12 and 5.13, as well as the initial pilot trials, it can be seen that the results for the LPA* algorithm were like those of $A^{*}$, in that the various h-value weightings did not appear to make a significant difference to the planned paths. The results gathered using the LPA* algorithm contained no surprises, with Figures 5.11, 5.12 and 5.13 all being similar to the corresponding ones of the $\mathrm{A}^{*}$ algorithm, which suggests that the LPA* algorithm was implemented correctly and without behavioural bugs. 
Config_combos_0 Completion Status Various h-value weighting for the LPA* Algorithm
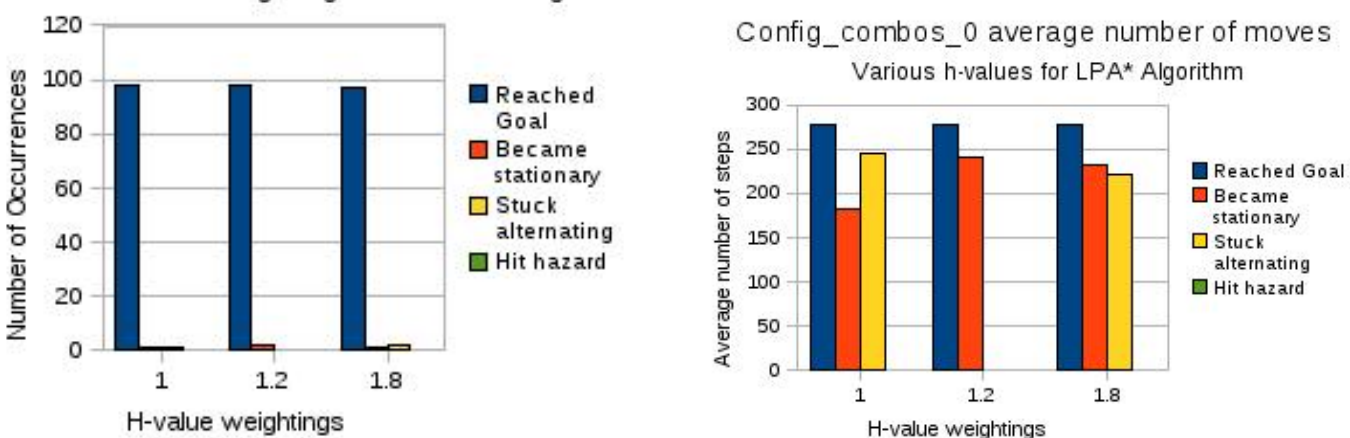

Figure 5.11: Left: Completion status of various h-value weightings for the LPA* algorithm, on Config_combos_0. Right: The average number of movements occurring for each completion status.

Config_combos_1Completion Status

Various h-value weighting for the LPA* Algorithm

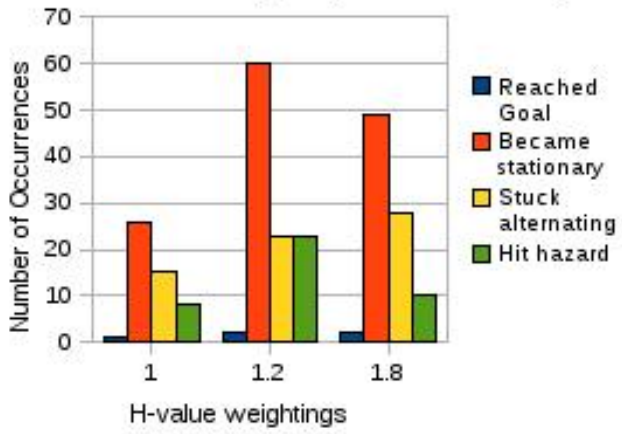

Config_combos_1 average number of moves Various h-values for LPA* Algorithm

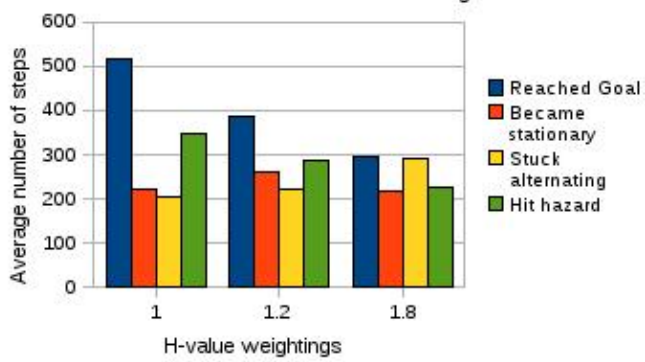

Figure 5.12: Left: Completion status of various h-value weightings for the LPA* algorithm, on Config_combos_1. Right: The average number of movements occurring for each completion status. 
Config_combos_2 Completion Status Various h-value weighting for the LPA* Algorithm
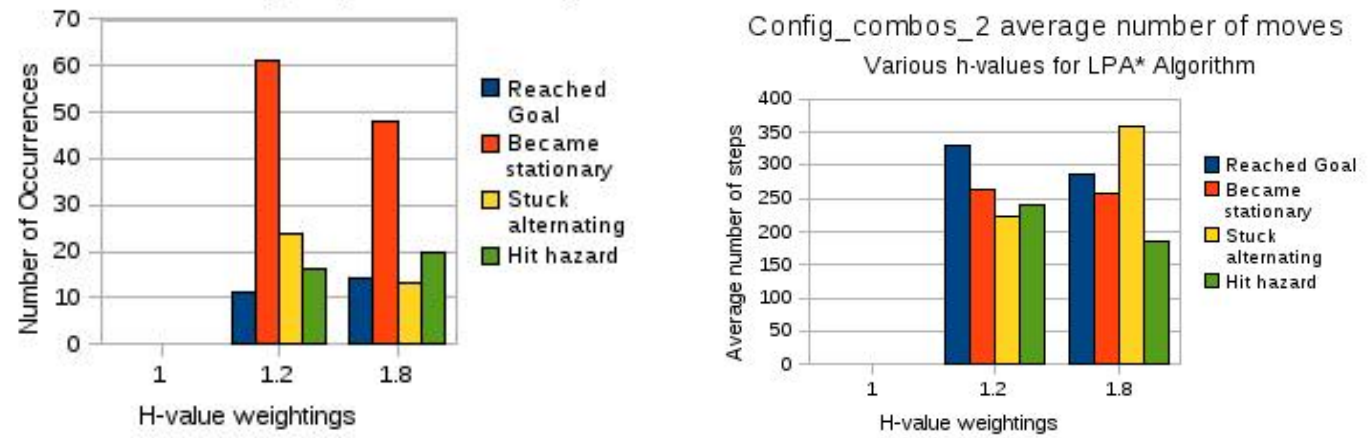

Figure 5.13: Left: Completion status of various h-value weightings for the LPA* algorithm, on Config_combos_2. Right: The average number of movements occurring for each completion status.

\subsection{A-Series Trial Sets}

Figure 5.14 contains the results across sets A0, A1 and A2, from Config_combos_0, with Figure 5.15 and Figure 5.16 showing the results from Config_combos_1 and Config_combos_2, respectively. One might note that the total number of completion results in Figure 5.14 is noticeably lower than in other cases, the reason is that the results of only one of the two scenarios in Config_combos_0 were gathered for each weighting of each set. The configuration file was accidentally edited during simulation and so for some weightings the first scenario was skipped. As the results displayed are the combination across all weightings, it was felt it was necessary to endeavour to have each weighting have a reasonably equal influence and thus omit the relevant data from those sets which had fully completed the scenarios of Config_combos_0. For the completion status which had a single line of data for each trial run removing the data was simple. However, the data for the sequence of steps taken for each trial run was significantly greater and more difficult to filter, which resulted in the re-inclusion of all data for Figure 5.14. For Figures $5.17,5.18$ and 5.19, it had been decided that all of the available paths were overlaid since the visual representation of the paths is more general and less definite 

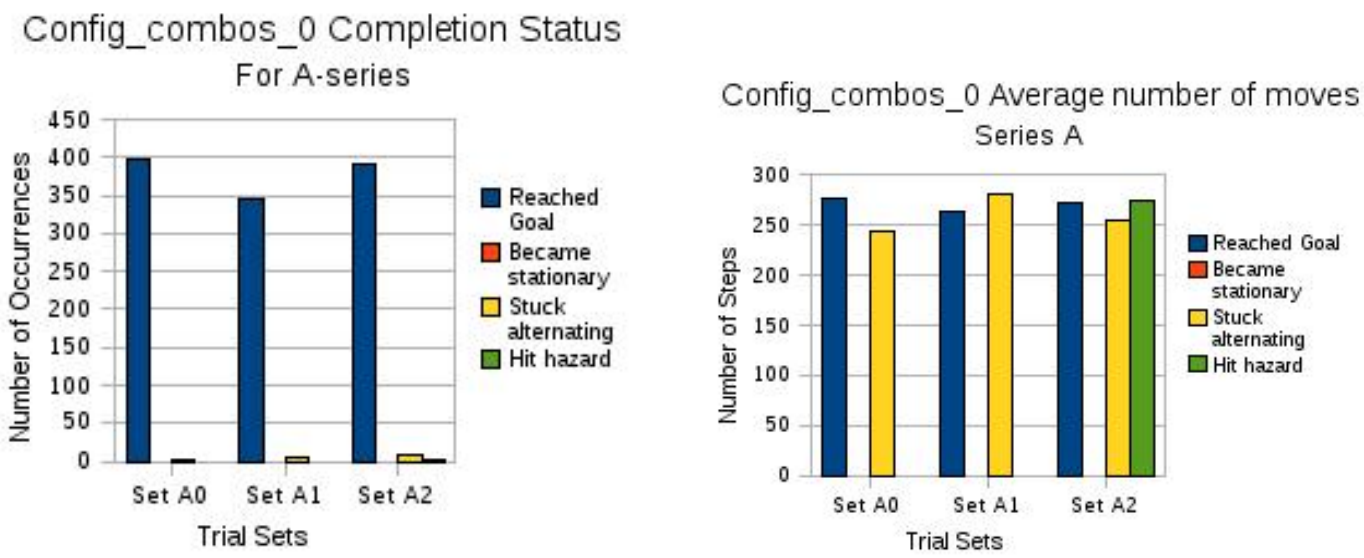

Figure 5.14: Left: Completion status for the A-series of alterations with the A* algorithm, on Config_combos_0. Right: The average number of movements occurring for each completion status.

than the statistical characteristics being shown in the charts, and this decision did not change. Across all three sets of trials, the results from the A-series produced paths which were very alike, as Figures 5.17, 5.18 and 5.19 show.

Once again the results for Config_combos_0, have resulted almost solely in successful runs being completed, as Figure 5.14 demonstrates. While the results shown in Figure 5.15 for Config_combos_ 1 are not particularly good, they do have a greater presence of successful runs and a lower incidence of encountering hazards than can be seen in Figure 5.21, for Sets D, E and H, which are presented later. Interestingly it would appear that each of the sets within the A-series managed to find a path down the slope located directly between start and finish, which did not exceed the maximum slope angle to which the robot was limited. This could be due to an error in the simulator's detection of hazardous traversals, however as the path appears in the same spot across all the sets this suggests it is an actual path, though it does not rule out it being a random error.

Note: The display/recreation of paths appears to show that the robot has travelled past the goals. This was originally thought to be due to not taking into account the affect of uphill/downhill travel, whereby the the absolute distance covered is actually further due to the z-component, while the recreation displays 

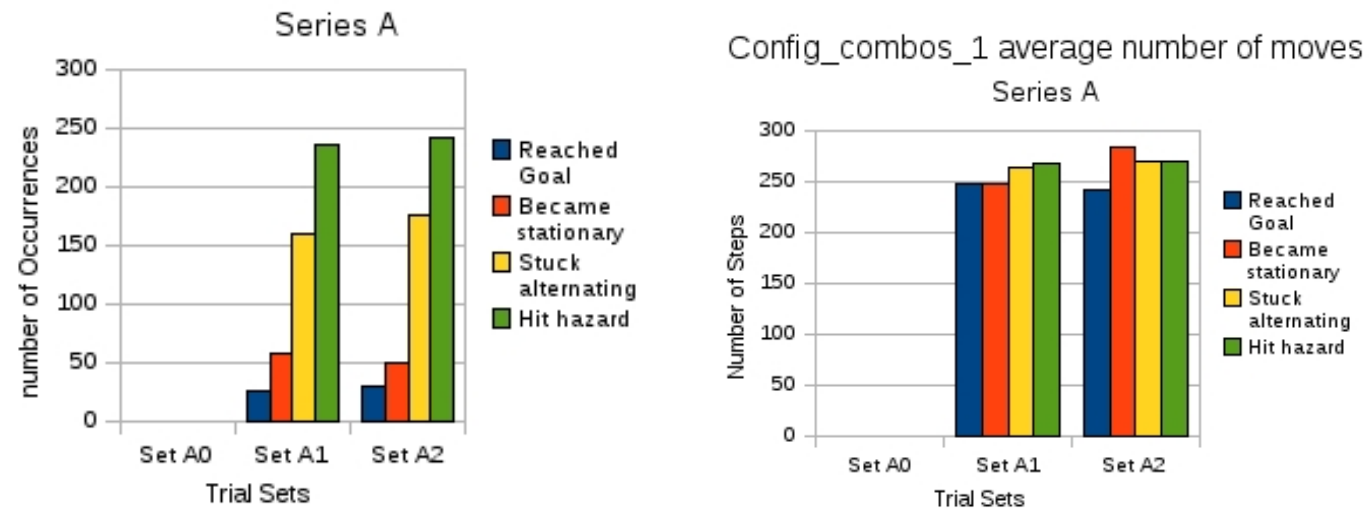

Figure 5.15: Left: Completion status for the A-series of alterations with the A* algorithm, on Config_combos_1. Right: The average number of movements occurring for each completion status.
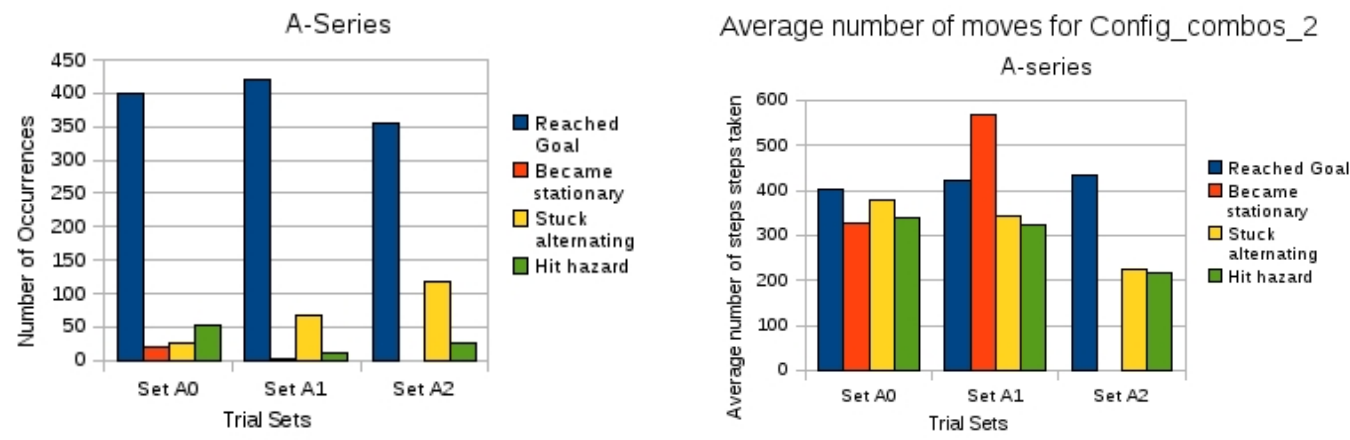

Figure 5.16: Left: Completion status for the A-series of alterations with the $\mathrm{A}^{*}$ algorithm, on Config_combos_2. Right: The average number of movements occurring for each completion status. 


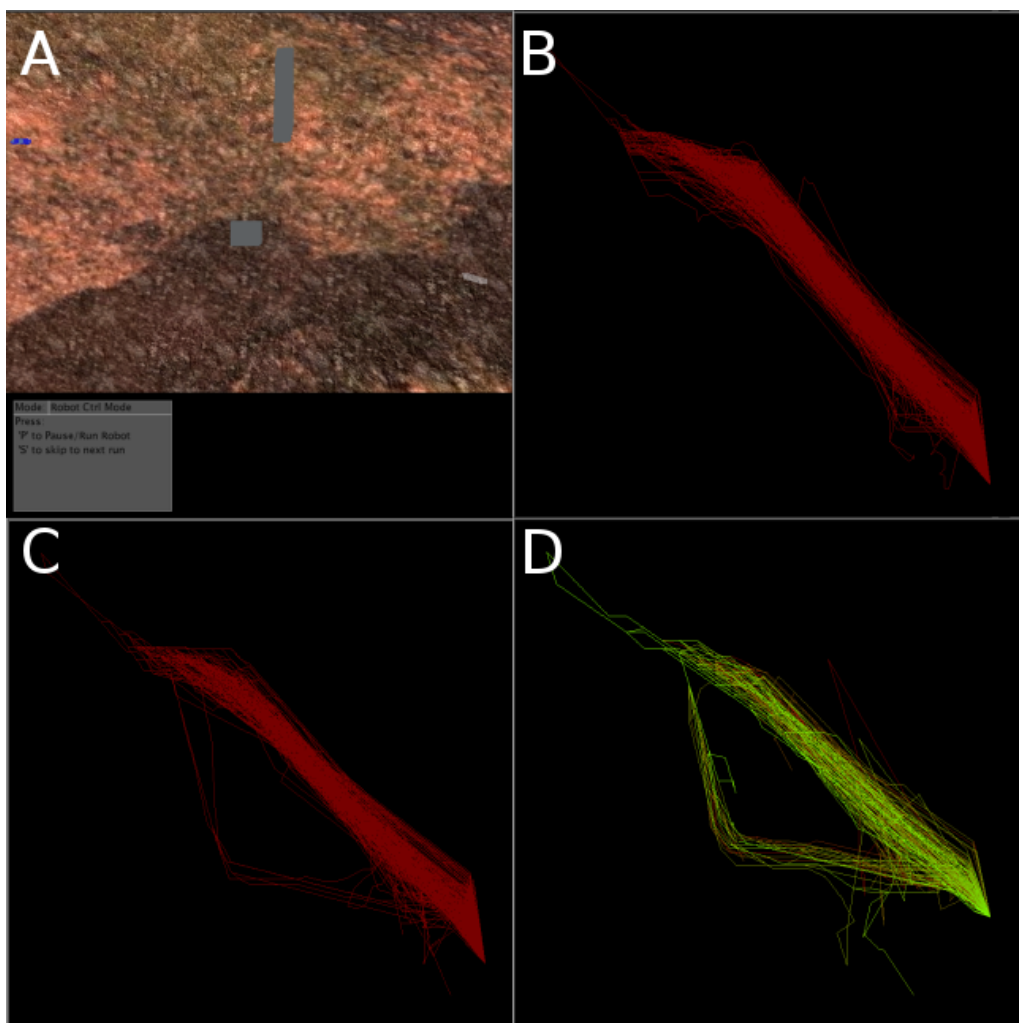

Figure 5.17: Overlaid paths generated by the A-series of variation, with the A* Algorithm on Config_combos_0. (A) Terrain overview (B) Set A0 paths, (C) Set A1 paths and (D) Set A2 paths. 


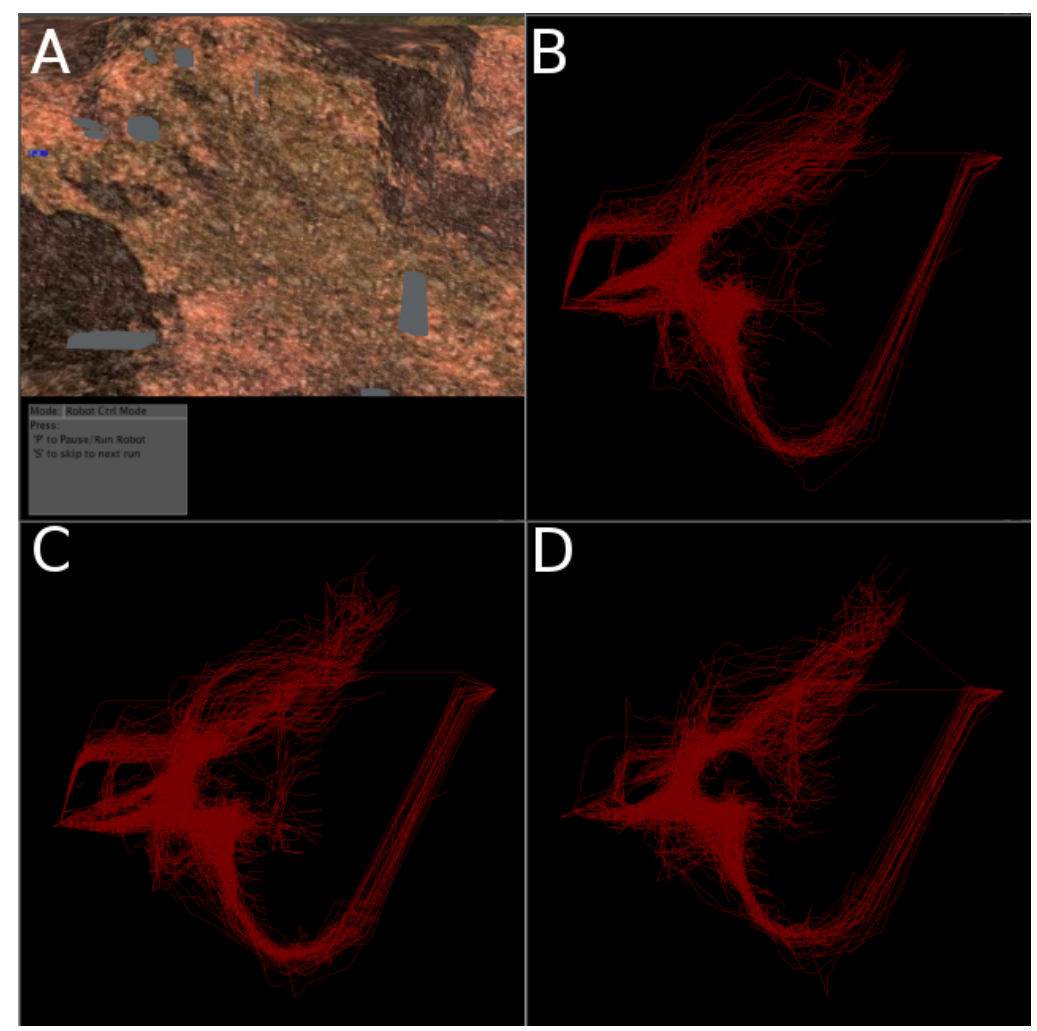

Figure 5.18: Overlaid paths generated by A-series variation with $A *$ Algorithm on Config_combos_1. (A) Terrain overview (B) Set A0 paths, (C) Set A1 paths and (D) Set A2 paths. 


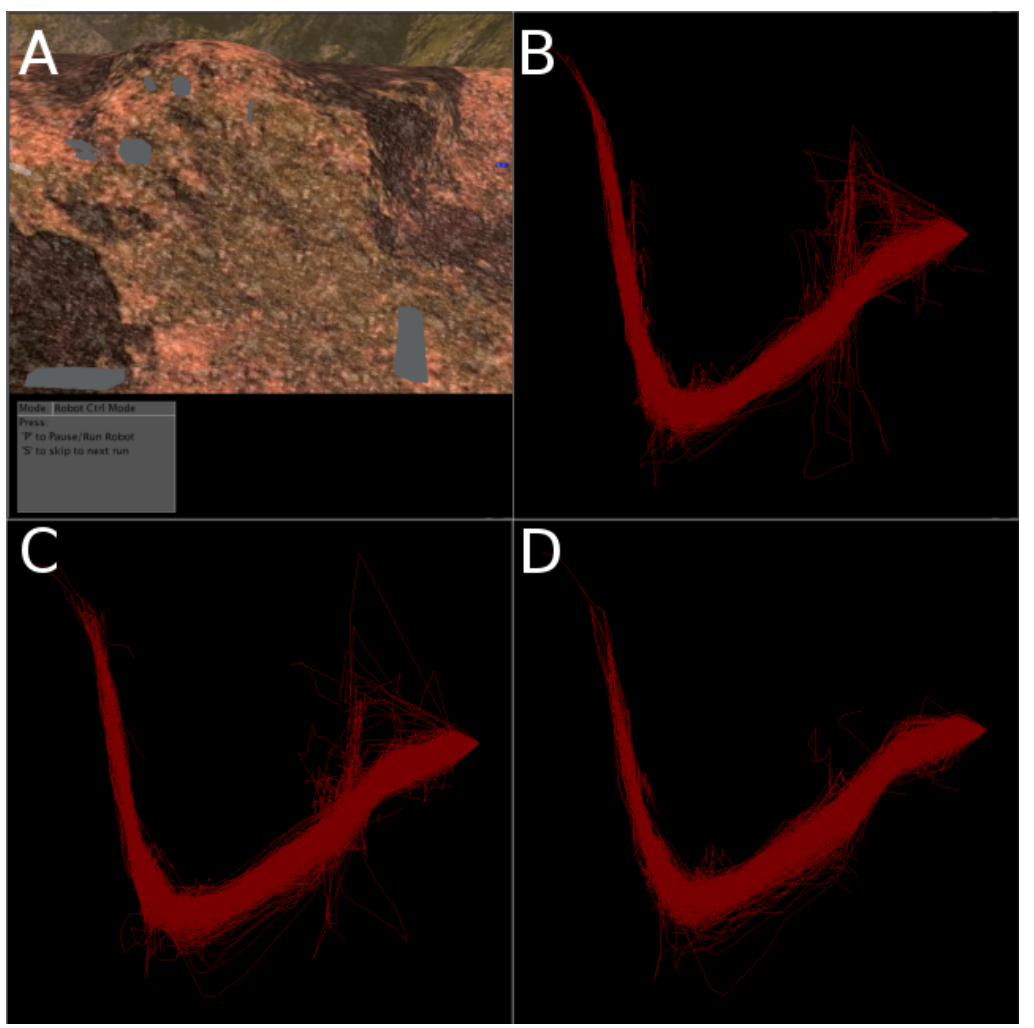

Figure 5.19: Overlaid paths generated by A-series variation with A* Algorithm on Config_combos_2. (A) Terrain overview (B) Set A0 paths, (C) Set A1 paths and (D) Set A2 paths. 
the movements as if derived purely from the XY-plane. Considering the paths of both Config_combos 1 and 2, the same overshoot appears to occur for both in spite of moving in opposite directions. As such, it may be that the display is twisted relative to the orientation at which it is believed to be presented.

\subsection{Trial Sets D, E,H}

Due to a numerical overflow occurring during the calculation of distances between points, for trial sets $\mathrm{D} \mathrm{E}$ and $\mathrm{H}$, the Config_combos_1 configuration file exited with an error. This was not detected during pilot testing, as not all potential combinations of trial sets and configuration files were tested, the aim having only been to ensure each trial set or configuration file was tested at least once. This bug was fixed through changing the data types used within the function. The original data type used were floats (floating point precision) and this was changed to using doubles (double floating point precision) to avoid the numerical overflow. With the bug fixed, the sets where then completed without further problems.

For Config_combos_0 the three sets of D, E and H, which all have the visible distances halved and the minimal distance between mapNodes doubled, prove to be in line with other trials set run on Config_combos_0 in having very high rates of success as can be seen in Figure 5.20. Of the instances in which the simulation did not end upon the successful arrival at the goal, the outcome was limited to not becoming stationary due to not finding further paths or due to getting stuck in a cycle of alternating between two positions, with no trials hitting any hazards. Figure 5.20 also shows that the number of steps taken in reaching the goal was homogeneous across the sets. It may be noted that Set $\mathrm{H}$ has a lower occurrence (but similar success rate) than the other two sets. This is because the simulation did not quite complete the full 500 trial runs, encountering a segmentation fault when creating and loading a new simulation environment. A bug/fault believed to be located in the code for the Irrlicht graphics engine as opposed to the code written for this project.

As with other trial sets, the results for D, E and $\mathrm{H}$ on Config_combos_1 have 

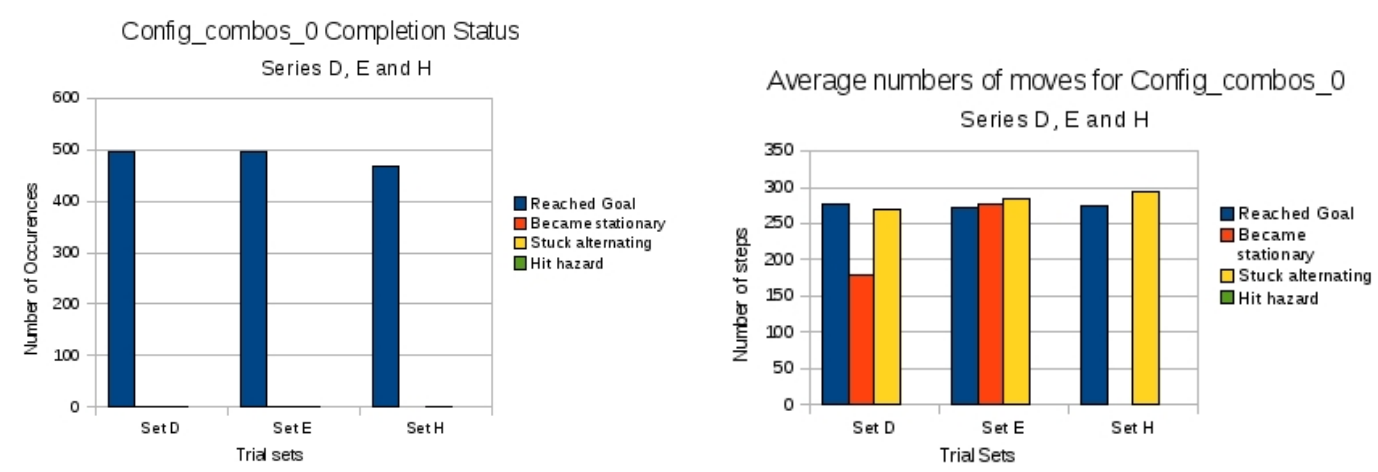

Figure 5.20: Left: Completion status for sets D, E and $\mathrm{H}$ with the $\mathrm{A}^{*}$ algorithm, on Config_combos_0. Right: The average number of movements occurring for each completion status.

a high failure rate consisting predominately of encountering hazards. The occurrence rates for the other non-successful status vary between the three sets, as evident in Figure 5.21. Sets D and E have a similar numbers of occurrences for the second and third most common status. However, which status is the second and third most common, is reversed between the two, with becoming stationary being the more common of the two while for set $\mathrm{E}$ it is being stuck alternating. Though the number/sample of successful runs is very low, it is still notable that the average number of steps taken in reaching the goal is much higher for set E than the results of any other set running on Config_combos_1. It is debatable whether this is a good result, indicating set E may find a path even in difficult circumstances, or whether it indicates that the paths chosen by set $\mathrm{E}$ are less efficient than the others. More testing across a variety of scenarios is necessary to make informed conclusions about the trial sets. The number of movements before trials terminated unsuccessfully, was reasonably similar both between the status and between the sets, though in each case set $\mathrm{H}$ appears to have encountered trouble earlier (within fewer moves than the others). Looking at Figure 5.24 it looks like the cause of the lower average number of steps before termination may be due to a greater tendency to attempt to traverse to the left which featured more obstacles.

As expected of Config_combos_2, the completion status across the trial sets 

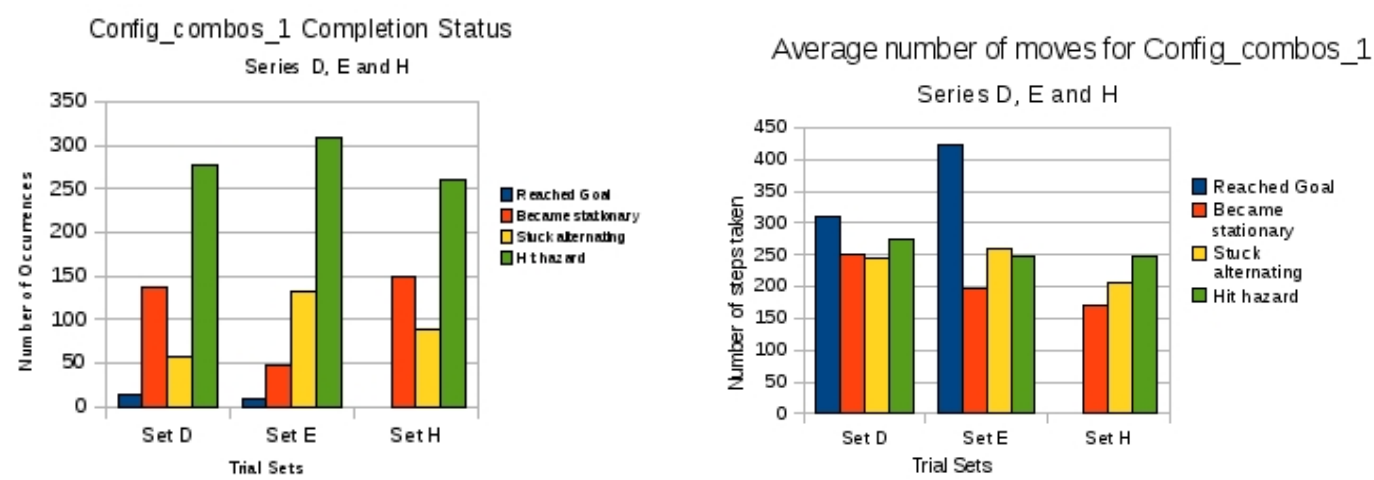

Figure 5.21: Left: Completion status for sets D, E and $\mathrm{H}$ with the $\mathrm{A}^{*}$ algorithm, on Config_combos_1. Right: The average number of movements occurring for each completion status.

was a lot better than with Config_combos_1. Looking at Figure 5.22 it appears that Set $\mathrm{H}$ did not perform as well as the others, with half as many successes as Set D and having the highest number of instances of hitting a hazard. Looking back upon Figures 5.20 and 5.20, it would appear that Set $\mathrm{H}$ has consistently performed the worst of these three variations based on reducing the resource requirements of the robot. With Set $\mathrm{H}$ having the distances across which nodes may be linked reduced further than the other two sets it had been expected the results would show greater performance due to a more simplified network of nodes being created with fewer links, however it would seem this instead handicapped the robot by reducing the options available to it for path planning. When looking at the paths produced by Sets D, E and H, shown in Figures 5.23, 5.24 and 5.25, the paths for Set H do not appear very dissimilar to the other two sets, in particular being quite like Set D in Figures 5.23 and 5.25, with the presence and spread of diffuse paths away from the most common channel of traverse.

Comparing the results of set D against the default settings to see the affect of reducing the number of nodes present in the potential path network, the first configuration file shows no difference, however looking at Figures 5.21 and 5.2, there is a difference in which completion status is the most common. For the D set of trials, the robot encountered a hazard more frequently while with the 
Config_combos_2Completion Status
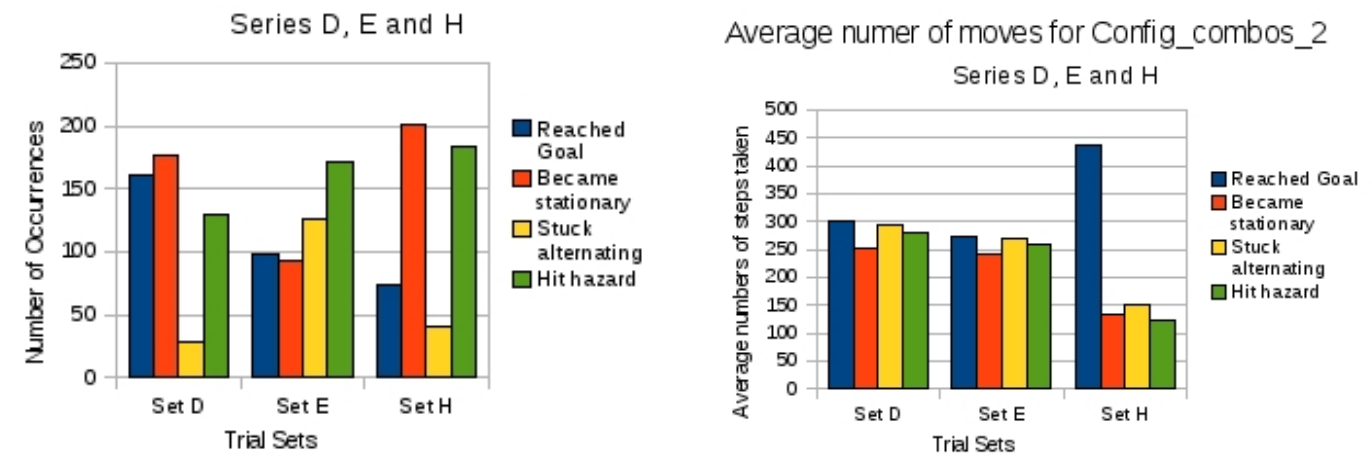

Figure 5.22: Left: Completion status for sets D, E and $\mathrm{H}$ with the $\mathrm{A}^{*}$ algorithm, on Config_combos_2. Right: The average number of movements occurring for each completion status.

default settings the robot had otherwise been exiting when it became stationary after an average of 70 moves and could not determine a new path. The most notable difference produced by the reduced node network is seen when the results show in Figures 5.22 and ?? are compared. The success rate of the robot was drastically diminished when using the reduced network, with the robot becoming stationary or hitting a hazard on a similar number of occasions to when it reached the goal. When sets A1 and E are compared, the affect of the reduced network is very similar, with Config_combos_0 and Config_combos_1 having very minor differences while the results of Config_combos_2, as shown in Figures 5.16 and 5.22 , show a significant change from a high rate of success to the other completion status become more dominant. The affect of the reduced node network is likely to be due to having fewer path options and hence with less information the robot is making poorer choices. Some method of reducing the node network selectively, to try and maintain the quality of decisions while reducing the resources required, would be beneficial. 


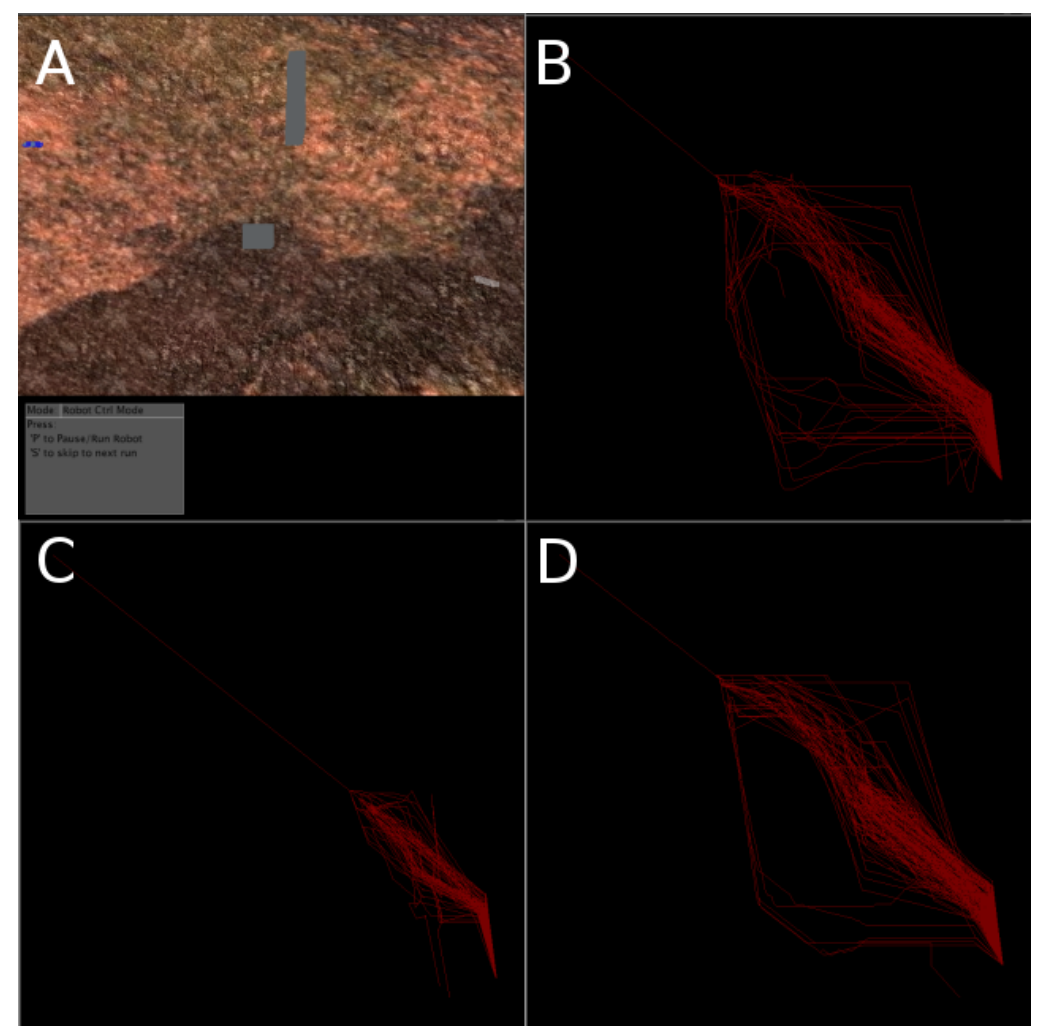

Figure 5.23: Overlaid paths generated by $A^{*}$ Algorithm in trial sets $\mathrm{D}, \mathrm{E}$ and $\mathrm{H}$, for Config_combos_0. (A) Terrain overview, (B) Set D paths, (C) Set E paths and (D) Set H paths. 


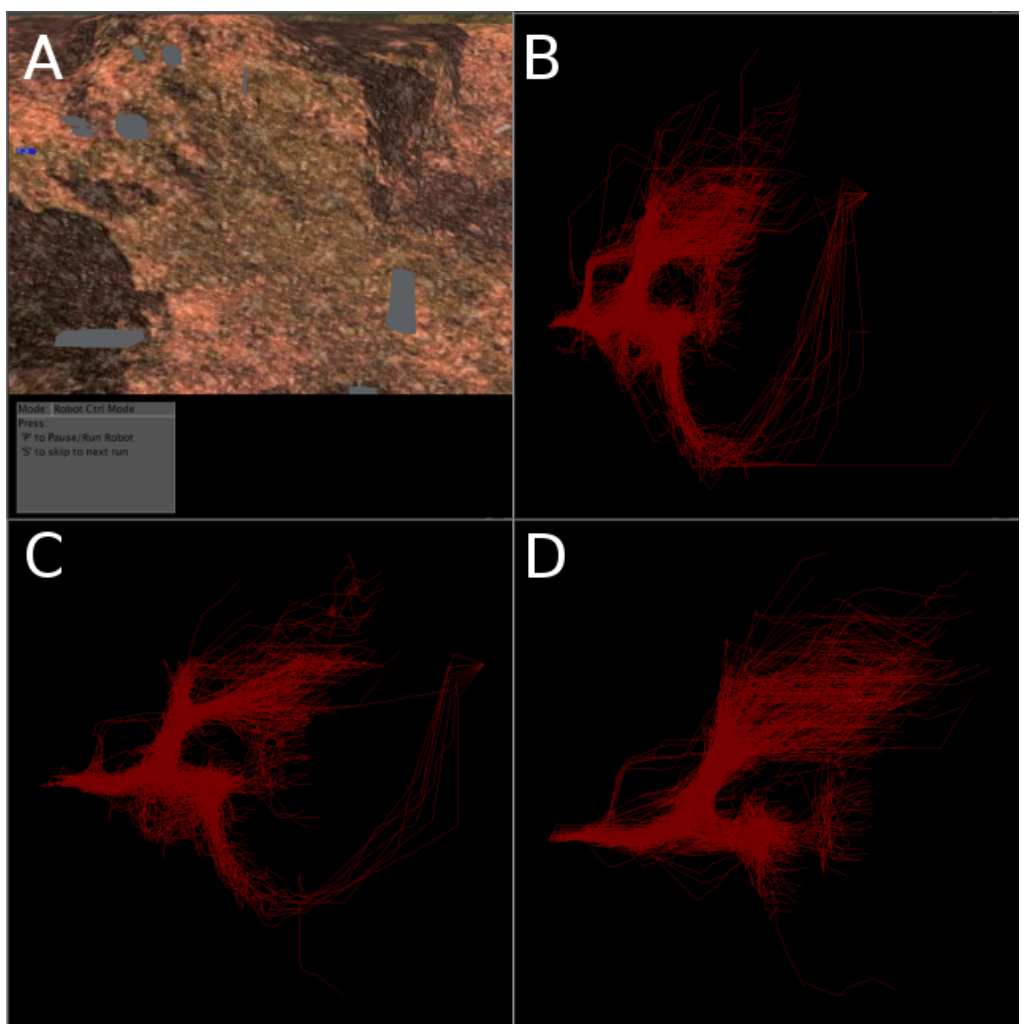

Figure 5.24: Overlaid paths generated by $\mathrm{A}^{*}$ Algorithm in trial sets $\mathrm{D}, \mathrm{E}$ and $\mathrm{H}$, for Config_combos_1. (A) Terrain overview, (B) Set D paths, (C) Set E paths and (D) Set H paths. 


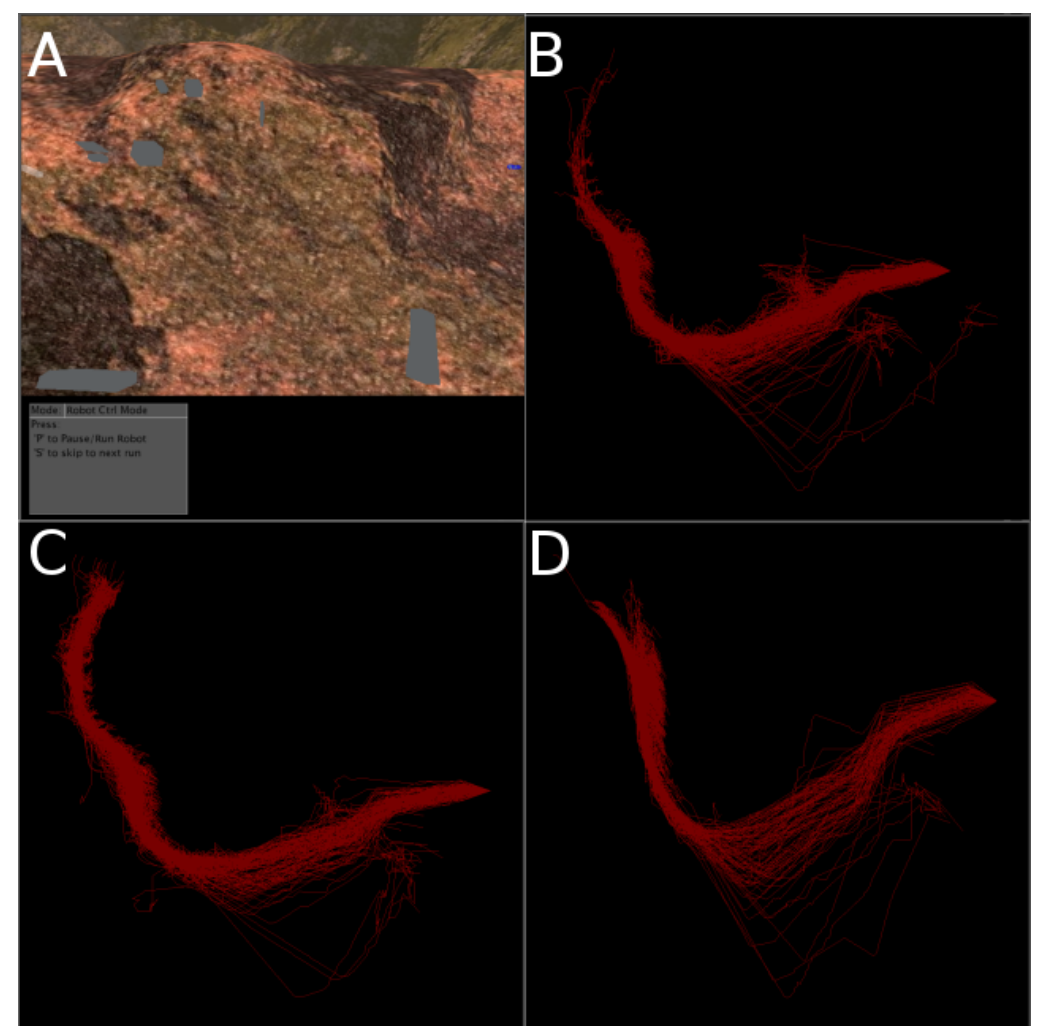

Figure 5.25: Overlaid paths generated by A* Algorithm in trial sets D, E and H, for Config_combos_2. (A) Terrain overview, (B) Set D paths, (C) Set E paths and (D) Set H paths. 


\subsection{Parameter comparisons}

\subsubsection{Trial sets A1 vs. C}

The success rates of trial sets A1 and C, as shown in Figures 5.26, 5.27 and 5.28, are quite interesting to compare. For set A1 the allowable maximum number of steps between re-analysis was five, with the step sizes being $100 \mathrm{~mm}$, while set $\mathrm{C}$ consisted of movements of $500 \mathrm{~mm}$ but with re-analysis occurring after each step. For the relatively simple scenarios of Config_combos_0 both sets produce a high number of successful traversals, though when adjusted to account for the step size of set $\mathrm{C}$ being five times that of set $\mathrm{A} 1$, it can be seen that the average number required to reach the goal is higher for set $\mathrm{C}$. While set $\mathrm{C}$ makes less moves, if adjusted to account for the difference in distance covered by the steps of set $\mathrm{A} 1$ and $\mathrm{C}$, set $\mathrm{C}$ could be considered to have travelled further in its trials which were unsuccessful. However, it should be taken into consideration that as the decision to exit due to alternating between two points, requires that a set number of consecutive iterations occur without finding a new path, the average number of steps given in the adjusted set $\mathrm{C}$ is slightly off/misleading.

In Config_combos_1, set $\mathrm{C}$ appears to fare better than set $\mathrm{A} 1$, with the number of occurrences for each status being closer than those of set A1 which differ dramatically with very few successes and a great number of instances of encountering a hazard. The average number of steps taken for each category of completion status, are fairly similar within each set, while set $\mathrm{C}$ once again appears to have moved further when adjusted to reflect step size.

Figure 5.28 shows that the results for Config_combos_2, are a reversal from the trend of set A1 producing paths which finished in fewer movements than the adjusted set $\mathrm{C}$ values. Set $\mathrm{A} 1$ has the average number of steps taken for each completion status being higher than those for the adjusted Set $\mathrm{C}$.

It is also notable that set $\mathrm{C}$ differs from the general trend across all sets. For the other sets the results of Config_combos_2 show a greater rate of success, while set $\mathrm{C}$ has there being a more balanced occurrence of all the completion status. Looking at the completion status of Config_combos_1, it appears that larger steps 

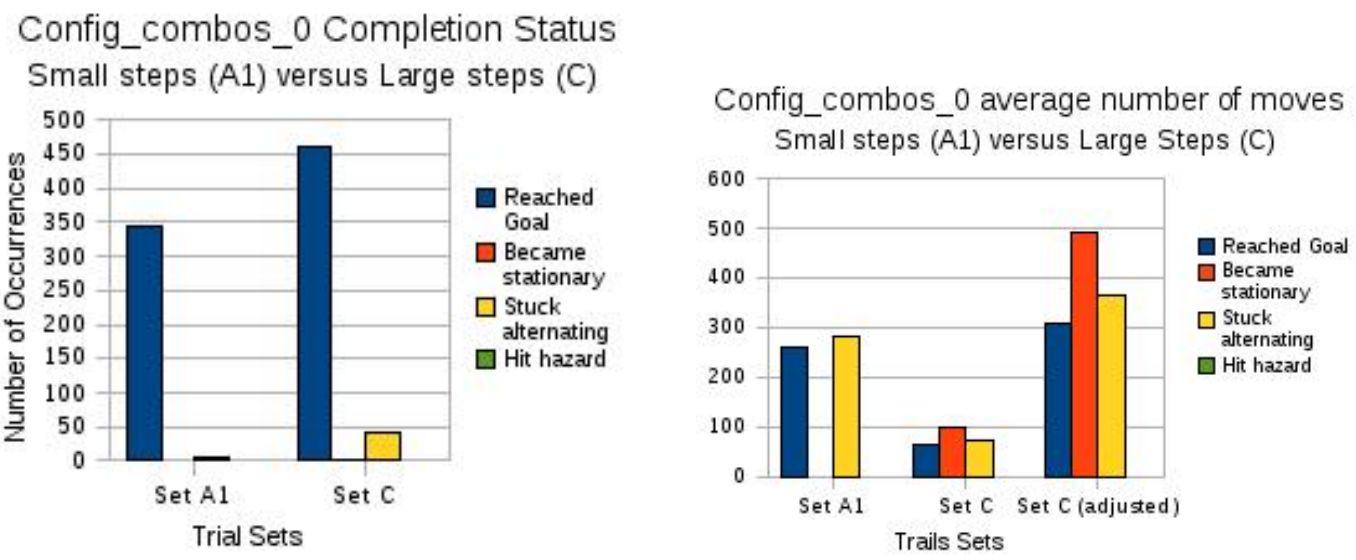

Figure 5.26: Left: Completion status for sets with small step sizes (set A1) and larger step sizes (set C), using the $\mathrm{A}^{*}$ algorithm on Config_combos_0. Right: The average number of movements occurring for each completion status of the sets A1 and $\mathrm{C}$ plus a set adjusted set to account for the movements of set $\mathrm{C}$ being 5 times larger than A1.
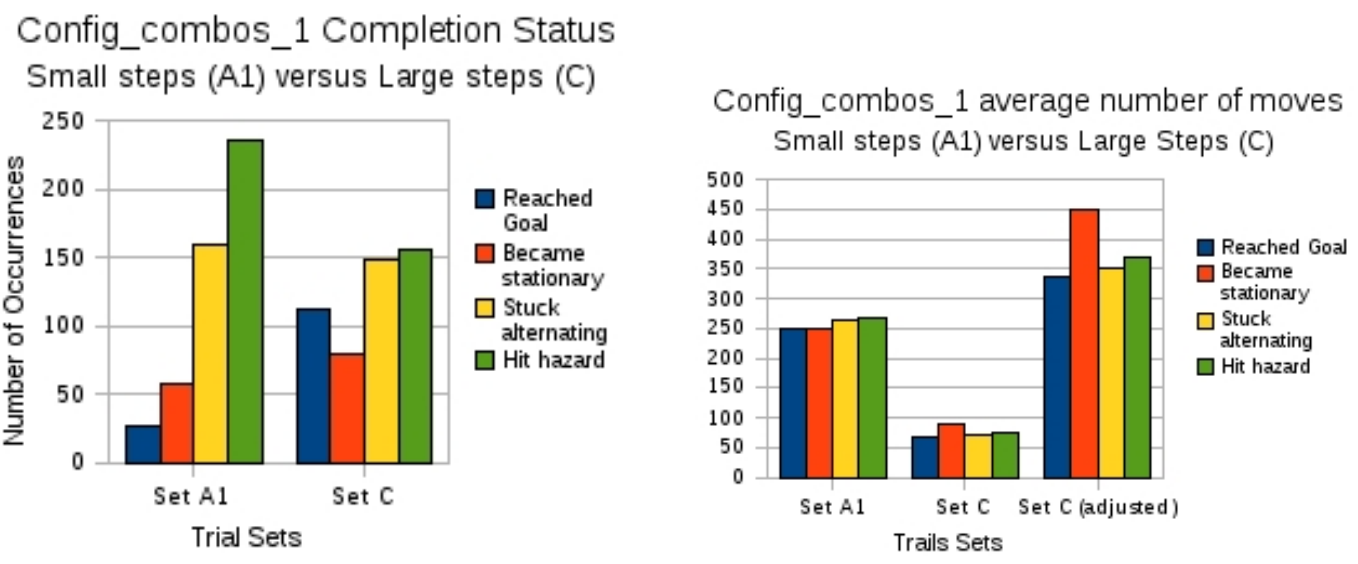

Figure 5.27: Left: Completion status for sets with small step sizes (A1) and larger step sizes (C), using the $A^{*}$ algorithm on Config_combos_1. Right: The average number of movements occurring for each completion status of the sets A1 and C plus a set adjusted set to account for the movements of set $\mathrm{C}$ being 5 times larger than A1. 

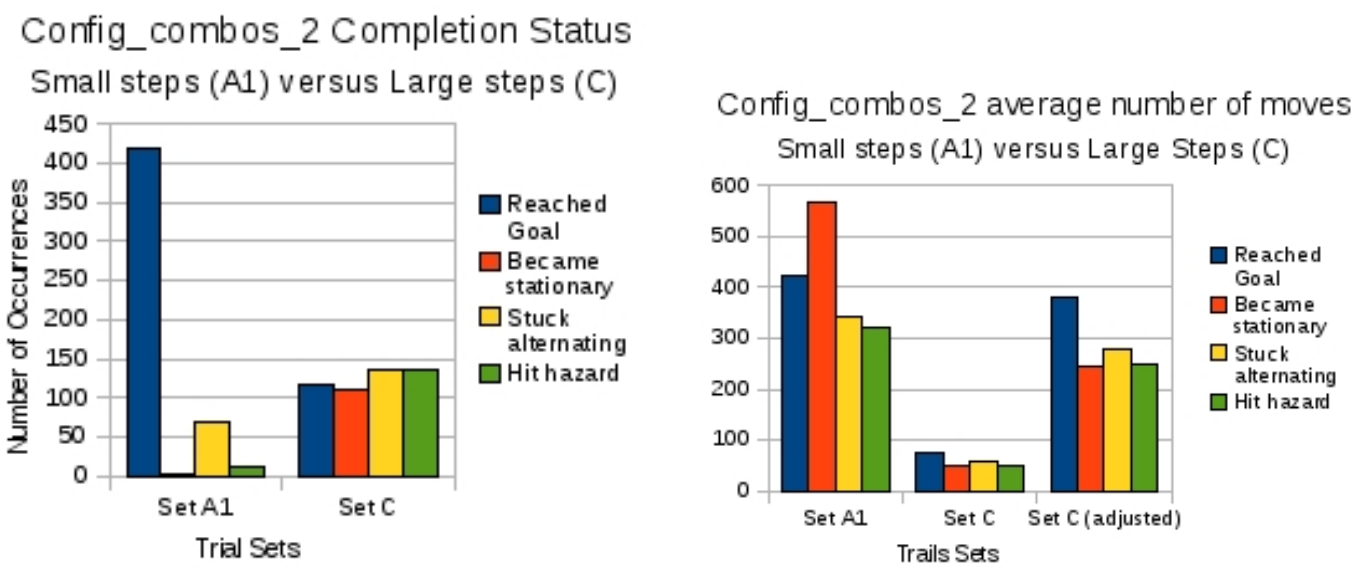

Figure 5.28: Left: Completion status for sets with small step sizes (A1) and larger step sizes (C), using the $A^{*}$ algorithm on Config_combos_2. Right: The average number of movements occurring for each completion status of the sets $\mathrm{A} 1$ and $\mathrm{C}$ plus a set adjusted set to account for the movements of set $\mathrm{C}$ being 5 times larger than A1.

sizes potentially have the effect of homogenising results, a beneficial characteristic for difficult terrains which would otherwise have few success, but less than desirable in other cases.

\subsection{Noise}

Initially the noise levels were set to have maximum levels of 5, 10 and 20\%. However, with relation to the maximum distances it was being applied to, this corresponded respectively to errors being up to $0.5,1$ or $2 \mathrm{~m}$. With these high values of noise the robot was very unsuccessful and averaged 6 movements on configuration file 2 before it was no longer able to determine a path to follow. Taking in to consideration the subsequent absolute values of the errors, the MAX_NOISE parameter was decreased to values of 0.5 and $1 \%$, to give a more realistic range of errors for the LIDAR sensing being simulated.

When the LPA* algorithm was accidentally run with noise present (at the decreased levels) the success rate was $0 \%$. Generally, the robot became stationary 
within ten moves, however on a few instances the robot managed to progress further. For these prolonged cases, the robot achieved median distances of about 40 moves before hitting a hazard.

The level of noise was in a potentially realistic range, however the distribution/frequency of it was not particularly realistic due to being based on the rand() function which gives an equal likelihood of occurrence for any value within the range. It had been hoped that this unrealistic and more extreme noise introduction may still have been manageable to some degree due to the tolerances and buffers within the design, however the potential for the noise to make a flat plane appear highly rugged and hazardous appears to have been too great. A better model for generating and introducing noise is necessary to examine the limits and performance of the path planning system in a realistic non-idealised environment. 


\section{Chapter 6}

\section{Discussion}

Through the design process of this simulation tool, many points of consideration necessary to develop successful path planning robots have been encountered. These points were encountered through the need to establish and model the internal modules of work which a robot must do. These sections are necessary in order to reach a stage at which it can make decisions about navigation. Most of these points of consideration, and their effects, have been explained during the design and implementation of the simulator. However, some facets were not obvious until experimental testing was undertaken, when the performance of the simulator and path planning systems could be better judged. During testing the level of flexibility in the settings and desired operation of path planning systems one wishes to simulate, were found to be significant. These factors having the potential to impact on a researcher's progress, through having too many options to choose from and hence encouraging a superfluous number of tests of various combinations.

\subsection{Design and Capabilities}

As evident by range of trials which were possible to run and the number of parameters which were untouched, this simulation tool provides a large and diverse variety of options for experimental trials and simulations. In addition to parameters and functionality already implemented, due to the modular design the variety 
of options can be even further increased through the introduction of other styles of input, control or path planning. Amongst the possible extensions to the current simulator, there is the addition of multiple robots within a terrain, the adaptation to replicate omnidirectional LIDAR input, introducing different forms of noise modelling/distribution and altering the manner of movement.

Modelling sensor types other than LIDAR has not been investigated, but any visual form of sensing should be fairly simple to implement and just draw from the functions available within the Irrlicht library. For other types of sensing and detection it may be necessary to first include modules to produce and model other signals, such as heat or noise and then in turn implement receivers to match such data generators.

Using configuration files in conjunction with shell scripts, it has been possible to run hundreds of unobserved and automated trials with a single command in the terminal. Replication of trials has also been possible, as was necessary in instances when data were lost or an error was encountered. Once the settings to be varied have been selected and altered, the simulation process has been very simple and allowed for multiple trials to be set up within a short period of time and left to progress without prompting or observation.

Though cross-platform usability has not been tested with Microsoft products, porting between different chipsets, structures and operating systems in Linux has been achieved and all of the components used are purported to have cross-platform capabilities. Installation of the simulator, though not yet automated, is fairly simple and without full administration/root privileges it is even possible to install and run a slightly restricted version, which runs minus the SDL output capabilities.

Amongst the capabilities of the simulation tool, is the ability to do basic statistical analysis of data produced as well as recreating/overlaying paths. These two features occur separately from simulation, with each requiring that the simulator is called with a different run option to select the appropriate mode of operation. 


\subsection{Comparison of Parameters}

Monitoring which parameters have been altered is important with a program as diverse and flexible as this one, especially when multiple instances are being run across a number of computers, as within the multitude of choices one can overlook the choices of past values or lose track of how each one interacts with the simulation. An instance of this occurred during testing of the implementation of the LPA* $^{*}$ algorithm, when noise inclusion was present following the setup of other trials which were aimed at investigating the effect of noise. The results from the LPA* algorithm when high levels of noise were incorporated, were very poor and initially believed to potentially be a fault or bug with how the LPA* algorithm had been implemented for use on the flexible node network. However, it was then noticed that the inclusion of noise had been left on in the settings. Some form of feedback appears to be necessary to make it easier to discern what variables have been altered and to which values, for a given executable file or running simulation. This is something which would be strongly recommended for future work.

\subsection{Flaws and Bugs}

At present there are a few bugs in the simulator, the most common of these appears to lie within the Irrlicht graphics engine. Upon creating and setting up a new simulation and graphical window, the simulator exits with an error which indicates that there has been an issue in creating a valid window and as such the GLX driver has terminated the simulation. Running the Valgrind memory leak checker, a couple of warnings are given about system calls with parameters which point to uninitialised addresses, originating within the createWindow function which calls functions from the GLX library. As previously mentioned, there is no feedback from the simulator about internal settings other than those relating to the visual interface, which is an oversight within the design of the simulator that needs to be rectified. The modification of the simulator to take the names and values of parameters it is desired to alter from a new settings input file would in part help 
to keep track of which variables were being varied. However, while running a shell script from the terminal window to do multiple trials, it would not possible to easily determine which input files were given. So, while the simulations are still running some form of feedback would still be beneficial, potentially giving the name of the settings file rather than listing all parameters and their values.

\subsection{Insight}

The usefulness and potential for improving path planning systems has been shown through the experimental trials undertaken. While the results for varying the values of some parameters showed no significant variation, there were cases where a distinct difference in the pattern of results could be observed. One idea which could be taken from an overview of the results gathered, is that for the robot and scenario types modelled, small steps proved to be better than larger steps. Through examining the graphical output at different stages through the traversal of the terrain, observations could also be made about what situations proved the easiest or hardiest in which to identify dangers and also how the network links and node positions affected the followed path.

\subsubsection{Config_combos}

From the results gathered, the success rates attained using the three Config_combos were distinctly different while the variance between the pair of scenarios within a Config_combos file was minimal in almost all cases, with the exception being the standard version of $A^{*}$ run on the Config_combos_1 setups. Each pair of scenarios had the same positional setup but with different numbers of obstacles inserted, which leads to the observation that for these cases the terrain surface/position appeared to provide the most impact. For the trials run on sim.config(??), the reasonably flat surface was easily traversed in a fairly direct path.

Config_combos_2 was the reversal of the start and goal positions used in Config_combos_1. Differences in results between the two configurations are of interest 
as the robot is tasked to cover the same ground but in the reverse direction. Looking at where the paths of Config_combos_1 failed in comparison to the successful chosen paths of Config_combos_2, highlights the importance of perspective as descending from a higher position the robot often runs into hazards. Viewing the steep hillside from the bottom, the robot was able to recognise it as a hazard much sooner and hence avoid it by a safe margin, whereas from the top, the robot struggled to gather enough information about the slope until it was very close and at risk of crossing a dangerously steep region.

As the robot builds up its terrain model based on the presence of data, hazards which are best identified by the absence of data (such as steep drops) are not detected as early as they could be. Some form of record of regions the robot has faced but not acquired data from could compliment the model with respect to hazard avoidance. As the altitude of the goal can not be known until it is within visible range and is not occluded by any obstacles, the robot may find itself rising above or descending below the goal due to only being able to base movement on approximate distance in the XY-plane, thus choosing poorly and leading to approaching insurmountable rises or dangerous drops

\subsubsection{Movement/Step size}

Moving between points, the location of nodes within the network may change to match the alterations within the model due to the gathering of further data about a region as it was approached. The distance travelled between new sets of data being gathered and the frequency with which the planned paths are reanalysed, may affect the speed at which approaching hazards are detected, the quality of path determined and/or the amount of time spent on processing data and planning paths. The setup of the A series of trials examined the effect of varying the maximum number of steps which may be taken between re-analysis (this counter being overridden in the event of nodes changing due to the presence of a hazard). The results across the three sets within the A-series of tests, had very little variation, which may indicate that the number of steps between path reanalysis has little influence or that for the scenarios encountered in the trials, 
the detection of new hazards regularly forced early re-analysis for the cases. The recording to file of the count between re-analysis would be a useful addition to determine how often the trials reached the maximum limit for steps before reanalysis.

The trials of $\mathrm{A} 1$ and $\mathrm{C}$, further explored the relations of steps through comparing 5 steps of $100 \mathrm{~mm}$ to individual steps of $500 \mathrm{~mm}$. While C-trials, for which the maximum number of steps between re-evaluation of paths were low and the steps sizes large, were much faster to run simulations of, they provided poorer results than those from the A series which had smaller steps but more of them potentially occurring between re-evaluation. The large steps resulted in the robot hitting hazards which it had not had a chance to identify yet or overshooting points at which divergence of potential paths may have occurred. In general the large steps did not produce the refined paths exhibited by smaller movement increments, which allow for more curvature within the terrain as opposed to needing long straight regions to traverse. Smaller steps show an increased flexibility in potential paths and greater safety through the increased frequency of hazard checks. This result follows the expectation that restricted/limited movement positions are suboptimal and that a greater flexibility is required. It is felt that this behaviour indicates that flexible networks are better than grid-based networks, which also produce less flexible paths, involving moving regular distances from node to node at the set resolution of the grid.

\subsubsection{View/input}

The physical qualities of a robotic unit such as its dimensions or the maximum angle it is able to traverse without danger, are obvious factors in affecting potential paths due to defining what is and is not traversable. What can be less obvious is the affect different aspects of the robot's field of view can have. During both the design and testing of the simulation program, the field of vision of the robot was found to have significant affect on the terrain model in a number of ways, and hence the node networks and planned paths as well. The three facets which have a combined affect on the view are the angular range and the maximum distance 
within which points were detected, in conjunction with the spread of points within that field. These characteristics of the robot's input view are dependant on the attributes of the sensors, such as the type, location and mounting.

For simplicity and robustness, sensors are often mounted in fixed directions and as such if one is travelling purely in that general direction for the whole trip then the data received should be sufficient to get from start to finish. However, if a deviation is required then very little is known about the regions which it is now necessary to face, although they may have been passed already. Humans have the benefit of interpolating some information about the environment from their peripheral vision and having the capability to look around without needing to stop and change orientation if something interesting is noticed. This difference between man and machine means that the model for an obstacle or hazard encountered in passing may be poorly formed.

For robots with fix mounted sensors the limited angle of view can prove particularly problematic for algorithms such as $\mathrm{A}^{*}$ which lack any specific exploratory behaviour as they assume/expect knowledge to have been acquired in all directions. Through having an omnidirectional view, such algorithms can select a drastic change in direction which may lead to moving backwards and away from the goal/direction being faced initially and thus enable passing around an obstacle. When given only a fixed view, the rate at which they may expand their knowledge of peripheral regions is slow and can be hampered by nearby obstacles and hence special cases to re-orient the robot would be highly recommended.

The dispersal of input points received from the sensors is another way in which the sensors can have a strong influence on the quality of the terrain modelling. With the input needing to be discrete rather than continuous, as a unit moves forward along a plane it will tend to get new sets of data which are equally spaced from the previous set. The ratio of the intervals at which the robot moves forwards and the horizontal dispersal of inputs affects the quality/shape of the triangles which constitute the terrain mesh. Even with point culling occurring, the movement's effect on the tessellation can be seen in Figure 6.1 which depicts a sequence of four terrain meshes produced by the robot as it moved. New rows 
of points can be seen to be introduced at regular intervals as the robot progresses towards the bottom right corner (sequence $\mathrm{A} \rightarrow \mathrm{D}$ ).

The relative angles between where a robot is situated and the surrounding terrain can also affect the model, as being on an upward or downward slope can limit the information gained if the rest of the terrain is in angled in the opposite direction. This loss of information can be dangerous as it hinders the detection of hazards, which is further discussed in the next section (Section 6.4.4).

Having the robot pan its view across the terrain when it first begins, to get a wider view of its environment was a necessary addition to get the robot to begin to move and consider paths if it is initially faced with a nearby obstacle situated between itself and the destination. For a fixed-vision robot, regularly having it look around itself in directions unrelated to, or perhaps distinctly varied from, the chosen paths may help improve the robot's ability to pick paths through difficult terrain. Similar situations may arise where all the robot's vision is currently filled by a looming obstacle and due to the path of approach the peripheral regions may not have been viewed. The peripheral regions may be occluded by the obstacle being approached, other obstacles or the angle of incline which the robot crossed. Without having viewed a region there can not be network nodes present to draw it into exploring and expanding its field of knowledge and thus regions which may hold potential paths can be missed.

\subsubsection{Hazard identification}

The hazard identification process becomes more difficult once it is desired that hazardous regions, where applicable, are identified as potentially traversable in certain directions. From identifying potentially traversable hazards, judging at which angles they are safe to approach and cross from, and then representing this information in a useful manner for the path planning system, all processes associated with traversable hazards are laborious. The long term risk of not being able to re-ascend a slope is something which a path planning algorithm must weigh up as it risks becoming locked into a region from which it can not reach its goal, but that is not the only risk involved. While traversing a hazard in a safe direction or 


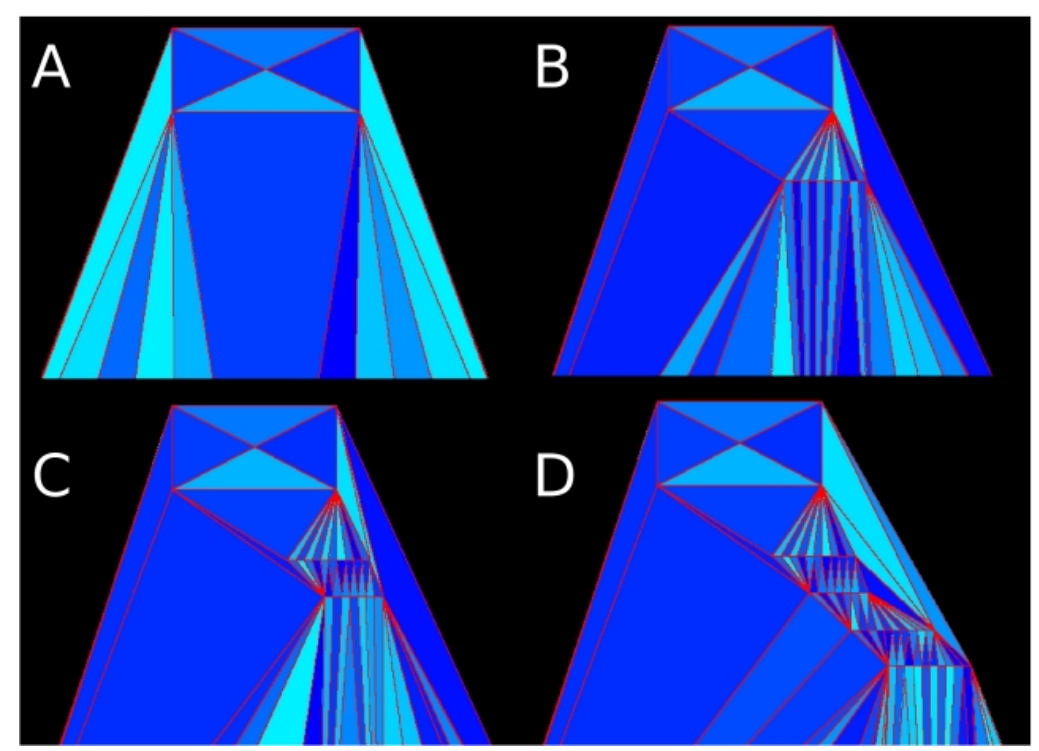

Figure 6.1: Altering Terrain Tessellation as the Robot moves towards bottom right corner (sequence $\mathrm{A} \rightarrow \mathrm{B} \rightarrow \mathrm{C} \rightarrow \mathrm{D}$ ).

position, the path planning system will have to remember the nature of the region being crossed such that it does not attempt to alter direction in order to pursue a newly identified path, as it risks going from being safely orientated to finding itself toppling down a slope or stuck in a crevasse.

Traversable slopes and crevasses both present problems later in the process, when networks of traversable points are formed for use by the path planning algorithms. The basis for forming nodes which link across traversable regions does not apply well when crevasses or directionally traversable slopes are involved and needs some adaptation. The Nodes themselves are also affected in how the unidirectional links are to be represented, as they can be problematic for different algorithms since they use the network of nodes differently. With $\mathrm{D}^{*}$ for example, following links in reverse from destination to the current location.

The occurrence of areas which meet hazard identification criteria in all regards except for the size of the faces being too large and hence having low confidence about how accurate they model the terrain in that region, are generally caused through being at the peripheral regions of vision, though sometimes also due to 
occlusion by objects in the foreground. Orientating the robot to face the region and gathering data from directly viewing the area of interest provides a means to lift the level of knowledge and would be of benefit for systems which have a greater focus on general exploration, rather than path planning between two positions. The current technique of ignoring the hazard status of large regions works with current goal-driven approach, but does mean an alteration to an explorationdriven system would require significant modification. The removal or increase of the threshold for ignoring the status of a face would be necessary to begin with and then either the addition of functionality within the algorithms to prioritise orientation towards these regions before movement is considered or alternatively the node network sections creating nodes within these sparse areas. The second approach is likely to be more beneficial for pure exploration, whereas the former would work for systems attempting to balance the arrival at a destination, with maximising knowledge acquisition in the process.

Hazardous slopes have been found earlier and more reliably when approaching a steep incline from the base, such as occurred with Config_combos_2, than when on a plateau moving towards a steep drop, like the scenario in Config_combos_1. This is somewhat expected behaviour as a wall is obvious through the presence of data points whereas a drop is shown through an absence and hence may be mistaken for an area which is simply unknown/unscanned.

Curving regions are dangerous as they are modelled with high number of small triangles, which can be difficult to analyse the overall affect they have when they interact/combine as a group. Curves are more of a naturally occurring feature, and it is less likely that they will be found amongst the rubble of man made infrastructure, but one still needs to be aware of their effects. This is potentially why the rectangular obstacles proved less issue than the slopes and rises of the terrain.

\subsubsection{Network}

The ability to give the robot pre-made/positioned network nodes allows firstly for the testing of the network linking and then secondly the interaction of the path planning algorithm with an idealised network. It is hard for an individual to 
discern from a terrain what the correct position for nodes will necessarily be based on the location of hazards and the triangle faces which they are made up of, so this feature allows one to confirm the implementation of an algorithm is working as expected in a theoretical/idealised situation, so that the behaviour upon a system aimed at realism, can be attributed to the more realistic environment and input as opposed to intrinsic to the algorithms implementation.

Further refinement of the node creation and linking, is expected to reduce resources and processing time. However, this must be monitored and a gradual process as it may come at the cost of the quality of the paths produced by the algorithms. Reduction of the distances links span was expected to have minimal impact on the paths, however from comparison of Set $\mathrm{H}$ of the experimental results it would appear that the longer links produce better results, although this maybe due to the number and positioning of the nodes. With a modified basis of creation or relative positioning and proximity thresholds, the shorter links may increase to a similar level as networks with links spanning greater distances. Considering the discussion points made earlier in Section 6.4.3, about the effect of the spread of input points received from the sensors, the techniques for creating and linking nodes will also rely on their interaction with the dispersal of input points and the size of the faces created during tessellation. Smaller triangles result in more edges bordering the hazardous and traversable regions, thus increasing the number of hazard nodes and links between them.

In sparse environments, the basis for creating the node network may lead to obstacles being skirted by large distances. The creation of multiple nodes along a line crossing the traversable region between two hazard nodes, may provide an improvement in sparse regions and allow for tighter paths. Creating a fixed number of points upon the lines between hazard nodes does not provide any benefit over a single point as they may lie too close or far apart and as such it would be necessary to make individual calculations based on the distance between specific hazard nodes. Alternatively, allowing a higher number of links and the connection to far off nodes might provide the same behaviour as cutting corners closely. However, it is felt that this would come at the cost of processing and resources. 


\subsection{Algorithms}

In porting the $\mathrm{A}^{*}$ and LPA* algorithms for use on a flexible node network, as opposed to the grid-based systems they appear to primarily be used upon, it was found that the re-plan nature of $\mathrm{A}^{*}$ was much simpler. For the LPA* algorithm, which follows a re-use approach, the process of updating the node network was difficult due to the potential for nodes to be removed or inserted through improved data for modelling the terrain or identifying hazards. With a grid-based system, updating the network involves polling the status of a node as to whether it is still traversable. However, with a flexible network where the existence of a node signifies that it is traversable it was necessary to instead check for the continued existence of predecessors.

If a predecessor had been removed it was then necessary to determine a new predecessor, however those nodes being considered may have also lost their predecessor and determining which ones to evaluate first is difficult, as they are not set distances apart and hence it is not possible to easily determining what tier or stage they are between the position and goal and hence their natural order. With minimal changes required to port the re-plan approach, one can be more confident about correctness and closeness of the behaviour to the original $\mathrm{A}^{*}$ algorithm.

The differences between re-use and re-plan are also likely to have largely been missed due to issues with monitoring the time taken for trials to run, as the main benefit of a re-use approach over a re-plan algorithm is in terms of minimising processing time upon successive iterations. So with this trouble quantifying the level of resource use, it is at present not as beneficial to run trials comparing the different styles of algorithms as it might otherwise be. 


\section{Chapter 7}

\section{Conclusion}

This project has produced a modular simulation tool which allows for the implementation and use of other input types, node networks, or algorithms. In addition to allowing alternative methods of doing core tasks, the simulator provides a variety of options by which the simulation can be altered to identify the affects of different design decisions. The graphical simulation allows users to view and follow the paths chosen by the robot and have a greater understanding of the environment in which it produces these.

For the fixed field of view simulated in the experimental trials, it was found that the role the path planning algorithms played was not the predominant factor but rather one of a set of equally important sections. The nature of the input gathering, the choices made during terrain modelling and the manner in which the node network of potential paths was created, are some other areas which were seen to affect the quality of planned paths.

Initially the intention had only been to allow the testing and comparison of different path planning algorithms, with the parameters being open to change simply due to not knowing the desired values. However, as the project progressed and the influence of other sections became apparent, the scope was expanded to enable the whole system (input/scan, modeling, network creation) to be tested.

The implications of a simulation tool such as this is that the process of design and testing can be made easier and faster, which will make this a valuable tool for 
researchers.

The incompatibility of unmodified $A *$ based algorithms with robots that have fixed position sensors with a restricted field of view, was shown. Modification of the behaviour of the algorithms in order to induce the gathering of more information is necessary if fixed view robots are to find paths in difficult terrains. This kind of insight and the ability to test new designs against the current behaviour, are what will make this a useful design tool to aid researchers in designing new systems and algorithms.

Inter-relation between most of the parameters as they combined to form a complete path planning system, makes it hard to identify specific parameters and values as better than others in general, only being able to judge them with respect to the combinations of values of all other parameters under which they were tested. With the number of parameters and the range of values each could potential have, the sheer number of combinations available is immense. As such, the decision of holding certain factors constant and predetermined is required to cut down the number of trials necessary to make design decisions. The level of flexibility provided by this simulation package is both beneficial in the freedom of design it allows but also can have a negative impact on progress as the range of choice in altering parameters entices one to do more comparisons than strictly necessary for one's purposes, and this must be kept in mind when being used.

Overall, the level to which this simulation platform has been developed, with the variety of features and the ability to easily be expanded for use with alternative lines of research, is very high and meets all the ambitions of the initial concept.

\subsection{Future work}

There is a variety of functionality which is not as at present available, requiring implementation, completion or debugging, which would be suggested as future endeavours.

For the benefit of users, it is highly recommended that some method of ascertaining which parameters had been altered and what the current values are, was 
included. This could be done through an option to output to a file the relevant information, or for it to be available and displayed within the GUI.

Functions for generating and using a grid-based node network have been implemented, but testing and improvement are still required as there are bugs present within the code. The completion of this section would enable path planning algorithms such as $A^{*}$, which were designed to use a limited grid network, to run on their native system.

With regards to the retention and removal of data there are two areas of work which it would be recommended to get to a functional level. Firstly, the use of Hazard objects to reduce the memory requirements of storing distant hazard, is not fully implemented. It is required that the functions for re-inserting old hazards when re-approached, are completed. Having the ability to join and continue hazard objects to previous objects when they cross the threshold/border of retention, is also necessary and should be tested.

The other technique for improving the decisions of keeping or removing data, is the use of confidence levels which are boosted when new data matches the terrain and the application of distance or time based decay upon these confidence levels. With confidence and decay implemented, it would be possible to base the decision to remove data based on how relevant and current it is, by setting a minimum threshold of acceptable confidence values.

One of the most difficult modification which would be recommended, is altering the terrain model from being a single layer of triangle faces, to a model built from isohedrons. Using isohedrons allows for the presence of holes within the terrain model, likely features of a disaster site. Adapting the terrain model is quite simple, primarily requiring a change in the types of CGAL objects used. However, the other modules of the path planning system will most likely require the adaptation of many functions in addition to the creation of some new ones. Having to identify holes, determine whether they are hazardous and then judging where to create mapNode and how to link them will require much thought. The simple methods of displaying information or regions of interest, as two dimensional images will also no longer be sufficient. 
A final suggestion would be to create some sort of technique or process by which, the robot can use the absence of data for a region to detect the presence of a dangerous slope or cliff. The inclination of the robot, previous path of approach and the dispersal of scan points would have to be considered in order to not erroneously label a region as a hazard when the lack of data was due to not having viewed a region or due to being occluded. Having a special type of node object for representing regions where there is a lack of information would be a potential way to combine this with the current node network of potential paths. 


\section{References}

[1] A. Davids, "Urban search and rescue robots: from tragedy to technology," IEEE Intelligent Systems, vol. 17, no. 2, pp. 81-83, 2002.

[2] D. Stormont, "Autonomous rescue robot swarms for first responders," in Computational Intelligence for Homeland Security and Personal Safety, 2005. CIHSPS 2005. Proceedings of the 2005 IEEE International Conference on, pp. 151-157, 2005.

[3] R. Murphy, "Rescue robotics for homeland security," 2004.

[4] J. Borenstein, G. Granosik, and M. Hansen, "The OmniTread serpentine robot: design and field performance," in Proceedings of SPIE, vol. 5804, p. 324, 2005.

[5] D. Hobbelen, T. de Boer, and M. Wisse, "System overview of bipedal robots flame and tulip: Tailor-made for limit cycle walking," in IEEE/RSJ Int. Conf. on Intelligent Robots and Systems, IROS2008, Nice, France, pp. 2486-2491, 2008 .

[6] LynxMotion, "Beginners guide to pathfinding algorithms." http://www.lynxmotion.com/Category.aspx?CategoryID=100, last visited on 19/12/2009.

[7] C. Ye, S. Ma, and B. Li, "Design and Basic Experiments of a Shape-shifting Mobile Robot for Urban Search and Rescue," in 2006 IEEE/RSJ International Conference on Intelligent Robots and Systems, pp. 3994-3999, 2006. 
[8] R. Murphy, "Marsupial and shape-shifting robots for urban search and rescue," IEEE Intelligent Systems and Their Applications, vol. 15, no. 2, pp. 14 19, 2000.

[9] A. Kamimura, H. Kurokawa, E. Yoshida, S. Murata, K. Tomita, and S. Kokaji, "Automatic locomotion design and experiments for a modular robotic system," IEEE/ASME Transactions on Mechatronics, vol. 10, no. 3, pp. 314-325, 2005.

[10] D. Williamson and D. Carnegie, "Toward Hierarchical Multi-Robot Urban Search and Rescue: Development of a MotherAgent," Autonomous robots and agents, 2007.

[11] H. Ishida, K. Nagatani, and Y. Tanaka, "Three-dimensional localization and mapping for a crawler-type mobile robot in an occluded area using the scan matching method," in 2004 IEEE/RSJ International Conference on Intelligent Robots and Systems, 2004.(IROS 2004). Proceedings, vol. 1.

[12] J. Carlson, R. Murphy, and A. Nelson, "Follow-up analysis of mobile robot failures," in 2004 IEEE International Conference on Robotics and Automation, 2004. Proceedings. ICRA'04, vol. 5.

[13] R. Bostelman, T. Hong, R. Madhavan, and B. Weiss, "3D range imaging for urban search and rescue robotics research," in 2005 IEEE International Safety, Security and Rescue Robotics, Workshop, pp. 164-169, 2005.

[14] A. Jacoff, E. Messina, B. Weiss, S. Tadokoro, and Y. Nakagawa, "Test arenas and performance metrics for urban search and rescue robots," in 2003 IEEE/RSJ International Conference on Intelligent Robots and Systems, 2003.(IROS 2003). Proceedings, vol. 4, 2003.

[15] Defense Advanced Research Projects Agency, "Darpa grand challenge." http://www.darpa.mil/grandchallenge/index.asp. 
[16] S. Wirth and J. Pellenz, "Exploration Transform: A stable exploring algorithm for robots in rescue environments," in Workshop on Safety, Security, and Rescue Robotics, 2007.

[17] J. Craighead, R. Murphy, J. Burke, and B. Goldiez, "A Survey of Commercial \& Open Source Unmanned Vehicle Simulators," in 2007 IEEE International Conference on Robotics and Automation, pp. 852-857, 2007.

[18] B. Balaguer, S. Balakirsky, S. Carpin, M. Lewis, and C. Scrapper, "USARSim: a validated simulator for research in robotics and automation," in Workshop on Robot Simulators: Available Software, Scientific Applications, and Future Trends at IEEE/RSJ, 2008.

[19] B. Delaunay, "Sur la sphere vide," Izv. Akad. Nauk SSSR, Otdelenie Matematicheskii i Estestvennyka Nauk, vol. 7, pp. 793-800, 1934.

[20] S. Rebay, "Efficient unstructured mesh generation by means of Delaunay triangulation and Bowyer-Watson algorithm," Journal of Computational Physics, vol. 106, no. 1, pp. 125-138, 1993.

[21] J. Lee and R. Dyczij-Edlinger, "Automatic mesh generation using a modified Delaunay tessellation," IEEE Antennas and Propagation Magazine, vol. 39, no. 1 , pp. 34-45, 1997.

[22] T. Gerbaud, V. Polotski, and P. Cohen, "Simultaneous exploration and 3D mapping of unstructured environments," in 2004 IEEE International Conference on Systems, Man and Cybernetics, vol. 6, 2004.

[23] H. Durrant-Whyte and T. Bailey, "Simultaneous Localisation and Mapping (SLAM): Part I The Essential Algorithms," Robotics and Automation Magazine, vol. 13, no. 2, pp. 99-110, 2006.

[24] J. M. Holland, Designing mobile autonomous robots. Elsevier Newnes, 2004. 
[25] A. T. de.Almeida and O. Khatib, Autonomous robotic systems: - Advanced Research Workshop on Autonomous Robotic Systems. Springer, 1998.

[26] M. K. Miller, N. Winkless, and J. Bosworth, The personal robot navigator. Robot Press, 1998.

[27] S. Iyengar and A. Elfes, Autonomous mobile robots. IEEE Computer Society Press, 1991.

[28] R. M. Jensen, R. E. Bryant, and M. M. Veloso, "SetA*: An efficient BDDbased heuristic search algorithm," tech. rep., Computer Science Department, Carnegie Mellon University, 5000 Forbes Ave, Pittsburgh,PA, August 2002.

[29] "Beginners guide to pathfinding algorithms." http://aidepot.com/Tutorial/PathFinding.html, visited on 3/9/2006.

[30] J. Pearl, Heuristics-intelligent search strategies for computer problem solving. Addison-Wesley Publishing Co., Reading, MA, 1984.

[31] T. Cormen, C. Leiserson, R. Rivest, and C. Stein, Introduction to algorithms. The MIT press, 2001.

[32] P. Hart, N. Nilsson, and B. Raphael, "A formal basis for the heuristic determination of minimum cost paths," IEEE transactions on Systems Science and Cybernetics, vol. 4, no. 2, pp. 100-107, 1968.

[33] K. Manley, "Pathfinding: from A* to LPA," University of Minnesota, 2003.

[34] A. Stentz, "Optimal and efficient path planning for partially-known environments," in Robotics and Automation, 1994. Proceedings., 1994 IEEE International Conference on, pp. 3310-3317 vol.4, May 1994.

[35] S. Koenig and M. Likhachev, "D* Lite," in Proceedings of the National Conference on Artificial Intelligence, pp. 476-483, Menlo Park, CA; Cambridge, MA; London; AAAI Press; MIT Press; 1999, 2002. 
[36] D. Ferguson and A. Stentz, "Multi-resolution Field D*" in Proceedings of the International Conference on Intelligent Autonomous Systems (IAS), 2006.

[37] J. Wang, M. Lewis, and J. Gennari, "Interactive simulation of the NIST USAR arenas," in IEEE International Conference on Systems, Man and Cybernetics, 2003, vol. 2, 2003.

[38] M. Kadous, R. Sheh, and C. Sammut, "Controlling Heterogeneous Semiautonomous Rescue Robot Teams," in IEEE International Conference on Systems, Man and Cybernetics, 2006. SMC'06, vol. 4, 2006.

[39] Nikolaus Gebhardt \& Development Team, "Irrlicht 3d graphics engine." http://irrlicht.sourceforge.net/.

[40] D. Garlan and M. Shaw, "An introduction to software architecture," Tech. Rep. CMU-CS-94-166, Carnegie Mellon University, 5000 Forbes Ave, Pittsburgh,PA, January 1994.

[41] B. Chazelle, "Triangulating a simple polygon in linear time," Discrete and Computational Geometry, vol. 6, no. 1, pp. 485-524, 1991.

[42] R. Gilberg and B. Forouzan, Data structures: a pseudocode approach with $\mathrm{C}++$. Brooks/Cole Publishing Co. Pacific Grove, CA, USA, 2001.

[43] CGAL Development Team, "Computational geometry algorithms library." http://www.cgal.org/.

[44] J. hoey, "Wheelchair obstacle avoidance helper (woah) system." http://www.cs.toronto.edu/jhoey/wheel/mapping.html. 


\section{Appendix A}

\section{Source Code for Simulation System}

\section{A.1 Algorithms}

\section{A.2 Algorithms.h}

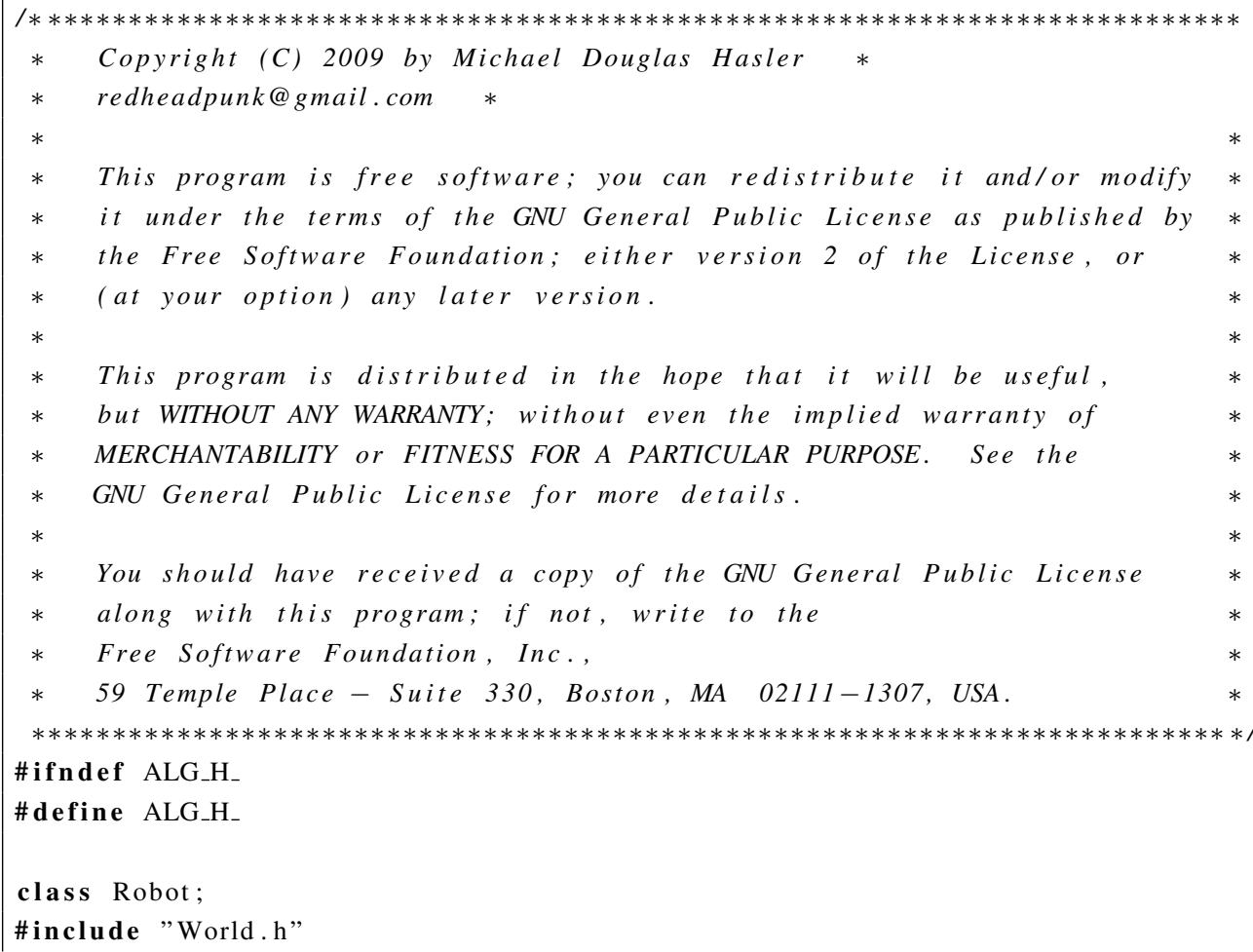




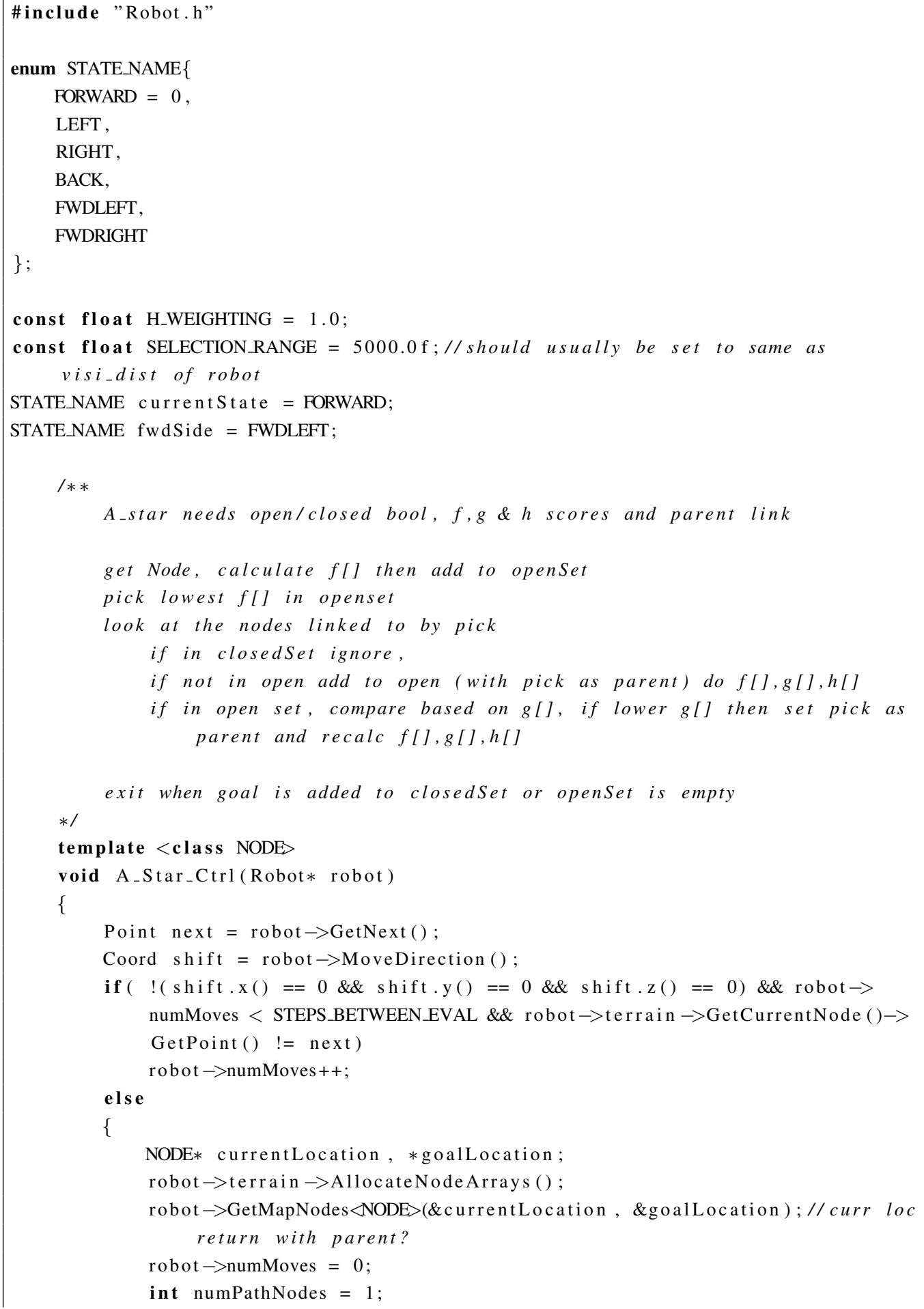




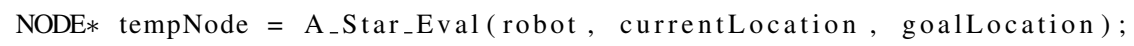
NODE* tNode $=$ tempNode;

while(tNode $!=$ currentLocation) // tNode $\rightarrow$ Parent $!=$ NULL

\{

tNode $=$ tNode $\rightarrow$ predecessor $;$

numPathNodes ++;

\}

robot $\rightarrow$ SetNumSteps (numPathNodes);

Point $*$ path $=($ Point $*)$ calloc (numPathNodes, sizeof(Point));/ this affects the currentPos how/why?

for $($ int $1=$ numPathNodes $-1 ; 1>=0 ; 1--)$

\{

path $[1]=$ tempNode $\rightarrow$ GetPoint () ;

tempNode $=$ tempNode $\rightarrow$ predecessor $;$

\}

robot $\rightarrow$ SetPath (path);

free ( path);

path = NULL;

\}

\}

/** Have each algorithm having its own node type which extends mapNode?

A_star needs open/closed bool, $f, g \& h$ scores and parent link

//get Node, calculate f[] then add to openSet

//pick lowest $f[]$ in openset

//look at the nodes linked to by pick

// if in closedSet ignore,

// if not in open add to open (with pick as parent) do f[],g[],h[]

// if in open set, compare based on $g[]$, if lower $g[]$ then set pick as parent and recalc $f[], g[], h[]$

// exit when goal is added to closedSet or openSet is empty

$* /$

template <class NODE $>$

NODE* A_Star_Eval (Robot* robot, NODE* currentLocation, NODE* goallocation) \{

in $\mathbf{t}$ numNodes $=0$

int openI $=0, \operatorname{closed} I=0$;

NODE $* *$ closedSet $=($ NODE $* *)$ calloc $($ ARRAY_CHUNK, $\quad$ size of $($ NODE $*))$;

NODE $* *$ openSet $=(\operatorname{NODE} * *)$ calloc $($ ARRAY_CHUNK, sizeof $(\mathrm{NODE} *))$;

NODE* goal = goalLocation;

NODE* start = currentLocation; 


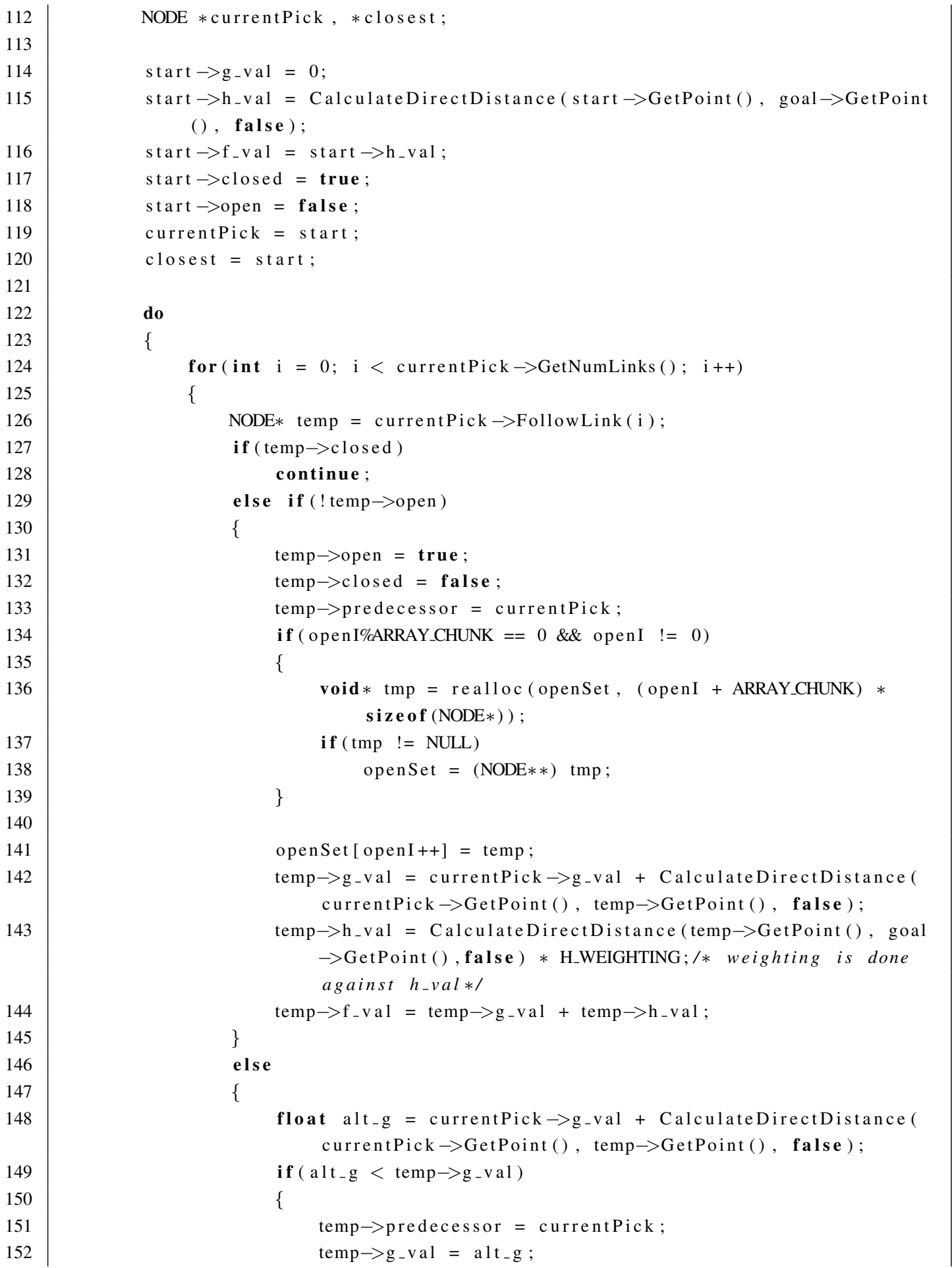




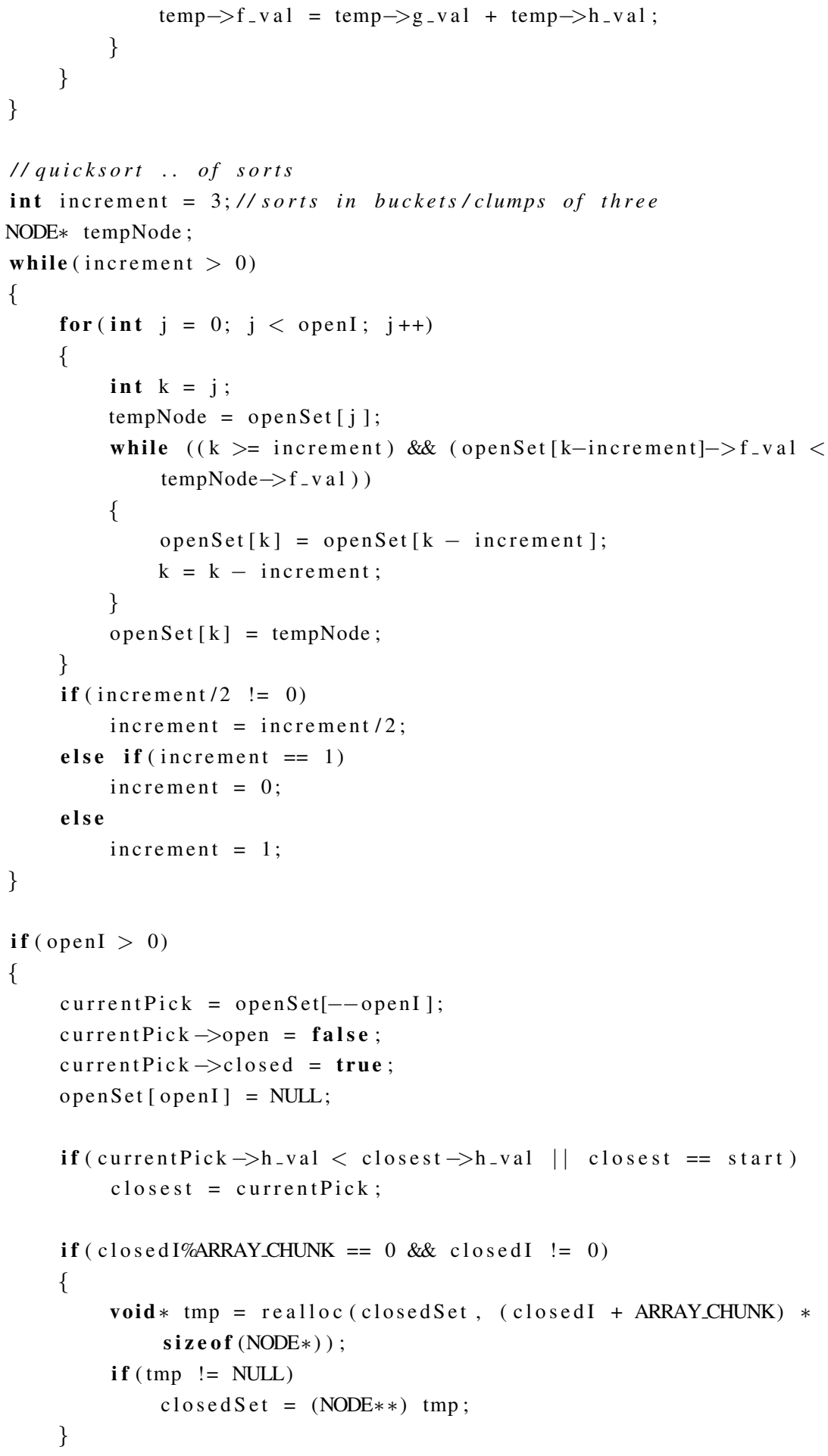




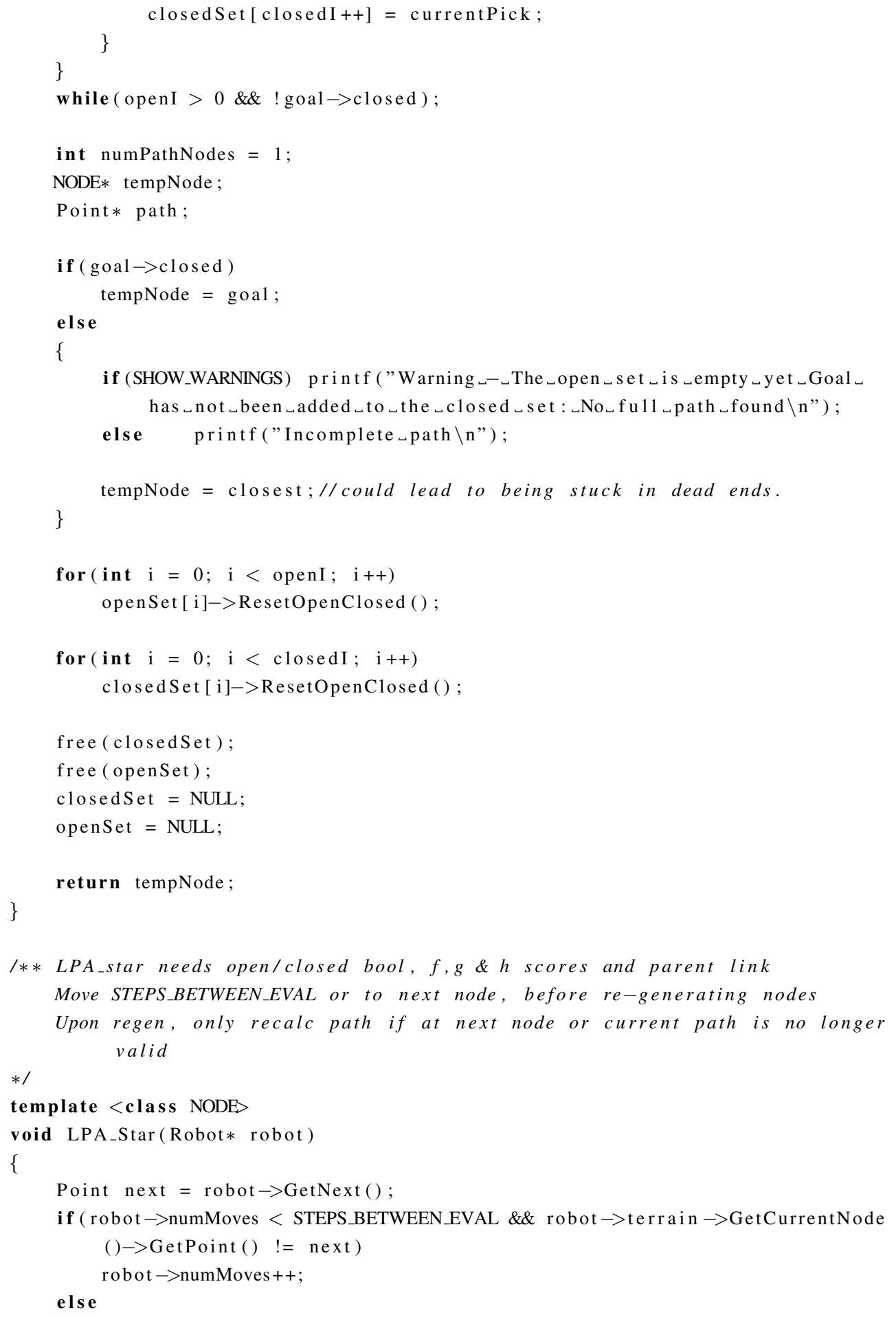




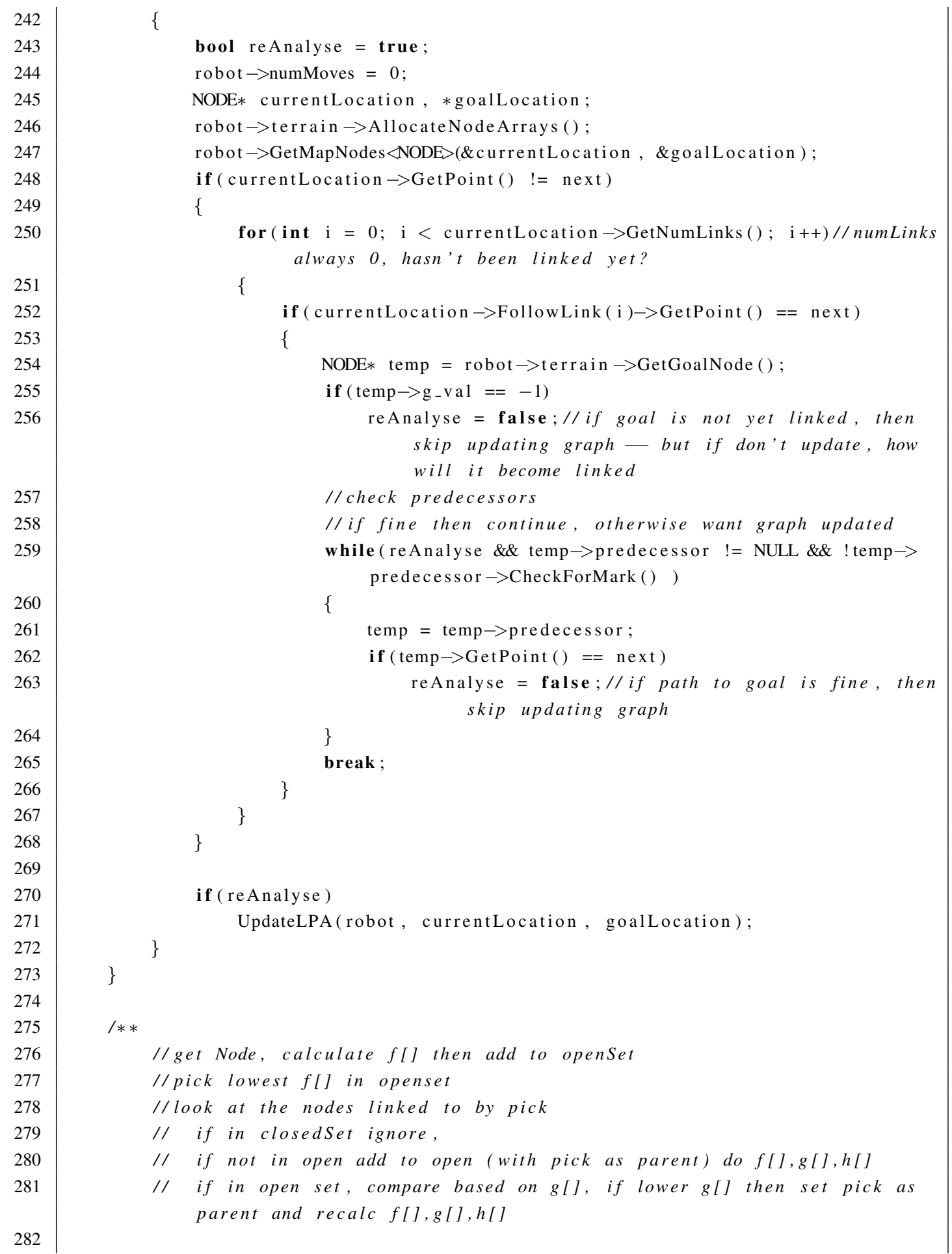




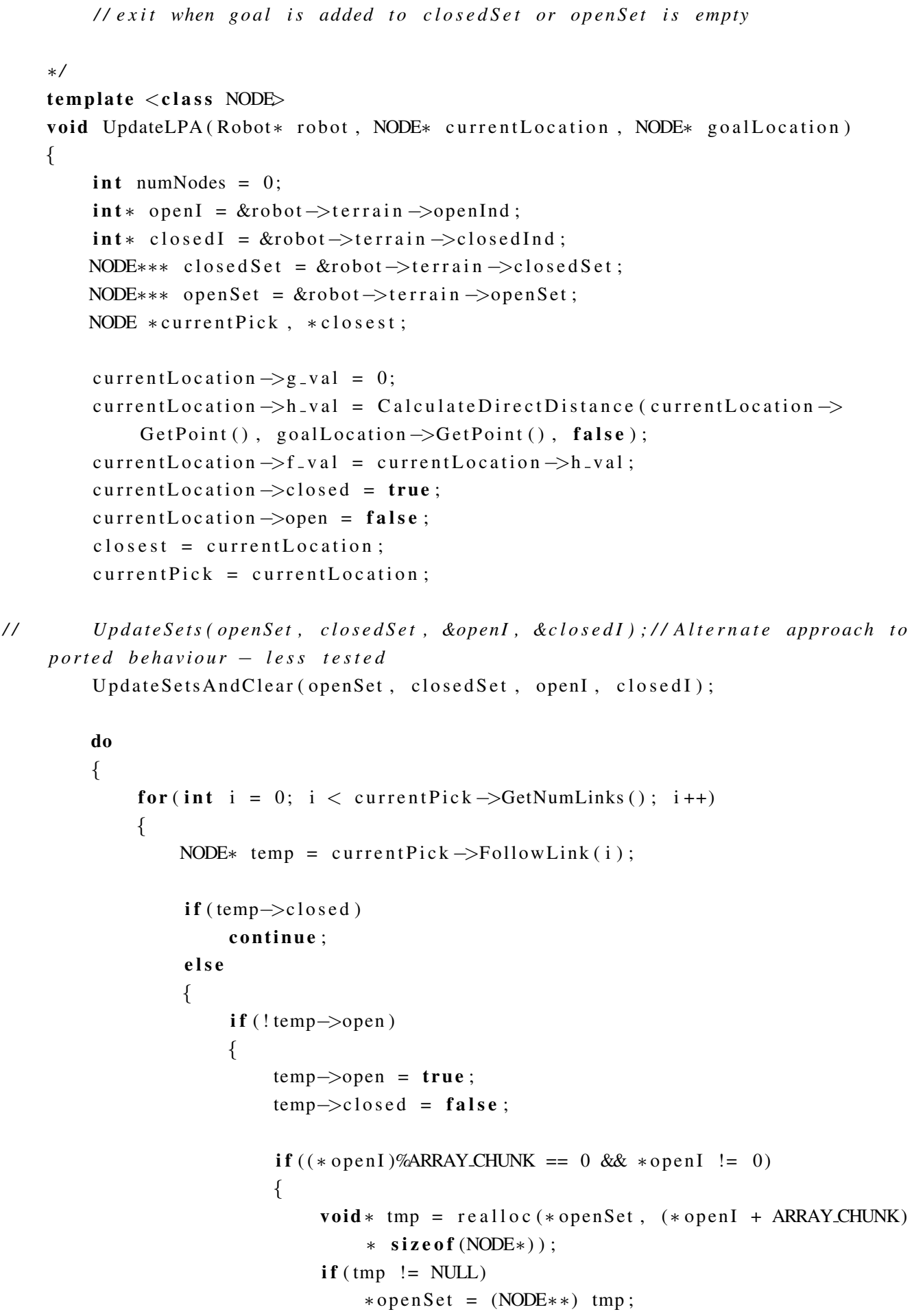




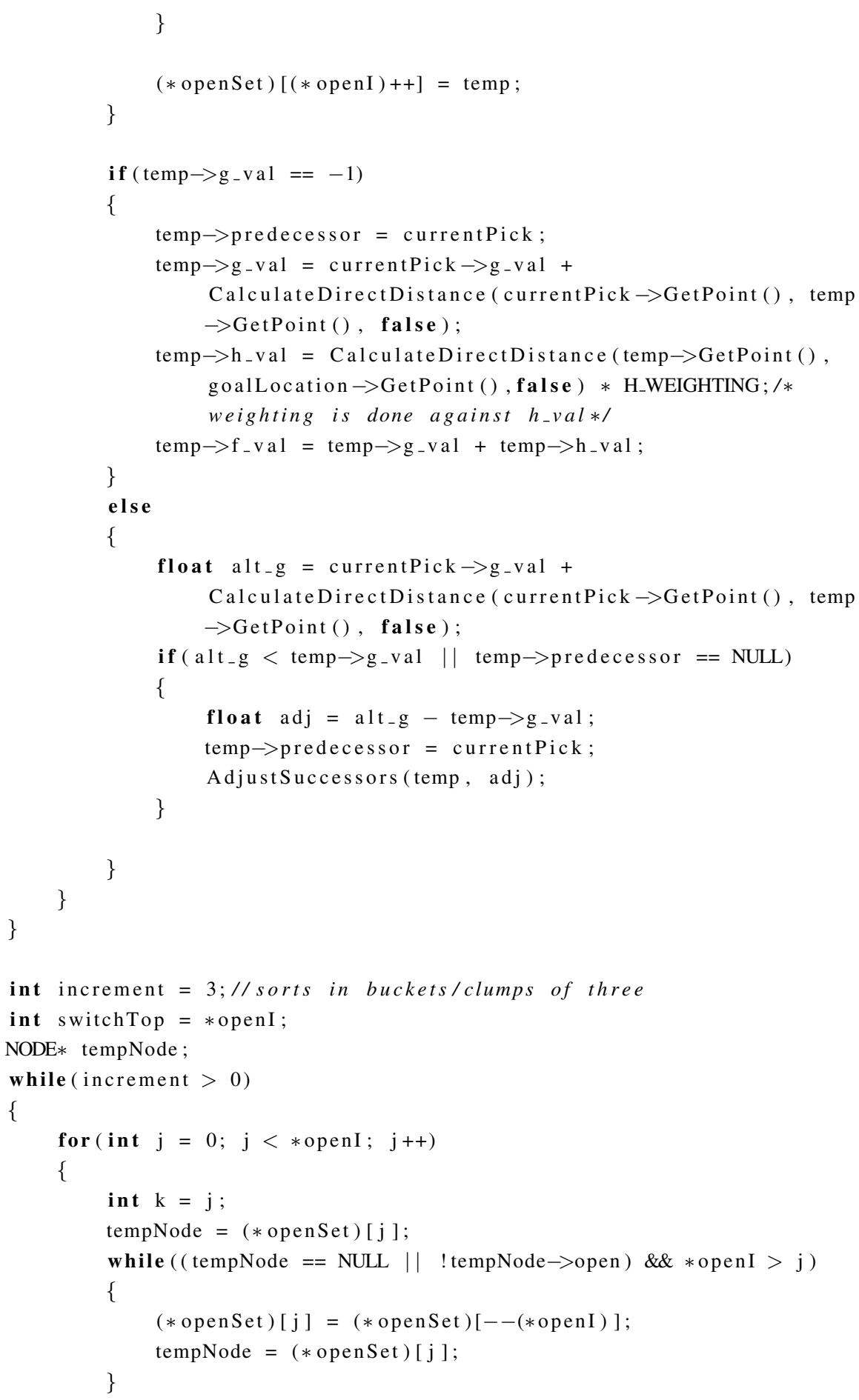




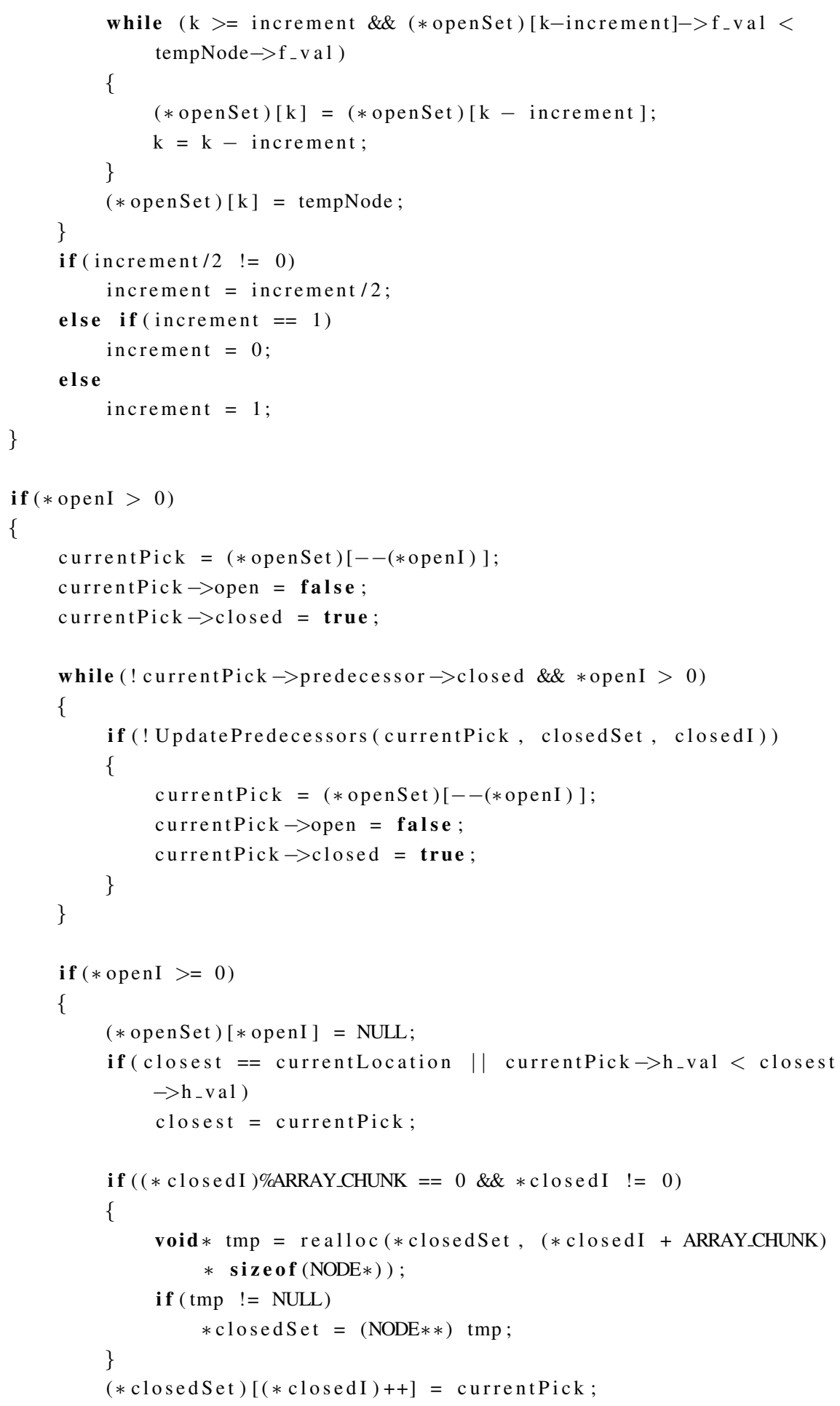




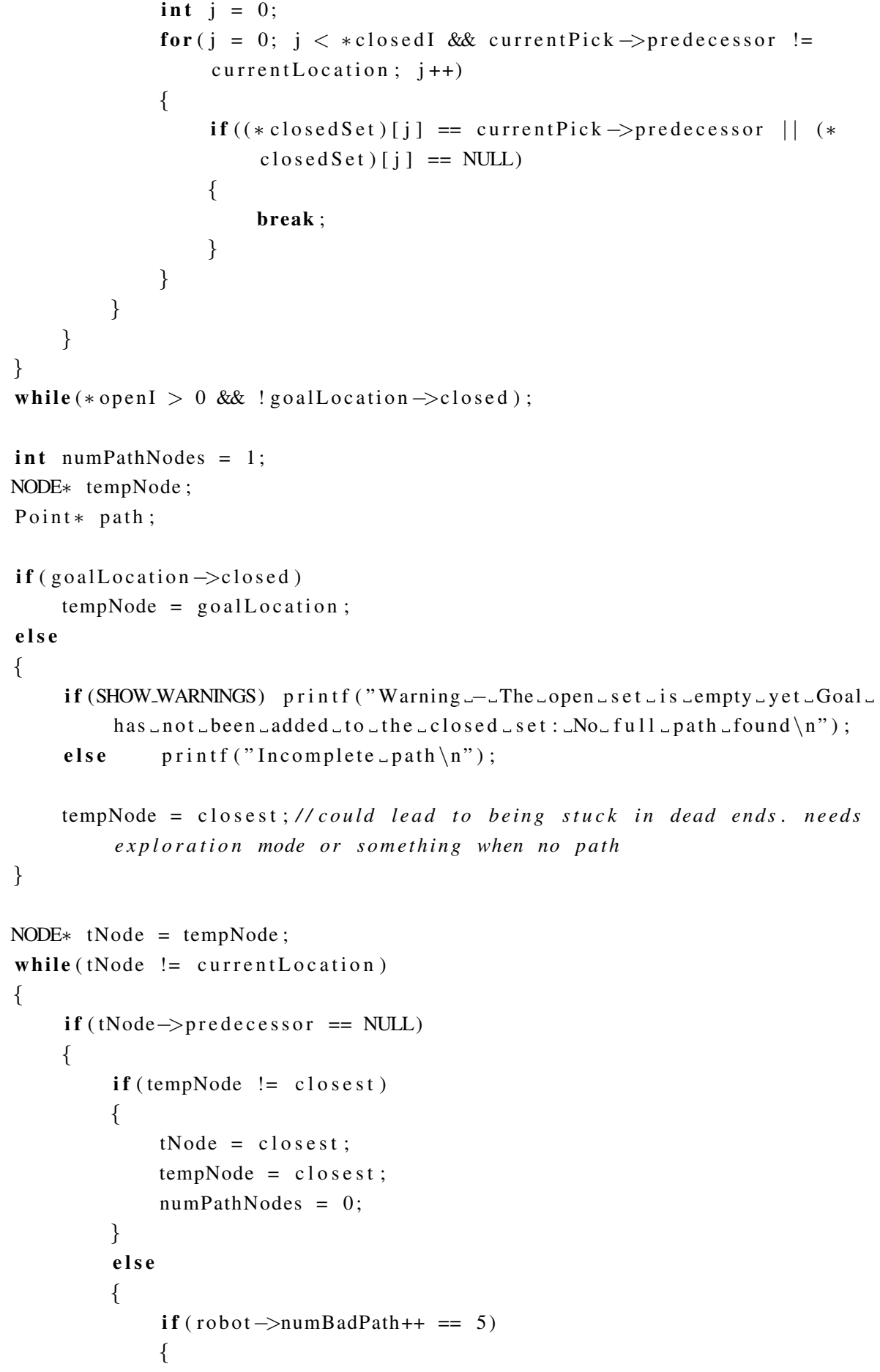


UpdateSetsAndClear (openSet, closedSet, openI, closedI); robot $\rightarrow$ numBadPath $=0$;

\}

return ;

\}

\}

else

\{

tNode $=$ tNode $\rightarrow$ predecessor

numPathNodes ++;

\}

\}

robot $\rightarrow$ SetNumSteps (numPathNodes);

path $=($ Point $*)$ calloc $($ numPathNodes, sizeof $($ Point $))$;

for ( int $1=$ numPathNodes $-1 ; 1>=0 ; 1--)$

\{

path [1] $=$ tempNode $\rightarrow$ GetPoint ();

tempNode $=$ tempNode $\rightarrow$ predecessor $;$

\}

robot $\rightarrow$ SetPath (path);

free (path);

path = NULL;

\}

$/ * *$

//check nodes in open and closed sets

// if open set now has prec $=$ null, remove from open set

// if open set now has prec != null, no change

$/ /$ if closed set now has prec $=n u l l$, remove from closed set

$/ /$ if succ $!=$ null, recursively remove them from closed or open sets

$/ /$ if closed set now has succ $=$ null, move to open set

// compact sets and sort/order the open one

$* /$

template <class NODE>

void UpdateSets (NODE*** openSet, NODE*** closedSet, int $*$ openI, int $*$ closedI) \{

int openInsertInd $=0$;

int closedinsertInd $=0$;

for (int $\mathrm{i}=0 ; \mathrm{i}<* \operatorname{closedI} ; \mathrm{i}++)$

\{

if $\left((*\right.$ closedSet $)[\mathrm{i}]==\operatorname{NULL}||(*$ closedSet $)[\mathrm{i}]->\mathrm{g}_{-}$val $\left.==0\right)$ continue ;

else if $((*$ closedSet $)[i]->$ predecessor $==$ NULL $)$

\{ 


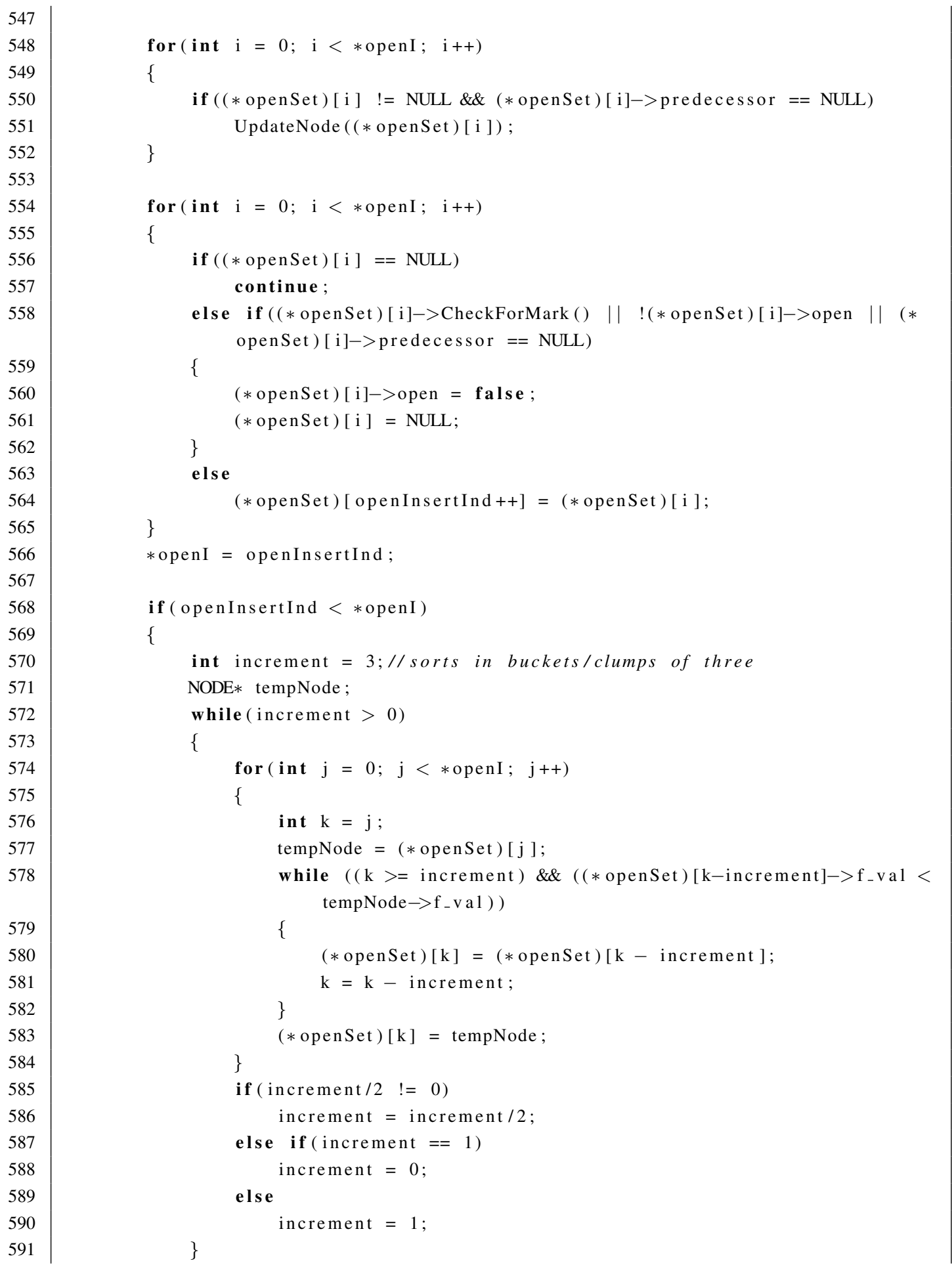




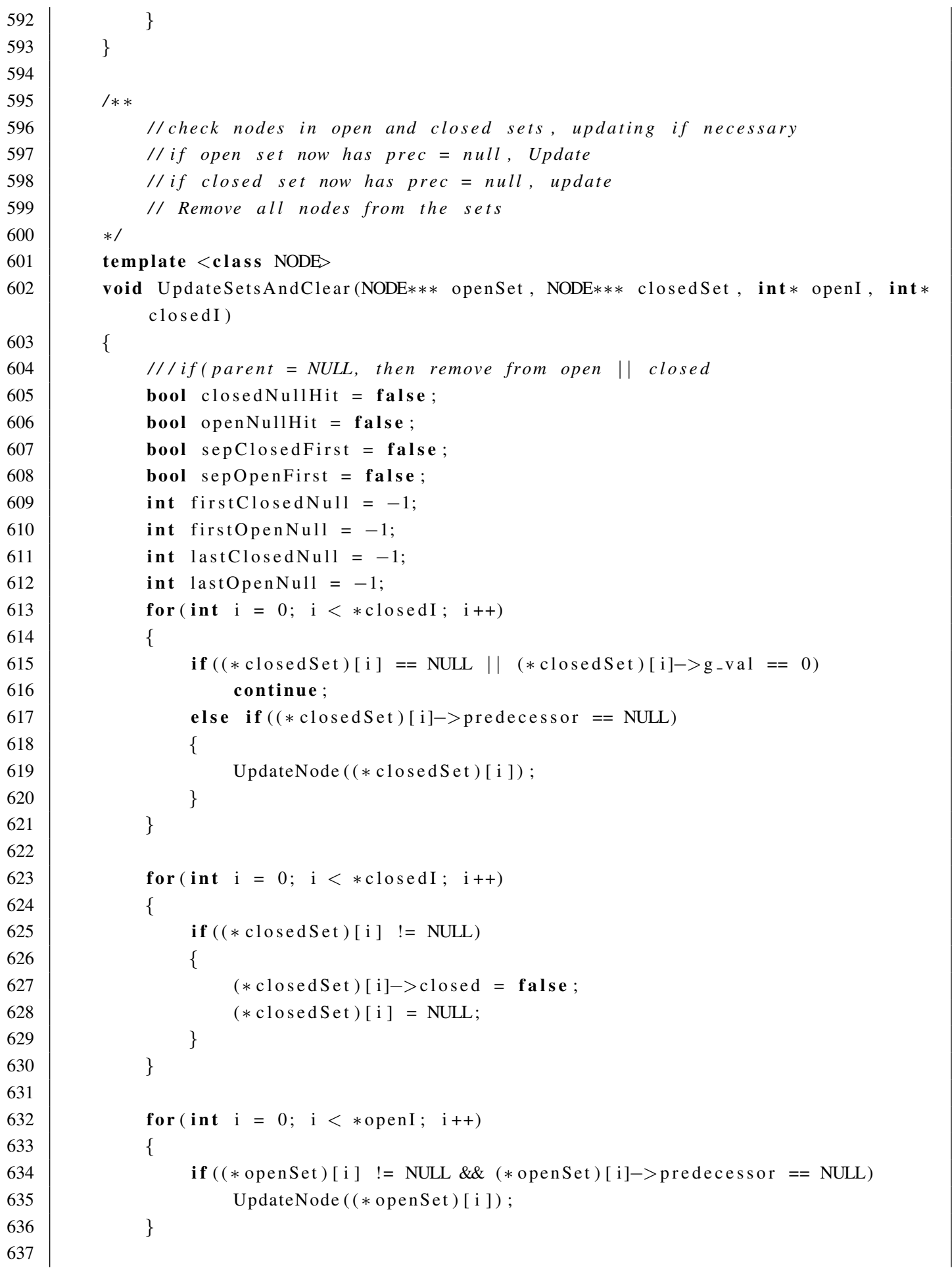




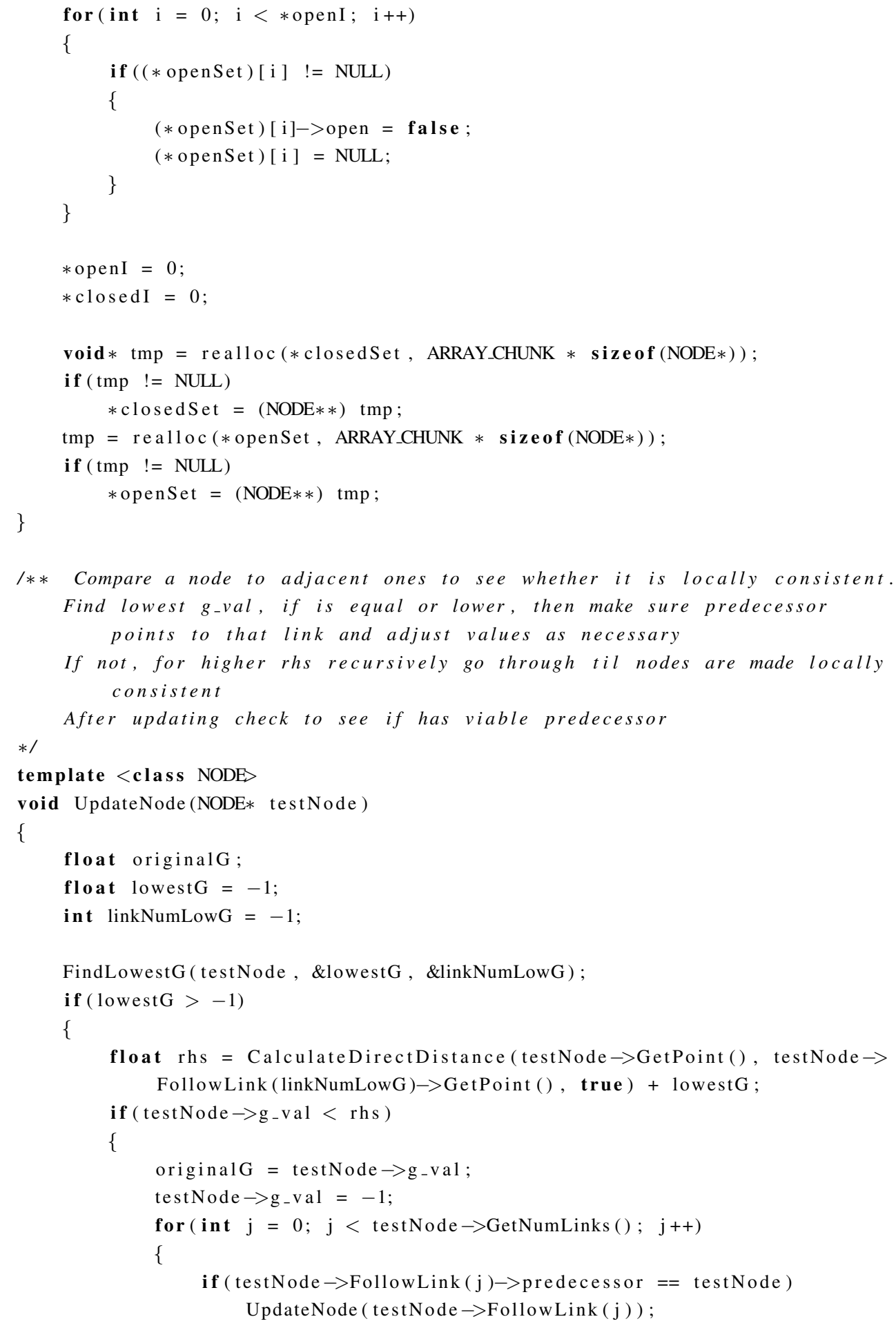




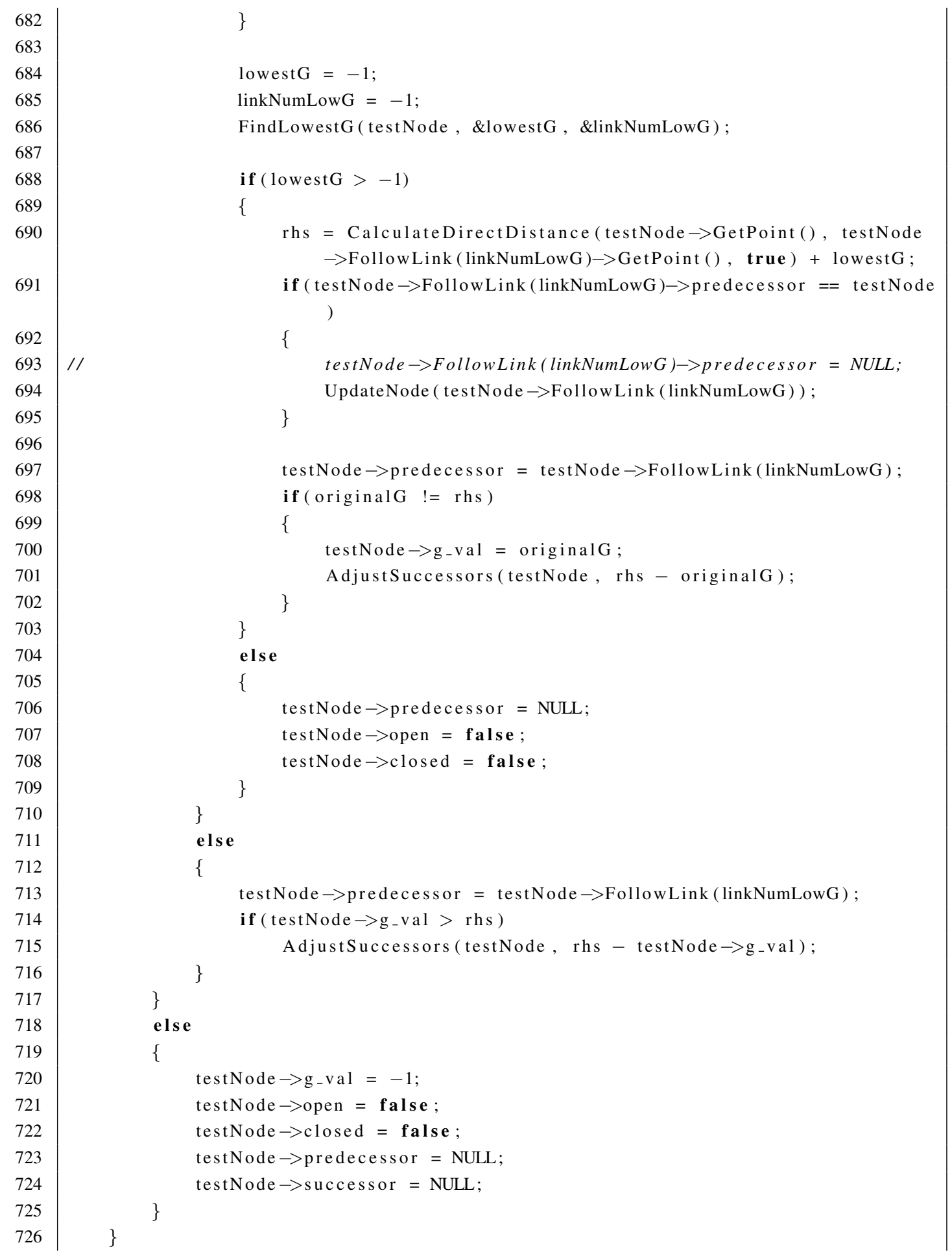




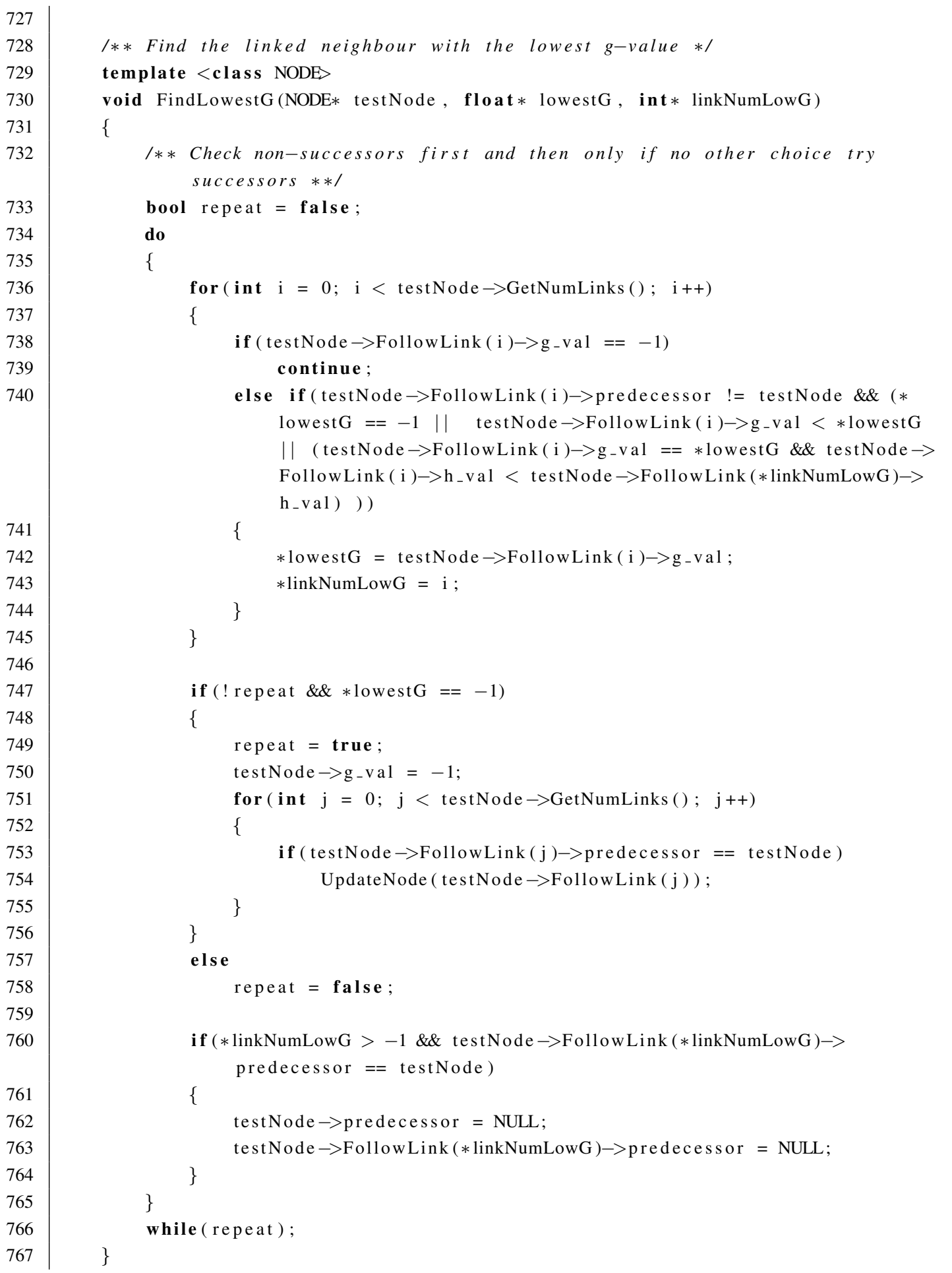




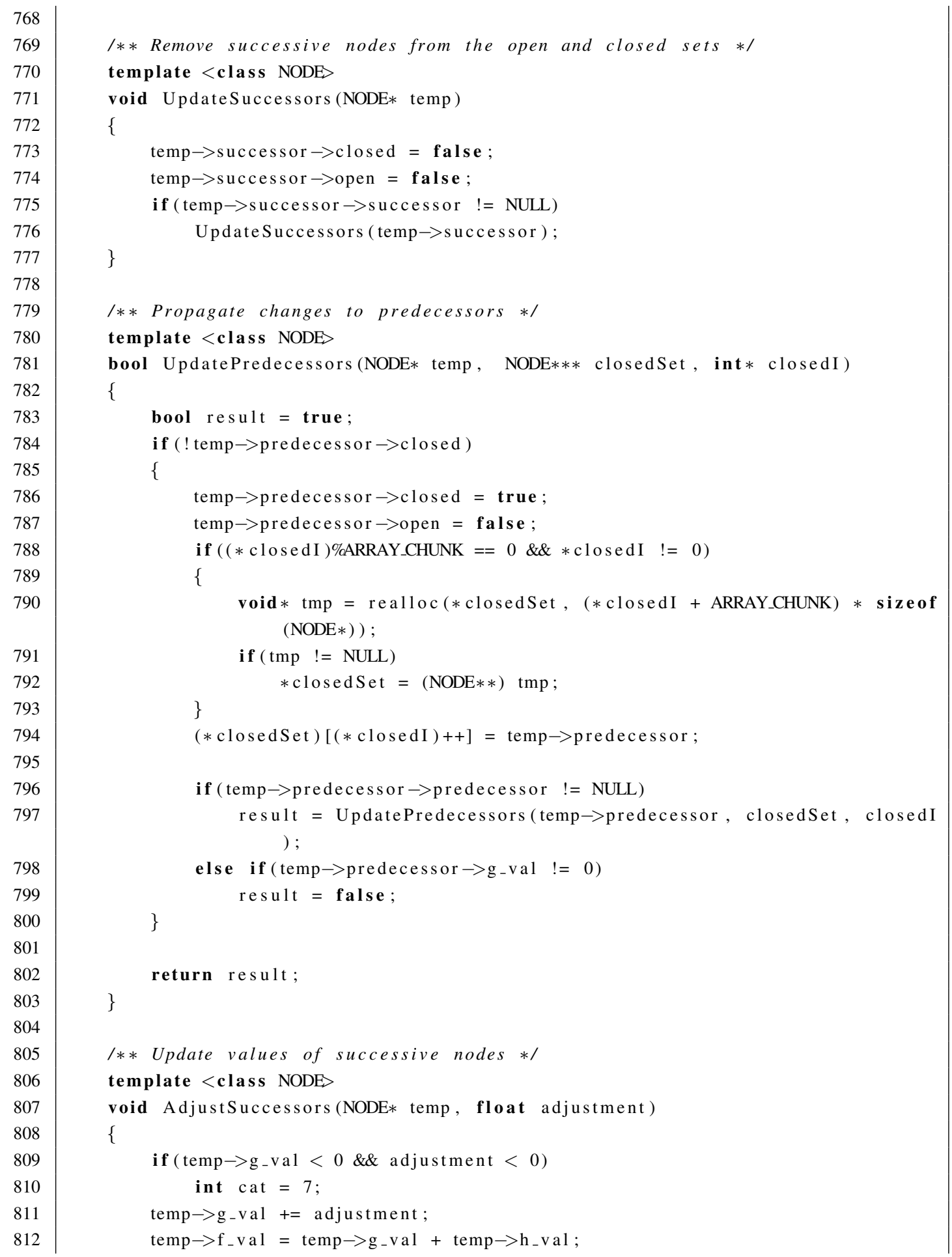




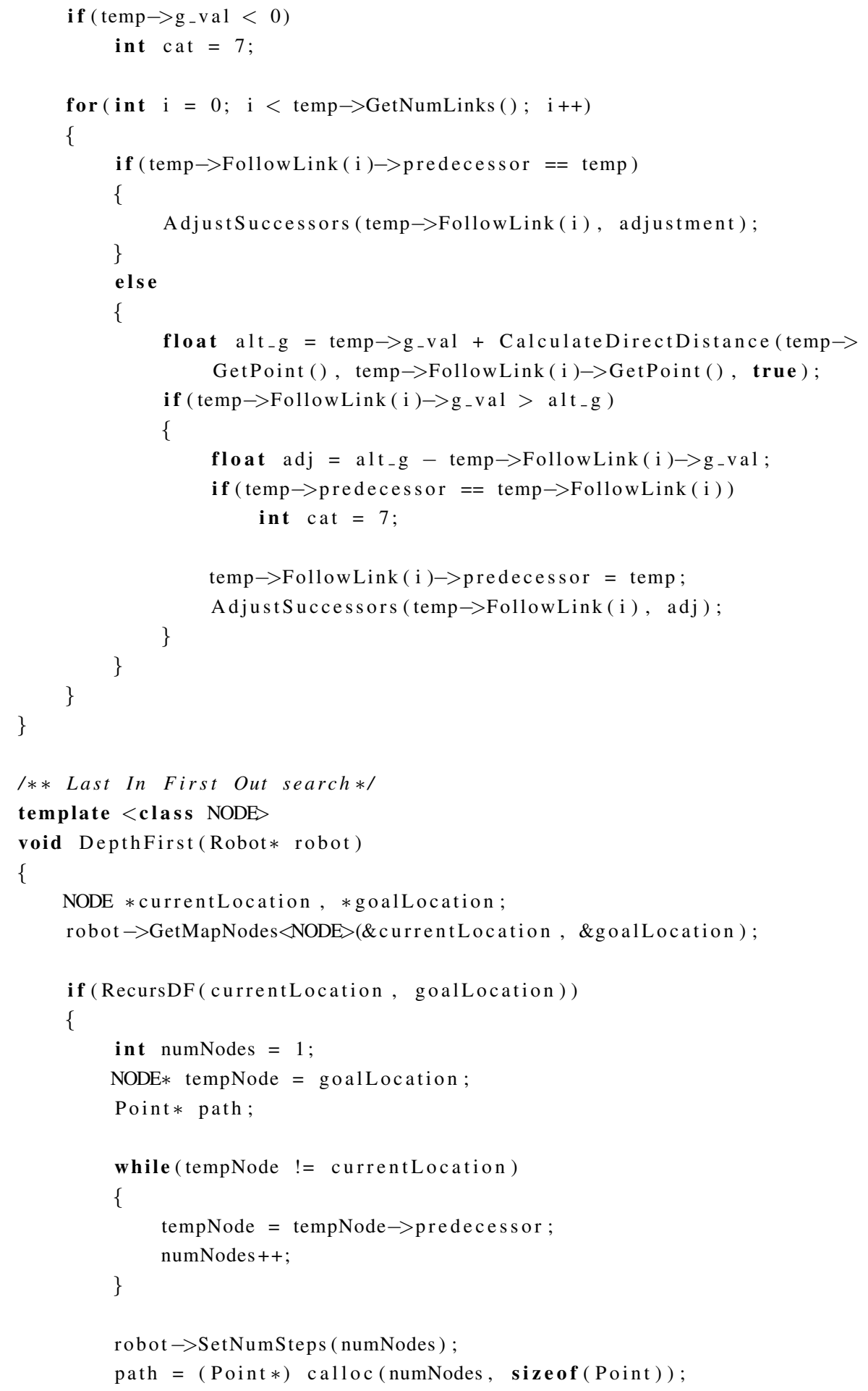




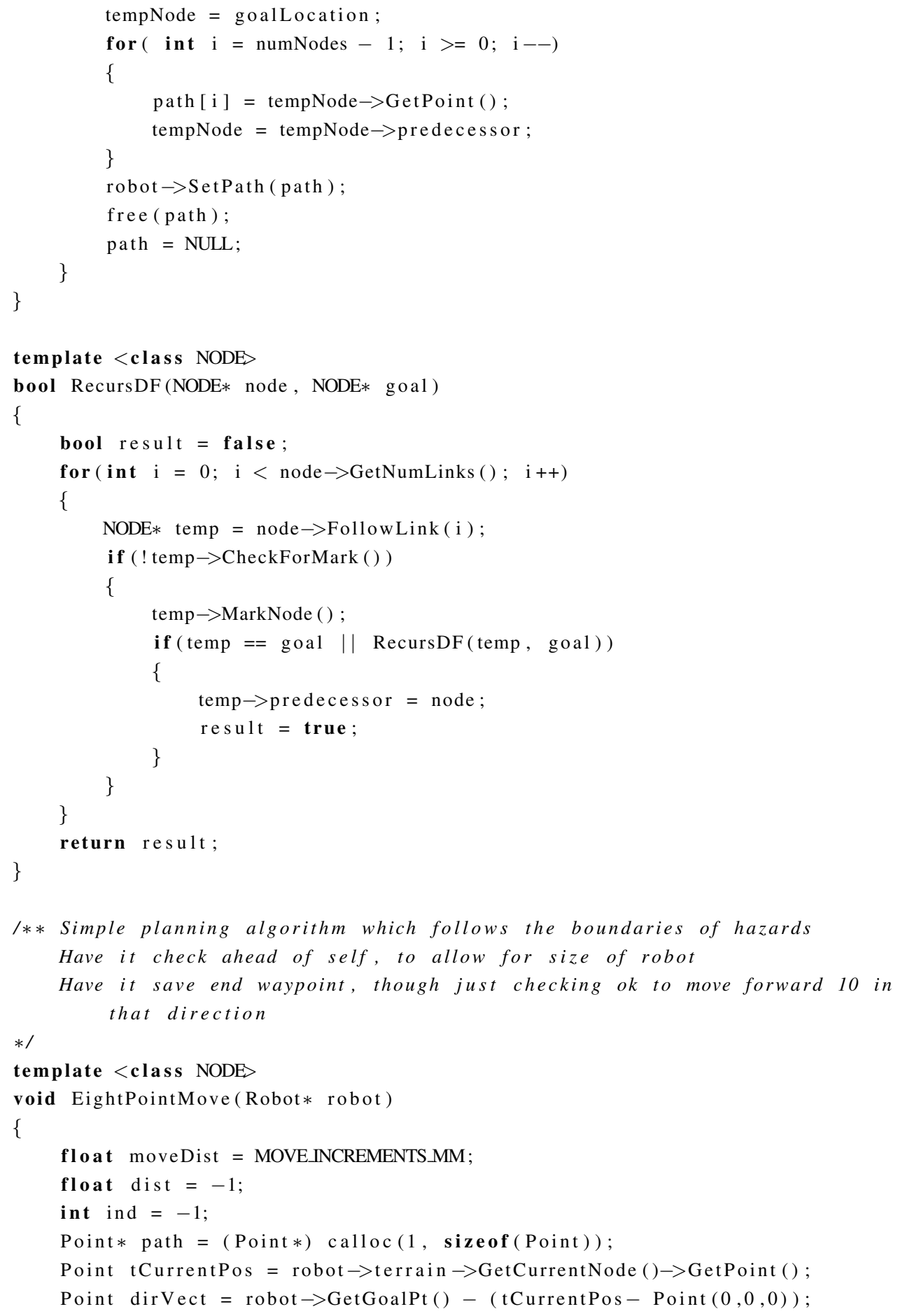


Point moves $[8]=\{$ Point $($ moveDist $, 0,0)$, Point $($ moveDist, 0, moveDist $)$, Point ( moveDist $, 0,-$ moveDist ),

Point (0,0, moveDist), Point $(0,0,-$ moveDist $)$,

Point (-moveDist $, 0,-$ moveDist ), Point (- moveDist, 0 , moveDist ), Point $(-$ moveDist $, 0,0)\}$;

for (int i $=0 ;$ i $<8 ; \quad \mathrm{i}++)$

\{

Point tNewPos $=$ tCurrentPos $+(\operatorname{moves}[\mathrm{i}]-\operatorname{Point}(0,0,0))$;

Face_handle newTri;

if $($ robot $\rightarrow$ terrain $\rightarrow$ GetTri(\&newTri, \&tNewPos $))$

\{

if (newTri $\rightarrow$ CheckValidTri () \&\& !newTri $\rightarrow$ hazardType )

\{

//add check for clearance

float tDist $=$ CalculateDirectDistance (tCurrentPos, tNewPos, true ) ;

if $(\mathrm{d}$ ist $<0 \quad||$ t Dist $<$ dist $)$

\{

dist $=$ tDist

ind $=\mathrm{i}$;

\}

\}

\}

\}

if ( ind $!=-1)$

path $[0]=\operatorname{tCurrentPos}+(\operatorname{moves}[$ ind $]-\operatorname{Point}(0,0,0))$;

else

path $[0]=$ tCurrentPos;

robot $\rightarrow$ SetNumSteps (1);

robot $\rightarrow$ SetPath ( path);

\}

I** Simple state-model based algorithm, which cycles through the directions of forward, left, back and right.

Attempts to move until a hazard is encounter in which case next direction is tried

Or if an obstacle has been successfully skirted then return to moving in previous direction */

template <class NODE>

void StateAlg (Robot* robot)

\{

Point dirVect, normVect, frontLeft, frontRight, backRight, backLeft, currentPos: 


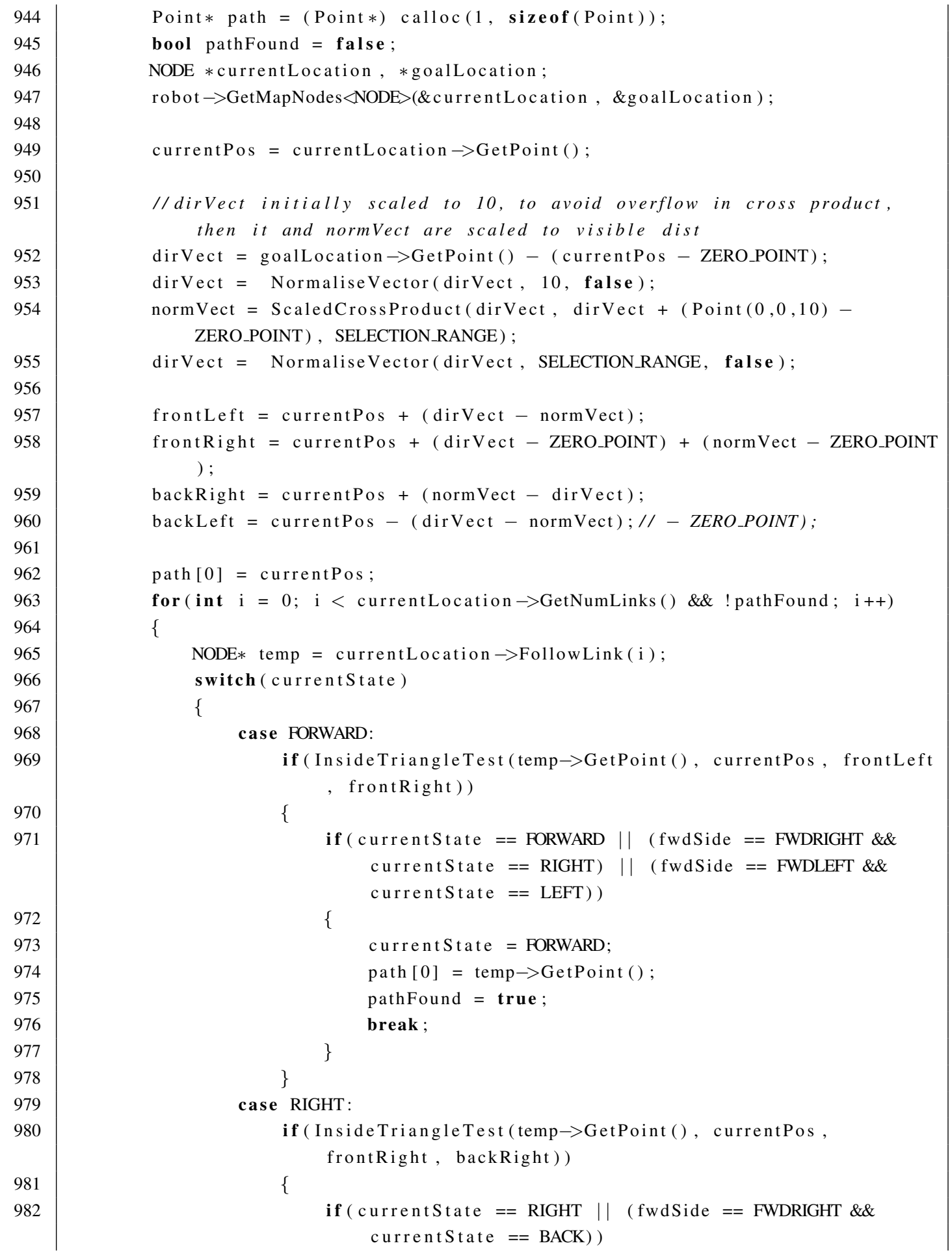




\author{
\{
}

currentstate = RIGHT;

path $[0]=$ temp $\rightarrow$ GetPoint () ;

pathFound = true;

break ;

\}

\}

case BACK:

if (InsideTriangleTest (temp->GetPoint (), currentPos, backLeft, backRight))

\{

if ( currentstate $==$ BACK $\|($ fwdSide $==$ FWDRIGHT $\& \&$ currentstate $==$ LEFT $) \|($ fwdSide $==$ FWDLEFT $\& \&$ currentstate $==$ RIGHT) )

\{

currentstate = BACK;

path $[0]=$ temp $\rightarrow$ GetPoint () ;

pathFound = true;

break ;

\}

\}

case LEFT:

if (InsideTriangleTest (temp->GetPoint (), currentPos, frontLeft , frontRight))

\{

if $($ currentstate $==$ LEFT $\|($ fwdSide $==$ FWDLEFT \& \& currentState $==$ BACK) )

\{

currentstate = LEFT;

path $[0]=$ temp $\rightarrow$ GetPoint () ;

pathFound = true;

break ;

\}

\}

\}

if (! pathFound)

\{

if $($ fwdSide $==$ FWDLEFT $)$

\{

if ( currentstate $==$ FORWARD)

currentstate = LEFT;

else if (currentstate $==$ LEFT)

currentstate = BACK;

else if (currentstate == BACK)

currentstate = RIGHT; 


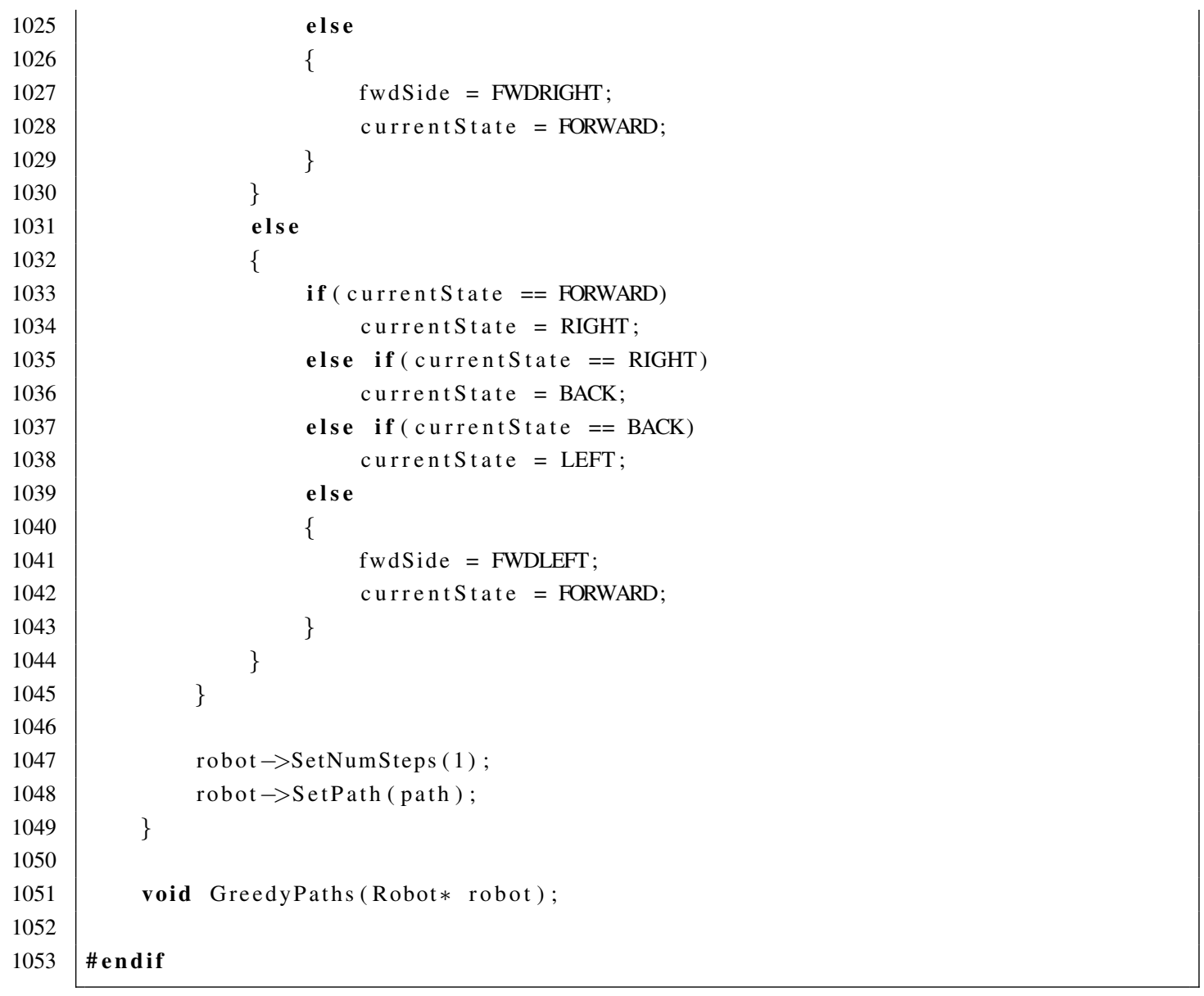

\section{A.3 Display}

\section{A.3.1 Display.h}

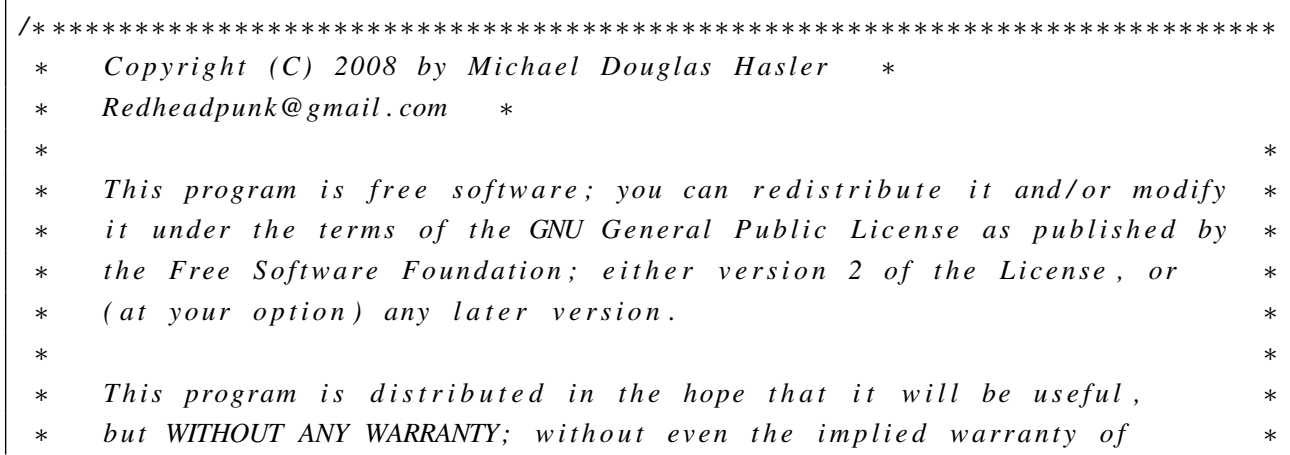




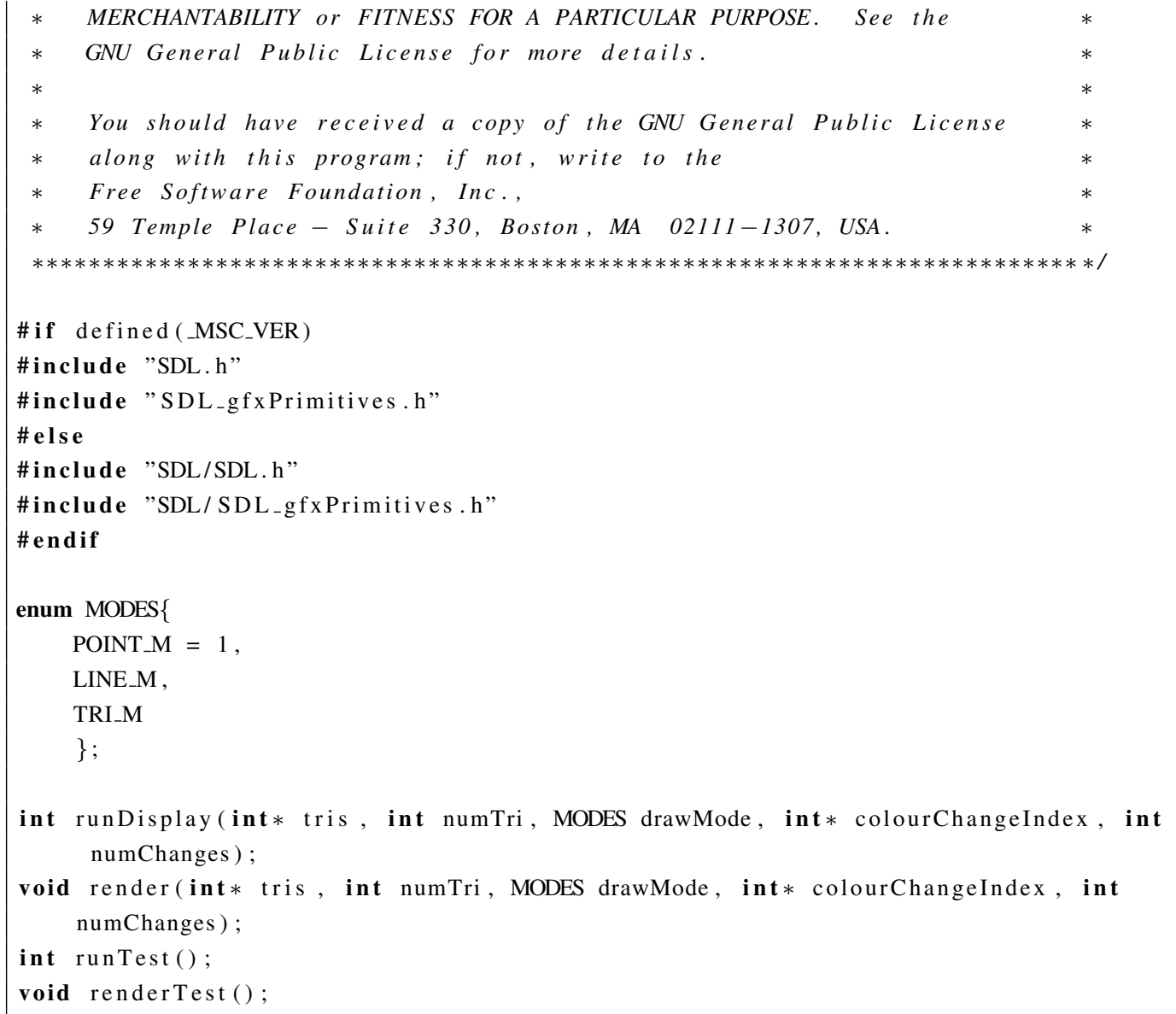

\section{A.3.2 Display.cpp}

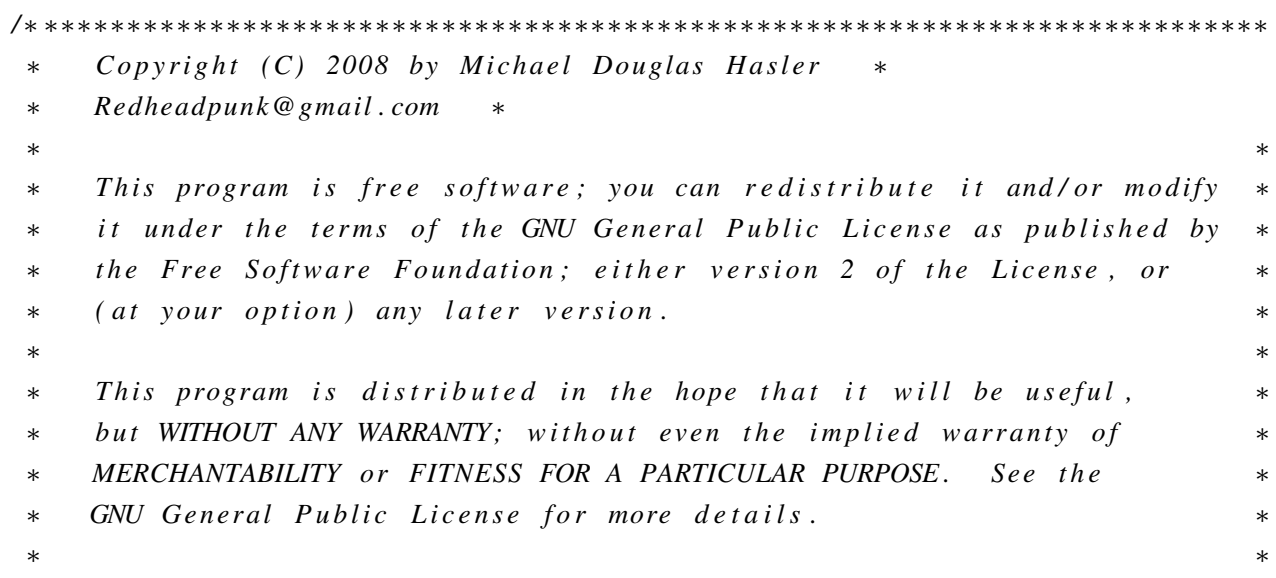




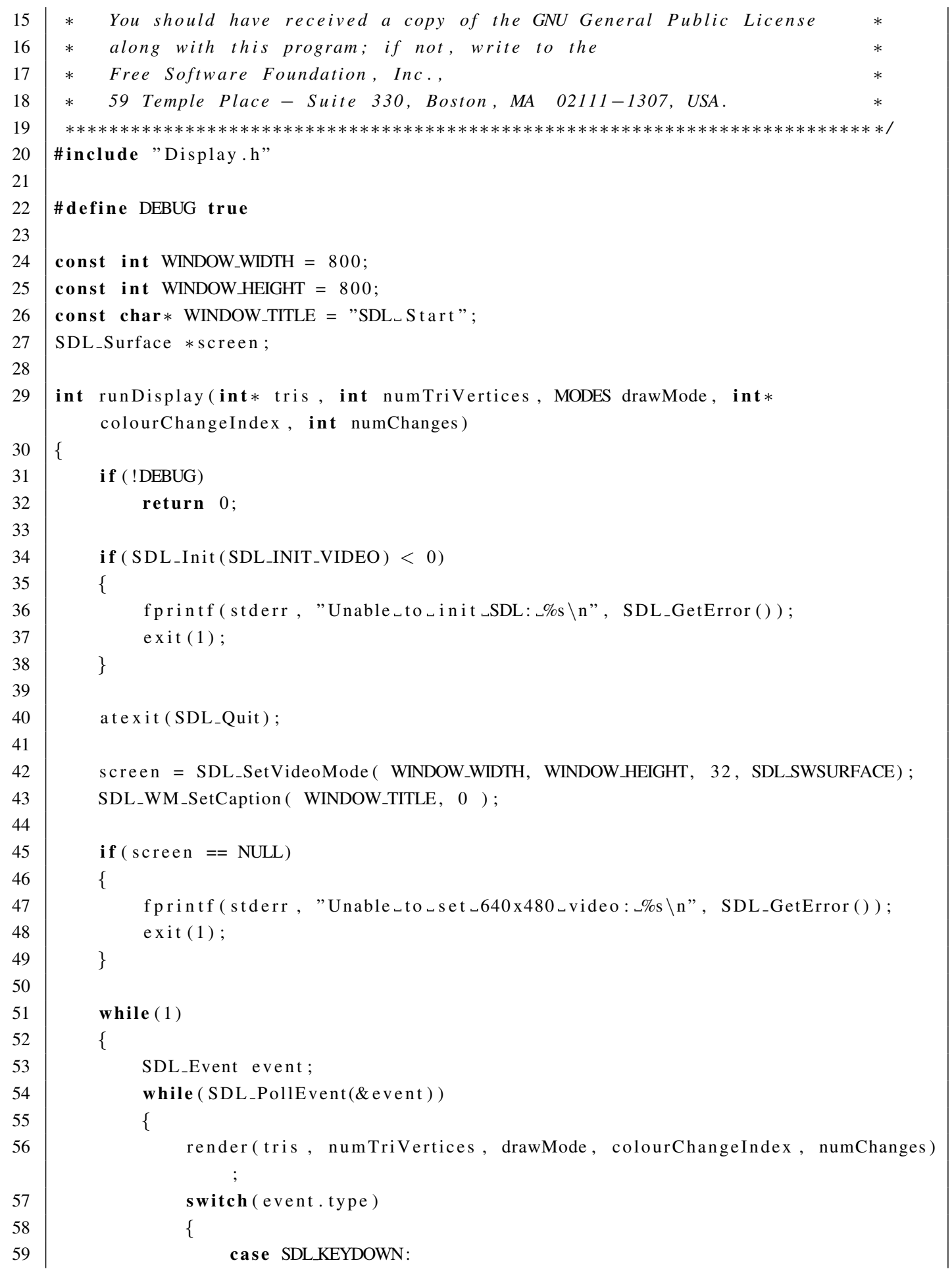


break ; case SDL_KEYUP:

// If escape is pressed, return (and thus, quit)

if ( event. key. keysym.sym == SDLK_ESCAPE) return 0 ;

break ;

case SDL_QUIT :

\{

SDL_Quit ( ) ;

return ( 0$)$; 


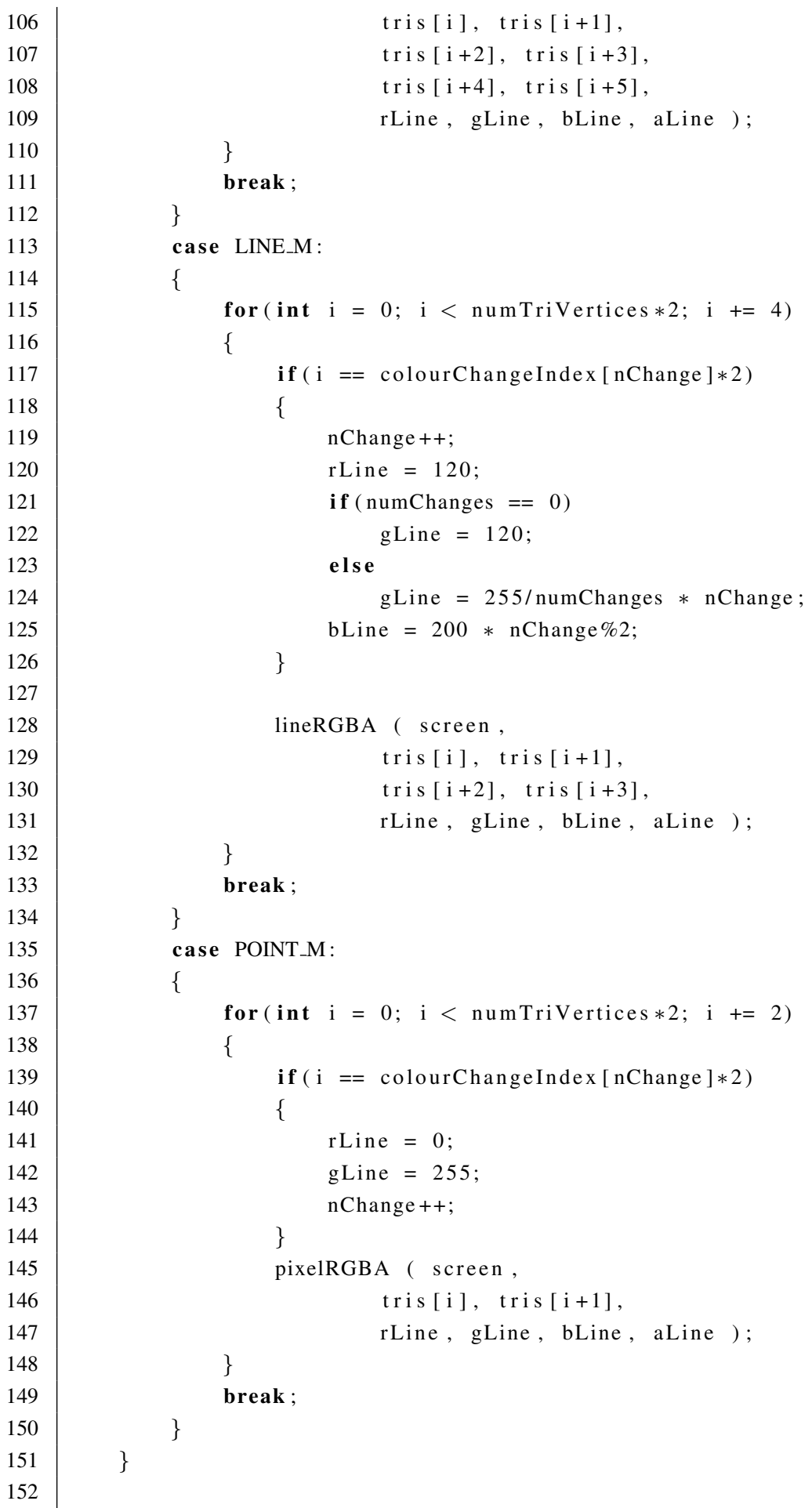




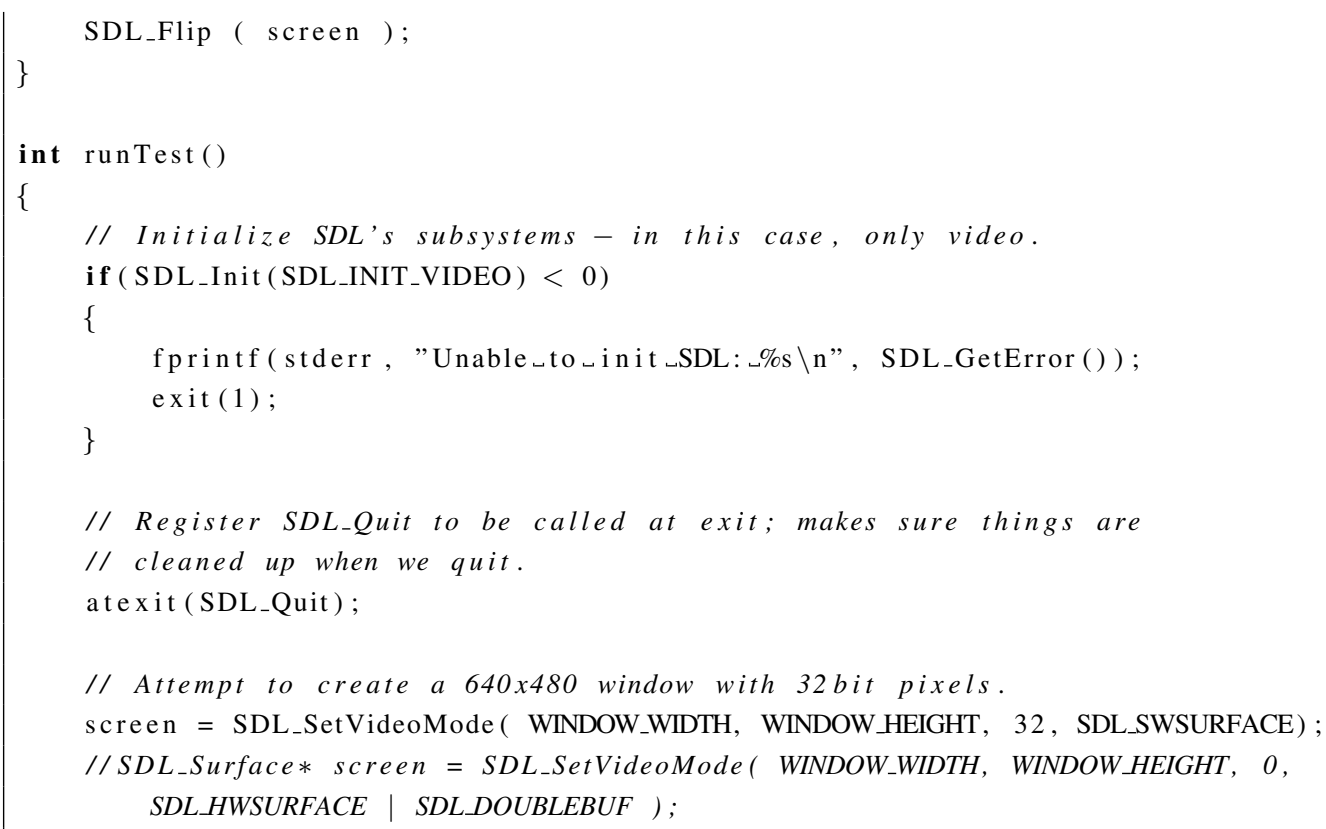




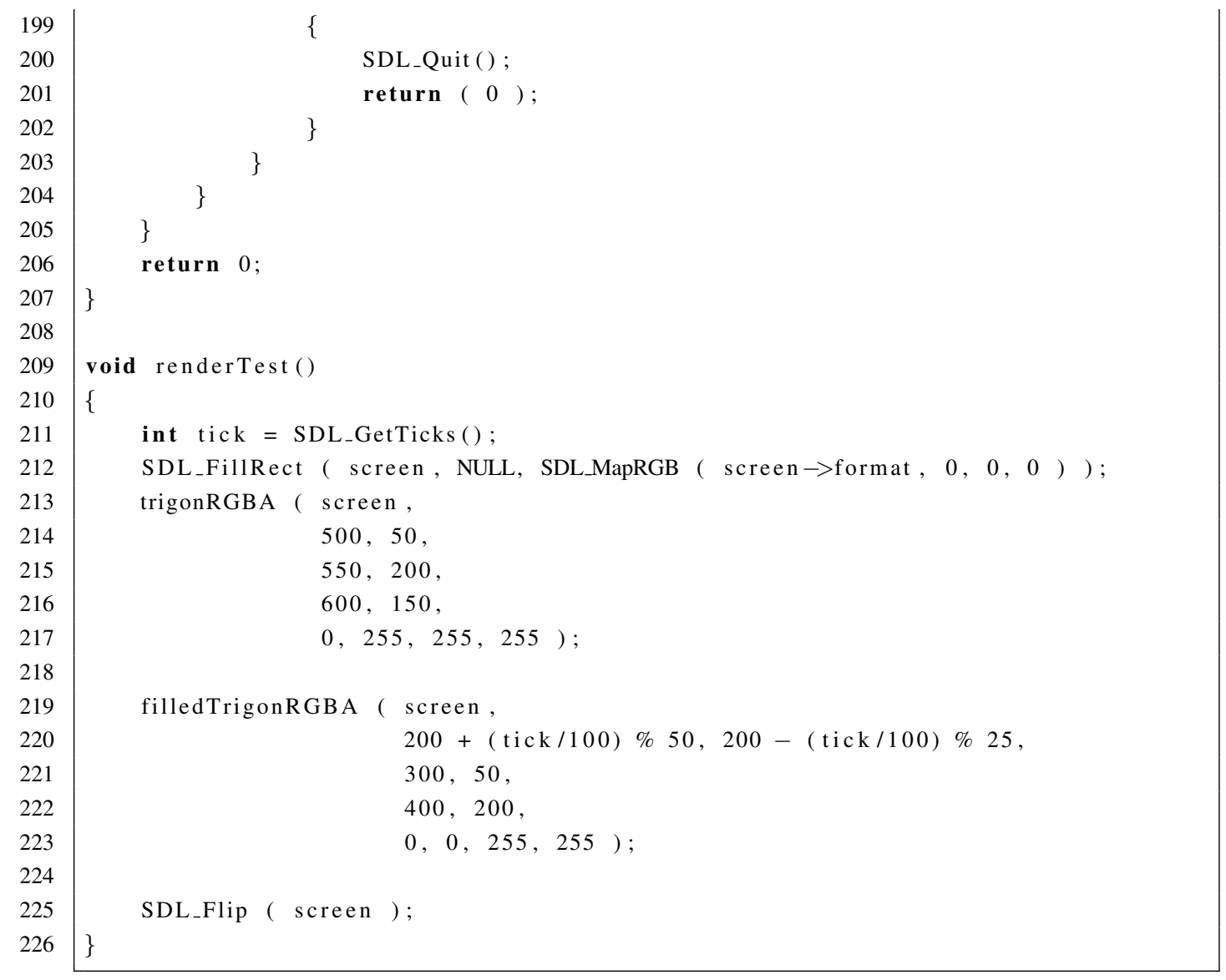

\section{A.4 Hier_Triangulation}

\section{A.4.1 Hier_Triangulation.h}

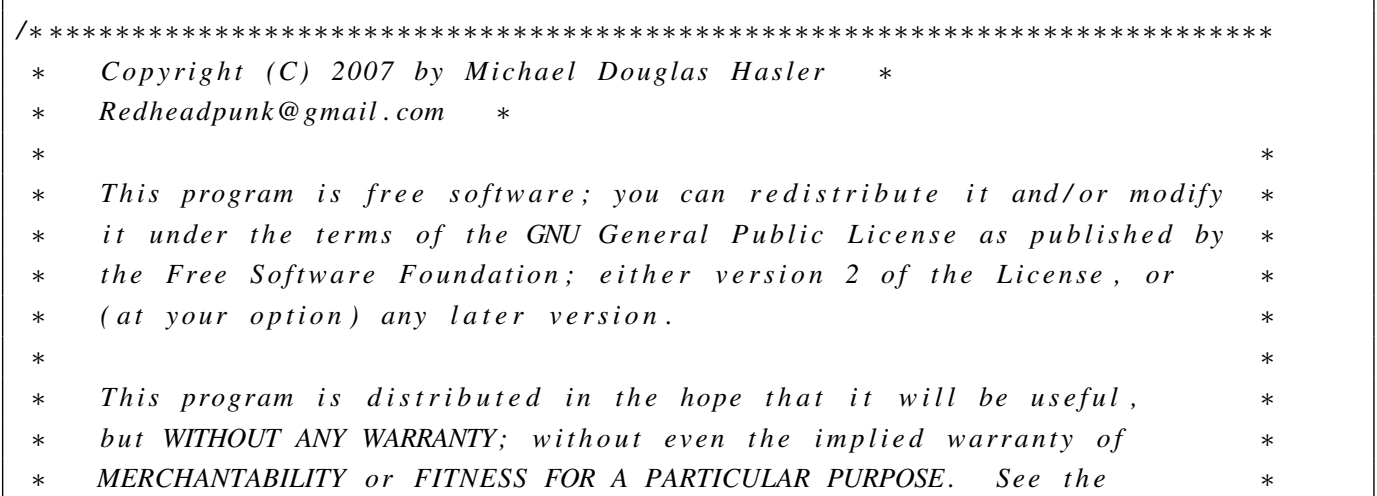




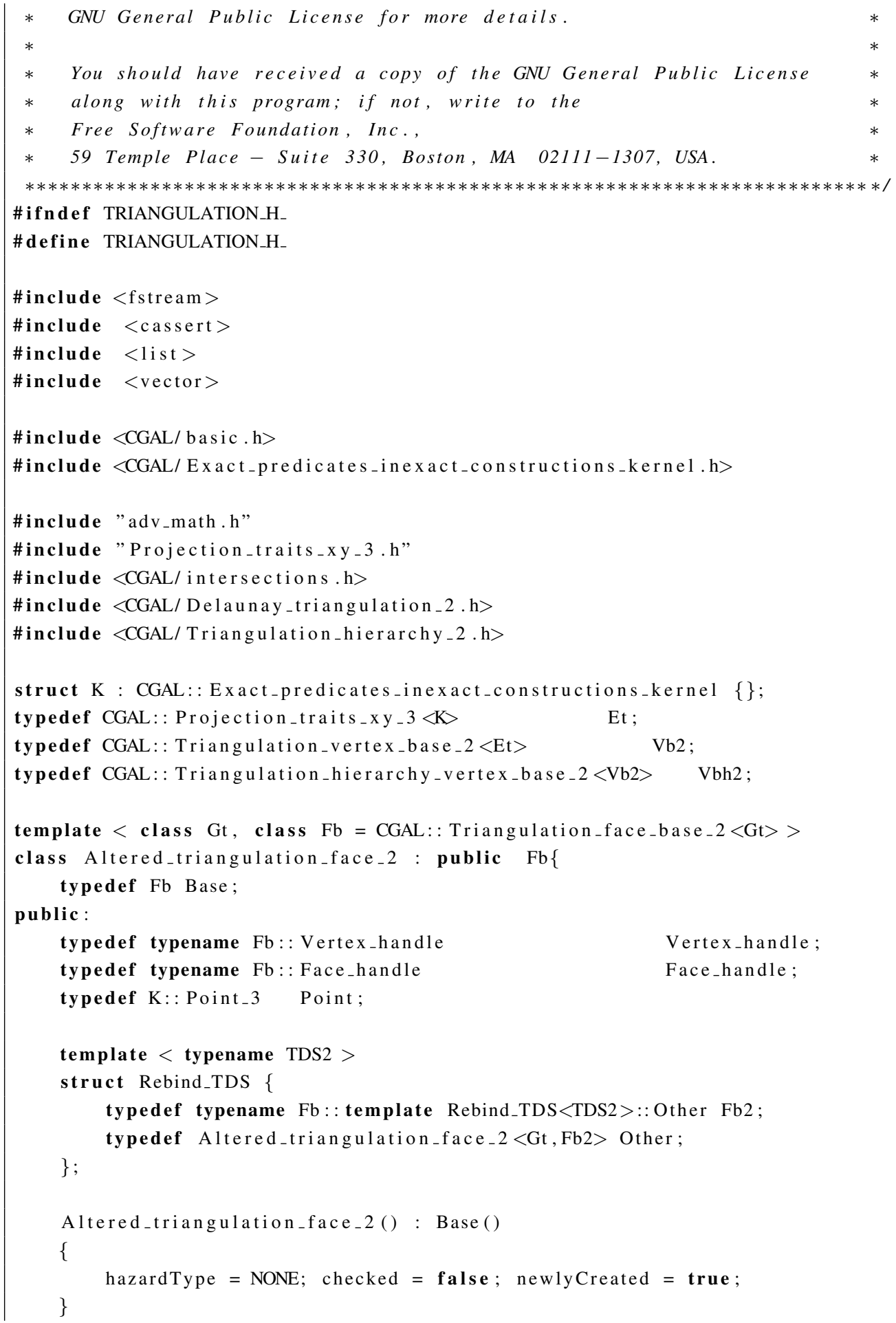




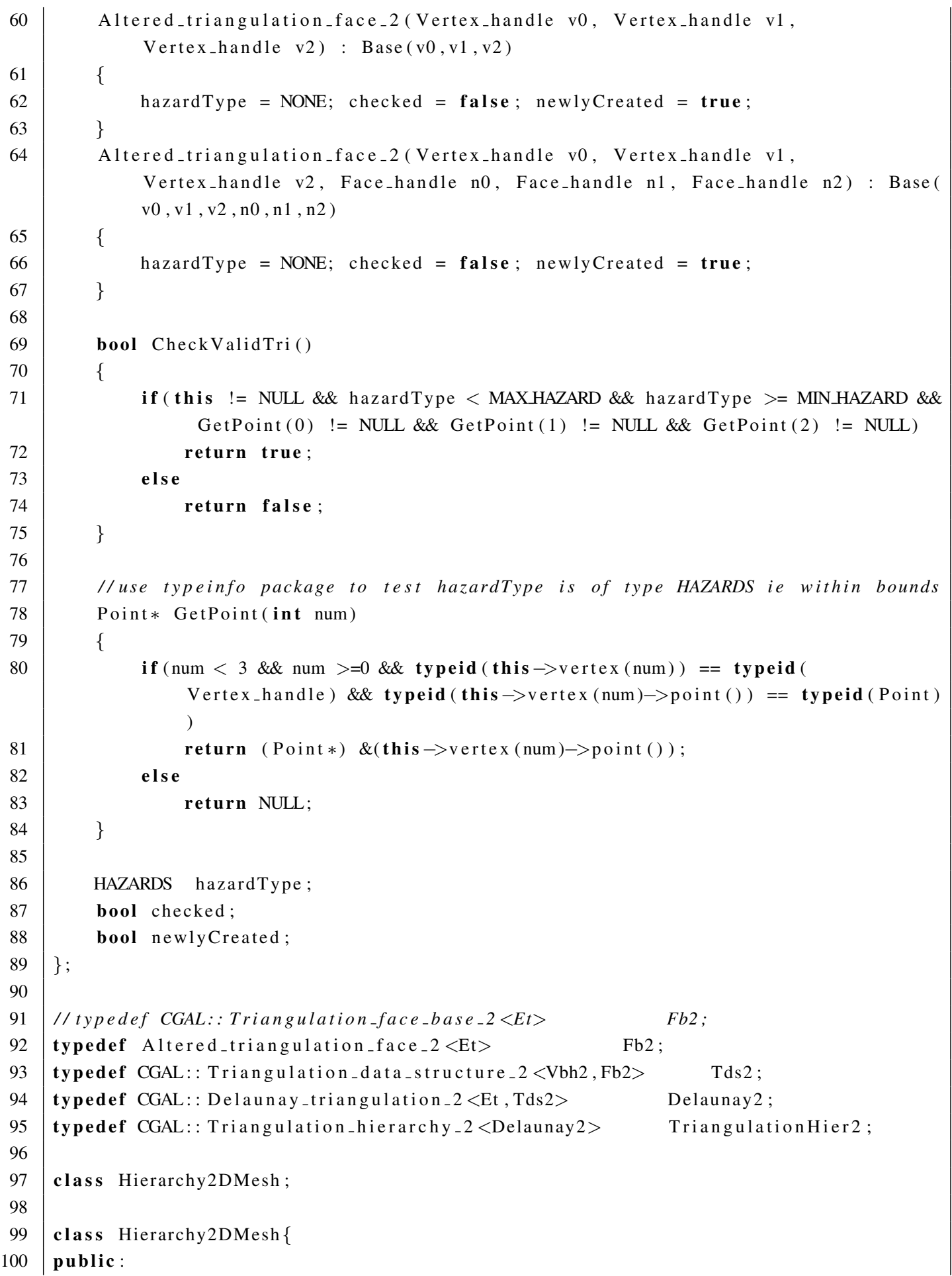




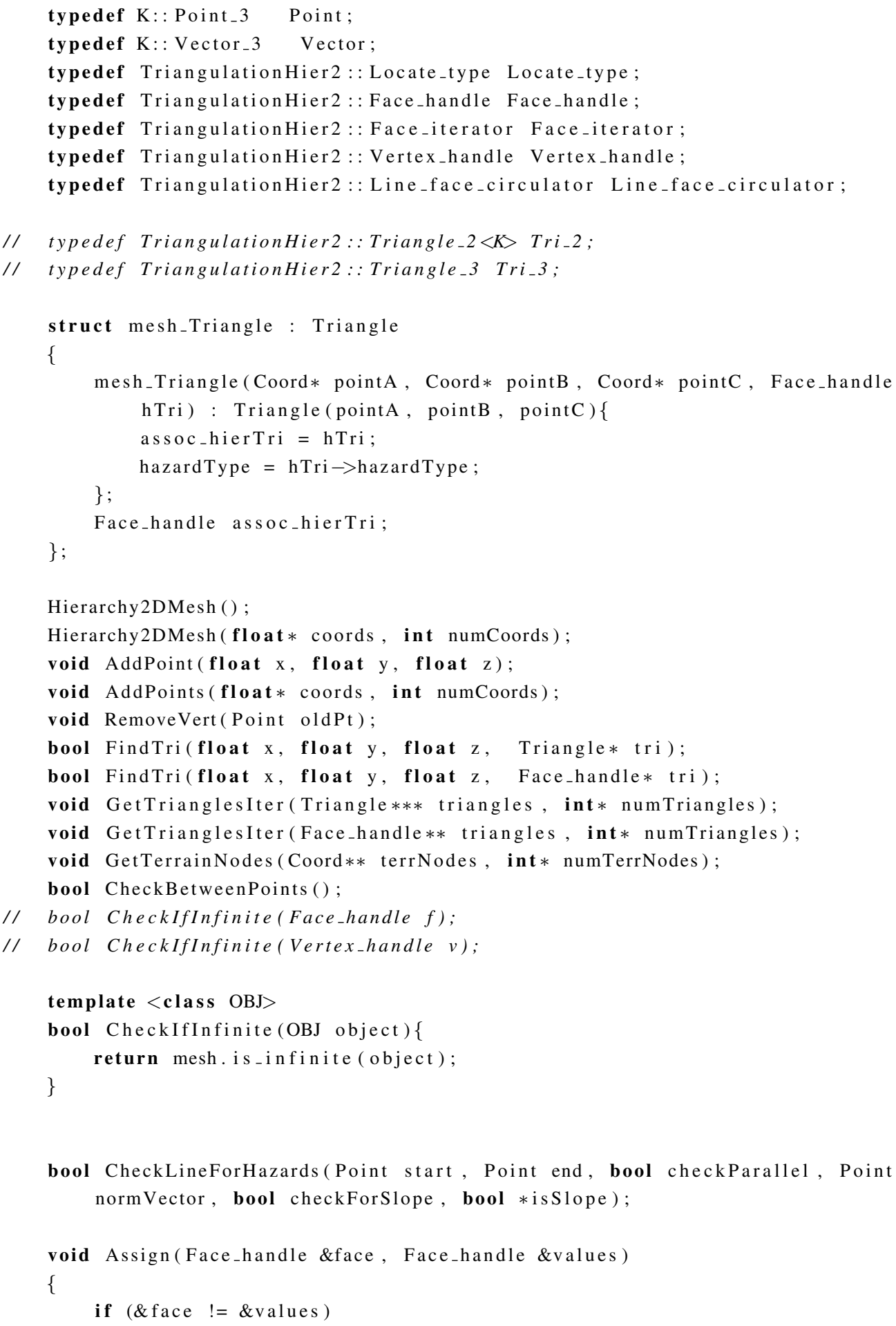




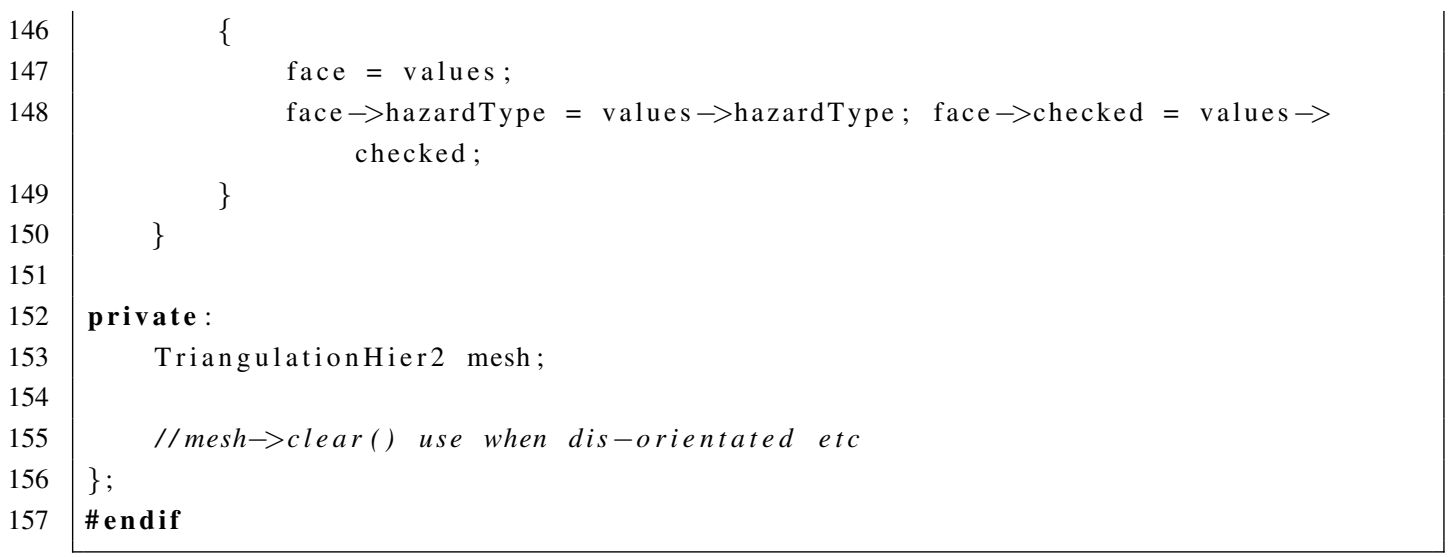

\section{A.4.2 Hier_Triangulation.cpp}

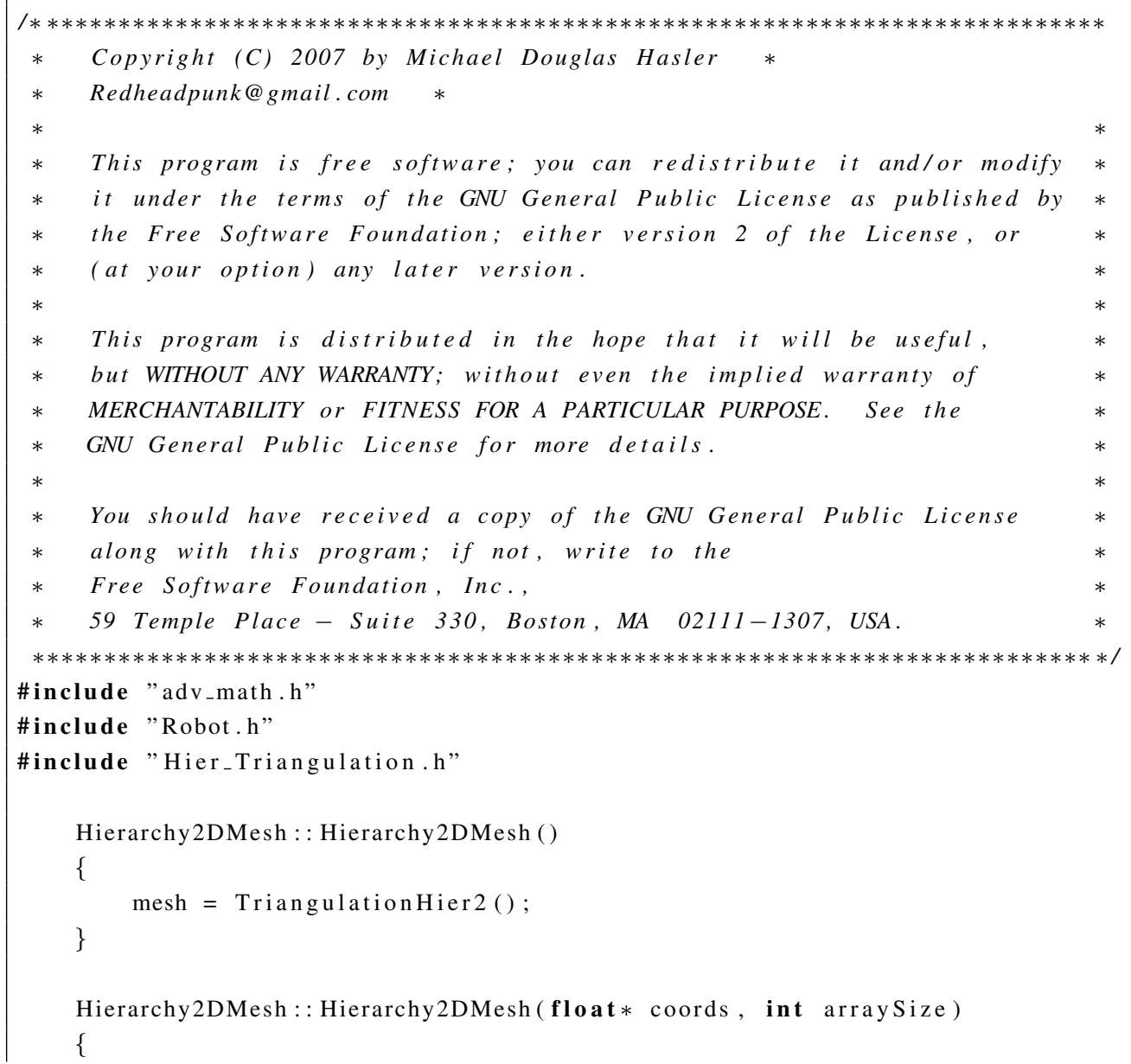




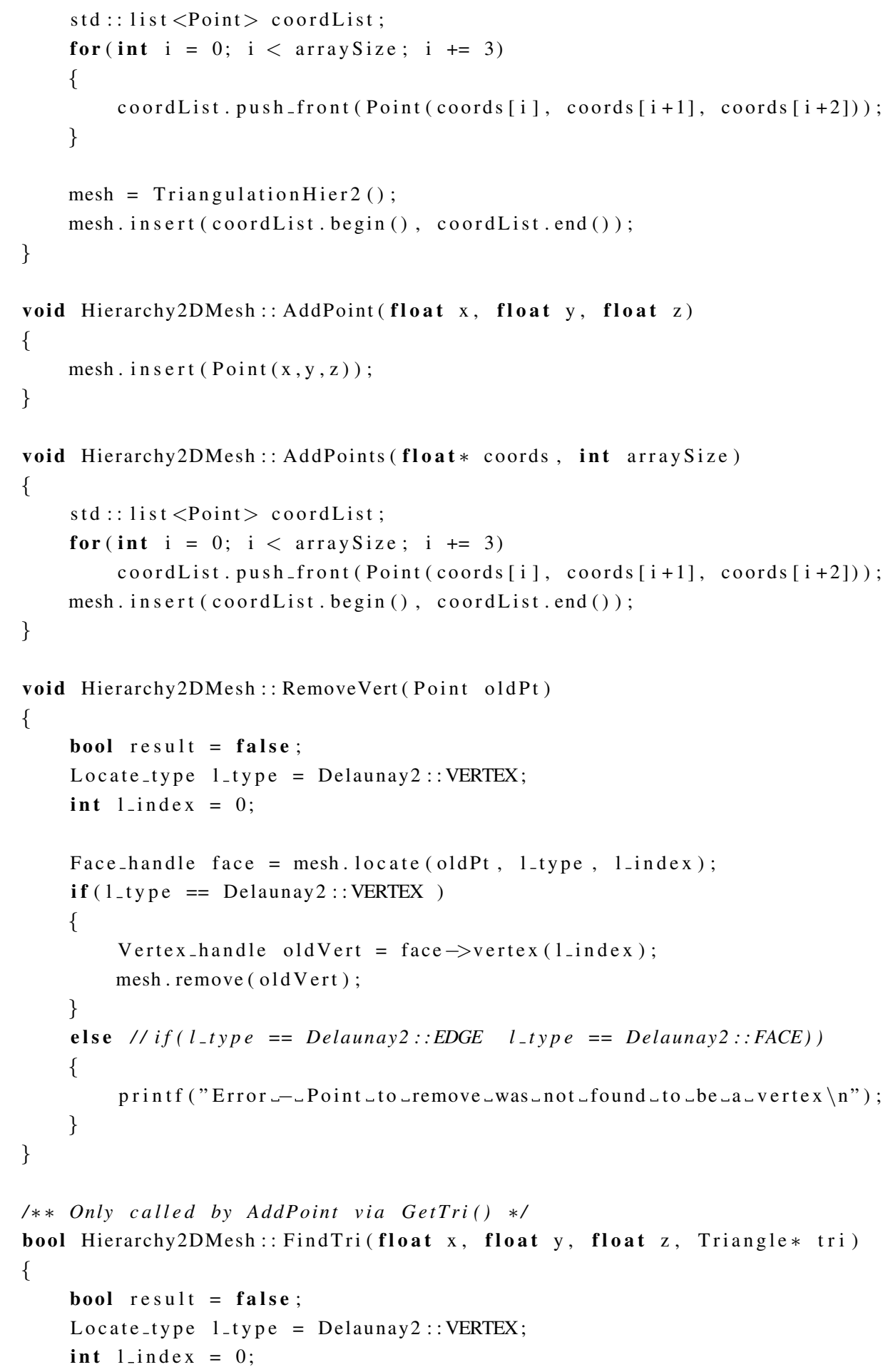


Point pt $(x, y, z)$;

Face_handle face $=$ mesh. 1ocate (pt, 1_type, 1_index);

if $\left(1_{-}\right.$type $==$Delaunay $2::$ FACE $\| 1_{-}$type $==$Delaunay $2::$ VERTEX $\| 1_{-}$type == Delaunay $2:$ : EDGE) //Edge

\{

result $=$ true

tri $\rightarrow$ point $1 \rightarrow$ xVal $=$ face $\rightarrow$ vertex $(0) \rightarrow$ point ()$. x()$;

tri $\rightarrow$ point $1 \rightarrow$ yVal $=$ face $\rightarrow$ vertex $(0) \rightarrow$ point ()$\cdot y() ;$

tri $\rightarrow$ point $1 \rightarrow$ zVal $=$ face $\rightarrow$ vertex $(0) \rightarrow$ point ()$. z()$;

tri $\rightarrow$ point $2 \rightarrow$ xVal $=$ face $\rightarrow$ vertex (1) $\rightarrow$ point (). $x()$

tri $\rightarrow$ point $2 \rightarrow$ yVal $=$ face $\rightarrow$ vertex $(1) \rightarrow$ point ()$\cdot y()$

tri $\rightarrow$ point $2 \rightarrow$ zVal $=$ face $\rightarrow$ vertex $(1) \rightarrow$ point ()$. z()$;

tri $\rightarrow$ point $3 \rightarrow$ xVal $=$ face $\rightarrow$ vertex $(2) \rightarrow$ point ()$. x() ;$

tri $\rightarrow$ point $3 \rightarrow$ yVal $=$ face $\rightarrow$ vertex $(2) \rightarrow$ point ()$\cdot y()$;

tri $\rightarrow$ point $3 \rightarrow$ zVal $=$ face $\rightarrow$ vertex $(2) \rightarrow$ point ()$. z() ;$

tri $\rightarrow$ checked $=$ false ;

tri $\rightarrow$ hazardType $=$ face $\rightarrow$ hazardType

\}

else if ( $1_{-}$type $==$Delaunay $2::$ OUTSIDE_CONVEX_HULL $)$

\{

int $\mathrm{a}=0, \mathrm{~b}=1$;

if (CheckIfInfinite (face $\rightarrow$ vertex (1)))

$\mathrm{b}=2$;

else if (CheckIfInfinite (face $\rightarrow$ vertex (0)))

$\mathrm{a}=2$;

if ( face $\rightarrow$ vertex (a) != NULL)

\{

$\operatorname{tri} \rightarrow$ point $1 \rightarrow$ xVal $=$ face $\rightarrow$ vertex (a) $\rightarrow$ point (). $x() ;$

tri $\rightarrow$ point $1 \rightarrow$ yVal $=$ face $\rightarrow$ vertex $($ a $) \rightarrow$ point ()$\cdot y() ;$

$\operatorname{tri} \rightarrow$ point $1 \rightarrow$ zVal $=$ face $\rightarrow$ vertex $($ a $) \rightarrow$ point ()$. z() ;$

\}

if $($ face $\rightarrow$ vertex (b) $!=$ NULL)

\{

$\operatorname{tri} \rightarrow$ point $2 \rightarrow$ xVal $=$ face $\rightarrow$ vertex $($ b $) \rightarrow$ point ()$. x() ;$

$\operatorname{tri} \rightarrow$ point $2 \rightarrow$ yVal $=$ face $\rightarrow$ vertex $(b) \rightarrow$ point ()$\cdot y() ;$

$\operatorname{tri} \rightarrow$ point $2 \rightarrow$ zVal $=$ face $\rightarrow$ vertex (b) $\rightarrow$ point ()$. z() ;$

\}

\}

return result; 
bool Hierarchy2DMesh:: FindTri(float x, float y, float $z$, Face_handle* tri) \{ 


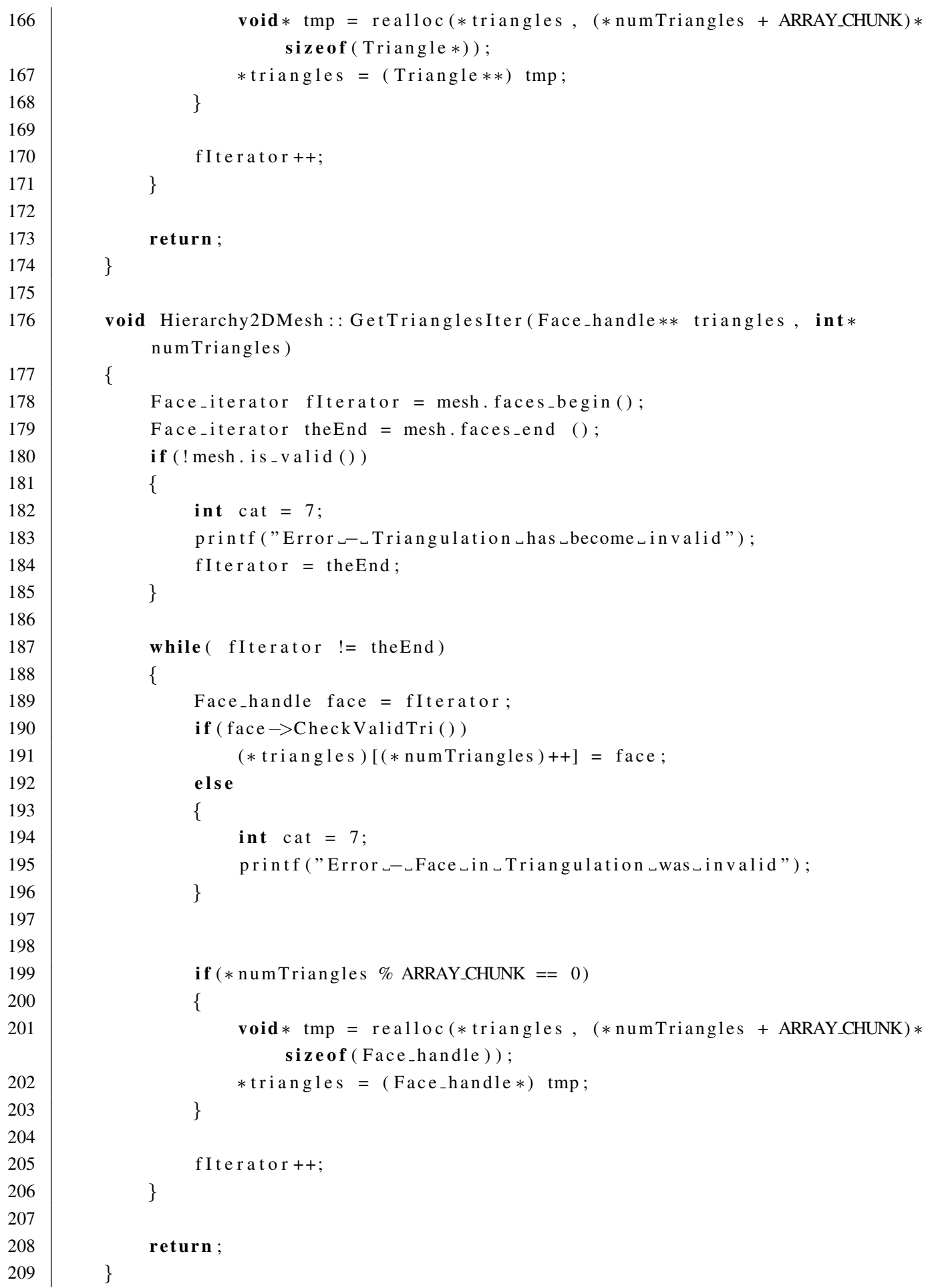




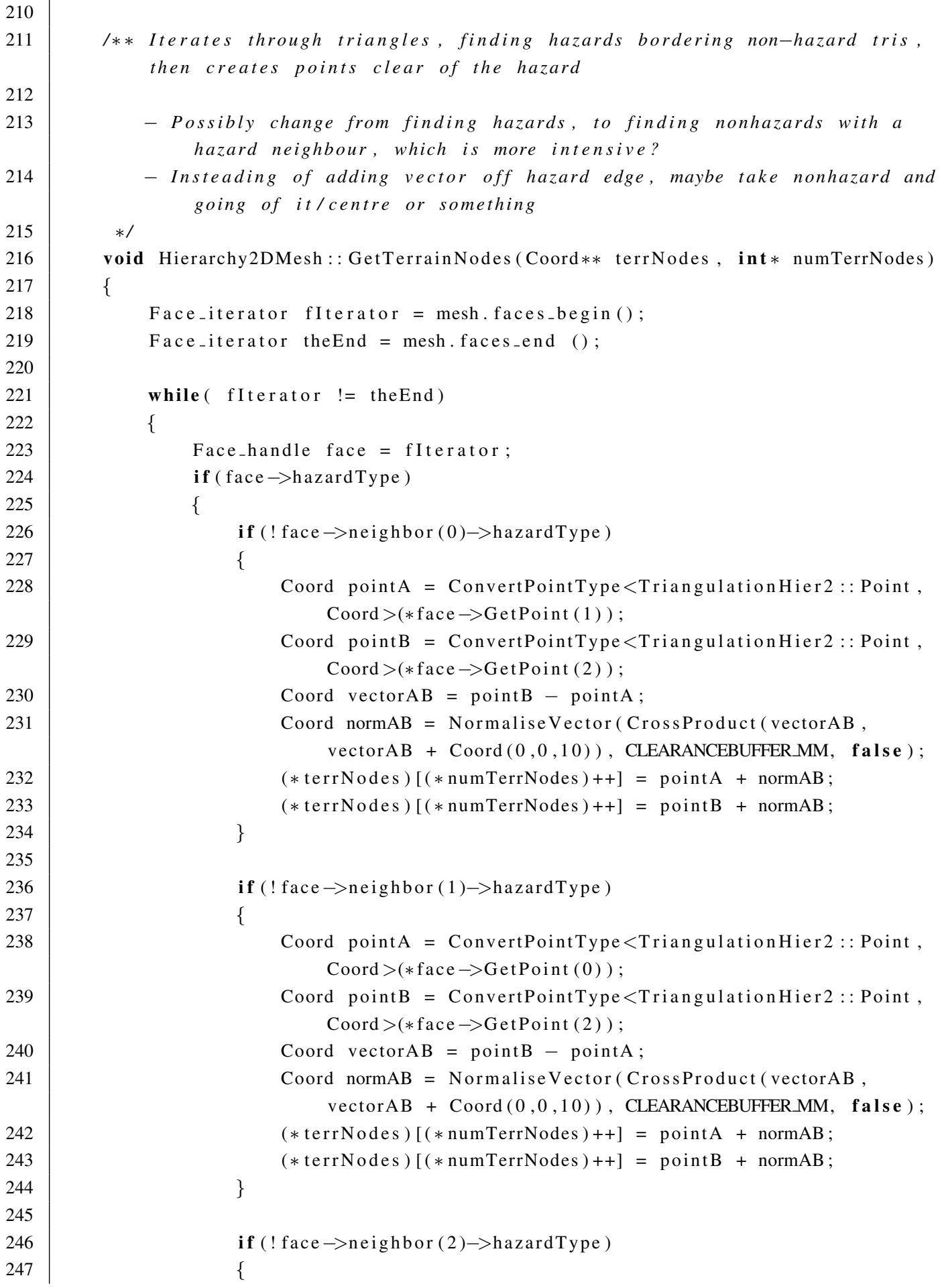

I** Iterates through triangles, finding hazards bordering non-hazard tris, then creates points clear of the hazard

- Possibly change from finding hazards, to finding nonhazards with a hazard neighbour, which is more intensive?

- Insteading of adding vector off hazard edge, maybe take nonhazard and going of it/centre or something 
Coord point $\mathrm{A}=$ ConvertPointType $<$ TriangulationHier $2::$ Point Coord $>(*$ face $\rightarrow$ GetPoint $(0))$;

Coord pointB $=$ ConvertPointType $<$ TriangulationHier $2:$ : Point, Coord $>(*$ face $\rightarrow$ GetPoint $(1))$;

Coord vector $\mathrm{AB}=$ point $\mathrm{B}-$ point $\mathrm{A}$;

Coord normAB $=$ NormaliseVector $($ CrossProduct (vector $A B$, vectorAB + Coord $(0,0,10))$, CLEARANCEBUFFER_MM, false );

$(*$ terrNodes $)[(*$ numTerrNodes $)++]=$ point $\mathrm{A}+$ normAB;

$(*$ terrNodes $)[(*$ numTerrNodes $)++]=$ point $B+$ normAB;

\} \}

if $(*$ numTerrNodes $\%$ ARRAY_CHUNK $==0)$

\{

void $* \operatorname{tmp}=$ realloc $(* \operatorname{terrNodes},(*$ numTerrNodes + ARRAY_CHUNK $) *$

size of (Coord) );

$*$ terrNodes $=(\operatorname{Coord} *)$ tmp;

\}

fIterator ++ ;

\}

return ;

\}

//Maybe inverse result or change name as they seem at odds

/** Returns false if a hazard is encountered. Uses two line_face_circulators (reverse one needed for faces bordering line but lying to the left, refer CGAL manual) to get all faces intersected by a line, then cycles through so only the ones between start and end are checked, then looks whether

they are hazards. Faces which are intersected at vertex are omitted, thus the lines are offset to overlap coverage slightly to ensure all triangles are checked.

272

273

Infinite check is for when reaches end of faces, to avoid looping through again.

is Slope is used to signify if is traversable in one direction. Requires that no other hazard types be encountered. pOffset); 
Line_face_circulator reverseCirclator = mesh. line_walk(end - pOffset, start - pOffset);

Face_handle begin = fCirclator

Face_handle stop = reverseCirclator;

bool result = true;

int fLoops = 1 ;

int $\mathrm{rLoops}=1$;

while(fCirclator != NULL \&\& fCirclator $!=$ stop)

\{

Face_handle face $=$ fCirclator

if (CheckIfInfinite $<$ Face_handle $>$ (face) || InsideTriangleTest (start, * face $\rightarrow$ GetPoint $(0), *$ face $\rightarrow$ GetPoint $(1), *$ face $\rightarrow$ GetPoint (2))) break ;

fCirclator ++ ;

fLoops ++ ;

\}

if $($ fCirclator $==$ stop)

fCirclator $=$ mesh. line - walk $($ start + pOffset, end + pOffset $)$;

while (reverseCirclator $!=$ NULL \&\& reverseCirclator $!=$ begin)

\{

Face_handle face $=$ reverseCirclator;

Point $\mathrm{p} 0=*$ face $\rightarrow$ GetPoint $(0)$;

Point $\mathrm{p} 1=*$ face $\rightarrow$ GetPoint $(1)$;

Point $\mathrm{p} 2=*$ face $\rightarrow$ GetPoint $(2)$;

if (CheckIfInfinite $<$ Face_handle $>$ (face) $\|$ InsideTriangleTest (end, * face $\rightarrow$ GetPoint $(0), *$ face $\rightarrow$ GetPoint (1), *face $\rightarrow$ GetPoint (2)))

break;

reverseCirclator ++ ;

rLoops ++;

\}

if $($ reverseCirclator $==$ begin)

reverseCirclator $=$ mesh. line - walk $($ end - pOffset, start - pOffset $)$;

begin = fCirclator;

stop = reverseCirclator;

if (begin != NULL)

\{

Face_handle face = fCirclator;

while (result $\& \&$ fCirclator $!=$ stop $\& \&$ ! CheckIfInfinite $<$ Face_handle $>$ ( face ) )

for (int $i=0 ; i<$ fLoops; $i++)$

\{

face $=$ fCirclator ++ ;

Point p0 $=*$ face $\rightarrow$ GetPoint $(0)$; 


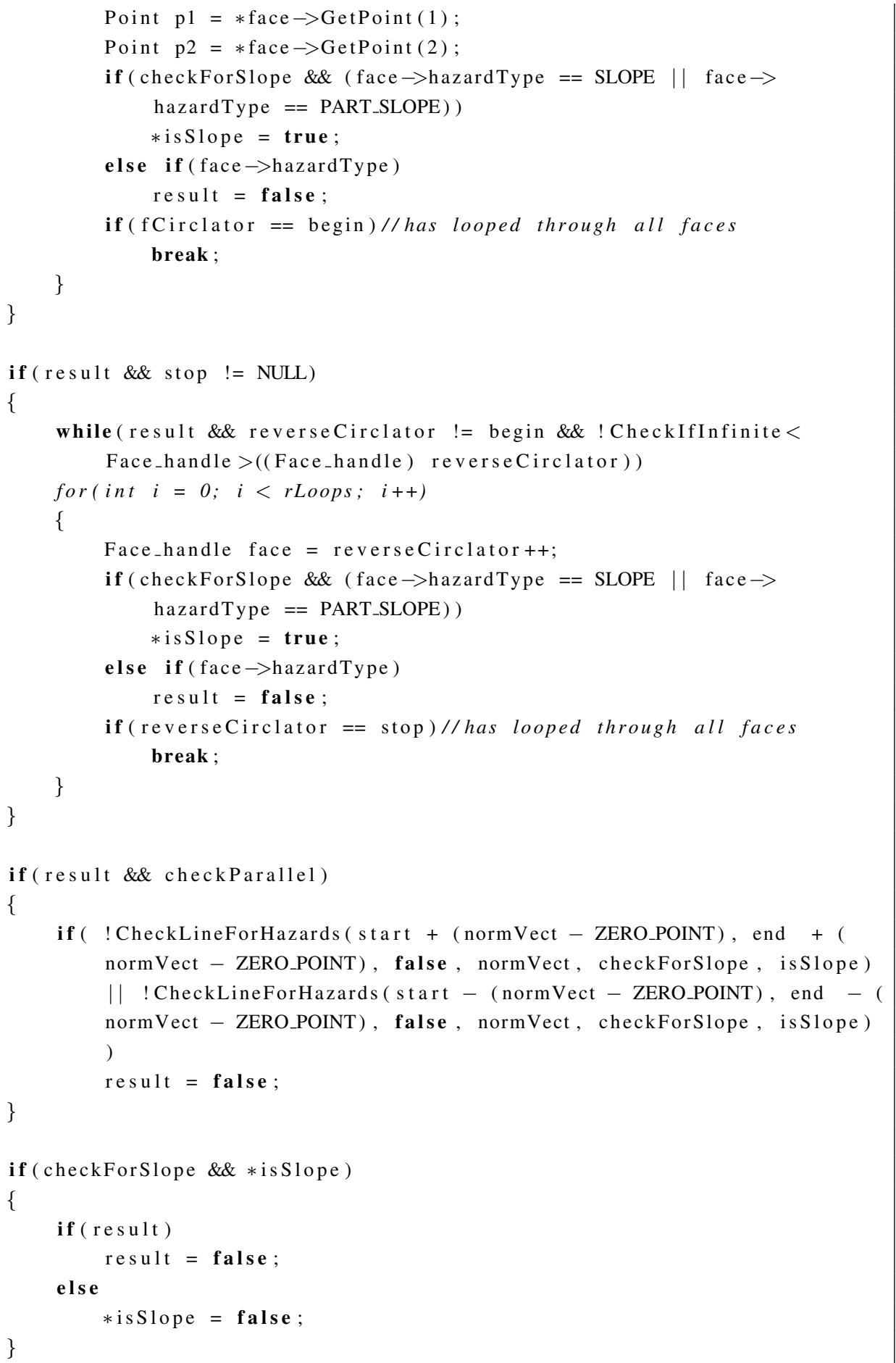




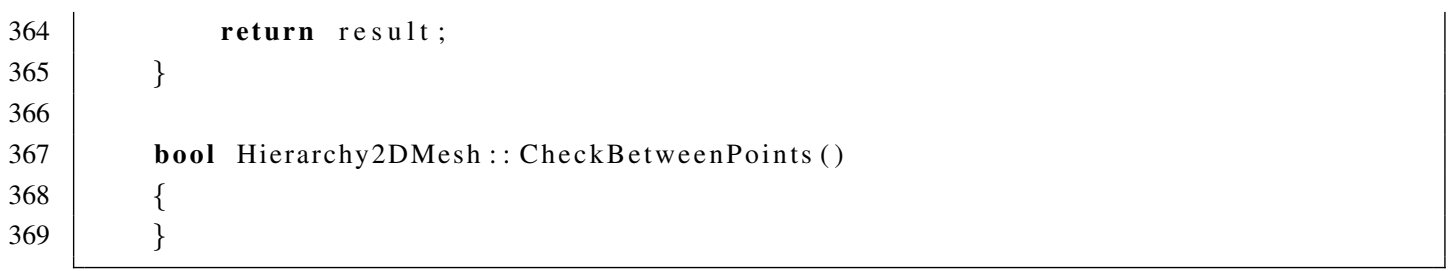

\section{A.5 Nodes}

\section{A.5.1 Nodes.h}

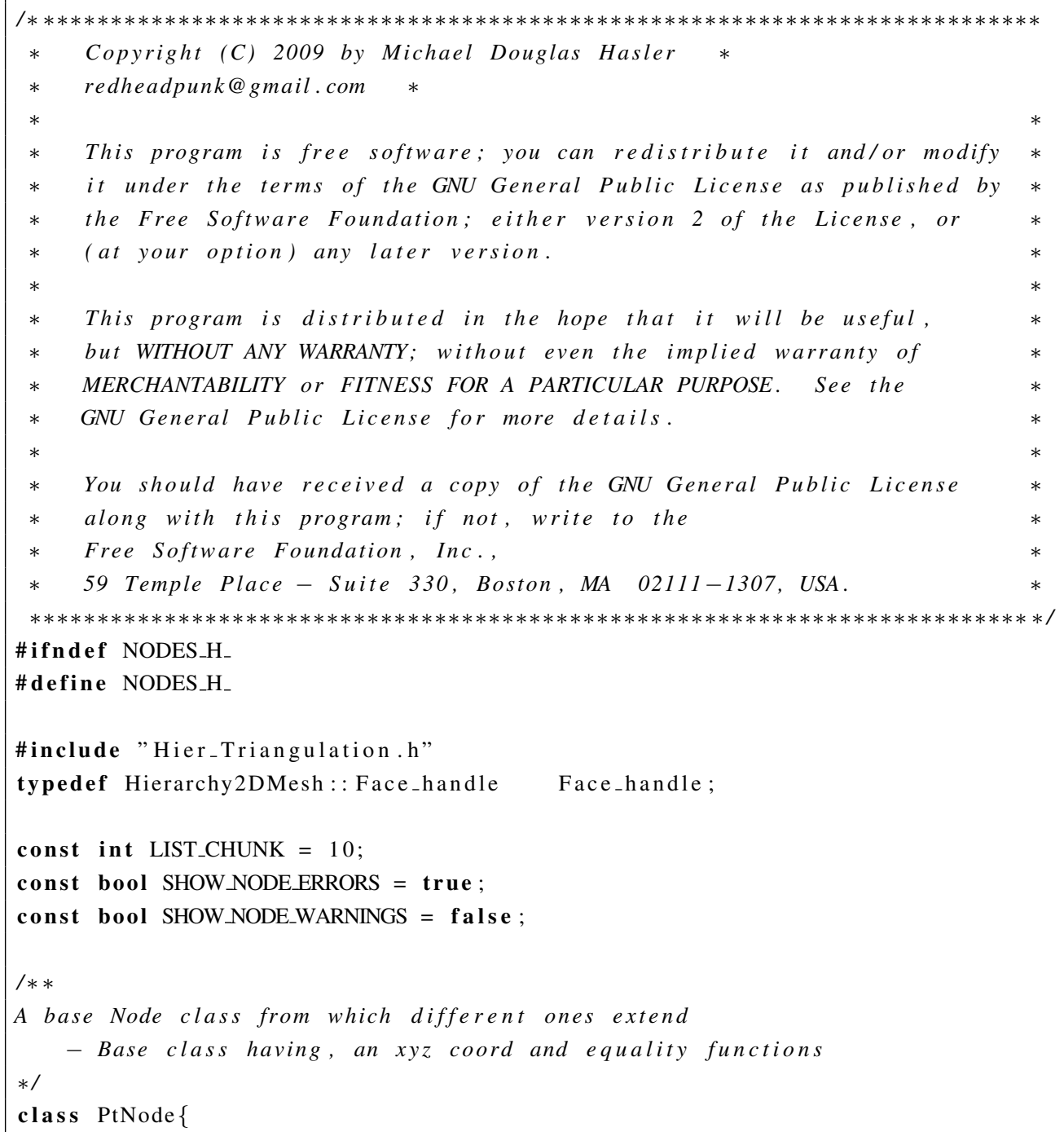




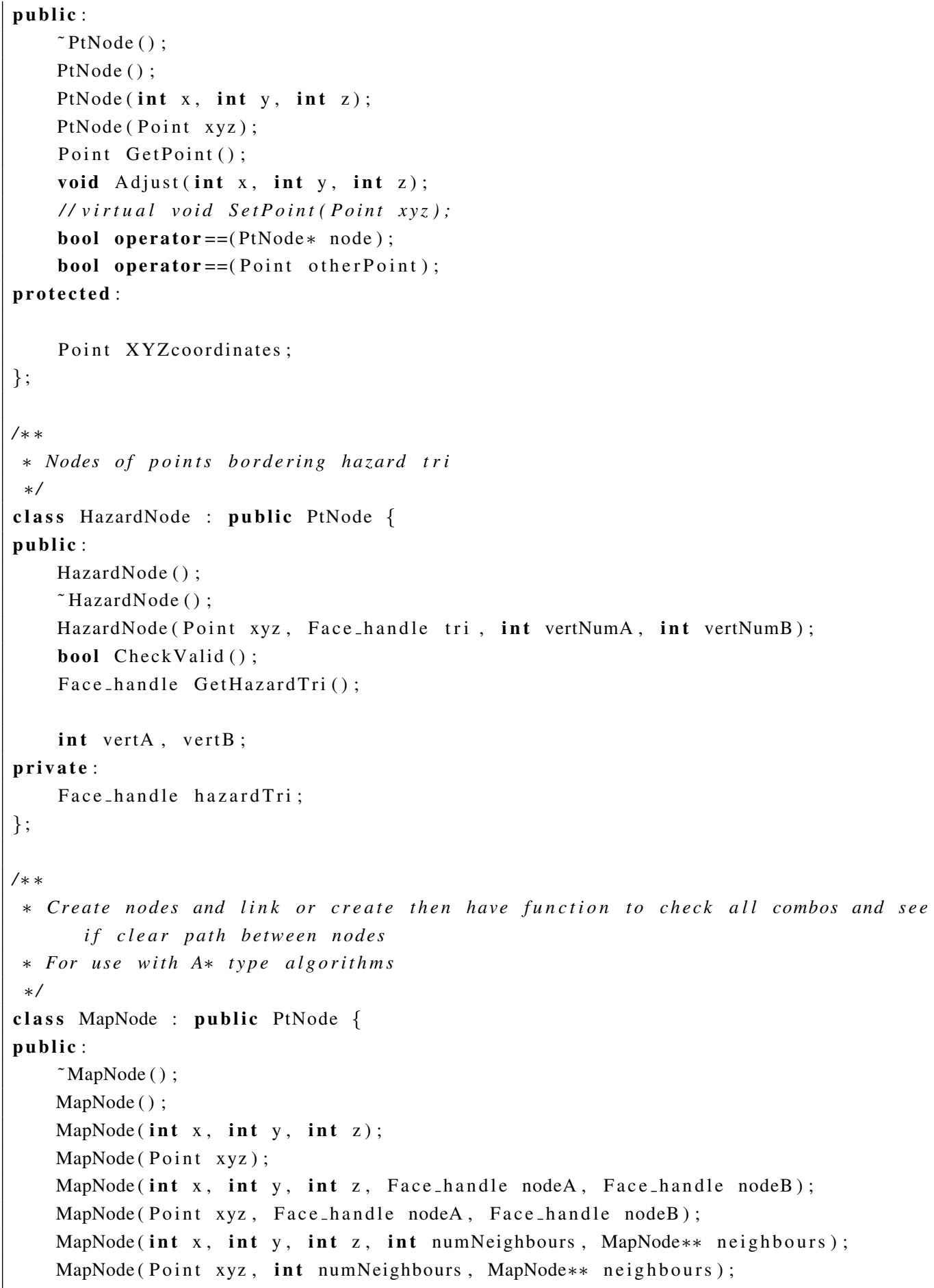




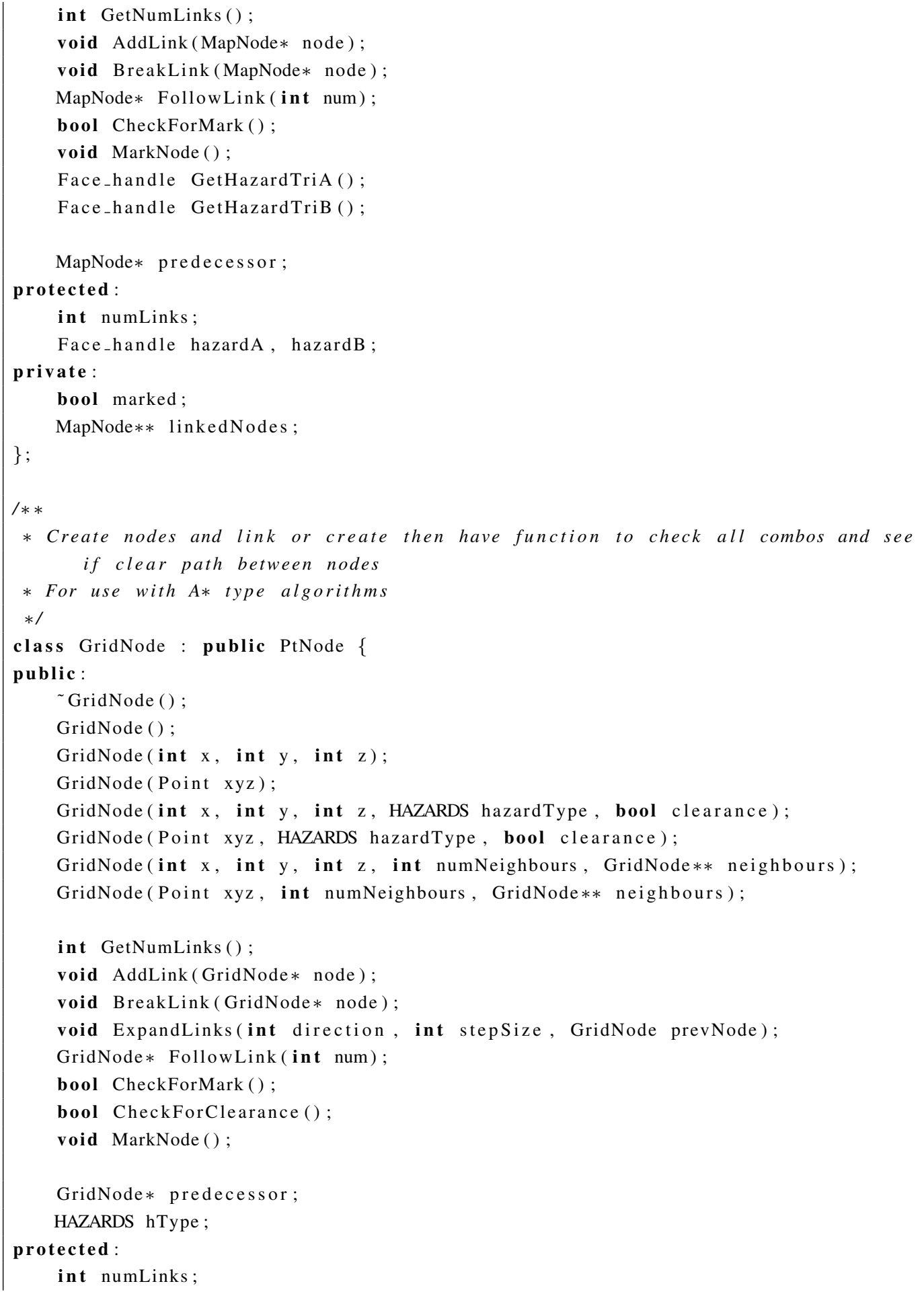


bool marked;

bool clearance;

GridNode $* *$ linkedNodes ;

\}

$/ * *$

* For use with A* type algorithms based on graph of mapNodes formed from hNodes

*/

class ASTAR_MapNode : public MapNode \{

public :

ASTAR_MapNode () ;

ASTAR_MapNode ( ) ;

ASTAR_MapNode( int $\mathrm{x}$, int $\mathrm{y}$, int $\mathrm{z})$;

ASTAR_MapNode ( Point xyz);

ASTAR_MapNode(int $\mathrm{x}$, int $\mathrm{y}$, int $\mathrm{z}$, Face_handle nodeA, Face_handle nodeB);

ASTAR_MapNode(Point xyz, Face_handle nodeA, Face_handle nodeB);

void AddLink (ASTAR_MapNode* node) ;

void BreakLink (ASTAR_MapNode* node) ;

ASTAR_MapNode* FollowLink (int num);

void ResetOpenClosed ();

bool open, closed;

float f_val, g_val, h_val;

ASTAR_MapNode* predecessor ;

ASTAR_MapNode* successor ;

private :

ASTAR_MapNode** linkedNodes ;

\}

$1 * *$

* For use with A* type algorithms based on gridNodes

$* 1$

class ASTAR_GridNode : public GridNode \{

public :

ASTAR_GridNode ();

ASTAR_GridNode () ;

ASTAR_GridNode( int $\mathrm{x}$, int $\mathrm{y}$, int $\mathrm{z}$ );

ASTAR_GridNode (Point xyz);

ASTAR_GridNode(int $\mathrm{x}$, int $\mathrm{y}$, int $\mathrm{z}$, HAZARDS hazardType, bool clearance);

ASTAR_GridNode(Point xyz, HAZARDS hazardType, bool clearance);

void AddLink (ASTAR_GridNode* node);

void BreakLink (ASTAR_GridNode* node); 
void ExpandLinks(int direction, int stepSize, ASTAR_GridNode prevNode); ASTAR_GridNode* FollowLink (int num); void ResetOpenClosed ();

bool open, closed;

float f_val, g_val, h_val;

ASTAR_GridNode $*$ predecessor ;

ASTAR_GridNode $*$ successor

private :

ASTAR_GridNode $* *$ linkedNodes ; \# endif

\section{A.5.2 Nodes.cpp}

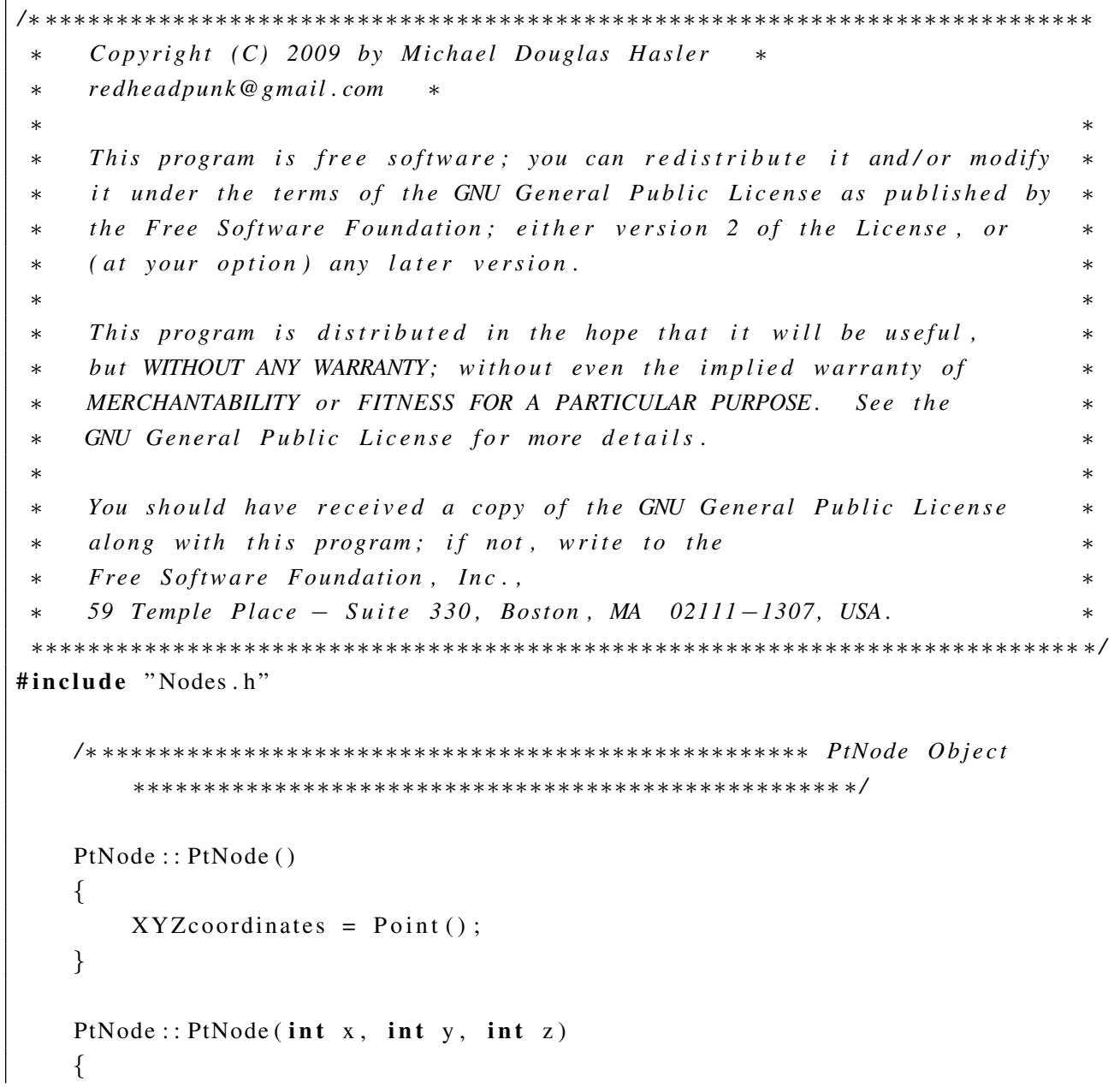




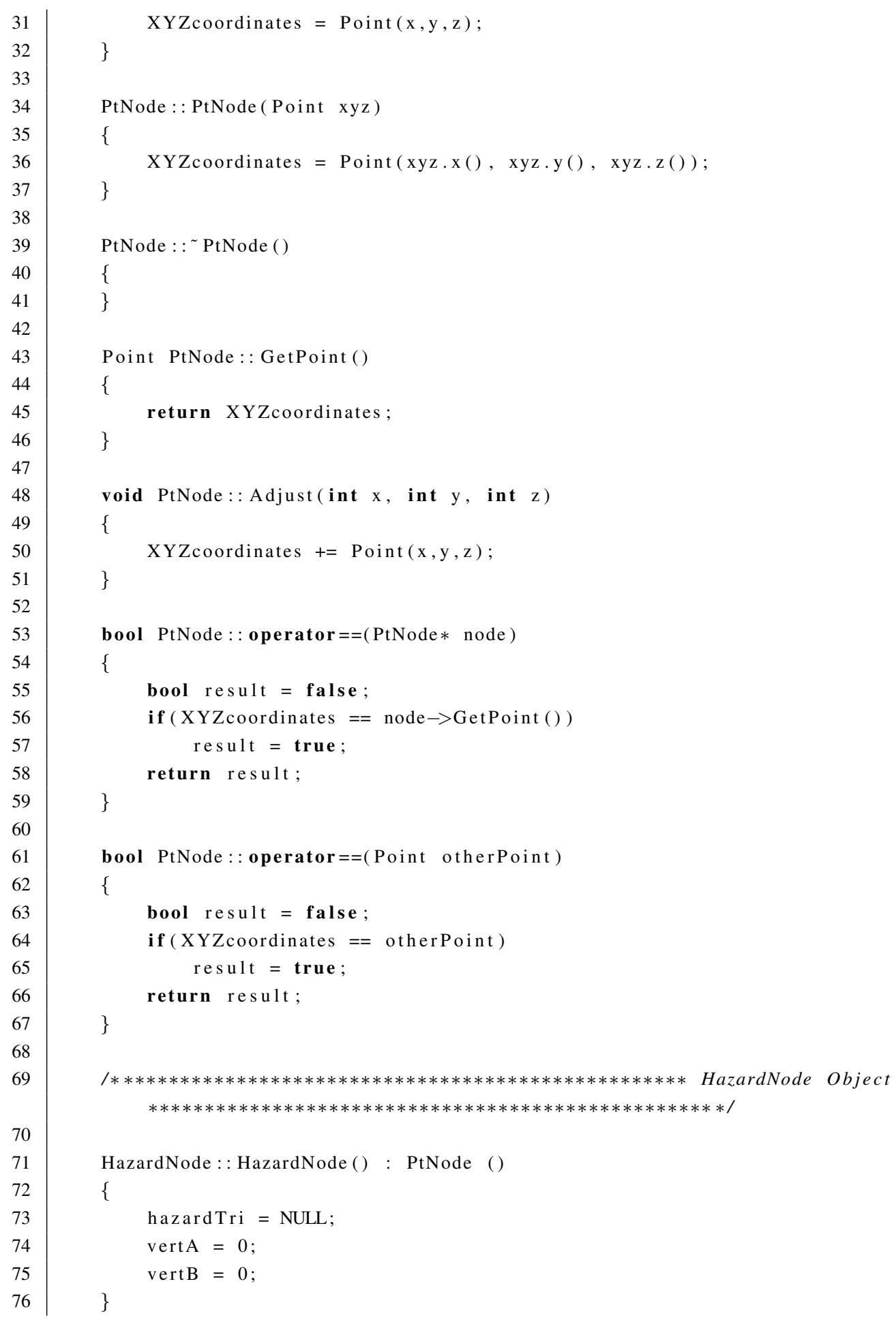




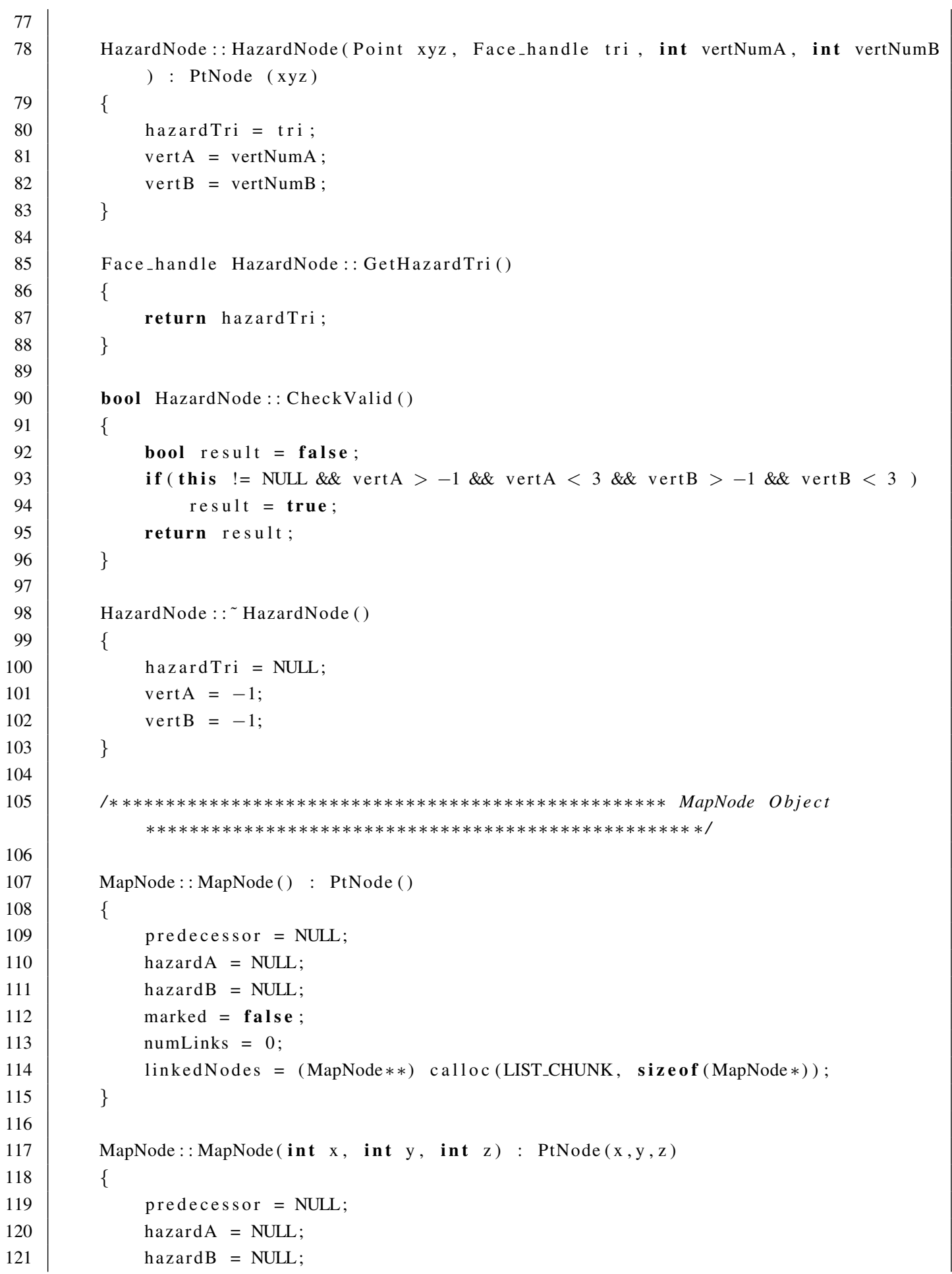




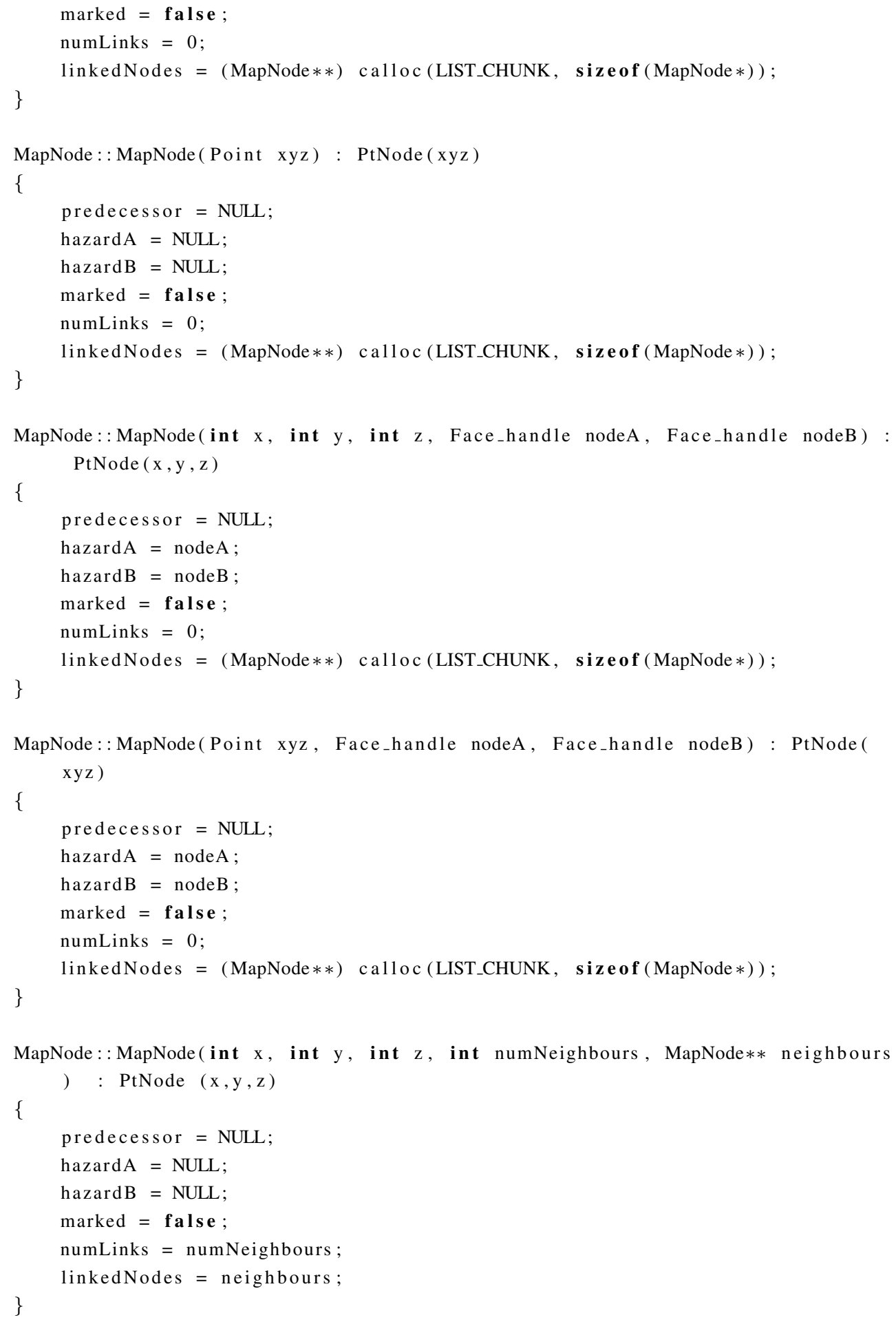




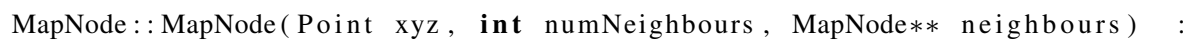




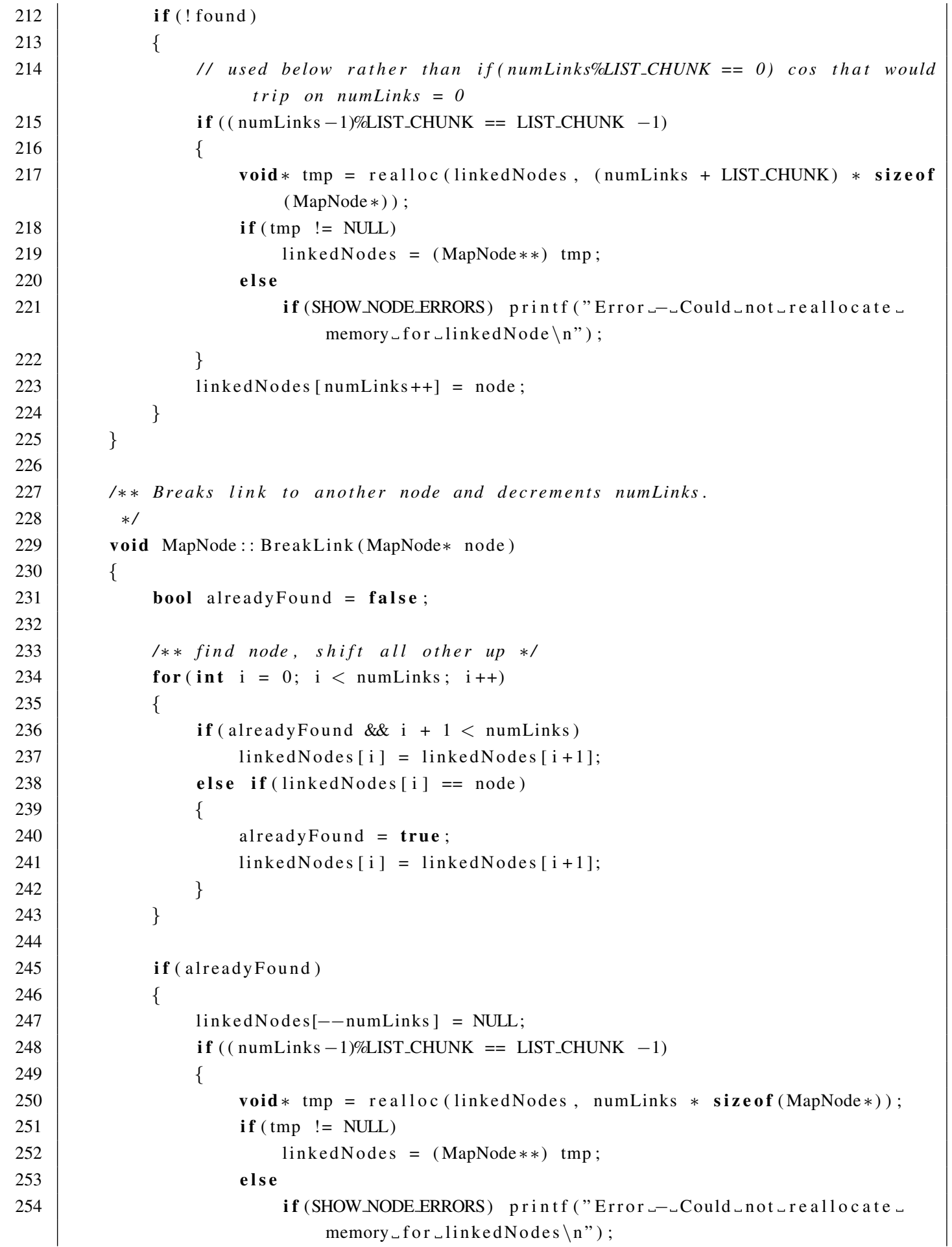




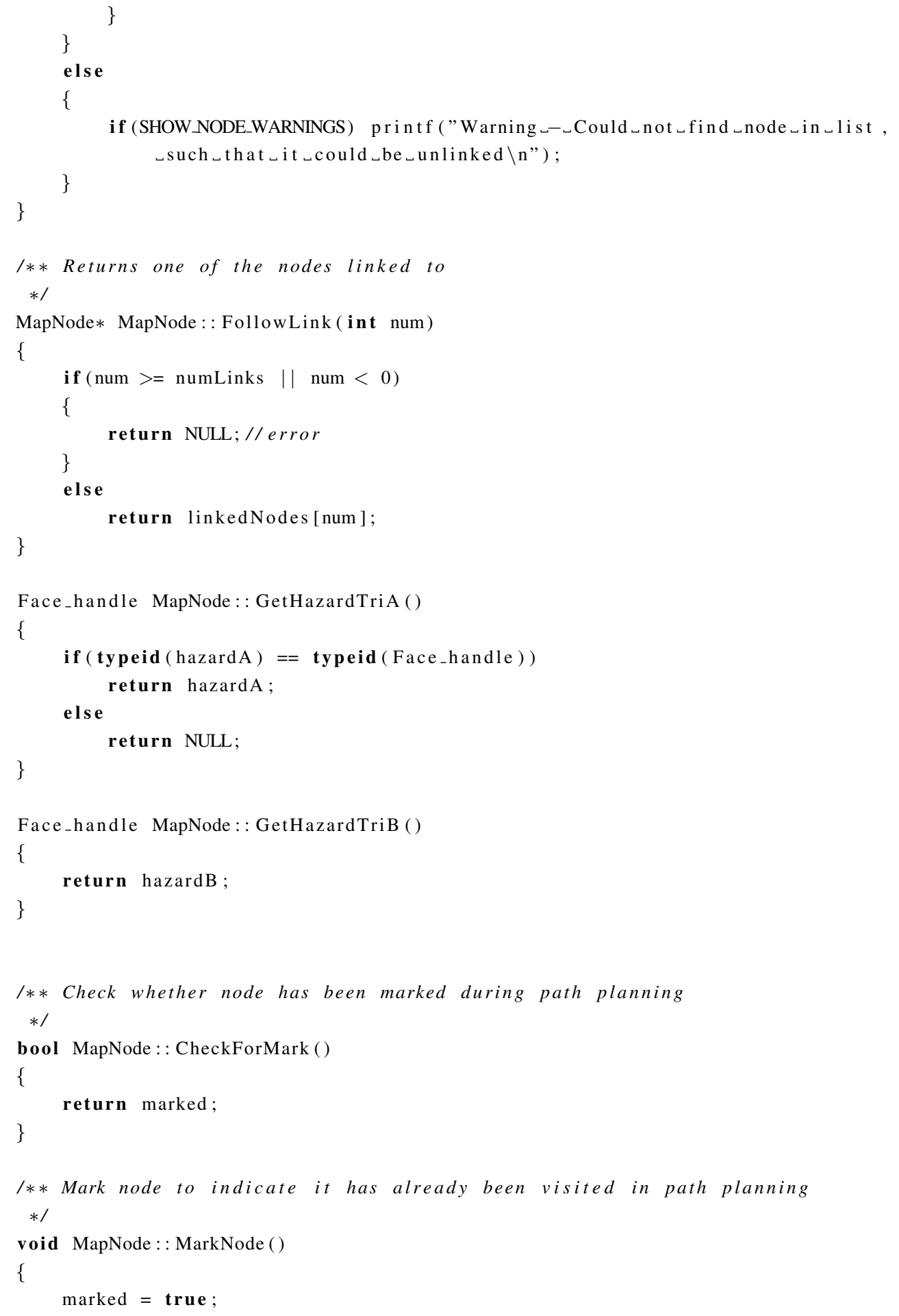




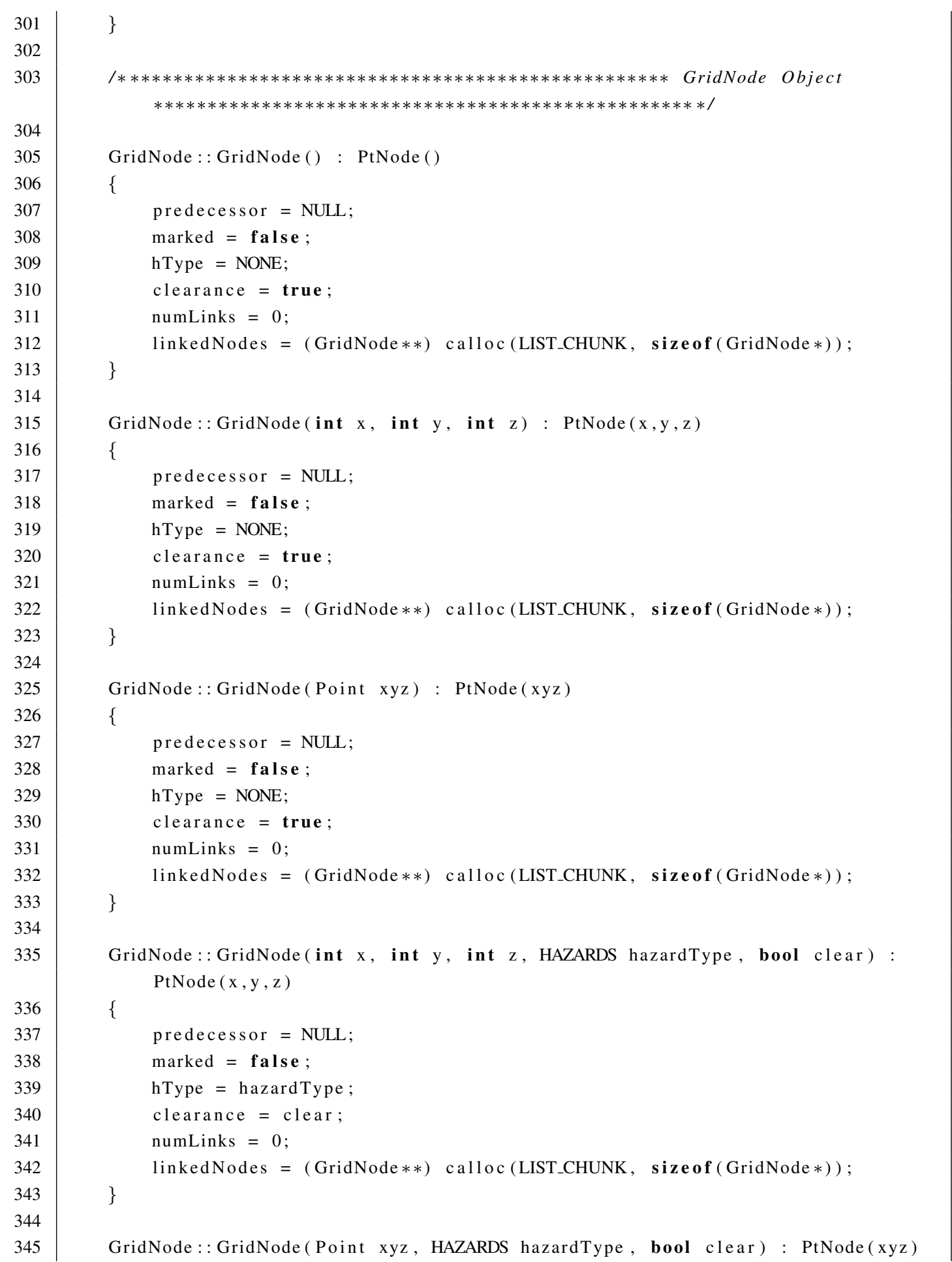




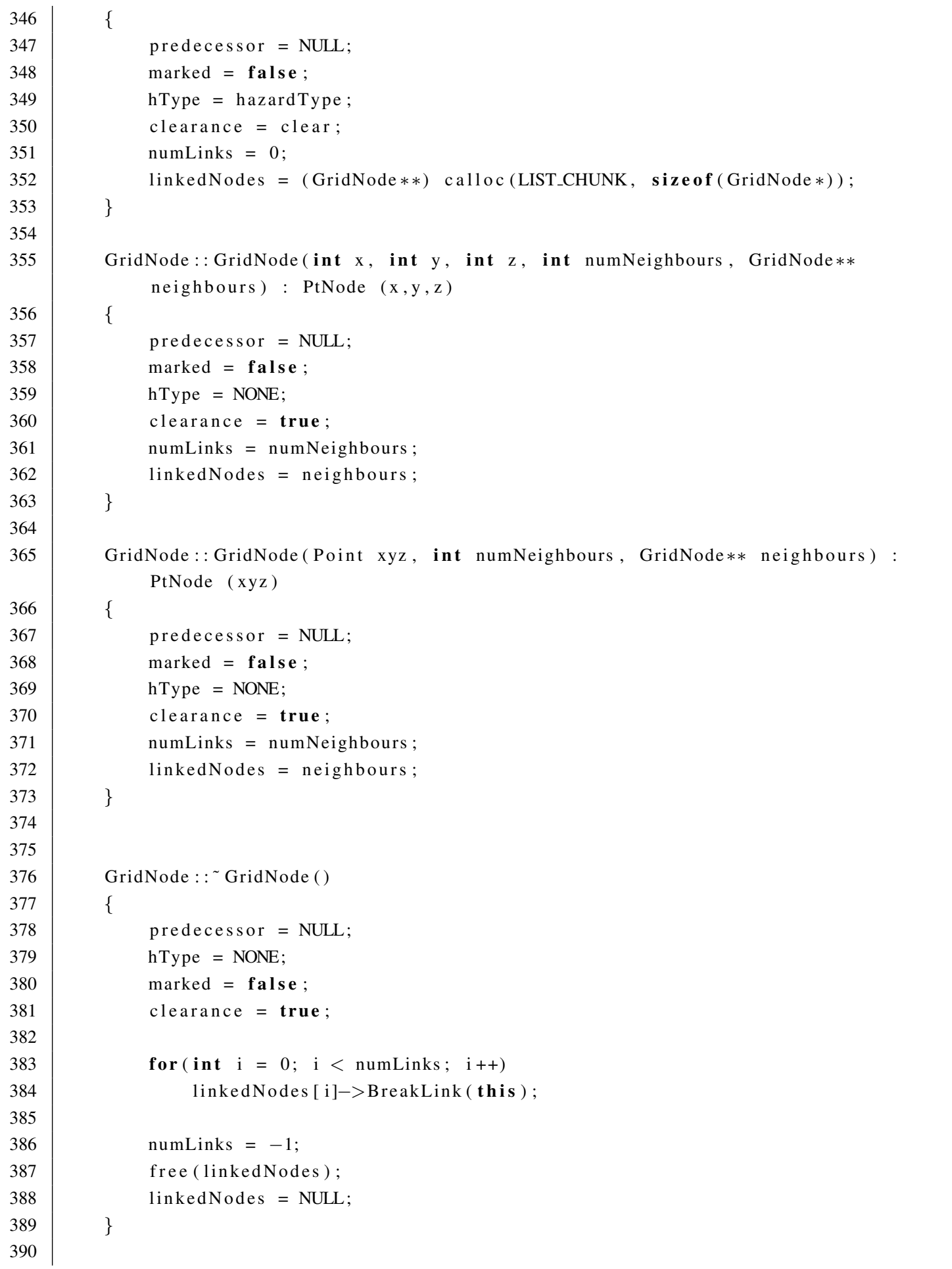




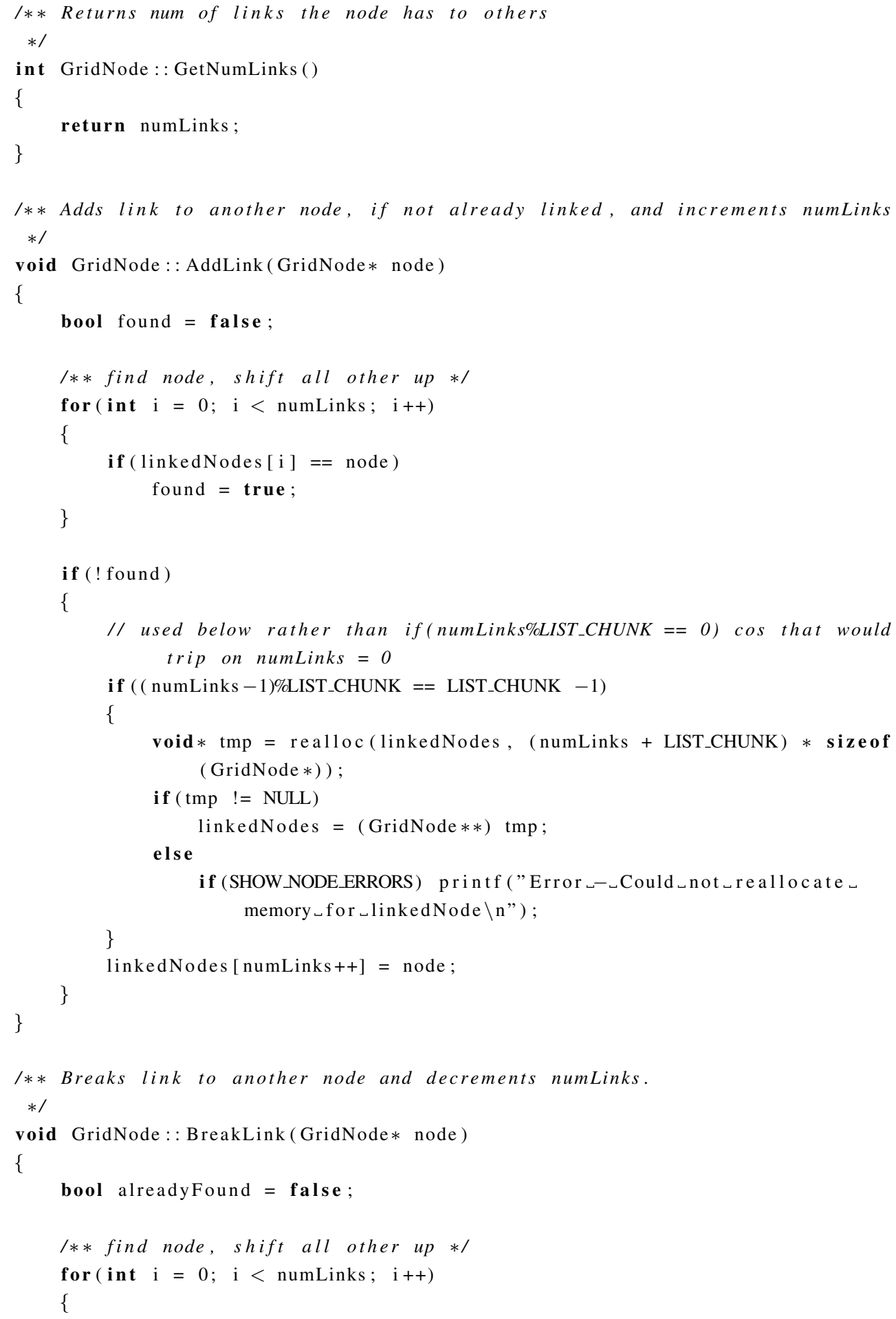




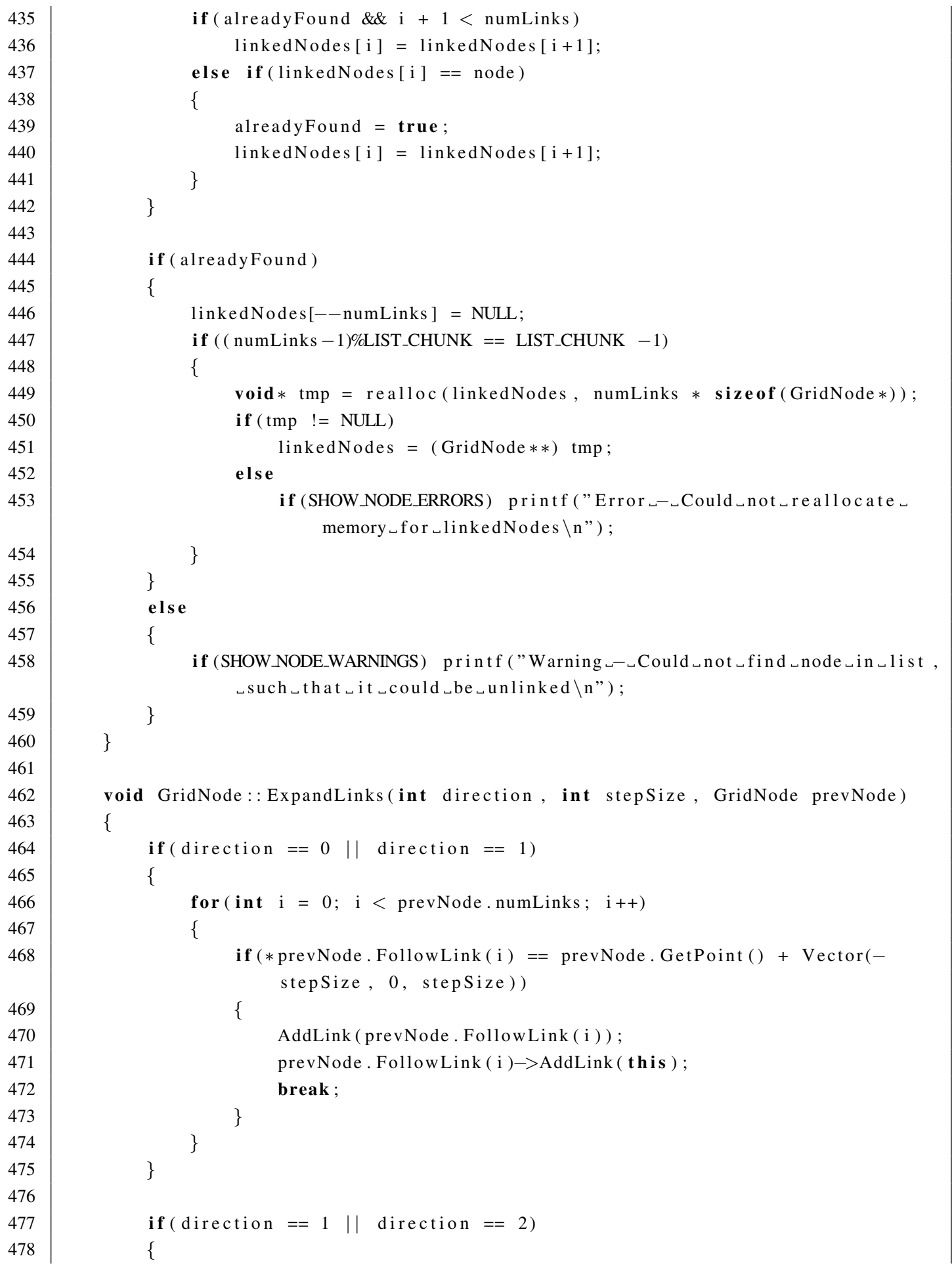




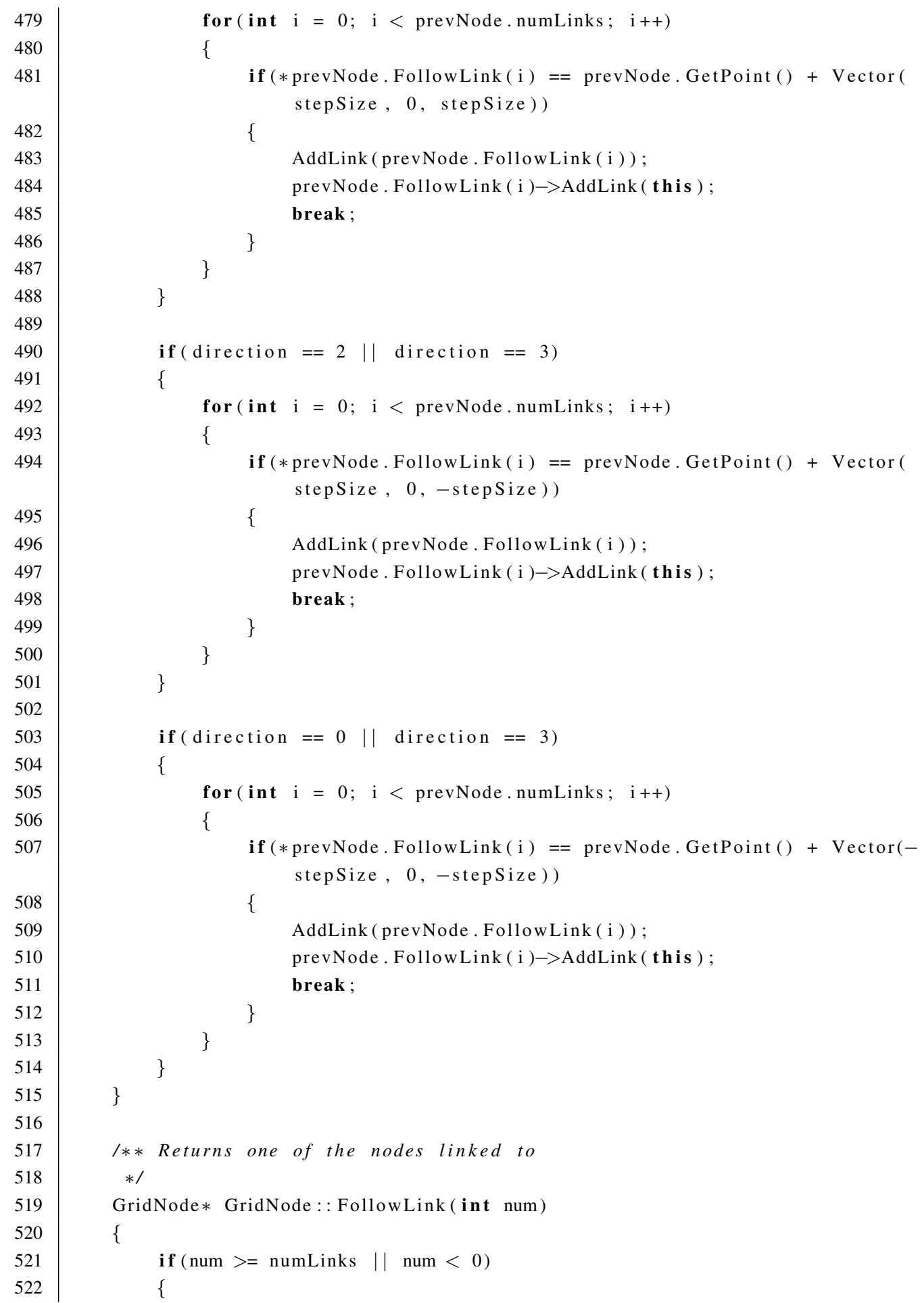




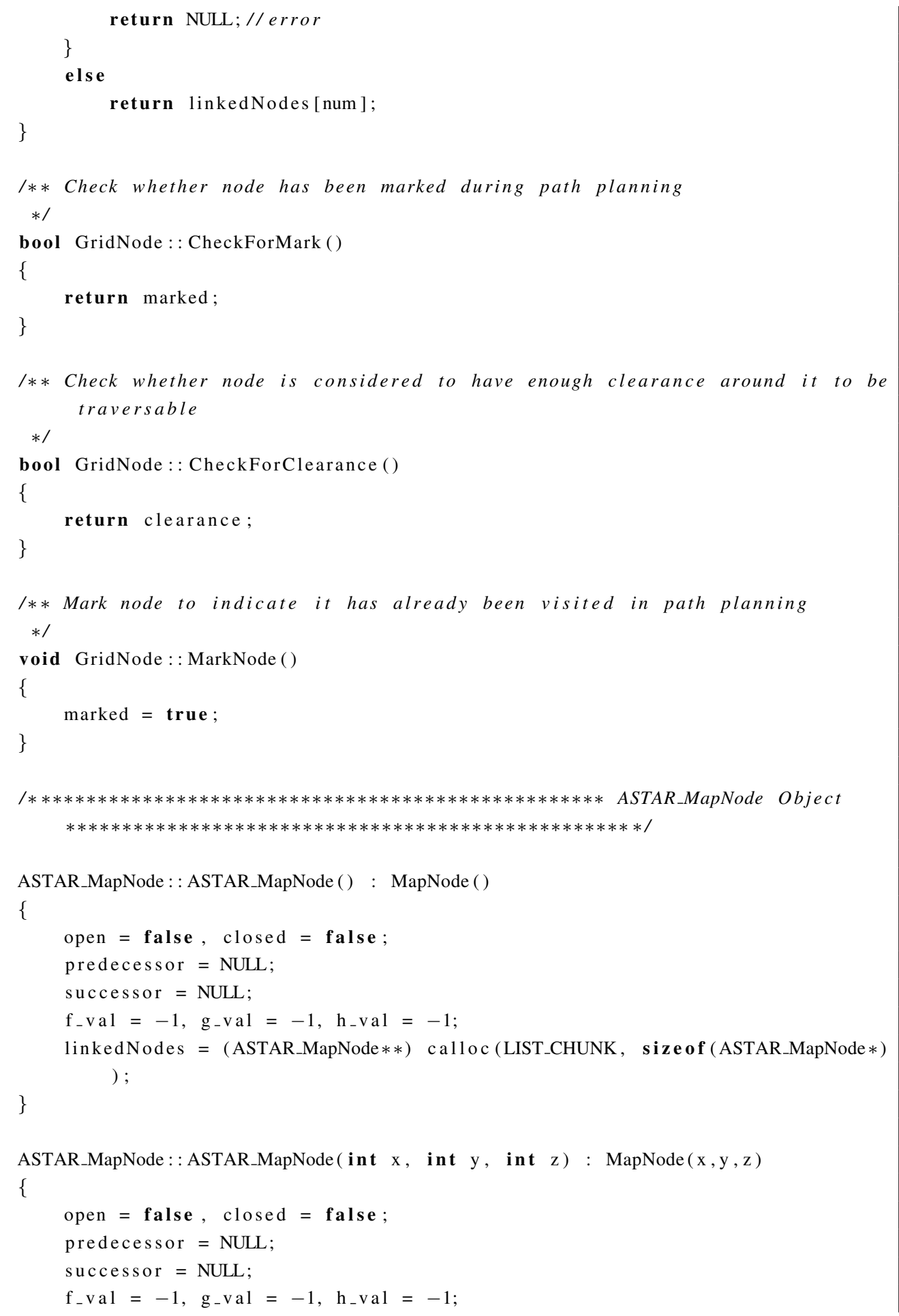




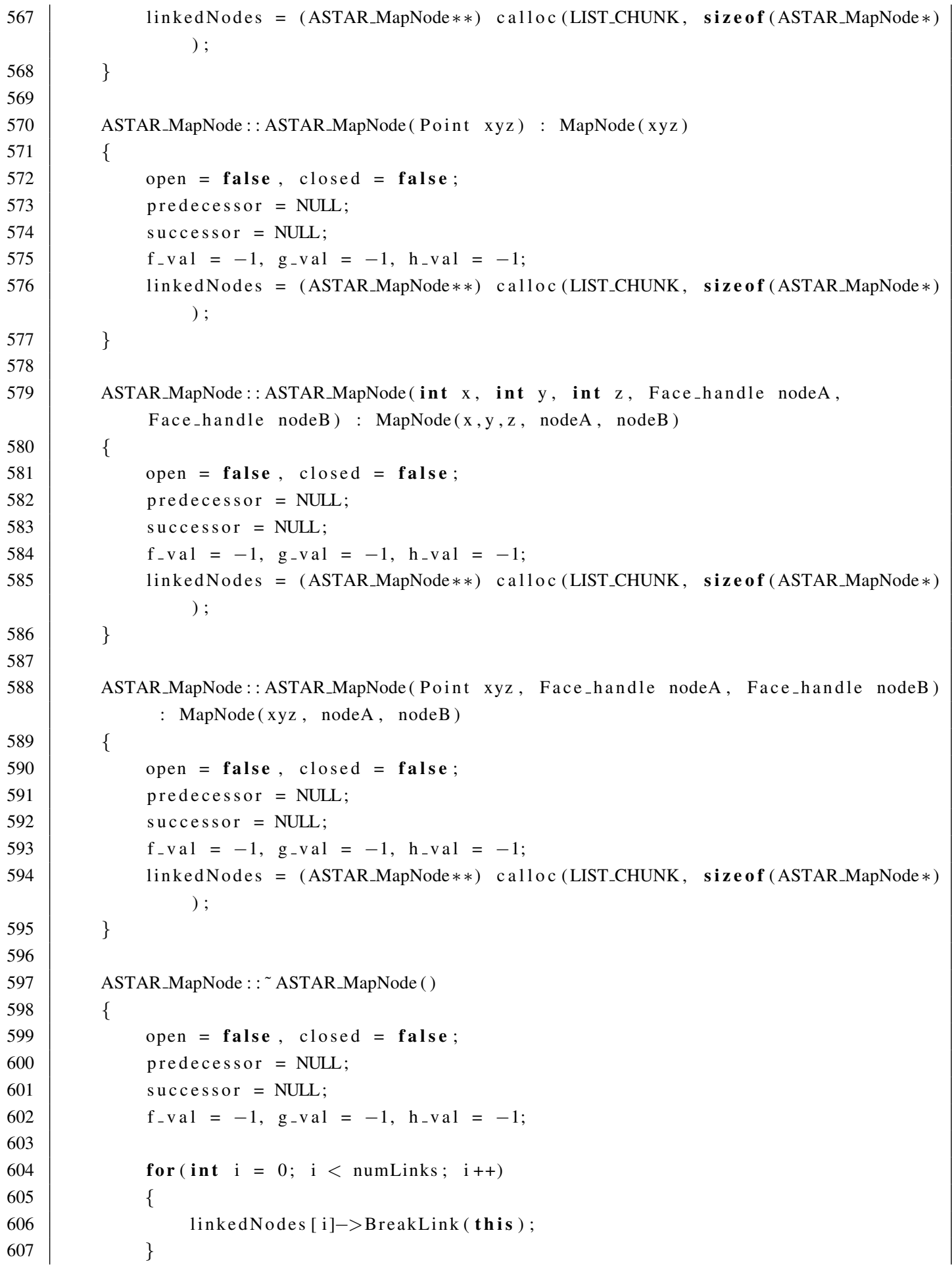




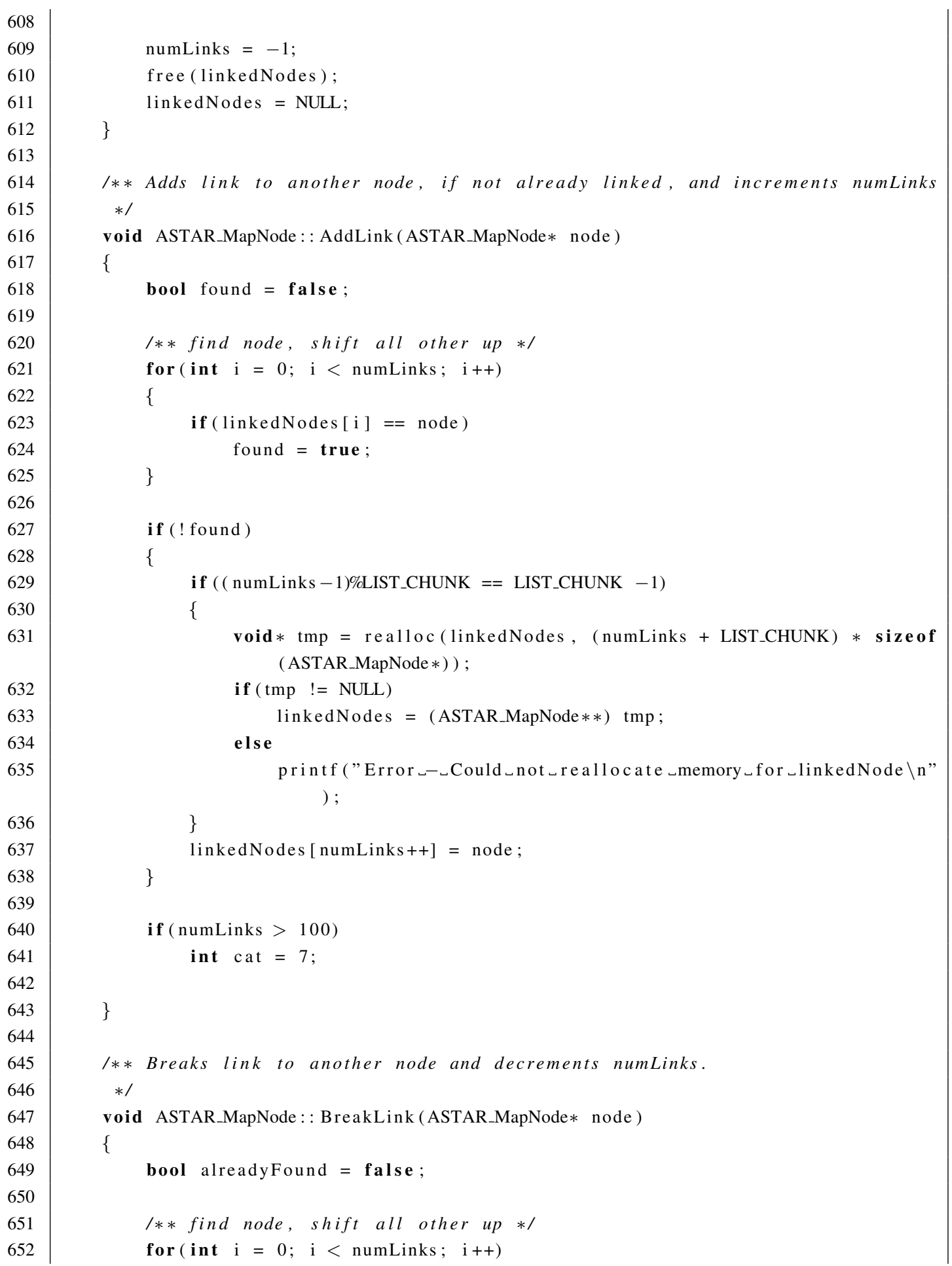


\{

if (alreadyFound $\& \& \mathrm{i}+1<$ numLinks) linkedNodes $[\mathrm{i}]=1$ inkedNodes $[\mathrm{i}+1]$;

else if (linkedNodes [i] = node $)$

\{

alreadyFound = true

if $(\mathrm{i}+1<$ numLinks $)$

linkedNodes $[\mathrm{i}]=1$ inkedNodes $[\mathrm{i}+1]$;

\}

\}

if (alreadyFound)

\{

linkedNodes[--numLinks ] = NULL;

if $(($ numLinks -1$) \%$ LIST_CHUNK == LIST_CHUNK -1$)$

\{

$\operatorname{void} * \operatorname{tmp}=$ realloc $($ linkedNodes, numLinks $*$ sizeof (ASTAR_MapNode *)) ;

if ( tmp $\quad !=$ NULL)

linkedNodes $=($ ASTAR_MapNode $* *)$ tmp ;

else

if (SHOW_NODE_ERRORS) printf ("Error ${ }_{-}{ }_{-}$Could $\_$not $\_$reallo c a te $\_$ memory for $_{\llcorner}$linkedNodes $\backslash \mathrm{n}$ ”);

\}

if $($ predecessor $==$ node $)$

\{

predecessor = NULL;

if (open)

int $\mathrm{cat}=7$

\}

if $($ successor $==$ node $)$

\{

successor = NULL;

if ( closed)

int $\mathrm{cat}=7$

\}

\}

else

\{

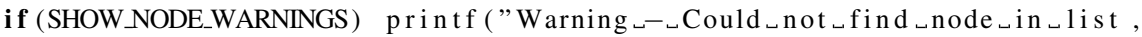

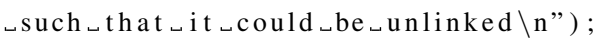

\}

/** Returns one of the nodes linked to

$* /$

ASTAR_MapNode* ASTAR_MapNode : :FollowLink (int num) 


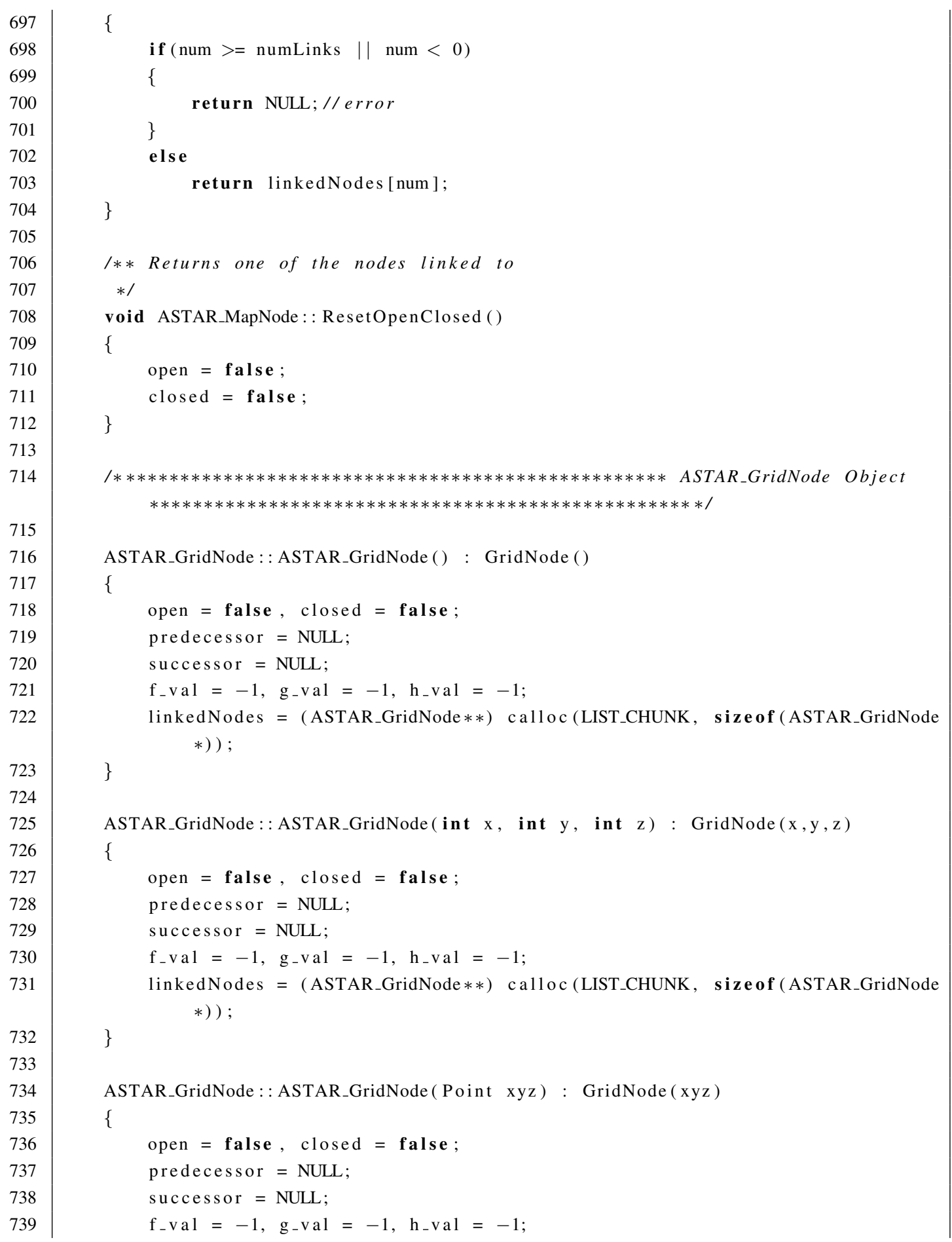




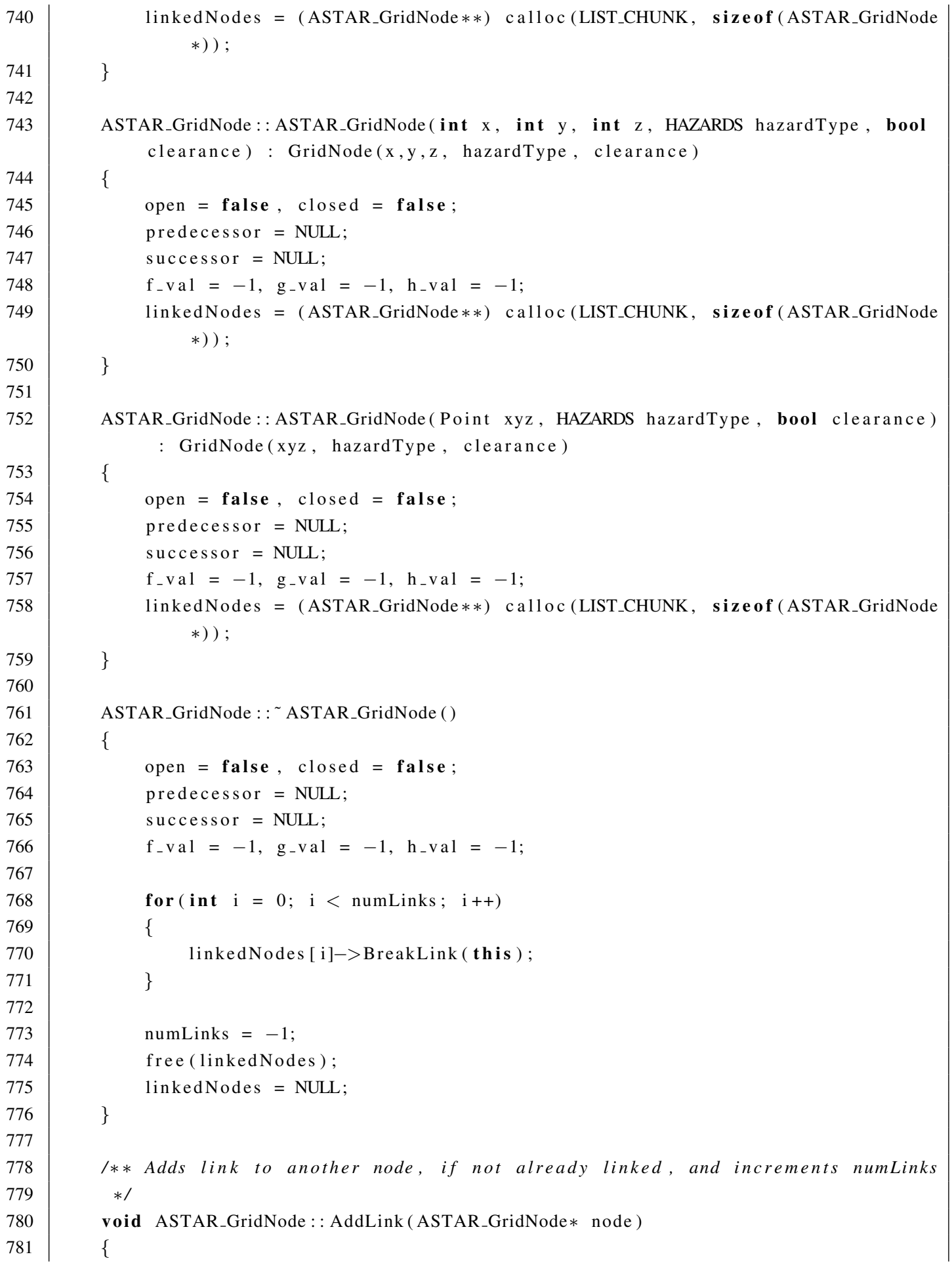




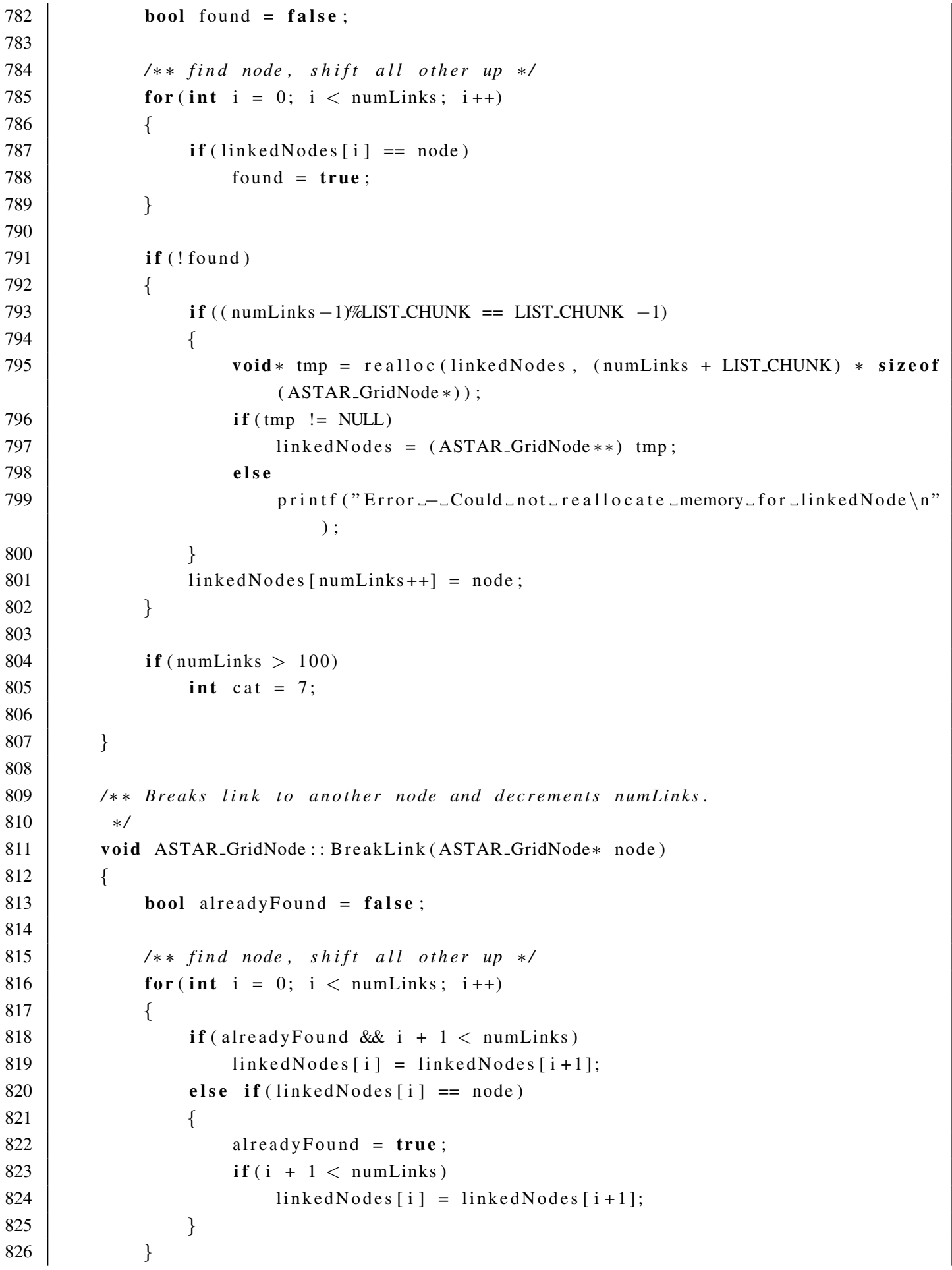




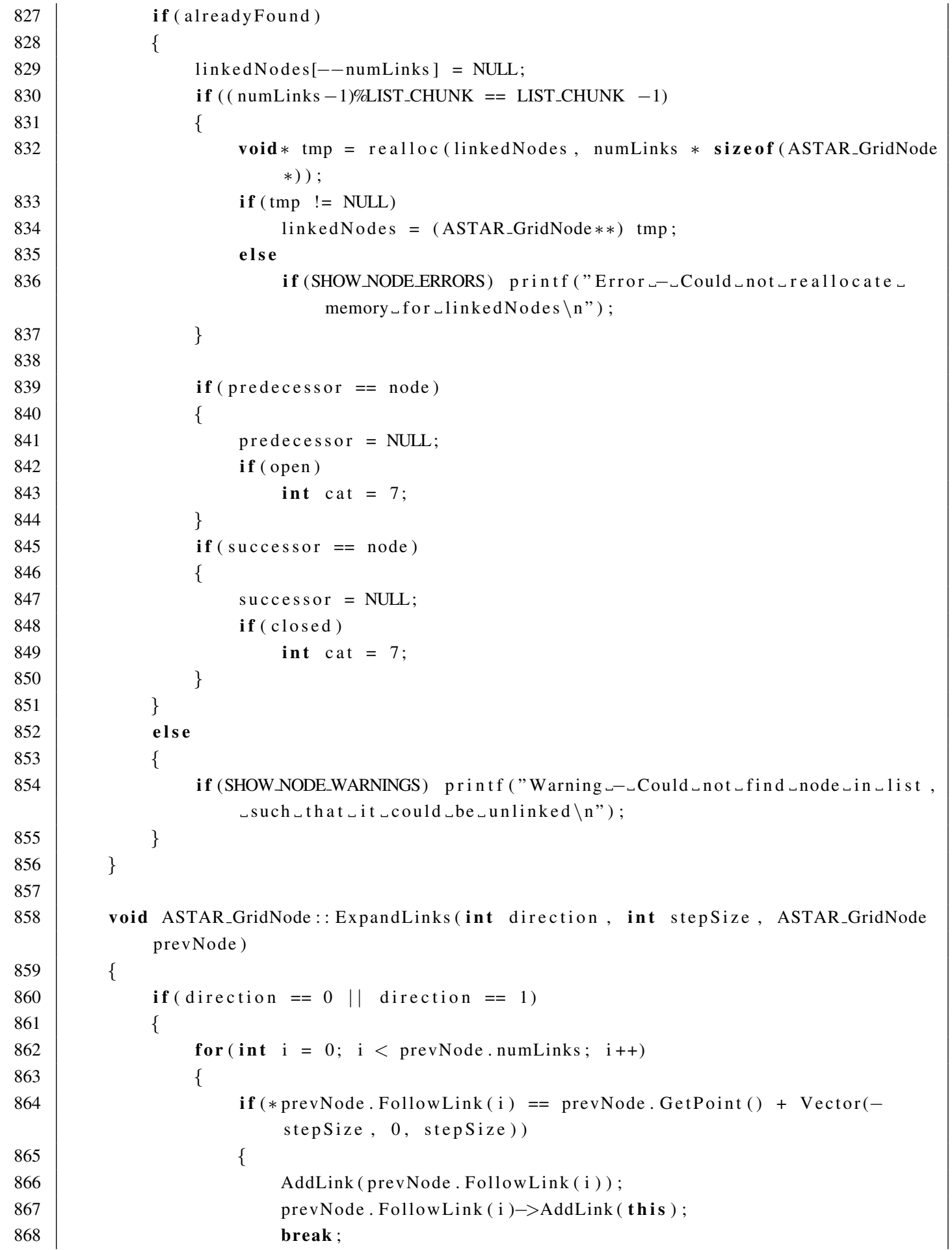




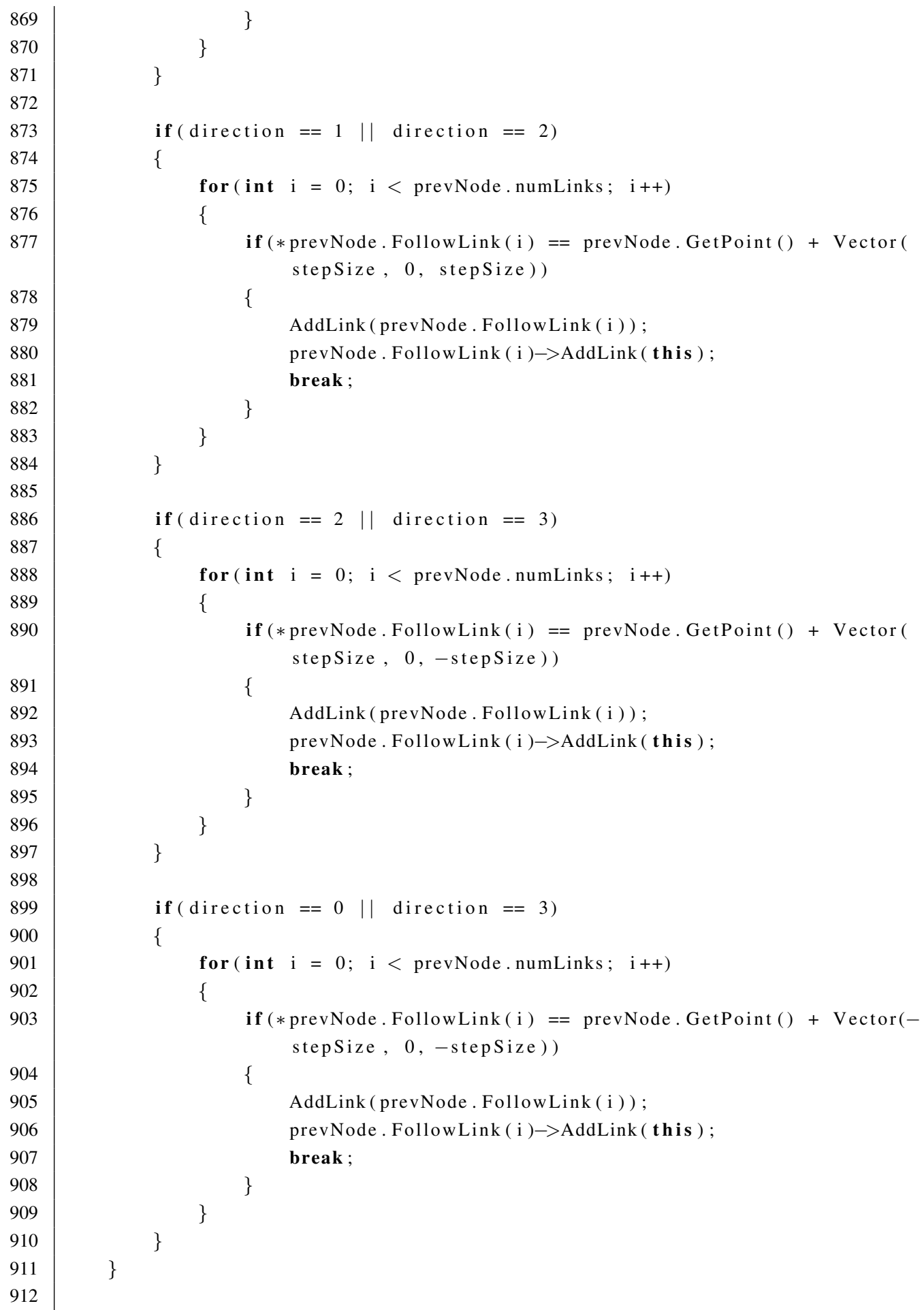




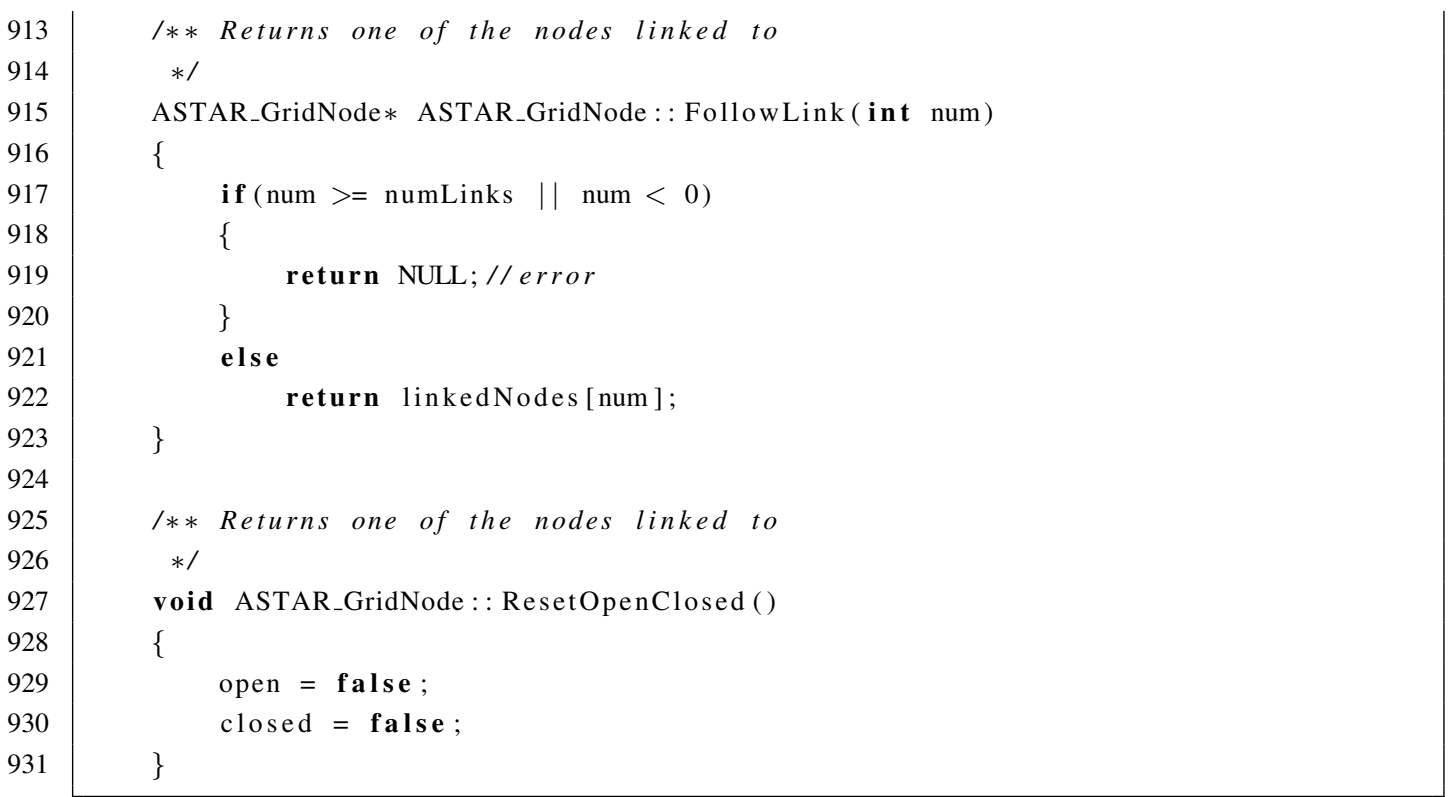

\section{A.6 Robot}

\section{A.6.1 Robot.h}

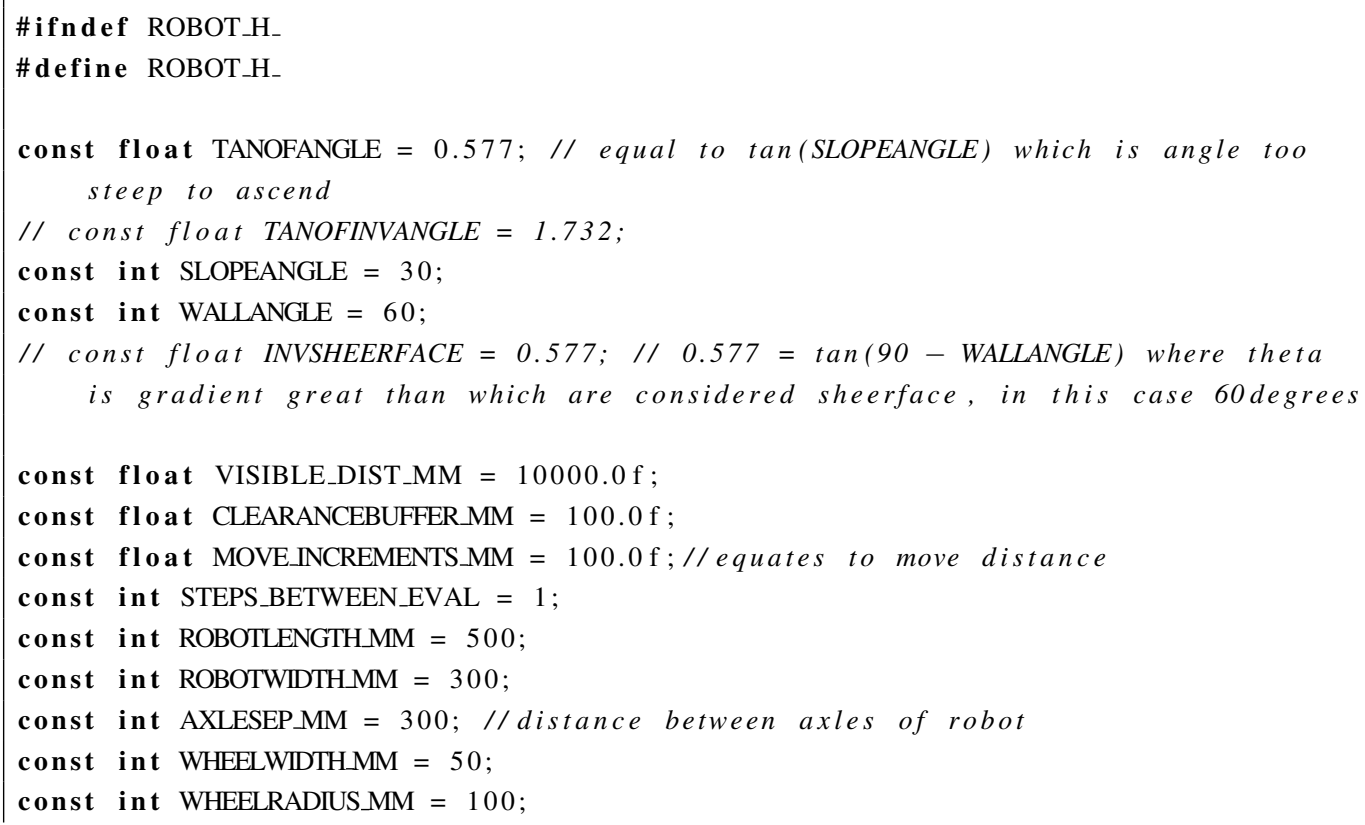


const int CAMERA_HEIGHT = WHEELRADIUS_MM;// if designed to run either way up. possibly higher if not designed to handle flipping. scan would have to be adjusted for that

const bool $\mathrm{KNOWN}=$ false

const bool KNOWNNODES = false;

class Robot

\#include $<$ time. $\mathrm{h}>$

\#include "Nodes. h"

\#include "World. h"

\#include "SimulationEnvironment.h"

enum ALG \{NO_ALG, HUGGER, STATE, EIGHT, DFS, BFS , GREEDY, ASTAR, LPASTAR, DSTAR ;

const ALG algorithm = LPASTAR;

// \# define ALG_NODE_TYPE MapNode

// \#define ALG_NODE_TYPE GridNode

\#define ALG_NODE_TYPE ASTAR_MapNode

// \#define ALG_NODE_TYPE ASTAR_GridNode

class Robot \{

public :

virtual Robot ( ) ;

/**

* gpsCoords are coords the world should start relative to

$* /$

Robot(Coord gpsCoords, Coord location, Coord destination);

Robot(Coord gpsCoords, Coord location, Coord destination,

SimulationEnvironment $*$ simEnviron );

void GatherData ();

void GatherData(Coord* scannedData, int numScanPoints);

void GatherData (SimulationEnvironment* simEnv, int inputQualityLevel);

template < class NODE> void GetMapNodes(NODE** currLoc, NODE** goal);

// template <class NODE> void GetGridNodes(NODE** currLoc, NODE** goal);

void PlanPath ();

template <class TRI, class PT> void EdgeHugger();

Coord CheckEdgePath (Coord start, Coord $*$ testPos, int numPoints, Coord* past TestPoints);

bool Halt ();

bool NaviDone ();

Coord MoveDirection(); 


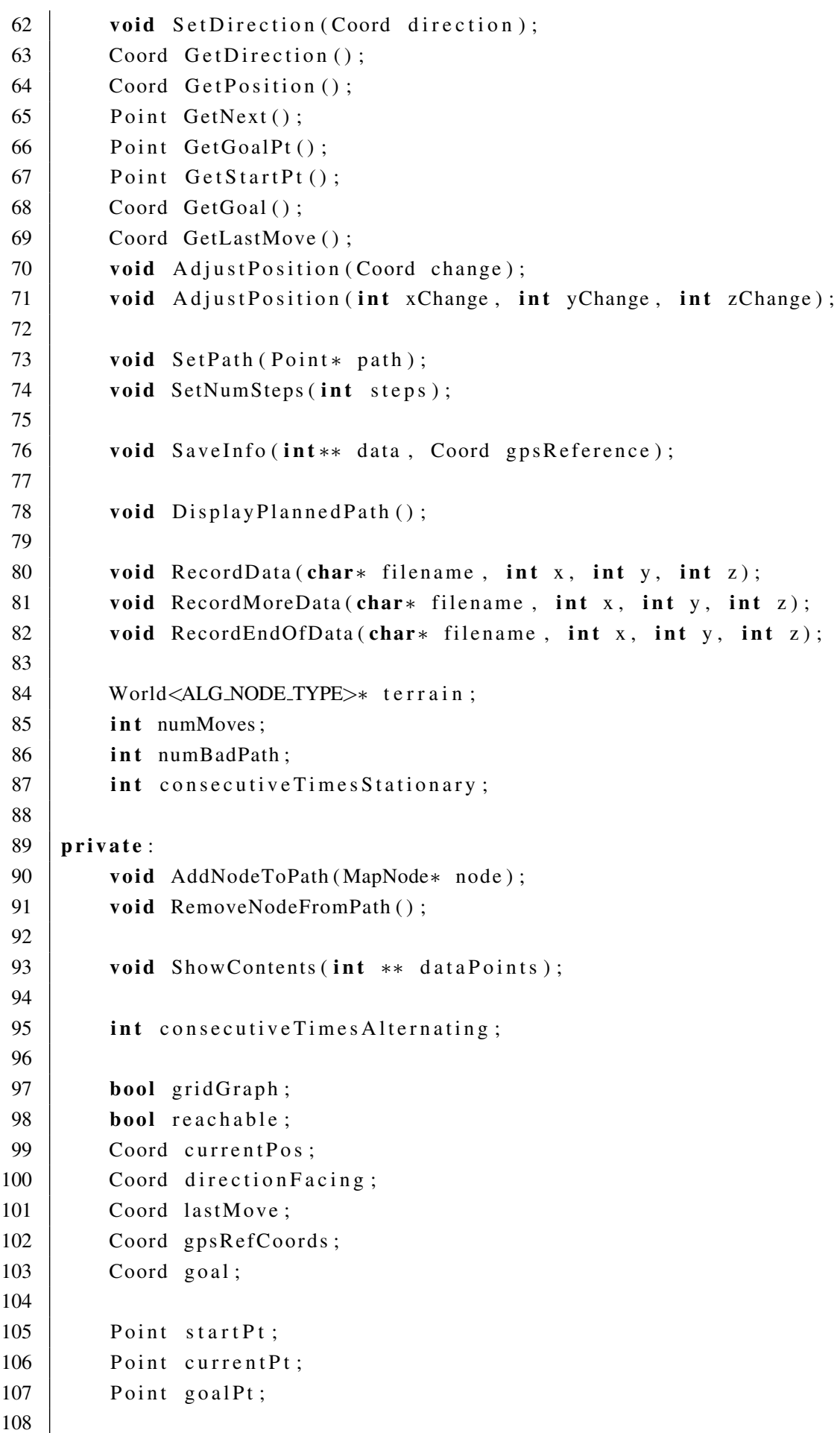




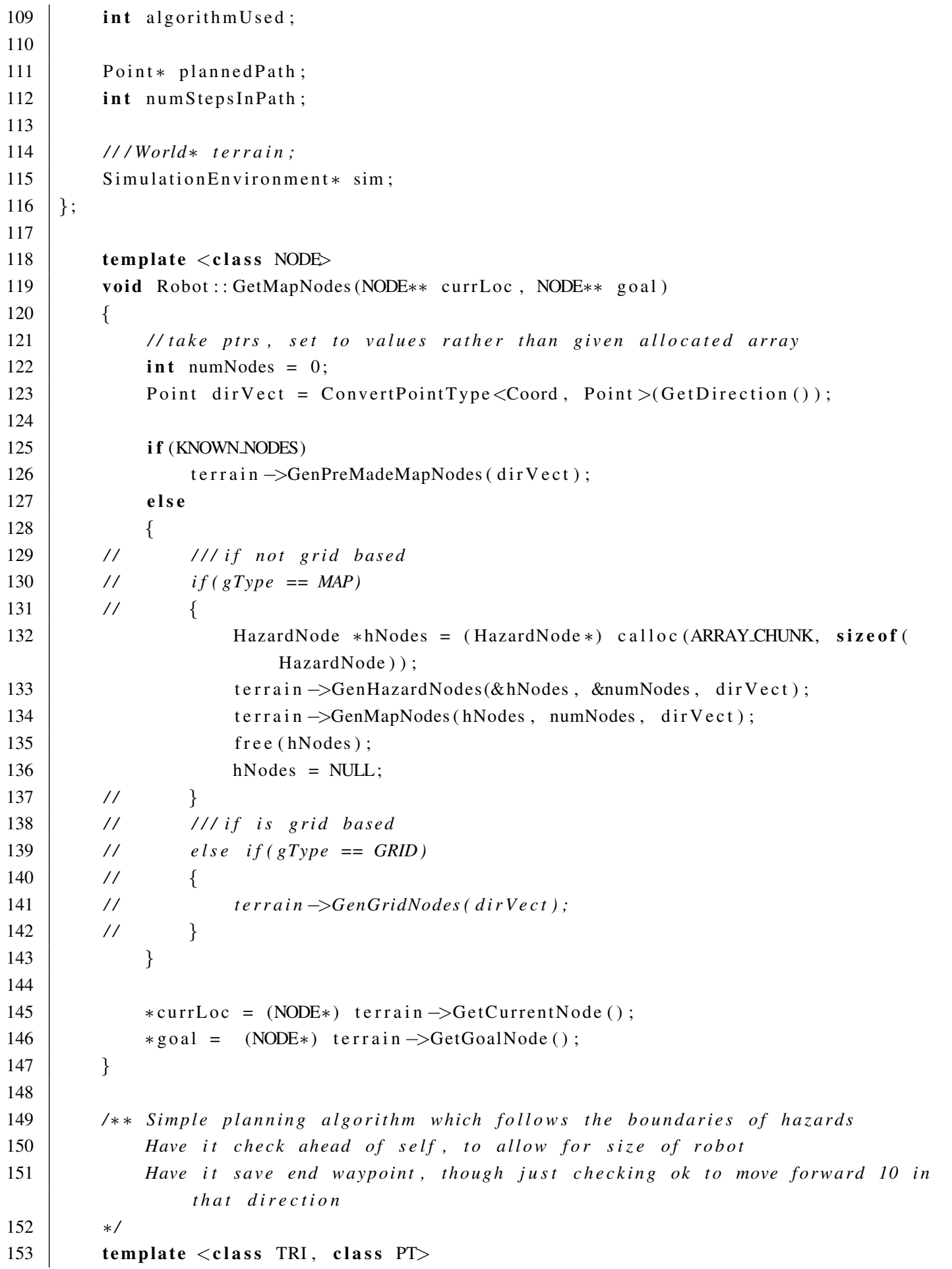


\{

int height $=$ goal.zVal;

PT tGoal = ConvertPointType $<$ Coord, PT $>$ (goal);

PT tCurrentPos $=$ ConvertPointType $<$ Coord, PT $>$ (currentPos);

PT directionVector $=$ tGoal $-($ tCurrentPos - PT $(0,0,0))$;

float magn $=$ CalculateDirectDistance(currentPos, goal, true);

directionVector $=$ NormaliseVector $<\mathrm{PT}>$ (directionVector,

MOVEINCREMENTS_MM, true ) ;

PT normVector $=$ CrossProduct $($ directionVector, directionVector $+(P T$ $(0,0,10)-\operatorname{PT}(0,0,0)))$;

normVector $=$ NormaliseVector $<$ PT $>$ (normVector, ROBOTWIDTHMM $/ 2$, false $)$;

PT tNewPos $=$ tCurrentPos $+($ directionVector - PT $(0,0,0))$;

Coord newPos $=$ ConvertPointType $<$ PT, Coord $>$ (tNewPos $)$;

TRI newTri;

numStepsInPath $=1$;

bool pathClear $=$ terrain $\rightarrow$ CheckBetweenPoints $($ tCurrentPos, tNewPos +( directionVector - PT $(0,0,0))$, true, normVector $)$;

if (terrain $\rightarrow$ GetTri(\&newTri, \&tNewPos))

if (pathClear \&\& newTri $\rightarrow$ CheckValidTri () \&\& !newTri $\rightarrow$ hazardType) plannedPath $[0]=$ ConvertPointType $<$ PT, Point $>$ (tNewPos $) ;$ else

\{

Coord $*$ testPts $=($ Coord $*)$ calloc (ARRAY_CHUNK, sizeof $($ Coord $))$; newPos $=$ CheckEdgePath (currentPos, \&(ConvertPointType $<$ PT, Coord $>($ tNewPos + (directionVector - PT $(0,0,0)))), 0$, testPts $)$; plannedPath $[0]=$ ConvertPointType $<$ Coord, Point $>$ (newPos); free (testPts); testPts = NULL;

\}

\}

else

\{

TRI currentTri $=$ TRI () ;

if ( pathClear)

plannedPath $[0]=$ ConvertPointType $<$ PT, Point $>($ tNewPos $) ;$

else if (terrain $\rightarrow$ GetTri(\&currentTri, \&PT(currentPos.x(), currentPos.y (), currentPos.z())) \&\& currentTri $\rightarrow$ CheckValidTri())

\{

int dist $1=999$, dist $2=999$, dist $3=999$; 


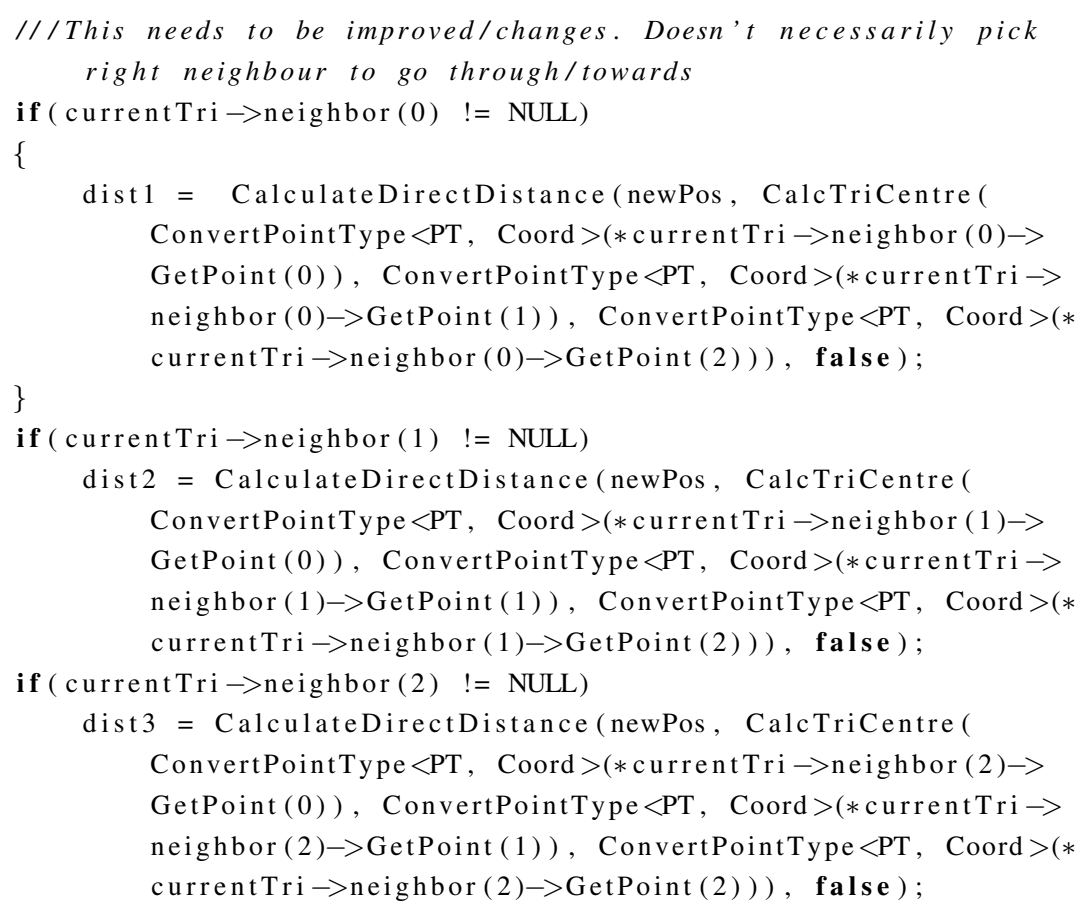




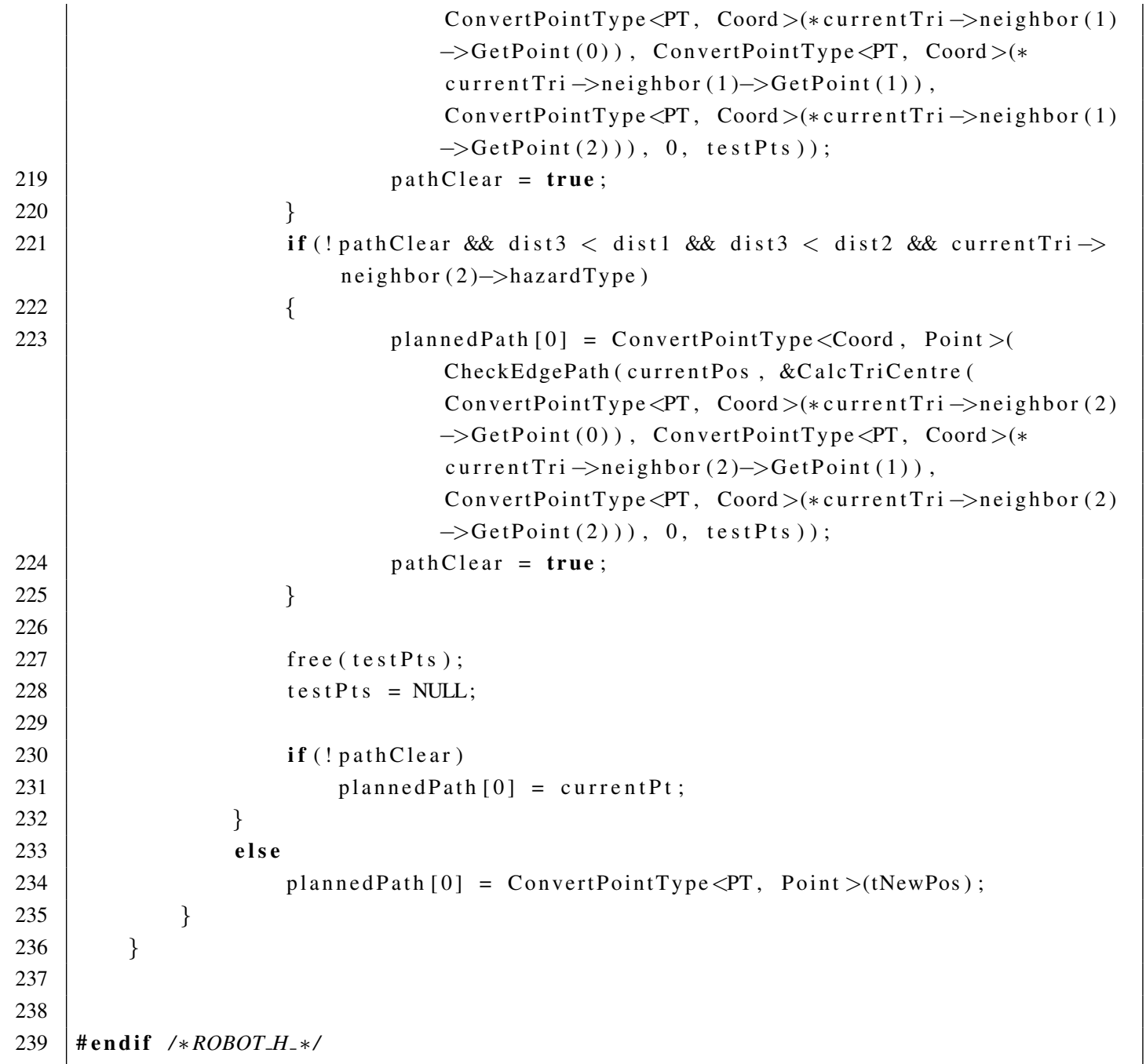

\section{A.6.2 Robot.cpp}

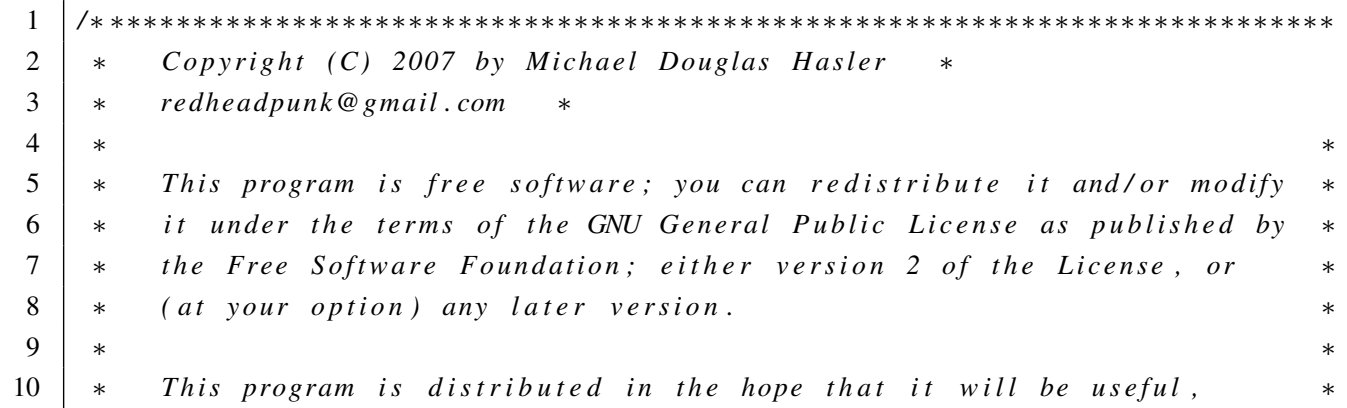




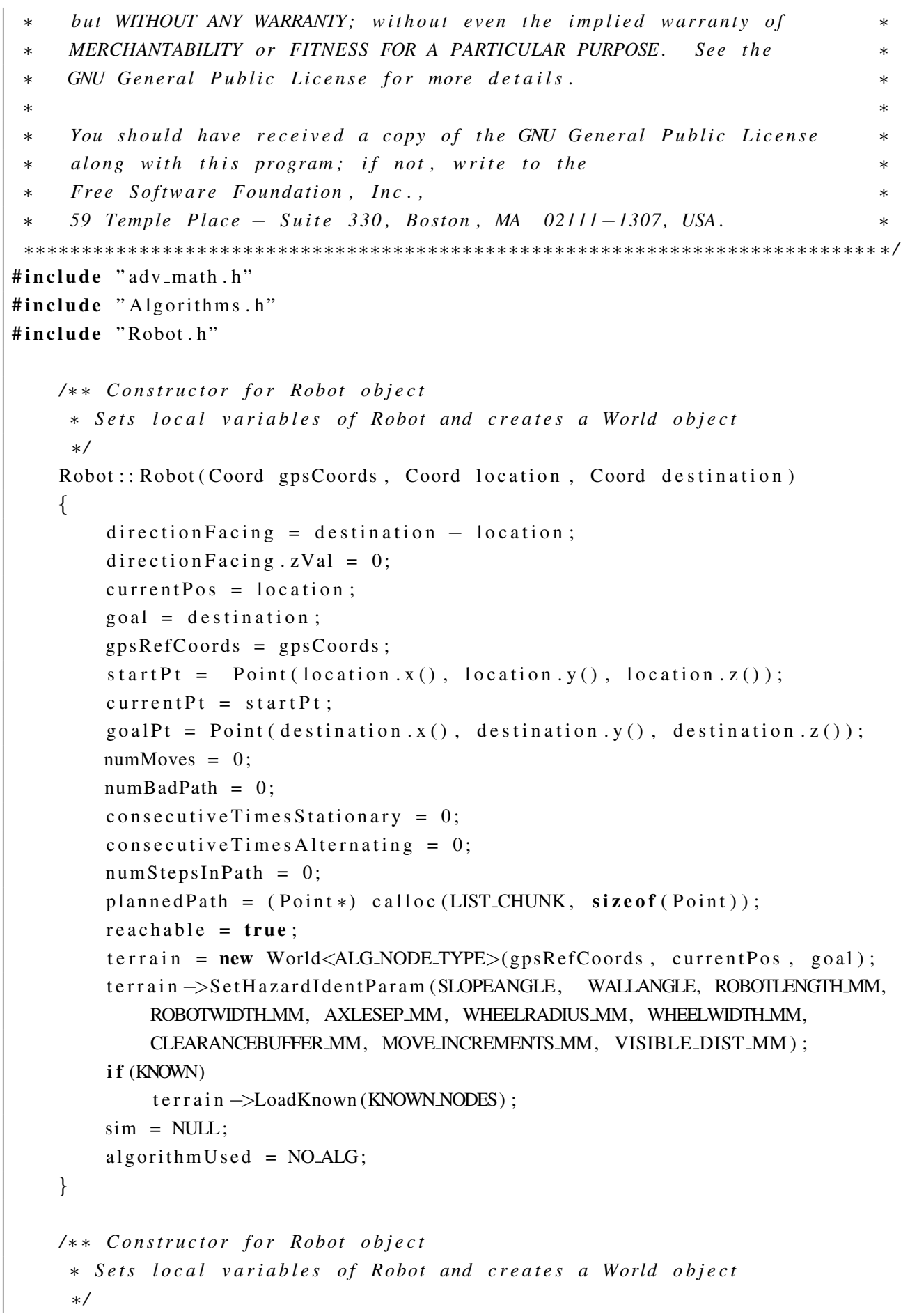




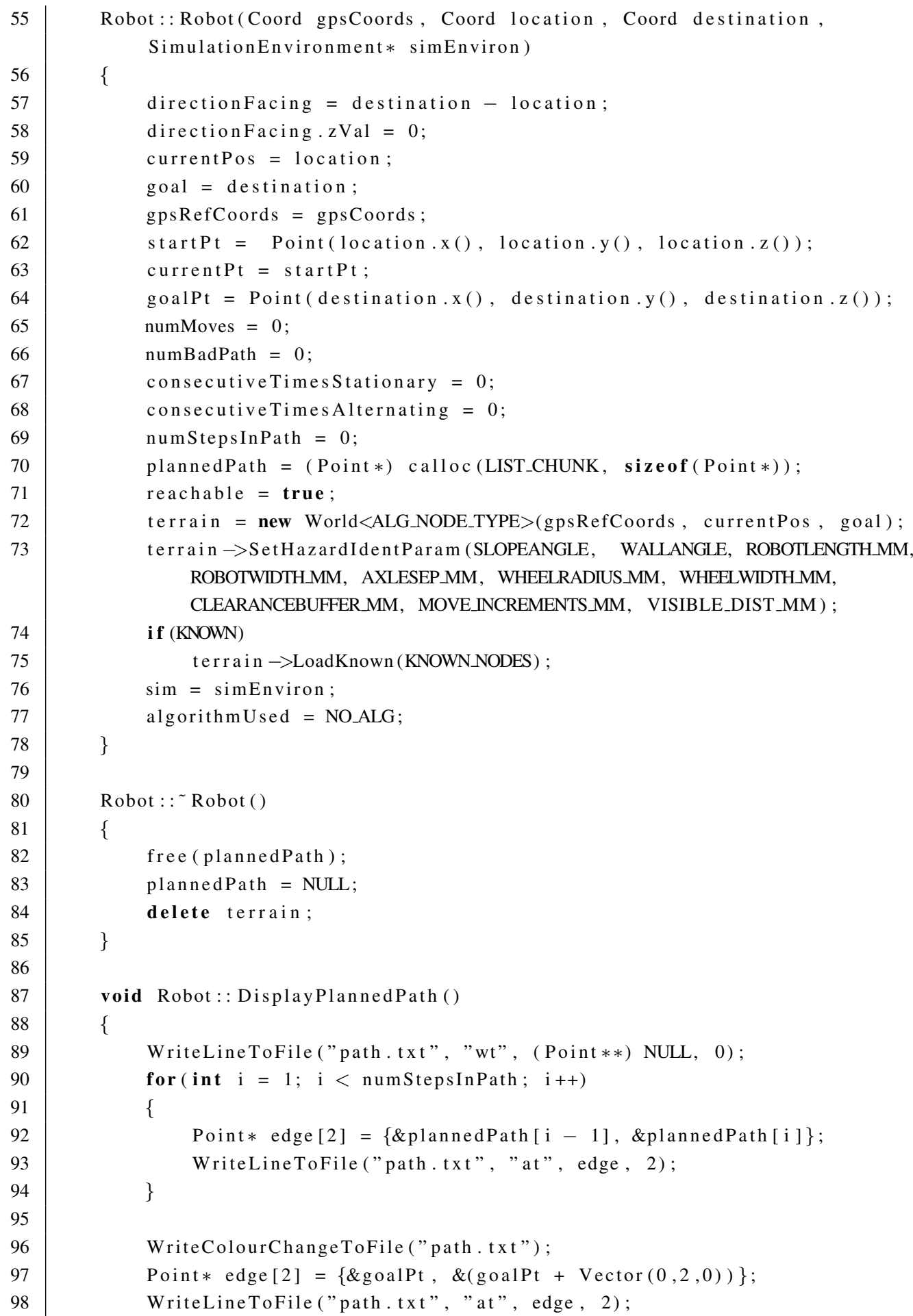




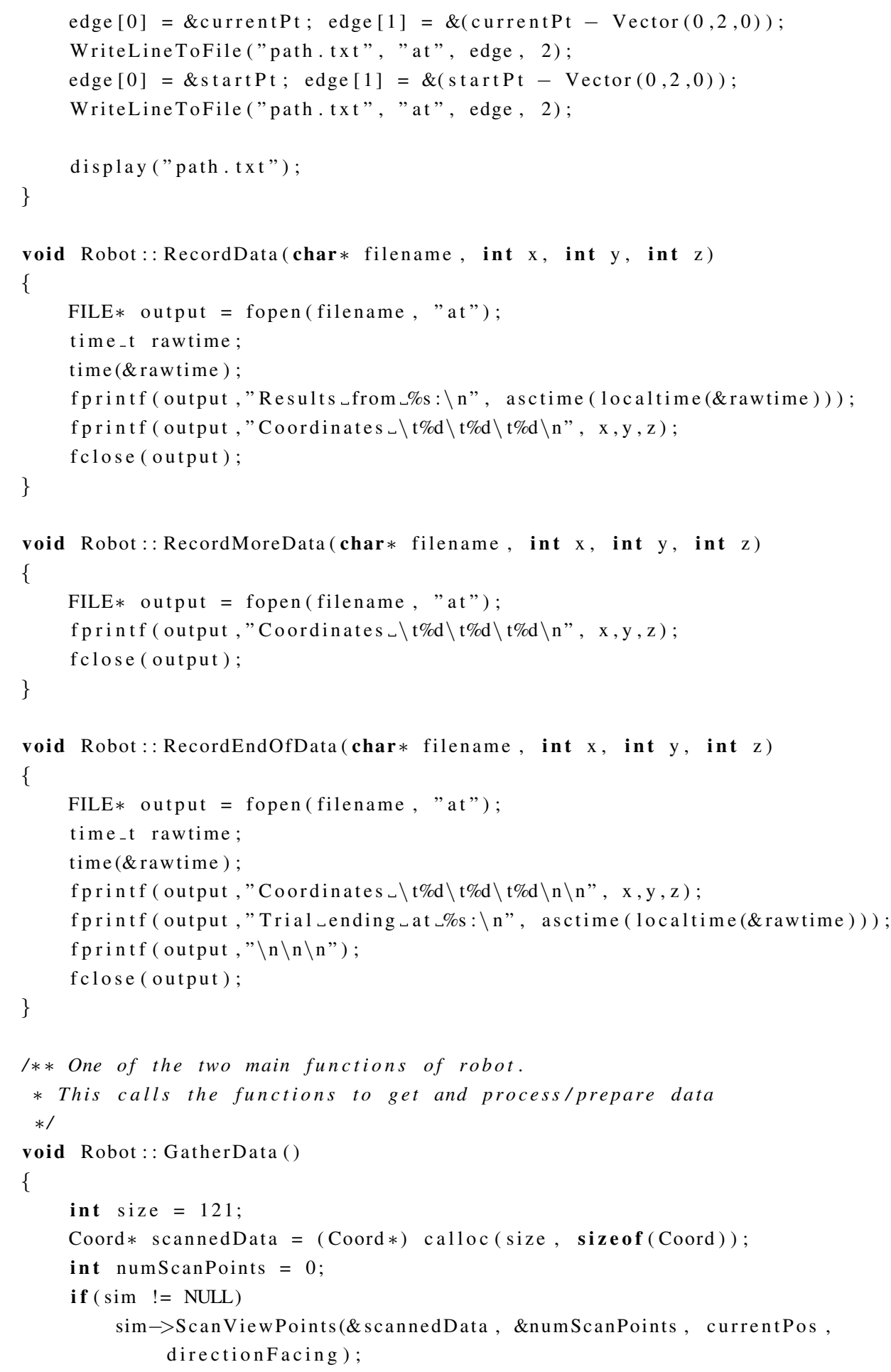


else

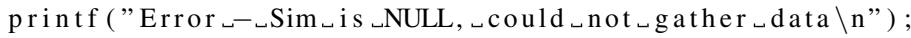

if ( numScanPoints $>0)$

terrain $\rightarrow$ AddPointsToWorld (scannedData, numScanPoints);

else if (numScanPoints $<0)$

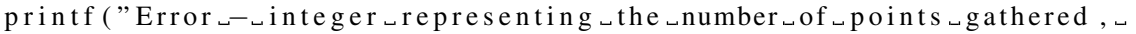
was $\_$negative $\backslash n$ ”);

free ( scannedData);

scannedData = NULL;

terrain $\rightarrow$ Process Points (KNOWN);

\}

/** Version for testing purposes only

$* /$

void Robot:: GatherData (Coord* scannedData, int numScanPoints)

\{

if ( scannedData $!=$ NULL \&\& numScanPoints $!=0$ )

terrain $\rightarrow$ AddPointsToWorld (scannedData, numScanPoints);

terrain $\rightarrow$ Process Points (KNOWN);

I** One of the two main functions of robot.

* This calls the functions to get and process/prepare data

*/

void Robot: : GatherData( SimulationEnvironment* simEnv, int inputQualityLevel) // could this just use already stored sim?

//perhaps use inputLevel for number of scanPoint in $x$ and $y$ axis?

\{

Coord $*$ scannedData ;

int numScanPoints $=0$;

if $($ sim $<$ RESERVED $)$

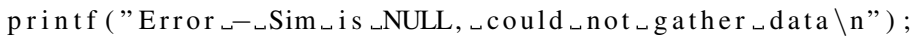

int size $=121$;

int size $=231$;

int $\mathrm{size}=441$;

scannedData $=($ Coord $*)$ calloc $($ size, sizeof $($ Coord $))$;

sim $\rightarrow$ ScanViewPoints (\&scannedData, \&numScanPoints, currentPos, directionFacing );

if ( numScanPoints $<0)$

printf ("Error $-_{-}$integer ${ }_{-}$representing the $_{\leftarrow}$ number of $_{\leftarrow}$ points gathered,, was $\_$negative $\backslash n "$ );

else

\{ 


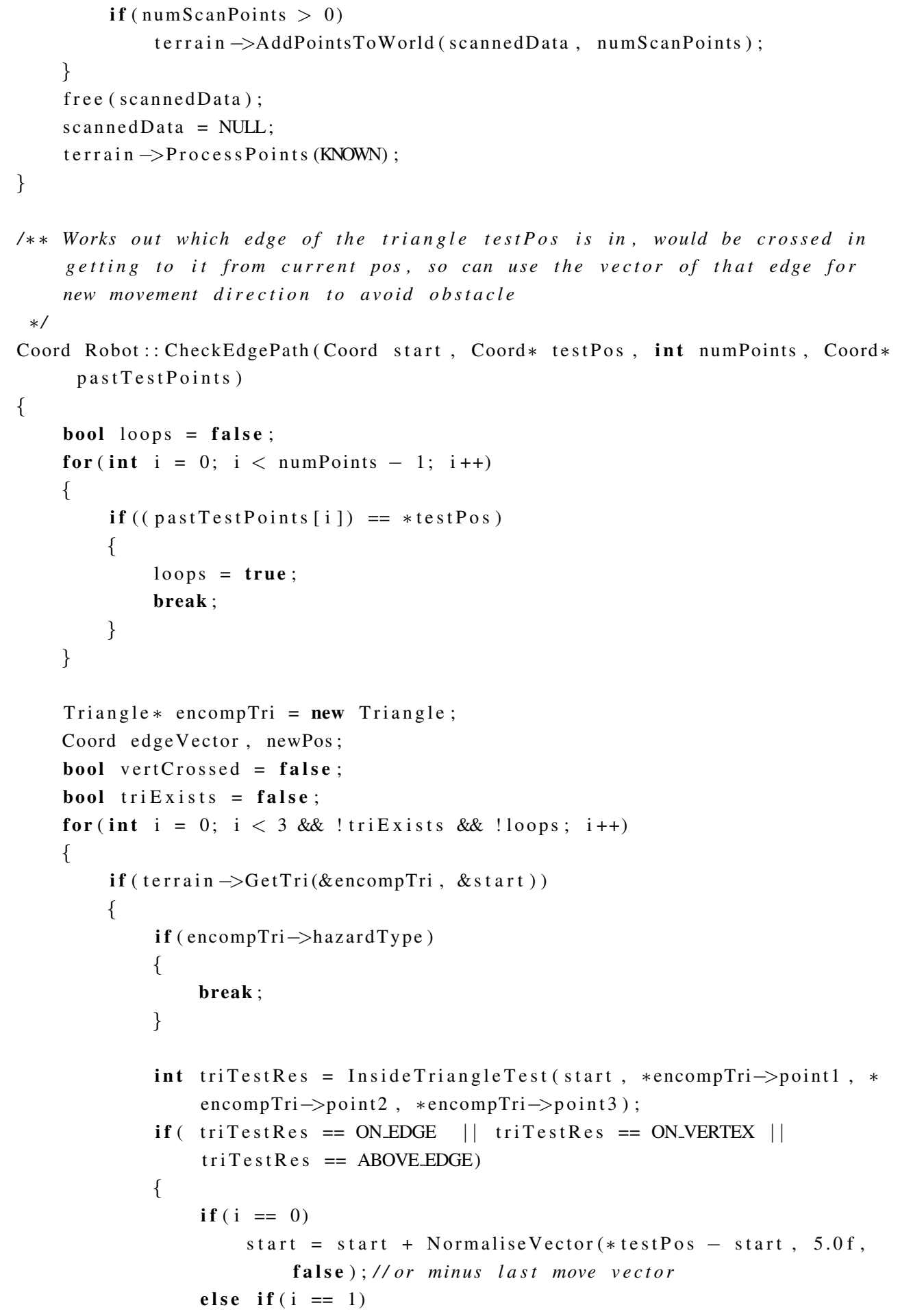




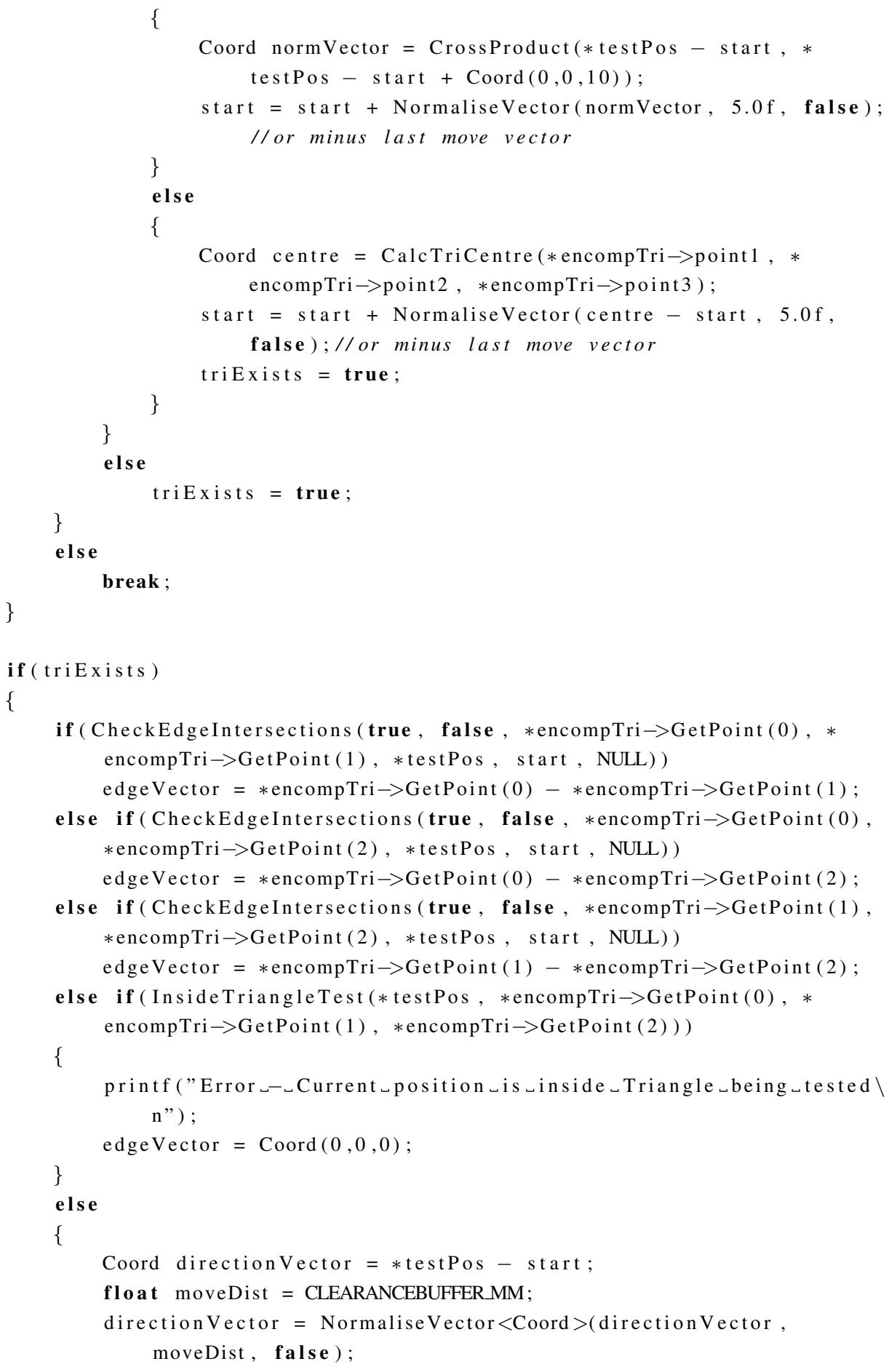


Coord normVector $=$ CrossProduct (directionVector, directionVector + Coord $(0,0,10))$;

pastTestPoints [ numPoints ++$]=(*$ testPos + NormaliseVector $<$ Coord $>($ normVector, $2.0 \mathrm{f}$, false));

newPos $=$ CheckEdgePath $($ start,$\&(*$ testPos + NormaliseVector $<$ Coord

$>($ normVector, $2.0 \mathrm{f}$, false)), numPoints, pastTestPoints);

vertCrossed = true;

\}

if (! vertCrossed)// as when vert cross new pos is all sorted

\{

edgeVector $=$ NormaliseVector $<$ Coord $>$ (edgeVector,

CLEARANCEBUFFERMM, fals e ) ;

if (edgeVector.xVal * (goal.yVal - currentPos.yVal) - (goal.xVal currentPos.xVal ) * edgeVector.yVal $>0$ )

newPos $=$ currentPos + edgeVector;

else

newPos $=$ currentPos - edgeVector

pastTestPoints [numPoints ++] = newPos;

if (terrain $\rightarrow$ GetTri(\&encompTri, \&newPos) \&\& encompTri->hazardType) newPos $=$ CheckEdgePath $($ start, \&newPos, numPoints, pastTestPoints);

\}

\}

delete encompTri;

return newPos;

\}

/** Second of the two main functions of robot.

* This calls/controls the functions which analyse the terrain and plan a path

$* 1$

void Robot: : PlanPath ()

\{

switch (algorithm)

\{

case NO_ALG:

break;

case HUGGER:

\{

algorithmUsed = HUGGER;

EdgeHugger $<$ Face_handle, Point $>()$;

break ;

\} 


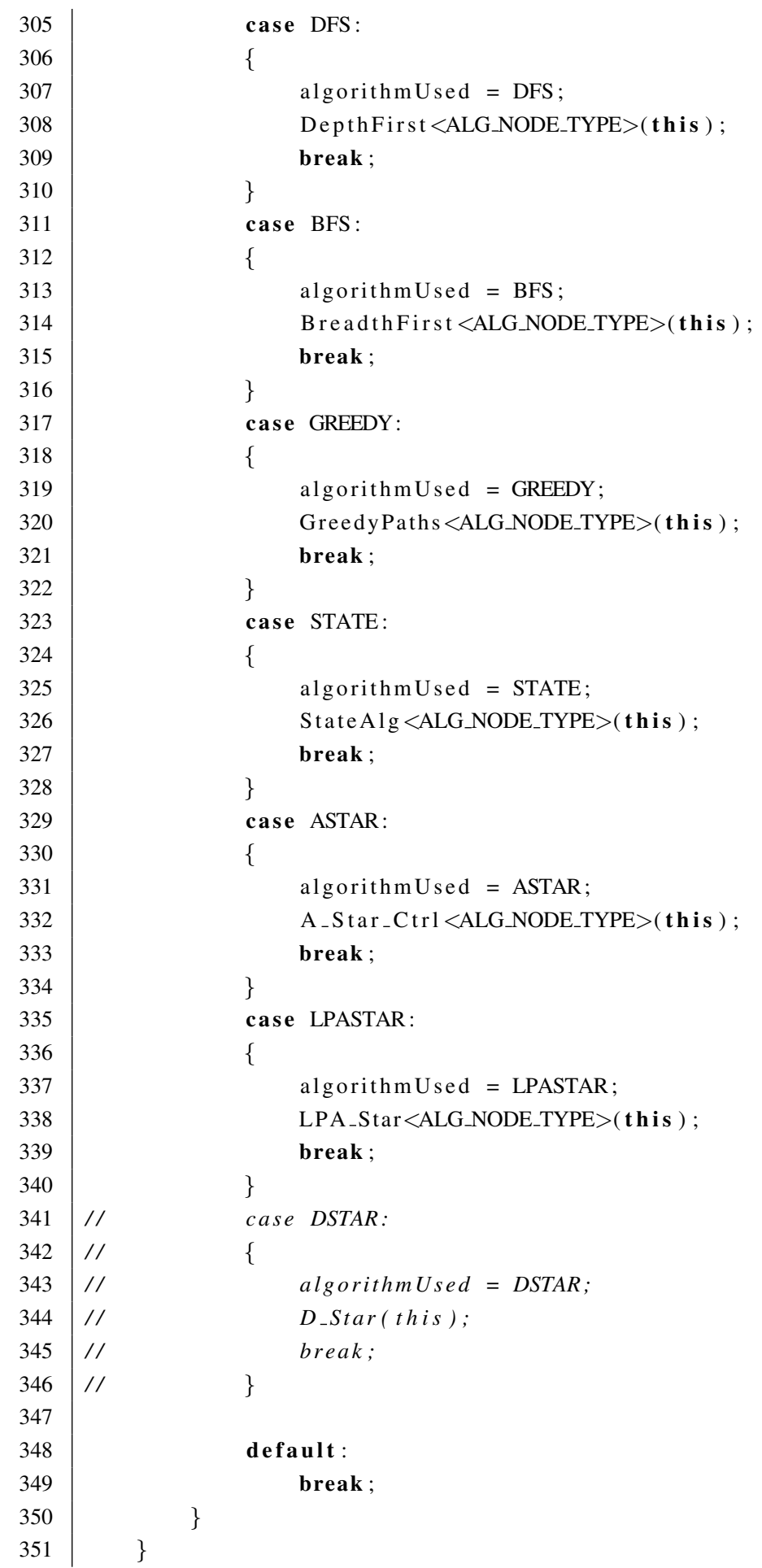




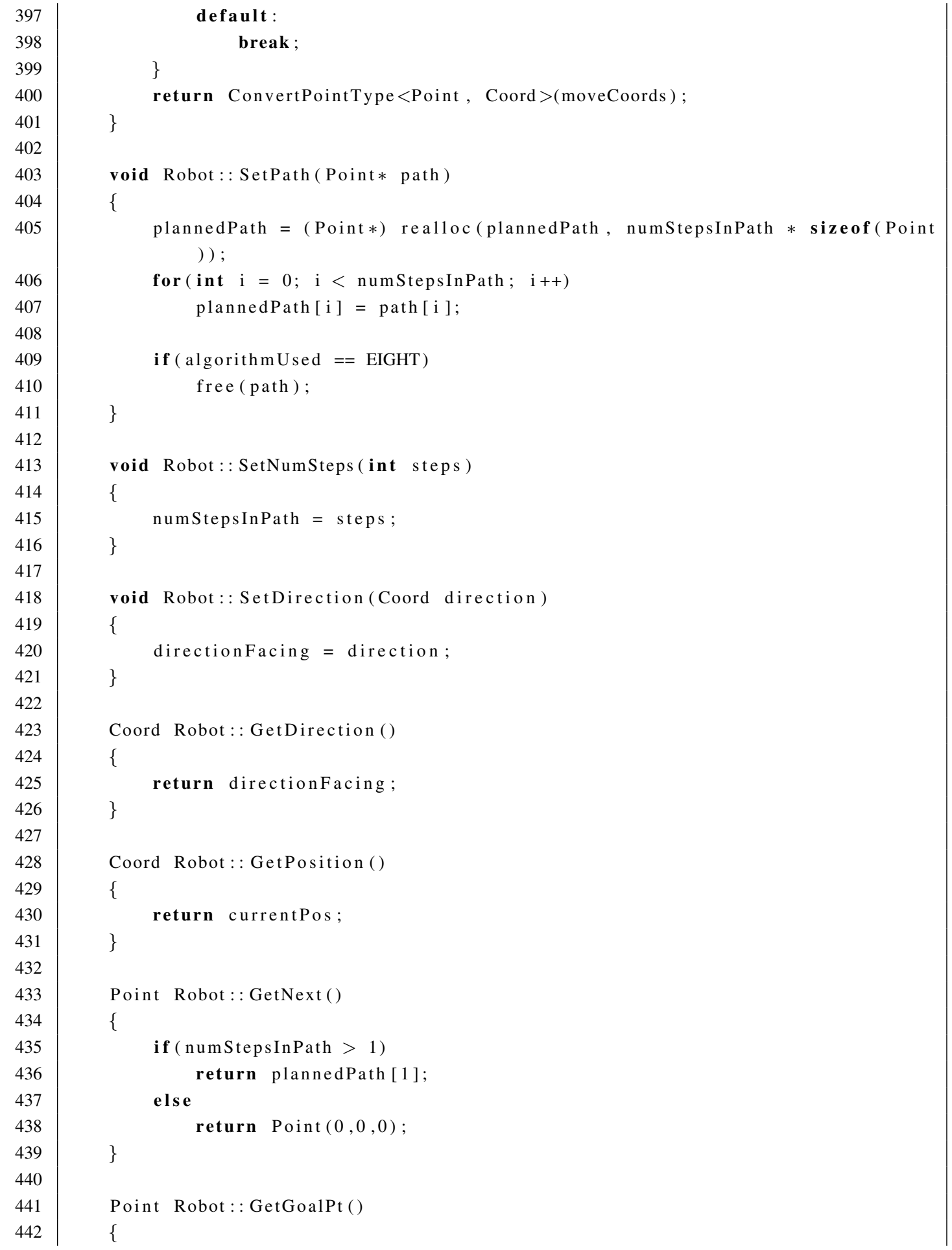




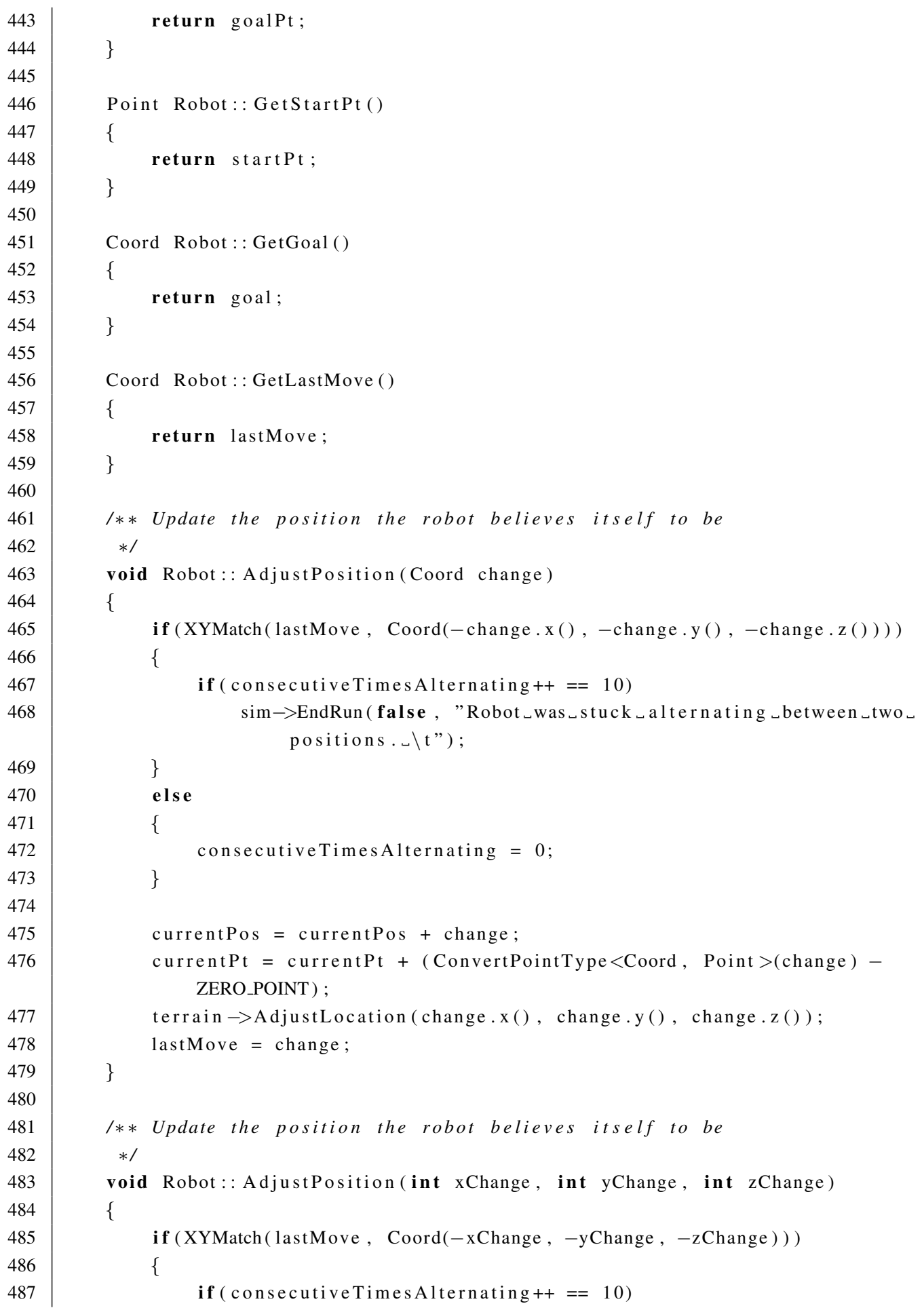




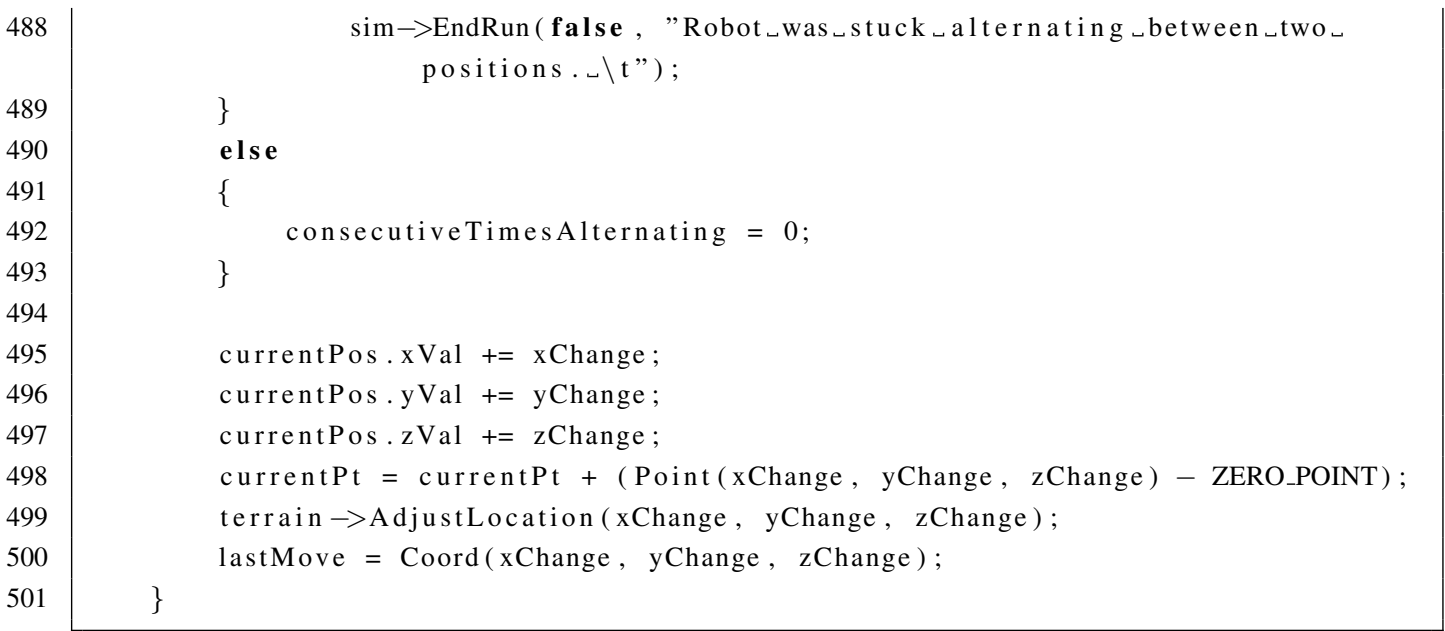

\section{A.7 SimulationEnvironment}

\section{A.7.1 SimulationEnvironment.h}

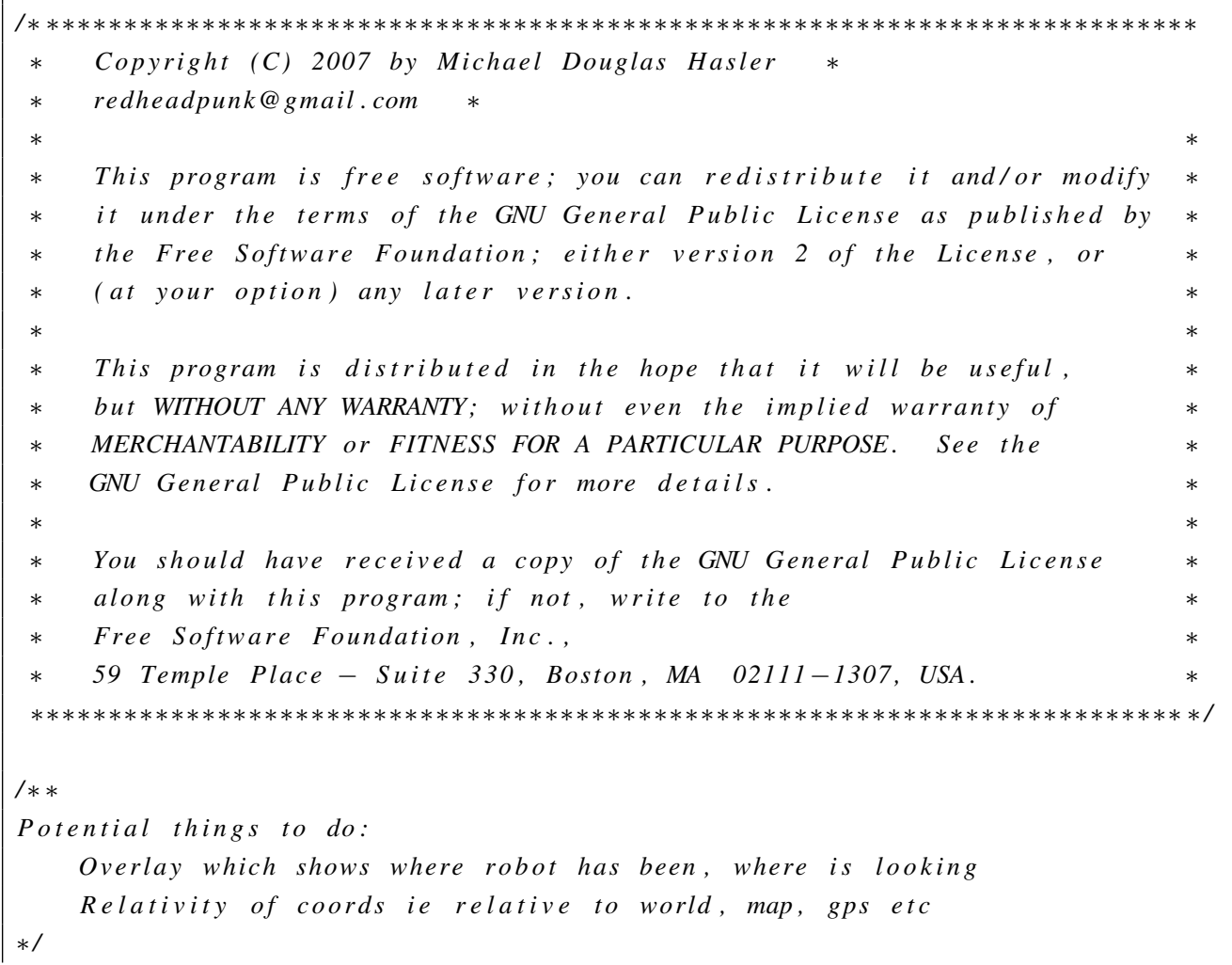




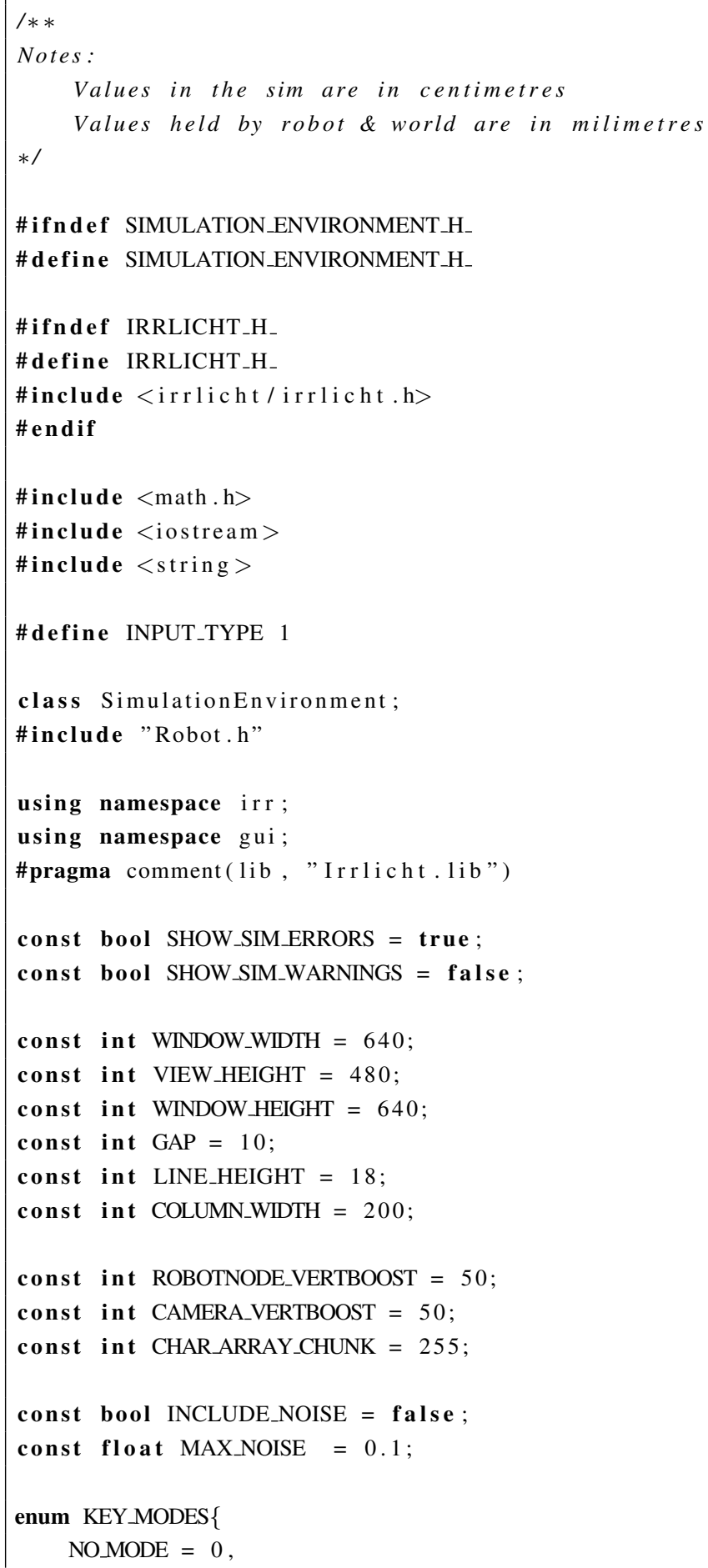




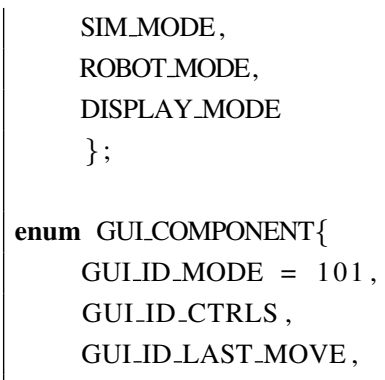




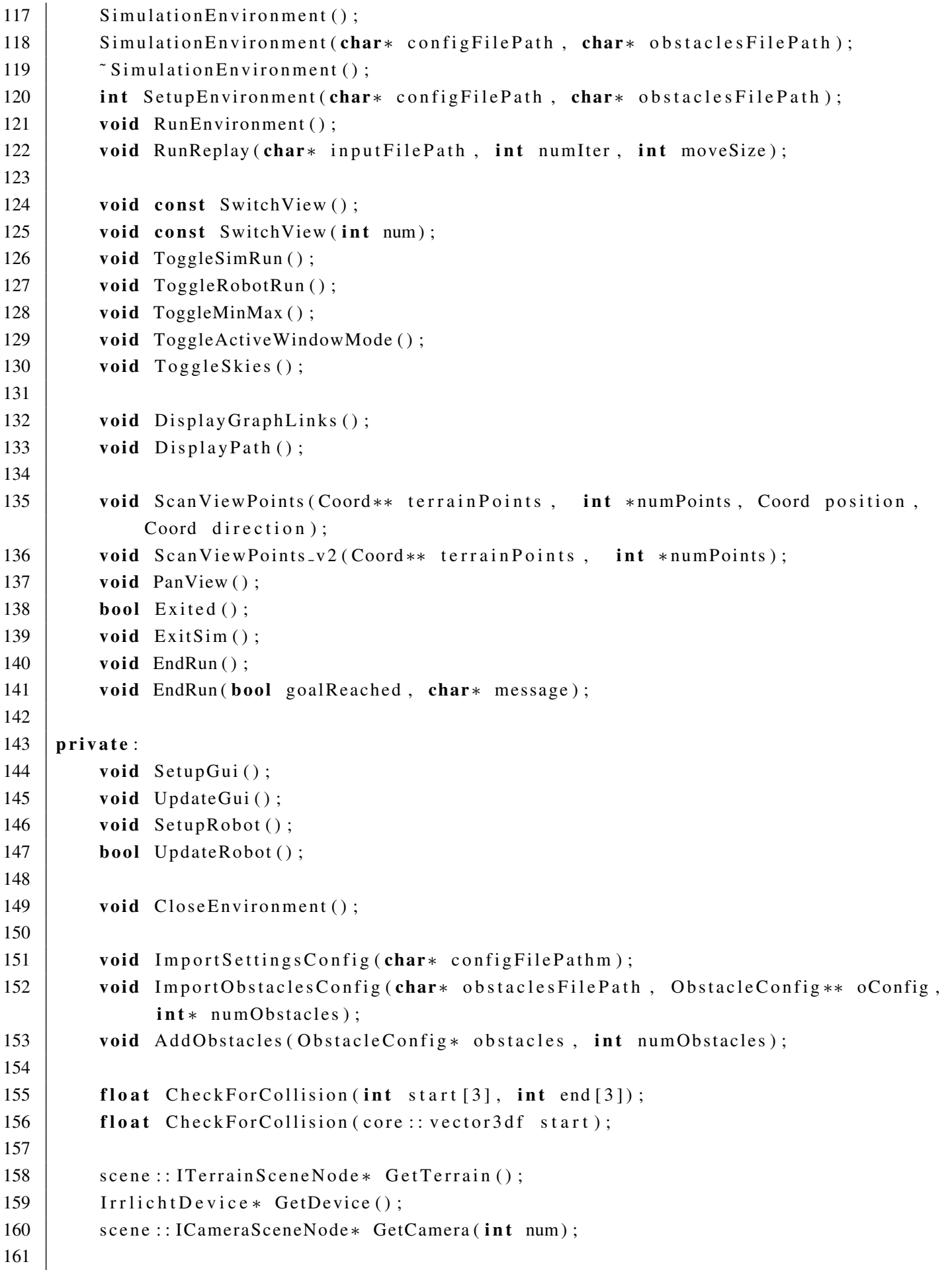




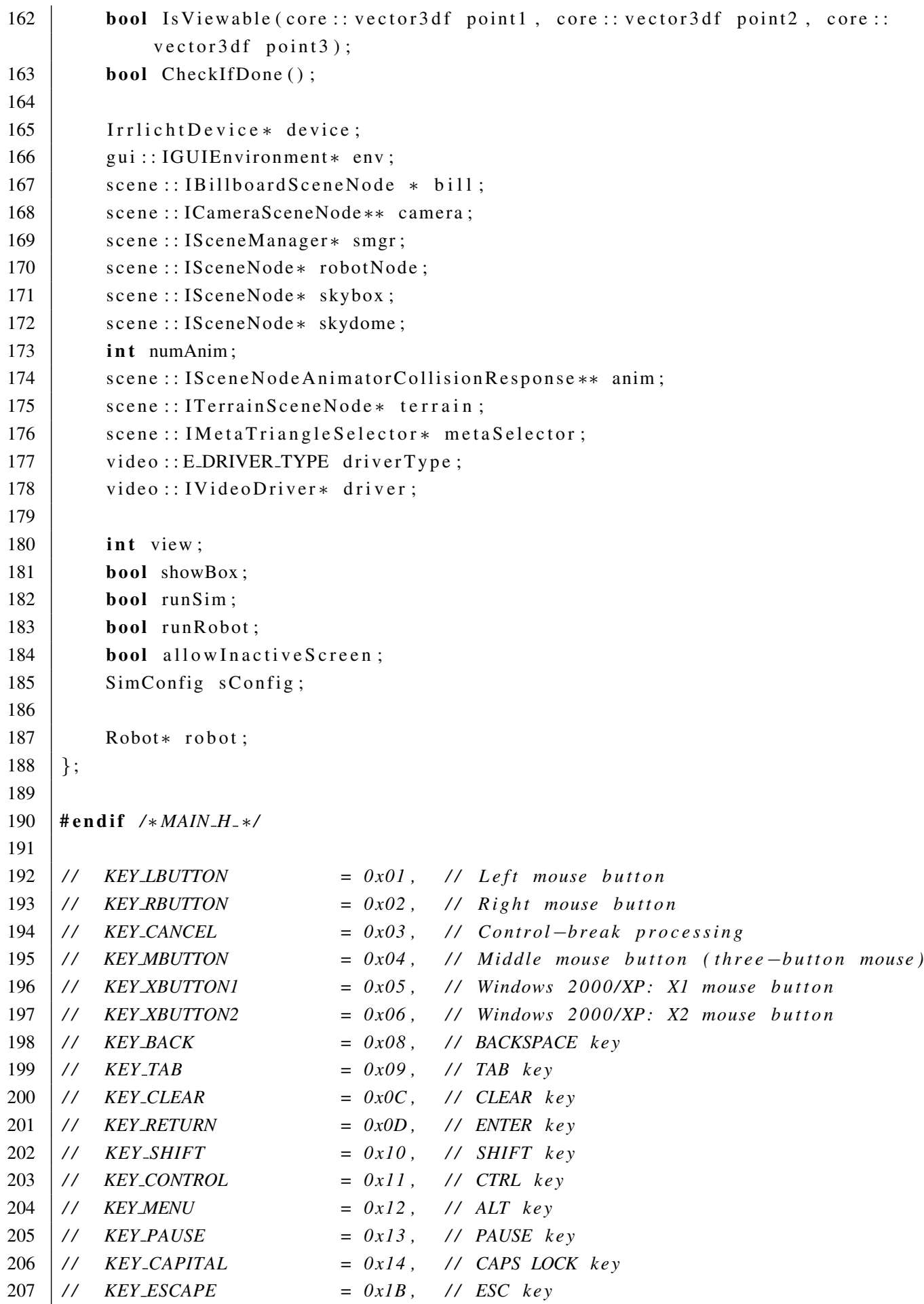




\begin{tabular}{|c|c|c|}
\hline 208 & // & KEY_SPACE \\
\hline 209 & // & KEY_PRIOR \\
\hline 10 & // & $K E Y \_N E X T$ \\
\hline 211 & // & KEY_END \\
\hline 212 & // & КЕY НОМЕ \\
\hline 213 & // & $K E Y_{-} L E F T$ \\
\hline 214 & $/ /$ & $K E Y_{-} U P$ \\
\hline 215 & // & $K E Y \_R I G H T$ \\
\hline 216 & $/ /$ & KEY DOWN \\
\hline 217 & // & KEY_SNAPSHO \\
\hline 218 & // & KEY_INSERT \\
\hline 219 & // & KEY_DELETE \\
\hline 220 & // & $K E Y_{-} K E Y_{-} O$ \\
\hline 221 & // & $K E Y_{-} K E Y_{-} l$ \\
\hline 222 & // & $K E Y_{-} K E Y_{-} 2$ \\
\hline 223 & // & $K E Y_{-} K E Y_{-} 3$ \\
\hline 224 & // & $K E Y_{-} K_{E} Y_{-} 4$ \\
\hline 225 & $/ /$ & $K E Y_{-} K E Y_{-} 5$ \\
\hline 226 & // & $K E Y_{-} K E Y_{-} 6$ \\
\hline 227 & $/ /$ & $K E Y_{-} K E Y_{-} 7$ \\
\hline 228 & // & $K E Y_{-} K E Y_{-} 8$ \\
\hline 229 & // & $K E Y_{-} K E Y_{-} 9$ \\
\hline 230 & // & $K E Y_{-} K E Y_{-} A$ \\
\hline 231 & // & $K E Y_{-} K E Y_{-} B$ \\
\hline 232 & // & $K E Y_{-} K_{E} Y_{-} C$ \\
\hline 233 & // & $K E Y_{-} K E Y_{-} D$ \\
\hline 234 & $/ /$ & $K E Y_{-} K E Y_{-} E$ \\
\hline 235 & // & $K E Y_{-} K E Y_{-} F$ \\
\hline 236 & $/ /$ & $K E Y_{-} K E Y_{-} G$ \\
\hline 237 & // & $K E Y_{-} K E Y_{-} H$ \\
\hline 238 & $/ /$ & $K E Y_{-} K E Y_{-} I$ \\
\hline 239 & // & $K E Y_{-} K E Y_{-} J$ \\
\hline 240 & // & $K E Y_{-} K E Y_{-} K^{\prime}$ \\
\hline 241 & // & $K E Y_{-} K E Y_{-} L$ \\
\hline 242 & // & $K E Y_{-} K E Y \_M$ \\
\hline 243 & // & $K E Y_{-} K E Y_{-} N$ \\
\hline 244 & // & $K_{E} Y_{-} \mathrm{KEY}_{-} \mathrm{O}$ \\
\hline 245 & $/ /$ & $K E Y_{-} K E Y_{-} P$ \\
\hline 246 & // & $K E Y_{-} K E Y_{-} Q$ \\
\hline 247 & $/ /$ & $K E Y_{-} K E Y_{-} R$ \\
\hline 248 & // & $K E Y_{-} K E Y_{-} S$ \\
\hline 249 & // & $K E Y_{-} K E Y_{-} T$ \\
\hline 250 & // & $K E Y_{-} K E Y_{-} U$ \\
\hline 251 & // & $K E Y_{-} K E Y_{-} V$ \\
\hline 252 & // & $K E Y_{-} K E Y_{-} W$ \\
\hline 253 & // & $K E Y_{-} K E Y_{-} X$ \\
\hline 254 & $/ /$ & $K E Y_{-} K E Y_{-} Y$ \\
\hline
\end{tabular}

$=0 \times 20, \quad / /$ SPACEBAR

$=0 \times 21, \quad / /$ PAGE UP key

$=0 x 22, \quad / /$ PAGE DOWN key

$=0 \times 23, \quad / / E N D$ key

$=0 \times 24, \quad / /$ HOME key

$=0 x 25, \quad / /$ LEFT ARROW key

$=0 x 26, \quad / /$ UP ARROW key

$=0 \times 27, \quad / /$ RIGHT ARROW key

$=0 x 28, \quad / /$ DOWN ARROW key

$=0 x 2 C, \quad / /$ PRINT SCREEN key

$=0 \times 2 D, \quad / / I N S$ key

$=0 x 2 E, \quad / / D E L$ key

$=0 \times 30, \quad / / \quad 0$ key

$=0 \times 31, \quad / / 1$ key

$=0 \times 32, \quad / / 2$ key

$=0 \times 33, \quad / / 3$ key

$=0 \times 34, \quad / / 4$ key

$=0 \times 35, / / 5$ key

$=0 \times 36, \quad / / 6$ key

$=0 \times 37, \quad / / 7$ key

$=0 \times 38, \quad / / 8$ key

$=0 \times 39, \quad / / 9$ key

$=0 x 41, \quad / /$ A key

$=0 x 42, \quad / / B$ key

$=0 x 43, \quad / / C$ key

$=0 x 44, \quad / /$ D key

$=0 x 45, / /$ E key

$=0 x 46, \quad / / F$ key

$=0 x 47, \quad / /$ G key

$=0 x 48, \quad / /$ H key

$=0 x 49, \quad / /$ I key

$=0 x 4 A, \quad / / J$ key

$=0 x 4 B, \quad / / K$ key

$=0 x 4 C, \quad / / L$ key

$=0 x 4 D, \quad / / M$ key

$=0 x 4 E, \quad / / N$ key

$=0 x 4 F, \quad / / O$ key

$=0 x 50, \quad / / P$ key

$=0 \times 51, \quad / / Q$ key

$=0 x 52, \quad / / \quad R$ key

$=0 x 53, \quad / /$ S key

$=0 \times 54, \quad / / T$ key

$=0 x 55, \quad / /$ U key

$=0 x 56, \quad / / V k e y$

$=0 \times 57, \quad / /$ W key

$=0 x 58, \quad / / X$ key

$=0 x 59, \quad / /$ Y key 


\begin{tabular}{l|ll}
255 & $/ /$ & KEY_KEY_Z \\
256 & $/ /$ & KEY_LWIN \\
& & Keyboard $)$ \\
257 & $/ /$ & KEY_RWIN \\
258 & $/ /$ & KEY_SLEEP \\
259 & $/ /$ & KEY_NUMPADO \\
260 & $/ /$ & KEY_NUMPAD1 \\
261 & $/ /$ & KEY_NUMPAD2 \\
262 & $/ /$ & KEY_NUMPAD3 \\
263 & $/ /$ & KEY_NUMPAD4 \\
264 & $/ /$ & KEY_NUMPAD5 \\
265 & $/ /$ & KEY_NUMPAD6 \\
266 & $/ /$ & KEY_NUMPAD7 \\
267 & $/ /$ & KEY_NUMPAD \\
268 & $/ /$ & KEY_NUMPAD \\
269 & $/ /$ & KEY_MULTIPLY \\
270 & $/ /$ & KEY_ADD \\
271 & $/ /$ & KEY_SEPARATOR \\
272 & $/ /$ & KEY_SUBTRACT \\
273 & $/ /$ & KEY_DECIMAL \\
274 & $/ /$ & KEY_DIVIDE \\
275 & $/ /$ & KEY_F1 \\
276 & $/ /$ & KEY_F2 \\
277 & $/ /$ & KEY_F3 \\
278 & $/ /$ & KEY_F4 \\
279 & $/ /$ & KEY_F5 \\
280 & $/ /$ & KEY_F6 \\
281 & $/ /$ & KEY_F7 \\
282 & $/ /$ & KEY_F8 \\
283 & $/ /$ & KEY_F9 \\
284 & $/ /$ & KEY_F1O \\
285 & $/ /$ & KEY_F11 \\
286 & $/ /$ & KEY_F12 \\
287 & $/ /$ & KEY_NUMLOCK \\
288 & $/ /$ & KEY_SCROLL \\
289 & $/ /$ & KEY_LSHIFT \\
290 & $/ /$ & KEY_RSHIFT \\
291 & $/ /$ & KEY_LCONTROL \\
292 & $/ /$ & KEY_RCONTROL \\
293 & $/ /$ & KEY_LMENU \\
294 & $/ /$ & KEY_RMENU \\
295 & $/ /$ & KEY_PLUS \\
296 & $/ /$ & KEY_COMMA \\
& $/ /$ & KEY_MINUS \\
\hline II & KEY_PERIOD \\
& & \\
\hline
\end{tabular}

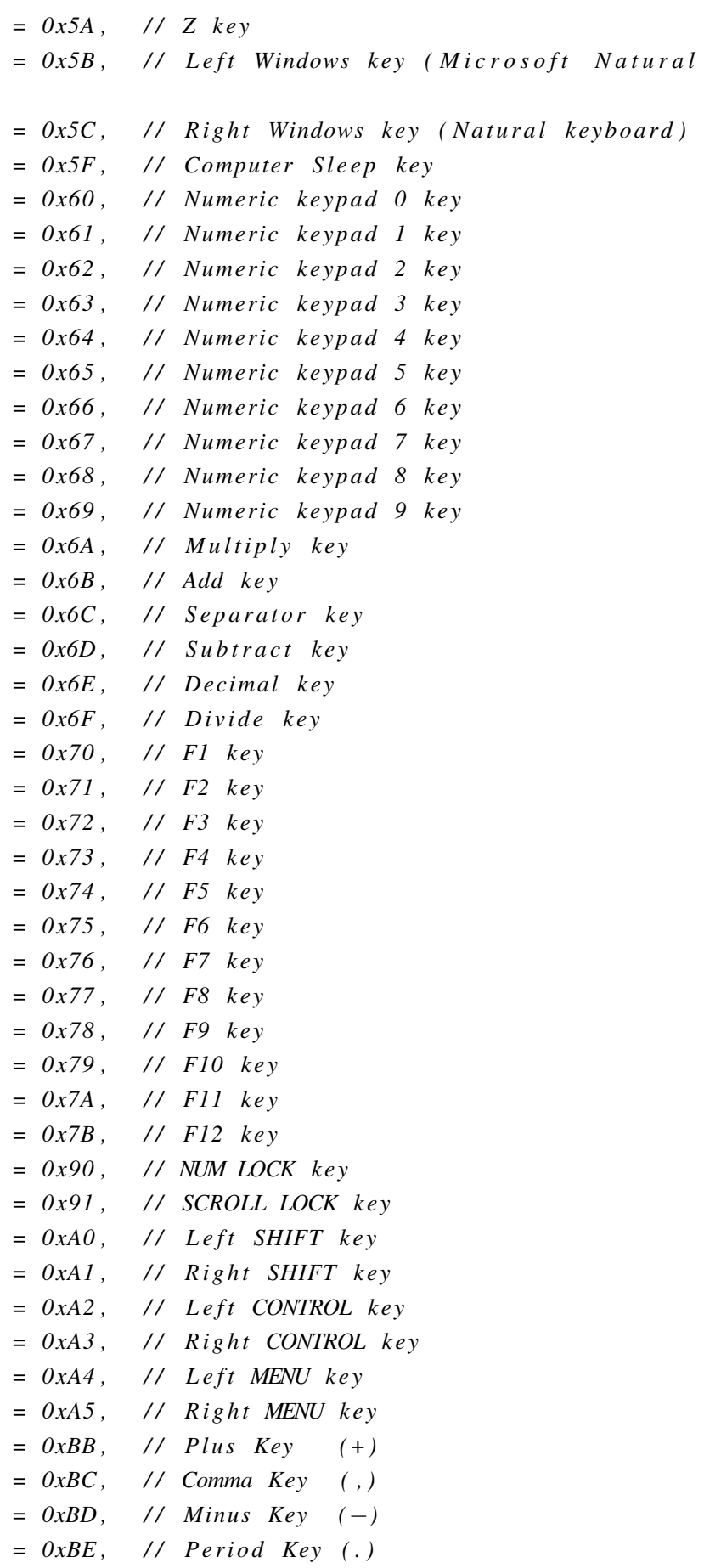



there are.

\section{A.7.2 SimEnv.cpp}

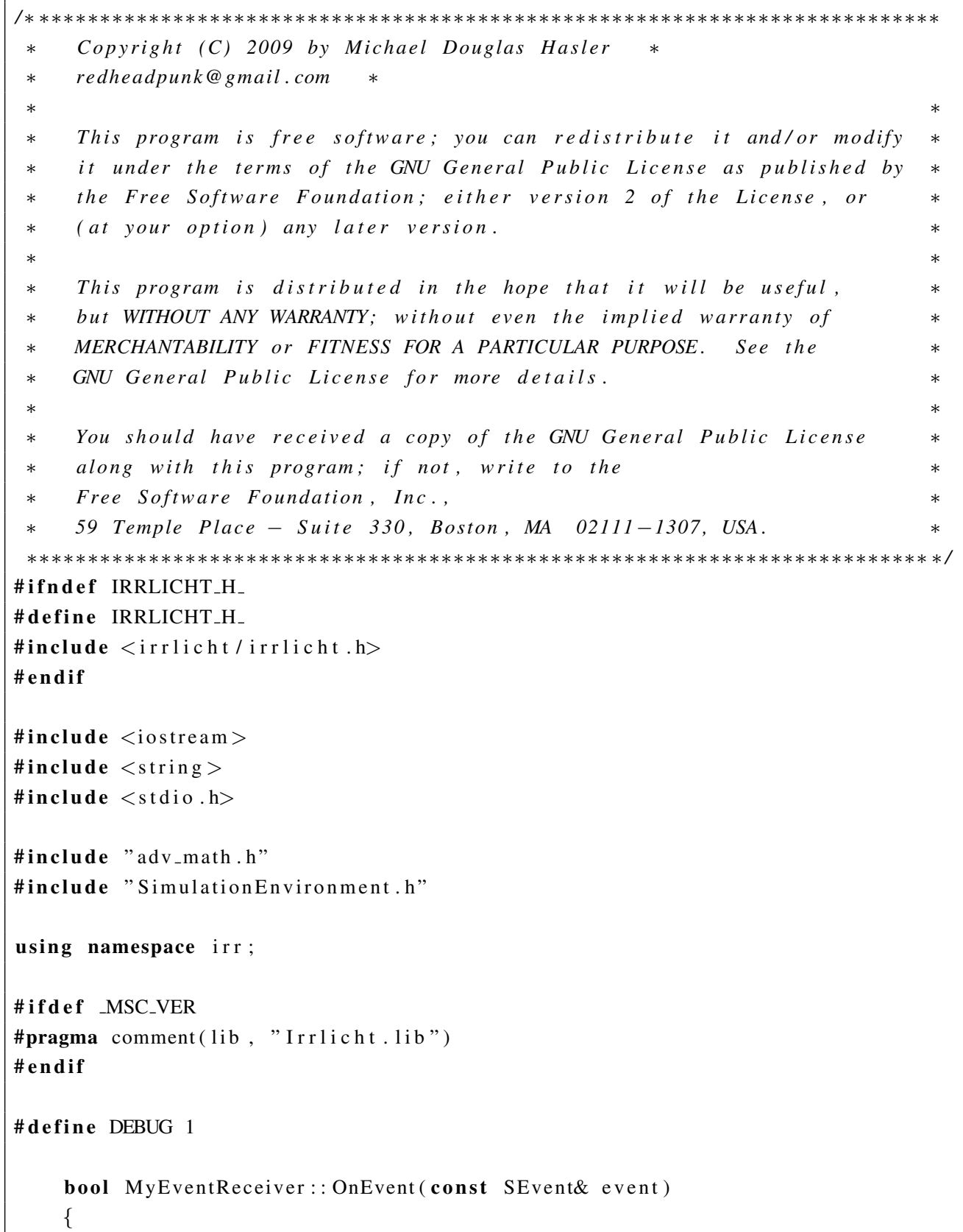




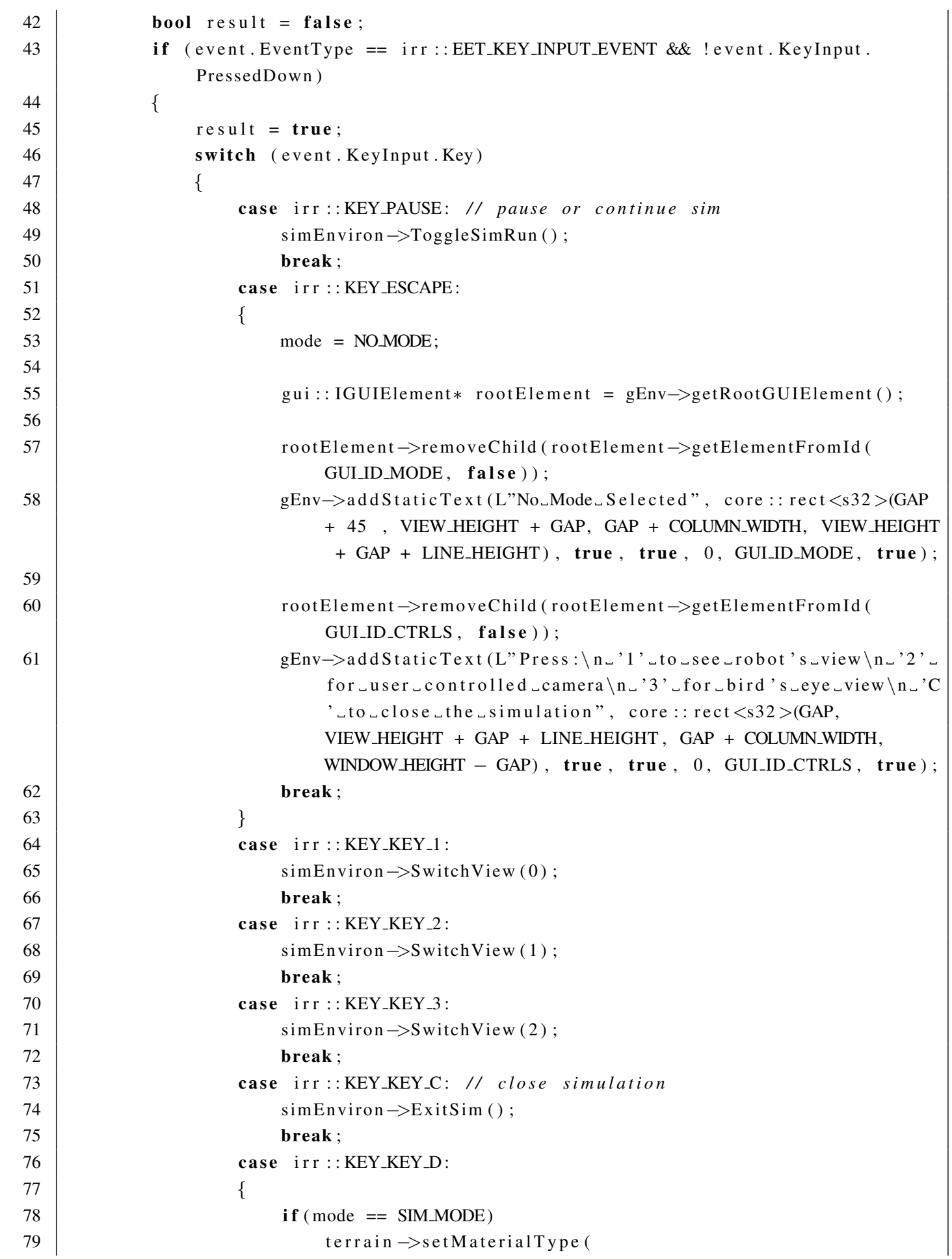




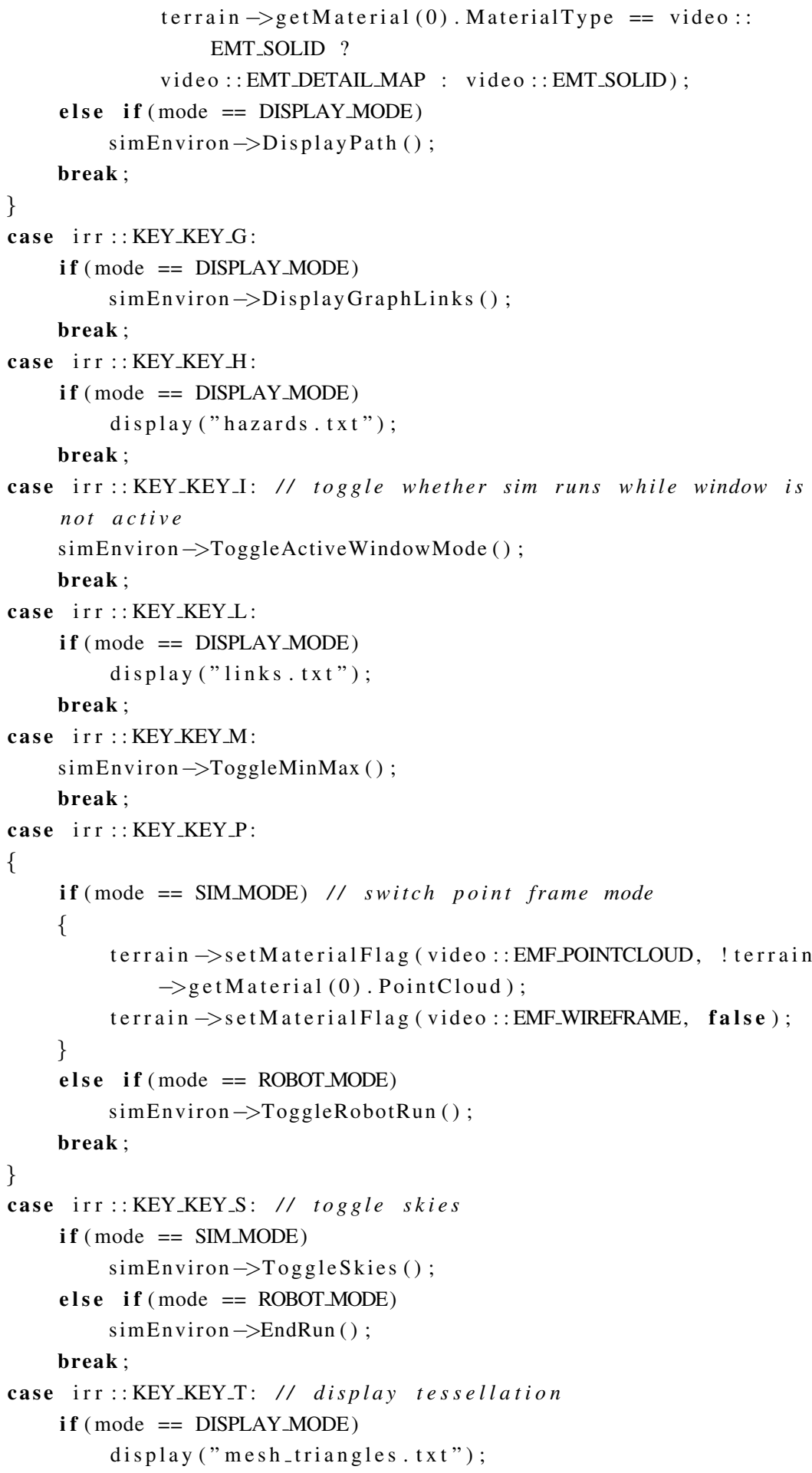


break ;

case ir $r:$ :KEY_KEY_W:

\{

if (mode == SIM_MODE) // switch wire frame mode

\{

terrain $\rightarrow$ setMaterialFlag (video : :EMF_WIREFRAME, ! terrain $\rightarrow$ getMaterial (0). Wireframe);

terrain $\rightarrow$ setMaterialFlag (video: :EMF_POINTCLOUD, false ); \}

break ;

\}

case irr::KEY_F1:

\{

mode $=$ SIM_MODE;

gui :: IGUIElement $*$ rootElement $=$ gEnv $\rightarrow$ getRootGUIElement () ;

rootElement $\rightarrow$ removeChild (rootElement $\rightarrow$ getElementFromId ( GUI_ID_MODE, false) ) ;

gEnv $\rightarrow$ addStatic Text (L"Sim_Ctrl_Mode", core $:$ : rect $<$ s32 $>($ GAP + 45 , VIEW_HEIGHT + GAP, GAP + COLUMN_WIDTH, VIEW_HEIGHT + GAP + LINE_HEIGHT), true, true , 0, GUI_ID_MODE, true);

rootElement $\rightarrow$ removeChild (rootElement $\rightarrow$ getElementFromId ( GUI_ID_CTRLS, false)) ;

gEnv $\rightarrow$ add Static Text (L"Press $: \backslash \mathrm{n}_{\sqcup}{ }^{\prime} \mathrm{D}^{\prime}{ }_{\lrcorner}$to ${ }_{\lrcorner}$toggle ${ }_{\sqcup}$ detail ${ }_{\lrcorner} \operatorname{map} \backslash \mathrm{n}_{\sqcup}{ }^{\prime} \mathrm{P}$ ' \lrcorner to $\lrcorner$ toggle $\lrcorner$ point $\lrcorner$ cloud $\lrcorner$ mode $\backslash \mathrm{n}_{\lrcorner}$'S $\left.{ }^{\prime}\right\lrcorner$ to ${ }_{\lrcorner}$toggle $\lrcorner$skybox $/$

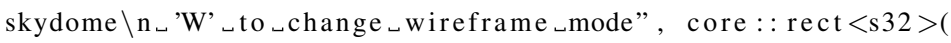
GAP, VIEW_HEIGHT + GAP + LINE_HEIGHT, GAP + COLUMN_WIDTH, WINDOW_HEIGHT - GAP), true, true, 0, GUI_ID_CTRLS, true) ;

break ;

\}

case irr::KEY_F2:

\{

mode $=$ ROBOT_MODE;

gui : : IGUIElement $*$ rootElement $=$ gEnv $\rightarrow$ getRootGUIElement () ;

rootElement $\rightarrow$ removeChild (rootElement $\rightarrow$ getElementFromId ( GUI_ID_MODE, false ) ) ;

gEnv $\rightarrow$ addStatic Text (L"Robot_Ctr1 „Mode”, core : : rect $<$ s32 $>$ (GAP + 45 , VIEW_HEIGHT + GAP, GAP + COLUMN_WIDTH, VIEW_HEIGHT

+ GAP + LINE_HEIGHT), true, true, 0 , GUI_ID_MODE, true );

rootElement $\rightarrow$ removeChild (rootElement $\rightarrow$ getElementFromId ( GUI_ID_CTRLS, false) );

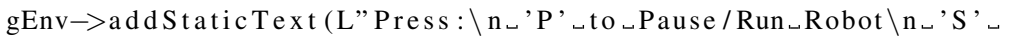
to_skip_to_next_run”, core : : rect $<$ s32 $>$ (GAP, VIEW_HEIGHT + 


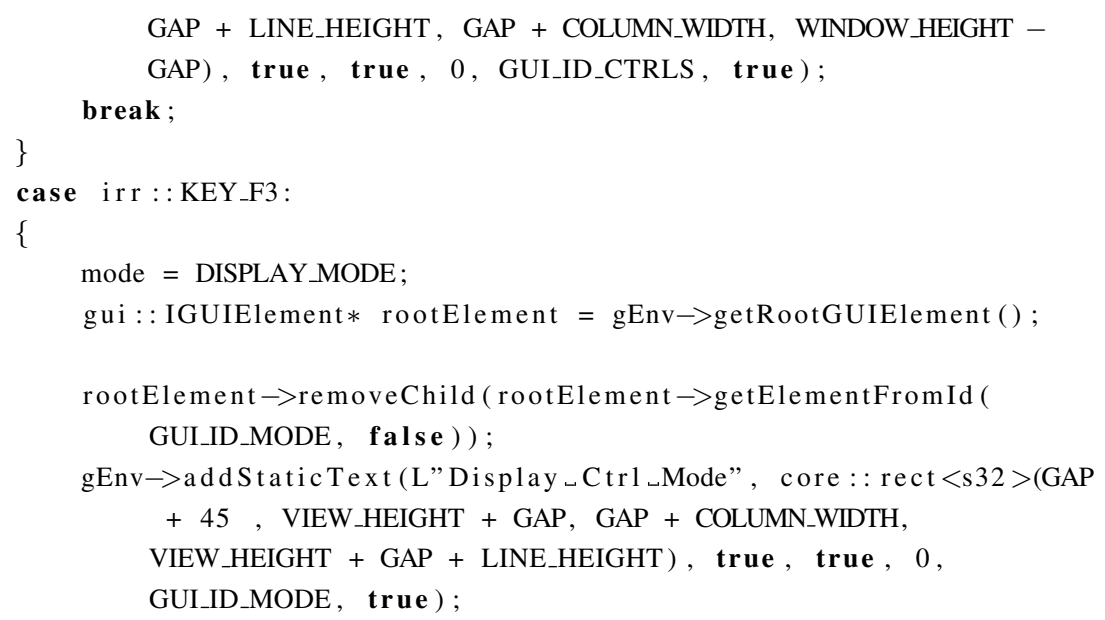




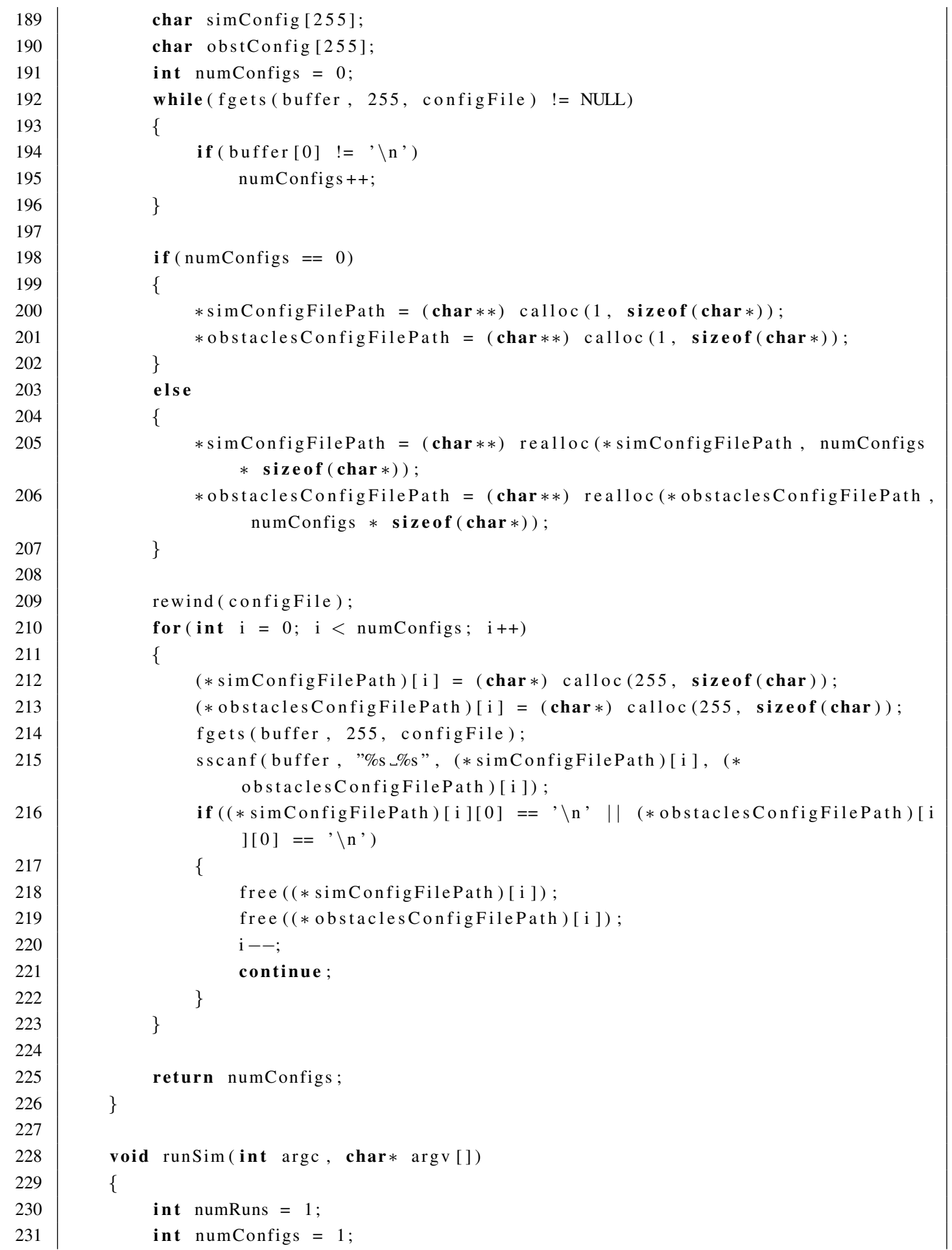




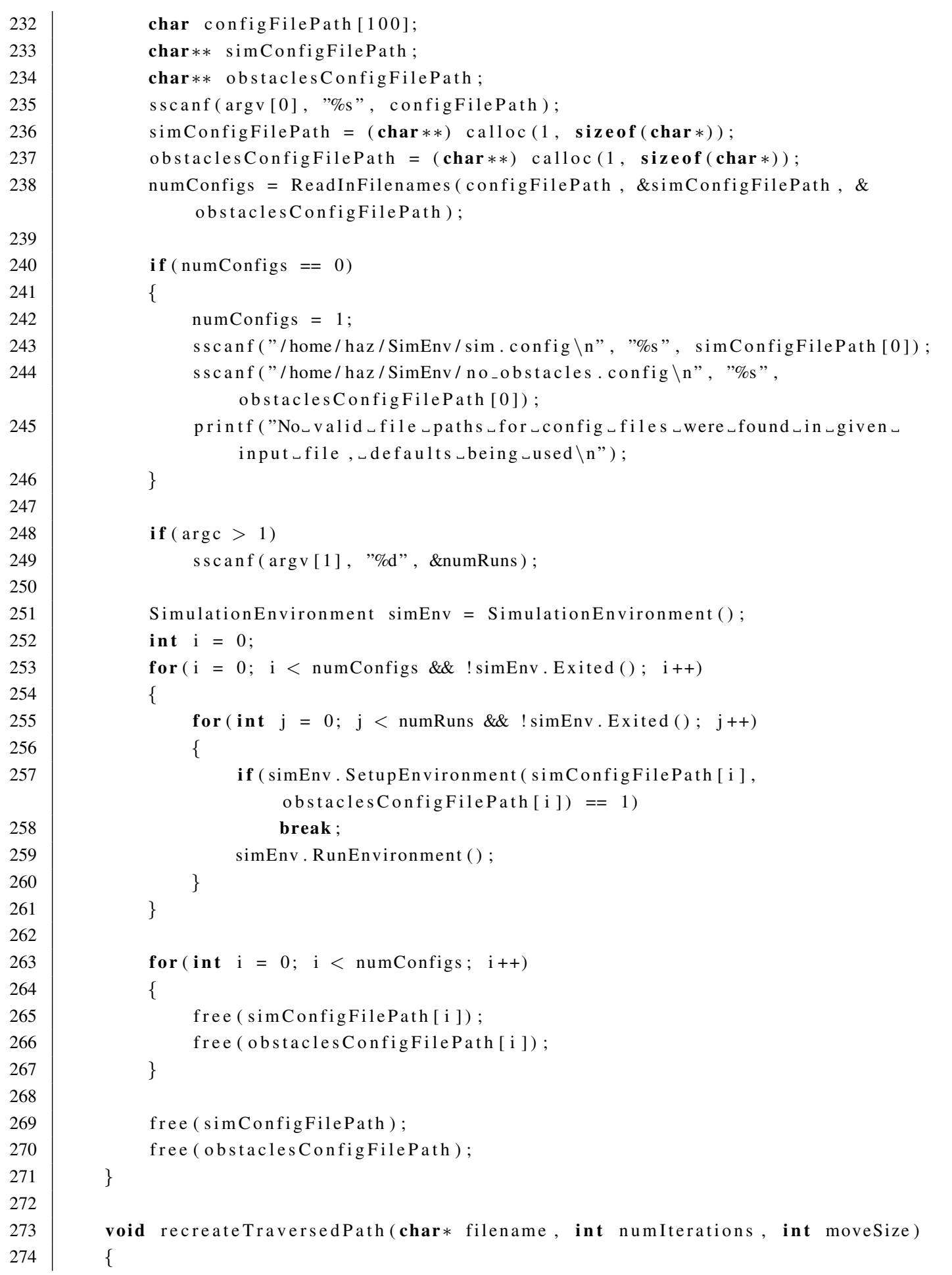




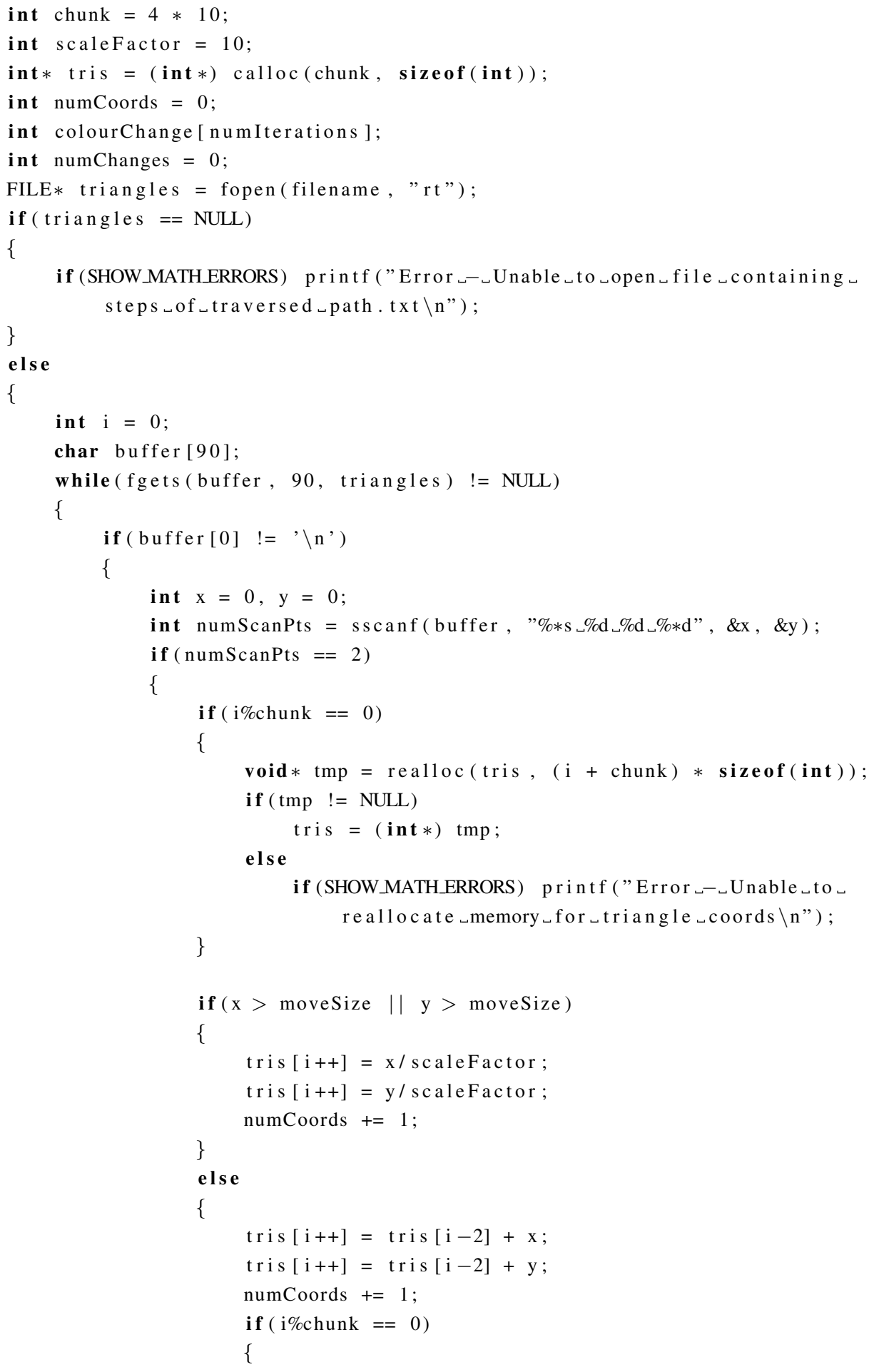




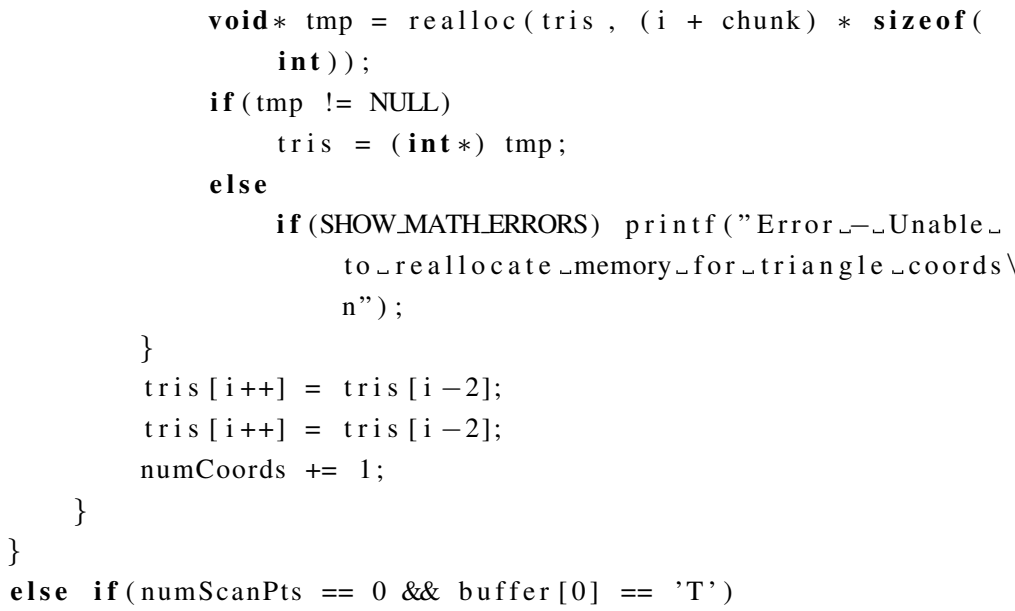




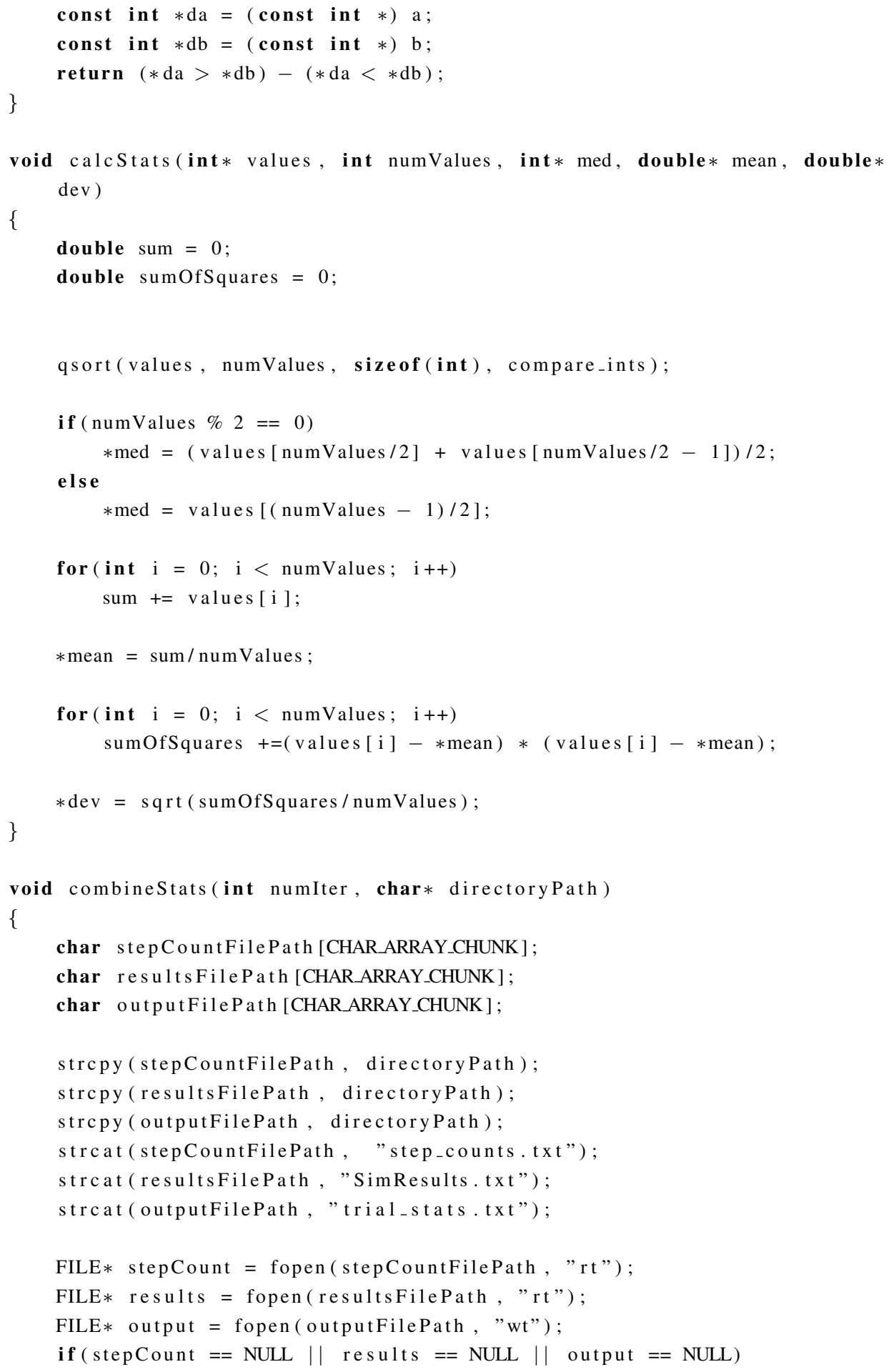




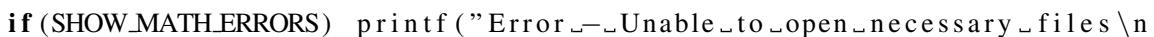
$"$; 
calcStats (stationary, numStation, \&stationMed, \& stationMean, \&stationDev);

if ( numAlt $\quad !=0$ )

calcStats (alternate, numAlt, \&altMed, \&altMean, \& altDev );

if ( numHaz $\quad !=0$ )

calcStats (hazard, numHaz, \&hazMed, \&hazMean, \&hazDev) ;

fprintf (output, "Success ${ }_{-} \backslash$ n");

fprintf (output, "Reached_Goal_-_Occurrences: ${ }_{\smile} \% \mathrm{~d}_{-} \backslash$ tMedian : ヶ\%d $\backslash$ tMean : „\%.3f $\backslash \mathrm{tS}$.D: $\_\% .3 \mathrm{f} \backslash \mathrm{n}$ ”, numSucc, succMed, succMean, succDev);

fprintf (output, "Failure ${ }_{-} \backslash$ n");

fprintf (output, "Became $\_$stationary ${ }_{-} \_$Occurrences : $\left.{ }_{-} \% \mathrm{~d}_{-}\right)$ tMedian : „\%d $\backslash$ tMean : _\%.3f $\backslash \mathrm{tS} . \mathrm{D}$ : _\%.3f $\backslash \mathrm{n}$ ”, numStation,

stationMed, stationMean, stationDev);

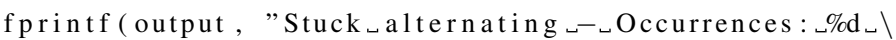

tMedian: $\%$ d $\backslash$ tMean : _\%.3f $\backslash \mathrm{tS} . \mathrm{D}: \_\% .3 \mathrm{f} \backslash \mathrm{n}$ ”, numAlt, altMed , altMean, altDev);

fprintf (output, "Hit $\lrcorner$ hazard $\_-\_$Occurrences : $\lrcorner \%_{\lrcorner} \backslash$ tMedian : $\lrcorner \%$ $\mathrm{d} \backslash \mathrm{tMean}: \_\% .3 \mathrm{f} \backslash \mathrm{tS} . \mathrm{D}: \_\% .3 \mathrm{f} \backslash \mathrm{n} \backslash \mathrm{n} \backslash \mathrm{n}$ ”, numHaz, hazMed, hazMean, hazDev);

succMed $=0 ;$ stationMed $=0 ;$ altMed $=0 ;$ hazMed $=0 ;$ succMean $=0 ;$ succDev $=0 ;$ stationMean $=0 ;$ stationDev $=$ 0

altMean $=0 ;$ altDev $=0 ;$ hazMean $=0 ;$ hazDev $=0 ;$

numSucc $=0 ;$ numStation $=0 ;$ numAlt $=0 ;$ numHaz $=0 ;$ \} \}

if ( numSucc $\quad !=0)$

calcStats (success, numSucc, \&succMed, \&succMean, \&succDev); if ( numStation $!=0)$

calcStats (stationary, numStation, \&stationMed, \&stationMean, \& stationDev);

if (numAlt $\quad !=0$ )

calcStats (alternate, numAlt, \&altMed, \&altMean, \&altDev);

if (numHaz $!=0$ )

calcStats (hazard, numHaz, \&hazMed, \&hazMean, \&hazDev);

fprintf (output, "Success ${ }_{-}-\backslash$ n") ;

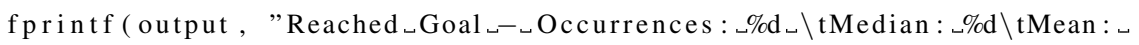
$\% .3 \mathrm{f} \backslash \mathrm{tS} . \mathrm{D}:$. \%.3f $\backslash \mathrm{n}$ ”, numSucc, succMed, succMean, succDev); 


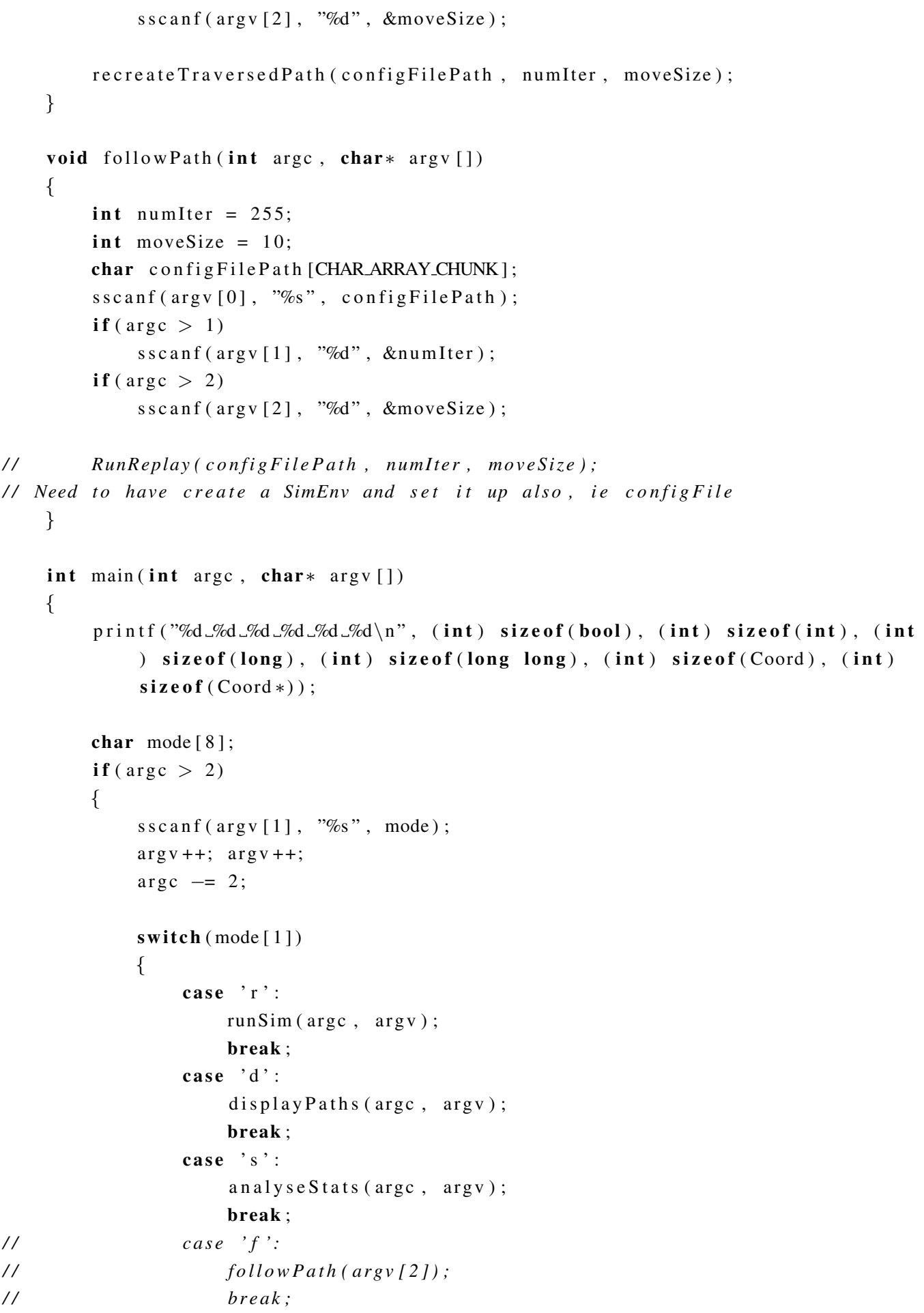




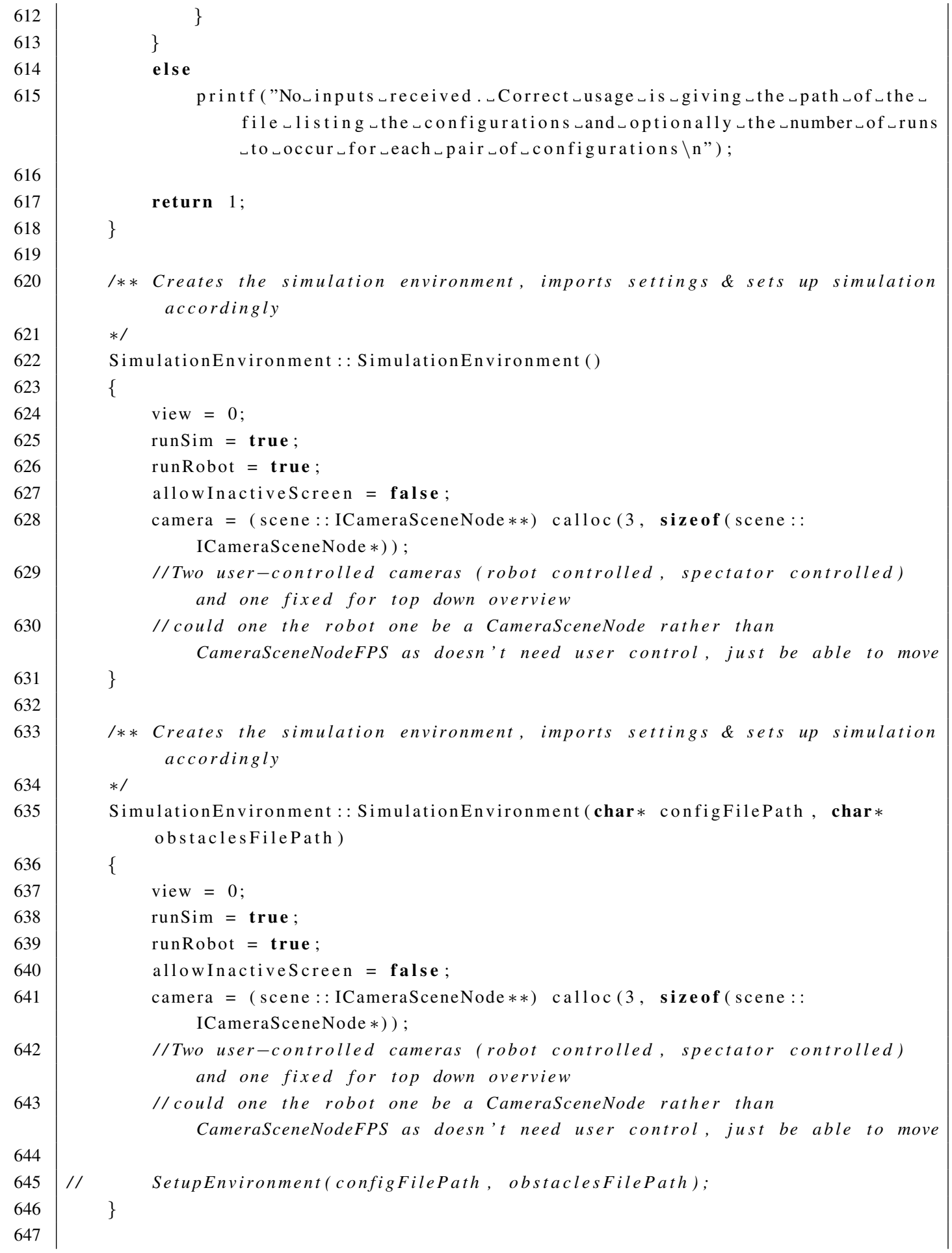




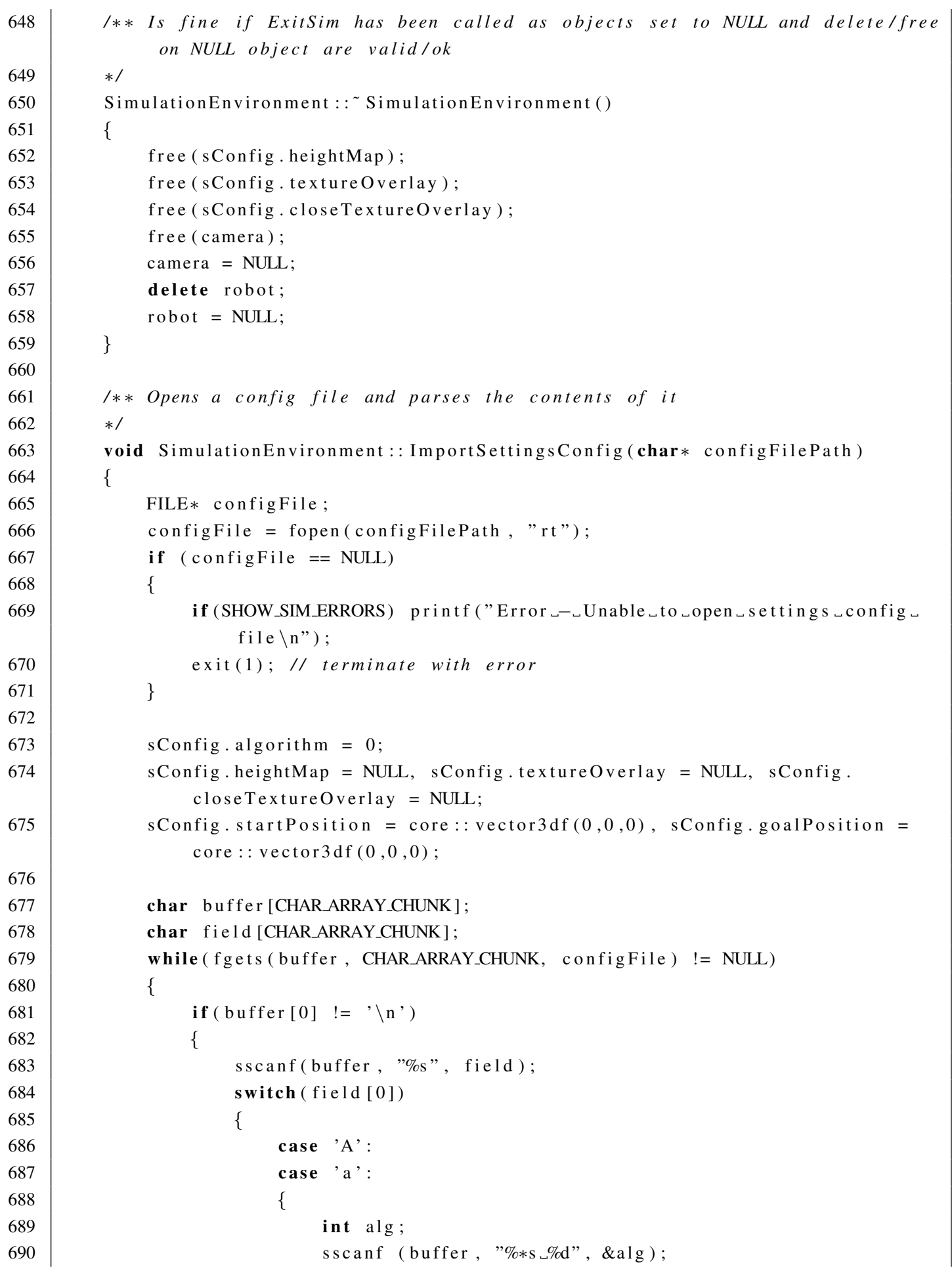




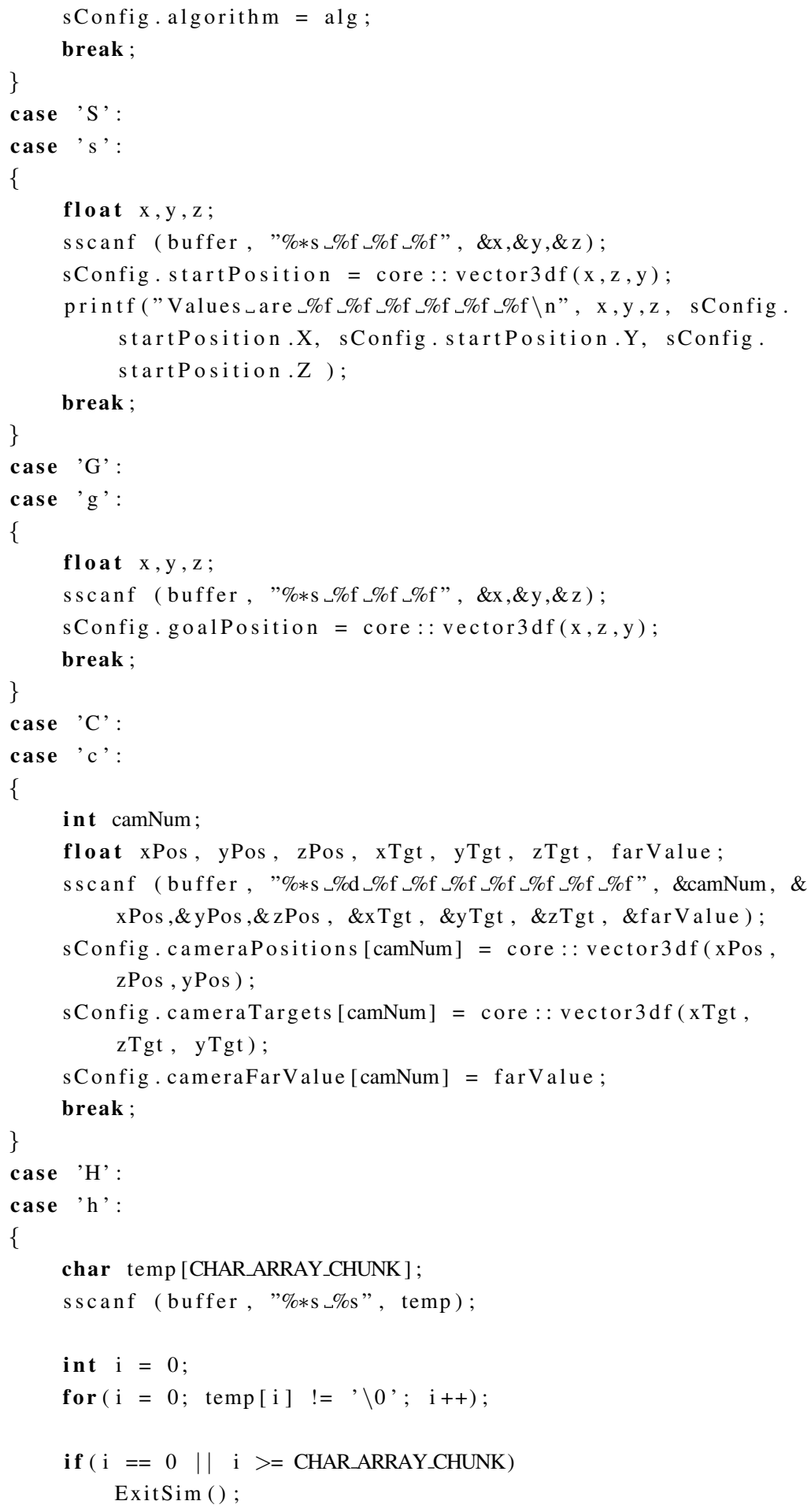


sConfig.heightMap $=(\operatorname{char} *) \operatorname{calloc}(i+1, \operatorname{sizeof}(\operatorname{char})) ; / / /$ add a free() somewhere

for (int $\mathrm{j}=0 ; \mathrm{j}<=\mathrm{i} ; \mathrm{j}++)$

$\operatorname{sConfig} \cdot \operatorname{heightMap}[j]=\operatorname{temp}[j]$;

break ;

\}

case ' $\mathrm{T}$ ':

case ' $t$ ':

\{

int textureNum;

char temp [CHAR_ARRAY_CHUNK];

sscanf (buffer, "\%*s $\_$d $\_$s", \&textureNum, temp) ;

int $\mathrm{i}=0$;

for $(i=0 ;$ temp $[i] !=, \backslash 0, ; i++)$;

if $(\mathrm{i}>=$ CHAR_ARRAY_CHUNK)

ExitSim ():

if $($ textureNum $==0)$

\{

sConfig. textureOverlay $=(\operatorname{char} *)$ calloc $(i+1, \operatorname{sizeof}($ char) );///add a free() somewhere

for (int $\mathrm{j}=0 ; \mathrm{j}<=\mathrm{i} ; \mathrm{j++}$ )

sConfig.textureOverlay $[j]=\operatorname{temp}[j]$;

\}

else

\{

sConfig. closeTextureOverlay $=(\operatorname{char} *)$ calloc $(\mathrm{i}+1$, sizeof(char));///add a free() somewhere

for (int $\mathrm{j}=0 ; \mathrm{j}<=\mathrm{i} ; \mathrm{j}++)$

sConfig.closeTextureOverlay $[j]=\operatorname{temp}[j]$;

\}

break ;

\}

case ' $\backslash \mathrm{n}$ ':

break ;

default :

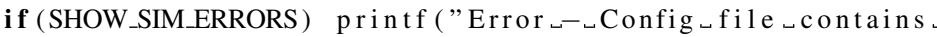

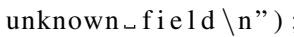

break ;

\}

\} 


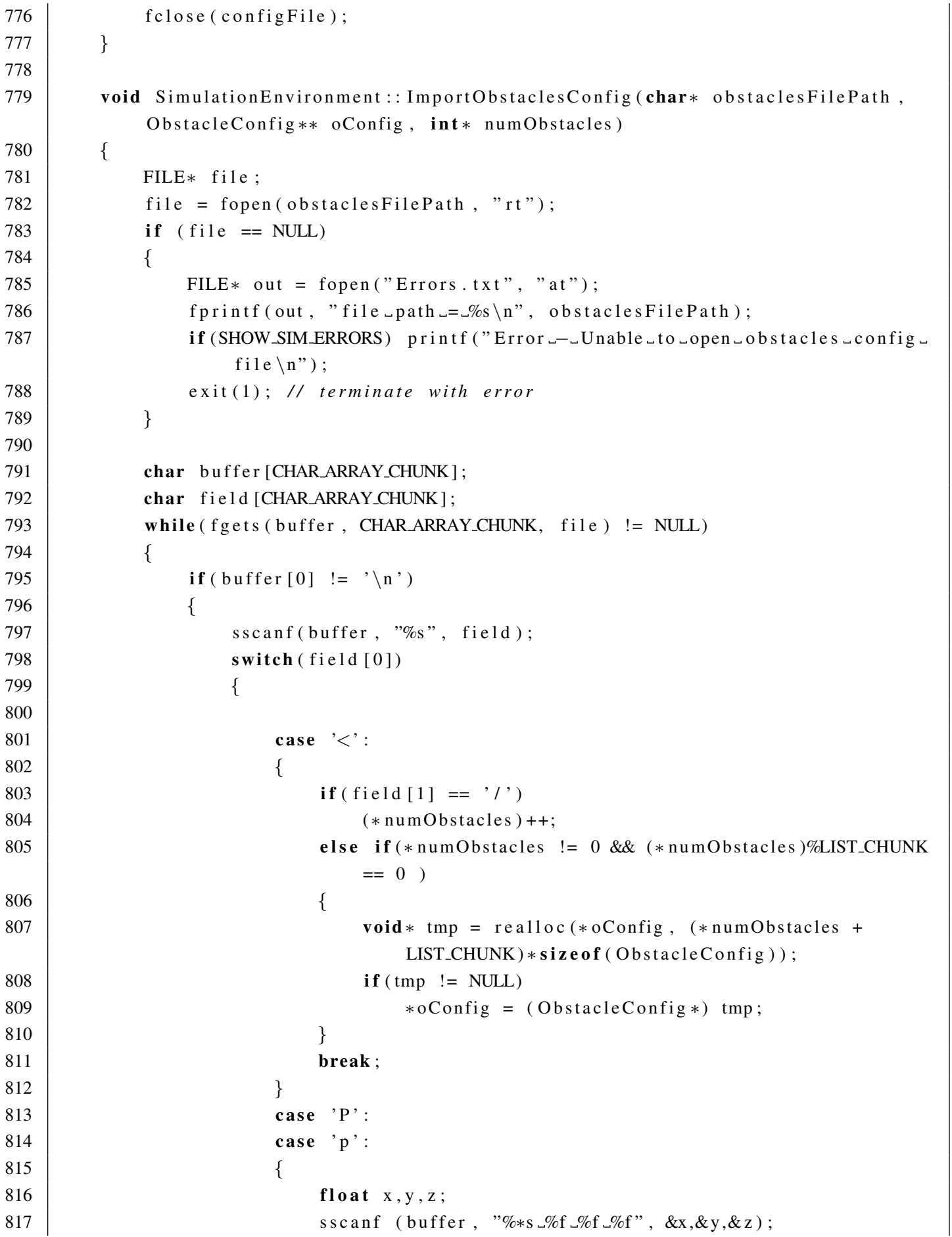




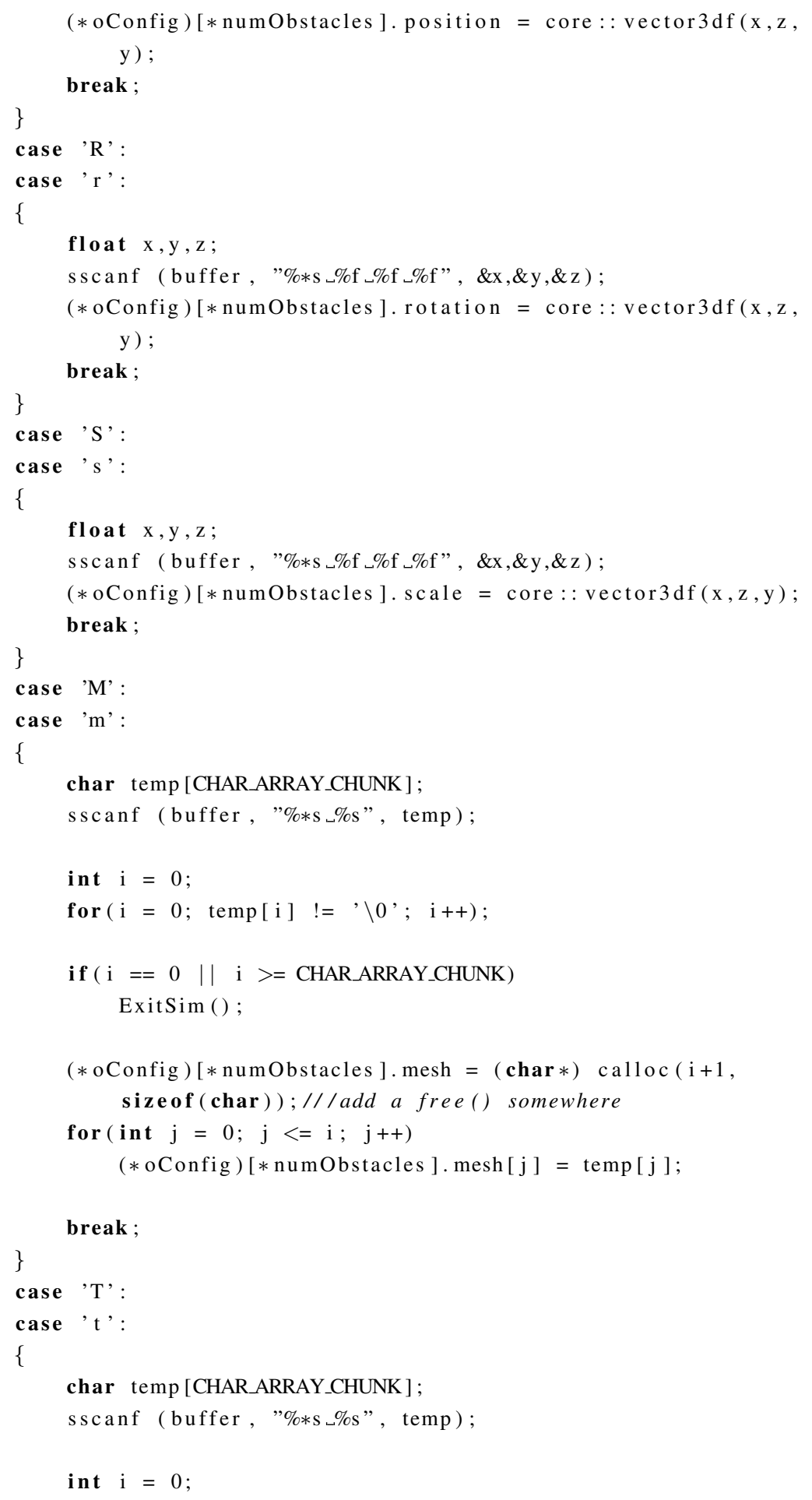




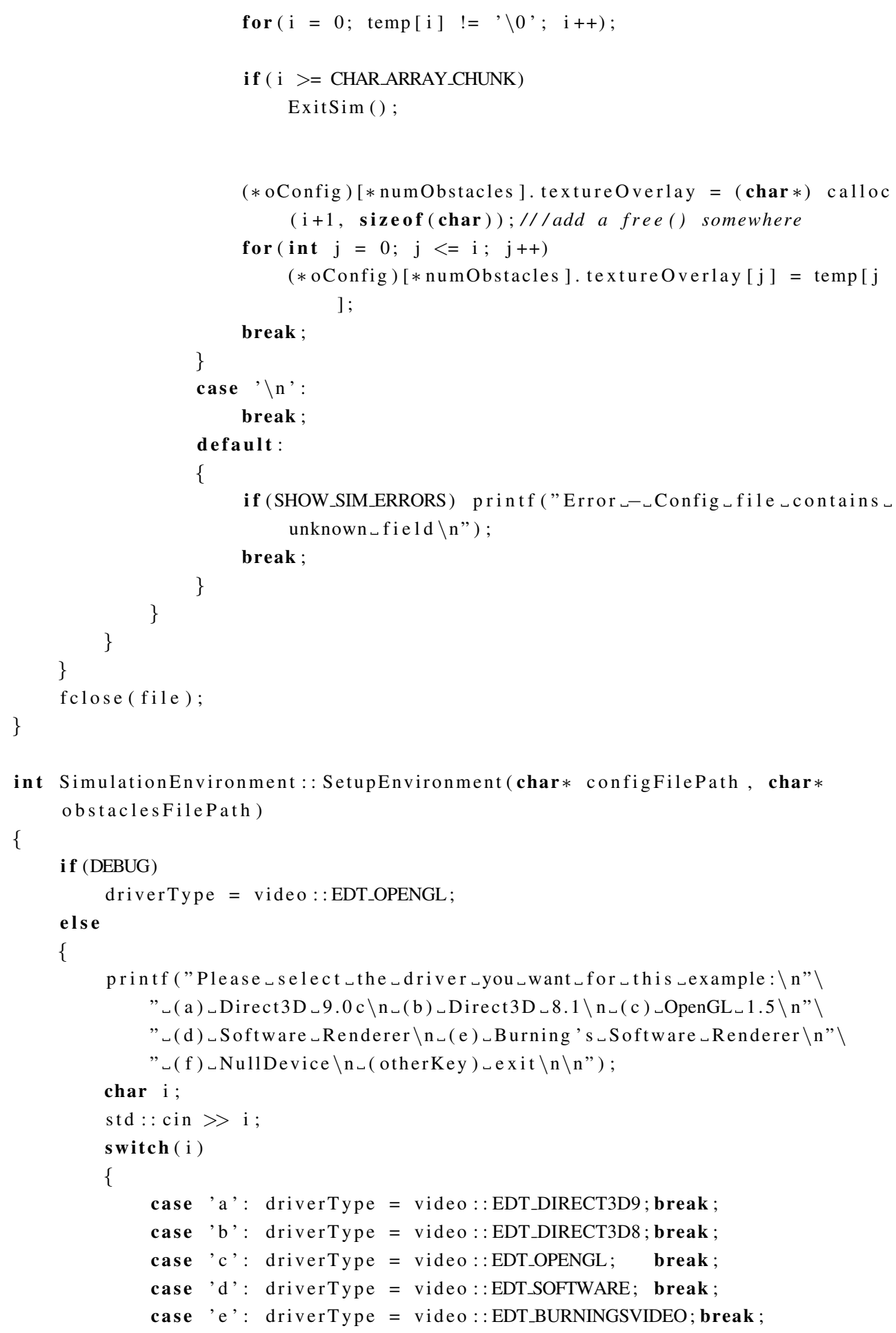




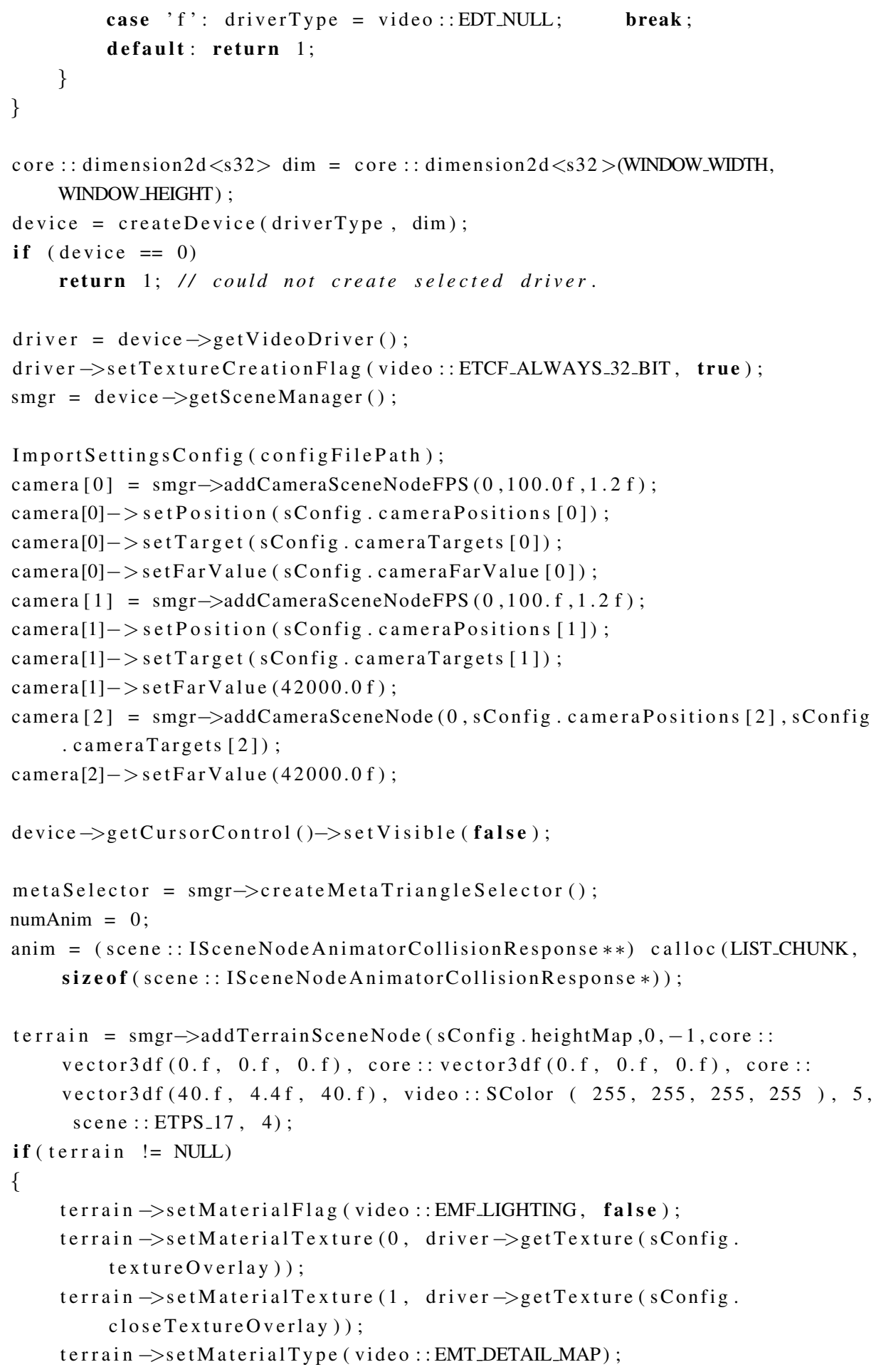




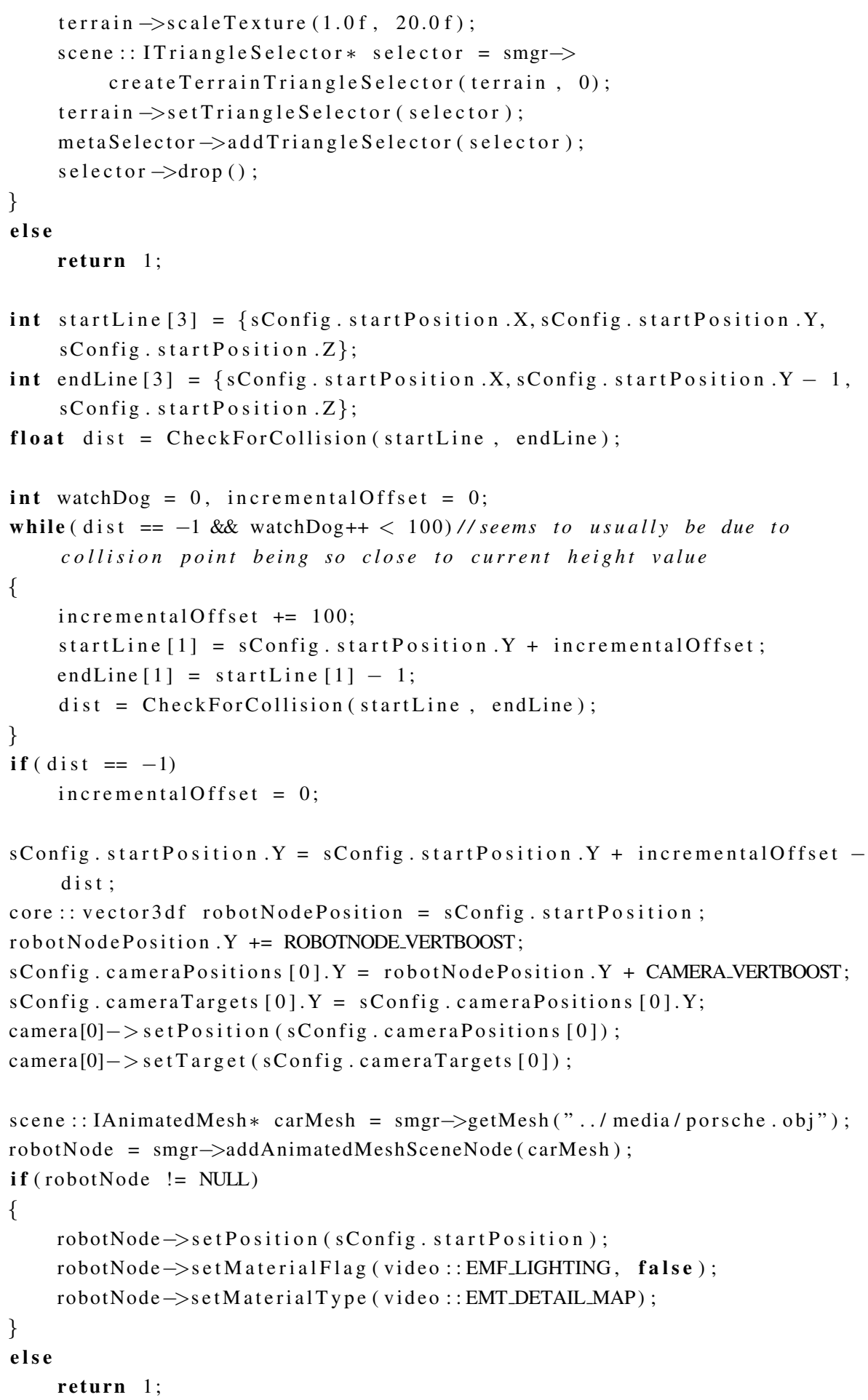




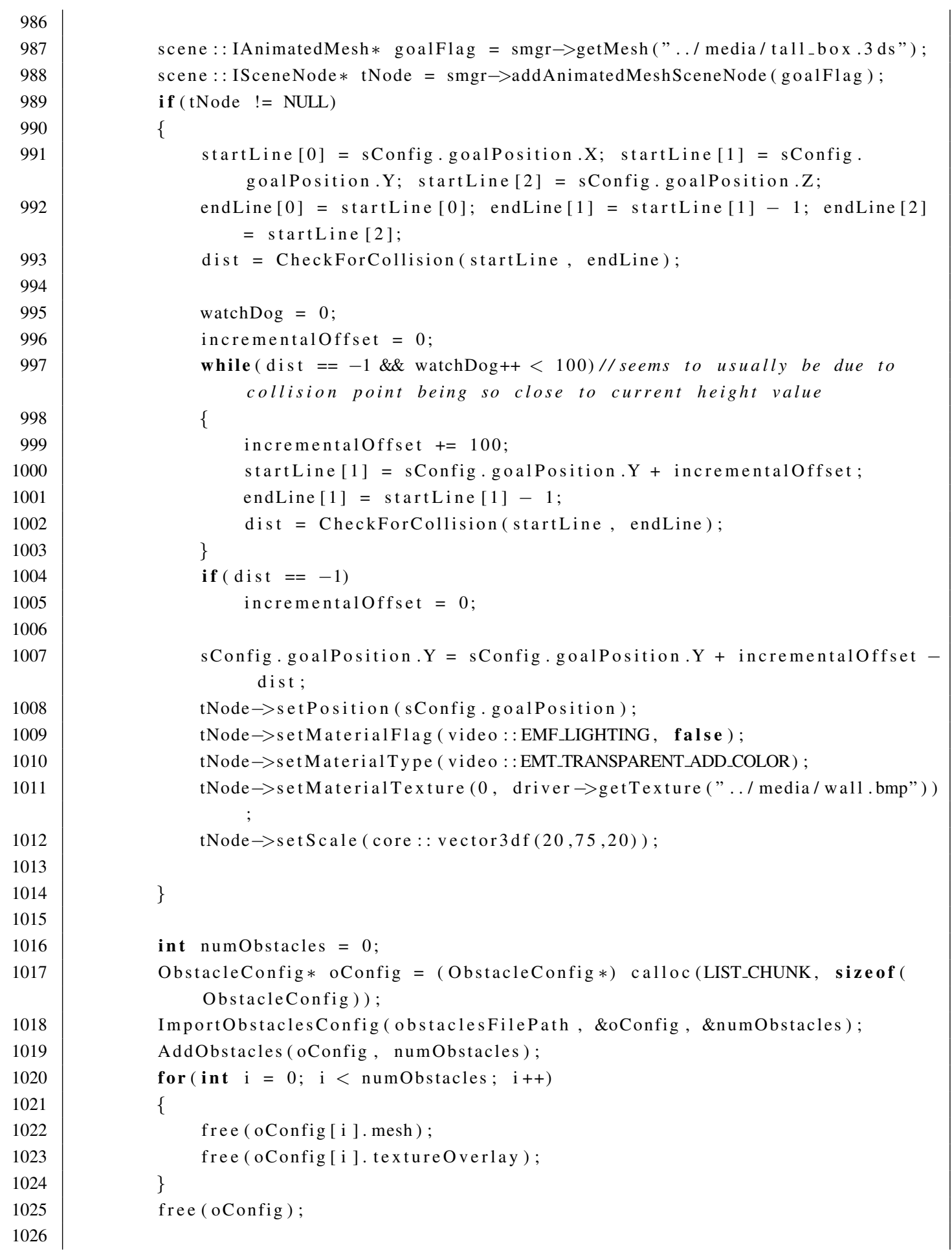




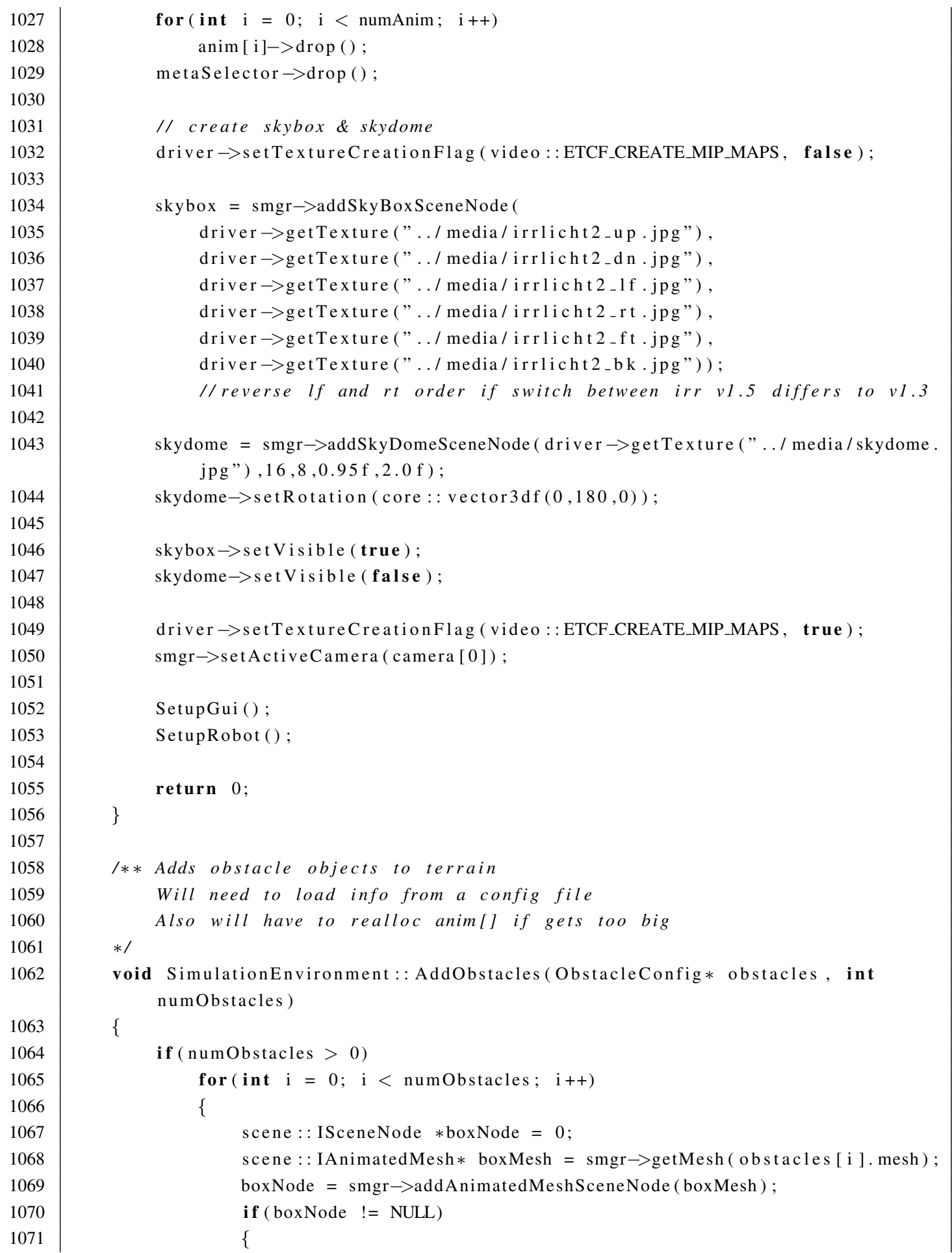


boxNode $\rightarrow$ setMaterialFlag (video::EMF_LIGHTING, false ); boxNode $\rightarrow$ setMaterialType (video : : EMT_DETAIL_MAP) ; boxNode $\rightarrow$ setMaterialTexture $(0$, driver $\rightarrow$ getTexture (obstacles [ i ]. textureOverlay));

boxNode $\rightarrow$ setScale (obstacles [i]. scale); boxNode $\rightarrow$ setRotation (obstacles [i]. rotation); 
core : : vector $3 \mathrm{df}(0,0,0))$; boxNode $\rightarrow$ addAnimator (anim [ numAnim ++$]$ );

if $($ numAnim\%LIST_CHUNK $==0)$

\{

void $* \operatorname{tmp}=$ realloc $($ anim, $\quad($ numAnim + LIST_CHUNK $) * \operatorname{sizeof}($ scene : : ISceneNodeAnimatorCollisionResponse $*)$ );

if ( $\mathrm{tmp} !=\mathrm{NULL})$

anim $=($ scene $:$ ISceneNodeAnimatorCollisionResponse $* *)$ tmp ; 


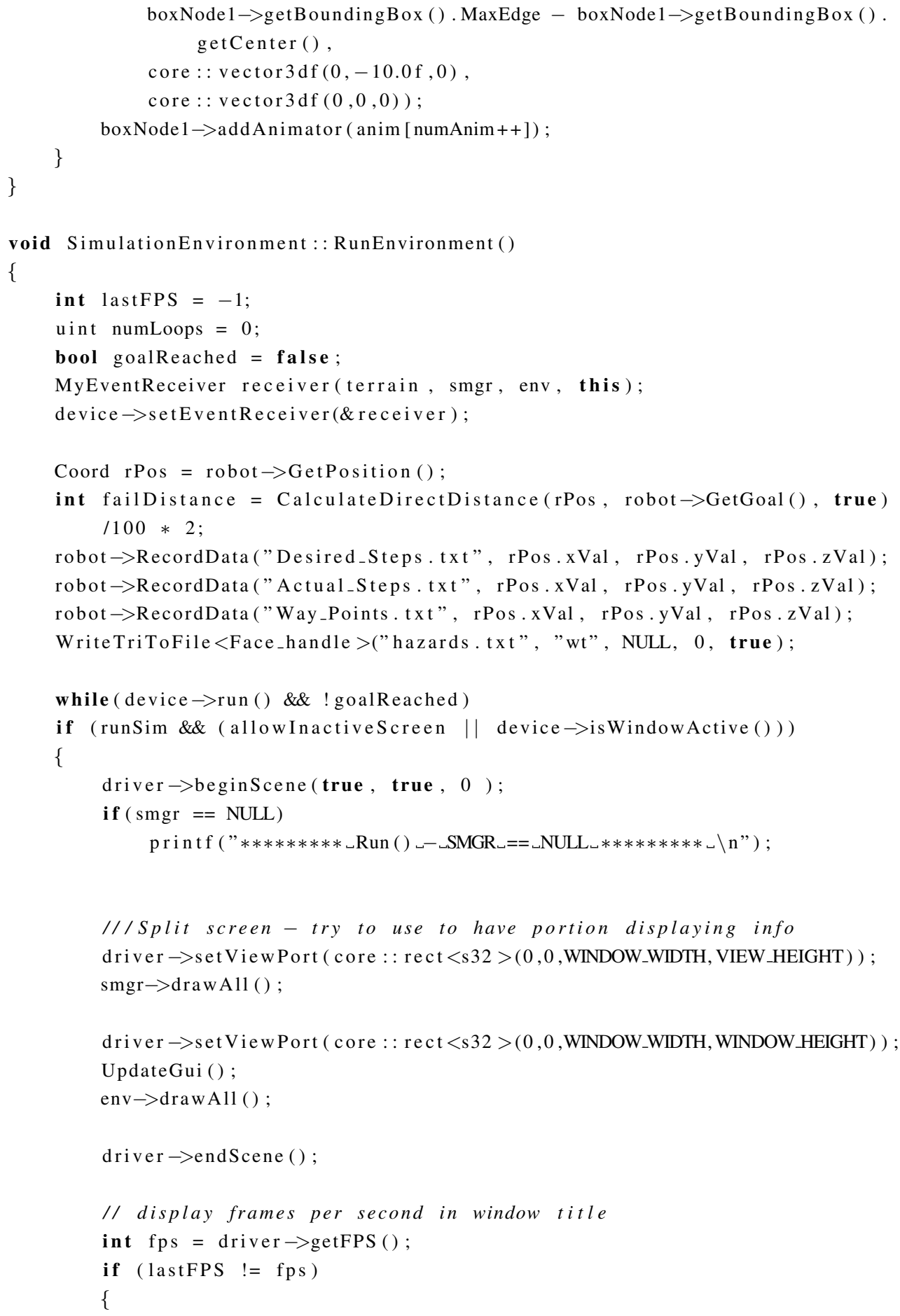




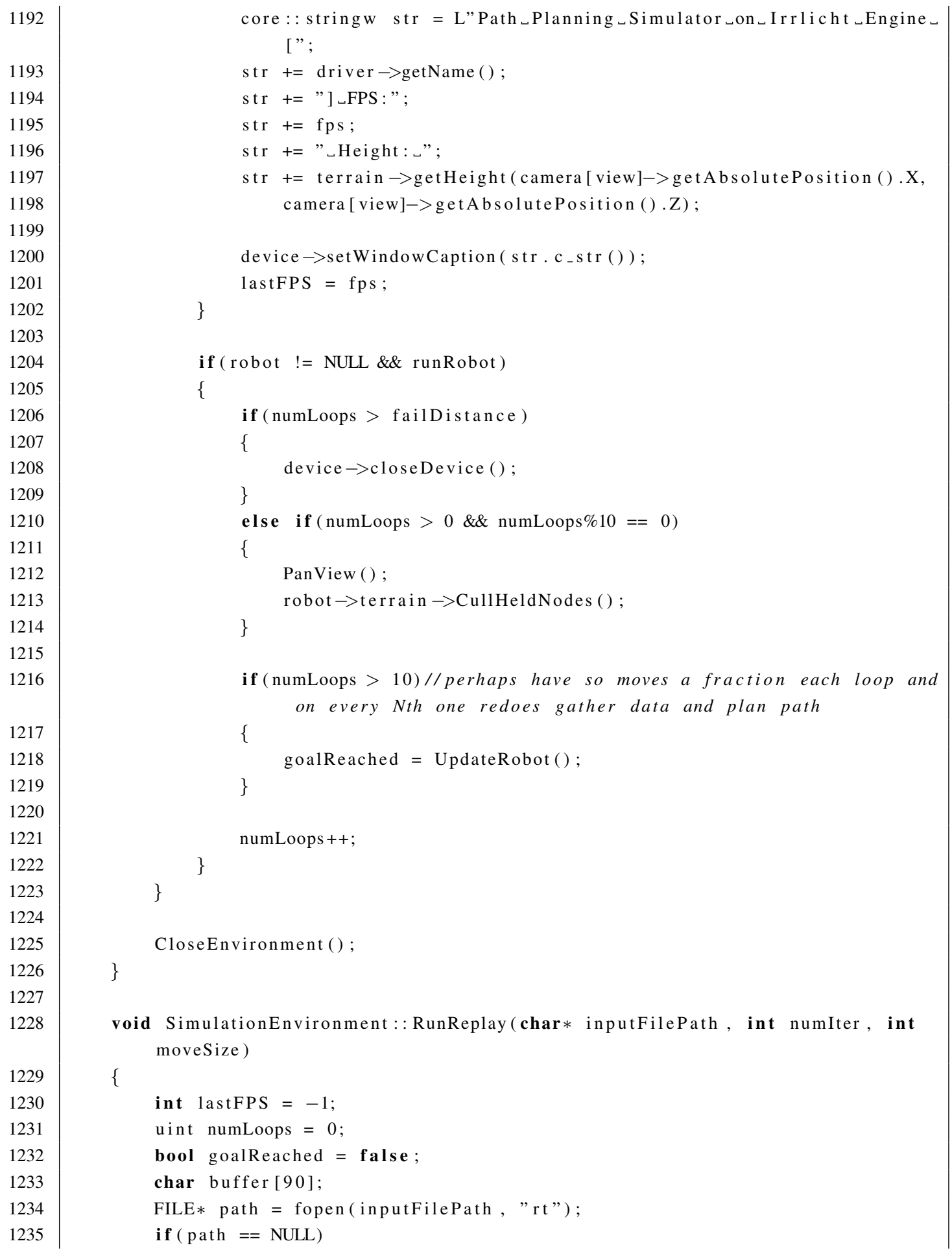


\{

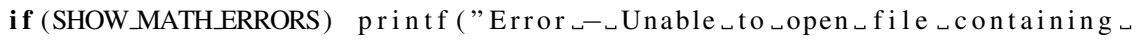
steps_of_traversed_path.txt $\backslash n ")$; return ;

\}

MyEventReceiver receiver(terrain, smgr, env, this); device $\rightarrow$ setEventReceiver(\&receiver);

while (device $\rightarrow$ run () \&\& ! goalReached)

if (runSim \&\& (allowInactiveScreen || device $\rightarrow$ isWindowActive()))

\{

driver $\rightarrow$ beginScene (true, true, 0 );

if $(\mathrm{smgr}==\mathrm{NULL})$

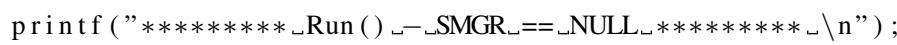

///Split screen - try to use to have portion displaying info driver $\rightarrow$ set View Port ( core $:$ : rect $<$ s32 $>(0,0$,WINDOW_WIDTH, VIEW_HEIGHT) );

smgr $\rightarrow$ drawAll ();

driver $\rightarrow$ setView Port ( core $:: \operatorname{rect}<\mathrm{s} 32>(0,0$, WINDOW_WIDTH,WINDOW_HEIGHT) $)$; UpdateGui () ;

env $\rightarrow$ draw All () ;

driver $\rightarrow$ endScene ();

// display frames per second in window title

int $\mathrm{fps}=$ driver $\rightarrow$ getFPS () ;

if (lastFPS $!=\mathrm{fps})$

\{

core: : stringw str $=$ L"Path $\lrcorner$ Planning $\lrcorner$ Simulator $\_$on $\lrcorner$Irrlicht ${ }_{\lrcorner}$Engine [";

str $+=$ driver $\rightarrow$ getName () ;

str $+=$ ] ]_FPS:";

str $+=$ fps ;

str $+=$ "Height: $: "$;

str $+=$ terrain $\rightarrow$ getHeight (camera[view] $->$ getAbsolutePosition().X, camera [view]->getAbsolutePosition ().Z);

device $\rightarrow$ setWindowCaption ( str. c-str ());

lastFPS $=$ fps;

\}

if (fgets (buffer, 90, path) != NULL)

\{

if (buffer [0] != ’’n')

\{

core: : vector $3 \mathrm{df}$ step, newPos;

int $\mathrm{x}=0, \mathrm{y}=0, \mathrm{z}=0$; 


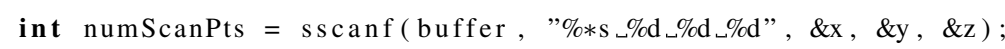
if $($ numScanPts $==3)$ \{ 
env $\rightarrow$ addStaticText (L”No־Mode־Selected”, core $:$ : rect $<$ s32 $>($ GAP +45 , VIEW_HEIGHT + GAP, GAP + COLUMN_WIDTH, VIEW_HEIGHT + GAP + LINE_HEIGHT), true , true , env $\rightarrow$ getRootGUIElement ( ) , GUI_ID_MODE, true );

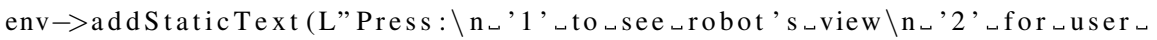

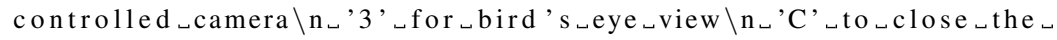
simulation", core : rect $<$ s32 $>$ (GAP, VIEW_HEIGHT + GAP + LINE_HEIGHT, GAP + COLUMN_WIDTH, WINDOW_HEIGHT - GAP), true, true , 0, GUI_ID_CTRLS , true ) ; 


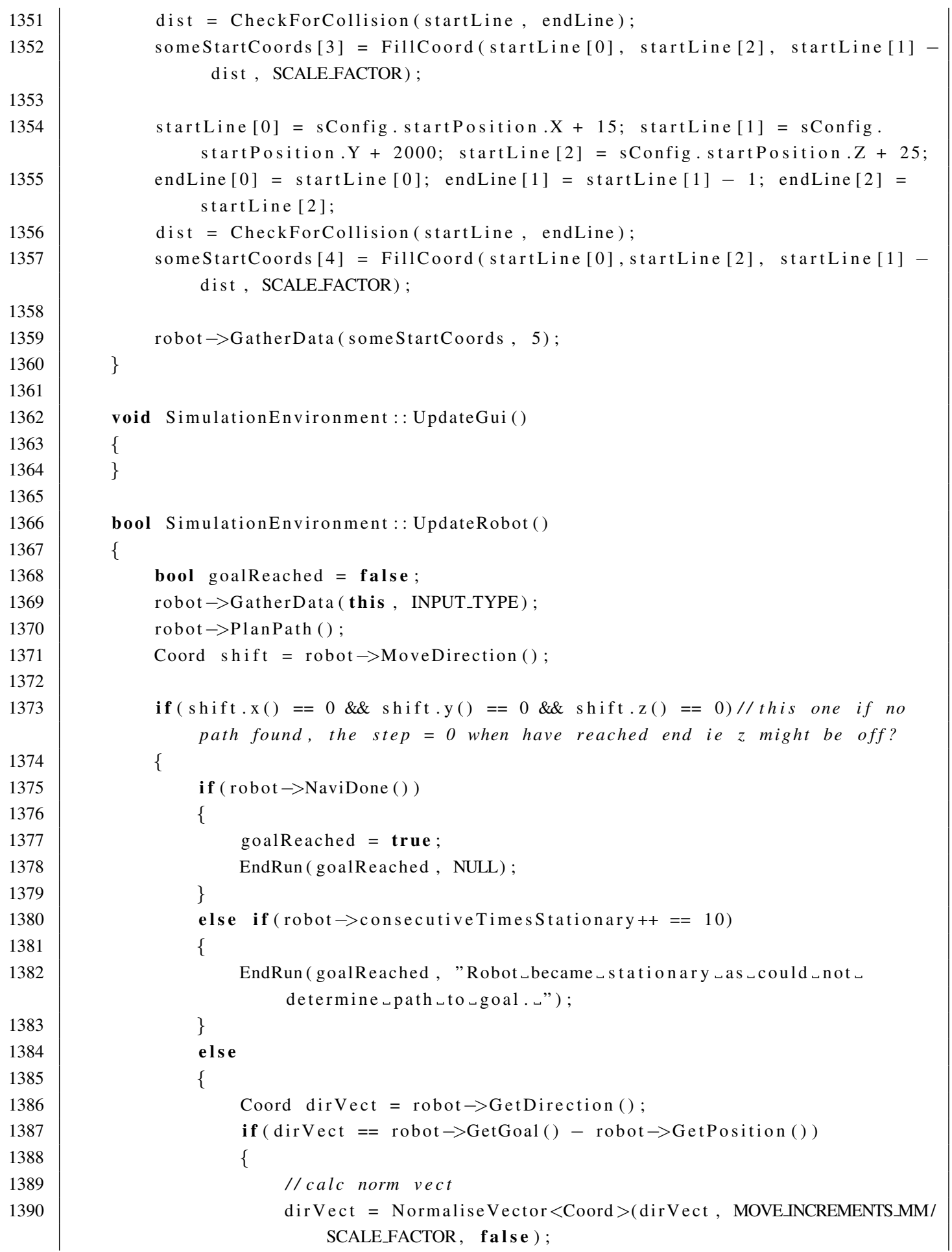


Coord normVect $=$ CrossProduct $($ dirVect, dirVect + Coord $(0,0$, MOVEINCREMENTS_MM/SCALE_FACTOR) ) ; normVect $=$ Normalise Vector $<$ Coord $>$ (normVect, MOVE_INCREMENTS_MM/SCALE_FACTOR, f a l s e ) ;

dirVect $=$ dirVect + normVect $; / /$ results in 45 degree angle change

\}

else

dirVect $=$ robot $\rightarrow$ GetGoal ()$-\operatorname{robot} \rightarrow$ GetPosition ()$;$

if (dirVect.z() > dirVect.y() \&\& dirVect.z() > dirVect.x()) //? or greater than 6 or -6 ?

dirVect.zVal $=0$;

if $(!(\operatorname{dirVect} . x()==0 \& \&$ dirVect.y( ()$==0))$

\{

robot $\rightarrow$ SetDirection (dirVect);

core: : vector3df newCamTarget $=$ camera[0]-> getPosition ()$+$ core:: vector3df(dirVect.xVal, dirVect.zVal, dirVect.yVal)

camera[0]-> setTarget (newCamTarget);

\}

else

int $\mathrm{cat}=7$;

\}

\}

else

\{

core : : vector $3 \mathrm{df}$ step = core : : vector $3 \mathrm{df}(\mathrm{shift} . \mathrm{xVal} /$ SCALEFACTOR, shift . zVal/SCALE_FACTOR, shift . yVal/SCALE_FACTOR);

\{

float moveDist $=$ MOVE_INCREMENTS_MM/SCALE_FACTOR;

if (CalculateDirectDistance (robot $\rightarrow$ GetPosition (), robot $\rightarrow$ GetGoal ()

, true)/SCALEFACTOR $<$ moveDist)// issue due to $z$ coordinate.

moveDist $=$ CalculateDirectDistance $($ robot $\rightarrow$ GetPosition () ,

robot $\rightarrow$ GetGoal (), true)/SCALE_FACTOR;

step $=$ core $:$ : vector $3 \mathrm{df}(\mathrm{shift} \cdot \mathrm{xVal}, 0$, shift.yVal $)$.normalize ();

if ( shift.xVal $!=0)$

step.Y $=$ shift.zVal $*$ step.X/shift. $x$ Val ;

else if (shift.yVal != 0 )

step.Y $=$ shift.zVal $*$ step.Z/ shift $\cdot$ yVal;

step $=$ step $*$ moveDist;

\}

robot $\rightarrow$ RecordMoreData("Desired_Steps.txt”, step.X, step.Z, step.Y);// * by scale? 


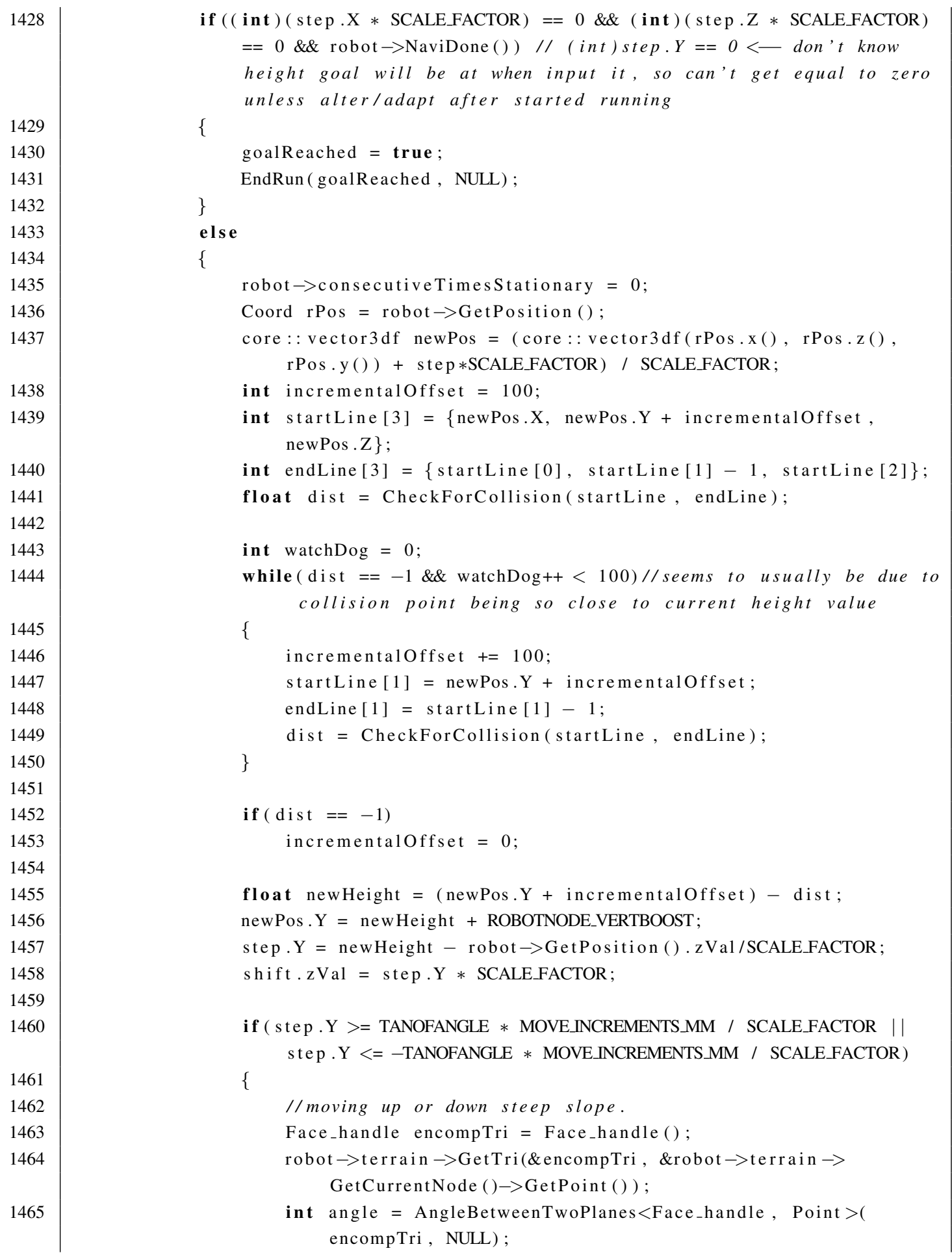


int angle $0=$ AngleBetweenTwoPlanes $<$ Face_handle, Point $>($ encompTri $\rightarrow$ neighbor $(0)$, NULL);

int angle 1 = AngleBetweenTwoPlanes $<$ Face_handle, Point $>($ encompTri $\rightarrow$ neighbor (1), NULL);

int angle 2 = AngleBetweenTwoPlanes $<$ Face_handle, Point $>$ ( encompTri $\rightarrow$ neighbor(2), NULL);

float dist $[2]=\{-1,-1\}$;

float dist $0[2]=\{-1,-1\}$;

float dist $1[2]=\{-1,-1\}$;

float dist $2[2]=\{-1,-1\}$

CalculateDistsToCentre $(*$ encompTri $\rightarrow$ GetPoint $(0), *$ encompTri $\rightarrow$ GetPoint (1), *encompTri $\rightarrow$ GetPoint (2), dist);

CalculateDistsToCentre (*encompTri $\rightarrow$ neighbor $(0) \rightarrow$ GetPoint $(0)$, *encompTri $\rightarrow$ neighbor $(0)->$ GetPoint $(1), *$ encompTri $\rightarrow$ neighbor (0)->GetPoint (2), dist 0$)$;

CalculateDistsToCentre (*encompTri $\rightarrow$ neighbor $(1) \rightarrow$ GetPoint $(0)$, *encompTri $\rightarrow$ neighbor (1)->GetPoint (1), *encompTri $\rightarrow$ neighbor(1) $\rightarrow$ GetPoint (2), dist 1$)$;

CalculateDistsToCentre (*encompTri $\rightarrow$ neighbor (2) $\rightarrow$ GetPoint $(0)$, *encompTri->neighbor (2)->GetPoint (1), *encompTri $\rightarrow$ neighbor(2) $\rightarrow$ GetPoint (2), dist 2$)$;

if $(($ angle $>$ SLOPEANGLE $\& \&$ angle $<180-$ SLOPEANGLE $\& \&$ dist $[0]$ $<$ HAZARD_TRI_DIST_THRESHOLD) $\|$ (angle $0>$ SLOPEANGLE \&\& angle $0<180-$ SLOPEANGLE \&\& dist $0[0]<$ HAZARD_TRI_DIST_THRESHOLD) $\|$ (angle $1>$ SLOPEANGLE \&\& angle $1<180-$ SLOPEANGLE \&\& dist $1[0]<$ HAZARD_TRI_DIST_THRESHOLD) $\|$ (angle $2>$ SLOPEANGLE $\& \&$ angle $2<180-$ SLOPEANGLE \&\& dist $2[0]<$ HAZARD_TRI_DIST_THRESHOLD ) )

\{

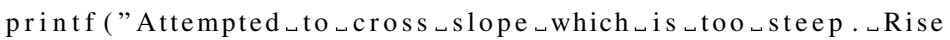
_of $\%$ d, _ angles_of $\%$ d $\backslash \mathrm{n} ”$, (int) step.Y, angle);

printf ("Angles $\lrcorner$ of $\lrcorner$ neighbours $\lrcorner$ are $\lrcorner \% d_{\lrcorner} \% d_{\lrcorner} \% d \backslash$ n”,,$\quad$ angle 0 , angle1, angle2);

EndRun (goalReached, "Simulation ended $_{\triangle}$ as $_{\triangle}$ robot tried $_{\lrcorner}$to traverse_hazard. .");

\}

else

\{

if (robot $\rightarrow$ terrain $\rightarrow$ GetTri(\&encompTri, \&Point (newPos.X, newPos.Z, newPos.Y)))

\{

angle $=$ AngleBetweenTwoPlanes $<$ Face_handle, Point $>($ encompTri, NULL);

angle $0=$ AngleBetweenTwoPlanes $<$ Face_handle, Point $>($ encompTri $\rightarrow$ neighbor (0), NULL); 
angle $1=$ AngleBetweenTwoPlanes $<$ Face_handle, Point $>$ ( encompTri $\rightarrow$ neighbor (1), NULL);

angle 2 = AngleBetweenTwoPlanes $<$ Face_handle, Point $>($ encompTri $\rightarrow$ neighbor (2), NULL);

CalculateDistsToCentre (*encompTri $\rightarrow$ GetPoint $(0)$, * encompTri $\rightarrow$ GetPoint (1), *encompTri $\rightarrow$ GetPoint (2), dist);

CalculateDistsToCentre (*encompTri $\rightarrow$ neighbor $(0) \rightarrow$ GetPoint $(0), *$ encompTri $\rightarrow$ neighbor $(0) \rightarrow$ GetPoint (1) , *encompTri $\rightarrow$ neighbor (0) $\rightarrow$ GetPoint (2), dist 0$)$;

CalculateDistsToCentre (*encompTri $\rightarrow$ neighbor (1) $\rightarrow$ GetPoint (0), *encompTri $\rightarrow$ neighbor (1) $\rightarrow$ GetPoint (1) , *encompTri $\rightarrow$ neighbor (1)->GetPoint (2), dist 1$)$;

CalculateDistsToCentre (*encompTri $\rightarrow$ neighbor $(2) \rightarrow$ GetPoint (0), *encompTri $\rightarrow$ neighbor (2) $\rightarrow$ GetPoint (1) , *encompTri->neighbor (2) $\rightarrow$ GetPoint (2), dist2); if $($ ( angle $>$ SLOPEANGLE $\& \&$ angle $<180-$ SLOPEANGLE \& \& dist $[0]<$ HAZARD_TRI_DIST_THRESHOLD) $\|$ (angle 0 $>$ SLOPEANGLE $\& \&$ angle $0<180-$ SLOPEANGLE $\& \&$ dist0 [0] < HAZARD_TRI_DIST_THRESHOLD) $\|$ (angle 1 $>$ SLOPEANGLE \&\& angle $1<180-$ SLOPEANGLE \& \& dist $1[0]<$ HAZARD_TRI_DIST_THRESHOLD) $\|$ (angle2 $>$ SLOPEANGLE $\& \&$ angle $2<180-$ SLOPEANGLE $\& \&$ dist $2[0]<$ HAZARD_TRI_DIST_THRESHOLD)

\{

printf ("Attempted $\sqcup$ to $\lrcorner$ cross $\sqcup$ slope $_{\sqcup}$ which $\sqcup$ is $_{\sqcup}$ too $\sqcup$

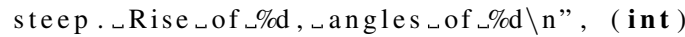
step.Y, angle);

printf ("Angles $\_$of $\lrcorner$neighbours $\lrcorner$are $\_$d $\_\%$ d $\_d \backslash$ n", angle0, angle 1 , angle 2$)$;

EndRun (goalReached, "Simulation $\_$ended $\_$as $\lrcorner$robot tried_to_traverse_hazard..”); \}

robot $\rightarrow$ RecordMoreData("Actual_Steps.txt", step.X, step.Z, step.Y) ; printf ("Moved_\%d_\%d_\%d $\backslash \mathrm{n} ", \quad$ (int) step.X, (int) step.Z, (int) step .Y) ;

robot $\rightarrow$ AdjustPosition ( (int) ( step.X $*$ SCALE_FACTOR), (int) ( step.Z

* SCALE_FACTOR), (int) ( step.Y * SCALE_FACTOR));//why use step over shift?

robotNode $\rightarrow$ setPosition (newPos); 


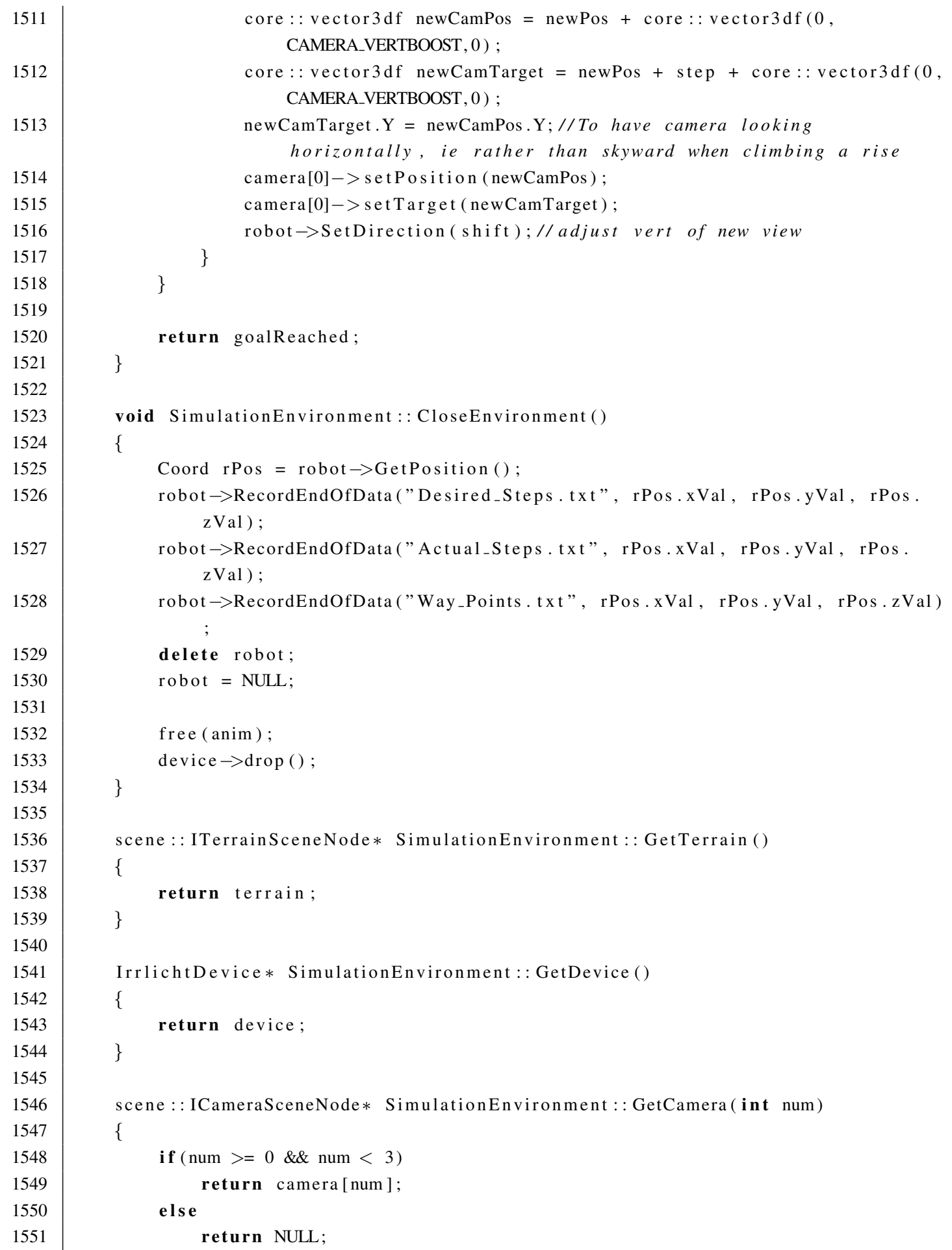




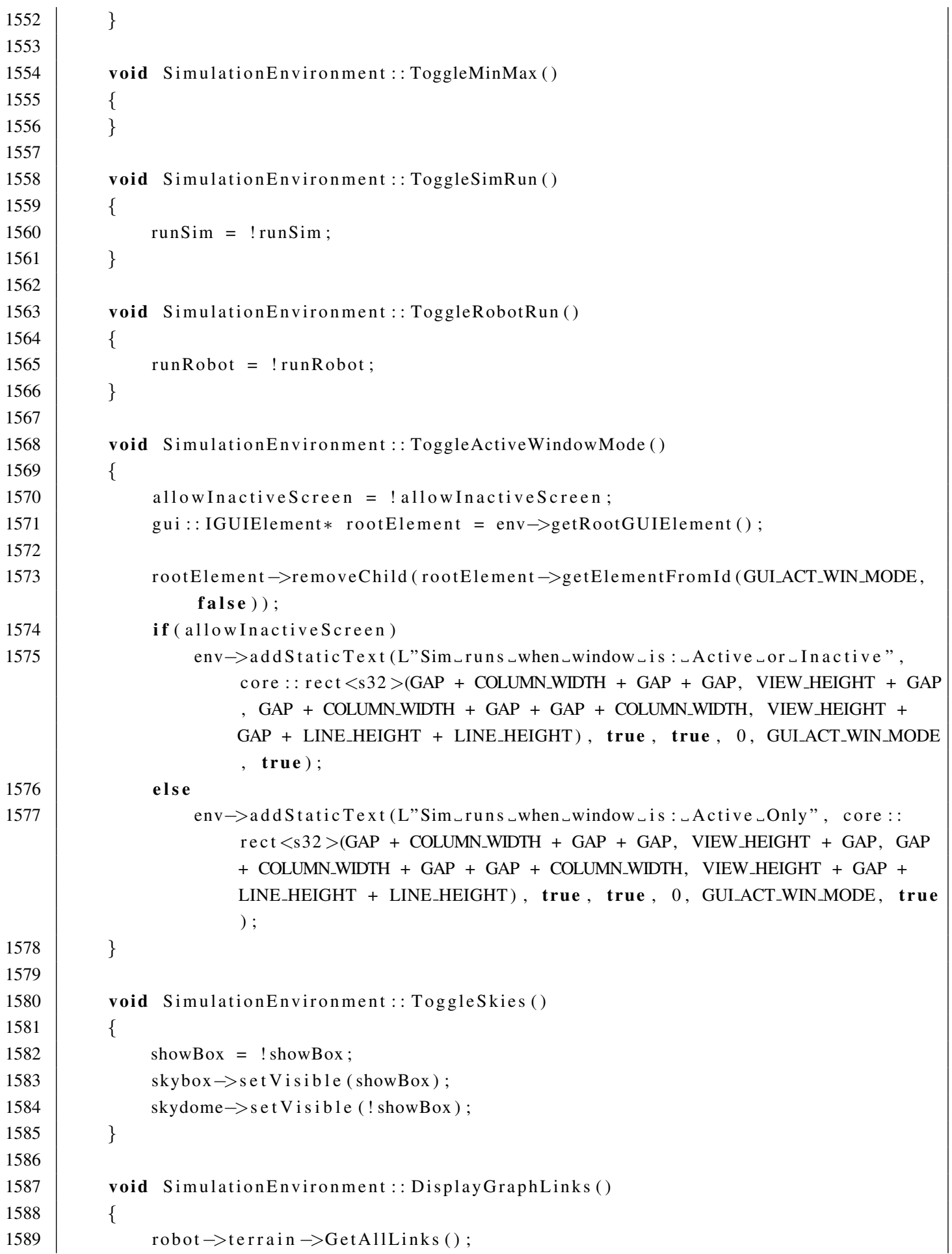




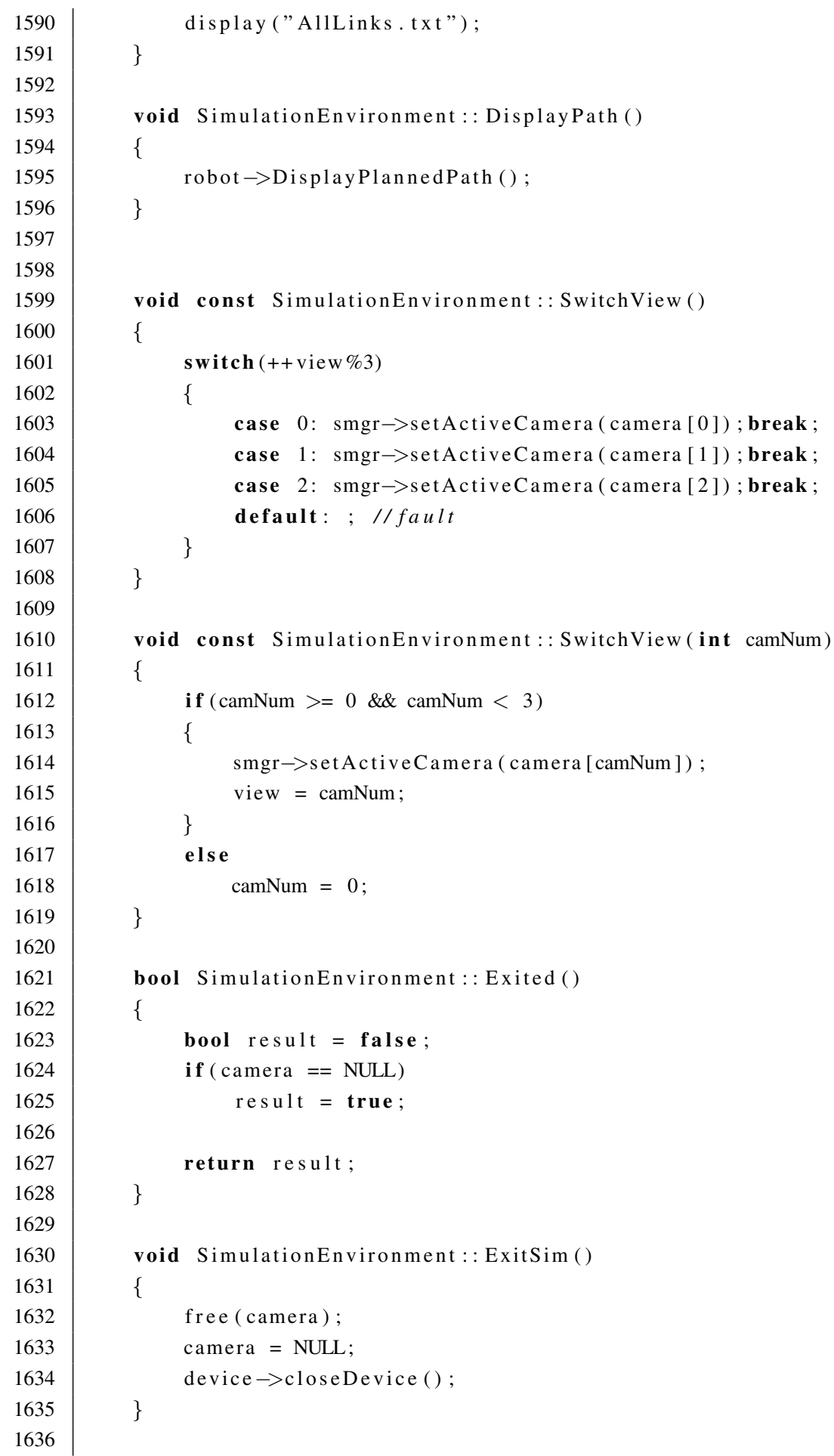




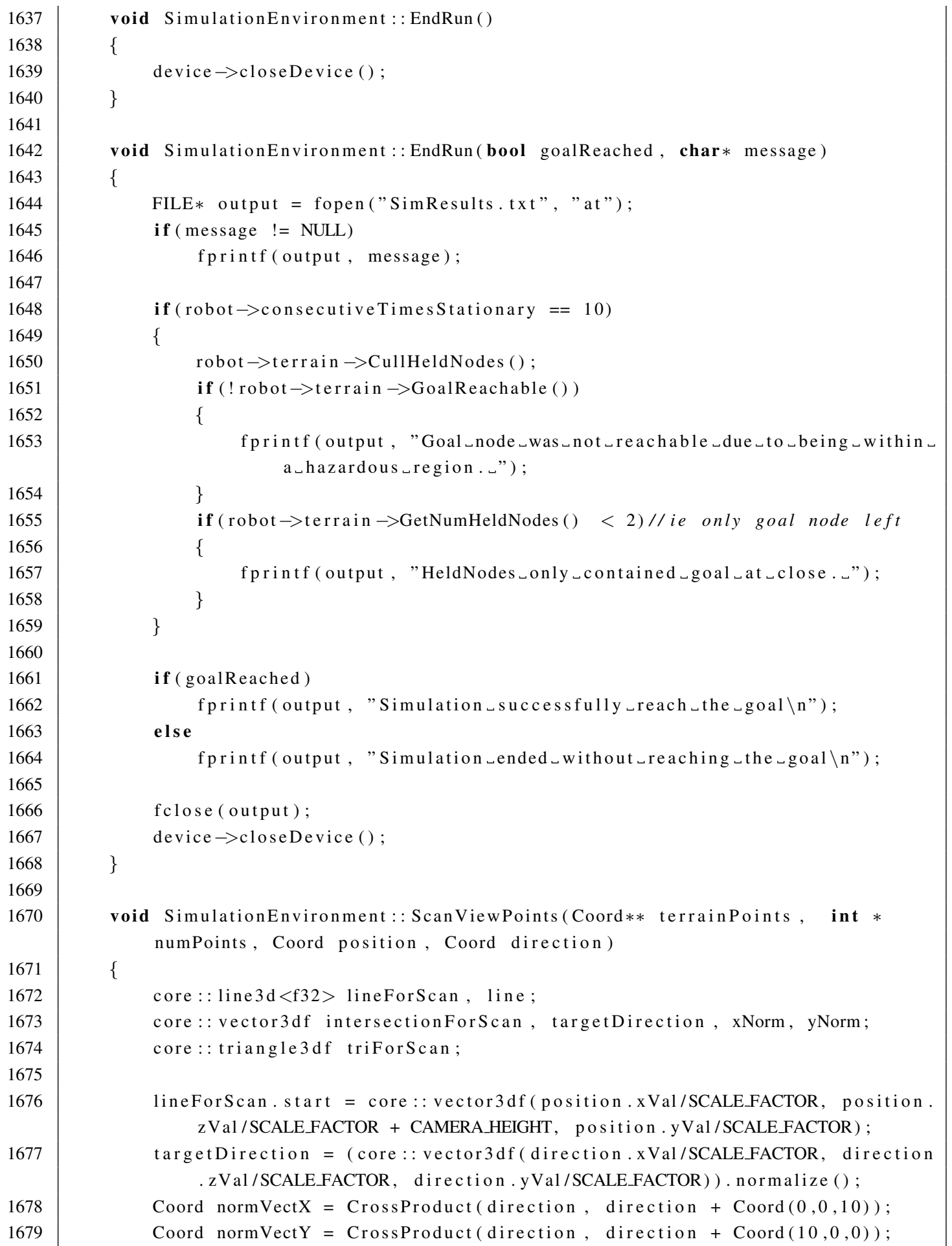




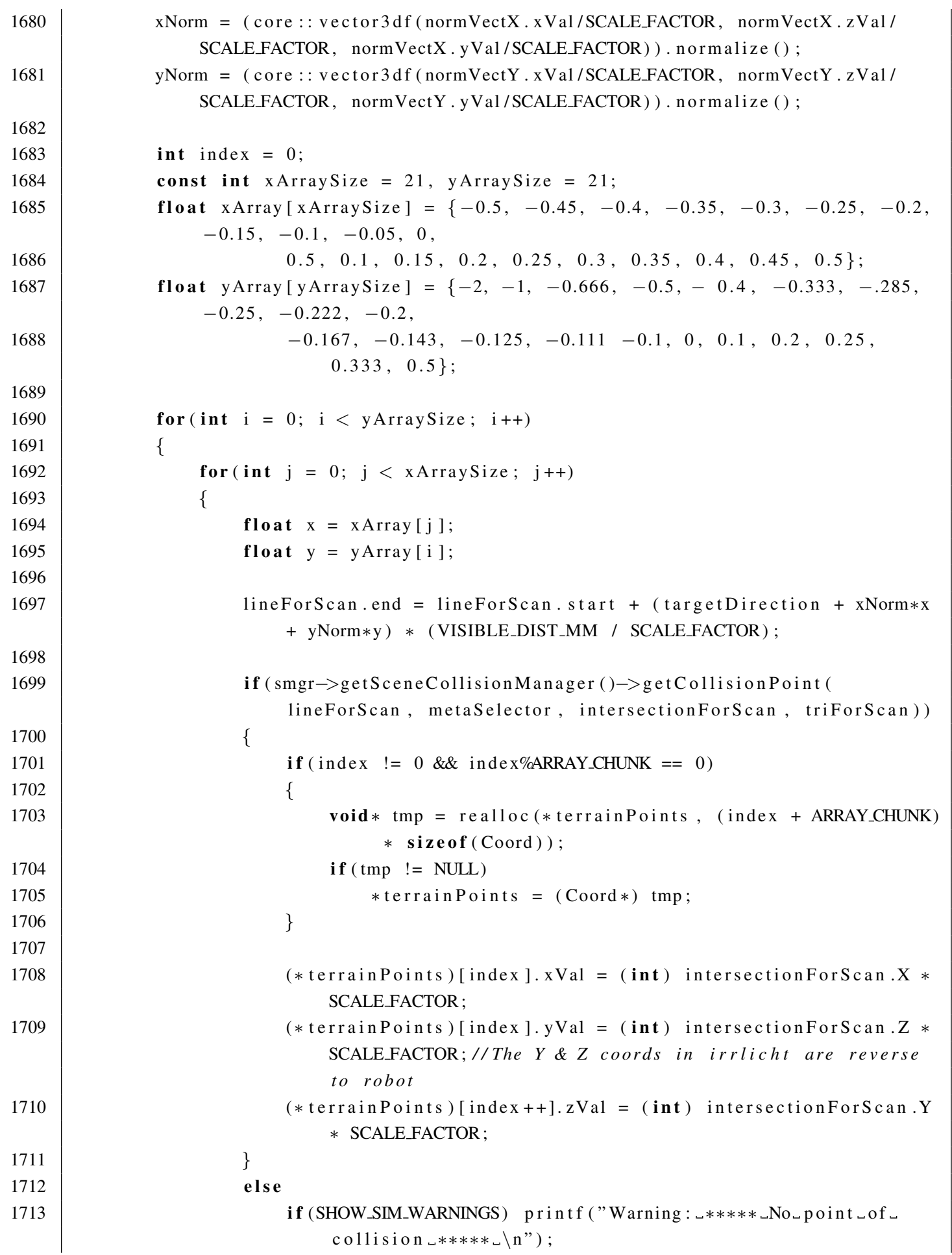




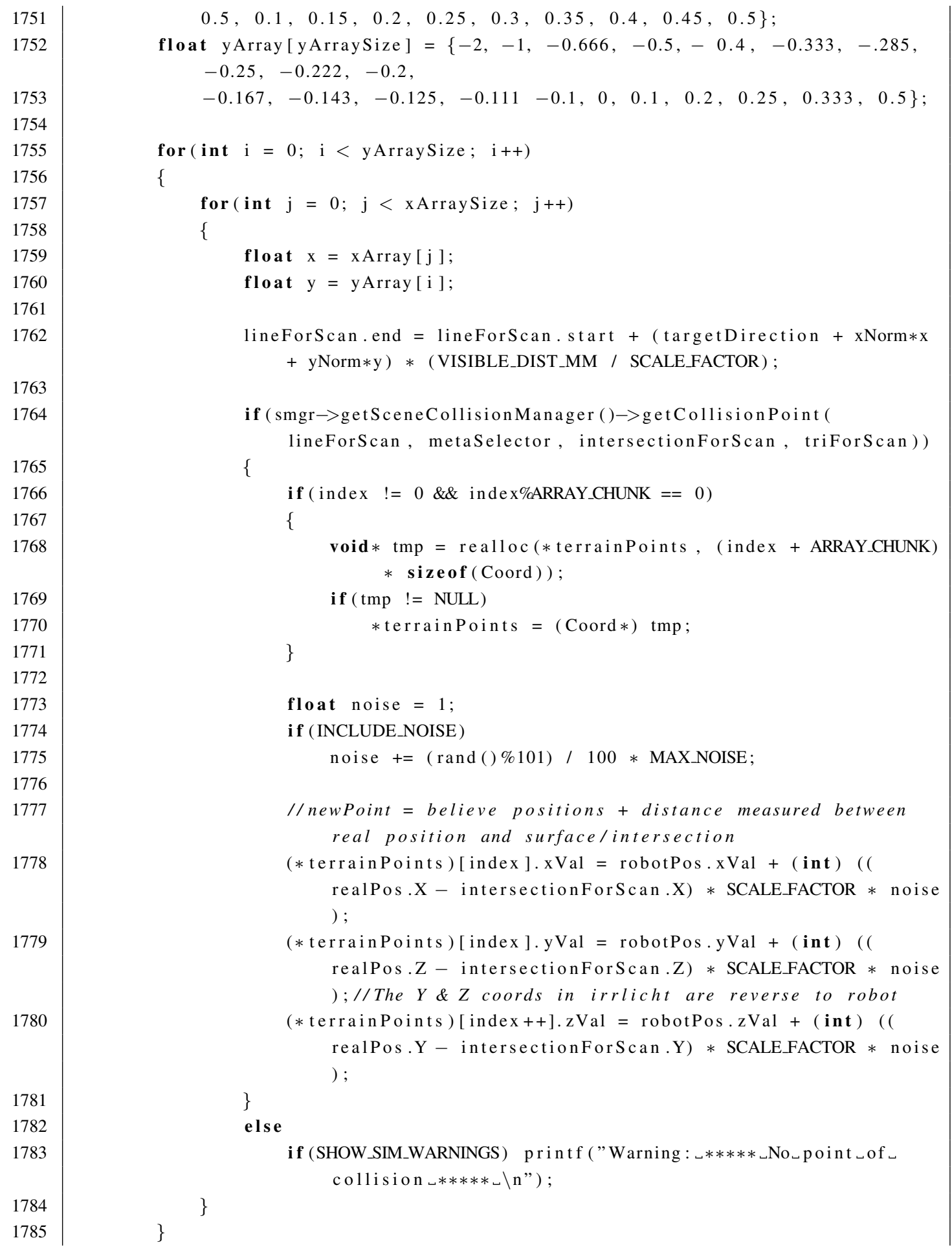




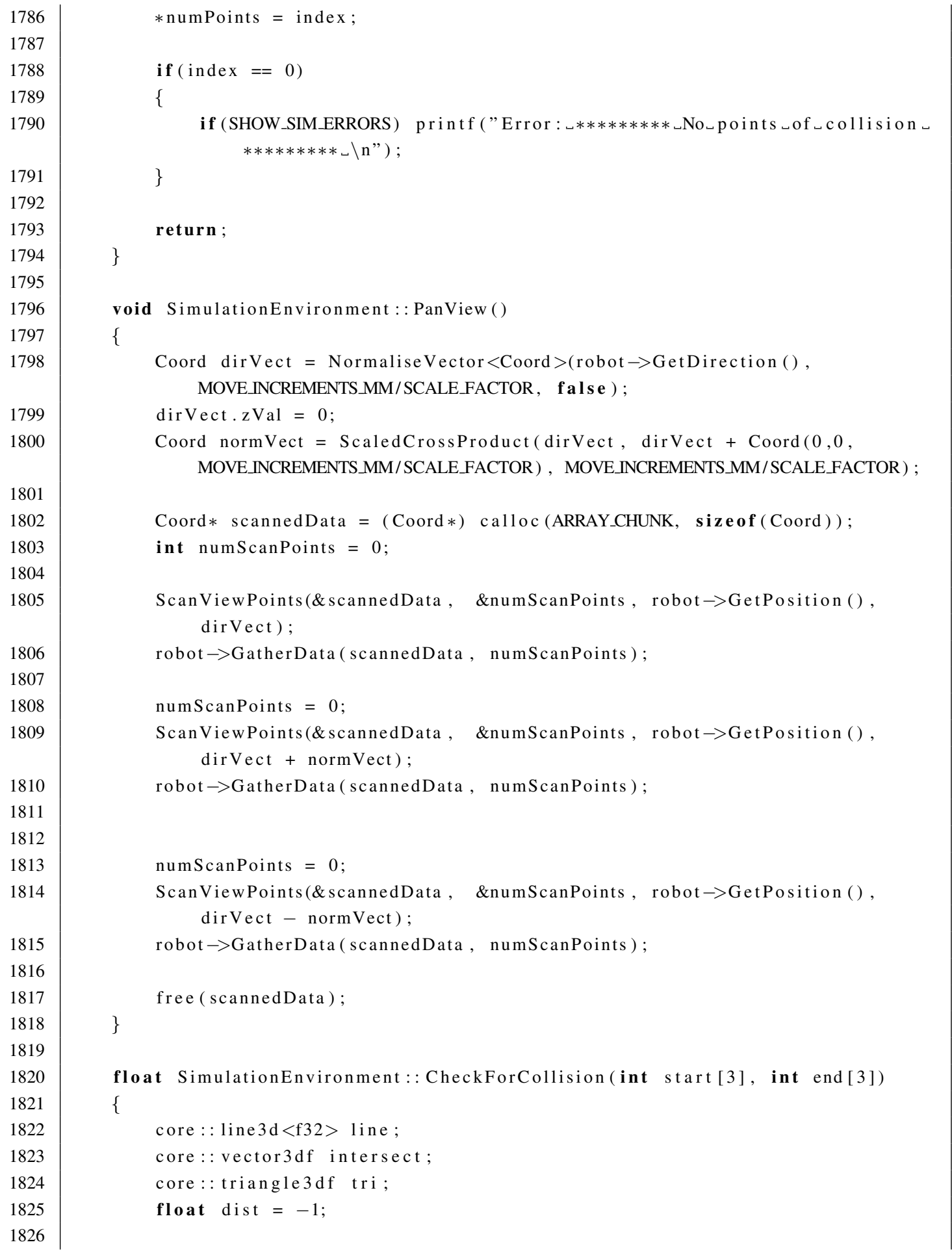




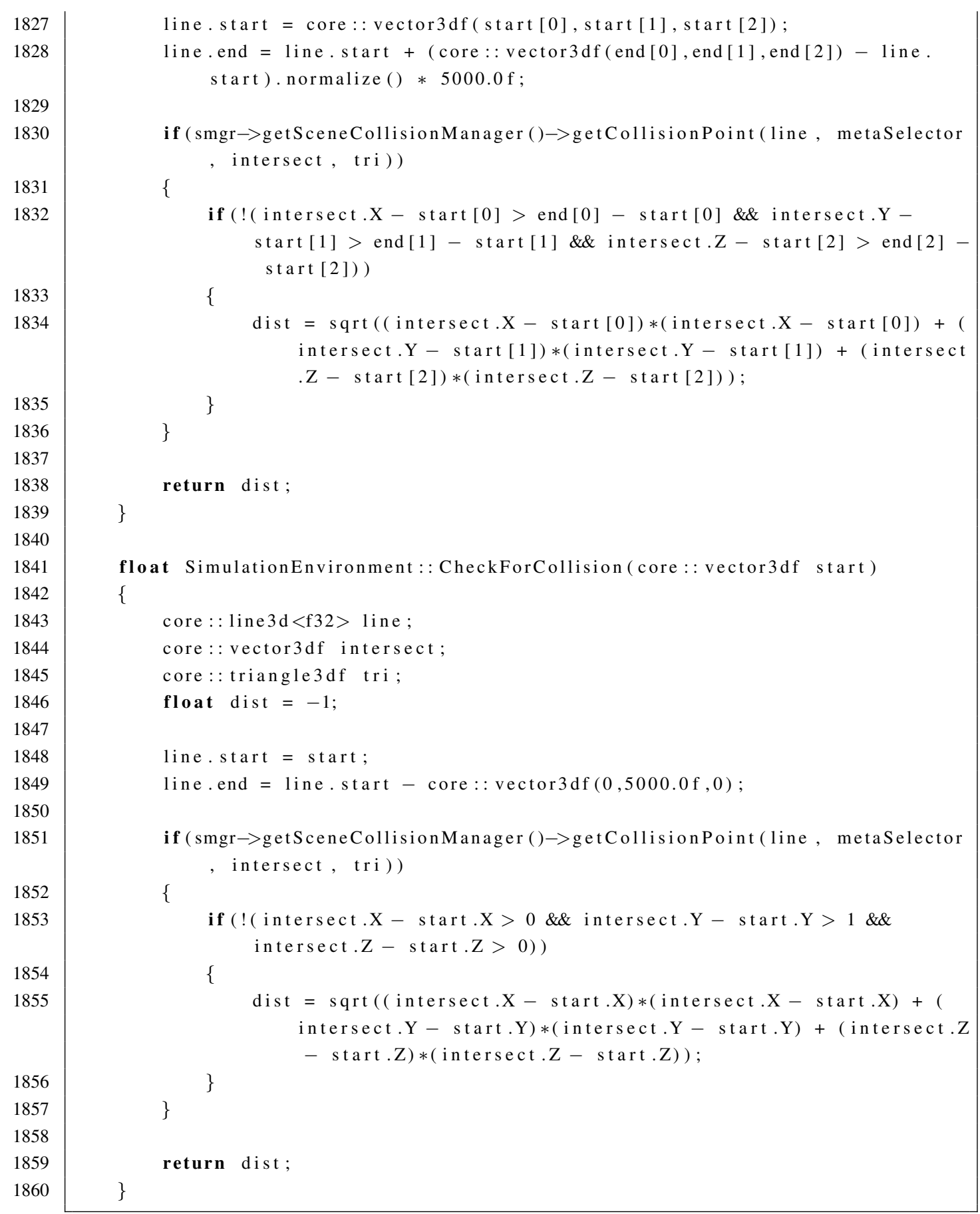




\section{A.8 World}

\section{A.8.1 World.h}

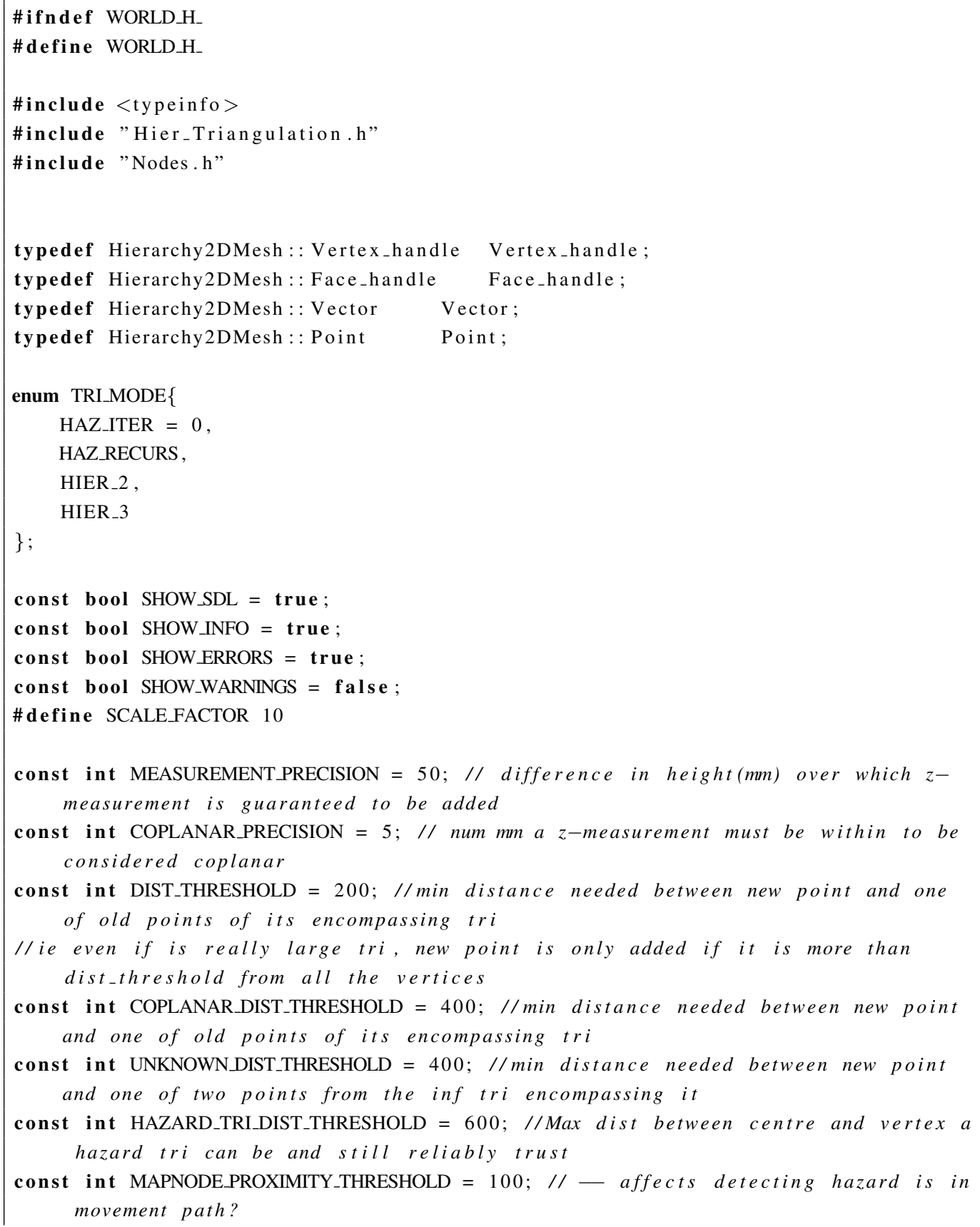




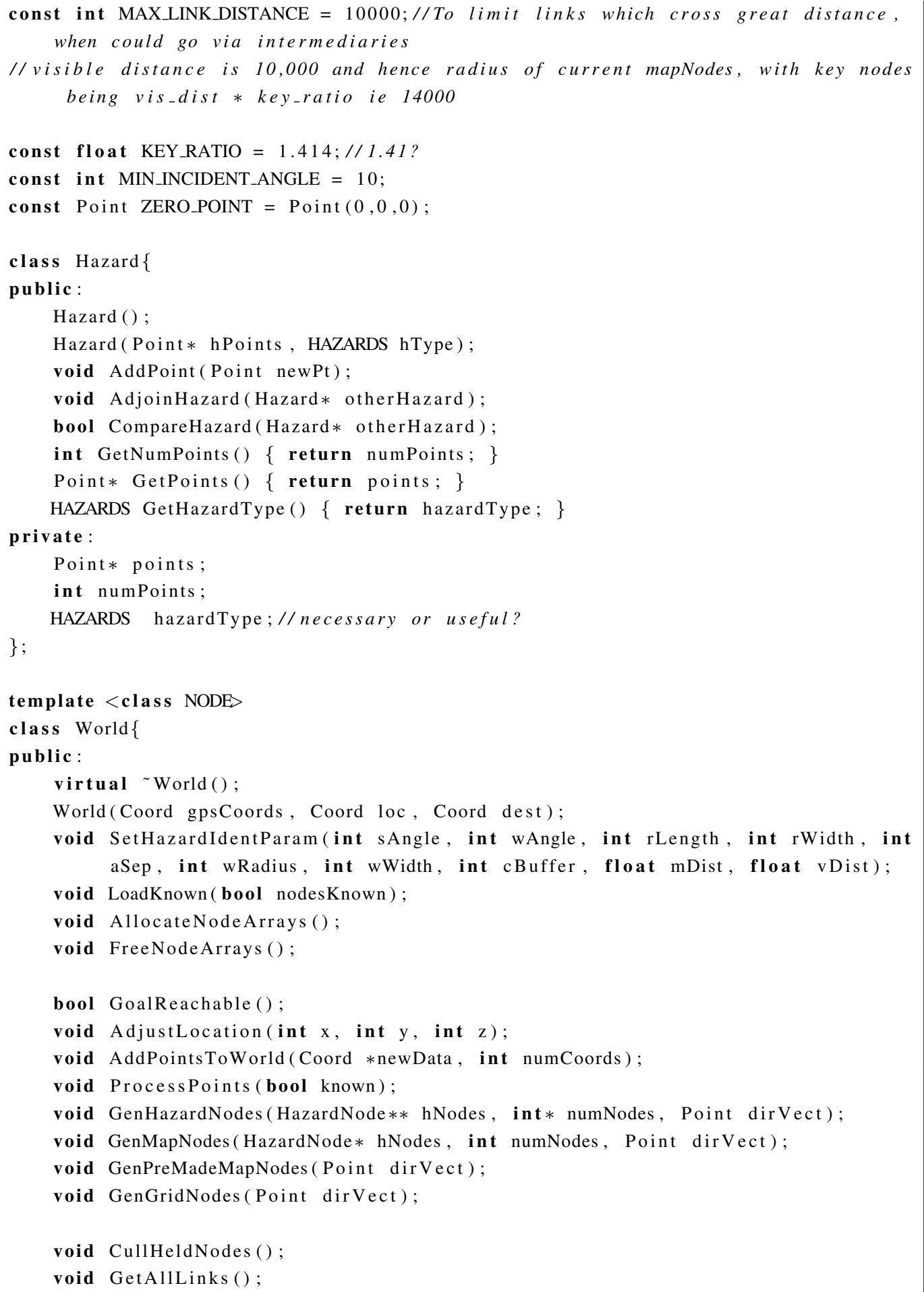


void GetAllKeyNodeLinks ();

int GetNumHeldNodes ( ) \{ return numHeldNodes; \}

bool CheckBetweenPoints (Point start, Point end, bool checkParallel, Point normVect )

NODE* GetCurrentNode ();

NODE* GetGoalNode () ;

void GarbageCollect ();

float CalculateUndulatingDistance (Point start, Point end);//obsolete?

bool GetTri(Triangle** tri, Coord * point);//Obsolete, remove along with CheckEdgePath from Edgehugger

bool GetTri(Face_handle*tri, Point * point);

void GetAllTriangles ( Triangle*** allTriangles, int *numTri);

void GetAllTriangles (Face_handle**allTriangles, int *numTri);

/** For incremental/life long path planning **/

NODE** closedSet;

NODE** openSet;

int openInd;

int closedind ;

/*********/

private :

void AddPoint (Coord* newPoint);

void AddHazard(Hazard hazard);

void DiscardPoints ();

bool RecursExpandHazardObject (Hazard *newHazard, Face_handle hazTri);

bool CheckBetweenHazardNodes (Point pt1, Point pt2, bool $*$ is Slope);

void CullKeyNodes (NODE** mNodes, int $*$ numMapNodes, Point dirVect);

void CullNodeSets ();

void CreateGrid ();

void ExpandNode(NODE* edgeNode, int eInd);

void CullGridNodes ( Point dirVect);

void FreeMarkedNodes ();

void LinkMapNodes (NODE** mNodes, int $*$ numMapNodes) ;

void LinkKeyNodes (NODE** mNodes, int $*$ numMapNodes, Point dirVect);

void SortNodes (NODE** mNodes, int $*$ numMapNodes) ;

void MergeWithKeyNodes(NODE** mNodes, int $*$ numMapNodes, bool preSorted); void LinkOtherNodes ();

template <class TRI, class PT> void IdentifyHazards(int numTri, TRI* surface) ;

template <class TRI, class PT> void IdentifyKnownHazards(int numTri, TRI* surface ); 


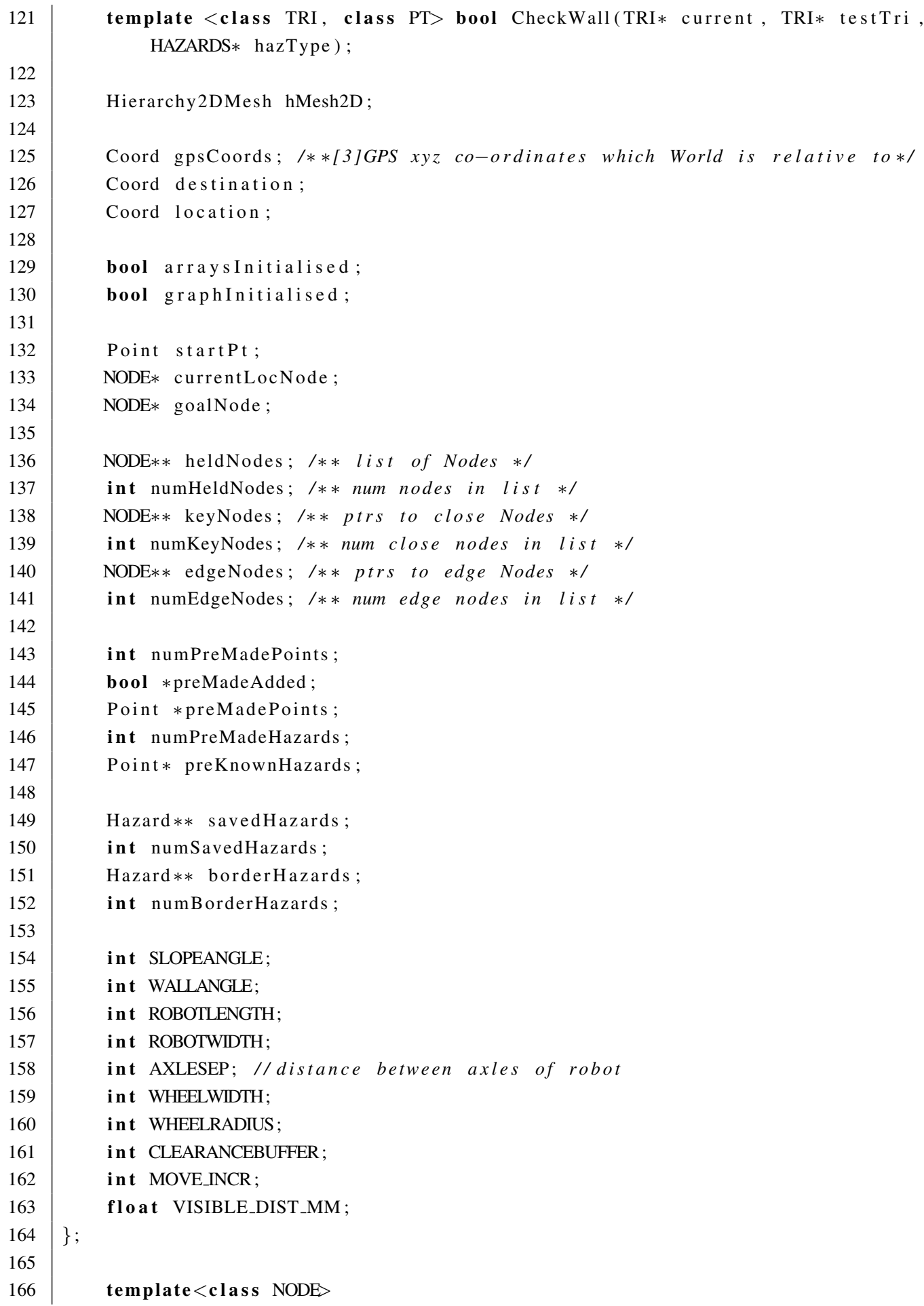




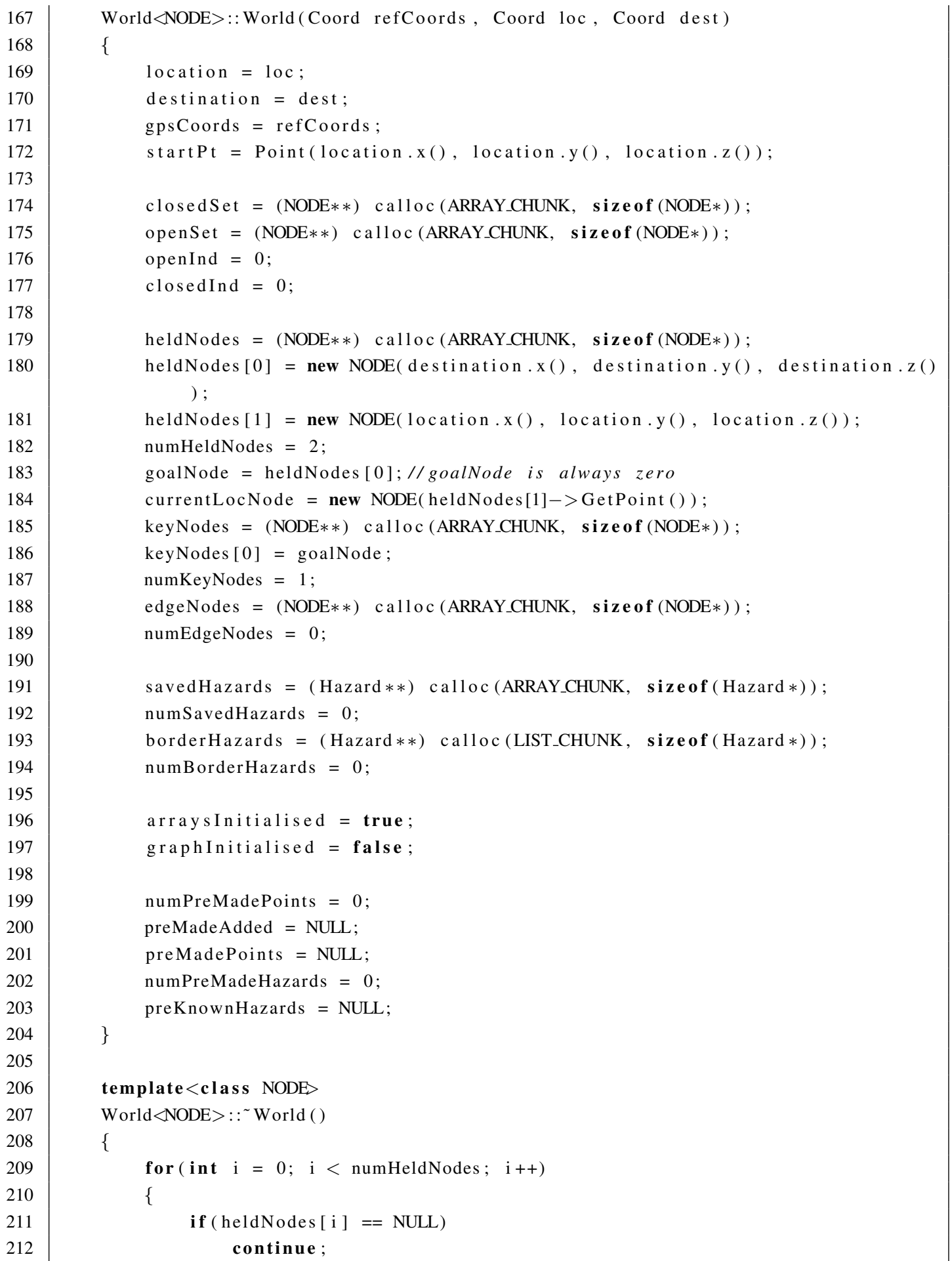




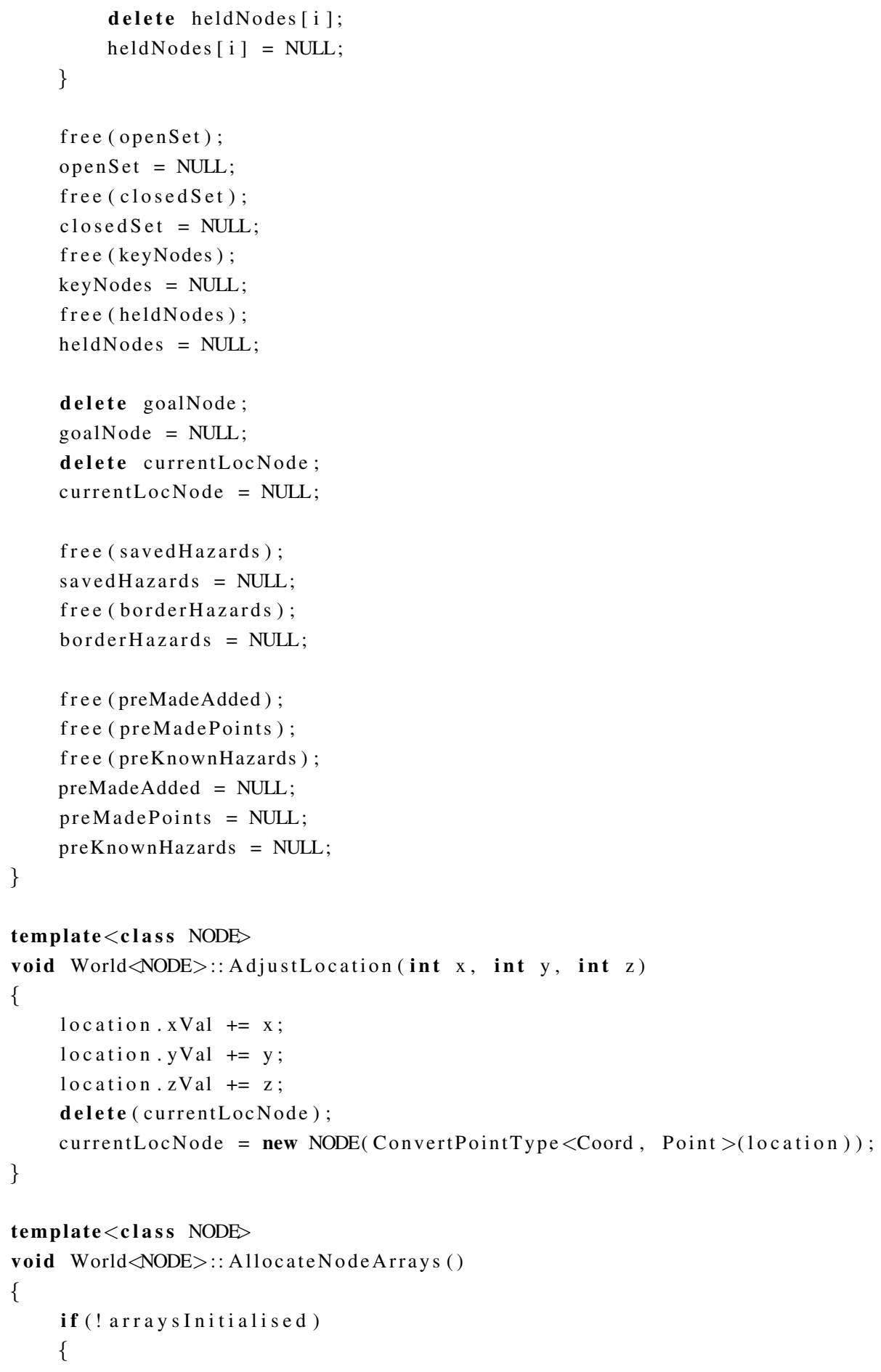




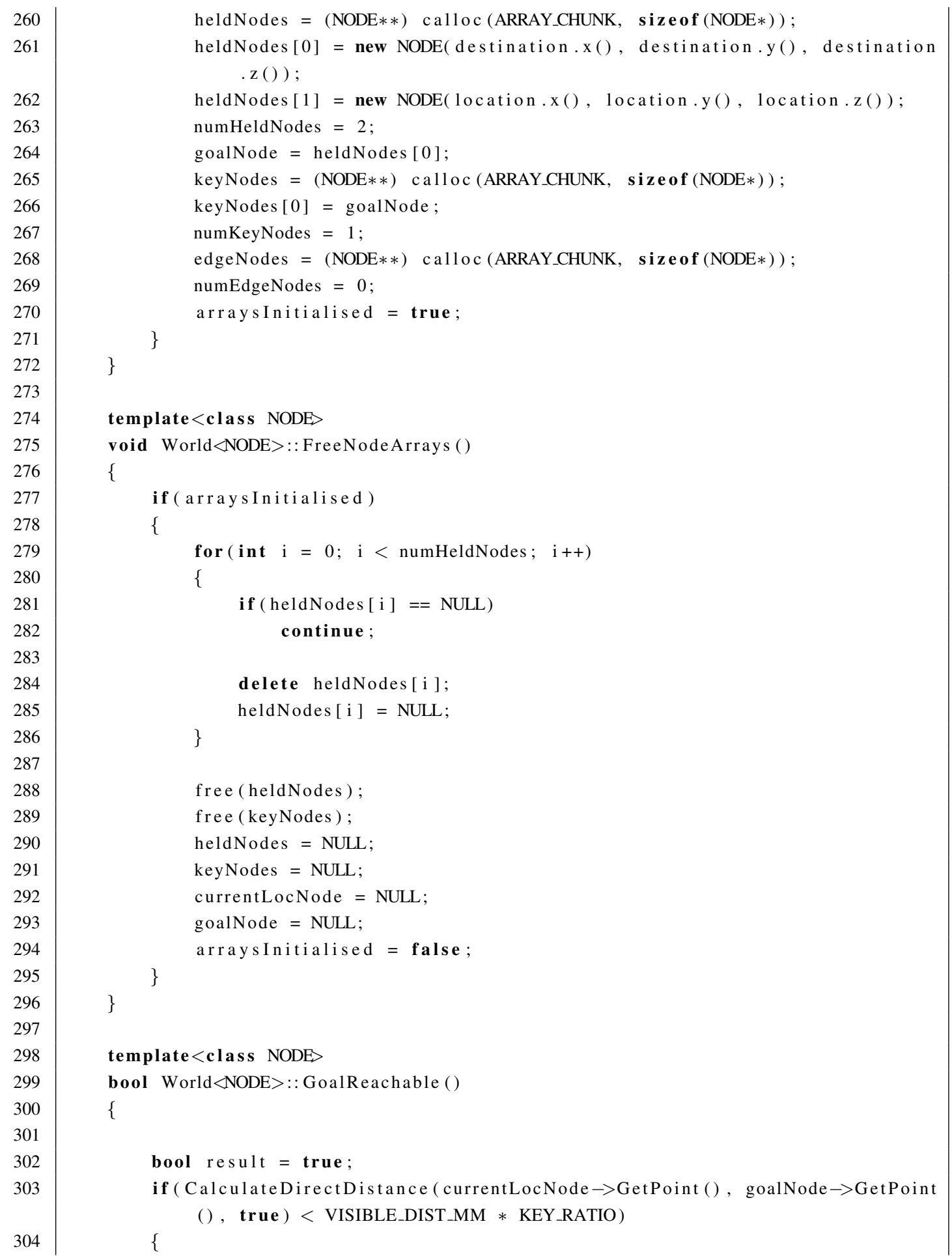


Face_handle encompTri $=$ Face_handle () ;

///If neighbours are hazards does that mean is unreachable?

//I If any neighbour is hazard, prob means goal is within clearance of hazard ie bad/unreachable

if (GetTri(\&encompTri , \&goalNode $\rightarrow$ GetPoint()) \&\& (encompTri $\rightarrow$ hazardType || (encompTri $\rightarrow$ neighbor $(0)->$ hazardType $\& \&$ encompTri $\rightarrow$ neighbor(1)->hazardType $\& \&$ encompTri->neighbor(2)->hazardType)))

\{

//can't reach goal

FILE* output $=$ fopen ("SimResults.txt", "at");

float dist $=$ CalculateDirectDistance(currentLocNode $\rightarrow$ GetPoint (), goalNode $\rightarrow$ GetPoint (), true);

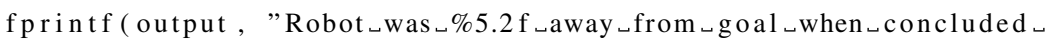
was $\lrcorner$ not $\lrcorner$ reachable. $\sqcup$, , dist );

fclose (output);

result = false ;

\}

\}

return result;

\}

$/ * *$

Add points to XYMatrix, for every new data point increment newPointCount or newFakePointCount

- If a number of points added are located close together, call identifyHazard on that region,

also interpolate

- If many points added, then call identifyHazards on entire MapSection, also call interpolation

$* 1$

template $<$ class NODE $>$

void World $\varangle$ NODE $>:$ : AddPointsToWorld (Coord $*$ newData, int numCoords)

\{

if (SHOW_INFO) printf ("Number $\_$of $\_$new $\_$data $\_$points $\_\% d_{\llcorner} \backslash \mathrm{n} "$, numCoords) ;

if (newData $!=$ NULL \&\& numCoords $>0$ )

\{

for $($ int $\mathrm{i}=0 ; \mathrm{i}<$ numCoords $; \mathrm{i}++)$

\{

AddPoint(\&newData [i ] ) ;

\}

Face_handle $*$ all Tri $=($ Face_handle $*)$ calloc (ARRAY_CHUNK, sizeof ( Face_handle));

int numTri $=0$;

GetAllTriangles(\&a11Tri, \&numTri); 
WriteTriToFile ("mesh_triangles.txt", "wt", allTri, numTri, false);

FILE* output $=$ fopen ("/home/haz/NumTriInTessellation.txt", "at $")$;

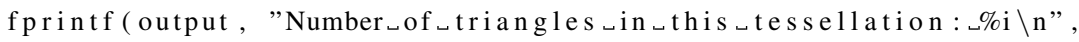
numTri);

fclose (output);

free (a11Tri);

a11Tri = NULL:

\}

template $<$ class NODE $>$

float World $<$ NODE $>$ : CalculateUndulatingDistance (Point start, Point end) \{

float distance $=0$;

Point dirVector $=$ end $-($ start - Point $(0,0,0))$;

bool triExists = false;

Point lineStart $=$ start, lineEnd;

Face_handle encompTri $=$ Face_handle () ;

while (CalculateDirectDistance(lineStart, end, true) > 15)

\{

if (GetTri(\&encompTri, \&lineStart) \&\& InsideTriangleTest (lineStart, * encompTri $\rightarrow$ GetPoint $(0)$, *encompTri $\rightarrow$ GetPoint (1), *encompTri $\rightarrow$ GetPoint (2)) == INSIDE)

\{

if (encompTri->hazardType)

\{

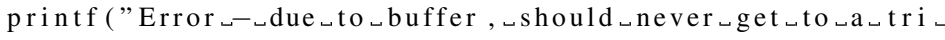
which $\lrcorner$ is $\lrcorner$ hazard $\lrcorner$ even $\lrcorner$ if $\lrcorner$ is $\lrcorner$ on $\lrcorner$ edge $\backslash n "$ "); break ;

\}

if (GetIntersection (*encompTri $\rightarrow$ GetPoint $(0), *$ encompTri $\rightarrow$ GetPoint (1), lineStart, end, \&lineEnd))

distance $+=$ CalculateDirectDistance(lineStart, lineEnd, false ) ;

else if (GetIntersection(*encompTri $\rightarrow$ GetPoint $(0)$, *encompTri $\rightarrow$ GetPoint(2), lineStart, end, \&lineEnd))

distance $+=$ CalculateDirectDistance(lineStart, lineEnd, false ) ;

else if(GetIntersection(*encompTri $\rightarrow$ GetPoint (1), *encompTri $\rightarrow$

GetPoint (2), lineStart, end, \&lineEnd))

distance $+=$ CalculateDirectDistance(lineStart, lineEnd, false ) ;

else if(InsideTriangleTest(end, *encompTri->GetPoint (0), * encompTri $\rightarrow$ GetPoint (1), *encompTri $\rightarrow$ GetPoint (2))) 


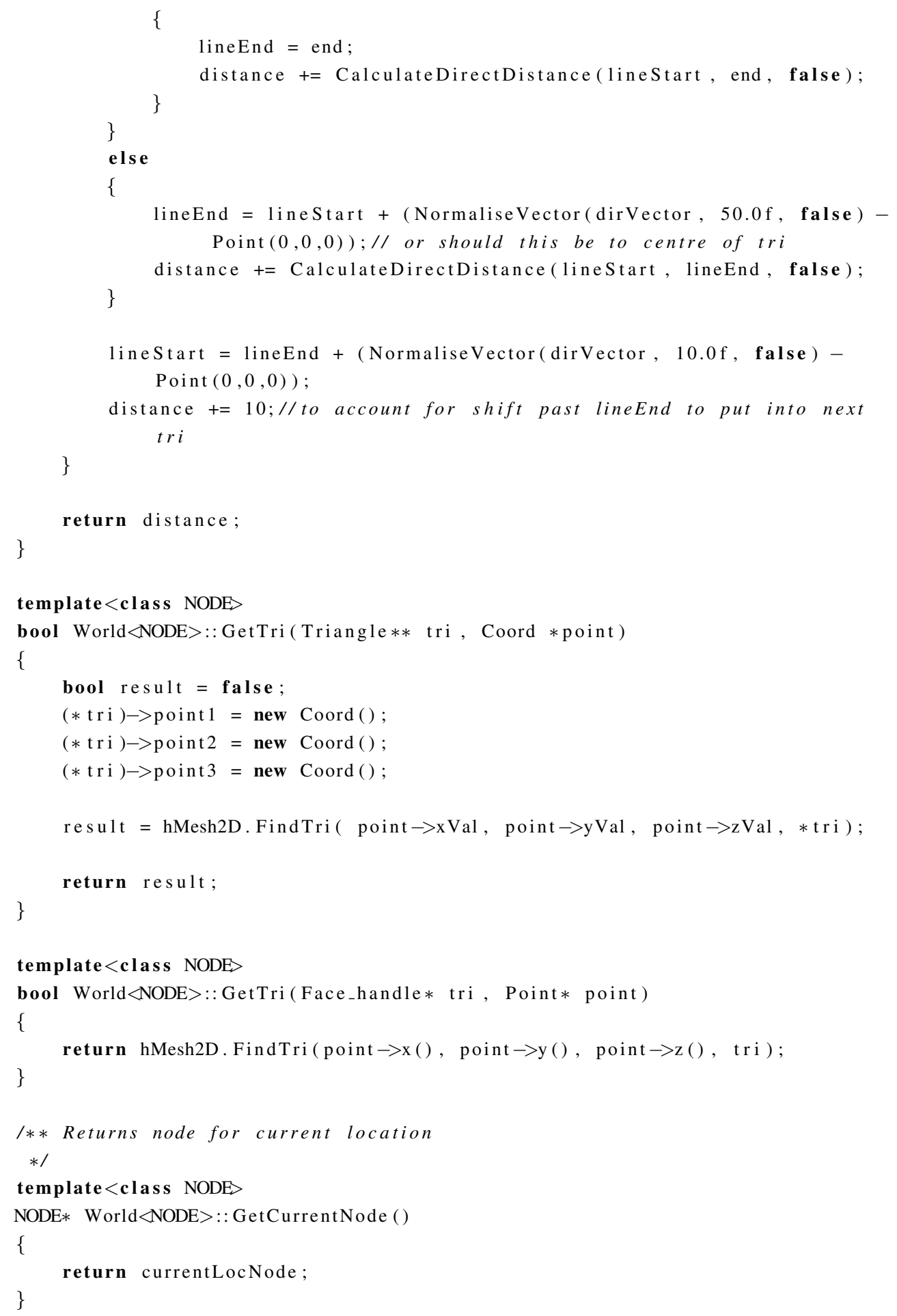




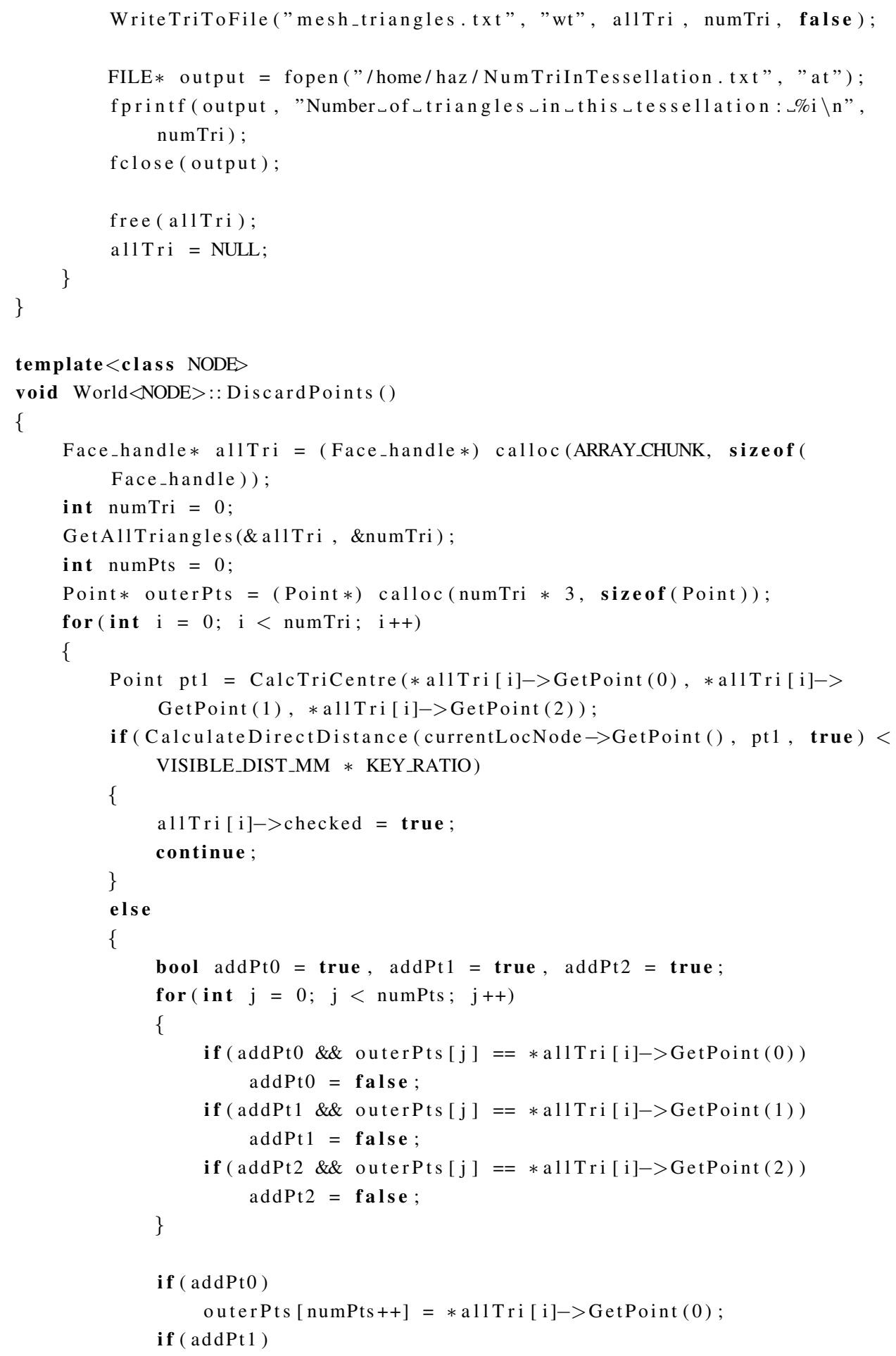




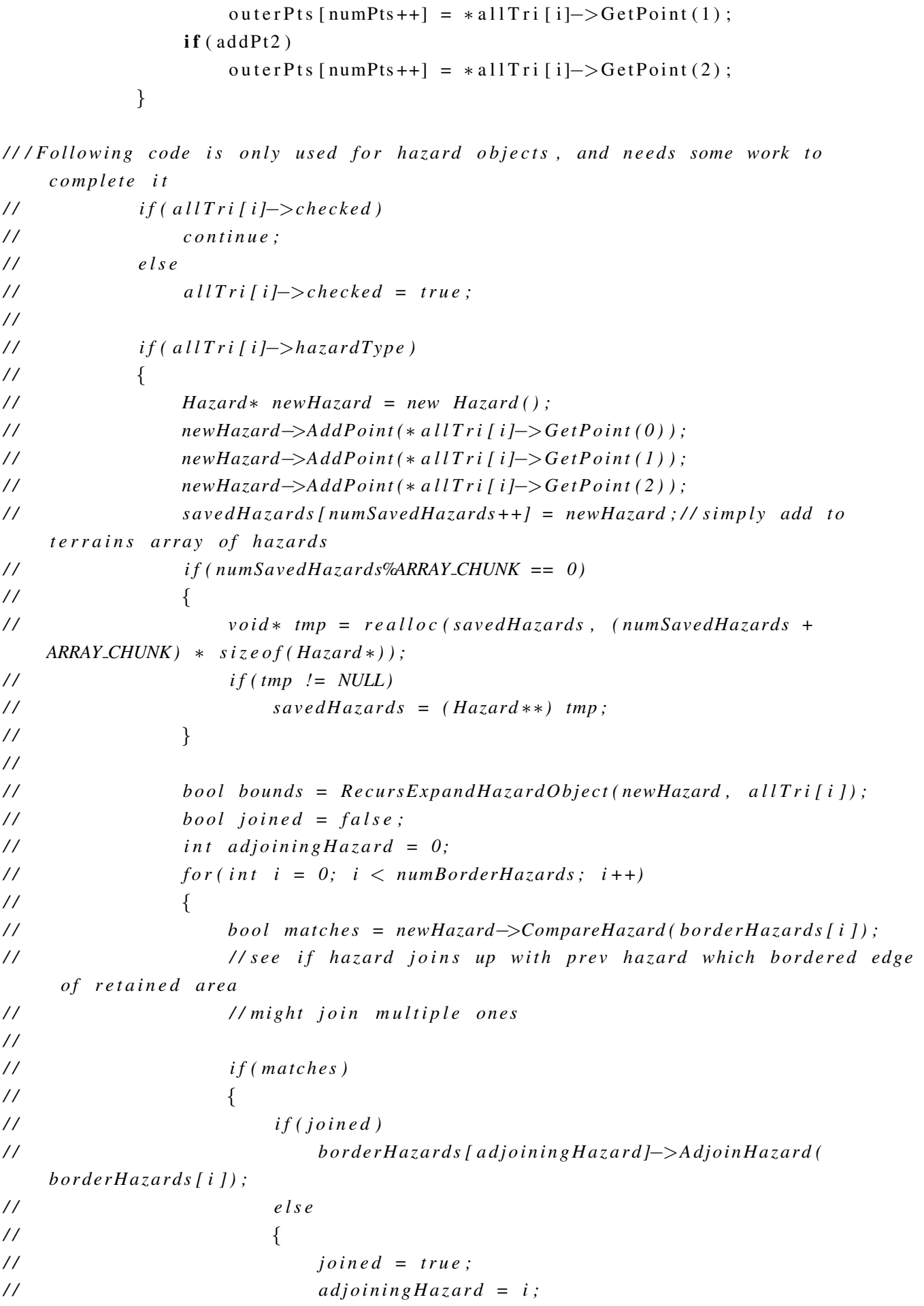


A.8. WORLD

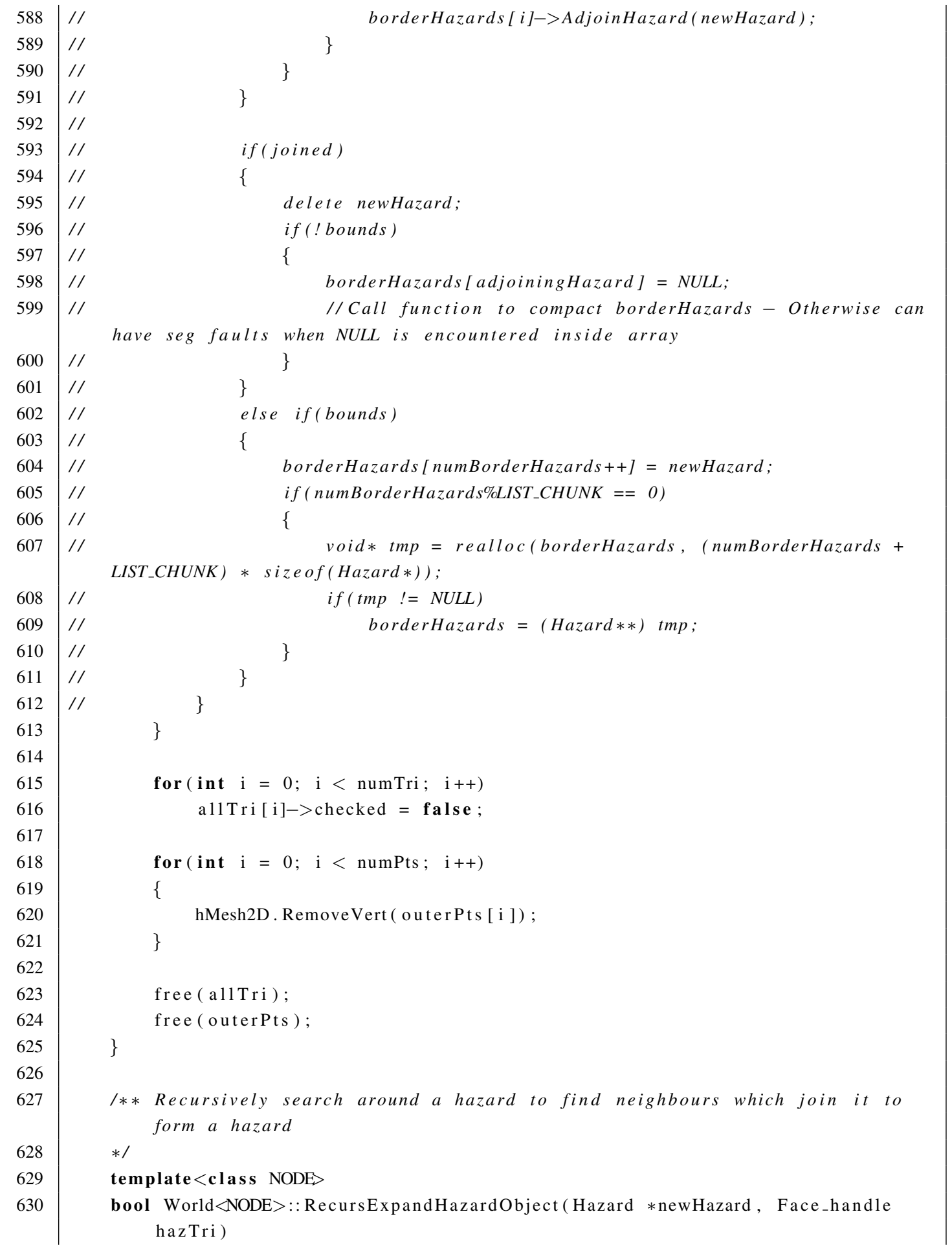




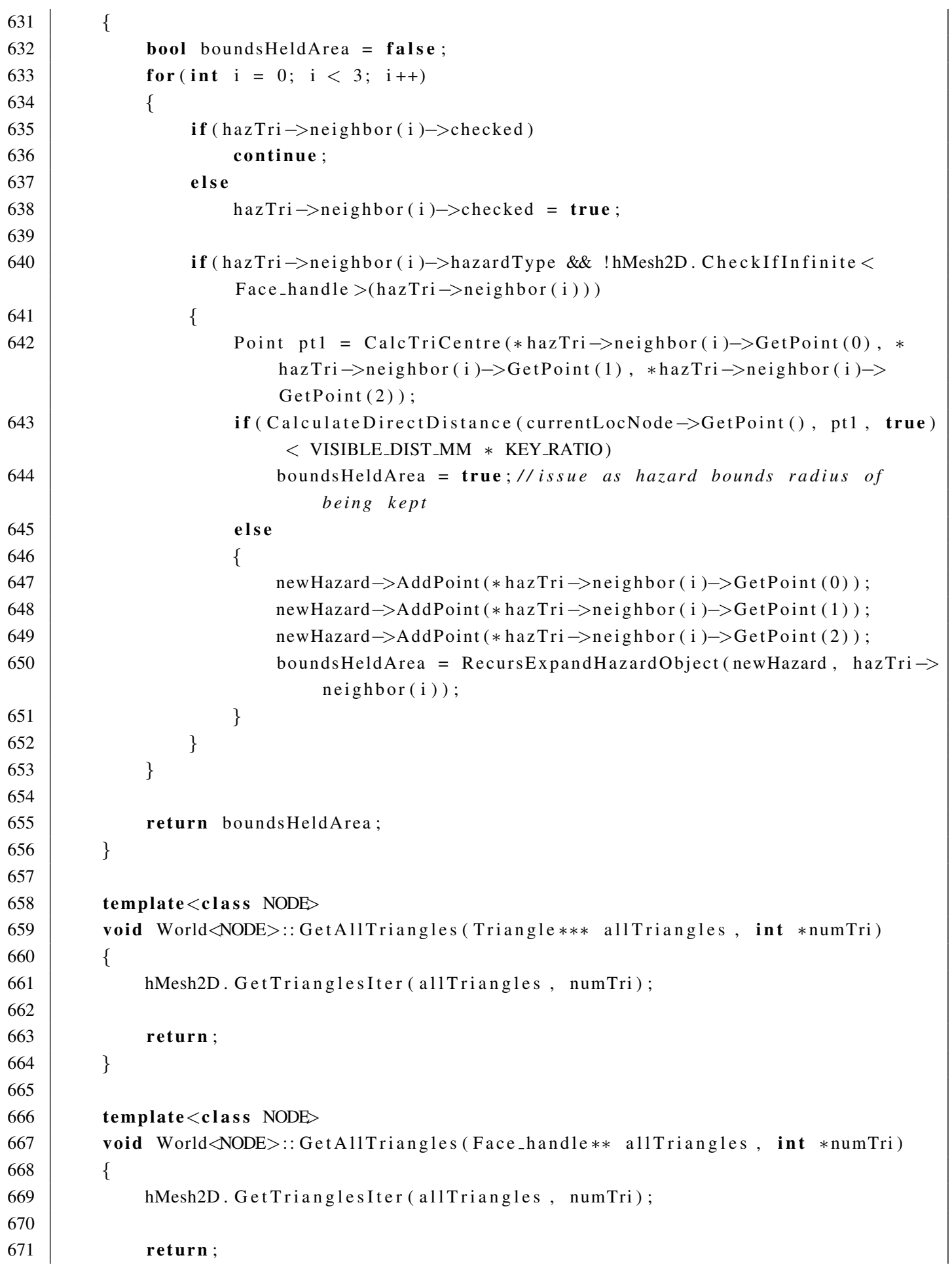




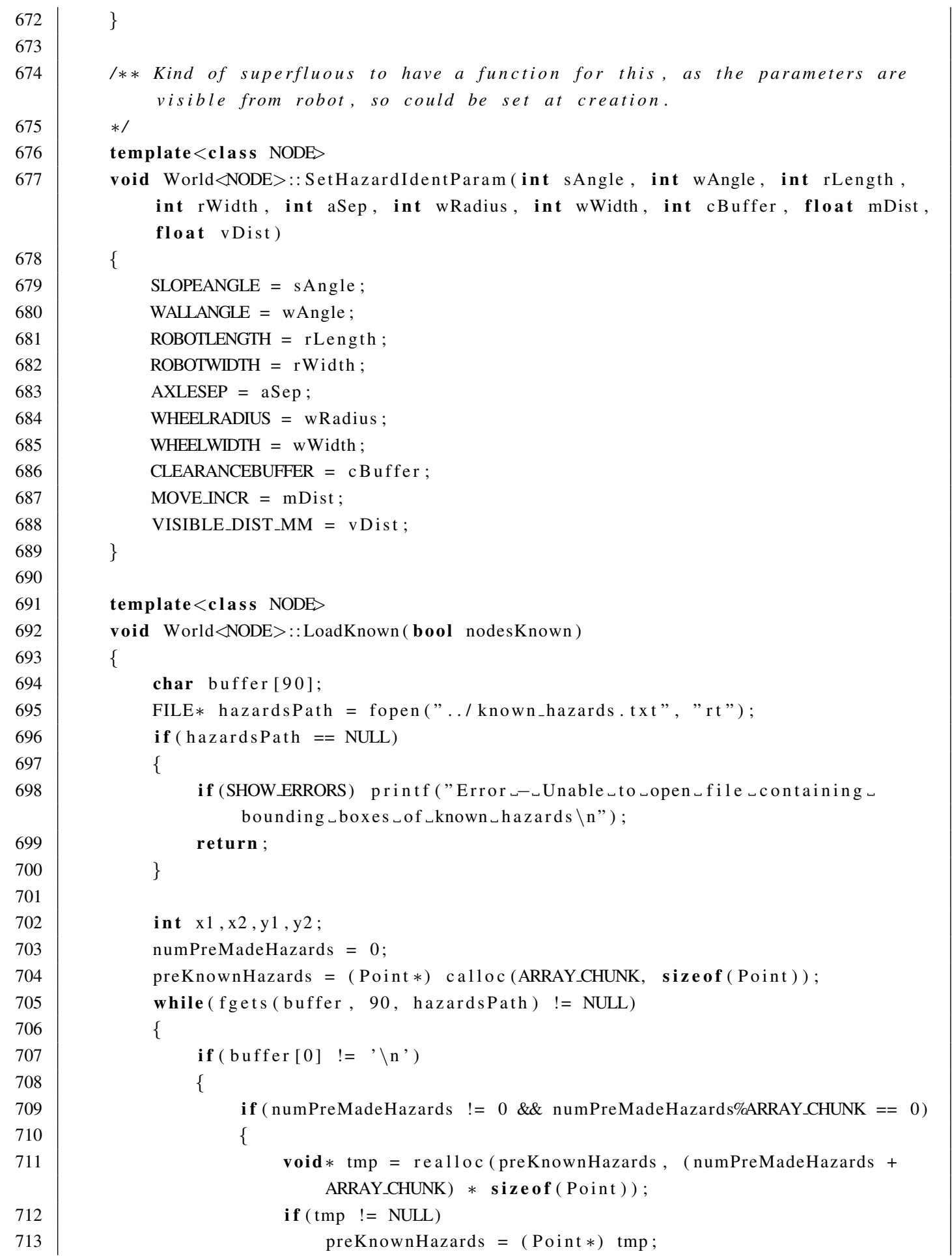




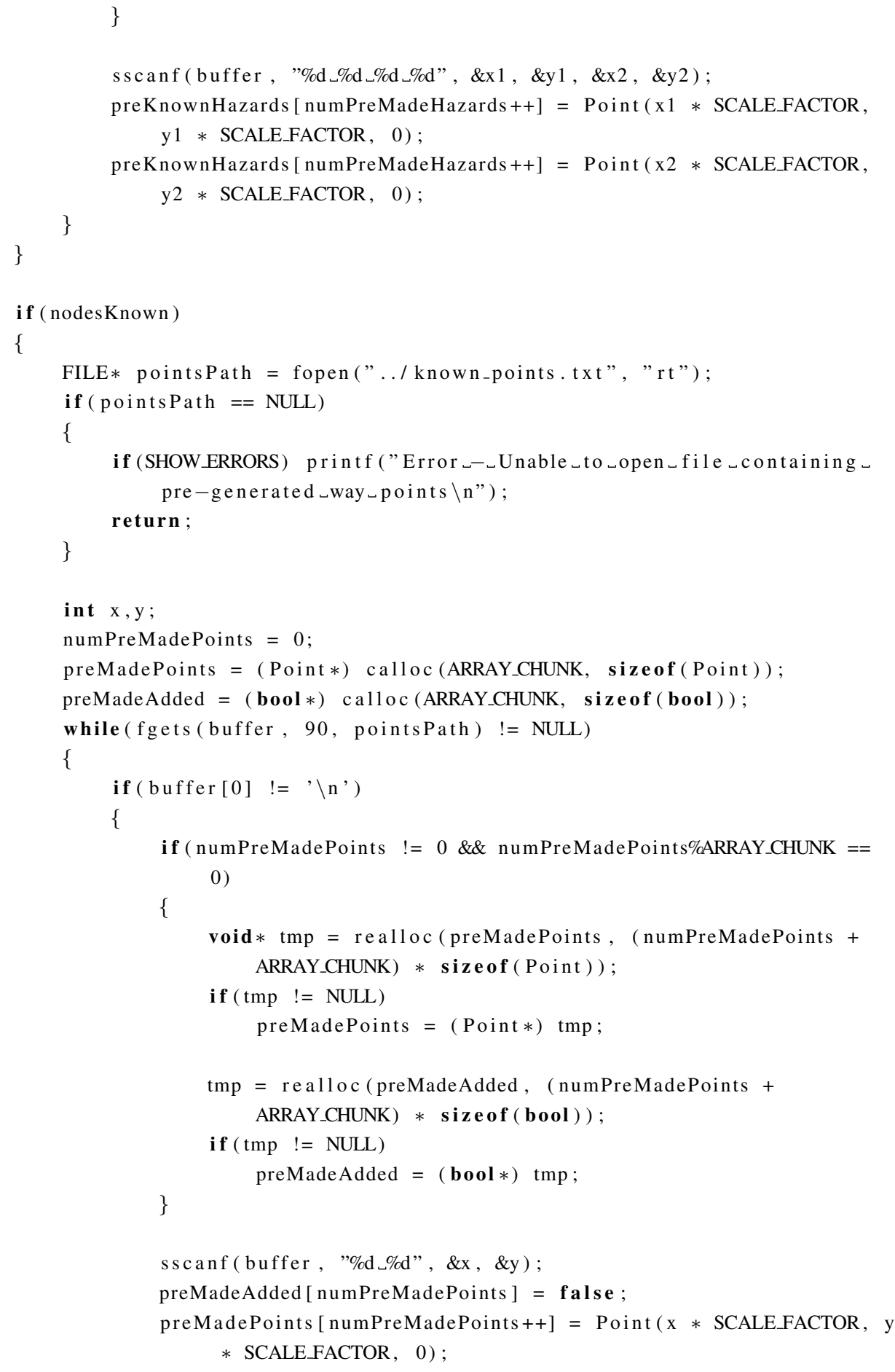




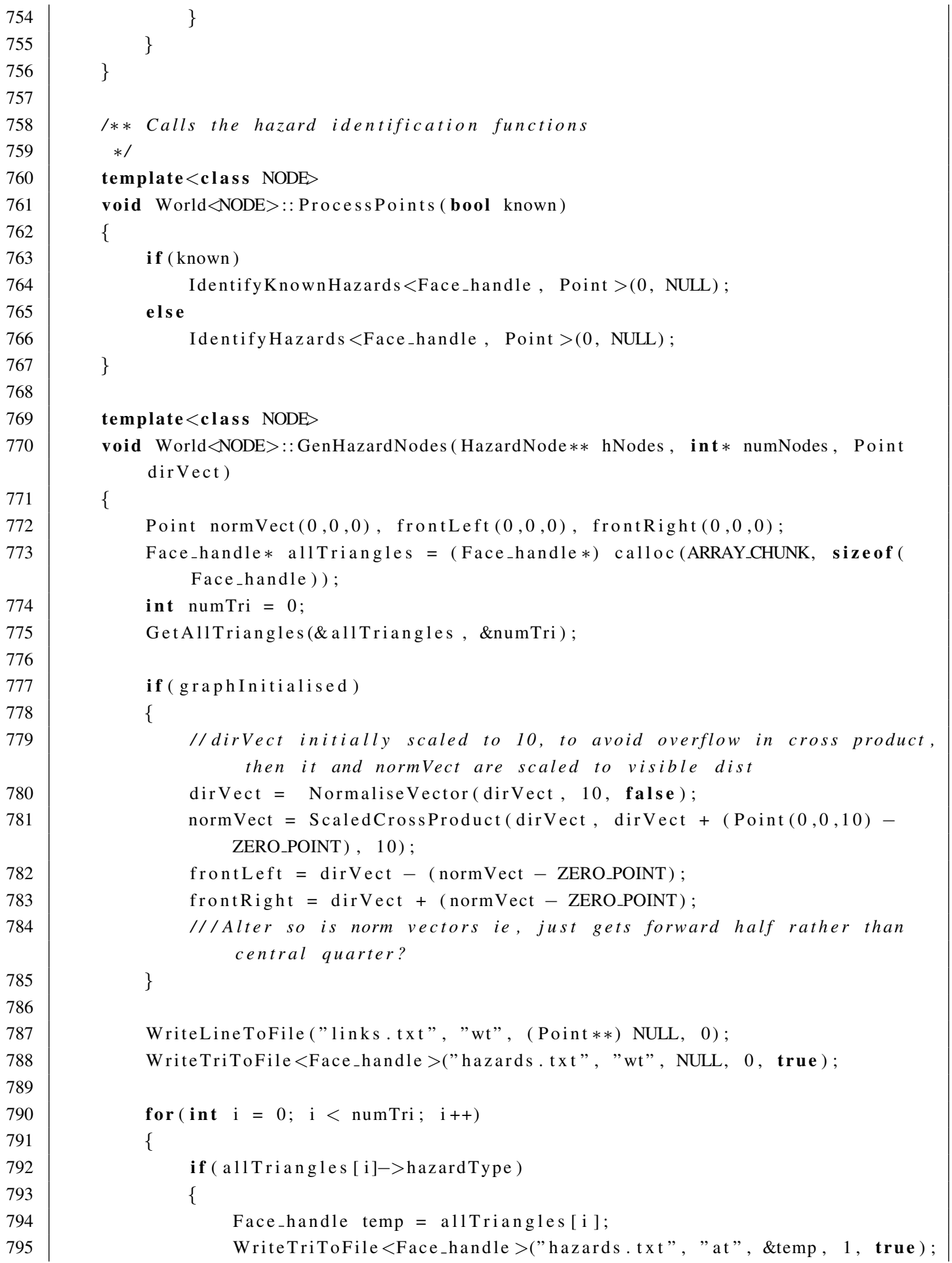




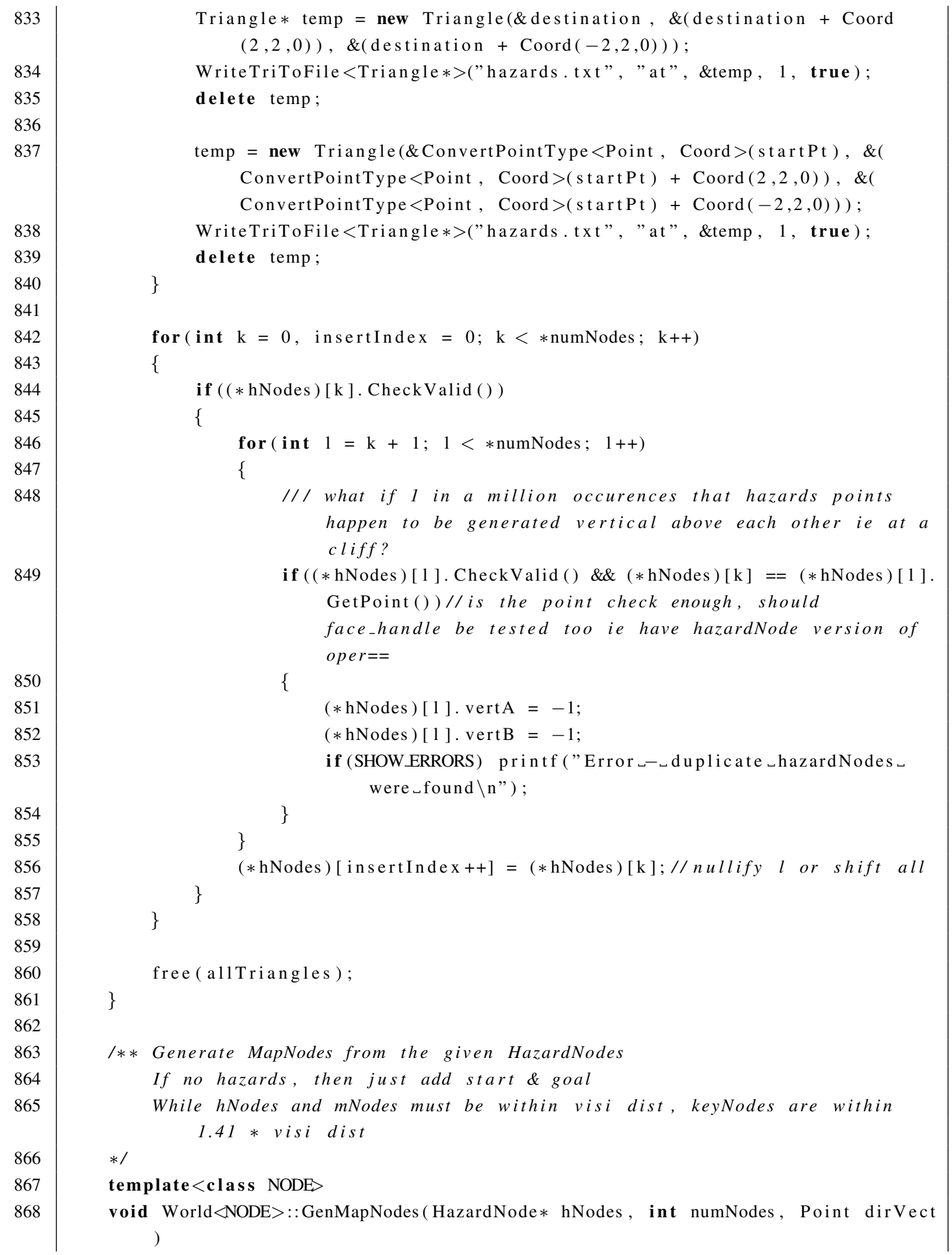




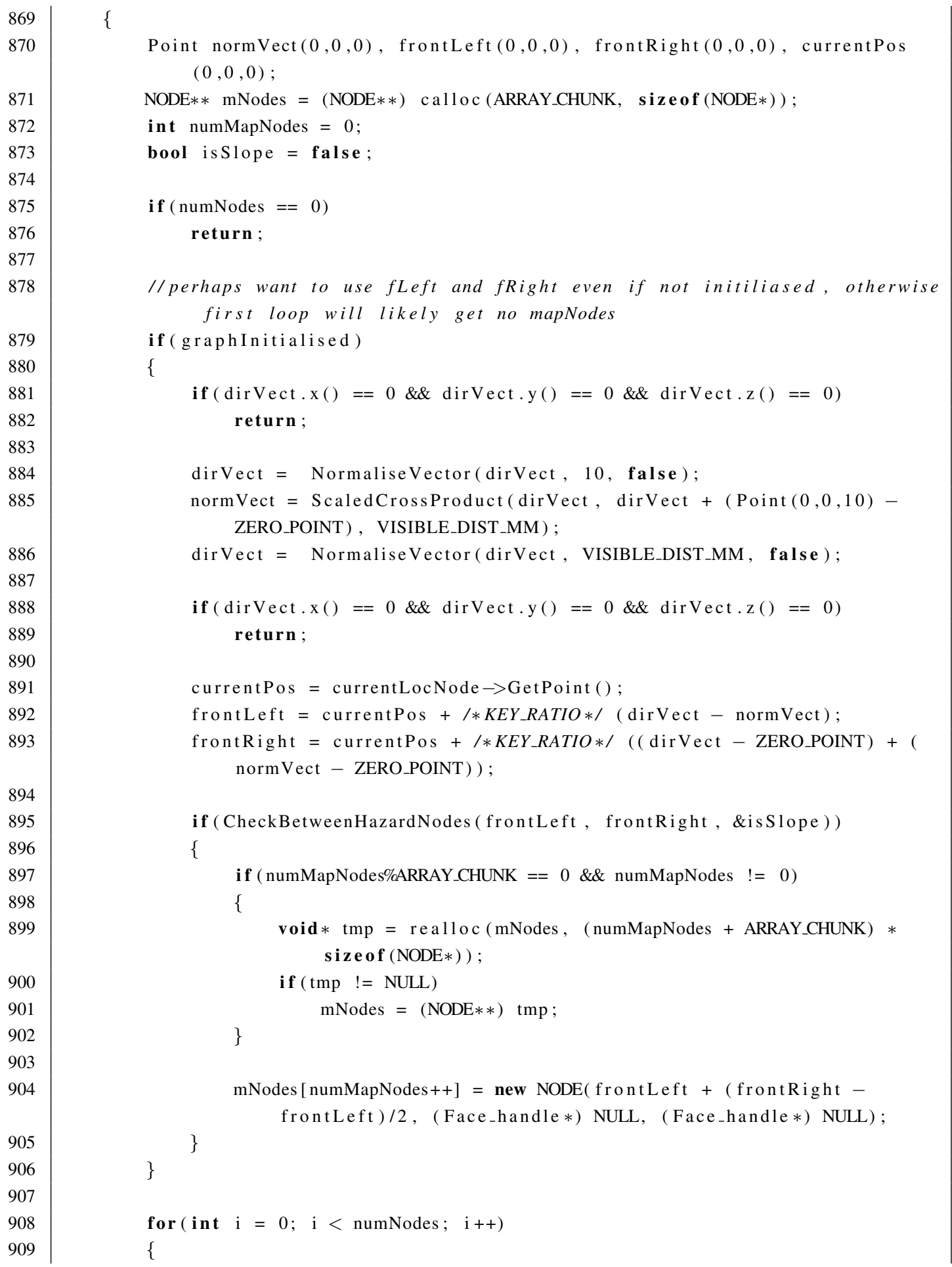


int startNumMapNodes = numMapNodes;

Point pt $1(0,0,0)$, pt1A $(0,0,0)$, pt1B $(0,0,0)$, pt1C $(0,0,0)$; bool nullTril = false;

pt 1 = hNodes[i]. GetPoint () ;

if (hNodes [ i ]. GetHazardTri () != NULL)

\{

pt1A $=*$ hNodes [i]. GetHazardTri ()$->$ GetPoint $($ hNodes [i ].vertA $)$;

pt1B $=*$ hNodes [i]. GetHazardTri ()$\rightarrow$ GetPoint (hNodes[i].vertB);

pt1C $=*$ hNodes [i ]. GetHazardTri ()$->$ GetPoint $(3-$ (hNodes[i].vertB + hNodes [i].vertA));

\}

else

nul1Tri $1=$ true

for (int $\mathrm{j}=\mathrm{i}+1 ; \mathrm{j}<$ numNodes $; \mathrm{j}++$ )

\{

//check relative position/sides

Point pt2(0,0,0), pt2A(0,0,0), pt2B $(0,0,0), \operatorname{pt} 2 \mathrm{C}(0,0,0)$;

pt2 $=$ hNodes $[j]$. GetPoint ()$;$

Point midPoint $=$ pt $1+(p t 2-p t 1) / 2$;

int angle $1=90$, angle $2=90$;

int res = InsideTriangleTest (midPoint, currentPos, frontLeft, frontRight);

if (graphInitialised \&\& !res)

continue :

$/ /$ if $>0$ then pt2 is same side, else if pt1 is same side if (! nul1Tri1)

\{

int $\mathrm{val}=((\mathrm{pt} 1 \mathrm{~B} \cdot \mathrm{x}()-\mathrm{pt} 1 \mathrm{~A} \cdot \mathrm{x}()) *(\mathrm{pt} 2 \cdot \mathrm{y}()-\mathrm{pt} 1 \mathrm{~A} \cdot \mathrm{y}())-($ pt2.x() - pt1A.x())*(pt1B.y() - pt1A.y()))*((pt1B.x() - pt1A.x()) * (pt1C.y() - pt1A.y()) - (pt1C.x() - pt1A.x ()) *(pt1B.y()-pt1A.y()));

if $(((\mathrm{pt} 1 \mathrm{~B} \cdot \mathrm{x}()-\mathrm{pt} 1 \mathrm{~A} \cdot \mathrm{x}()) *(\mathrm{pt} 2 \cdot \mathrm{y}()-\mathrm{pt} 1 \mathrm{~A} \cdot \mathrm{y}())-(\mathrm{pt} 2 \cdot \mathrm{x}()-$ pt1A.x()) *(pt1B.y() - pt1A.y())) *((pt1B.x() - pt1A.x ()) *(pt1C.y() - pt1A.y()) - (pt1C.x() - pt1A.x())*( pt1B.y() - pt1A.y())) >=0) continue ;

else angle $1=$ AngleBetweenTwoVectors $($ pt2 - pt1, pt1B - pt1A $)$

\}

if (hNodes $[\mathrm{j}]$. GetHazardTri () != NULL)

\{ 


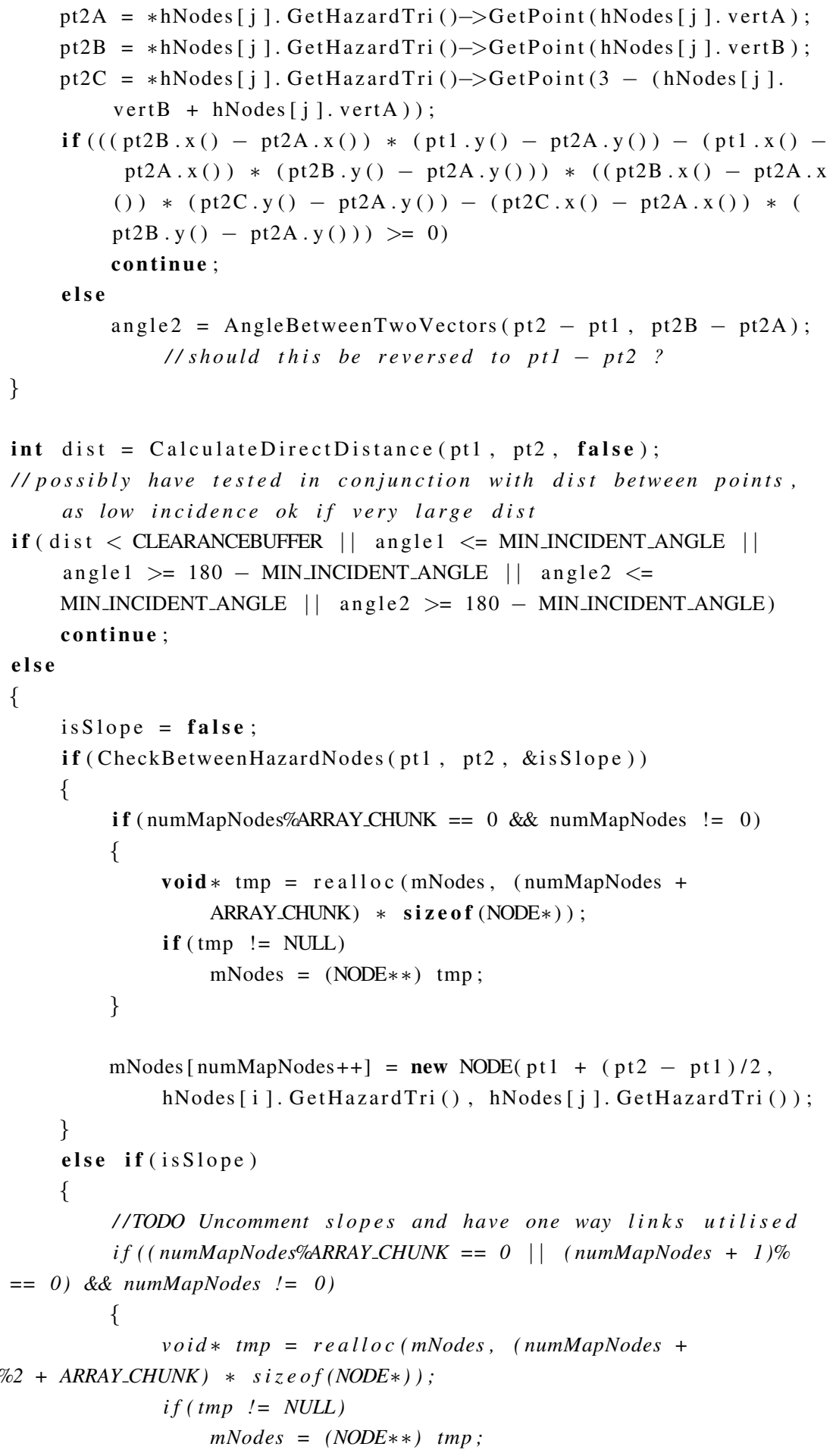




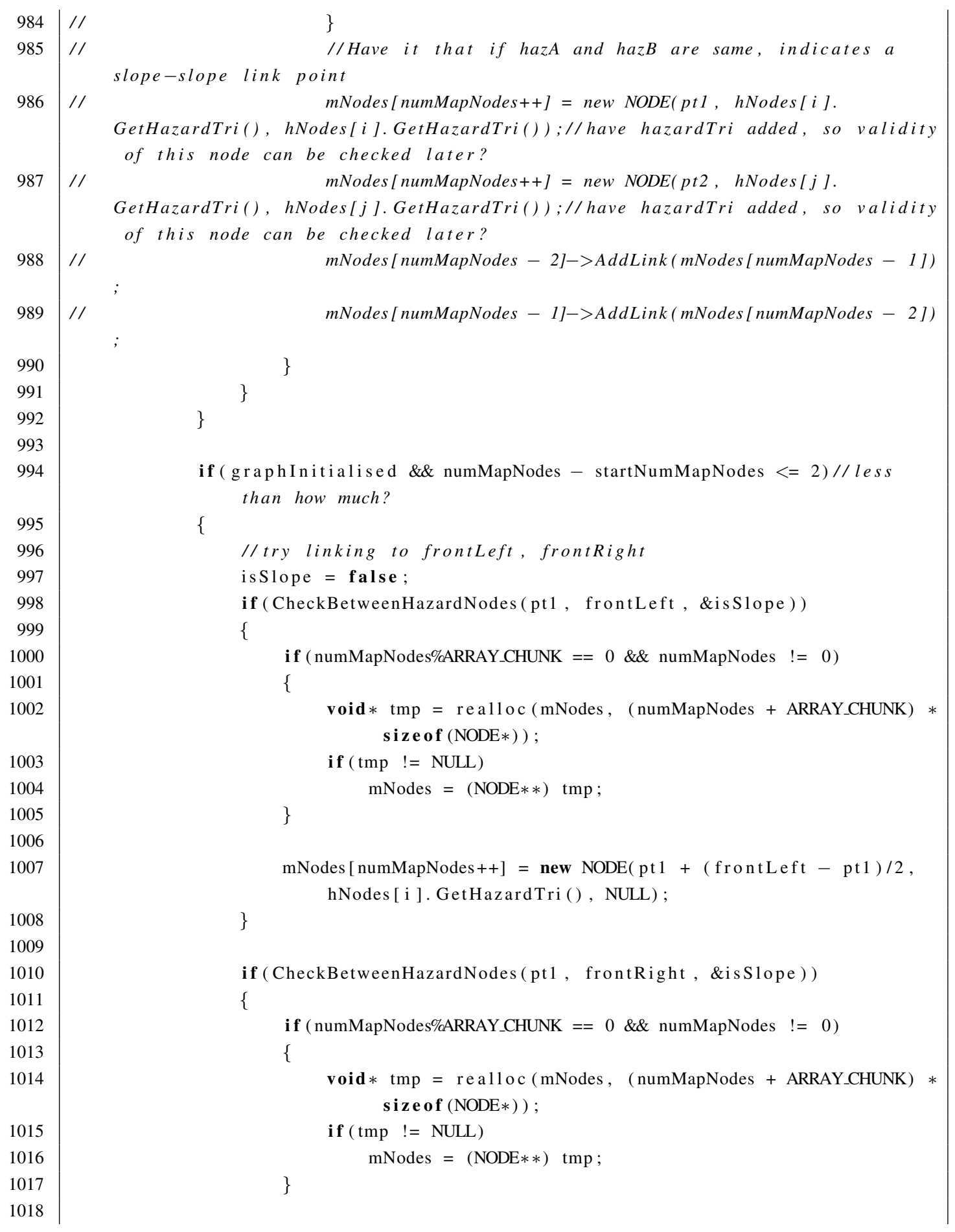




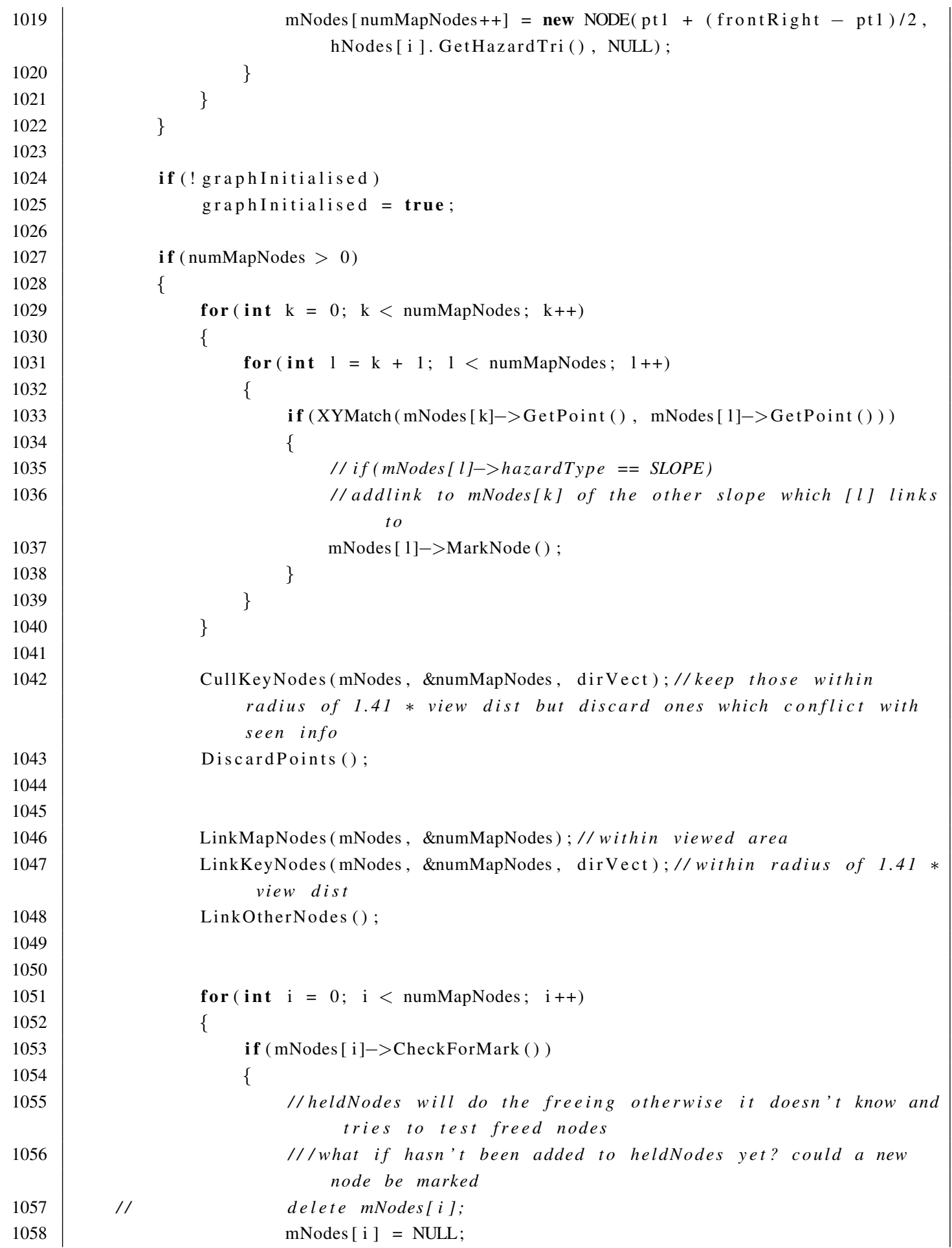




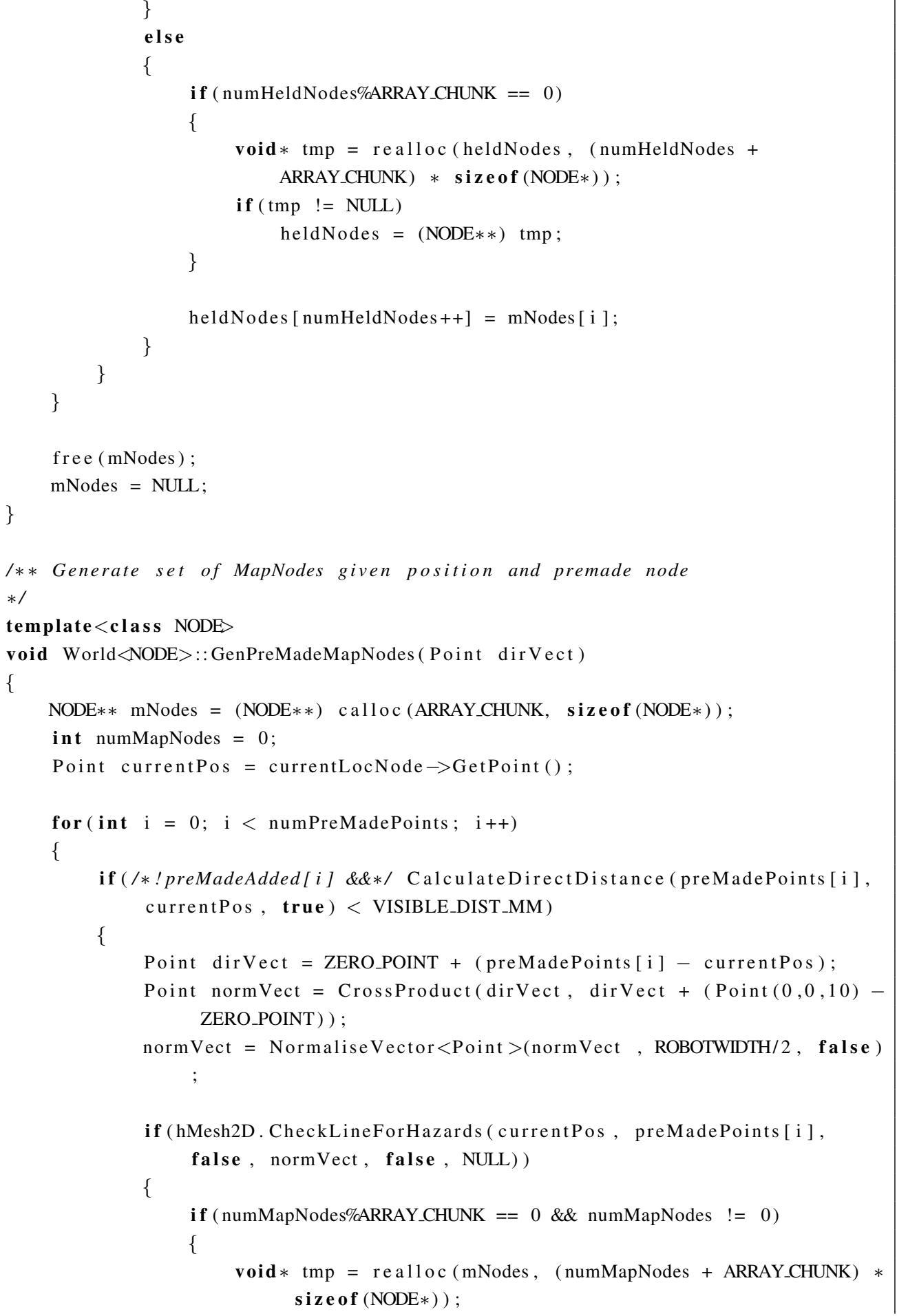




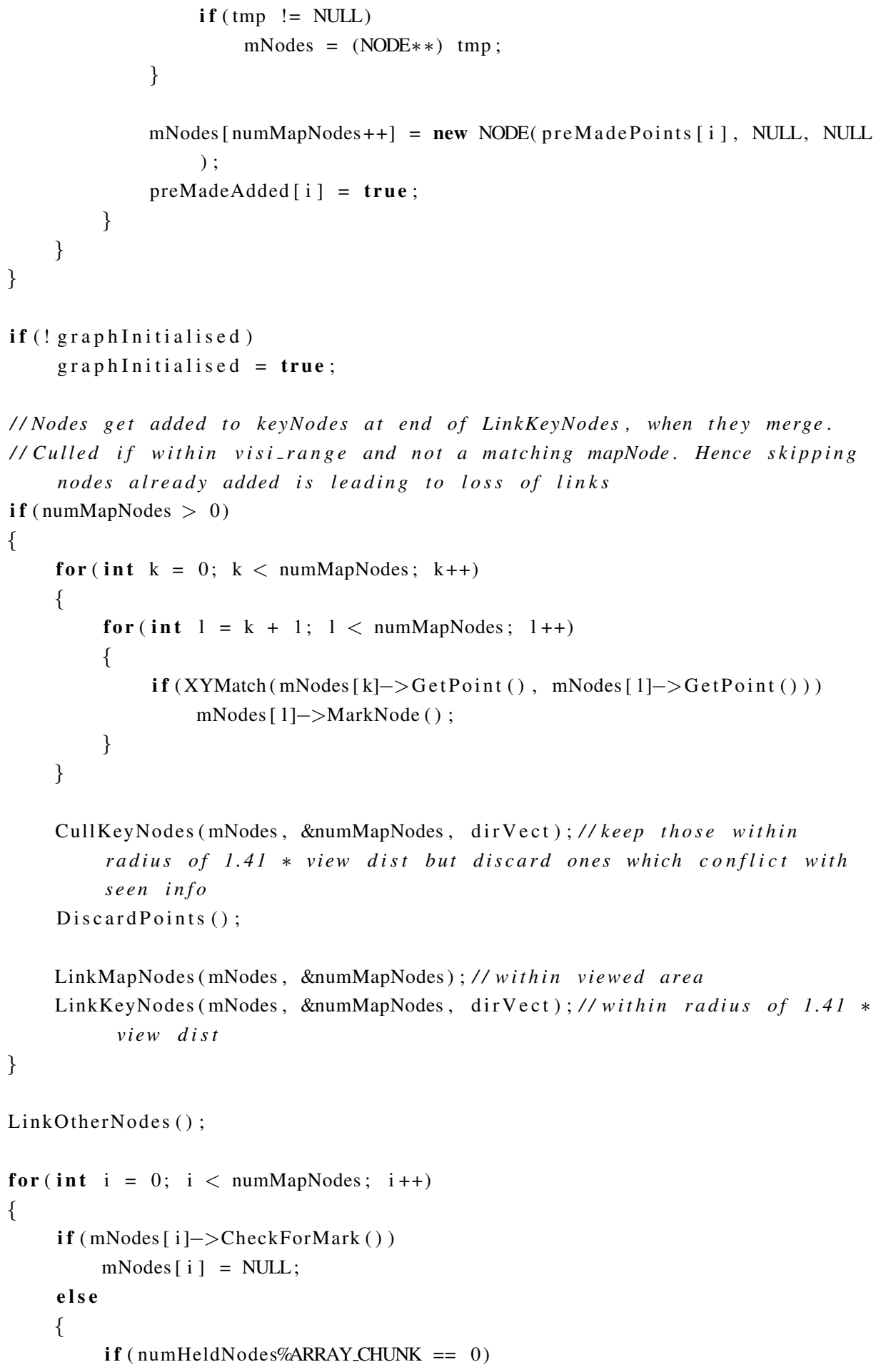




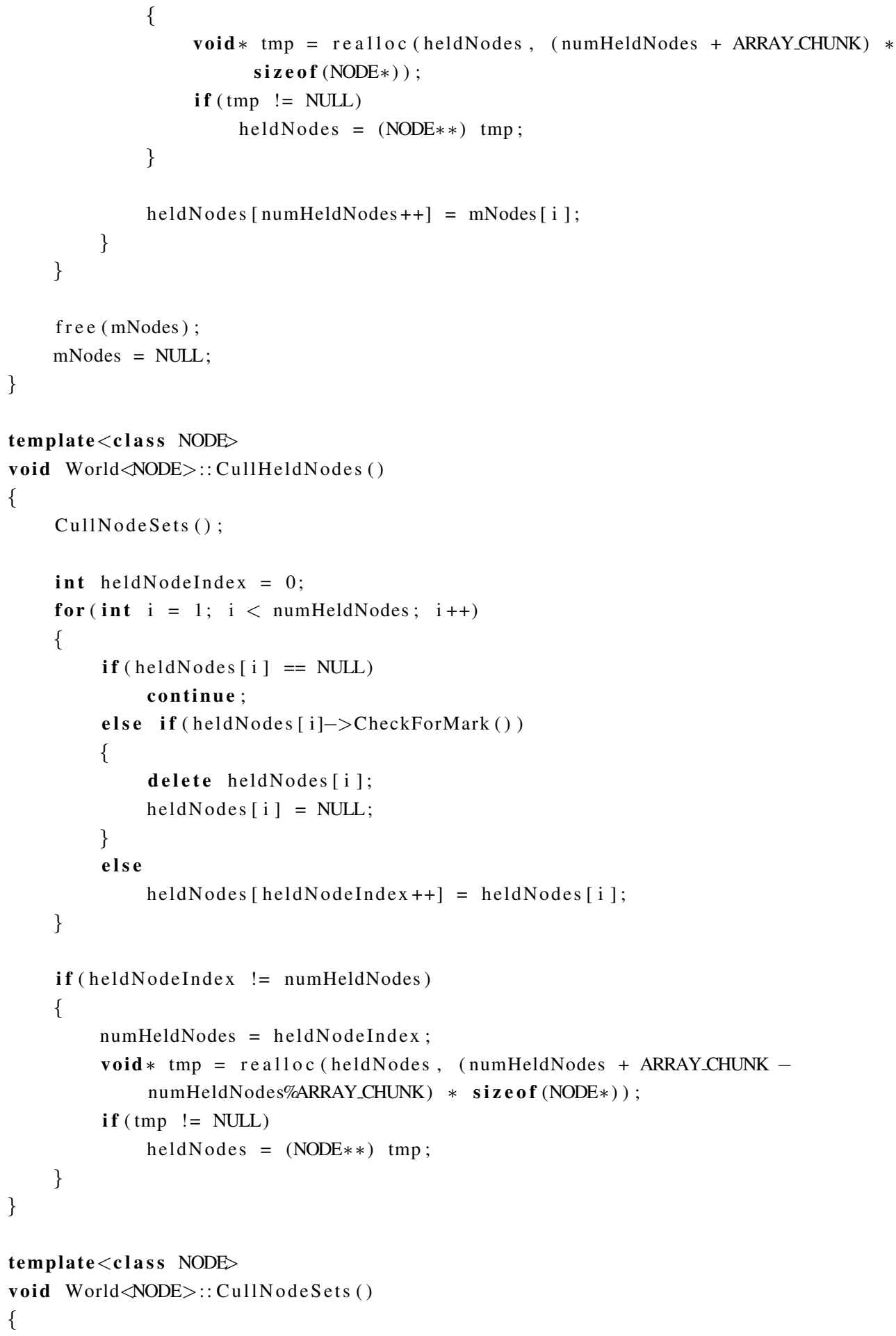




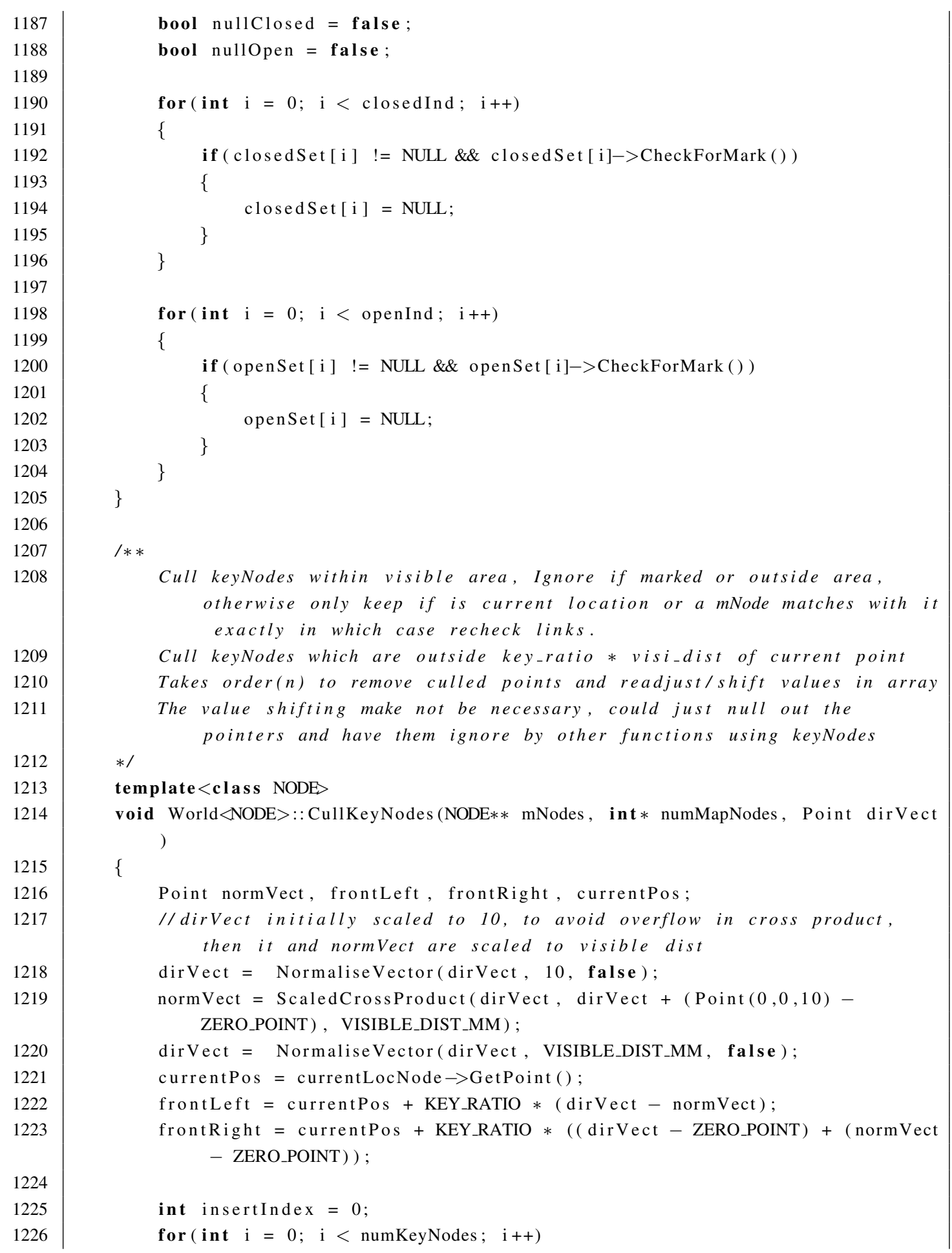




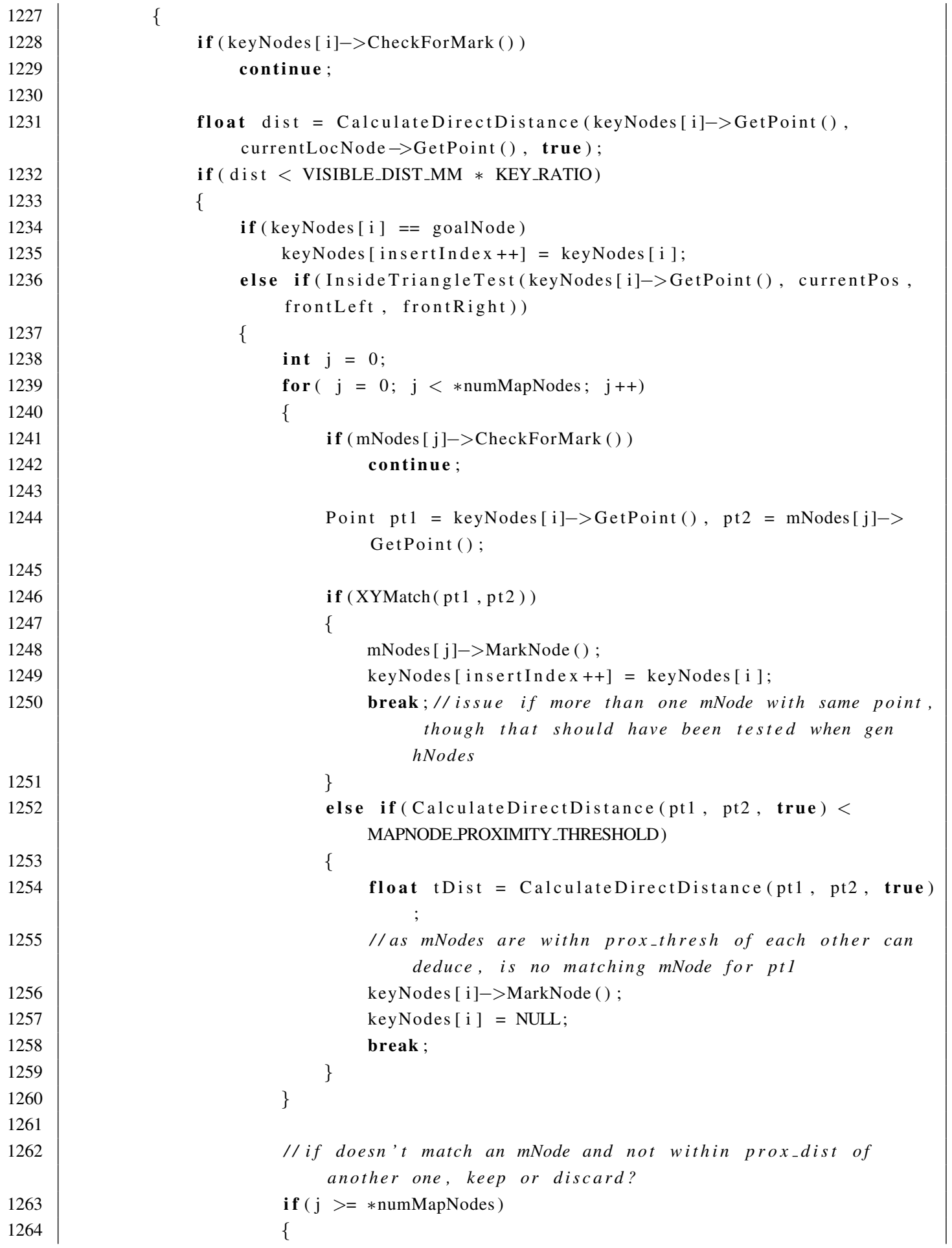




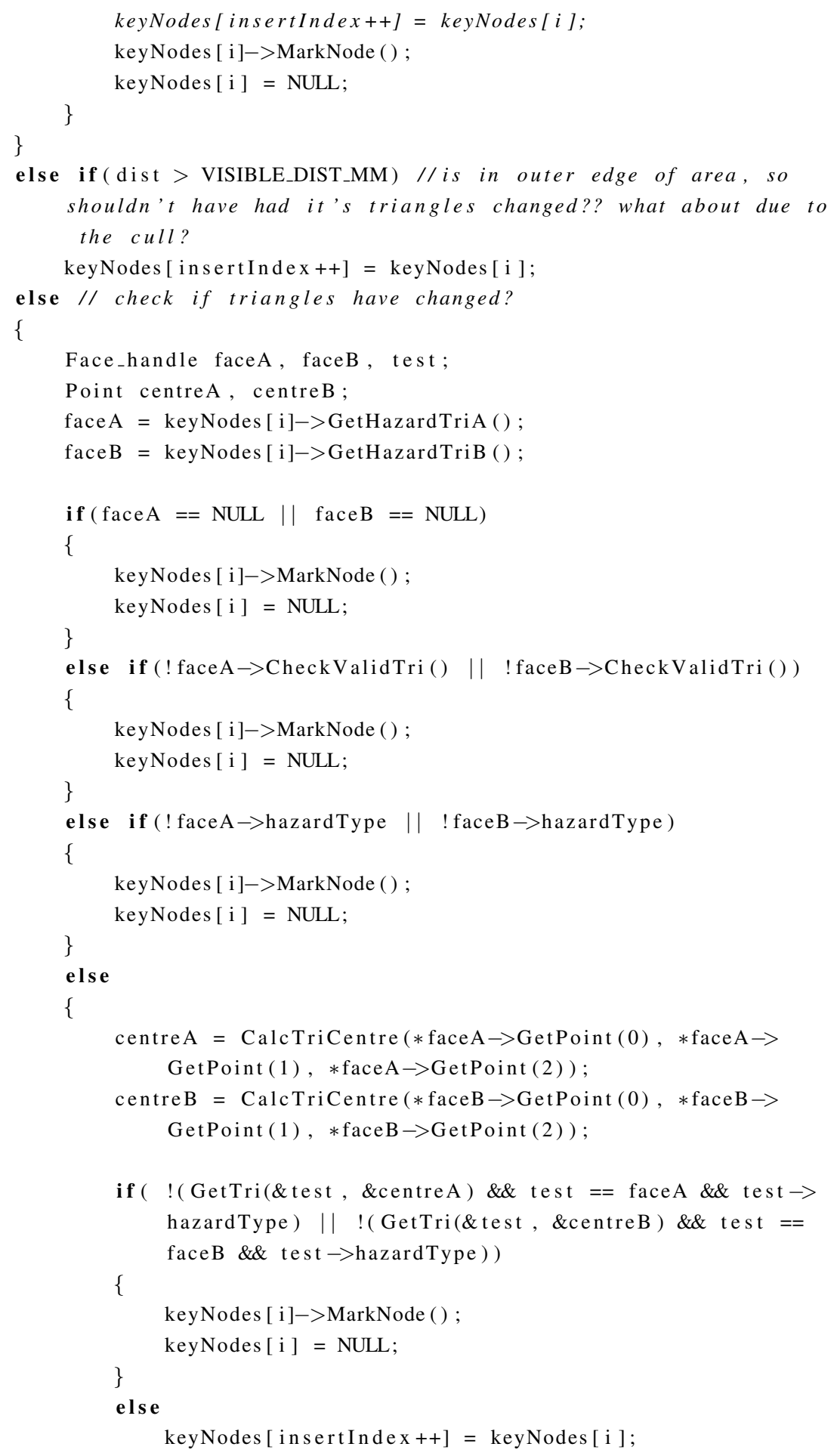


A.8. WORLD

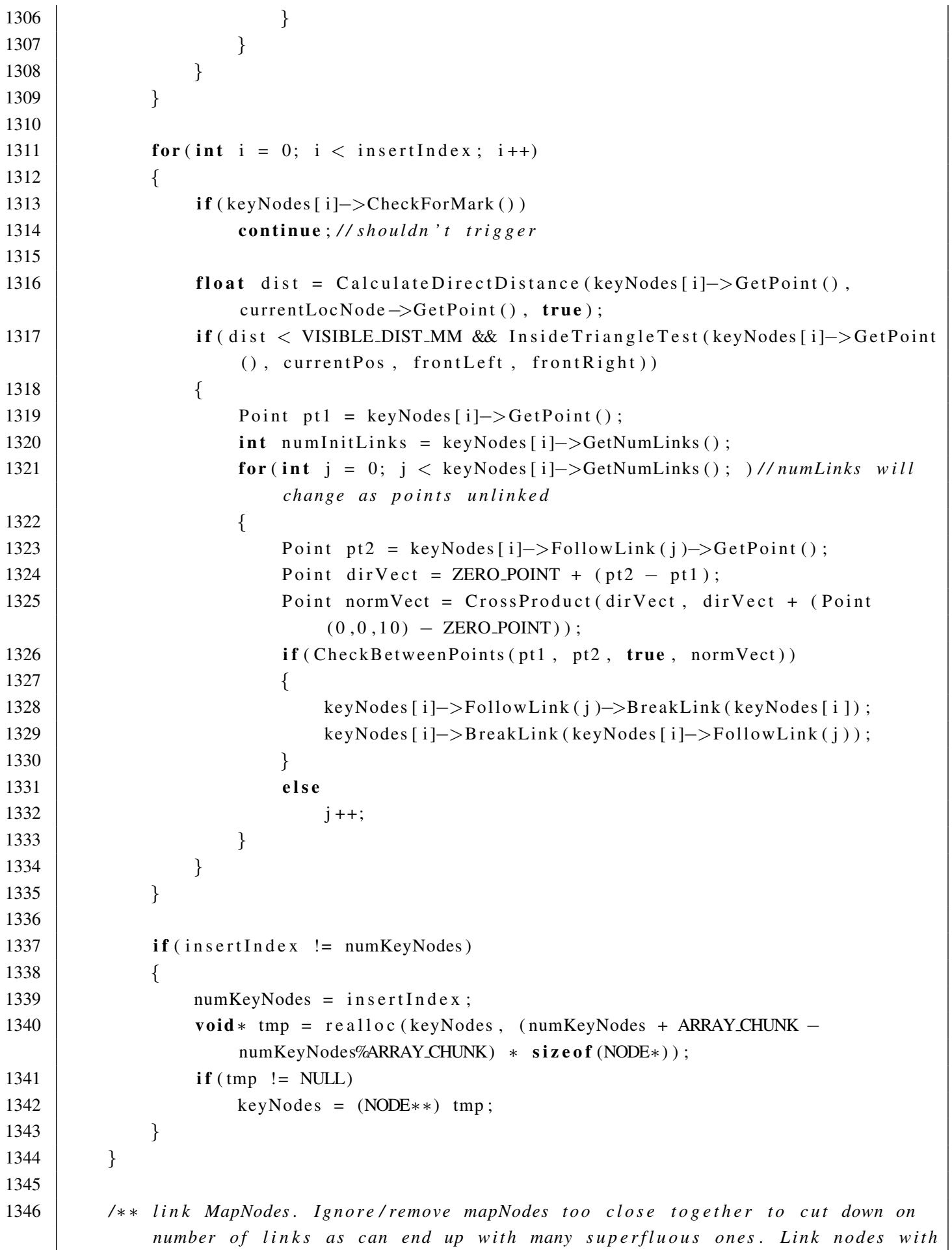




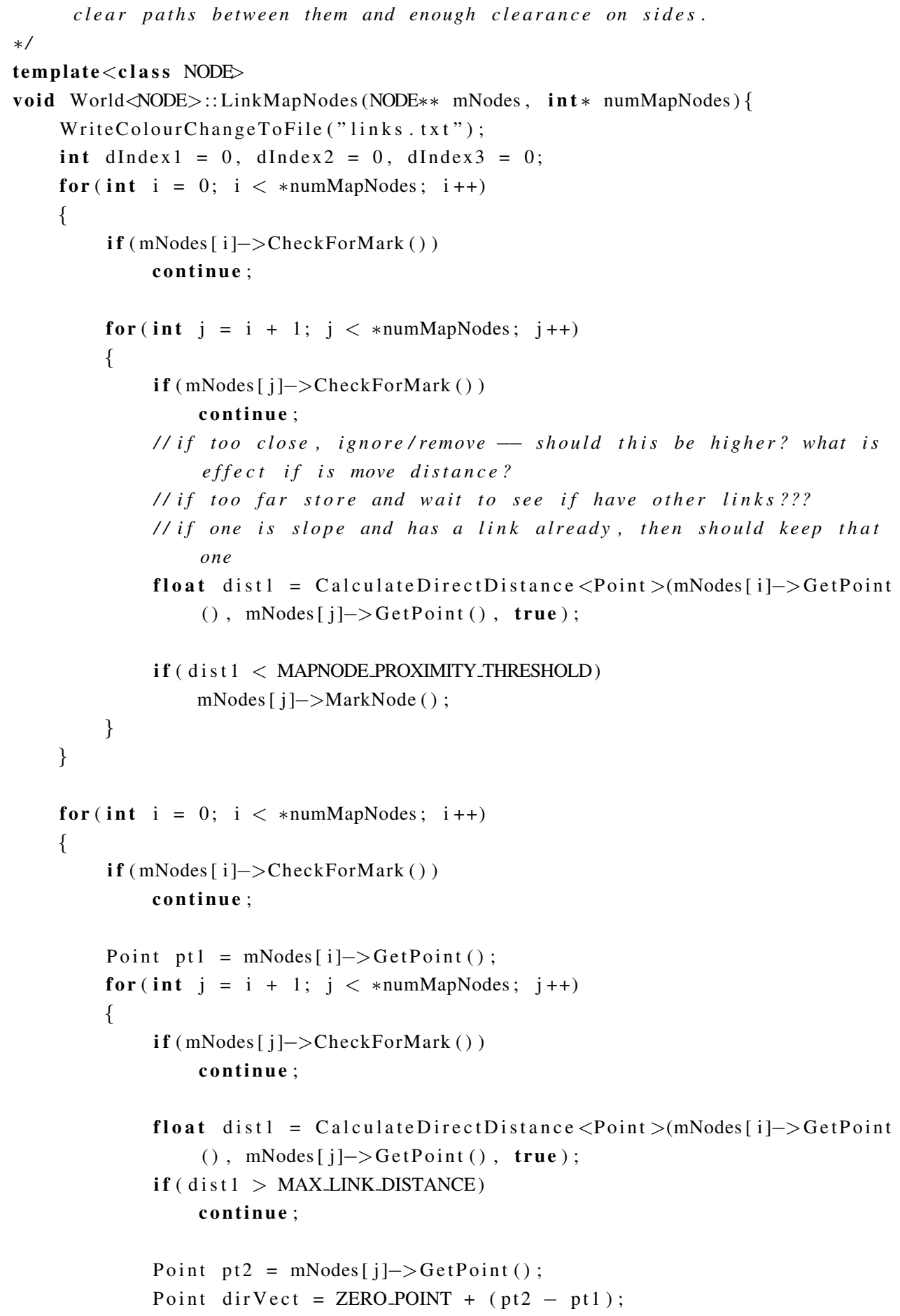


Point normVect $=$ CrossProduct $($ dirVect, dirVect $+(\operatorname{Point}(0,0,10)-$ ZERO_POINT) ) ; normVect $=$ NormaliseVector $<$ Point $>$ (normVect, ROBOTWIDTH $/ 2$, false $)$ ;

if (hMesh2D. CheckLineForHazards (pt1, pt2, true, normVect, false , NULL) )

\{

Point $*$ edge $[2]=\{\&$ pt $1, \&$ pt 2$\}$;

WriteLineToFile ("links.txt", "at", edge, 2);

mNodes [i]->AddLink (mNodes $[\mathrm{j}])$;

mNodes $[\mathrm{j}]->\operatorname{AddLink}(\operatorname{mNodes}[\mathrm{i}])$;

\}

$$
\}
$$

\}

\}

I** link freshly created mNodes with keyNodes (and thus the whole graph).

Links nodes with clear paths between them and enough clearance on sides.

Also appends the mNodes to the keyNodes list

*1

template $<$ class NODE $>$

void World $<$ NODE $>$ : LinkKeyNodes (NODE** mNodes, int $*$ numMapNodes, Point dirVect )

int $\mathrm{dIndex} 1=0, \operatorname{dIndex} 2=0, \operatorname{dIndex} 3=0$;

for (int $\mathrm{i}=0 ; \mathrm{i}<*$ numMapNodes $; \mathrm{i}++$ )

\{

if ( mNodes [ i ]->CheckForMark ())

continue ;

Point pt $1=\operatorname{mNodes}[\mathrm{i}]->$ GetPoint () ;

for (int $\mathrm{j}=0 ; \mathrm{j}<$ numKeyNodes $; \mathrm{j}++$ )

\{

if ( keyNodes $[\mathrm{j}]->$ CheckForMark ( ) )

continue ;

Point pt2 = keyNodes $[\mathrm{j}]->$ GetPoint ();

if $(\mathrm{pt} 1==\mathrm{pt} 2)$

\{

\}

else if (XYMatch $(p t 1, p t 2))$

\{

CullKeyNodes (mNodes, numMapNodes, dirVect);

\}

else

\{ 
float dist $1=$ CalculateDirectDistance $\langle$ Point $>(\operatorname{mNodes}[\mathrm{i}]->$ GetPoint (), keyNodes $[\mathrm{j}]->$ GetPoint (), true ); if ( $\mathrm{d}$ is t $1>$ MAX_LINK_DISTANCE) continue ;

Point dirVect $=$ ZERO_POINT $+($ pt2 - pt 1$)$;

Point normVect $=$ CrossProduct $($ dirVect, dirVect + (Point $(0,0,10)$ - ZERO_POINT) $)$;

Point tempNorm $=$ normVect;

normVect $=$ NormaliseVector $<$ Point $>$ (normVect , ROBOTWIDTH/2, false );

$/ /$ issue if pt1 and pt2 are the same. how does this occur modes created from crossing lines.?

if (hMesh2D. CheckLineForHazards (pt1, pt2, true, normVect, false, NULL) )

\{

Point $*$ edge $[2]=\{\&$ pt $1, \&$ pt 2$\} ;$

WriteLineToFile ("links . txt", "at", edge, 2);

mNodes [i] $\rightarrow$ AddLink ( (NODE*) keyNodes $[\mathrm{j}])$;

keyNodes $[\mathrm{j}]->\operatorname{AddLink}(\operatorname{mNodes}[\mathrm{i}])$;

\}

\}

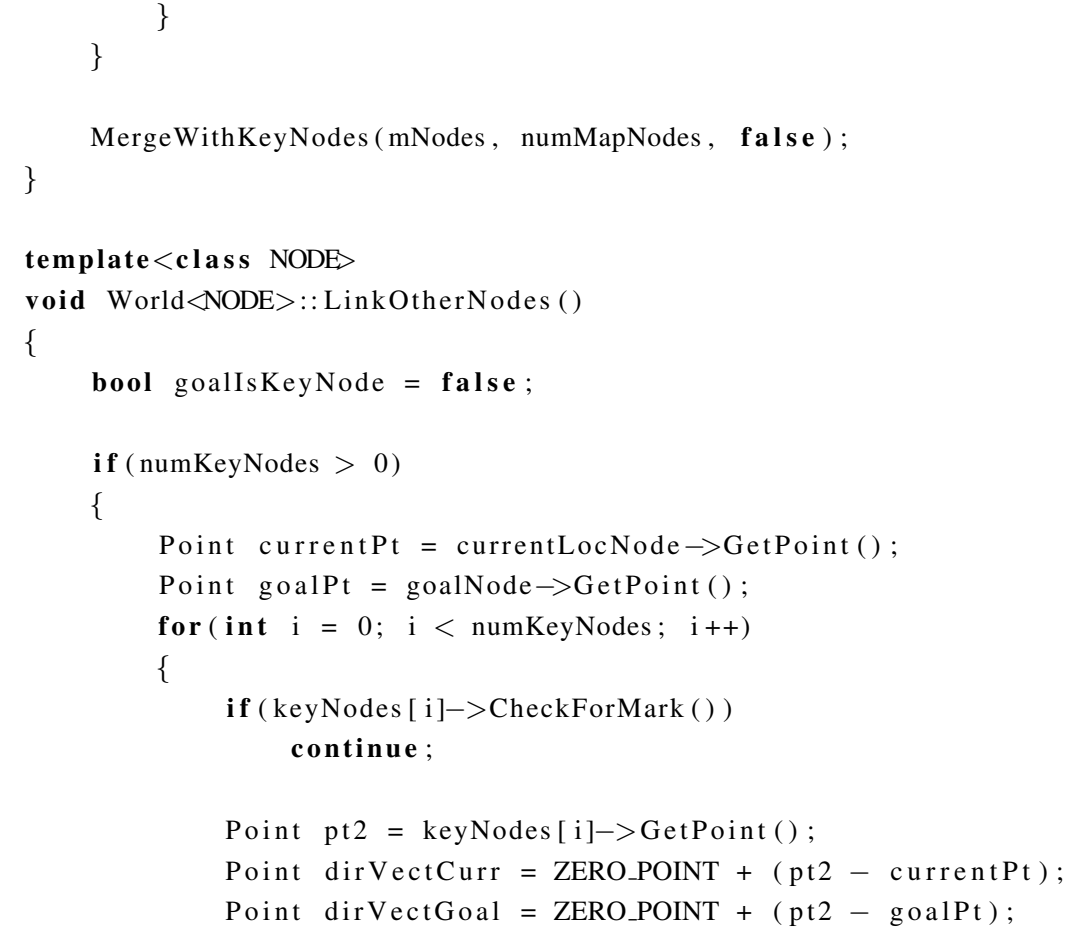




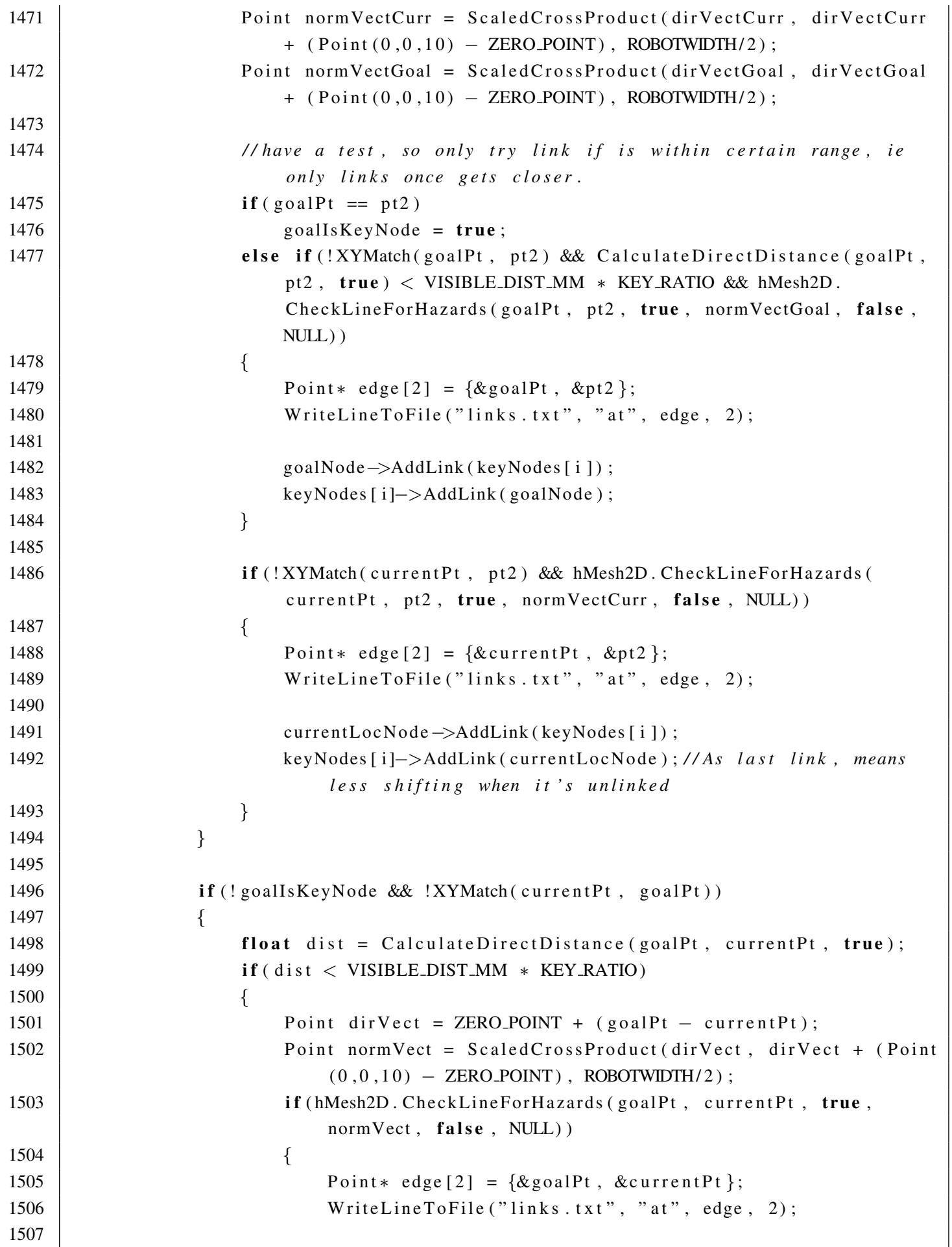




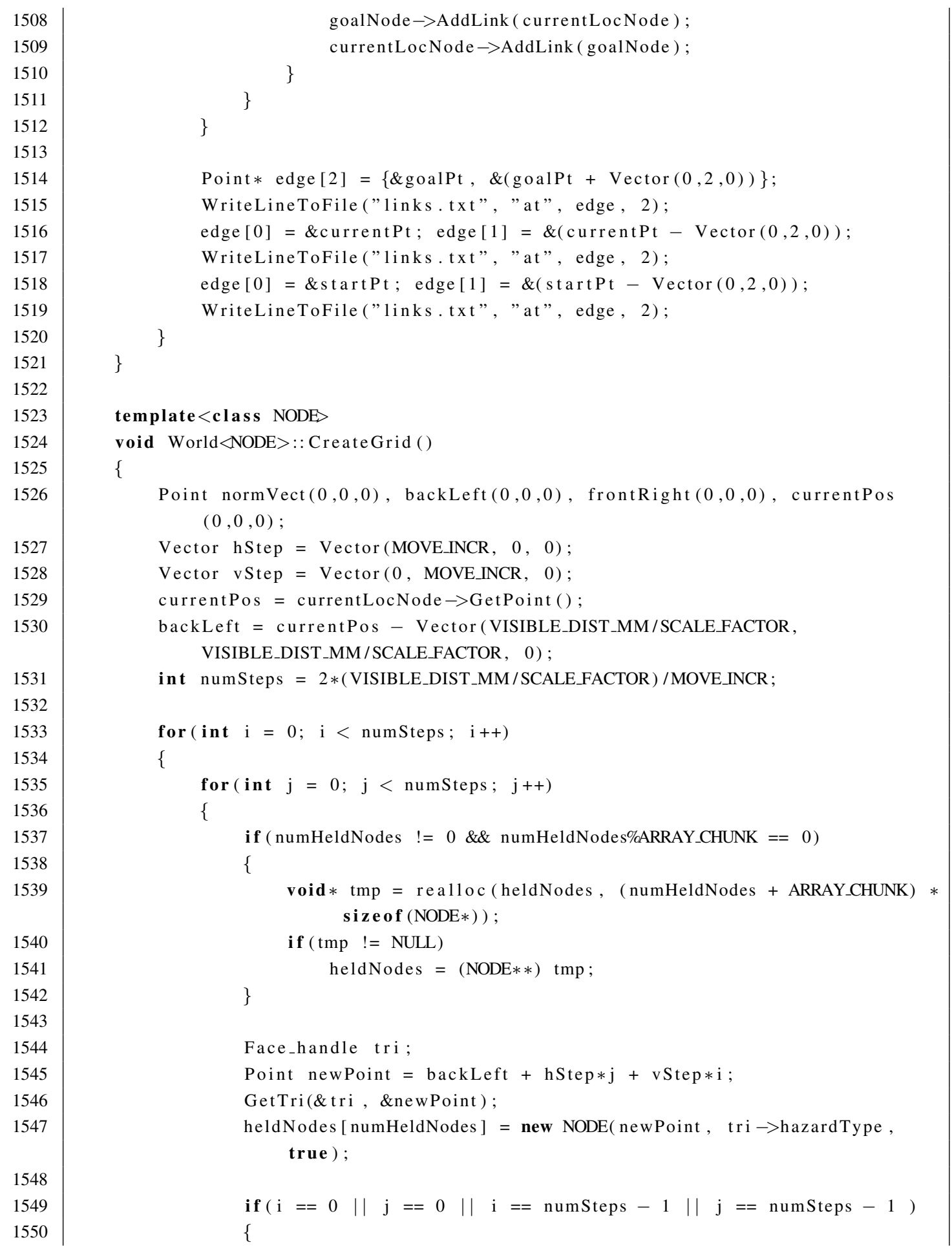


A.8. WORLD

1551
1552
1553
1554
1555
1556
1557
1558
1559
1560
1561
1562
1563
1564
1565
1566
1567
1568
1569
1570
1571
1572
1573
1574
1584
1585
1575
1576
1577
1587
1582

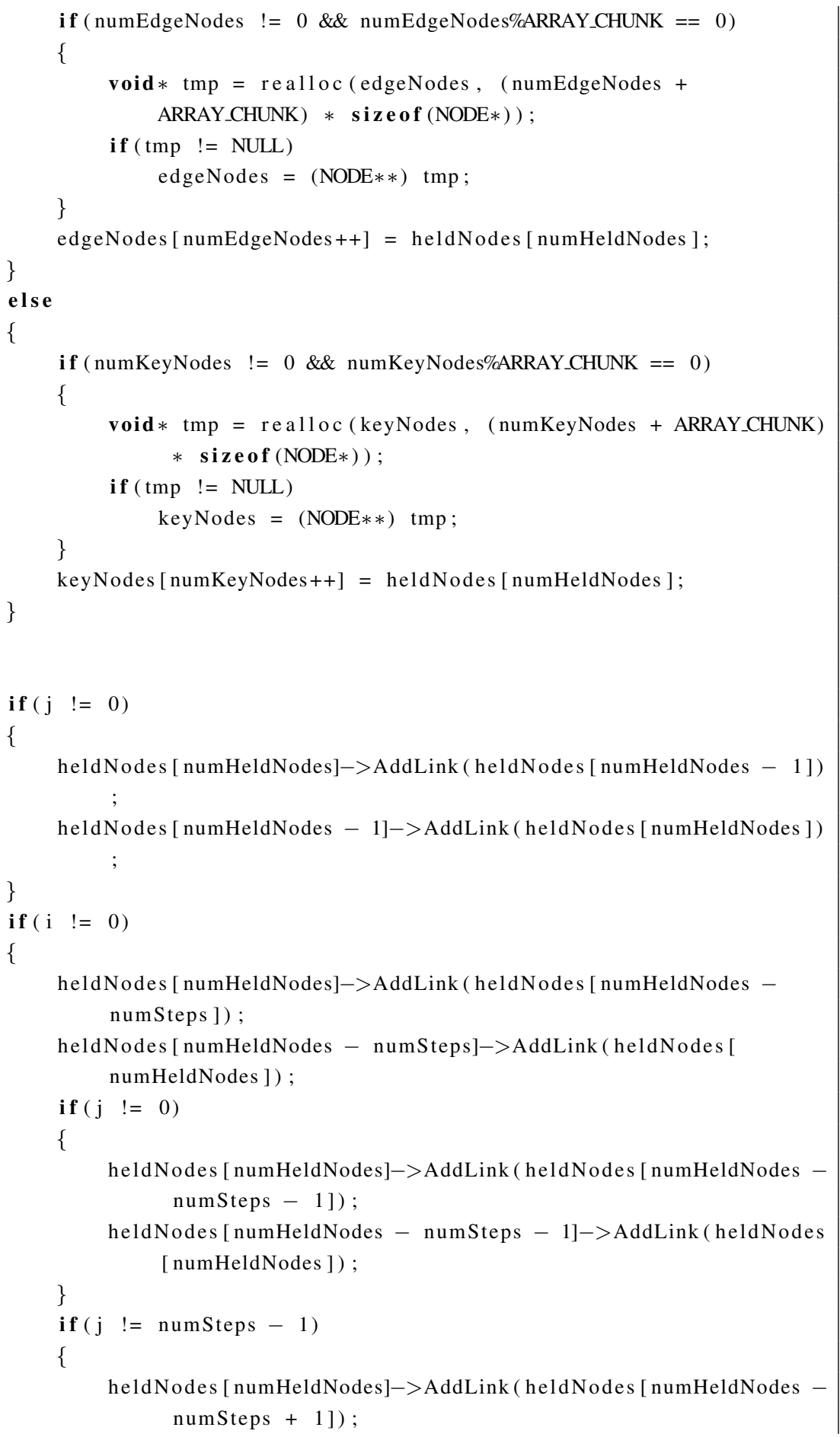




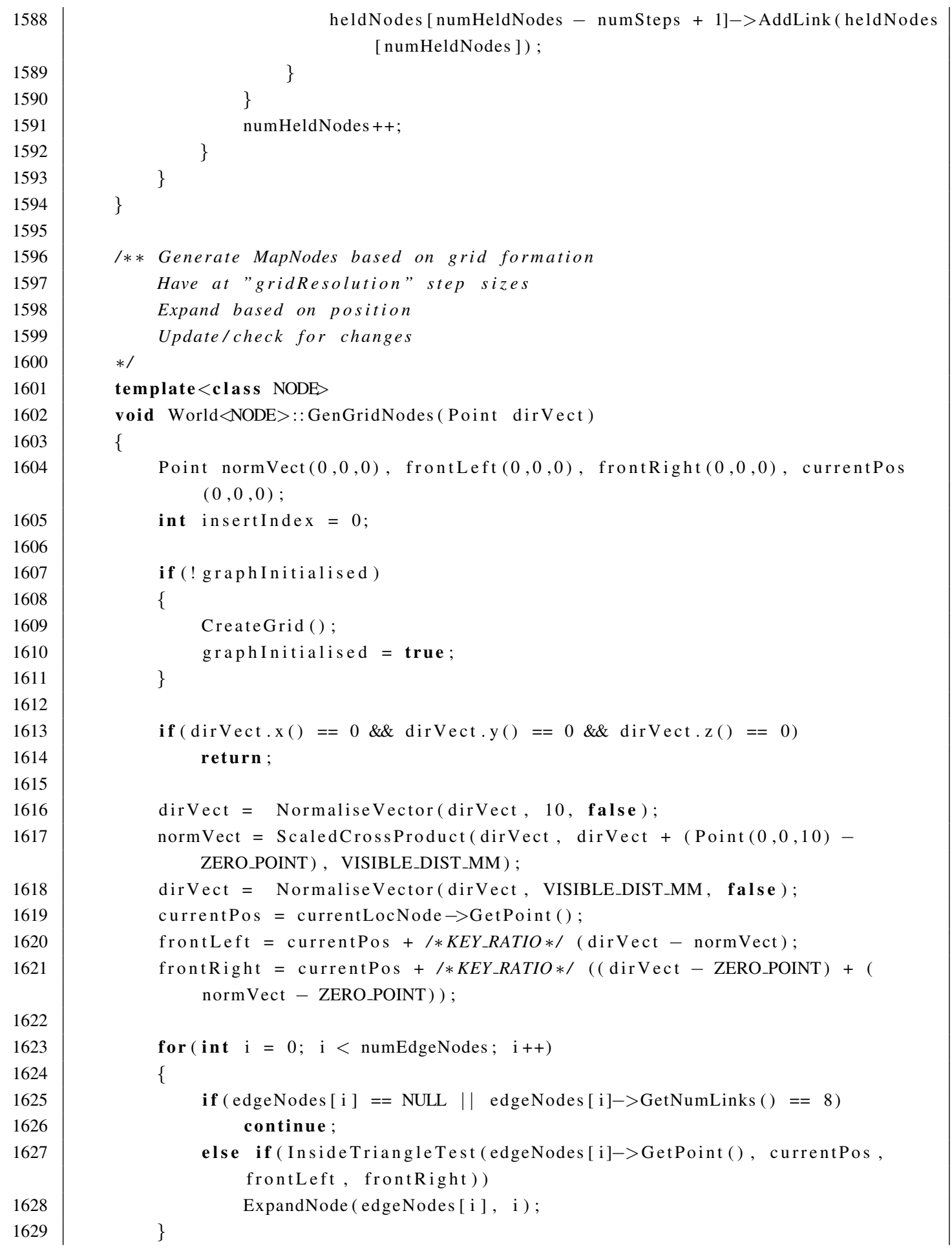




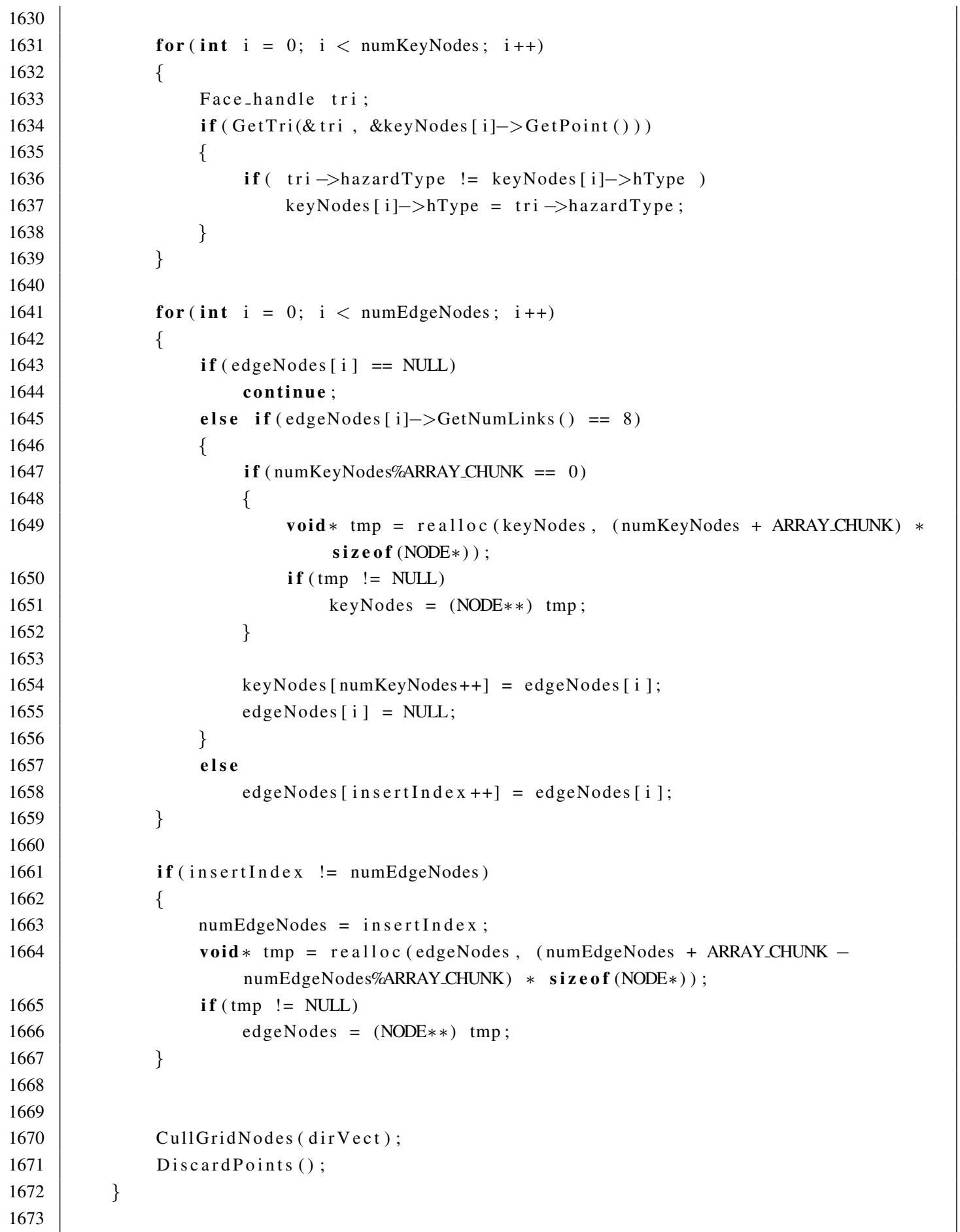




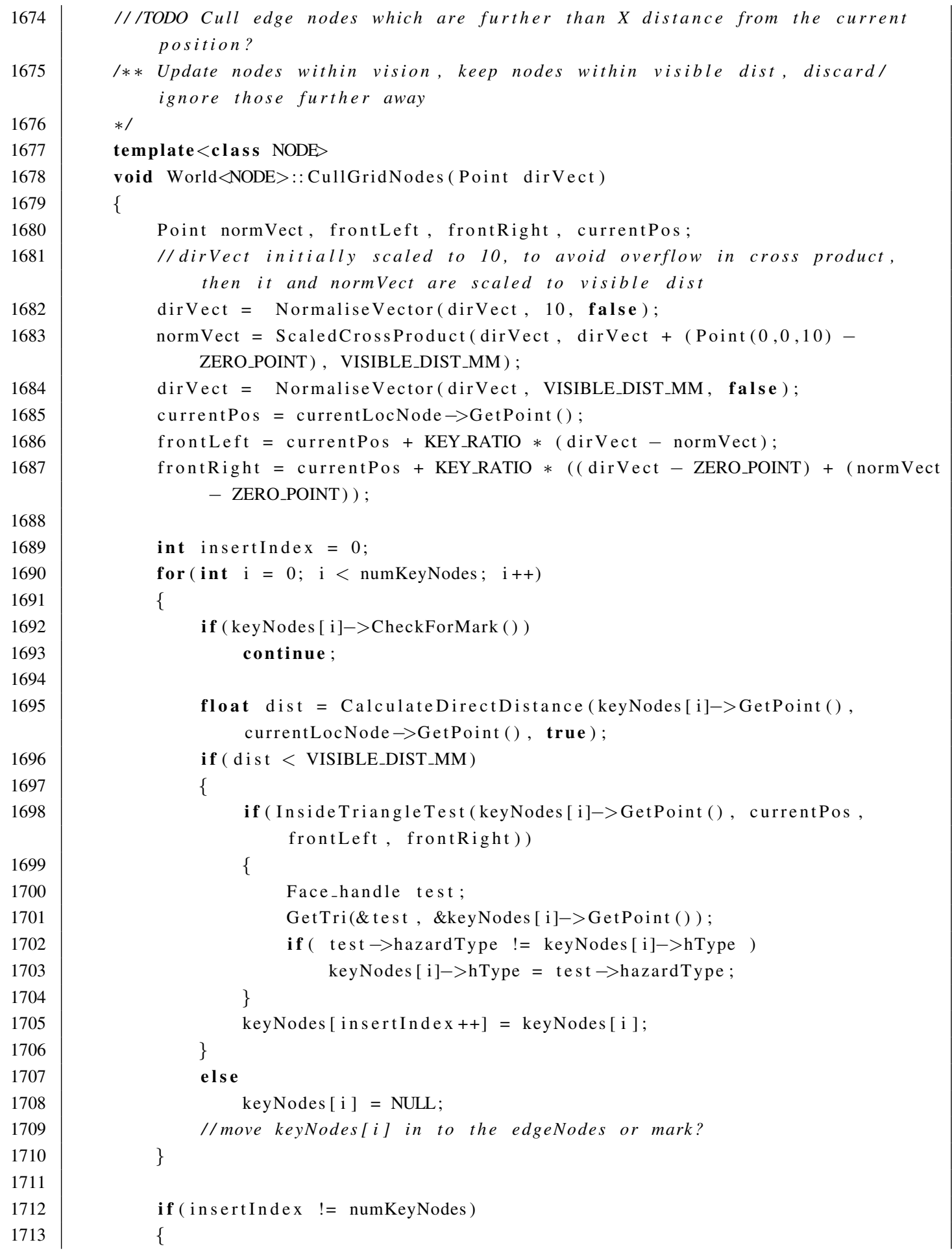




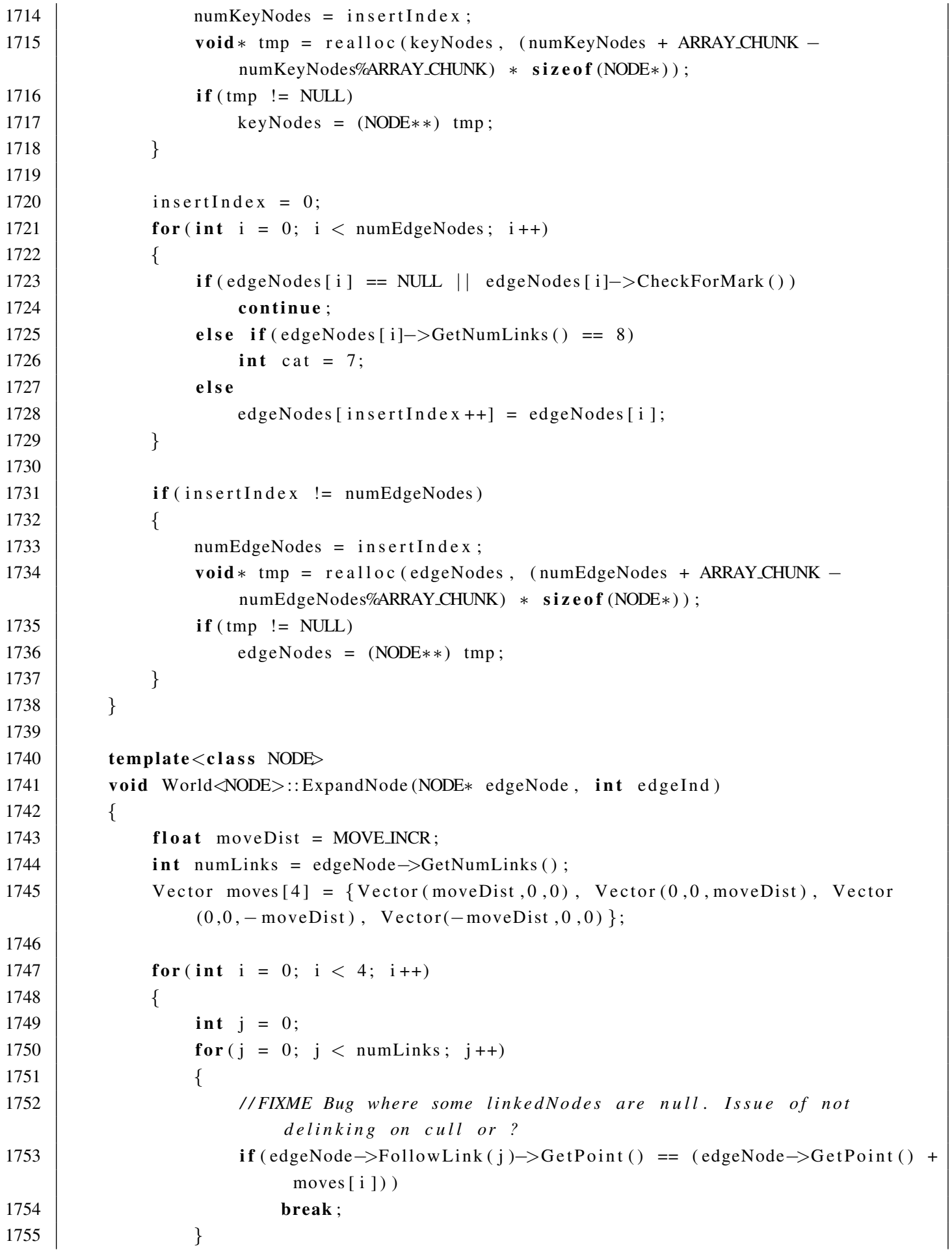




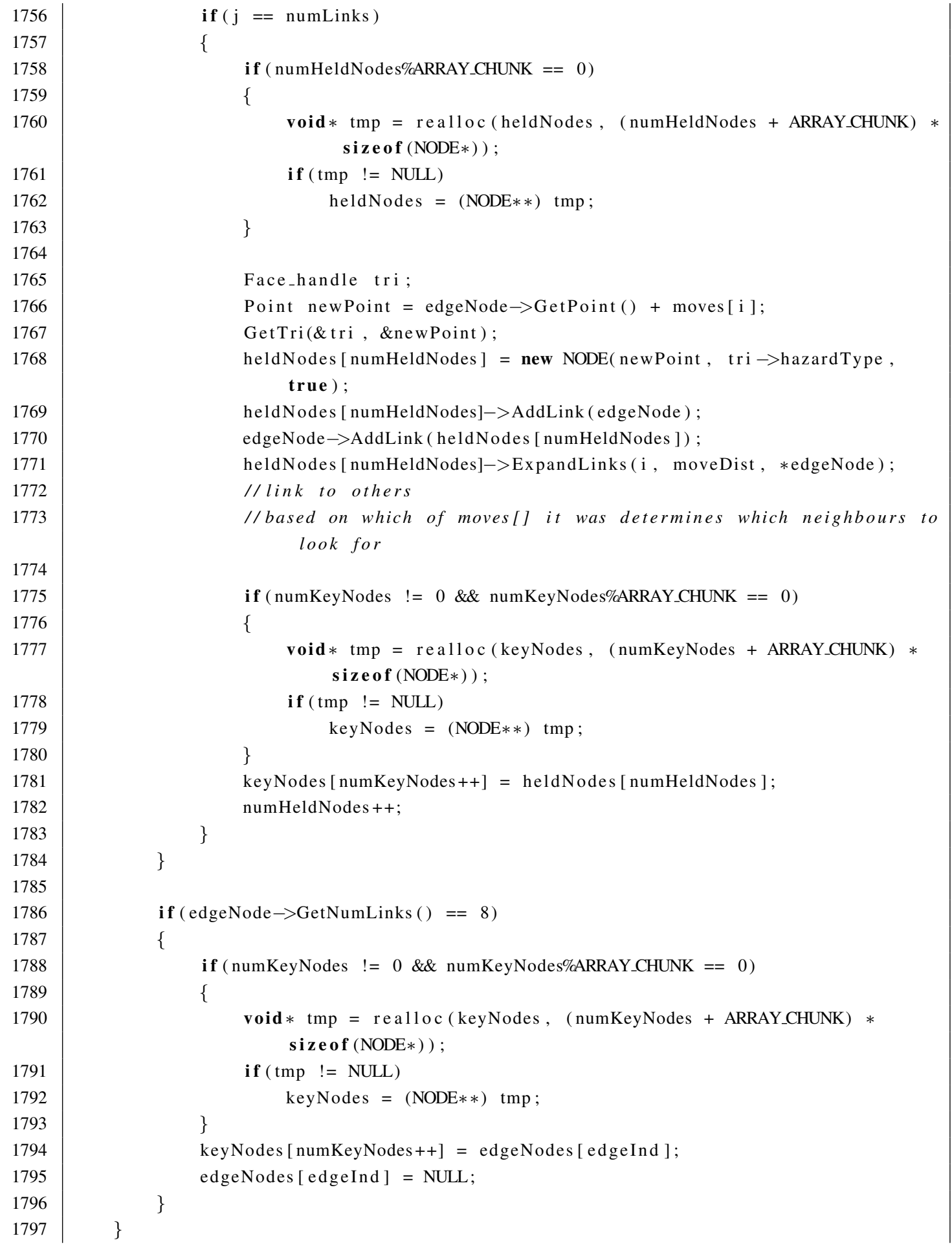




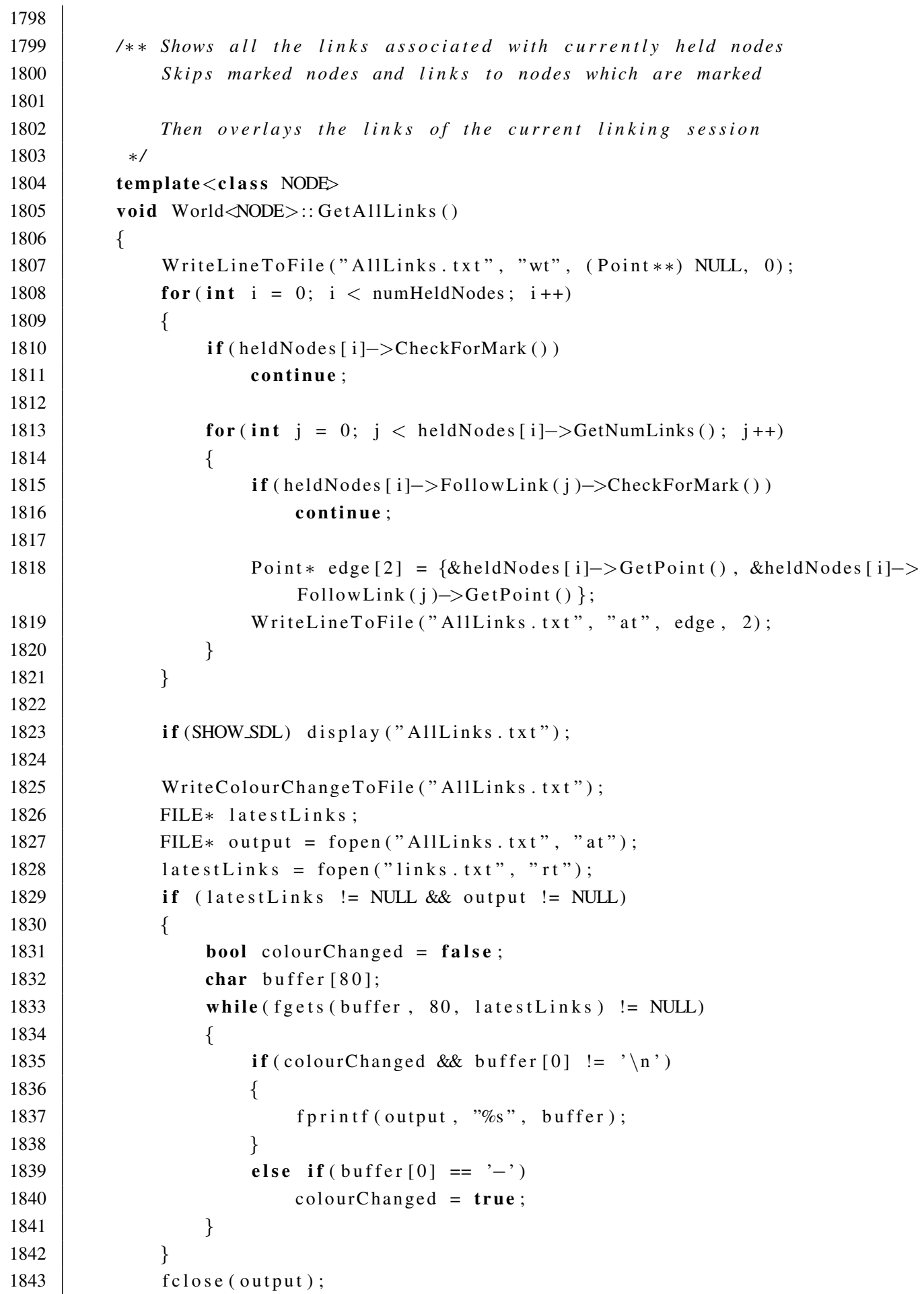




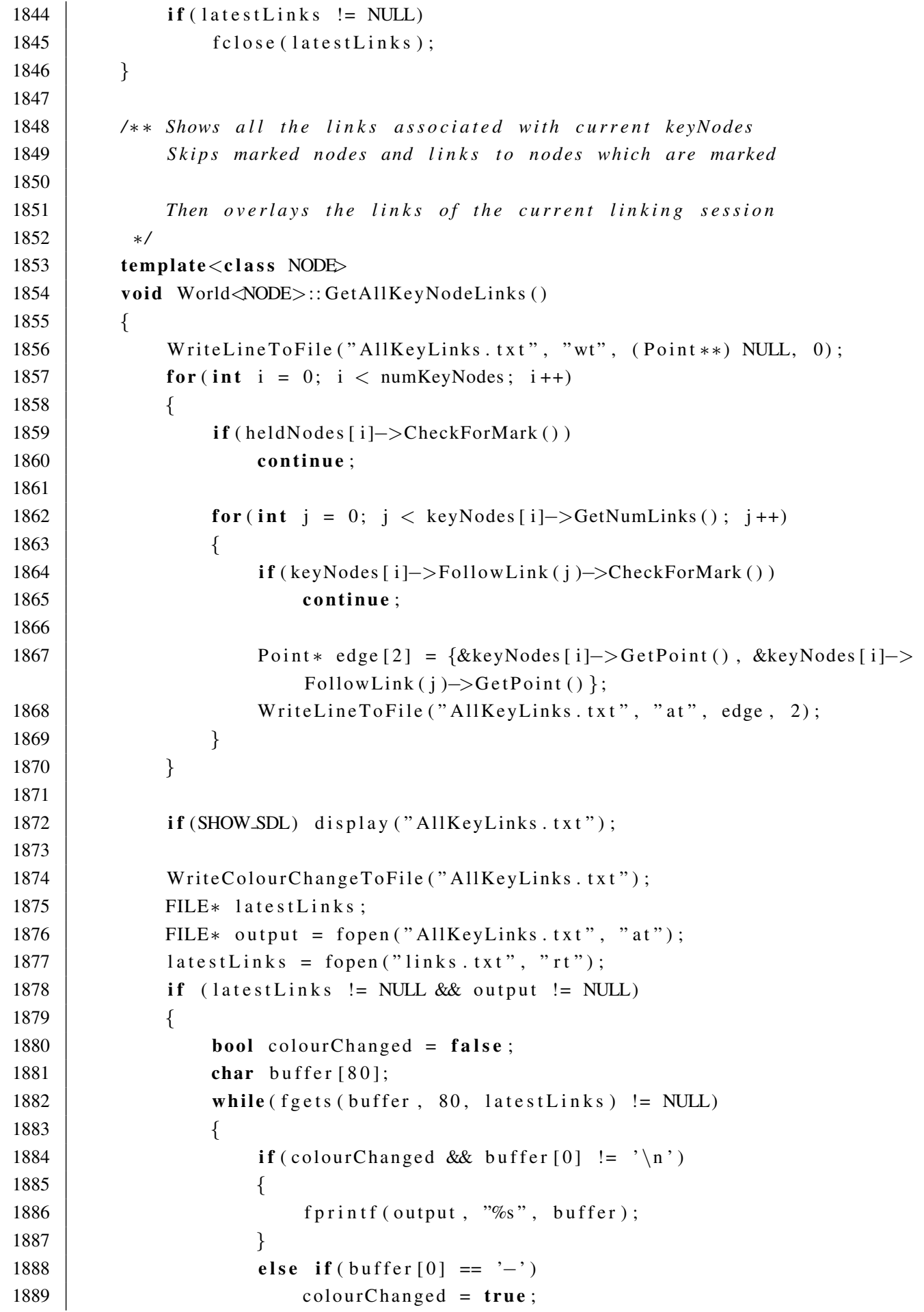




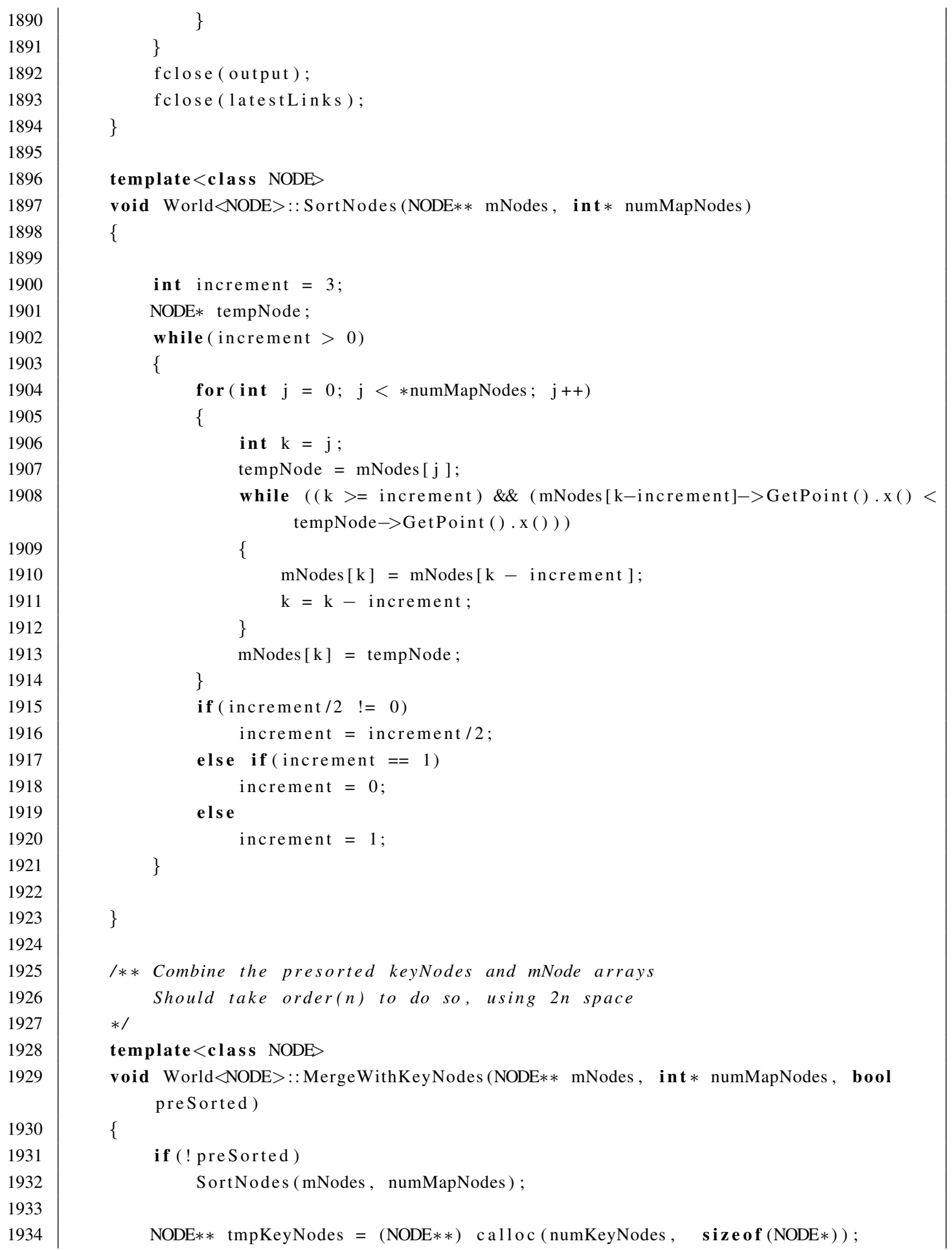




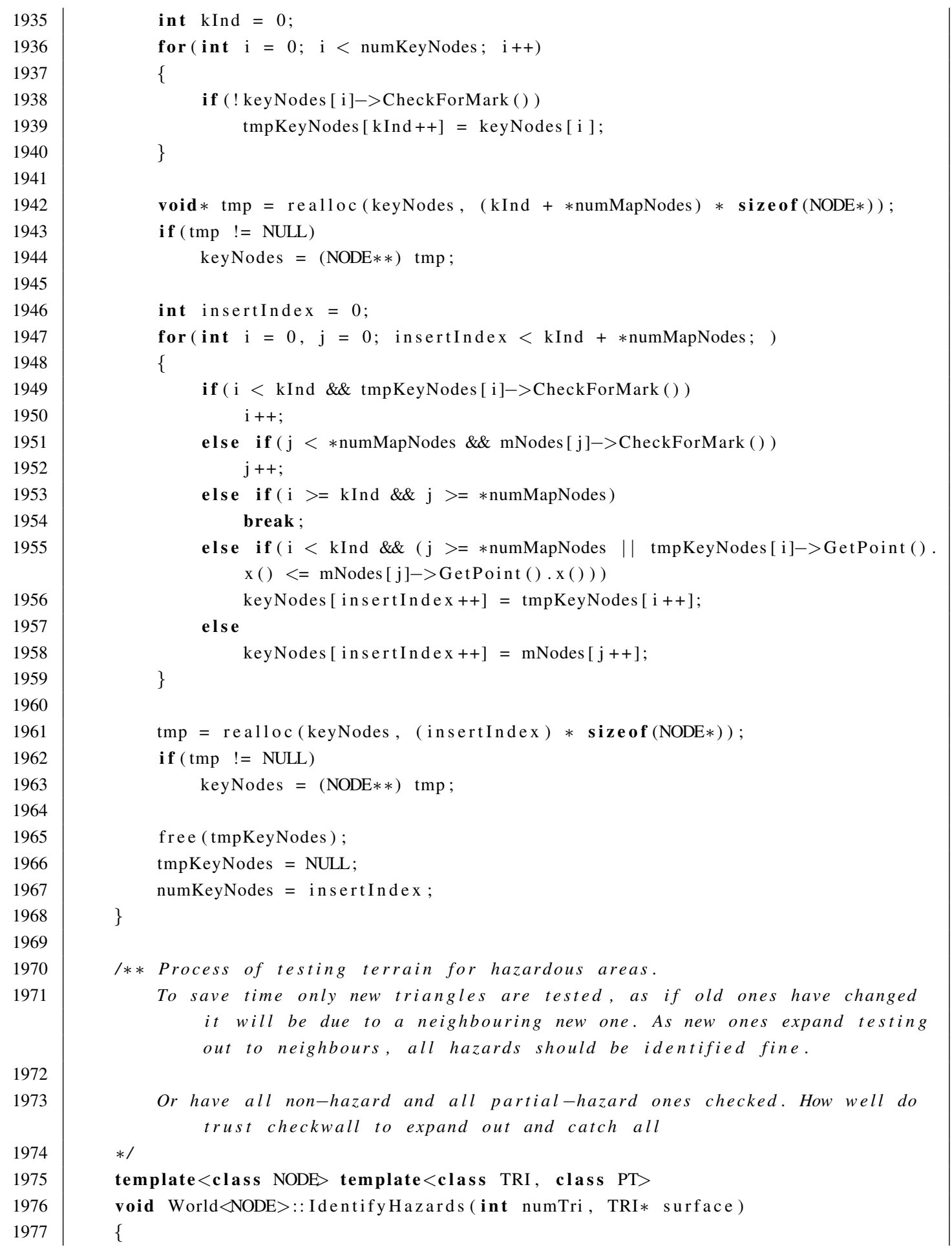




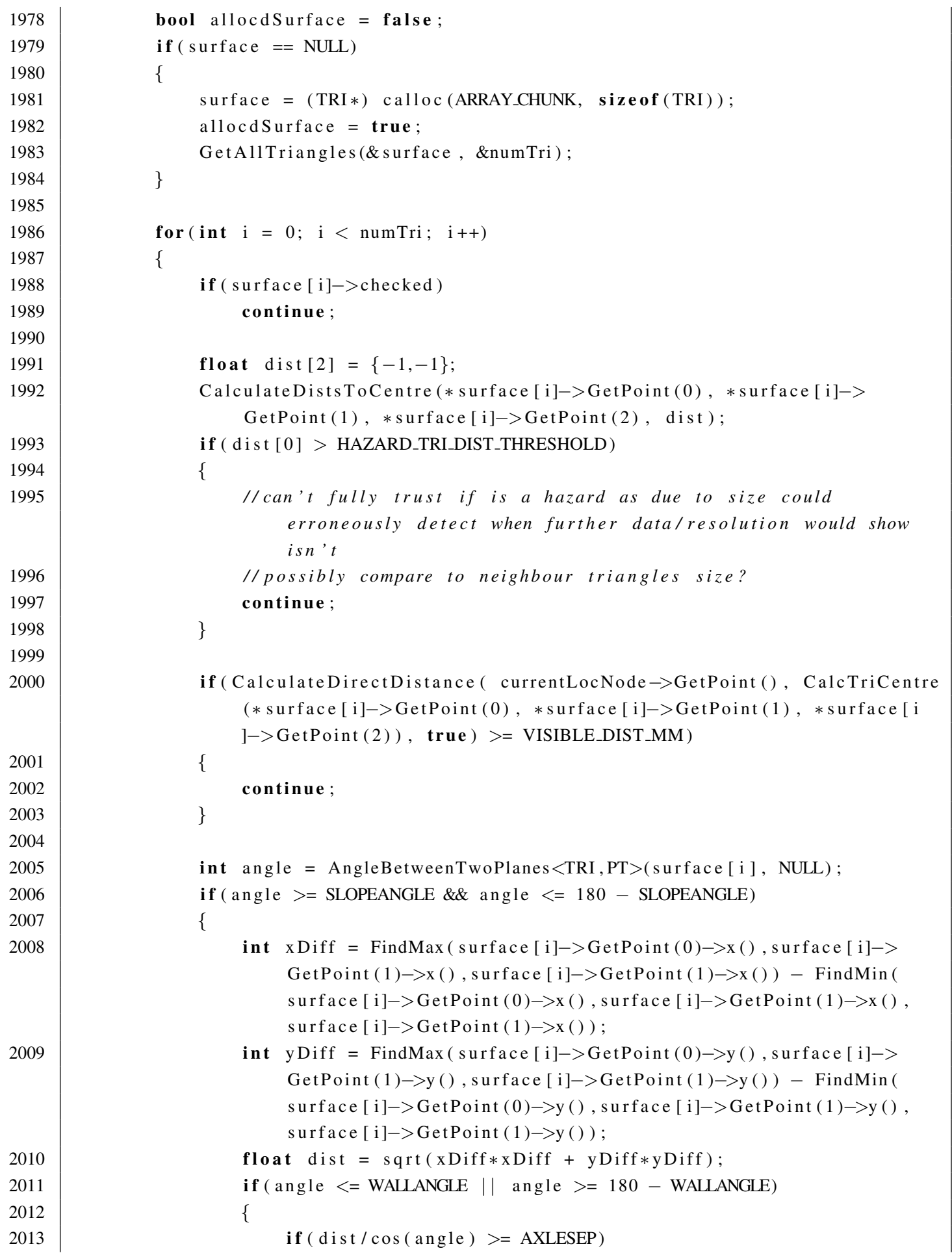




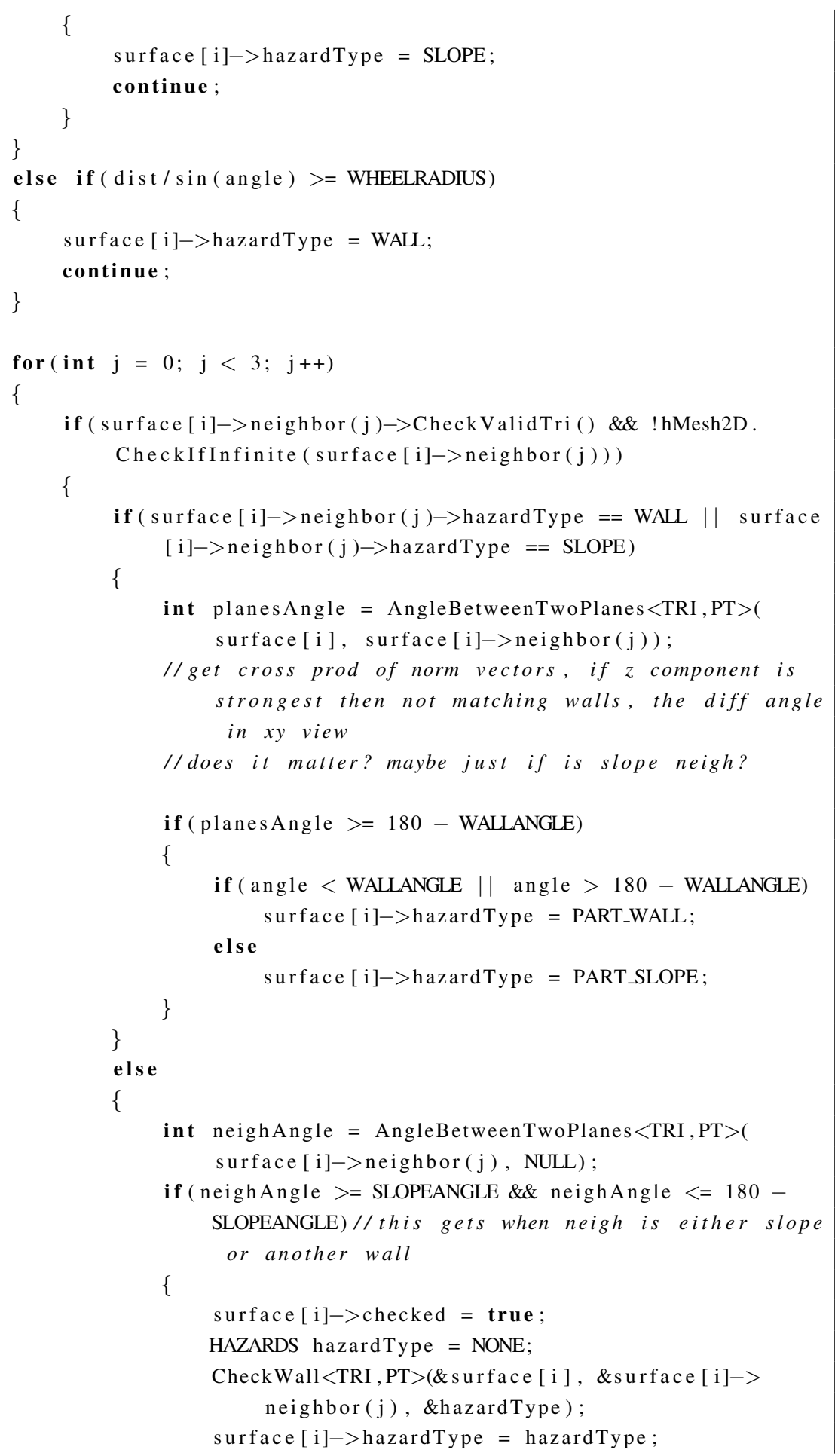


A.8. WORLD

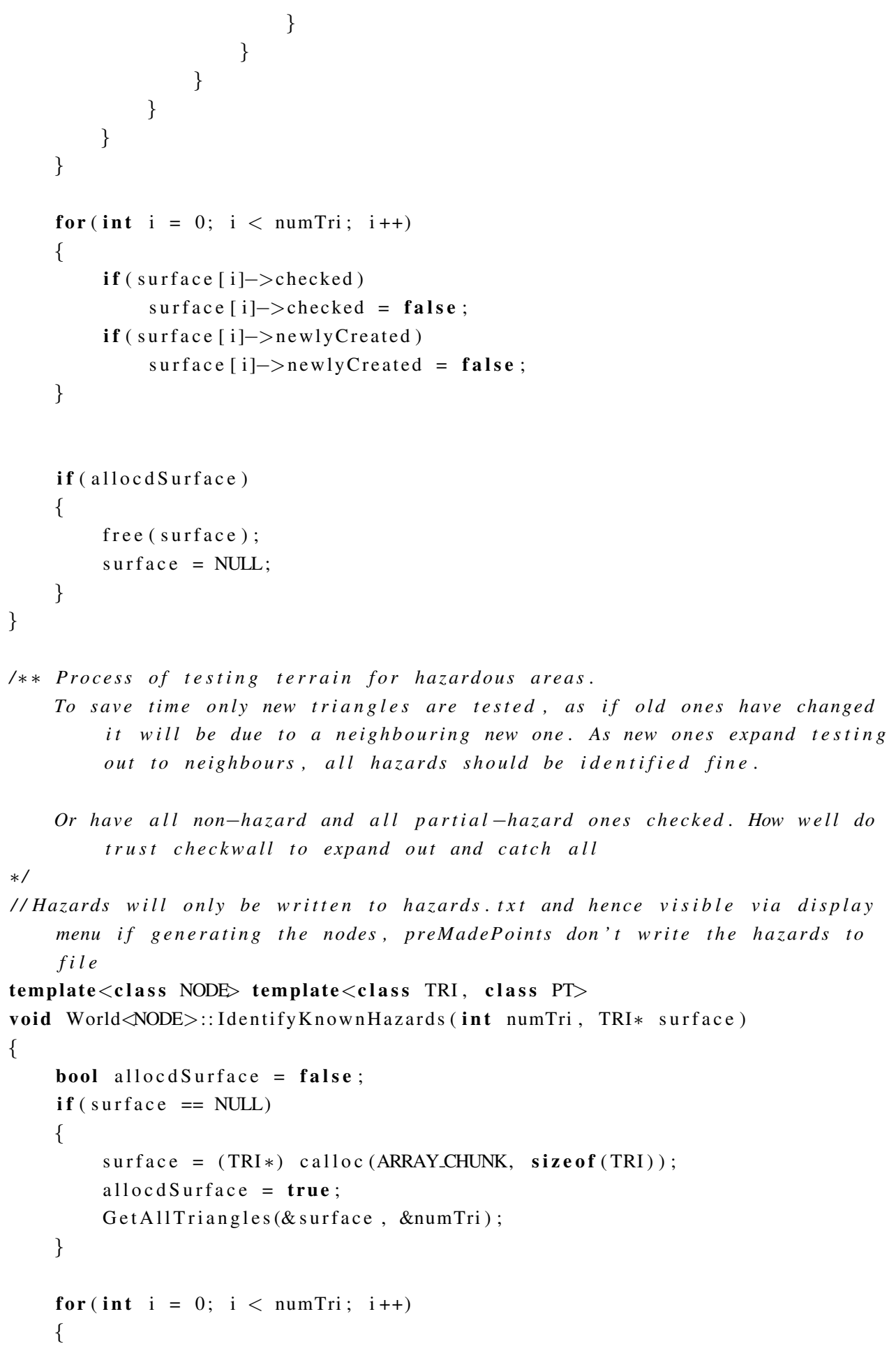




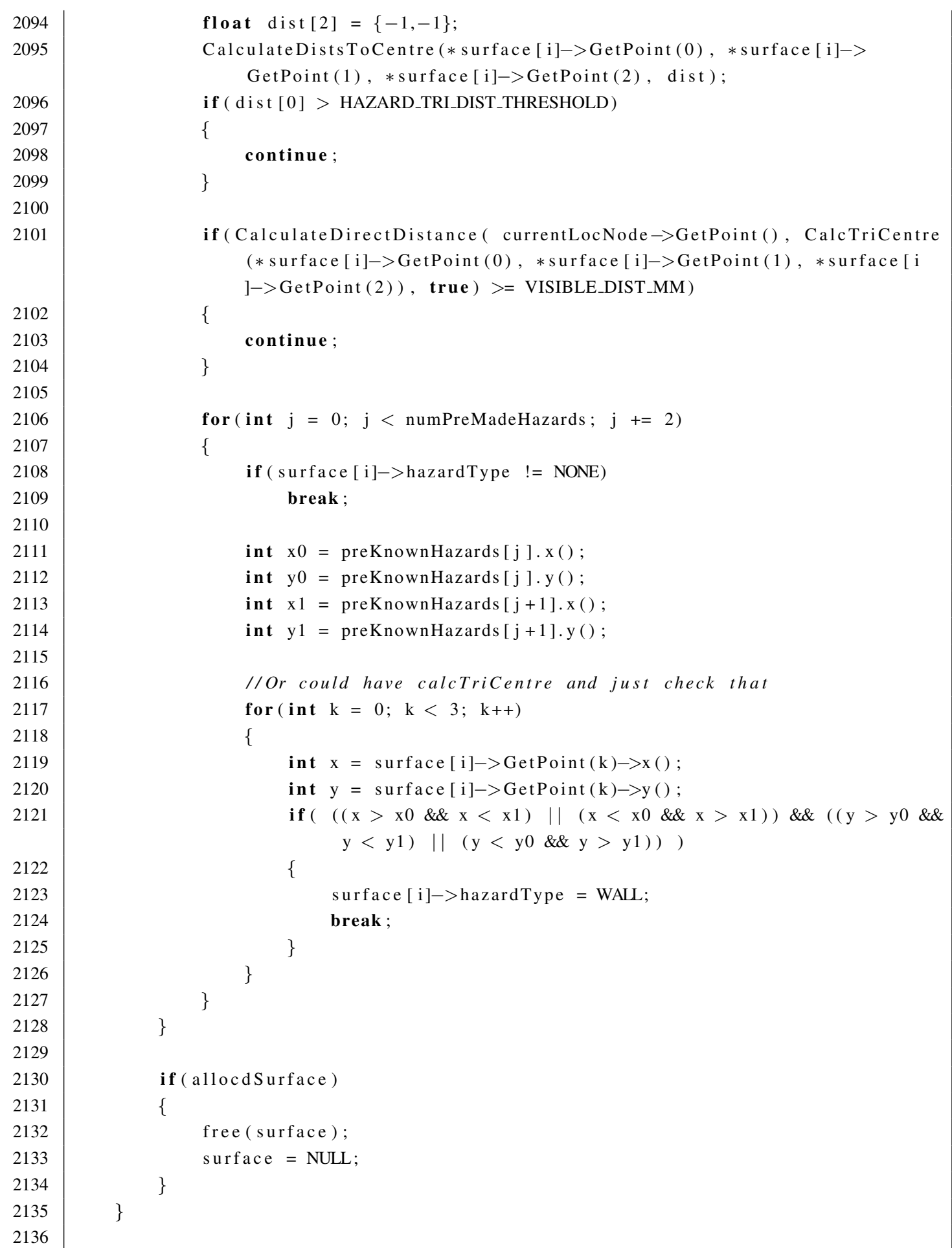

float dist $[2]=\{-1,-1\}$;

CalculateDistsToCentre (*surface $[i]->$ GetPoint $(0)$, *surface $[i]->$ GetPoint (1), *surface [i]->GetPoint (2), dist); if ( $\mathrm{d}$ i $\mathrm{st}[0]>$ HAZARD_TRI_DIST_THRESHOLD)

\{ continue ;

\}

if (CalculateDirectDistance ( currentLocNode $\rightarrow$ GetPoint (), CalcTriCentre $(* \operatorname{surface}[\mathrm{i}]->$ GetPoint $(0), * \operatorname{surface}[\mathrm{i}]->$ GetPoint $(1), * \operatorname{surface}[\mathrm{i}$ ]$->$ GetPoint (2)), true) $>=$ VISIBLE_DIST_MM) 


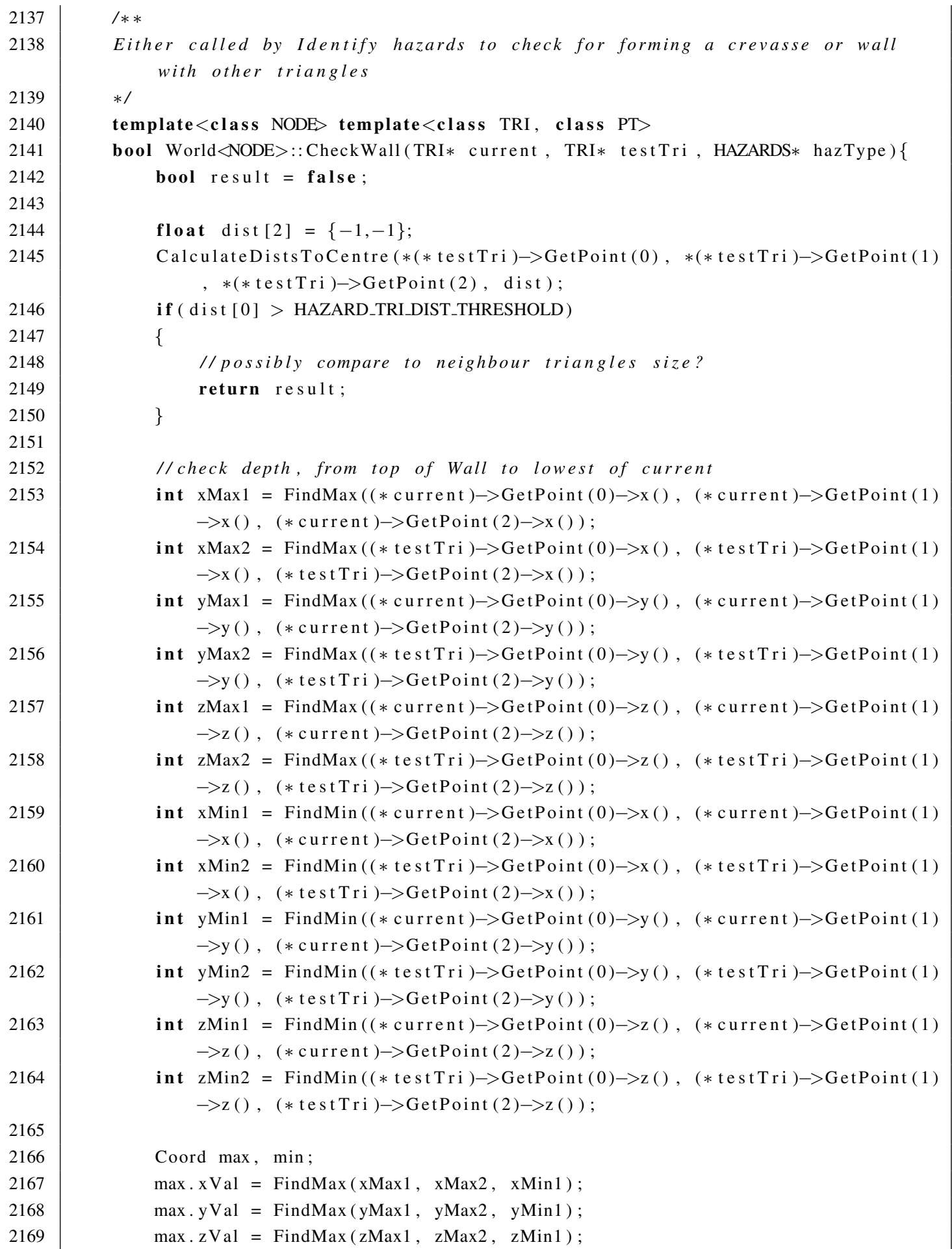




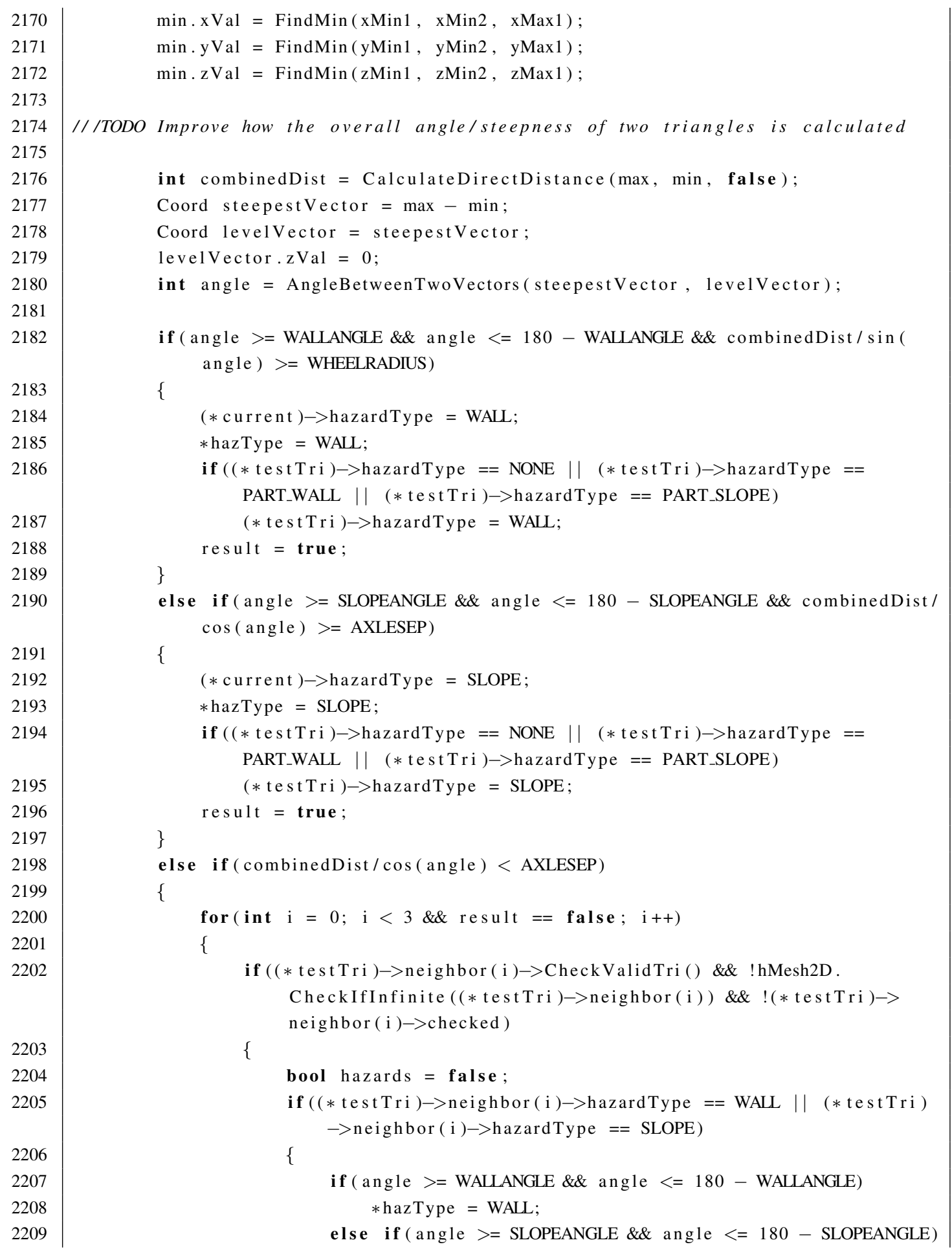


A.8. WORLD

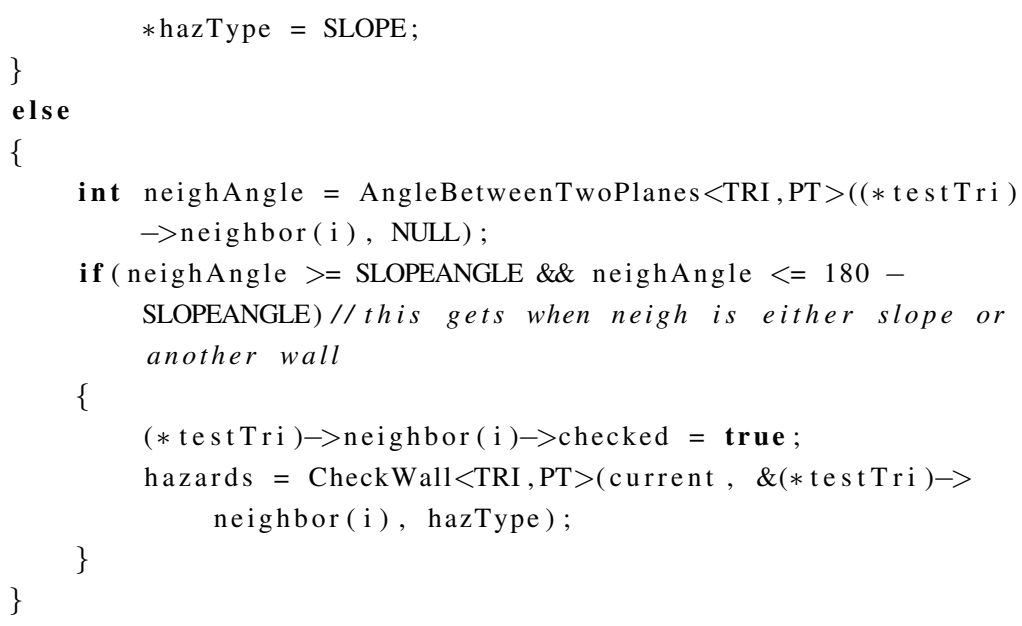




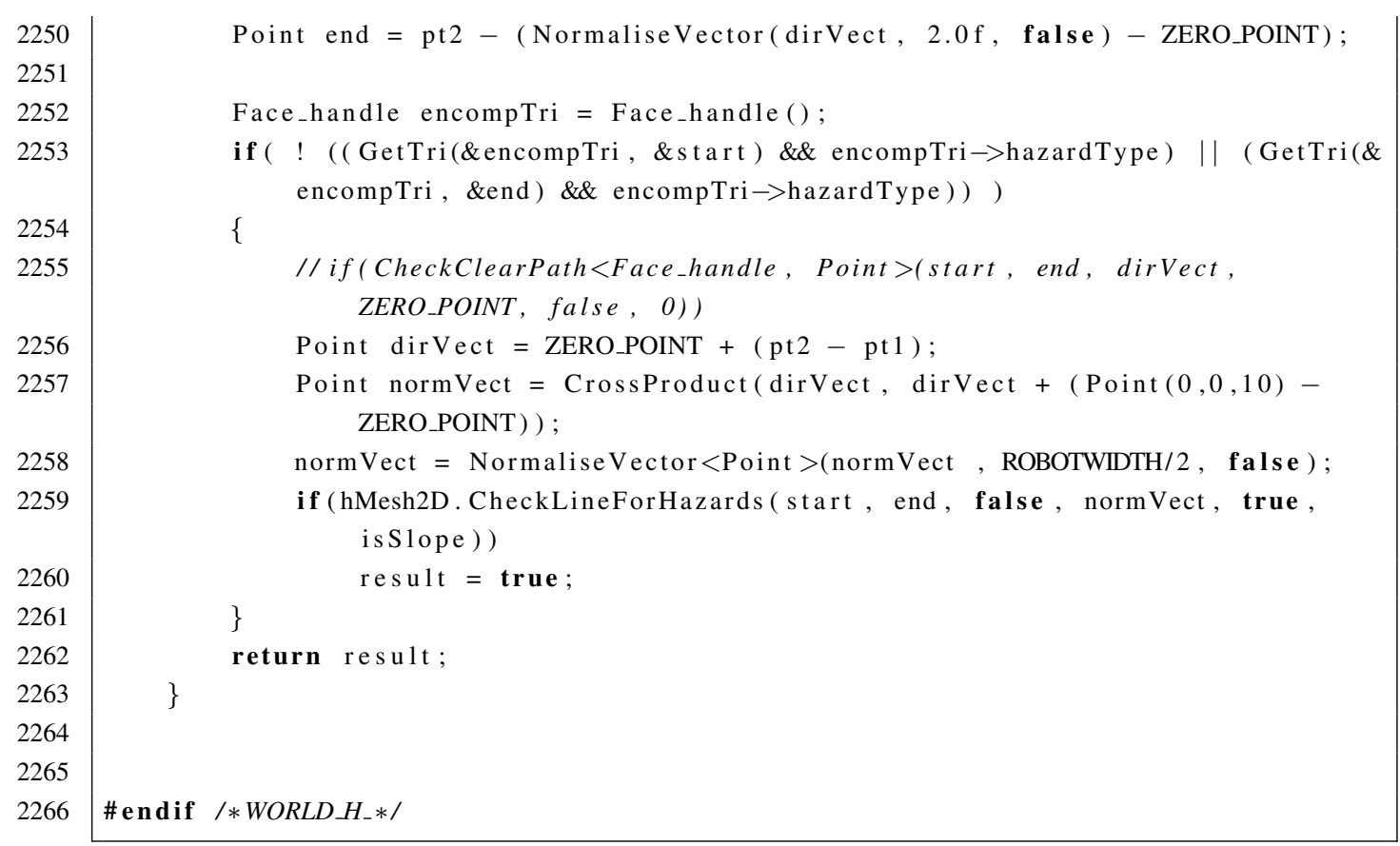

\section{A.8.2 World.cpp}

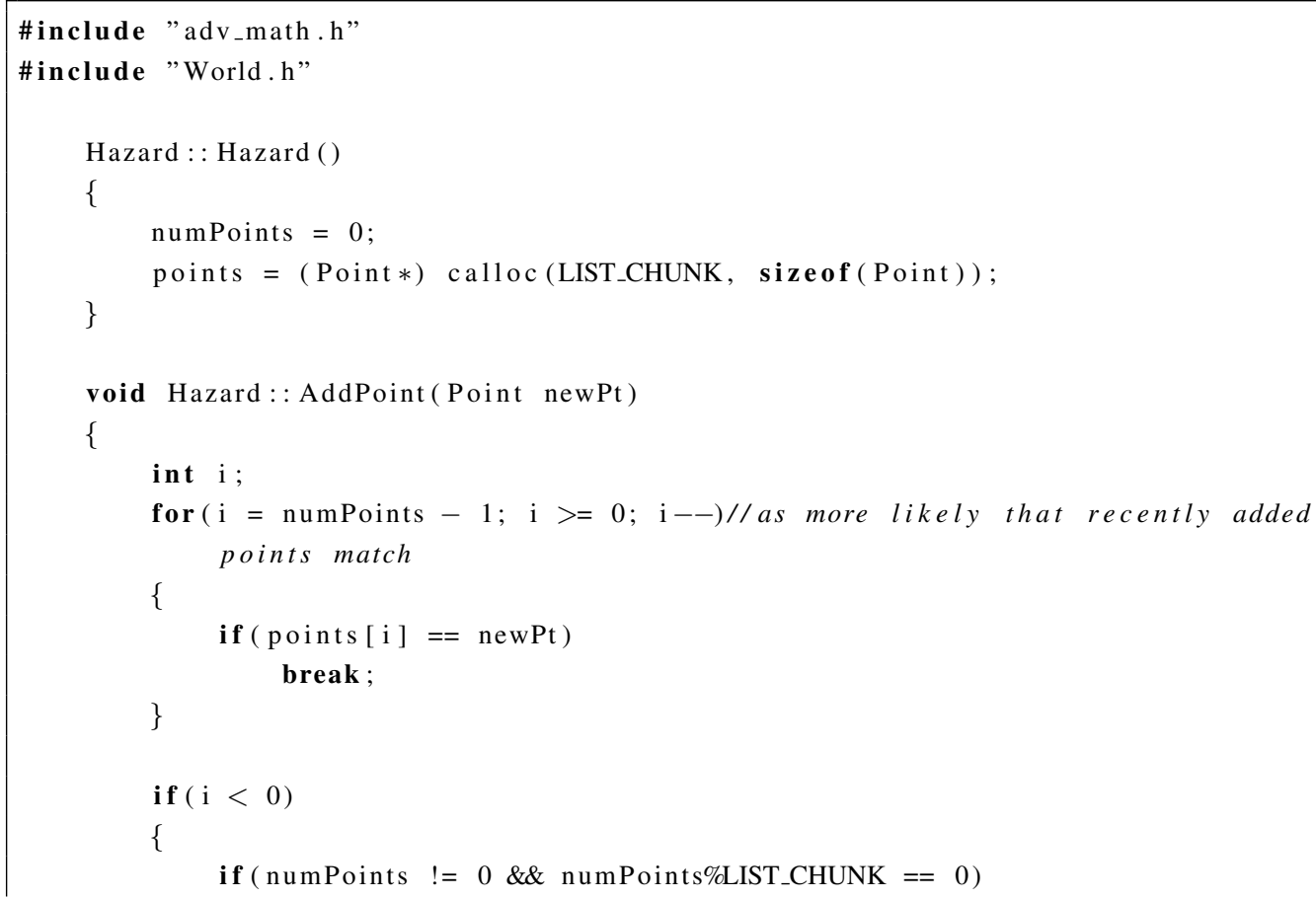




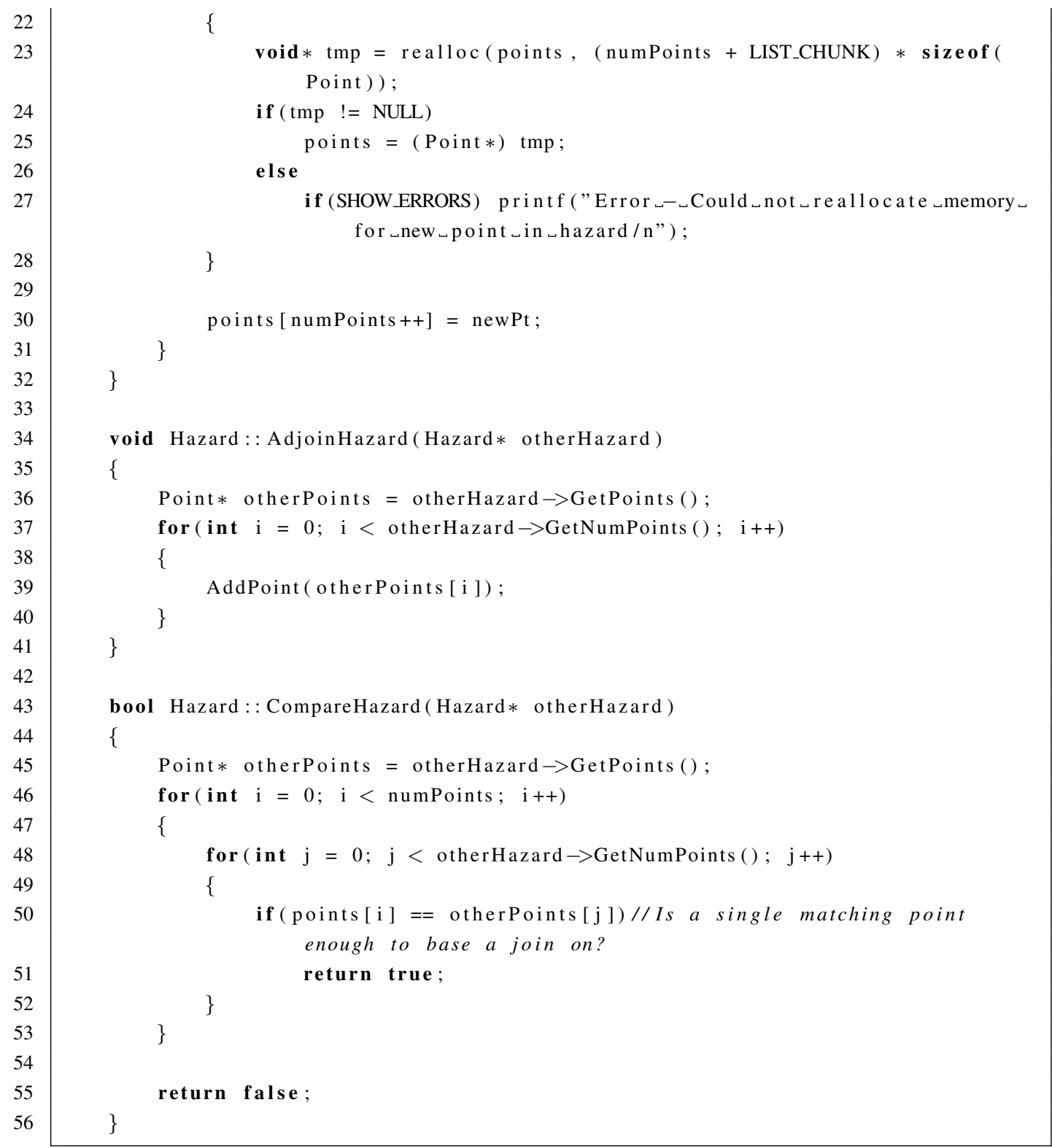

\section{A.9 adv math}

\section{A.9.1 adv math.h}

1 


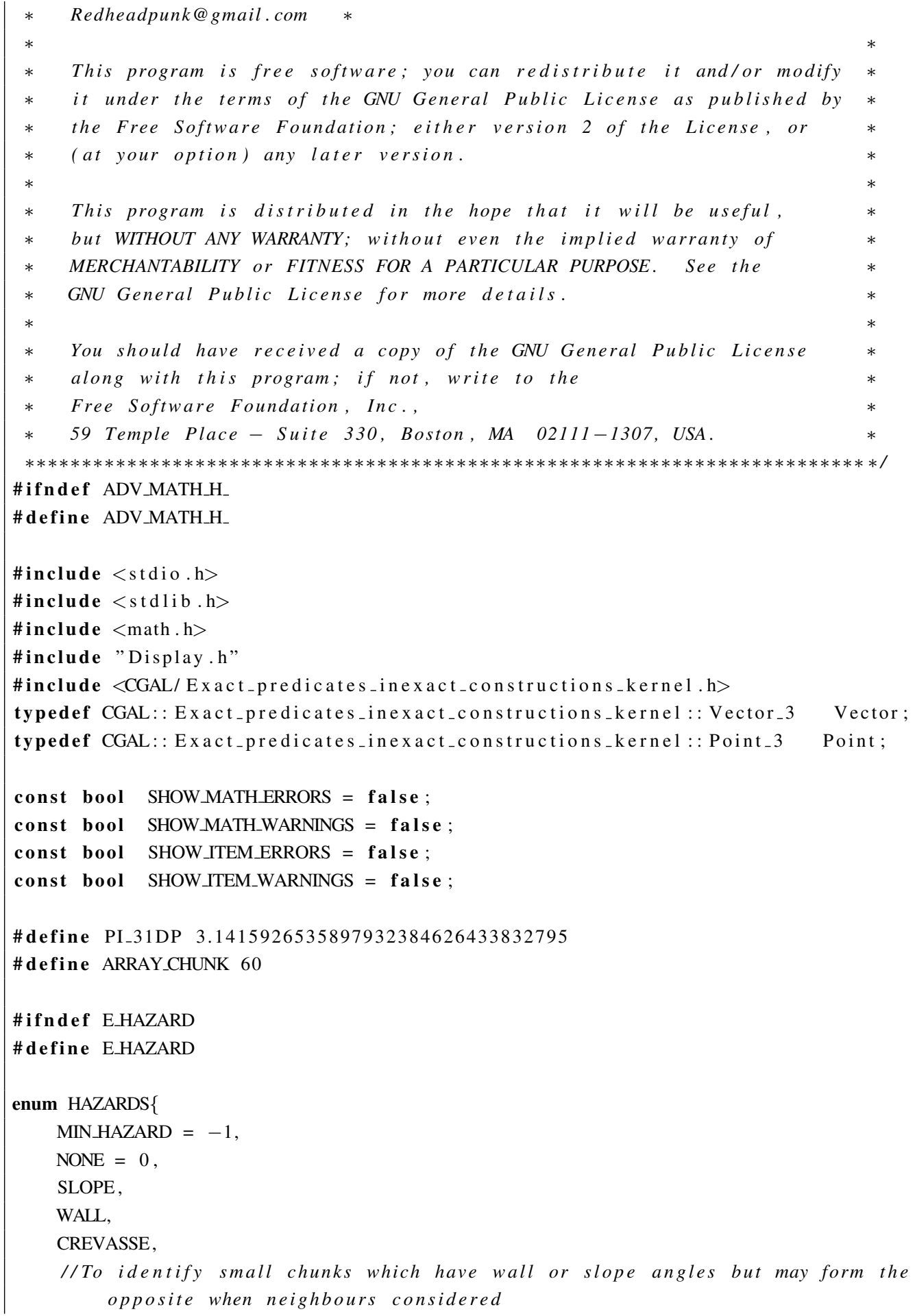




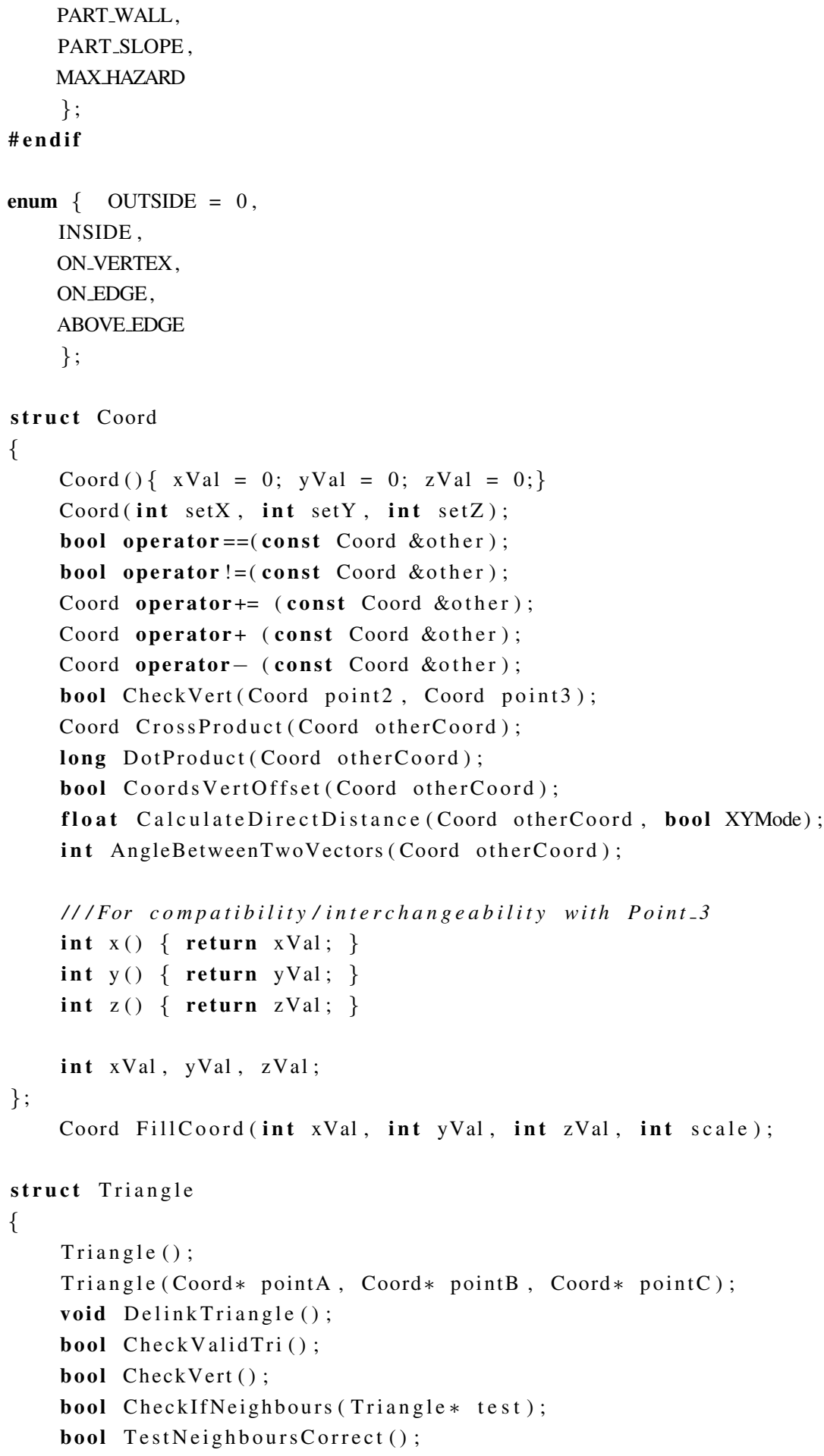


void SetNeighbour ( Triangle $*$ neigh, int num);

///For compatibility/interchangeability with faces

Triangle $*$ neighbor (int num);

Coord* GetPoint (int num);

void CommonPoints ( Triangle $*$ otherTri, int $*$ ptsA, int $*$ ptsB, int $* \operatorname{notCommonA,~}$ int $*$ notCommonB ) ;

void LinkTwoTriangles ( Triangle $*$ otherTri);

bool SameTri(Triangle* triToTest, bool identicalMatchesOnly, bool modDiffInstanceSameTri);

bool SameTriNeighboursCheck (Triangle $*$ triToTest);

bool SameTriSameNeighbourCheck (Triangle* triToTest, Triangle* triNeighbourToTest);

void RecursGetTri (Triangle*** allTriangles, int *numTri);

// int DistanceAcrossATriangle (Triangle* neighbour);

int AngleBetweenTwoPlanes( Triangle* neighbourTris);

int InterpolateZValue (Coord retrievalPoint);

Coord $*$ point 1 ;

Coord $*$ point 2 ;

Coord $*$ point 3 ;

Triangle $*$ neighbour 1 ;

Triangle $*$ neighbour 2 ;

Triangle $*$ neighbour 3 ;

HAZARDS hazardType;

bool checked;

bool vert;

\}

\section{struct Edge}

\{

Edge ()$\{$ point $=$ NULL; pointB = NULL; leftSideDone = false $;$ rightSideDone $=$ false ; \}

Edge $(\operatorname{Coord} *$ pointA, Coord $*$ pointB, bool left, bool right, Triangle* tri);

bool SameEdge(Edge $*$ newEdge, bool modEdges);

Coord *pointA, *pointB;

bool leftSideDone, rightSideDone;

Triangle $*$ sideTri;

\}

static const void $*$ RESERVED $=($ void $*)($ NULL $+0 \times 500)$;

// static const void $*$ RESERVED_UPPER $=($ void $*)($ NULL $+0 x 100000000000)$;

// Math etc 


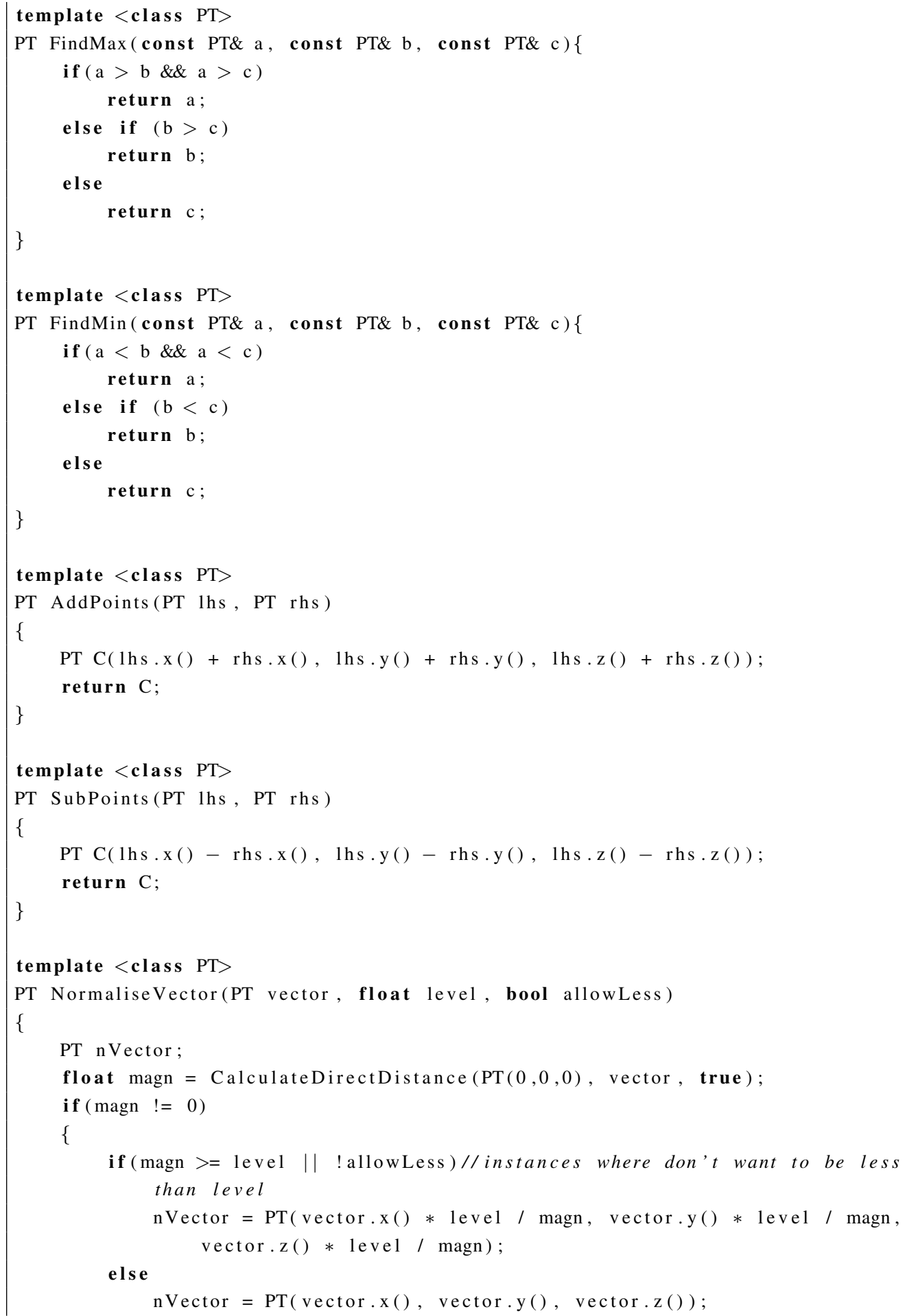




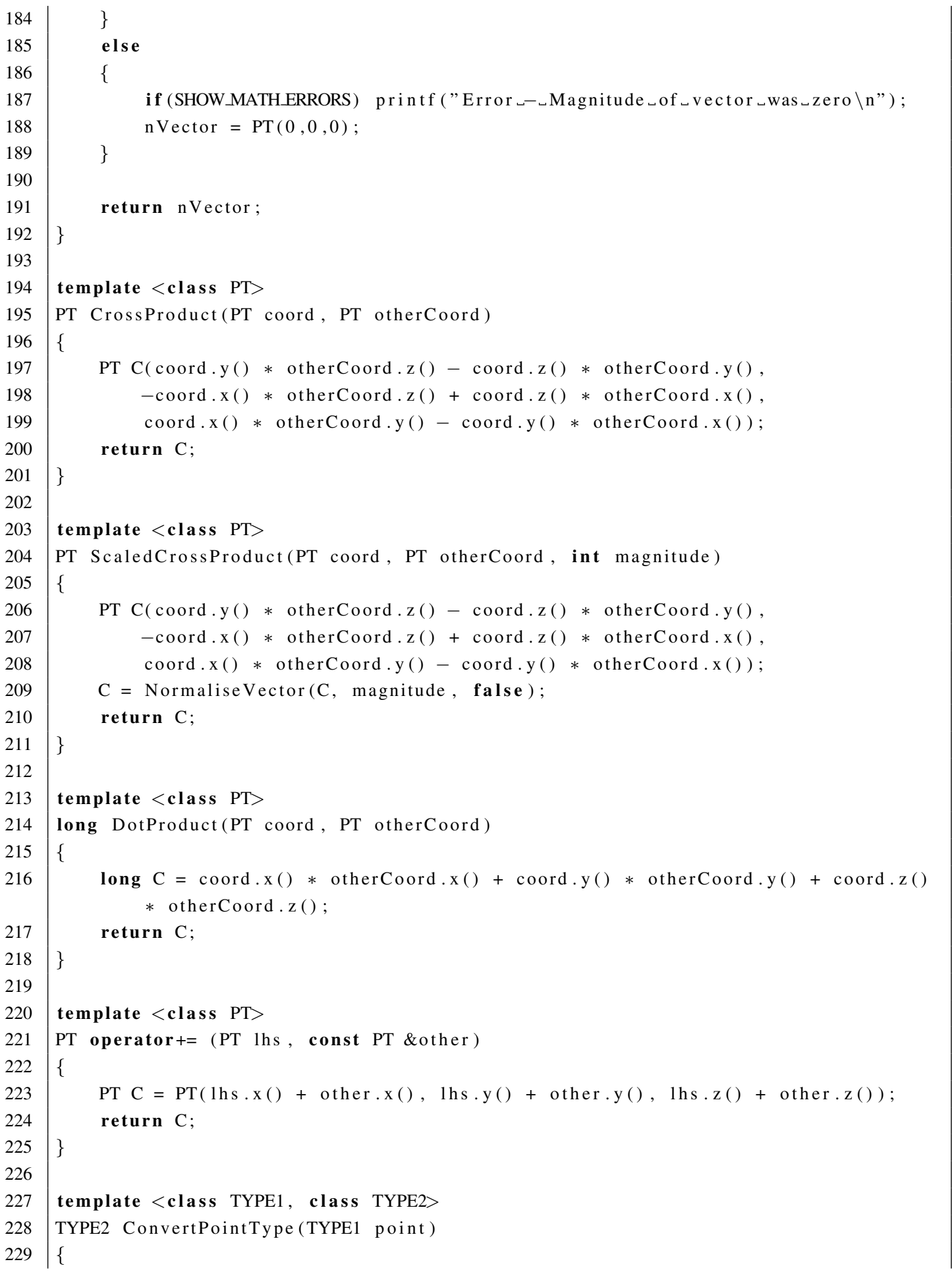




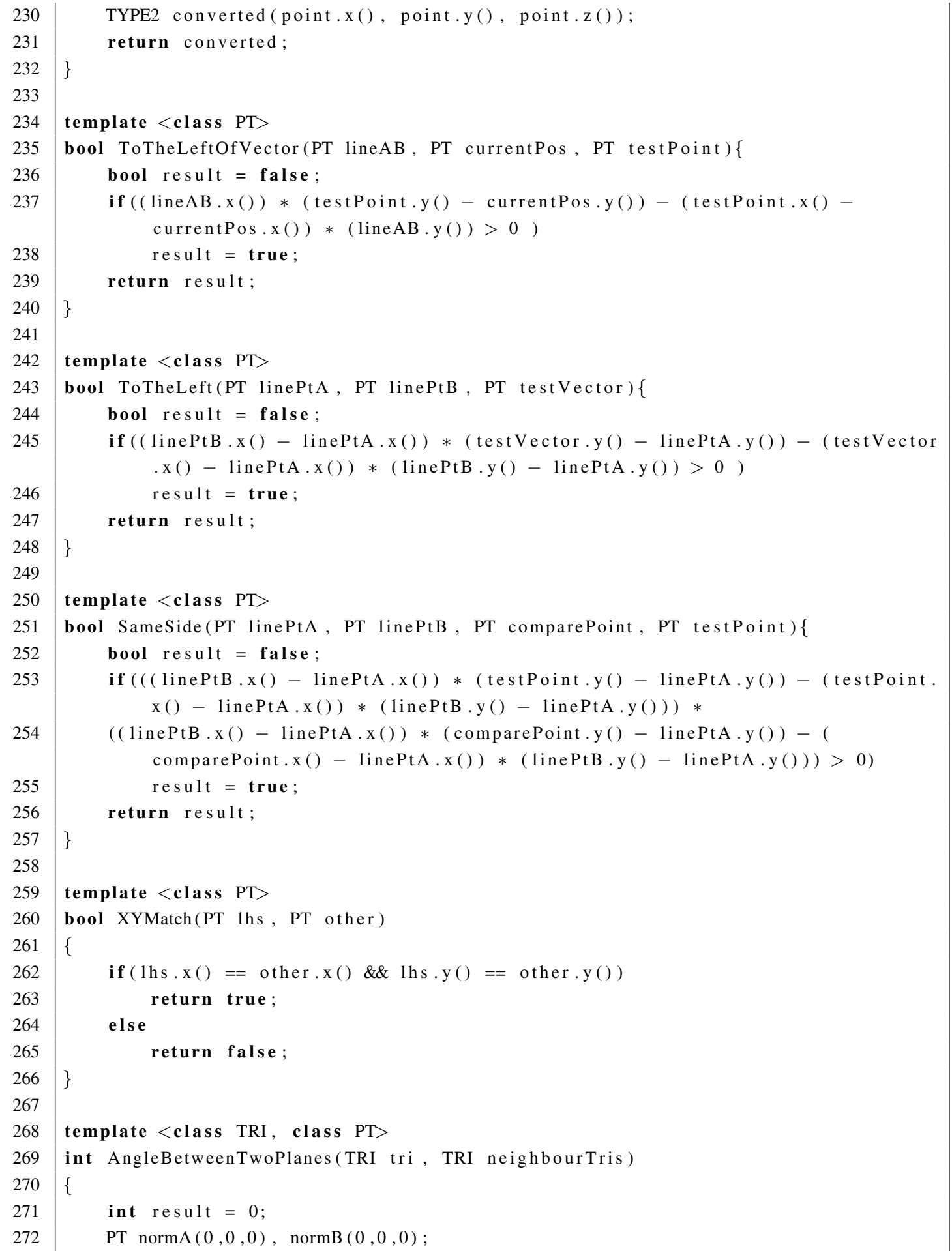




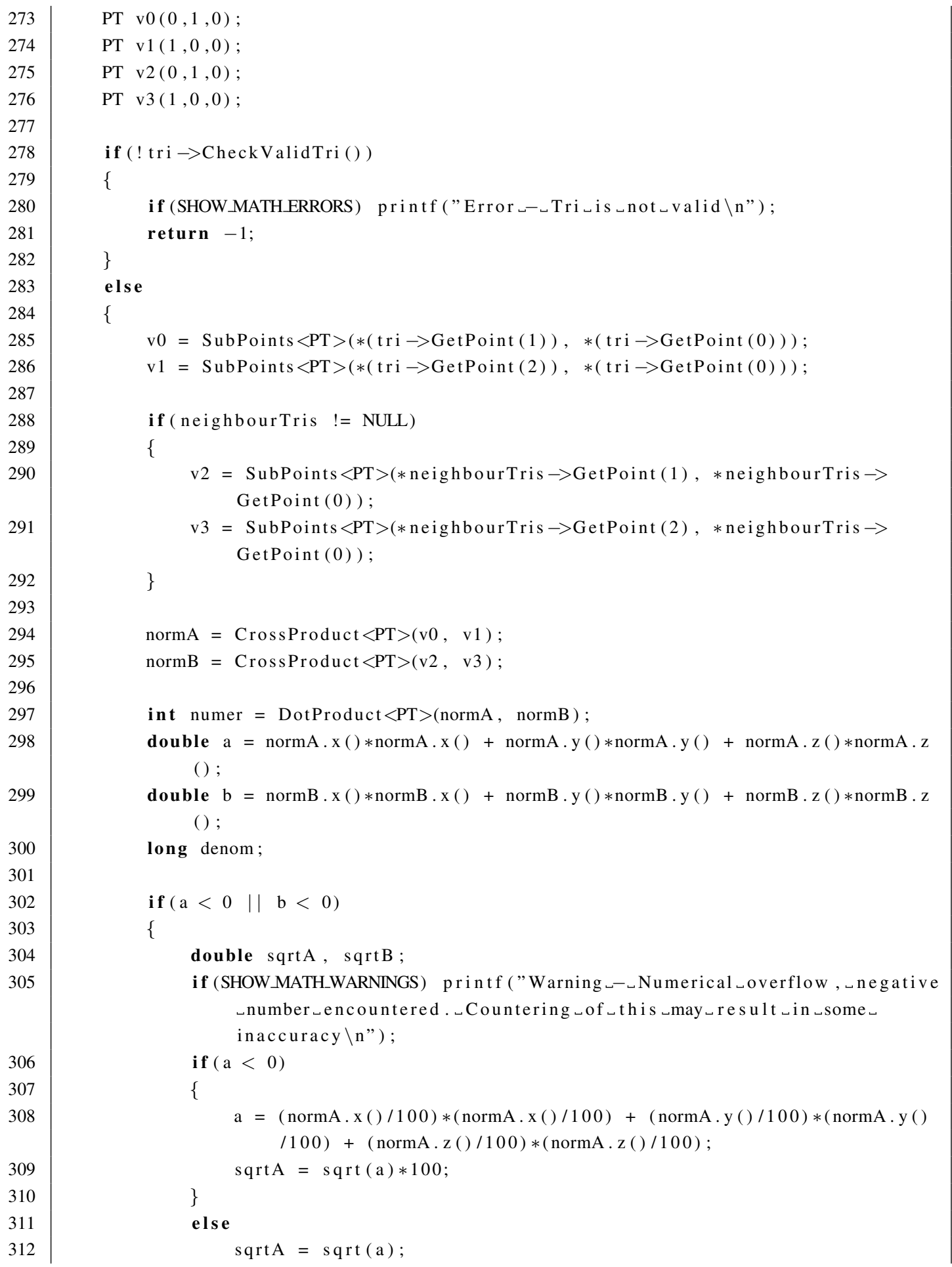




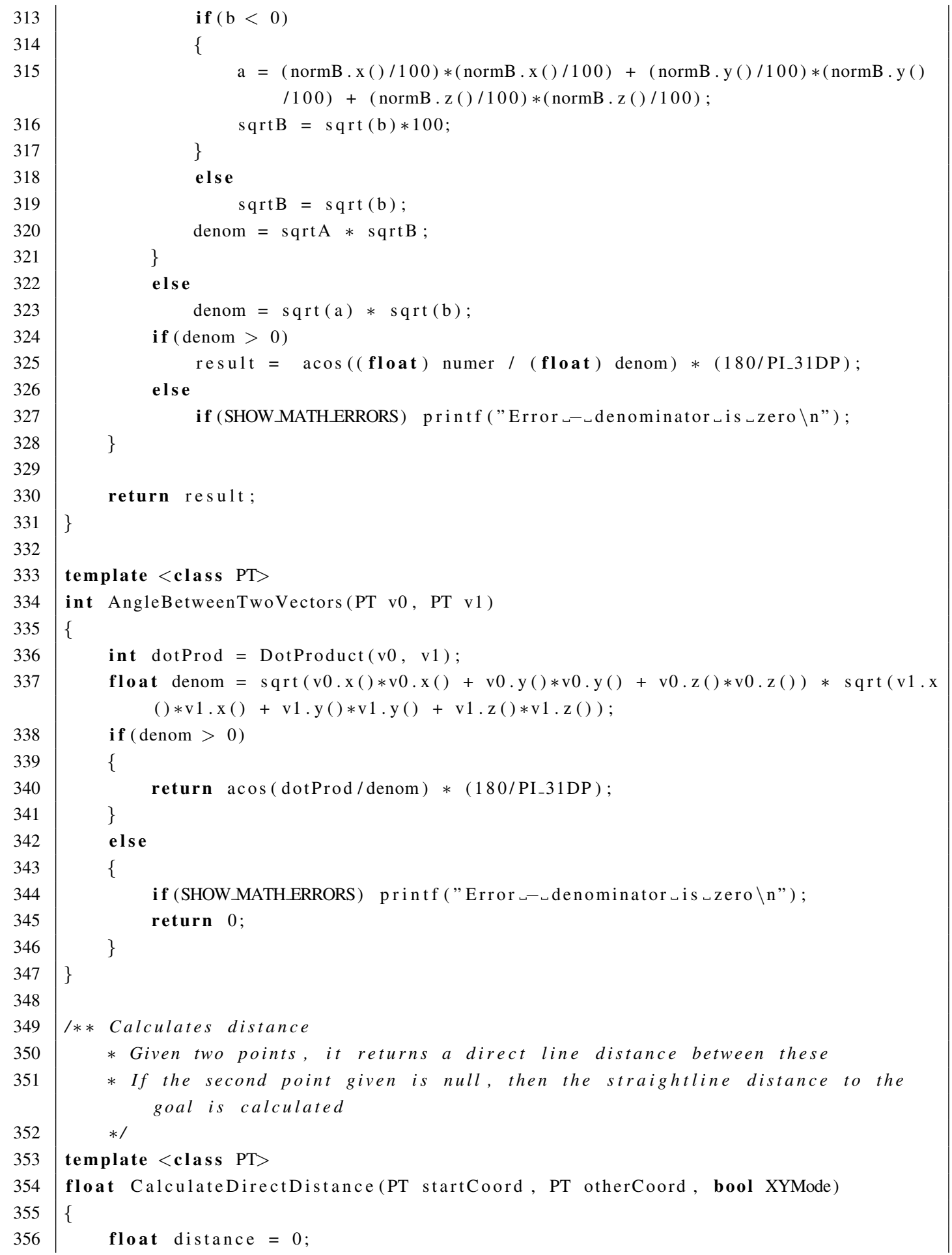


double sqrNum;

double $\mathrm{xDiff}=$ otherCoord. $\mathrm{x}()-$ startCoord.x();

double yDiff $=$ otherCoord.y()-startCoord.y();

double $\mathrm{zDiff}=$ otherCoord.z( $)-$ startCoord.z( );

if (XYMode)

sqrNum $=x$ Diff $* x$ Diff $+y$ Diff $* y$ Diff $;$

els e

sqrNum $=x$ Diff $* x D i f f+y D i f f * y D i f f+z D i f f * z D i f f ;$

distance $=$ sqrt (sqrNum);

if $($ distance $<=0 \quad \& \&$ startCoord $==$ otherCoord)

\{

return 0; //or less than one, as that indicates error \}

else

return distance

template <class TRI, class PT>

int DistanceAcrossATriangle(TRI tri, TRI neighbour)

\{

PT v0, v1;

PT *adjoiningEdge [2], pointB ;

int $\mathrm{a}=0$;

int theta, dist, hypot;

if $(\operatorname{tri} \rightarrow$ GetPoint $(0) !=$ neighbour $\rightarrow$ GetPoint $(0)$ \&\& tri $\rightarrow$ GetPoint $(0) \quad !=$ neighbour $\rightarrow$ GetPoint (1) $\& \&$ tri $\rightarrow$ GetPoint $(0) !=$ neighbour $\rightarrow$ GetPoint (2)) 


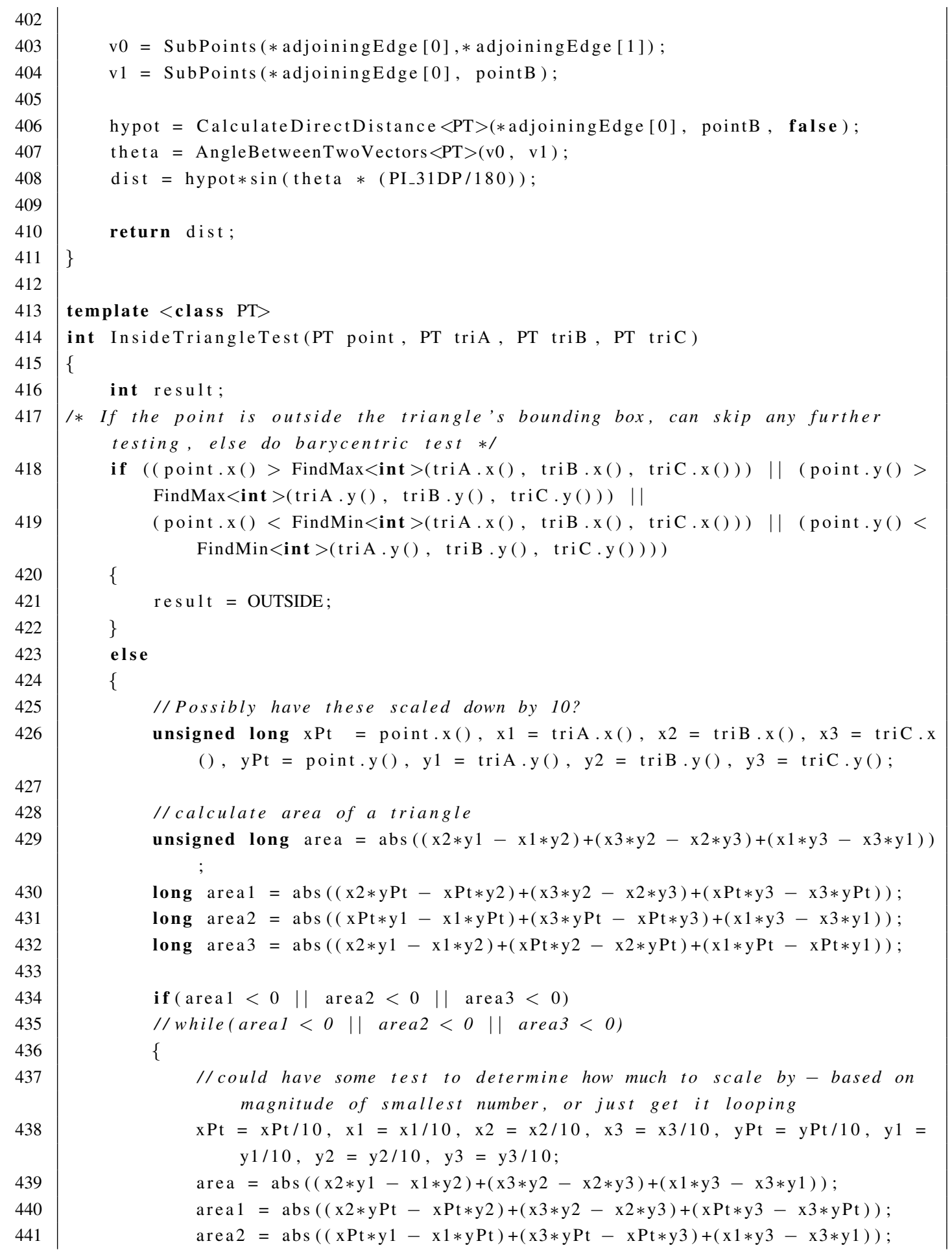




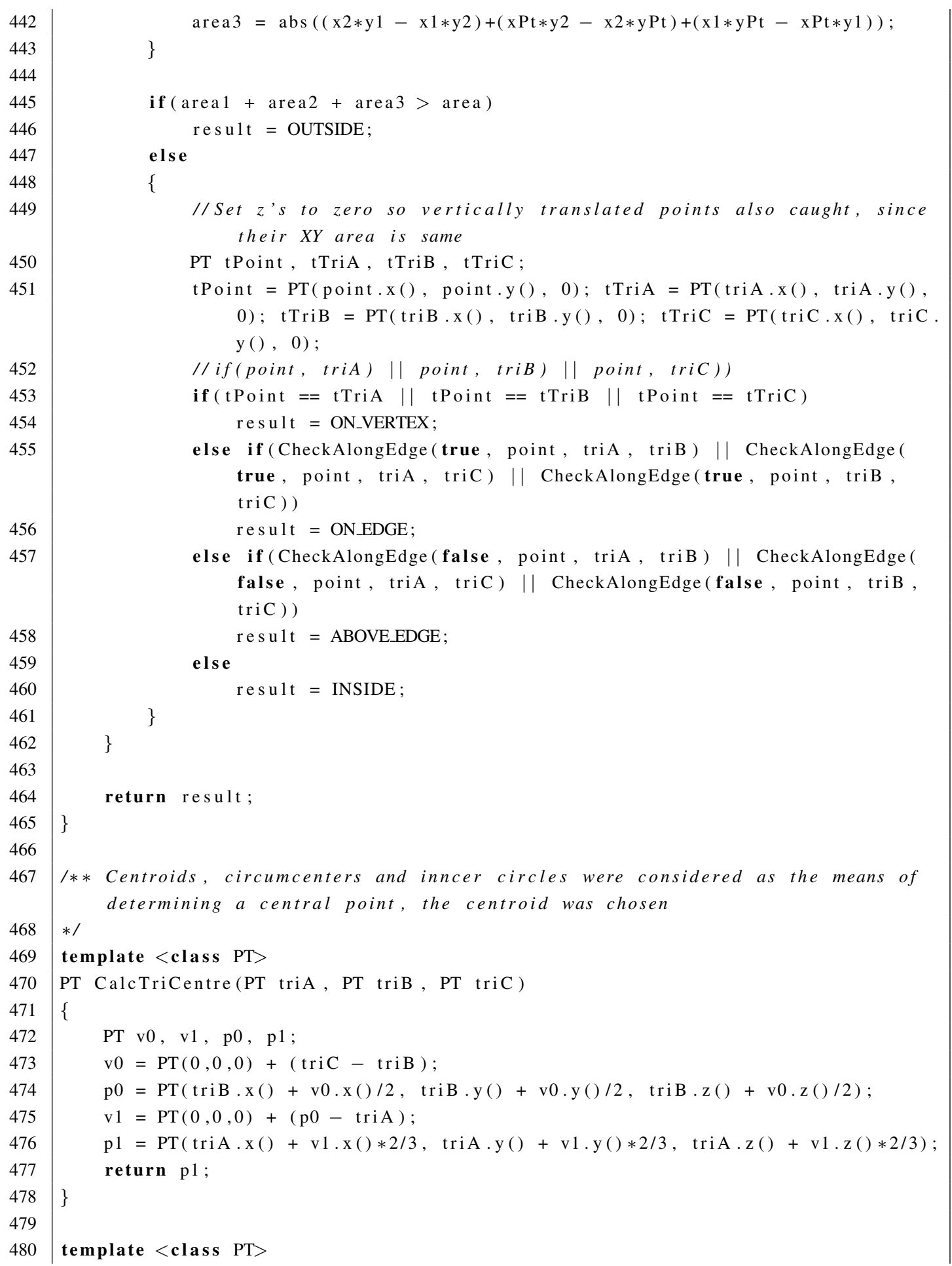




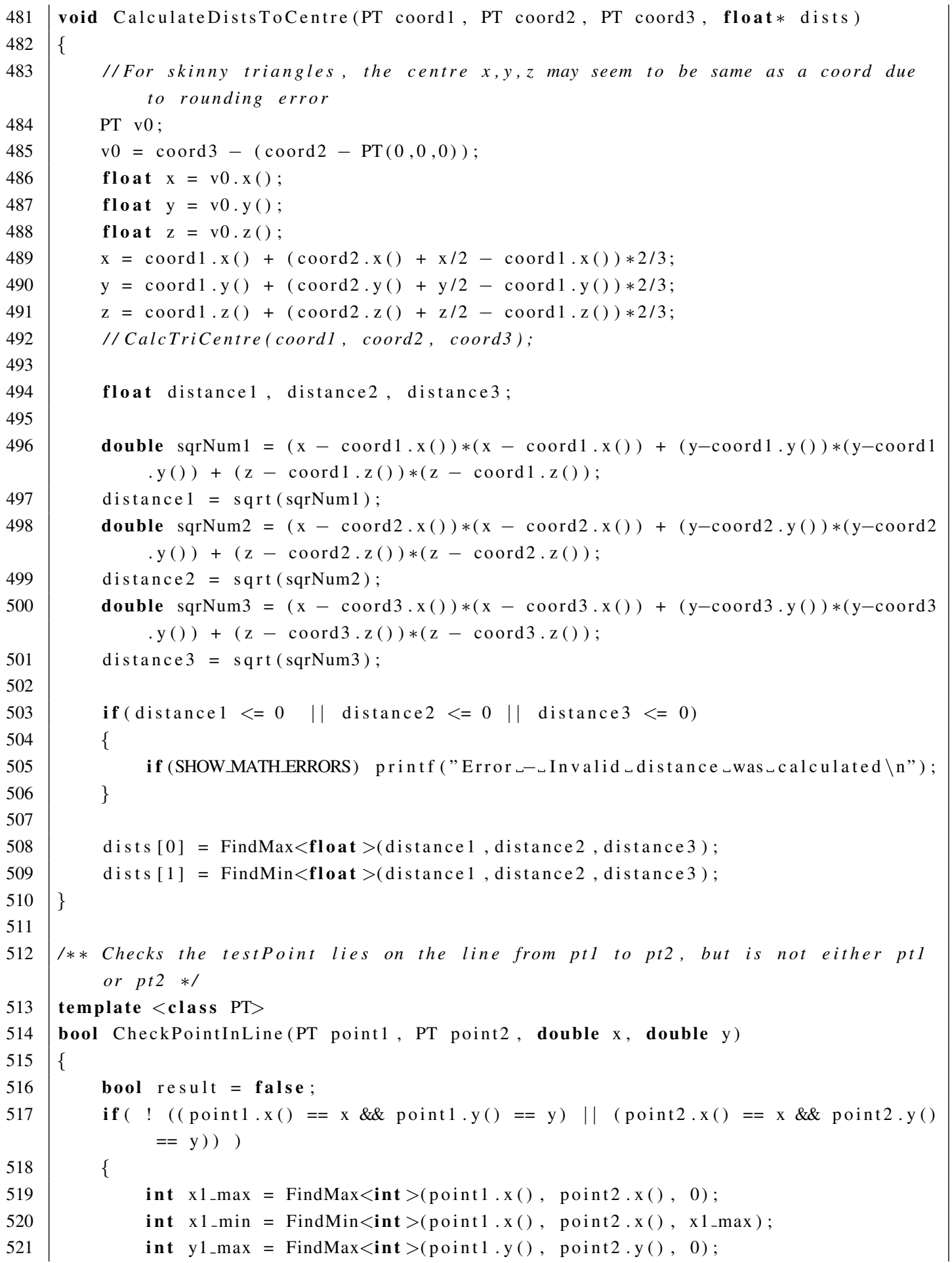




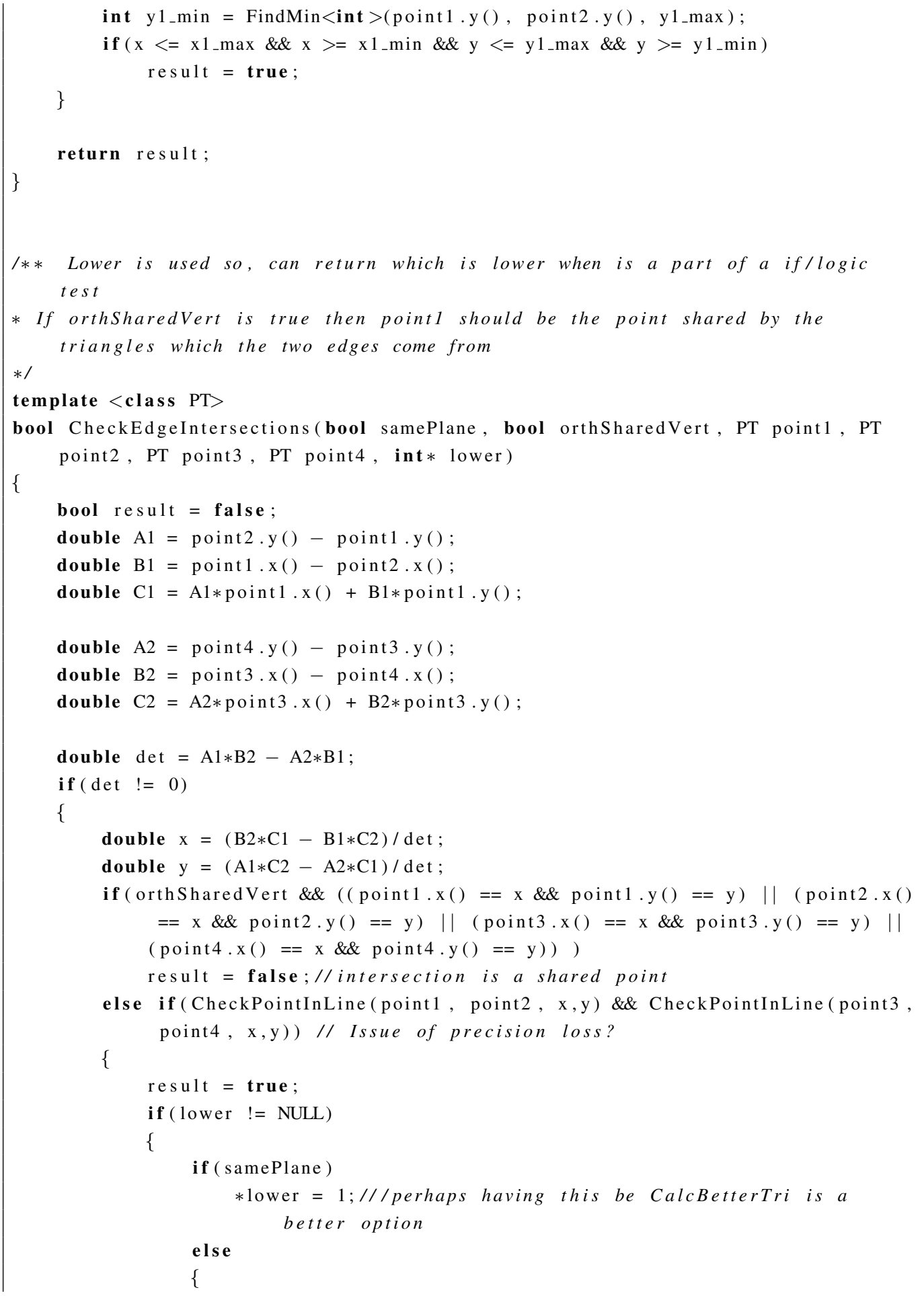




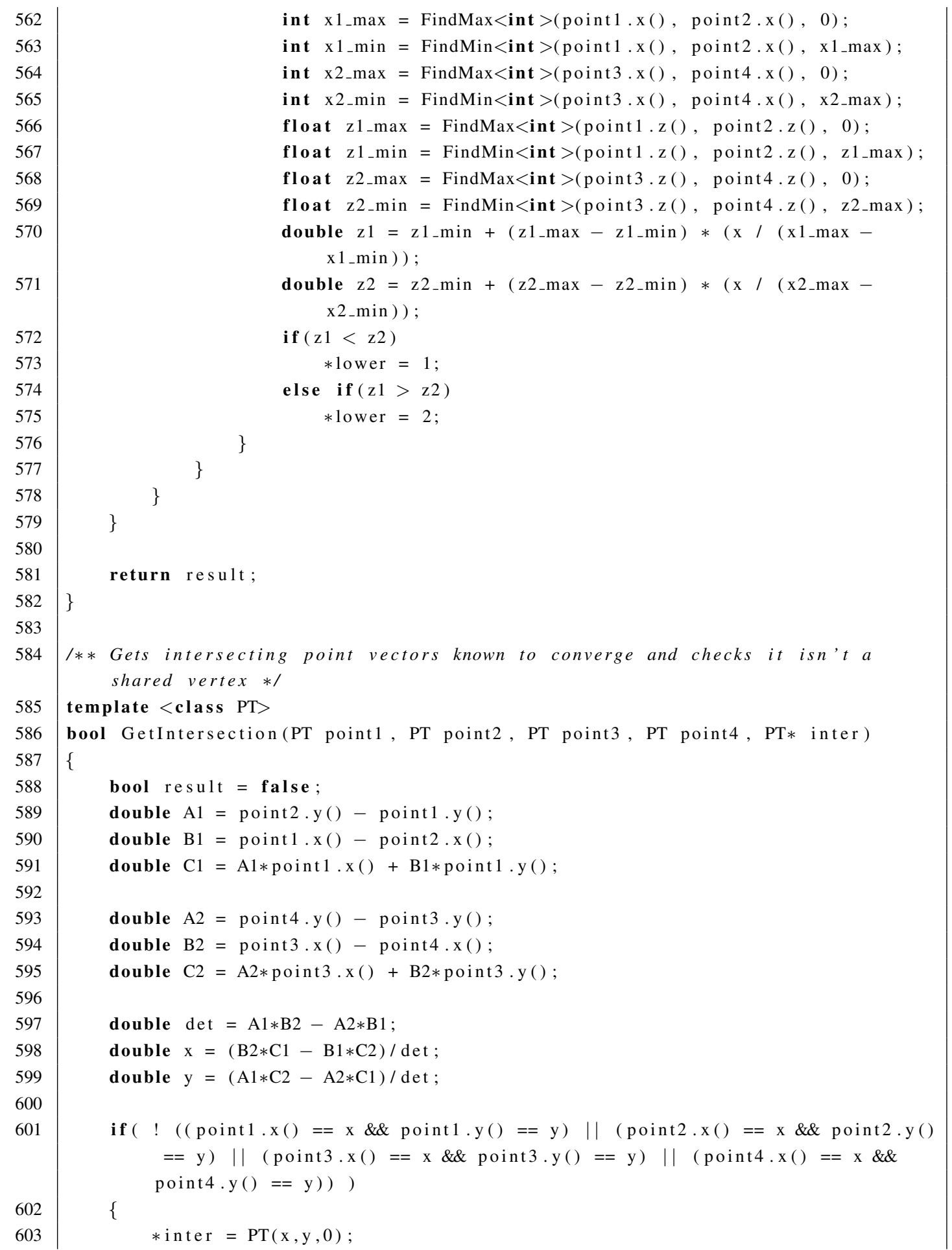




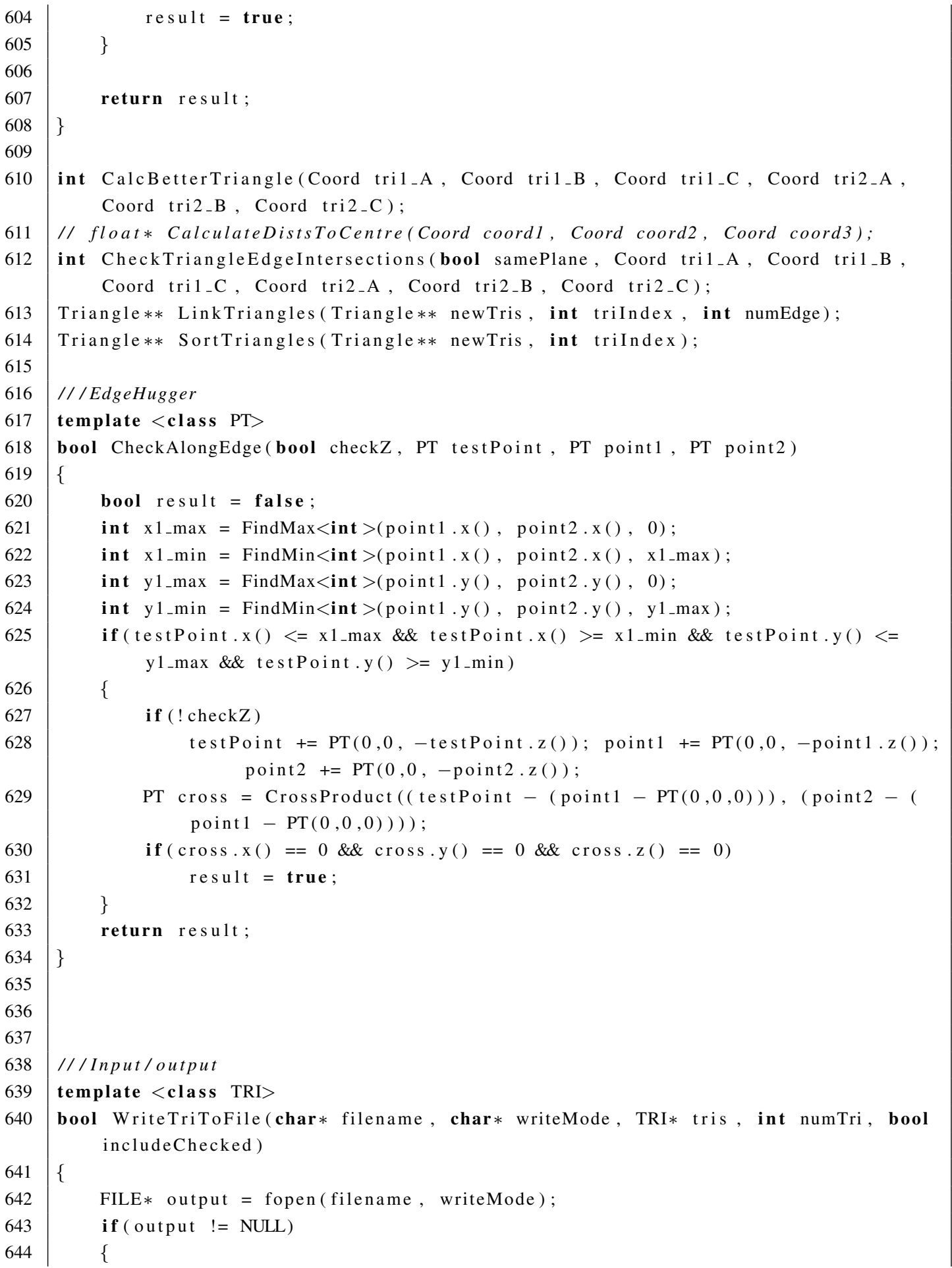




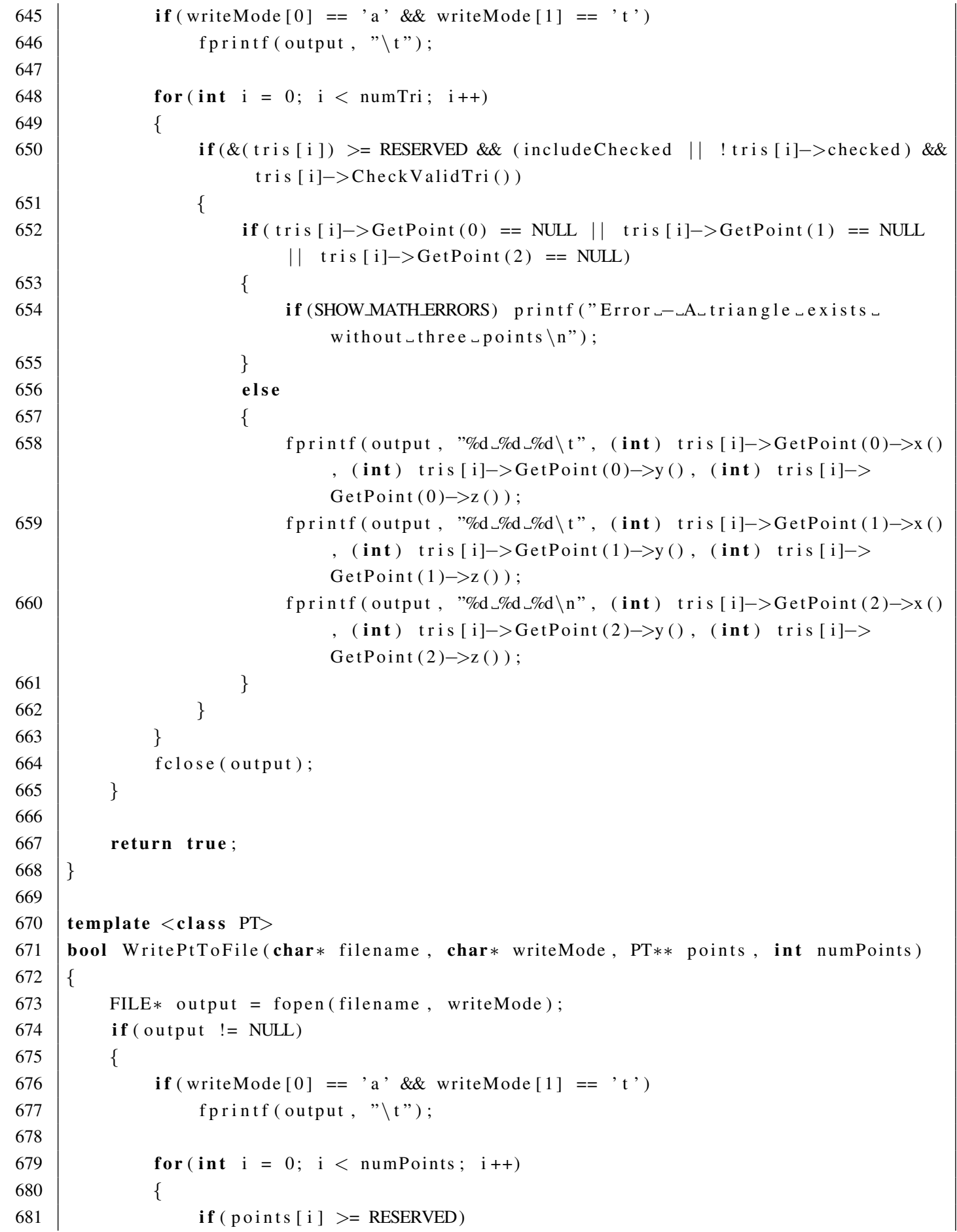




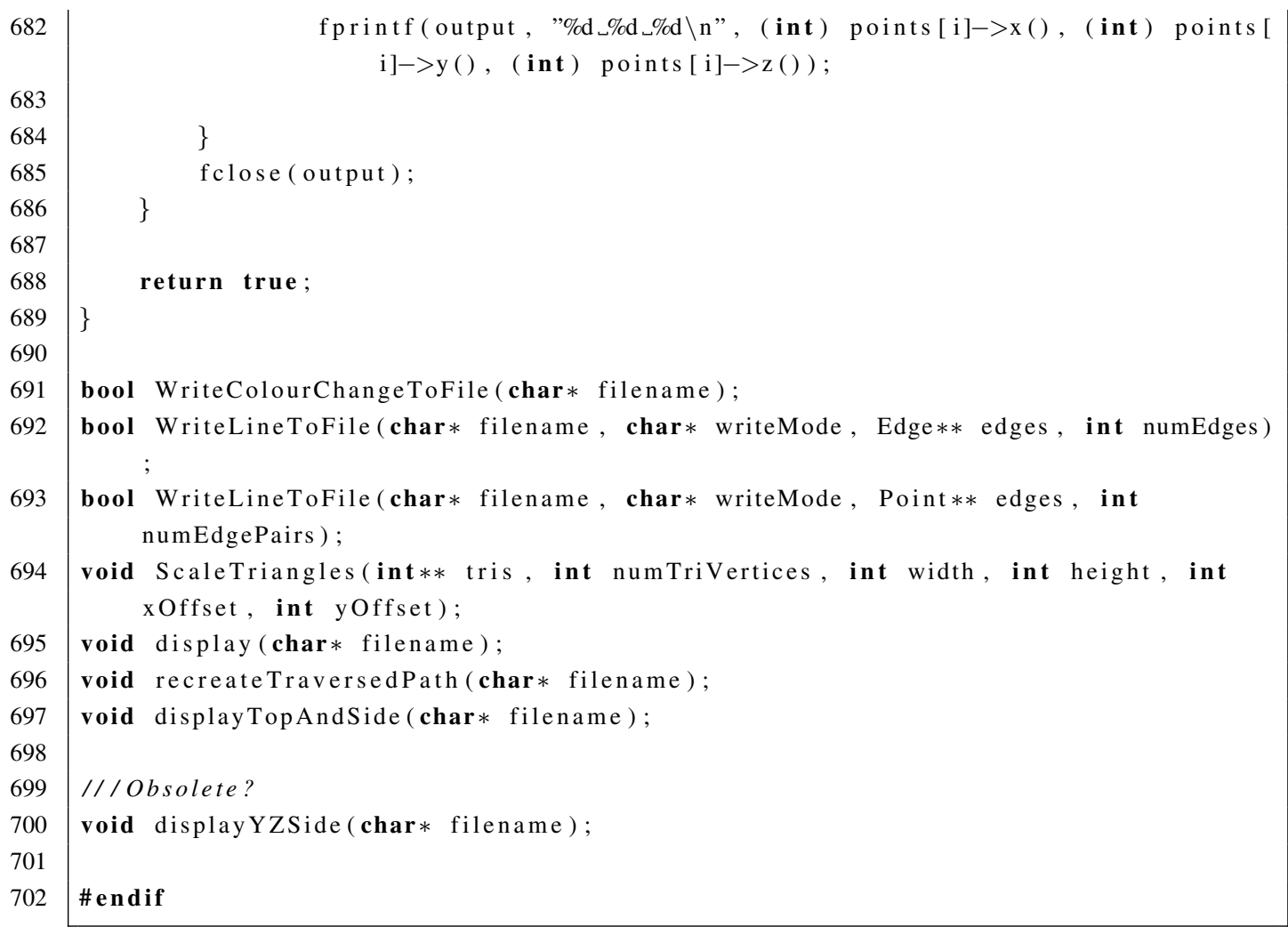

\section{A.9.2 adv math.cpp}

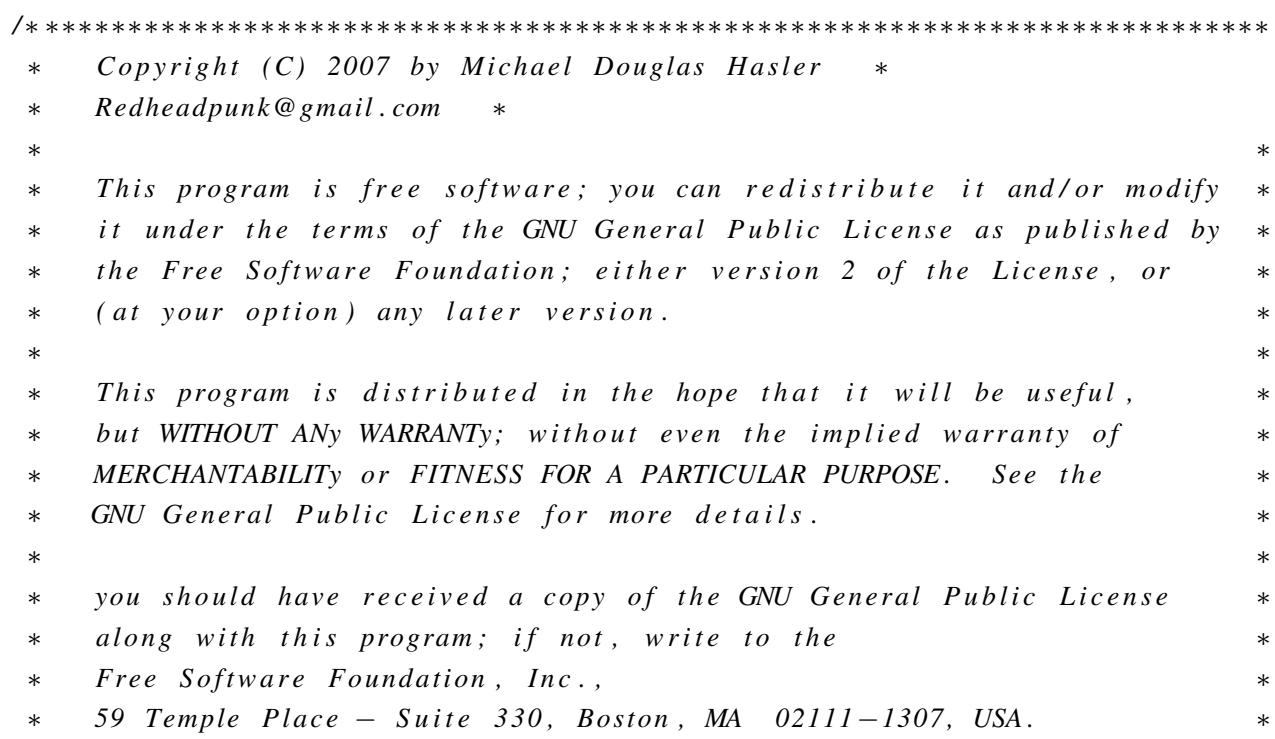




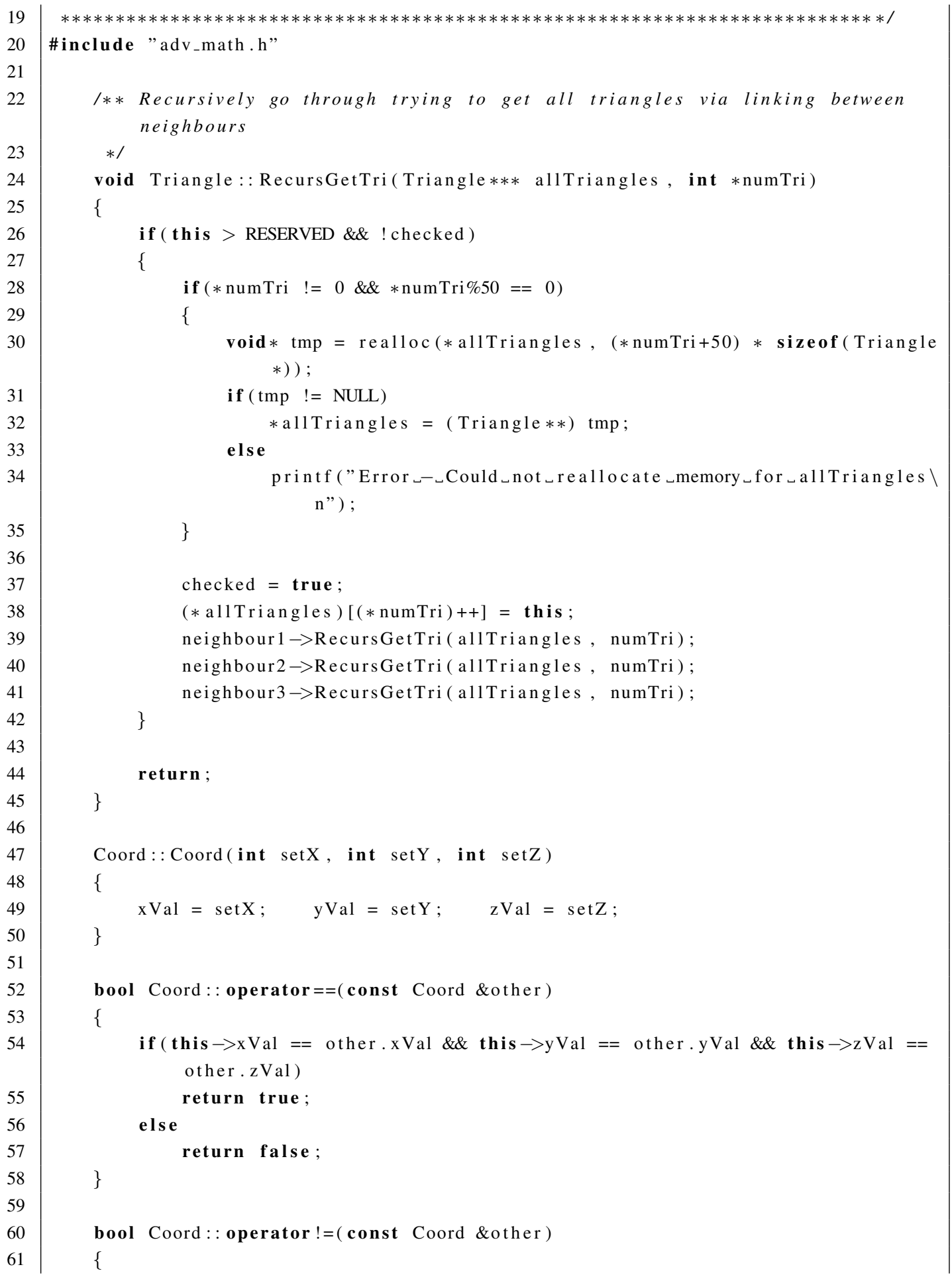




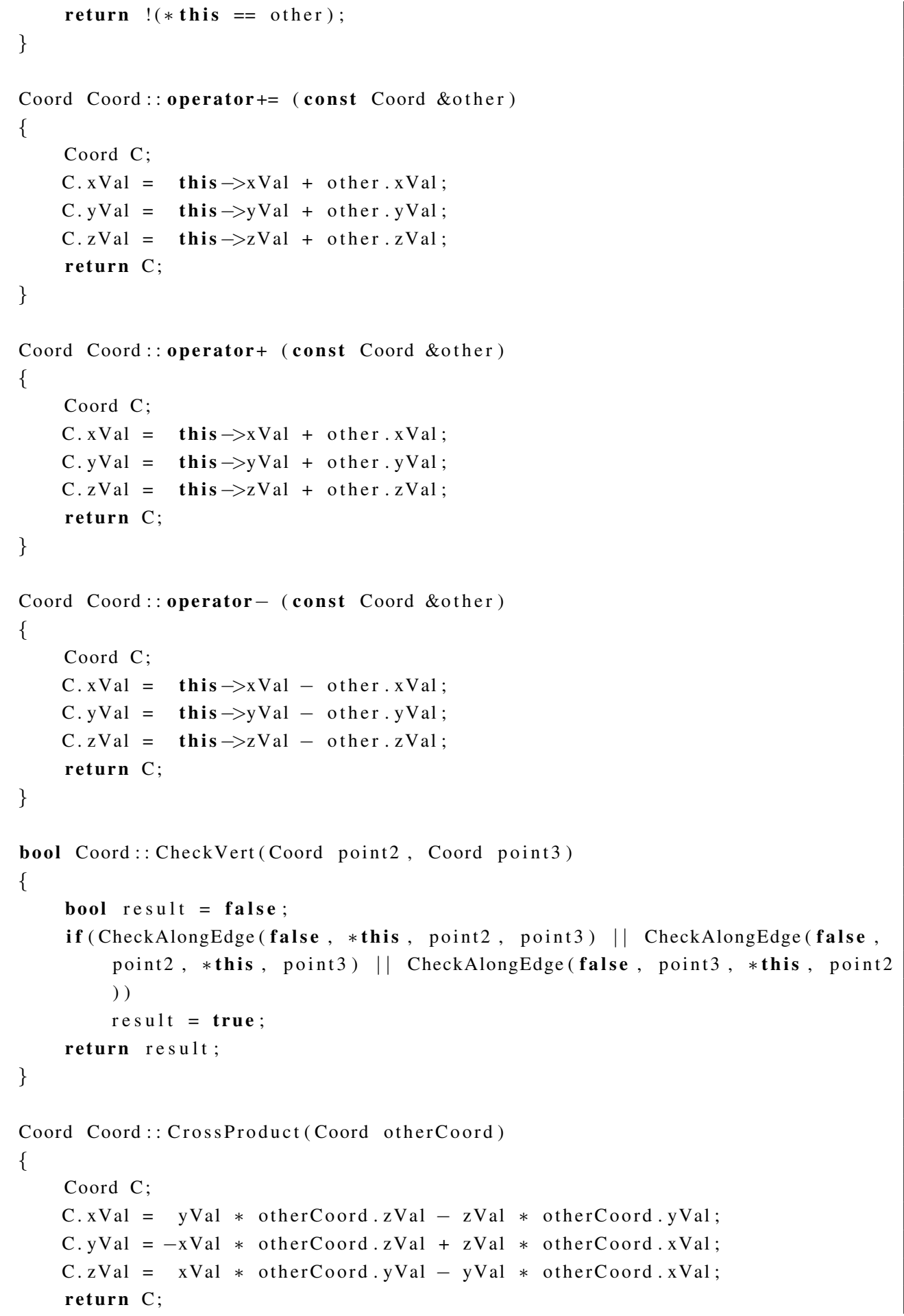




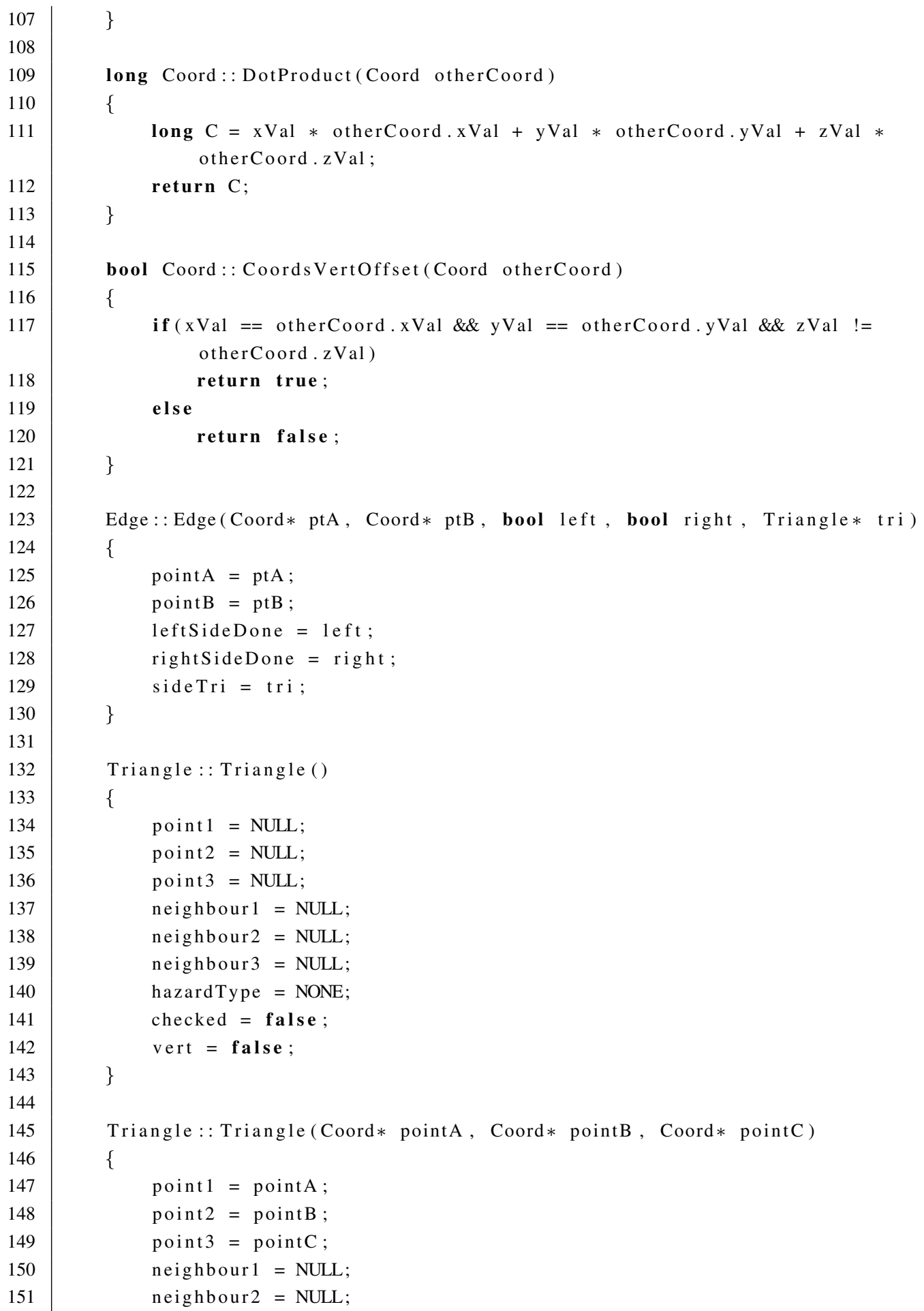




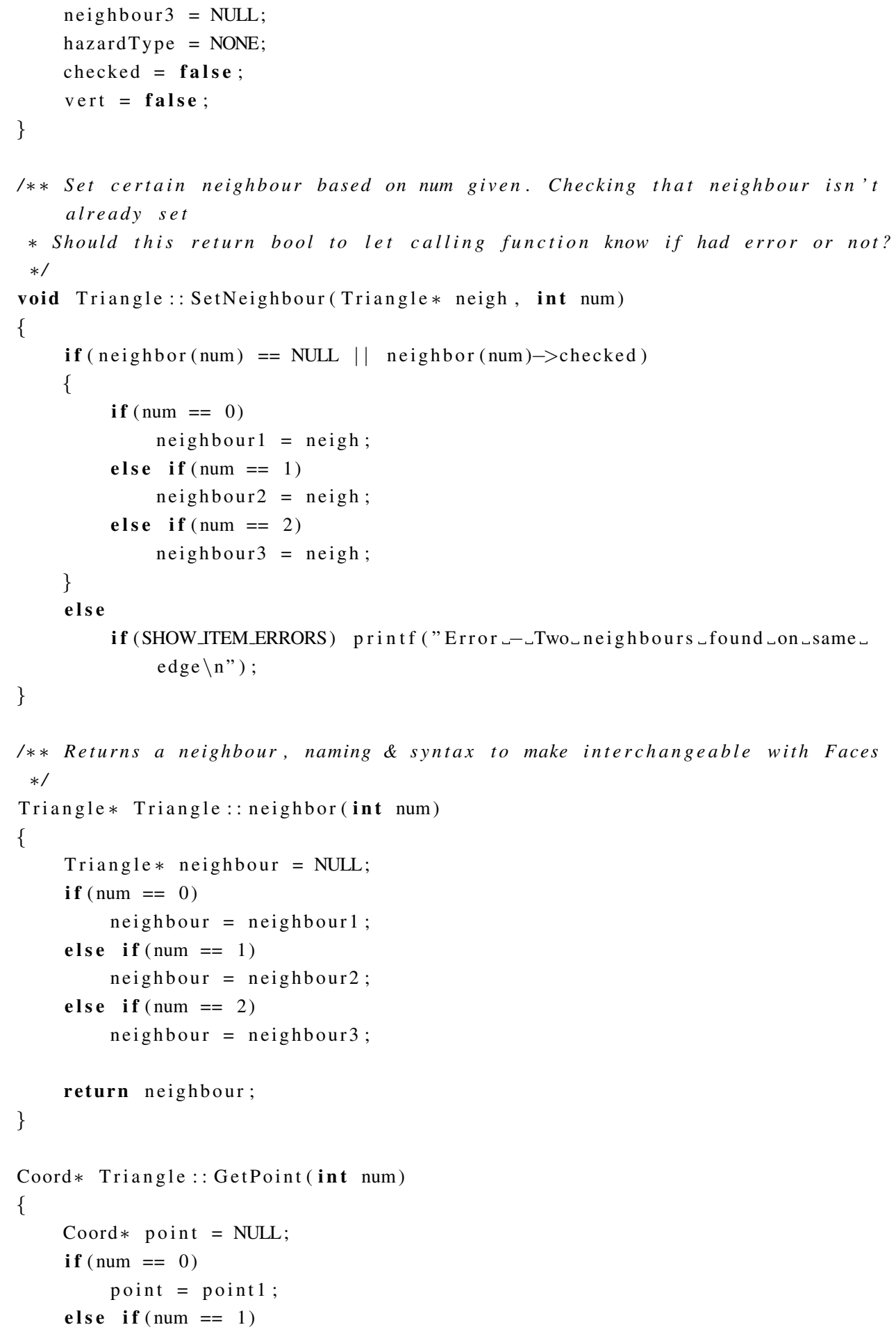




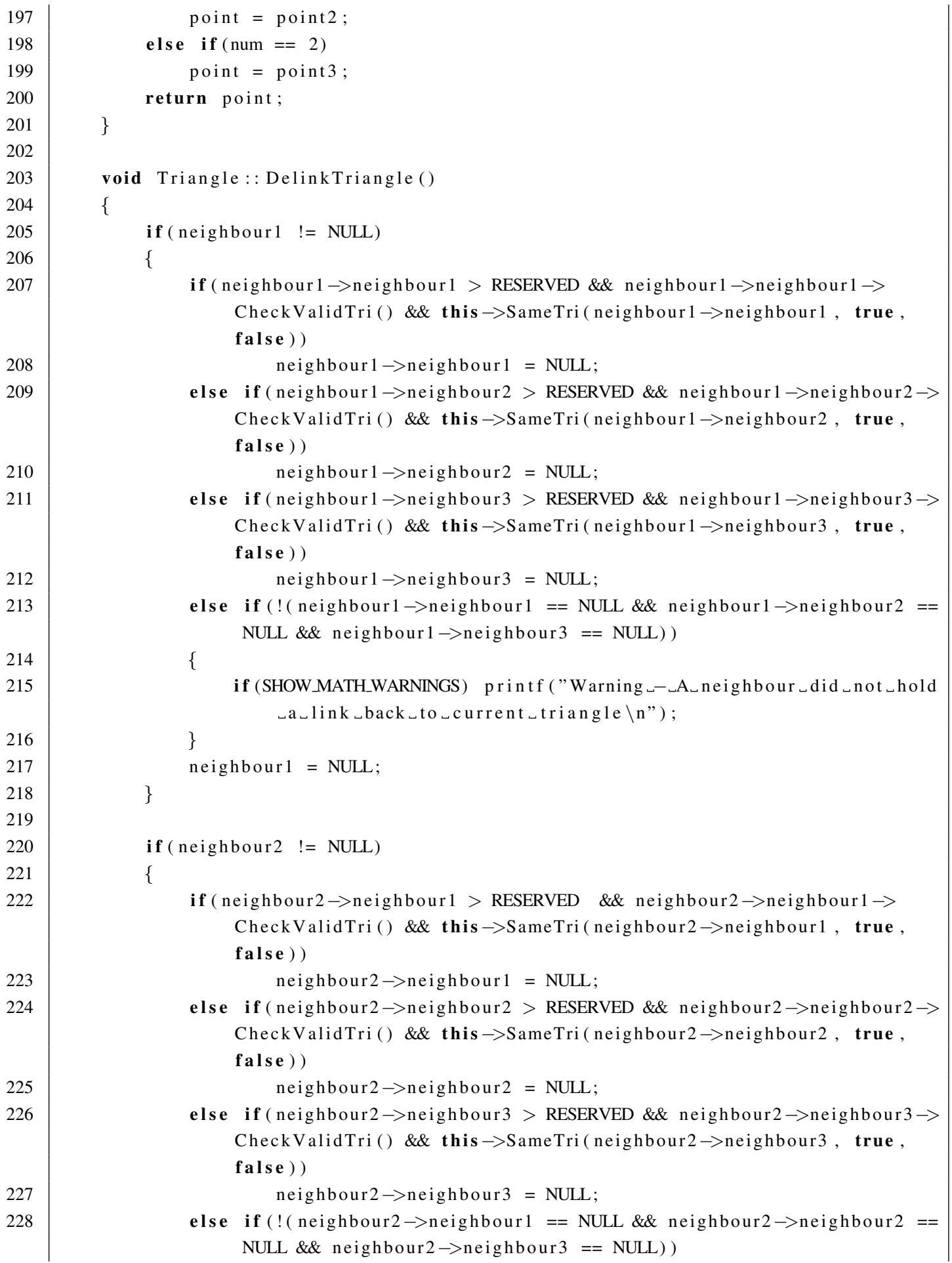




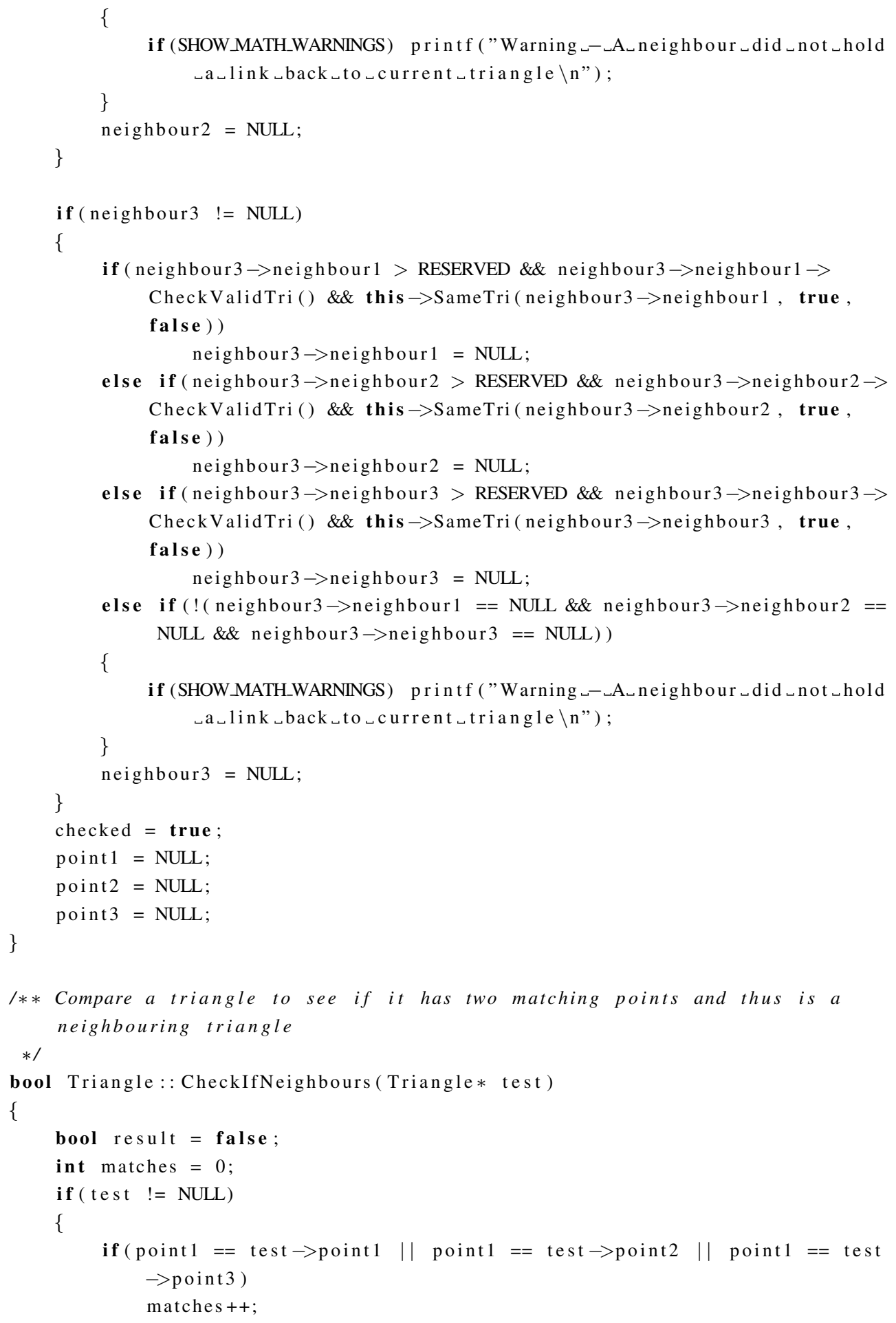




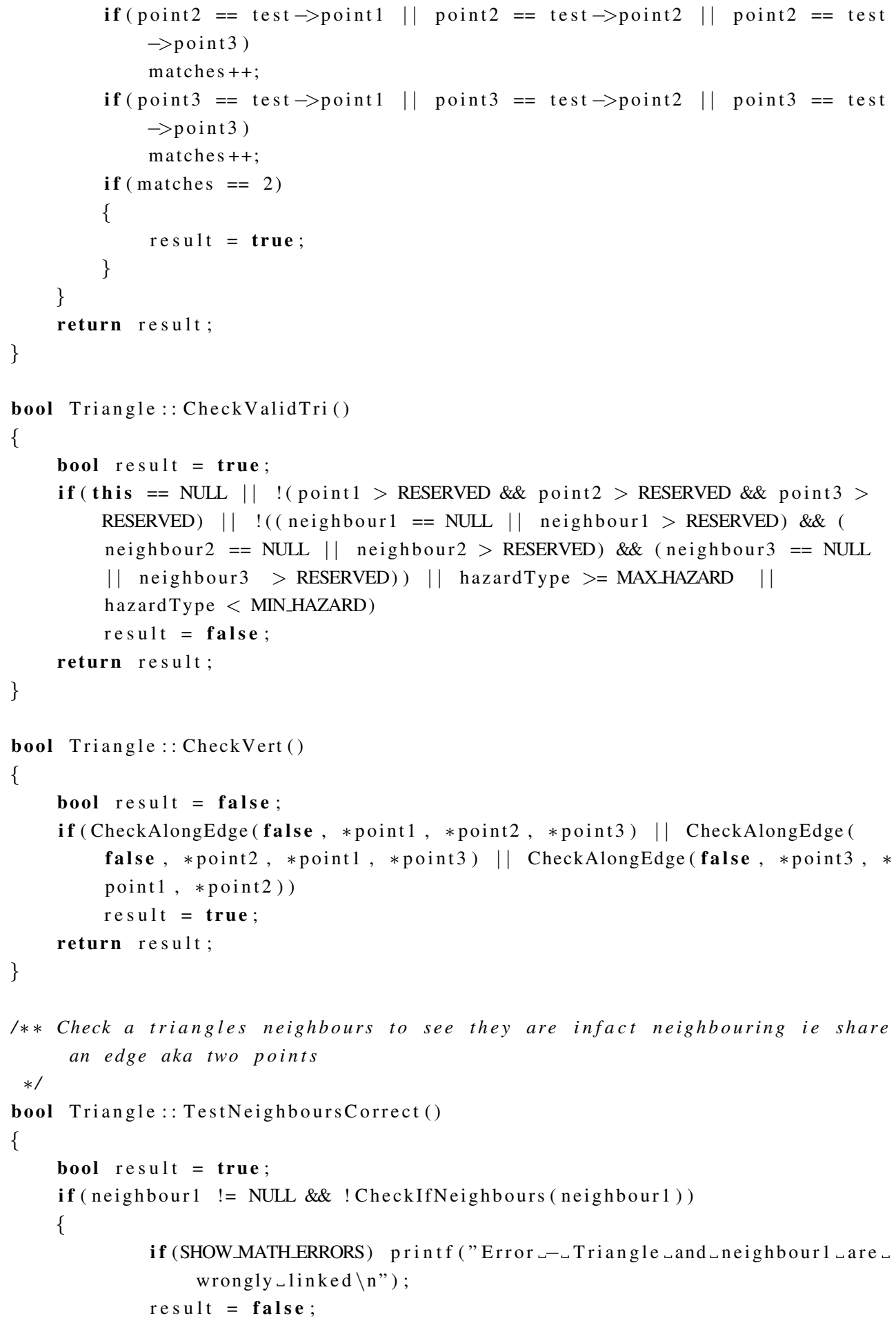




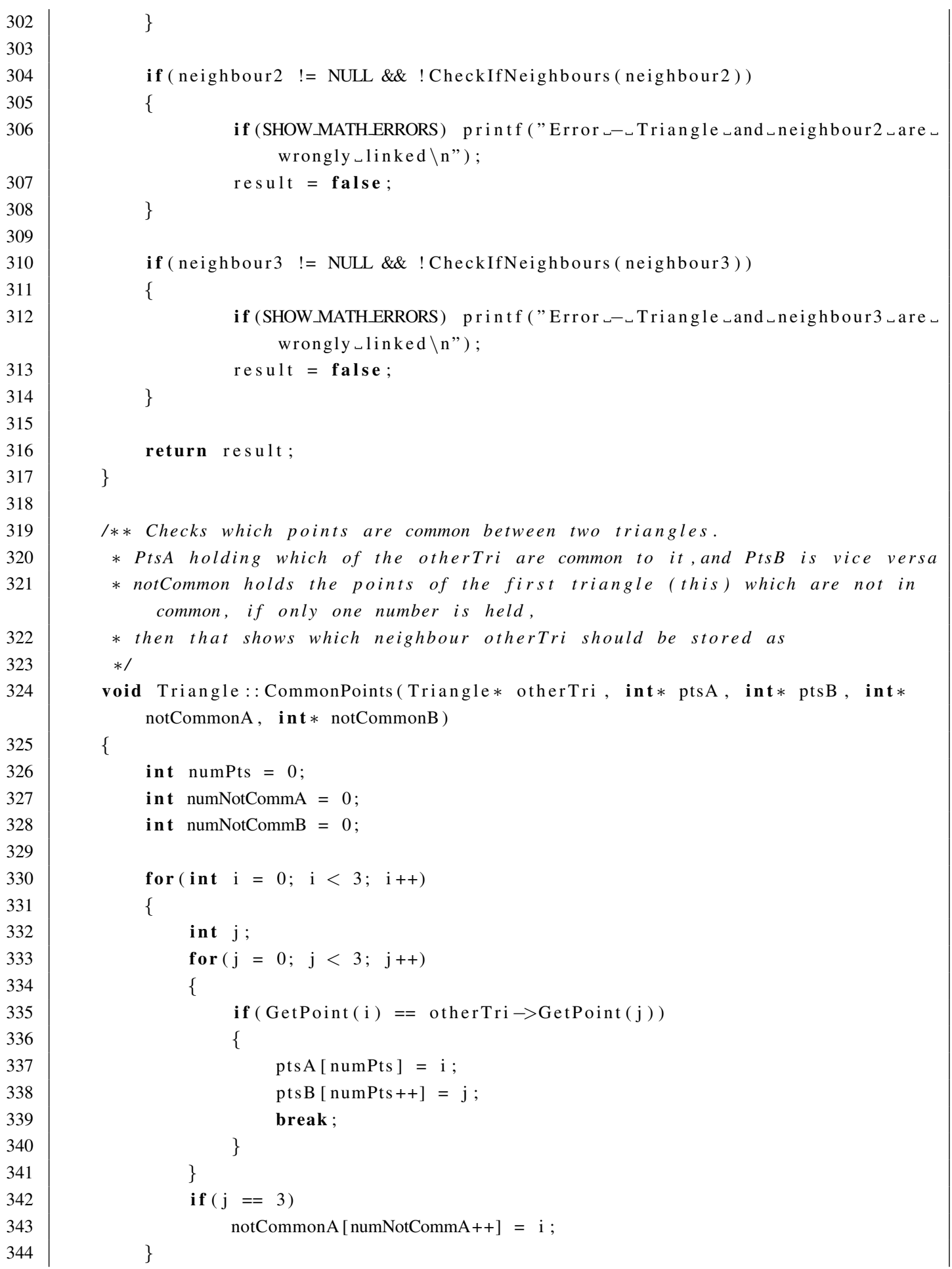




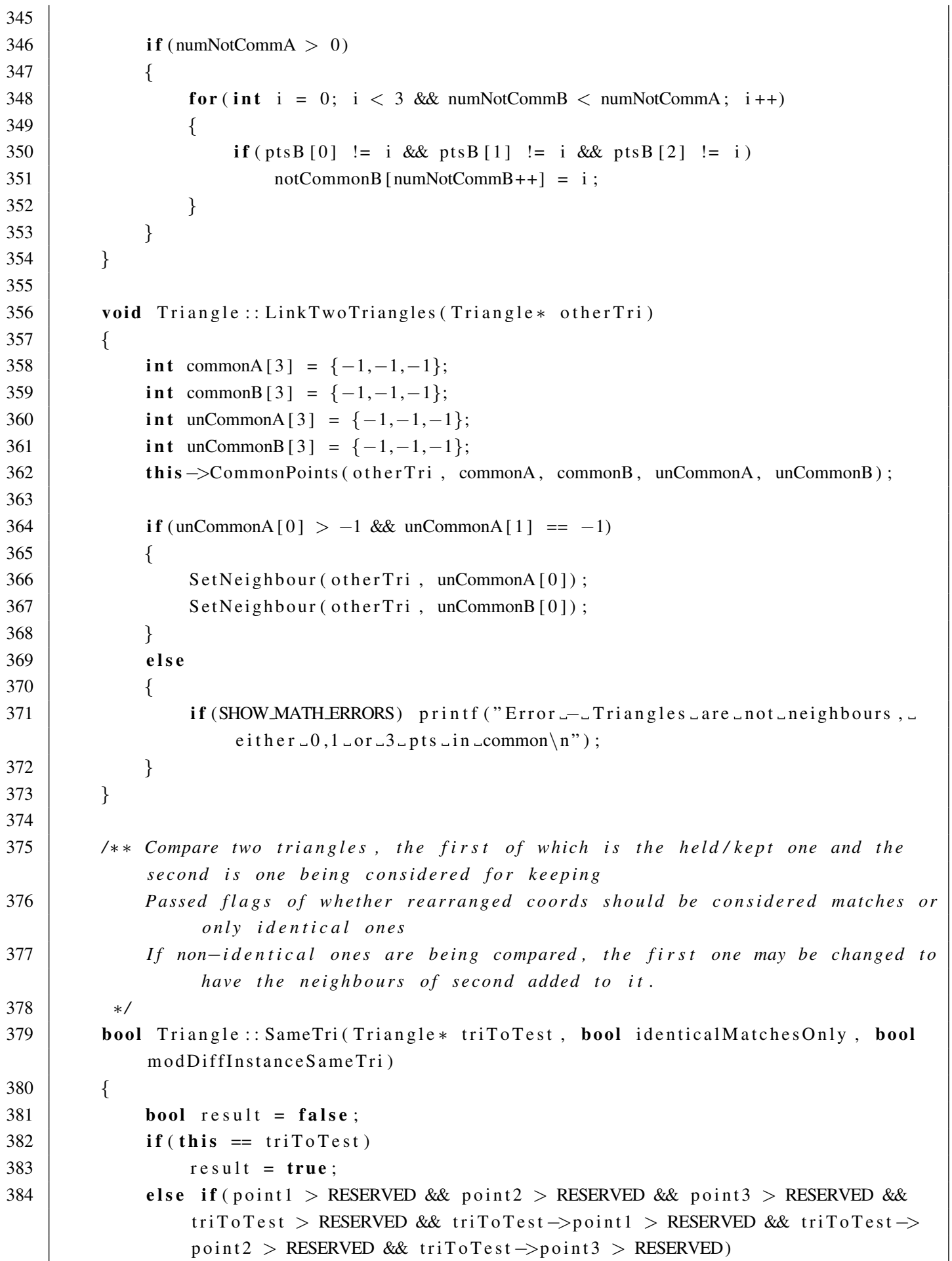


if ( unCommonA [0] $>-1 \& \&$ unCommonA [1] $==-1 \& \&$ SameTriSameNeighbourCheck(triToTest, triToTest $\rightarrow$ neighbor ( unCommonA [0])) ) result $=$ true

\}

return result ;

\}

I** Check if neighbours point to same tris, are same tris or if not, add unheld $t r i$

To be true, triNeighbourToTest must be the same as calling tri (this) and triToTest and this must be linked accordingly

Will there be problems of recursive calls or unneeded processing, which could be avoided buy not doing SametriNeighbour check on neighbours of neighbours etc?

$* /$

bool Triangle : SameTriSameNeighbourCheck (Triangle*triToTest, Triangle* triNeighbourToTest)

bool result = true ;

if (! ( triNeighbourToTest <= RESERVED || triNeighbourToTest $\rightarrow$ checked) \&\& ! checked )

\{

int commonA $[3]=\{-1,-1,-1\}$, commonB $[3]=\{-1,-1,-1\}$;

int unCommonA $[3]=\{-1,-1,-1\}$, unCommonB $[3]=\{-1,-1,-1\}$;

this $\rightarrow$ CommonPoints ( triNeighbourToTest, commonA, commonB, unCommonA, unCommonB ) ;

if ( unCommonA [0] $>-1 \& \&$ unCommonA [1] $==-1)$

\{

if $($ ! ( triNeighbourToTest == neighbor (unCommonA [0]) $\mid$ (neighbor ( unCommonA [0]) > RESERVED \&\& ! neighbor (unCommonA [0]) $\rightarrow$ checked $\& \&$ triNeighbourToTest $\rightarrow$ SameTri(neighbor (unCommonA [0]), false , true ) $\& \&$ triNeighbourToTest $\rightarrow$ SameTriNeighboursCheck (neighbor $($ unCommonA [0])))) )

\{

SetNeighbour (triNeighbourToTest, unCommonA [0]);

if (triNeighbourToTest $\rightarrow$ neighbour $1 !=$ triToTest $\& \&$

triNeighbourToTest $\rightarrow$ neighbour2 $!=$ triToTest $\& \&$

triNeighbourToTest $\rightarrow$ neighbour3 $!=$ triToTest)

if (SHOWMATHERRORS) printf ("Error-七triNeighbourToTest

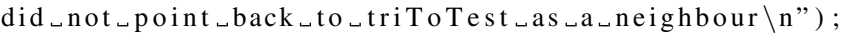

triNeighbourToTest $\rightarrow$ SetNeighbour (this, unCommonB [0]);

\} \} 


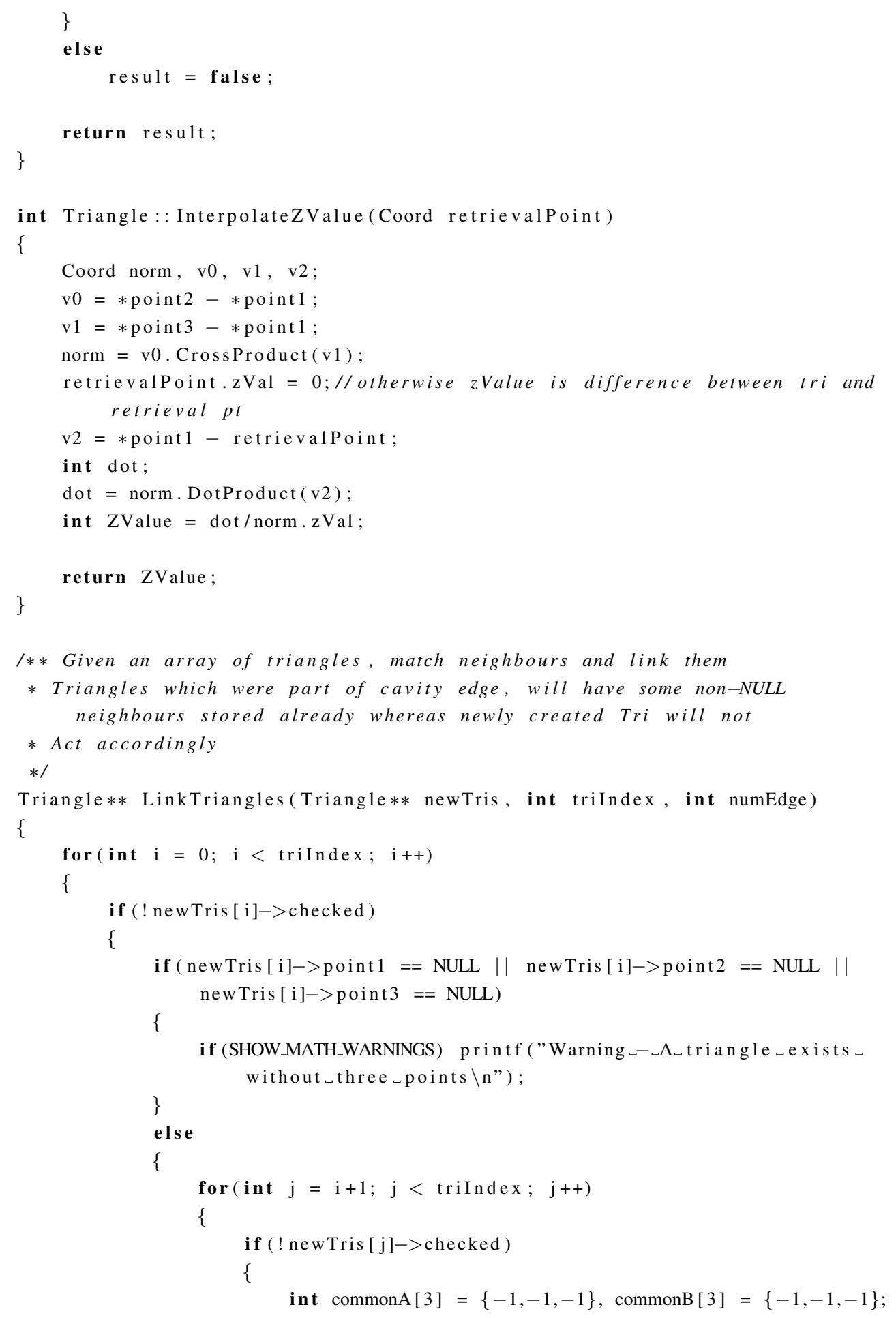


int unCommonA [3] $=\{-1,-1,-1\}$, unCommonB [3] $=$ $\{-1,-1,-1\}$ new Tris [i] $->$ CommonPoints (new Tris [j] , commonA, commonB , unCommonA, unCommonB);

if (unCommonA [0] $>-1 \& \&$ unCommonA [1] $==-1)$ \{

newTris [i] $->$ SetNeighbour (newTris [j], unCommonA $[0])$;

newTris $[j]->$ SetNeighbour (newTris [i ], unCommonB $[0])$;

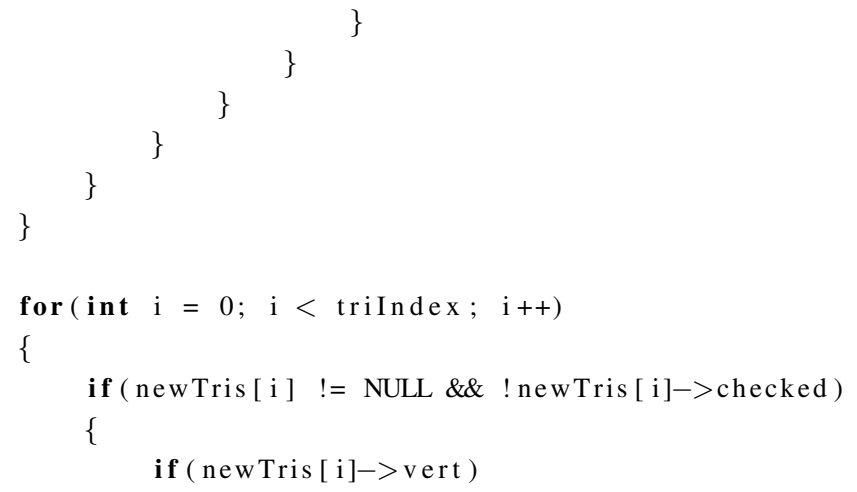


newTris [i] $->$ neighbour3 $\rightarrow$ checked) )

if (SHOWMATHERRORS) printf ("Error $\_-\_$After $\lrcorner$linking, , one $\lrcorner$non-

Vert॰triangle in $_{\llcorner}$newTris $\_$did $\_$not $\_$have $\_$neighbours $\backslash$ n”) ;

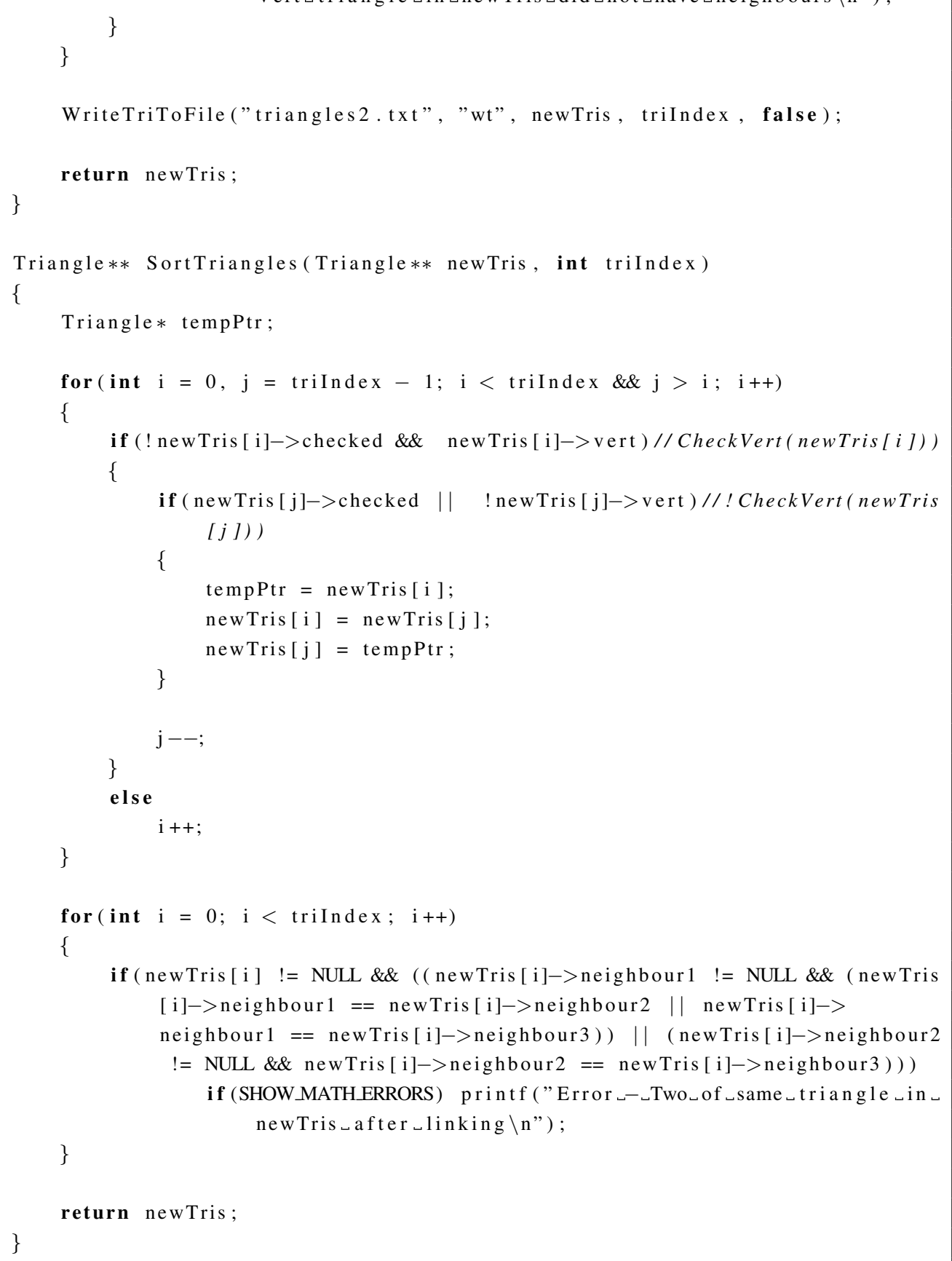




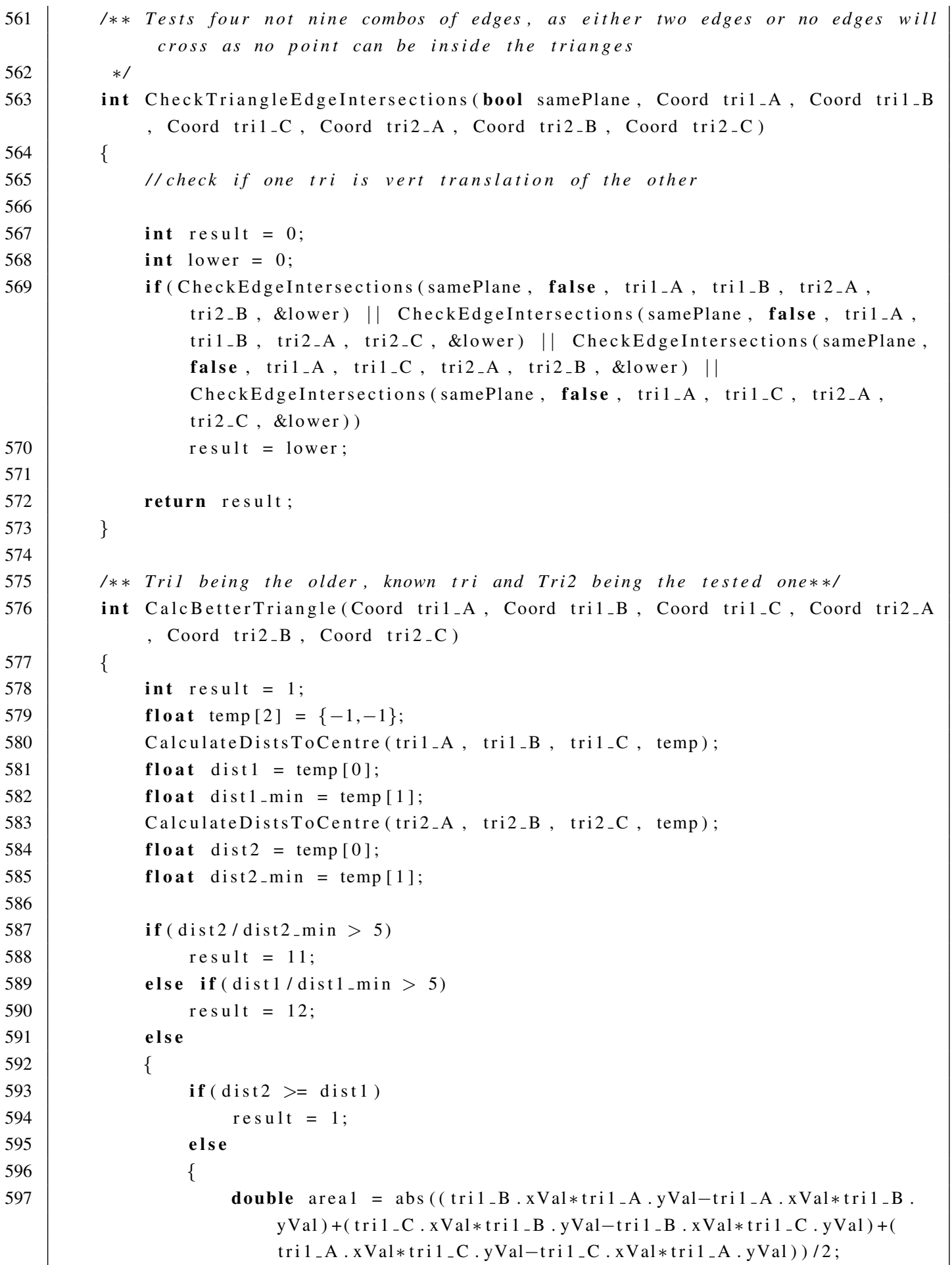




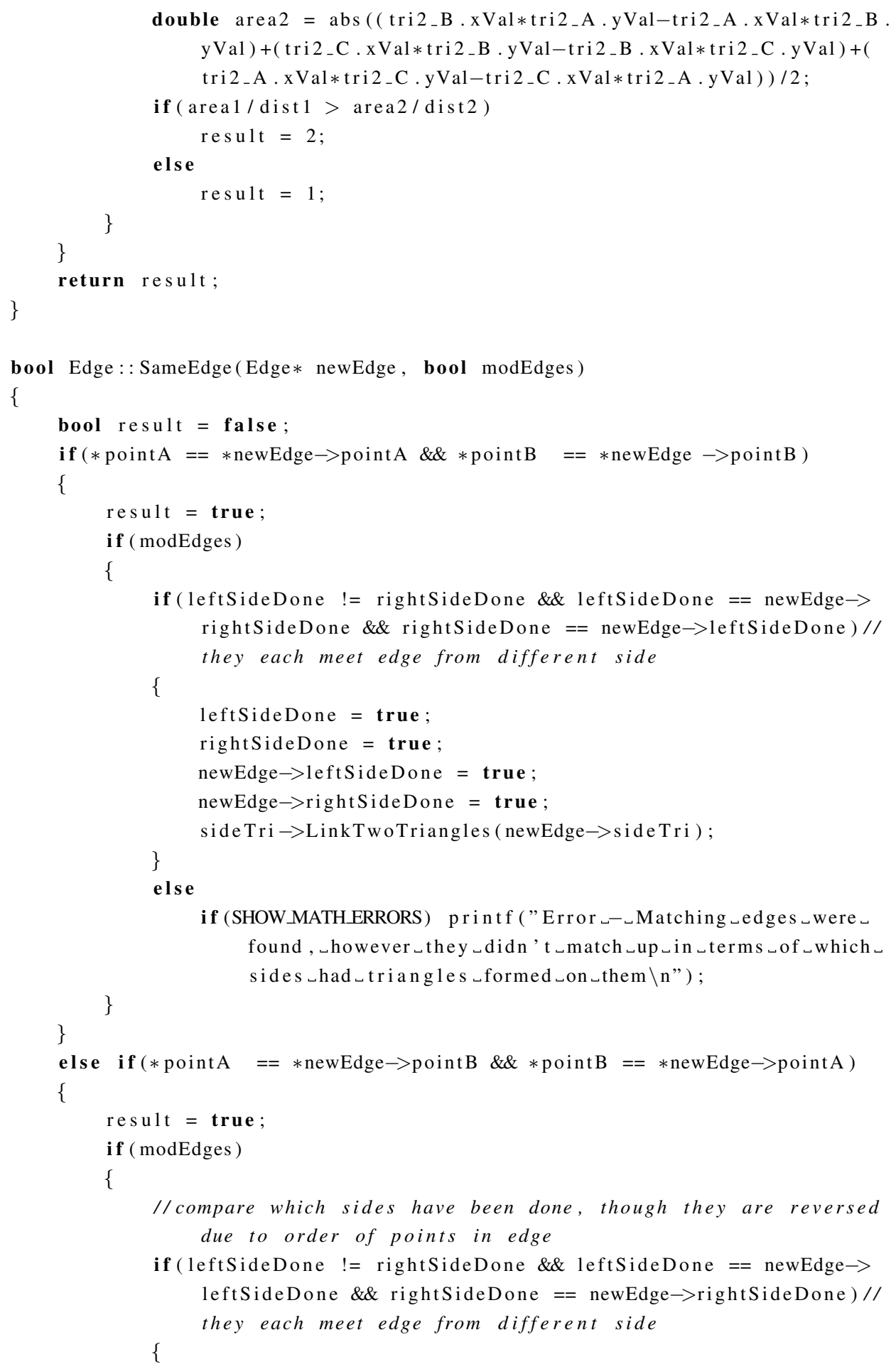




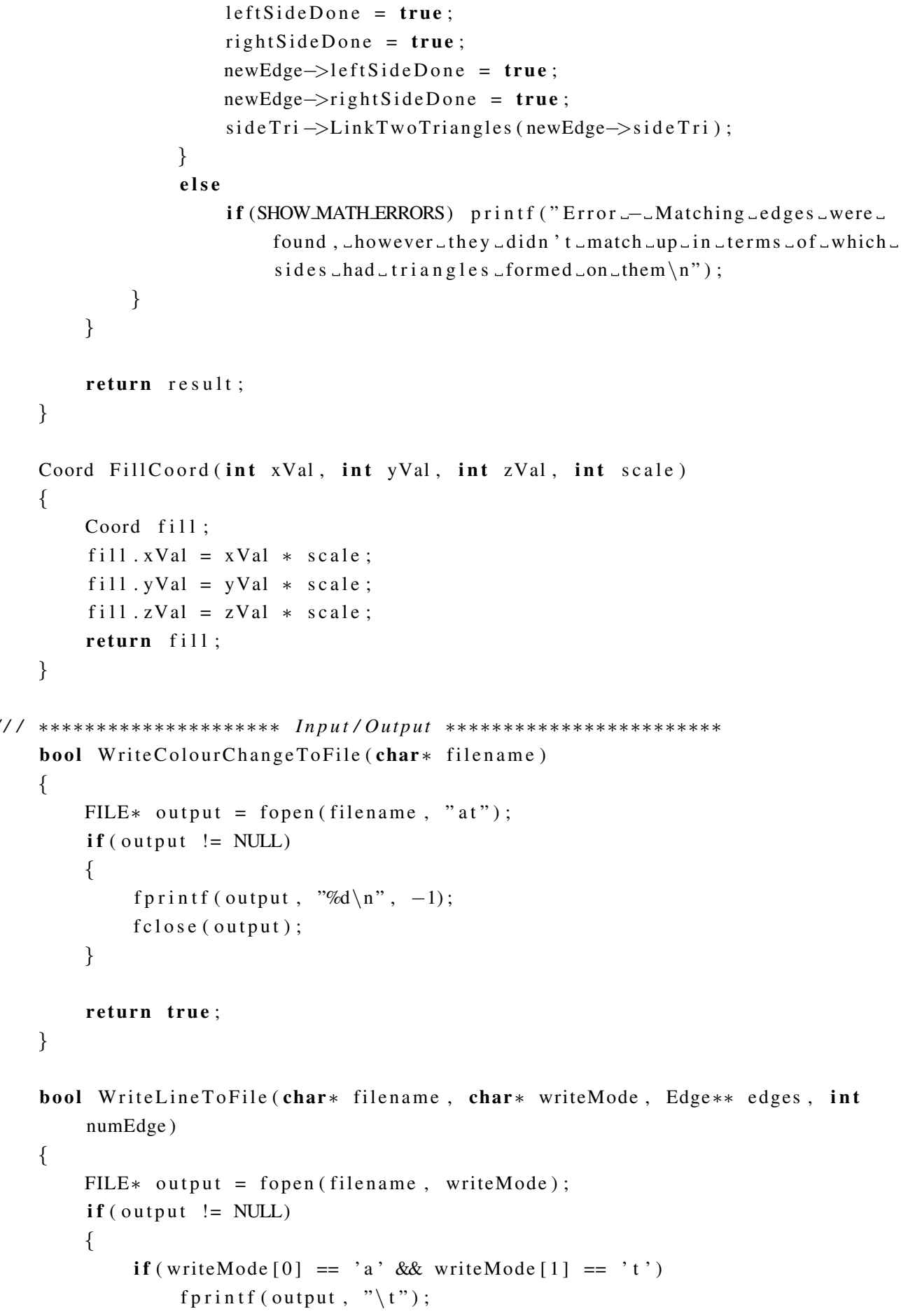




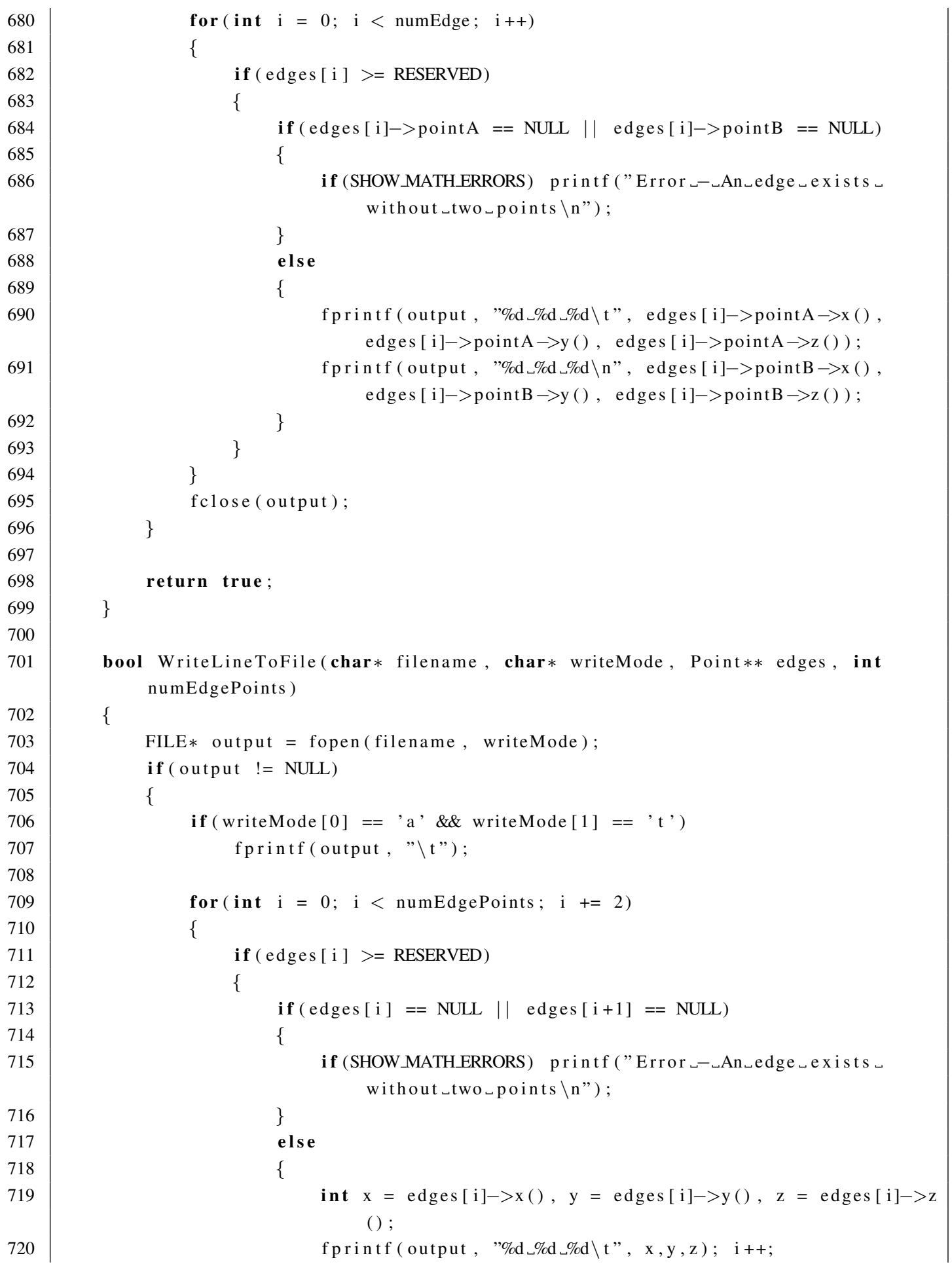




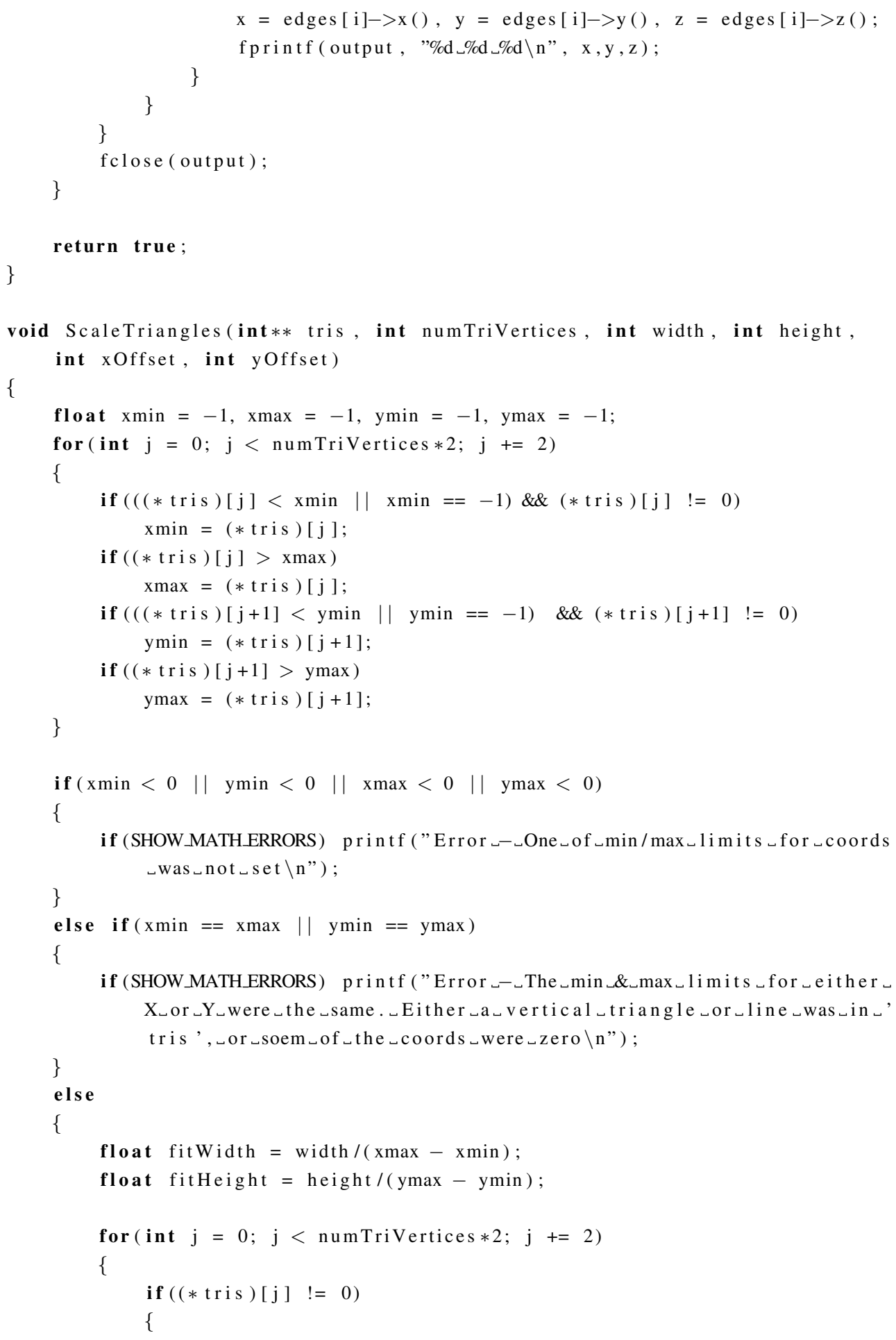


(*tris $)[\mathrm{j}]-=x \min$;

$(* \operatorname{tris})[\mathrm{j}]=(* \operatorname{tris})[\mathrm{j}] * \mathrm{fitWidth}+\mathrm{xOffset}$;

\}

if $((* \mathrm{tr}$ is $)[\mathrm{j}+1] \quad !=0)$

\{

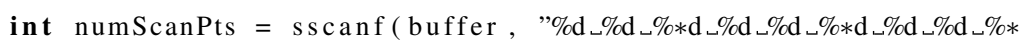
d" , \&tris $[i], \& \operatorname{tris}[i+1], \& \operatorname{tris}[i+2], \& \operatorname{tris}[i+3], \& \operatorname{tris}[\mathrm{i}$ 


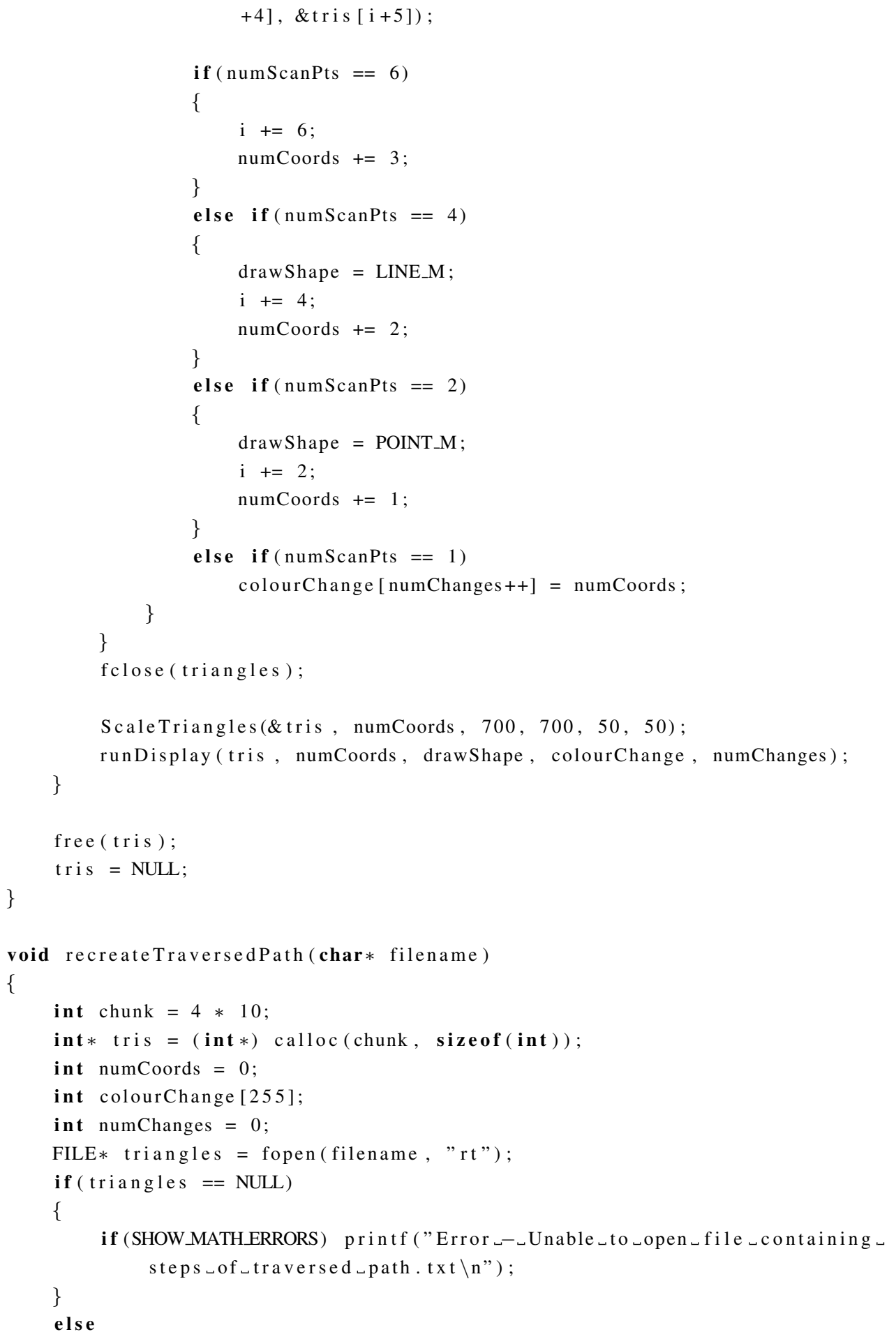




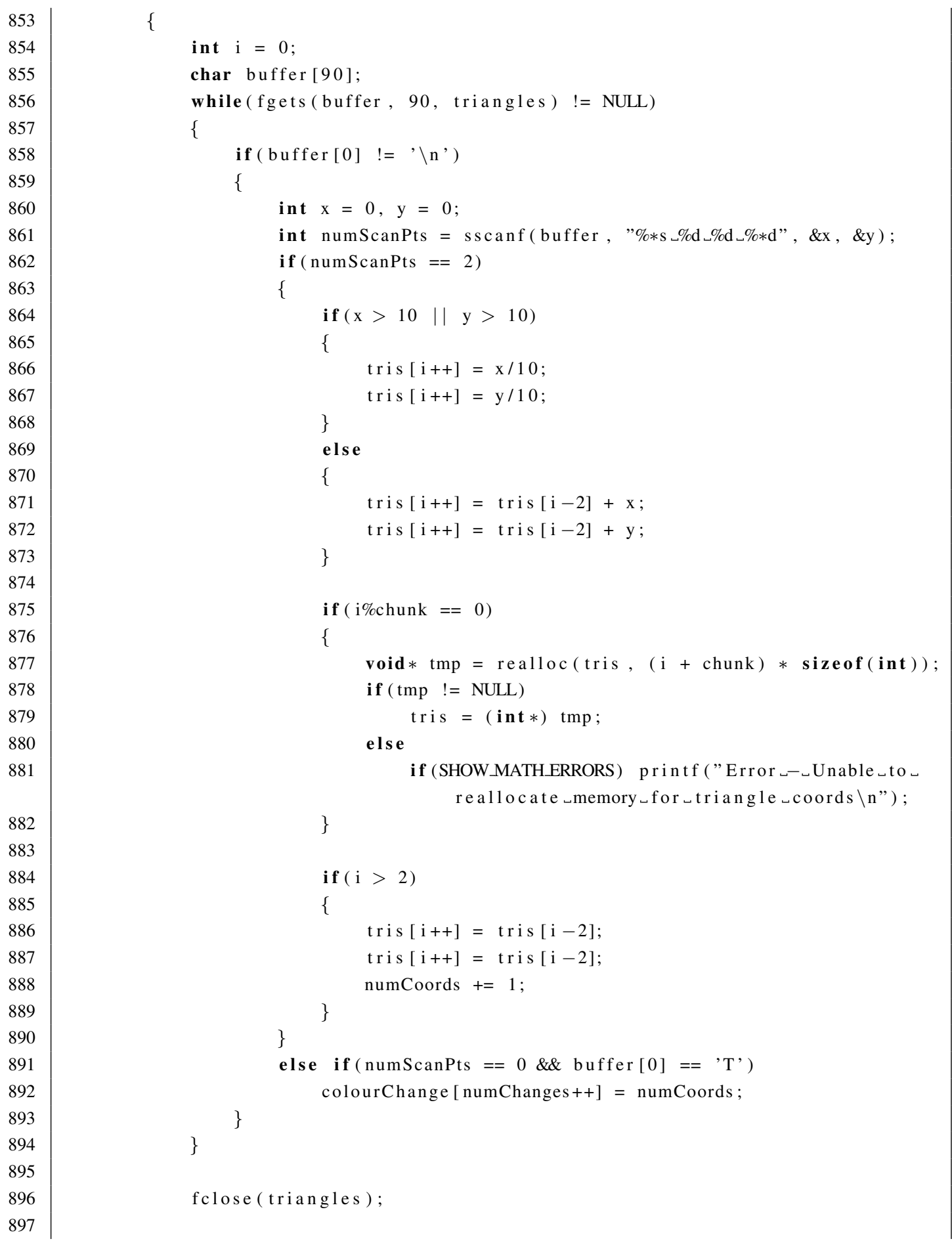


ScaleTriangles(\&tris , numCoords, 700, 700, 50, 50);//scaled a lot of values to zero

runDisplay ( $\mathrm{tr}$ is , numCoords, LINE_M, colourChange, numChanges);//make handle multiple colour changes? \}

free ( tris);

$\mathrm{tr}$ is = NULL;

\}

void displayTopAndSide (char $*$ filename)

\{

int $* \operatorname{tr}$ is $=($ int $*) \quad \operatorname{calloc}(6 * 10$, sizeof $($ int $))$;

int $* \operatorname{tris} 2=($ int $*) \operatorname{calloc}(6 * 10, \operatorname{sizeof}($ int $))$;

int $* \operatorname{tr}$ is $3=($ int $*) \quad \operatorname{calloc}(6 * 10, \operatorname{sizeof}($ int $))$;

int $* \operatorname{tr}$ is $4=($ int $*) \operatorname{calloc}(6 * 10, \operatorname{sizeof}($ int $))$;

int numTri $=0$;

FILE* triangles = fopen (filename, "rt");

if ( $\mathrm{t}$ riangle $\mathrm{s}==$ NULL)

\{

if (SHOW_MATHERRORS) printf ("Error $\_-\_$Unable to $_{\llcorner}$open $\_$triangles $2 . \mathrm{txt} \backslash \mathrm{n}$ " ) ;

exit (1); // terminate with error

\}

int $\mathrm{i}=0$;

char buffer [90];

bool scanfine = true;

fgets (buffer, 90, triangles); //get rid of first line

while (fgets (buffer, 90, triangles) $!=$ NULL)

\{

if ( buffer [0] $\left.!={ }^{\prime} \backslash \mathrm{n}^{\prime}\right)$

\{

if $\left(\mathrm{i} \% 60==\begin{array}{llll}0 & \& \& \text { i } \quad !=0)\end{array}\right.$

\{

void $* \operatorname{tmp}=\operatorname{realloc}(\mathrm{tris},(\mathrm{i}+6 * 10) * \operatorname{sizeof}($ int $))$;

$\operatorname{void} * \operatorname{tmp} 2=\operatorname{realloc}(\operatorname{tris} 2,(i+6 * 10) * \operatorname{sizeof}($ int $))$;

$\operatorname{void} * \operatorname{tmp} 3=\operatorname{realloc}(\operatorname{tris} 3,(i+6 * 10) * \operatorname{sizeof}($ int $))$;

$\operatorname{void} * \operatorname{tmp} 4=\operatorname{realloc}(\operatorname{tris} 4,(\mathrm{i}+6 * 10) * \operatorname{sizeof}($ int $))$;

if $(\mathrm{tmp} \quad !=$ NULL \&\& tmp $\quad !=$ NULL \&\& tmp3 $!=$ NULL \&\& tmp4 != NULL)

\{

$\operatorname{tris}=($ int $*)$ tmp;

$\operatorname{tr}$ is $2=($ int $*) \operatorname{tmp} 2$;

$\operatorname{tris} 3=($ int $*) \operatorname{tmp} 3$;

$\operatorname{tris} 4=($ int $*) \operatorname{tmp} 4$; 
else

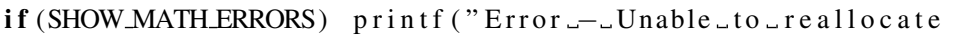
_memory _for $\_$triangle _coords $\backslash$ n”) ;

\}

if ( sscanf ( buffer, $\%$ d $\left.\left.\left.\left.\left.\left.\lrcorner \% \mathrm{~d}_{\lrcorner} \% * \mathrm{~d}\right\lrcorner \% \mathrm{~d}\right\lrcorner \% \mathrm{~d}\right\lrcorner \% * \mathrm{~d}\right\lrcorner \% \mathrm{~d}\right\lrcorner \% \mathrm{~d}\right\lrcorner \% * \mathrm{~d} ", \quad \&$ tris [ i ] , \& $\operatorname{tris}[i+1], \& \operatorname{tris}[i+2], \& \operatorname{tris}[i+3], \& \operatorname{tris}[i+4], \& \operatorname{tris}[i+5])==$ 6)

\{

$\operatorname{tris}[i]+=200$;

tris $[\mathrm{i}+2]+=200$;

$\operatorname{tris}[i+4]+=200$;

\}

else

scanFine $=$ false

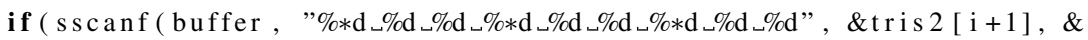
$\operatorname{tris} 2[i], \& \operatorname{tris} 2[i+3], \& \operatorname{tris} 2[i+2], \& \operatorname{tris} 2[i+5], \& \operatorname{tris} 2[i+4])$ $==6$ )

\{

$\operatorname{tris} 3[i]=\operatorname{tris} 2[i]+600$;

$\operatorname{tris} 3[i+1]=\operatorname{tris} 2[i+1]$

$\operatorname{tris} 3[i+2]=\operatorname{tris} 2[i+2]+600$;

$\operatorname{tris} 3[i+3]=\operatorname{tris} 2[i+3]$;

$\operatorname{tris} 3[i+4]=\operatorname{tris} 2[i+4]+600$;

$\operatorname{tris} 3[i+5]=\operatorname{tris} 2[i+5]$

$\operatorname{tris} 2[i]=200-\operatorname{tris} 2[i]$;

tris $2[i+2]=200-\operatorname{tris} 2[i+2]$;

$\operatorname{tris} 2[i+4]=200-\operatorname{tris} 2[i+4]$;

else

scanFine = false

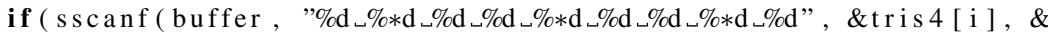
tris $4[i+1], \& \operatorname{tris} 4[i+2], \& \operatorname{tris} 4[i+3], \& \operatorname{tris} 4[i+4], \& \operatorname{tris} 4[i$

$+5])=6$

\{

$\operatorname{tris} 4[\mathrm{i}]+=200$

$\operatorname{tris} 4[\mathrm{i}+1]+=520$;

$\operatorname{tris} 4[i+2]+=200$;

$\operatorname{tris} 4[i+3]+=520$;

$\operatorname{tris} 4[i+4]+=200$;

$\operatorname{tris} 4[\mathrm{i}+5]+=520$;

else

scanFine = false 


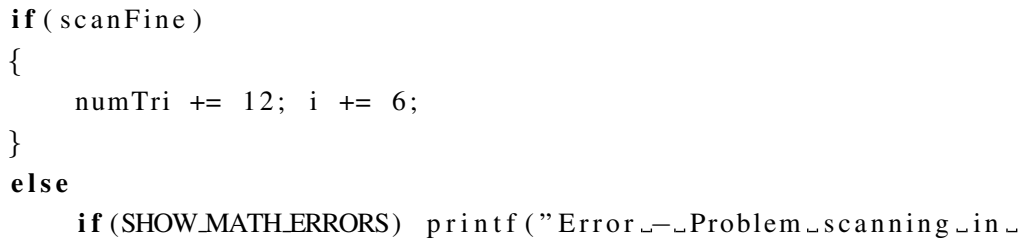




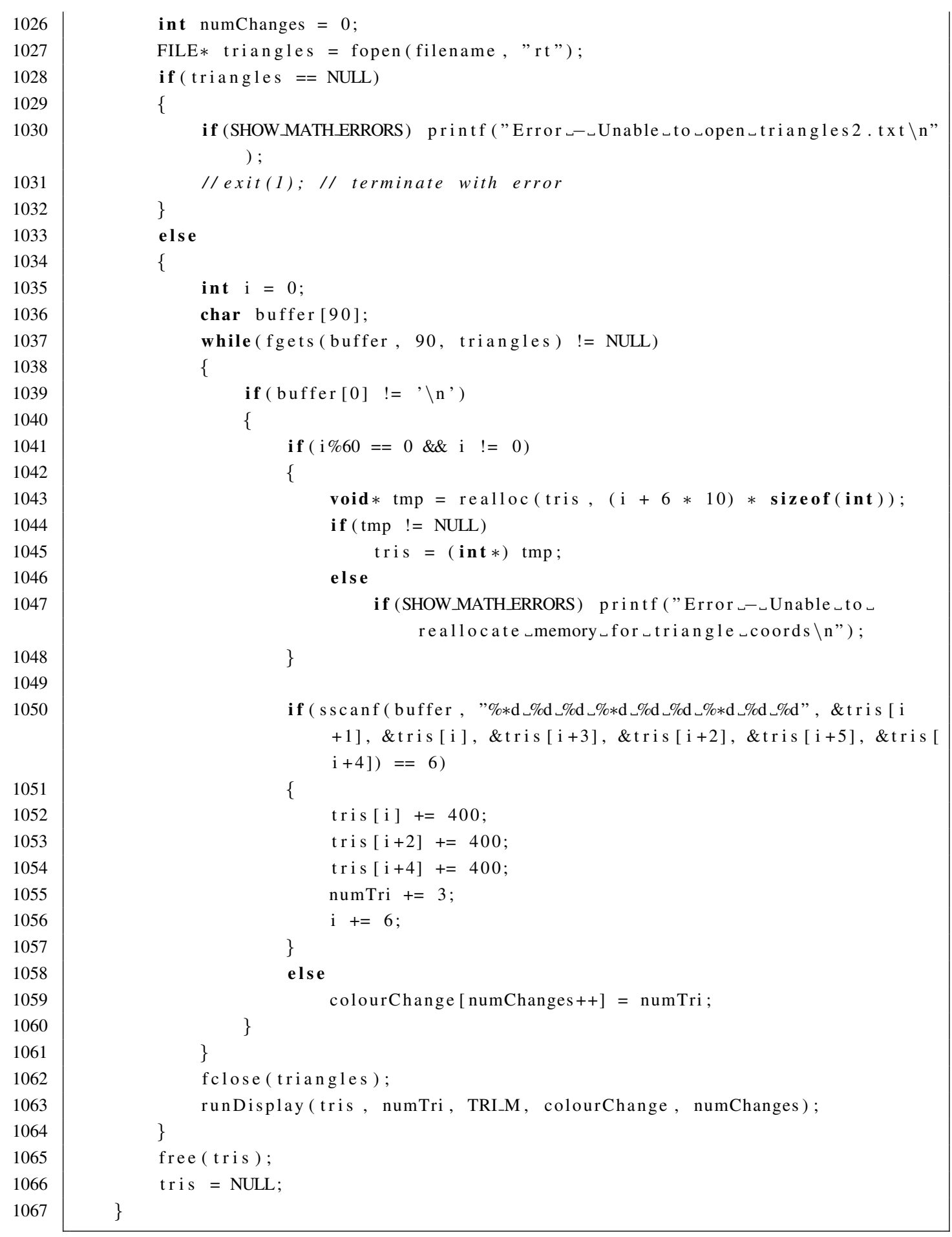




\section{Appendix B}

\section{Simulation Results}

\section{B.1 Statistical Data}

The statistical data and graphs are provided in spreadsheet form in the Results file of either .xls or .ods extension. 